\title{
Climatology and Forest Phenology during 1984-2013 around Western Lake Superior, USA.
}

\author{
By \\ Matthew Garcia \\ A dissertation submitted in partial fulfillment of \\ the requirements for the degree of \\ Doctor of Philosophy \\ (Forestry) \\ at the \\ UNIVERSITY OF WISCONSIN-MADISON \\ 2018
}

Date of final oral examination: 12/21/2017

The dissertation is approved by the following members of the Final Oral Committee: Philip A. Townsend, Professor, Forest \& Wildlife Ecology

Eric R. Kruger, Professor, Forest \& Wildlife Ecology

Volker Radeloff, Professor, Forest \& Wildlife Ecology

Ankur Desai, Professor, Atmospheric \& Oceanic Sciences

Brian Sturtevant, Research Scientist, USDA Forest Service 


\section{CONTENTS}

Abstract

Acknowledgements

Epigraph

List of Tables

List of Figures

1 Introduction and Background

1.1 Orientation on Land-Atmosphere Interactions and Modeling 3

1.2 Forest Phenology

1.3 Climate Change

1.4 Analytical Approach and Plan of Dissertation

1.4.1 Land-Atmosphere Modeling Example

1.4.2 Study Area, Data Sources, and Model (Pre-) Processing

iii

iv

V

$\mathrm{Vi}$

vii

1.4.3 Phenoclimatological Modeling Framework

1.4.4 Climatological Analysis

1.4.5 Remote Sensing and Phenological Modeling Analysis

2 Land-Atmosphere Modeling Example [Garcia et al., 2014] 13

2.1 Simulation Methods

2.2 Simulation Results

2.2.1 Surface Temperature and Wind Speed

2.2.2 Surface Fluxes and Energy Balance

3 Study Area and Data Sources

3.1 Study Area and Regional Hydroclimate 27

$\begin{array}{ll}\text { 3.2 Weather and Climate Data } & 31\end{array}$

3.3 Landsat TM/ETM+ Scenes $\quad 44$

3.4 Land Cover and Additional Datasets $\quad 50$

4 Model Formulation and Computation $\quad 52$

4.1 Borrowing from Climatology $\quad 52$

4.2 Mean Phenology by Regression $\quad 55$

$\begin{array}{ll}\text { 4.3 Phenological Variability by PLSR } & 60\end{array}$

4.4 Example Model Results for a Single Pixel $\quad 65$

4.5 Computational Implementation $\quad 71$

$\begin{array}{ll}\text { 4.5.1 Programming } & 72\end{array}$

$\begin{array}{ll}\text { 4.5.2 Domain Discretization } & 72\end{array}$

$\begin{array}{ll}\text { 4.5.3 Processing Implementation } & 73\end{array}$

5 Regional Climatology [Garcia and Townsend, 2016] 75

5.1 Temperature and Precipitation Indicators and Lake Superior Influences 75

5.2 Cold and Warm Season Indicators and Interseasonal Variability 89

5.3 Teleconnection Influences and a Climatological Regime Shift 97

5.4 Climatological and Growing Season Trends 102

$\begin{array}{ll}\text { 5.5 Seasonal Transitions } & 106\end{array}$

$\begin{array}{ll}\text { 5.6 Lake Effects } & 108\end{array}$

5.7 Summary and Discussion 109 
6 Mean Phenology and Interannual Variability 111

6.1 Results at the Footprint Scale using NDVI, EVI, and NDII 112

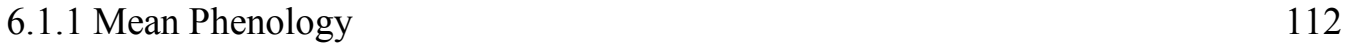

$\begin{array}{ll}\text { 6.1.2 Comparisons with Climatology } & 117\end{array}$

$\begin{array}{lr}\text { 6.1.3 Phenological Variability } & 122\end{array}$

$\begin{array}{ll}\text { 6.1.4 Phenological Prediction } & 131\end{array}$

6.2 Results at the Regional Scale using NDII 134

$\begin{array}{ll}\text { 6.2.1 Mean Phenology } & 134\end{array}$

$\begin{array}{ll}\text { 6.2.2 Mosaic Accuracy } & 142\end{array}$

6.2.3 Spatial Patterns and Lake Effects $\quad 145$

$\begin{array}{lr}\text { 6.2.4 Comparisons with Climatology } & 150\end{array}$

$\begin{array}{ll}\text { 6.2.5 Overall Variability } & 160\end{array}$

$\begin{array}{ll}\text { 6.2.6 Phenological Prediction } & 174\end{array}$

$\begin{array}{ll}\text { 6.3 Summary and Discussion } & 178\end{array}$

$\begin{array}{ll}\text { 6.3.1 Phenological Variability in Spring } & 181\end{array}$

$\begin{array}{ll}\text { 6.3.2 Phenological Variability in Autumn } & 183\end{array}$

7 Discussion and Conclusions $\quad 185$

$\begin{array}{ll}7.1 \text { Summary } & 185\end{array}$

$\begin{array}{ll}7.2 \text { Conclusions } & 187\end{array}$

$\begin{array}{ll}7.3 \text { Model Improvements } & 190\end{array}$

$\begin{array}{ll}7.4 \text { Applications } & 193\end{array}$

$\begin{array}{ll}\text { References } & 198\end{array}$

$\begin{array}{ll}\text { Appendix } & 239\end{array}$ 


\begin{abstract}
Landsat has a history of use in the diagnosis of land surface phenology, vegetation disturbance, and their impacts on numerous forest biological processes. Studies have connected remote sensing-based phenology to surface climatological patterns, often using average temperatures and derived growing degree day accumulations. I present a detailed examination of remotely sensed forest phenology in the region of western Lake Superior, USA, based on a comprehensive climatological assessment and 1984-2013 Landsat imagery. I use this climatology to explain both the mean annual land surface phenological cycle and its interannual variability in temperate mixed forests. I assess long-term climatological means, trends, and interannual variability for the study period using available weather station data, focusing on numerous basic and derived climate indicators: seasonal and annual temperature and precipitation, the traditionally defined frost-free growing season, and a newly defined metric of the climatological growing season: the warm-season plateau in accumulated chilling days. Results indicate $+0.56^{\circ} \mathrm{C}$ regional warming during the 30 -year study period, with cooler springs $\left(-1.26^{\circ} \mathrm{C}\right)$ and significant autumn warming $\left(+1.54^{\circ} \mathrm{C}\right)$. The duration of the climatological growing season has increased +0.27 days/y, extending primarily into autumn. Summer precipitation in my study area declined by an average $-0.34 \mathrm{~cm} / \mathrm{y}$, potentially leading to moisture stress that can impair vegetation carbon uptake rates and can render the forest more vulnerable to disturbance. Many changes in temperature, precipitation, and climatological growing season are most prominent in locations where Lake Superior exerts a strong hydroclimatological influence, especially the Minnesota shoreline and in forest areas downwind (southeast) of the lake. I then develop and demonstrate a novel phenoclimatological modeling method, applied over five Landsat footprints across my study area, that combines (1) diagnosis of the mean phenological cycle from remote sensing observations with (2) a partial-least-squares regression (PLSR) approach to modeling vegetation index residuals using these numerous meteorological and climatological observations. While the mean phenology often used to inform land surface models in meteorological and climate modeling systems may explain $50-70 \%$ of the observed phenological variability, this mixed modeling approach can explain more than $90 \%$ of the variability in phenological observations based on long-term Landsat records for this region.
\end{abstract}




\section{ACKNOWLEDGEMENTS}

Numerous sources of funding supported this work over the course of my dissertation program:

- the USDA Forest Service-Northern Research Station;

- USDA NIFA McIntire-Stennis funding to the University of Wisconsin-Madison (project WIS01621);

- the UW-Madison College of Agricultural and Life Sciences;

- the UW-Madison Department of Forest \& Wildlife Ecology;

- NASA Biodiversity and Ecological Forecasting Project NNX14AC36G;

- the author's 2015-2016 Wisconsin Space Grant Consortium Graduate Research Fellowship.

This work employed the computing resources and assistance of the UW-Madison Center for High Throughput Computing (CHTC) in the Department of Computer Sciences. I especially thank the CHTC Research Computing Facilitators, Christina Koch and Lauren Michael, for their guidance and support. The CHTC is supported by UW-Madison, the Advanced Computing Initiative, the Wisconsin Alumni Research Foundation, the Wisconsin Institutes for Discovery, and the National Science Foundation. The CHTC is an active member of the Open Science Grid (OSG), which is supported by the National Science Foundation and the US Department of Energy's Office of Science. This work represents more than 13 million hours of computing time, with approximately 5 million hours on UW-Madison CHTC systems, 2.5 million hours on affiliated UW-Madison computing resources, and more than 5.5 million hours on additional OSG hosts across the United States. 
But there is a limit to thinking about even a small piece of something monumental. You still see the shadow of the whole rearing up behind you, and you become lost in your thoughts in part from the panic of realizing the size of that imagined leviathan. I had to leave it there, compartmentalized, until I could write it all down, and seeing it on the page, begin to divine the true meaning. 


\section{LIST OF TABLES}

2.1 Noah LSM parameters for the simulation area land cover categories in Fig. 2.2. 16

2.2 Differences between experiment and control scenarios. 20

3.1 Temperature-based meteorological indicators calculated on a daily basis. 36

3.2 Temperature-based meteorological indicators calculated on a seasonal basis. 37

3.3 Precipitation-based meteorological indicators calculated on a daily basis. 37

3.4 Precipitation-based meteorological indicators calculated on seasonal/annual bases. 38

3.5 Temperature-based cold-, warm-, and growing-season indicators calculated on 39 daily/seasonal bases.

3.6 Landsat scene counts by footprint and sensor.

4.1 Mean-curve-based phenological indicators as illustrated in Fig. 4.2. 59

4.2 Model goodness-of-fit results for three VIs at a single pixel in P26R27. 71

5.1 Climatological indicator statistics for the study period 1984-2013. 77

5.2 Study area summarized significant teleconnection effects through the 1984-2013 98 study period.

5.3 Climatological indicator statistics for the periods 1984-1998 and 1999-2013. 100

6.1 Accuracy measures for three VIs averaged over footprint P26R27. 114

6.2 Statistics for comparison of VIs for the phenological indicators in Figs. 6.2 and 6.3. 121

6.3 Accuracy measures in areas of footprint overlaps for the NDII-based mean 148 phenological curve.

6.4 Minimum number of climatological indicators required to reach a threshold of 50\% 173 of the phenological variability explained by the PLSR model in each footprint.

$\begin{array}{ll}\text { A1 Collected P25R28 USGS Landsat scene descriptors. } & 239 \\ \text { A2 Collected P26R27 USGS Landsat scene descriptors. } & 240\end{array}$

A3 Collected P26R28 USGS Landsat scene descriptors.

A4 Collected P27R27 USGS Landsat scene descriptors. 242

A5 Collected P27R28 USGS Landsat scene descriptors. 243 


\section{LIST OF FIGURES}

2.1 The four innermost grids in the WRF simulation domain. $\quad 14$

2.2 Land cover classifications for the finest WRF simulation grid. $\quad 15$

2.3 Time series of surface temperature and near-surface wind speeds. $\quad 21$

2.4 Time series of $S H$ and $L H$ differences between control and experiment scenarios. 22

2.5 Mapped differences of surface SH difference between experiment and control 23 scenarios.

2.6 Energetic balance at the land surface for the three-day simulation period. 24

3.1 Study area topography with nominal Landsat footprint coverage. 28

3.2 Study area USGS NLCD 2011 land cover with USEPA ecoregion boundaries. 29

3.3 Locations of GHCND meteorological stations in and around the study area. 32

3.4 Meteorological station network interpolation accuracy. 34

3.5 Meteorological station network interpolation error maps. 35

3.6 Climatological processing and analysis procedure. $\quad 41$

3.7 Landsat scene dates, by footprint. 46

3.8 Distribution of collected Landsat scenes by day-of-year. $\quad 47$

3.9 Landsat image processing procedure. $\quad 48$

4.1 Phenological model block/flowchart diagram. 54

4.2 Schematic fitted 7-parameter ABC phenological curve. $\quad 58$

4.3 Illustration of PLSR model-based AIC $c$ vs. the number of model variables retained. 63

4.4 Example phenological model results for a single Landsat pixel. $\quad 66$

4.5 Time series of standardized Tasseled Cap residuals for a single Landsat pixel. 69

4.6 Goodness-of-fit results for the mean phenology fitted curve and the full phenological $\quad 70$ model.

4.7 Data merger and domain discretization process. 73

5.1 1984-2013 mean annual cycle of daily temperatures with climatological indicators. 76

5.2 1984-2013 mean annual cycle of $C D$ and $G D D$ accumulations. $\quad 78$

$\begin{array}{ll}5.31984-2013 \text { seasonal and annual precipitation. } & 79\end{array}$

$\begin{array}{ll}5.4 \text { 1984-2013 mean annual cycle of daily precipitation. } & 79\end{array}$

5.5 1984-2013 mean annual $T_{\min }, T_{\max }$, and $T_{\text {avg }}$ with trends. $\quad 80$

$\begin{array}{ll}5.61984-2013 \text { mean seasonal } T_{\min } \text { with trends. } & 82-83\end{array}$

5.7 1984-2013 mean seasonal $T_{\max }$ with trends. $\quad$ 84-85

$\begin{array}{lr}5.8 \text { 1984-2013 mean seasonal } T_{\text {avg }} \text { with trends. } & 86-87\end{array}$

5.9 1984-2013 mean annual $P$ with trends. $\quad 88$

5.10 1984-2013 mean seasonal $P$ with trends. $\quad 90-91$

5.11 1984-2013 mean derived cold season indicators with trends. 92

5.12 1984-2013 mean frost-based growing season start, end, and duration with trends. 94

5.13 1984-2013 mean $C D$-based growing season start, end, and duration with trends. 95

5.14 1984-2013 mean $C D$-based growing season indicators with trends. 96

5.15 1984-2013 intra- and interseasonal study area $T_{\text {avg }}$ and $P$ correlations. 97

5.16 1984-1998 and 1998-2013 trends in selected climatological indicators. 101

5.17 1984-1998 and 1999-2013 trends in mean derived cold season indicators. 102

5.18 1984-1998 and 1999-2013 trends in mean derived growing season indicators. 103

5.19 1984-1998 and 1999-2013 trends in mean derived warm season indicators. 104 
6.1 P26R27 fitted phenological curve RMSE and $r^{2}$ for 3 VIs.

6.2 P26R27 fitted Spring Inflection $(S I)$ and Autumn Inflection $(A I)$ dates for 3 VIs.

6.3 P26R27 fitted Durations of Spring (DOS) and Maturity $(D O M)$ for 3 VIs.

6.4 P26R27 NDVI-based phenology correlations with climatological mean indicators. and plateau duration.

6.33 Correlations between (a) D[SI-AI] and frost-free season GDD, and (b) D[SI-AI] and 153 plateau $G D D$.

6.34 Correlations between (a) DOS and Spring GDD, and (b) DOS and Spring $P$. plateau start date.

6.37 Correlations between (a) DOM and frost-free season $G D D$, and (b) $D O M$ and plateau $G D D$.

6.38 Correlations between (a) DOM and plateau duration, and (b) DOM and Summer $P$. 
6.47 Mosaic NDII-based phenological correlations with climatological StDev indicators. $\quad 169$

6.48 Mosaic PLSR-based RMSE based on NDII residuals. $\quad 170$

6.49 Mosaic PLSR-based $r^{2}$ based on NDII residuals. $\quad 170$

6.50 P25R28 PLSR overall effective coefficients using NDII. $\quad 171$

6.51 P26R28 PLSR overall effective coefficients using NDII. $\quad 171$

6.52 P27R27 PLSR overall effective coefficients using NDII. $\quad 172$

6.53 P27R28 PLSR overall effective coefficients using NDII. 172

6.54 Correlations between (a) DOS StdErr and Spring CDD StDev, and (b) DOS StdErr 175 and Spring $P$ StDev.

6.55 Correlations between (a) SOM StdErr and Spring CDD StDev, and (b) D[SI-AI] 176 StdErr and annual $P$ StDev.

6.56 Correlations between (a) DOM StdErr and Autumn GDD StDev, and (b) DOM 177 StdErr and Autumn $P$ StDev.

6.57 Mosaic NDII-based full phenological model RMSE. $\quad 178$

6.58 Mosaic NDII-based full phenological model $r^{2}$. 179 


\section{CHAPTER 1}

\section{INTRODUCTION AND BACKGROUND}

Forests cover vast areas of the Earth, serving as a vital natural resource while providing for carbon sequestration [Dixon et al., 1994; Bonan, 2008; Strassman et al., 2008; Pan et al., 2011] and mediating the exchange of energy and moisture between the land surface and atmosphere [Troen and Mahrt, 1986; André et al., 1989]. Temperature, photoperiod, and moisture availability influence the daily balance of forest gross primary productivity (carbon assimilation via photosynthesis) with respiration [Laube et al., 2014a] and determine through phenological phases the overall magnitude of the forest carbon sink over the growing season [Desai, 2010]. Vegetation and forest phenology refer in this work to the seasonal timing of events in a tree's annual physiological cycle, including leaf budburst and growth, senescence, and (for deciduous species) abscission. Phenology also includes flowering and seed production, the generation of annual growth rings in stemwood, winter hardening and spring sap flush, and other less visible processes. Seasonal progression through the phenological cycle is driven by a combination of internal and environmental processes [Morisette et al., 2009] with solar, meteorological, and climatological cues often marking the beginning and end of the vegetation growing season [Jolly et al., 2005; Ceccherini et al., 2014; Hwang et al., 2014; Koster et al., 2014; Xie et al., 2015]. A principal indicator of vegetation phenological phase is leaf area available for photosynthesis, and leaf area index ( $L A I)$ remains a key parameter in land surface representations for meteorological and climate modeling [Dickinson et al., 2008].

Regional and global climate both affect, and are affected by, forest phenology [Peñuelas et al., 2009; Richardson et al., 2013]. Hydroclimatology and its anomalies [Ceccherini et al., 2014; Hwang et al., 2014; Koster et al., 2014] can be used to explain a large fraction of the observed year-to-year variability in annual growing season start, intensity, and duration [Fisher et al., 2007; Marchin et al., 2015], as I will explore in this work. With such sensitivity to environmental conditions, overt signals of the vegetation life cycle such as budburst, flowering, and leaf coloration dates have become highly visible 
indicators of climate change in many locations [Wolkovich and Ettinger, 2014]. It is expected that climate change will lead to altered phenological timing [Saxe et al., 2001; Nemani et al., 2003; Schwartz et al., 2006; Peñuelas et al., 2009]. Changes in the beginning, end, and duration of the vegetation growing season will be among the most obvious effects of climate change due to long-term temperature increases, especially around spring and autumn transitions at the most visible of phenological phases. Given strong correspondence between phenological phases and the seasonal temperature cycle [Cannell and Smith, 2003], climatological analysis is a necessary component of any phenological analysis [ White et al., 1997; Zhang et al., 2004; Fisher et al., 2007; Augspurger, 2013; Ceccherini et al., 2014; Koster et al., 2014; Marchin et al., 2015].

I approach the examination of forest phenology from a combination of climatological and remote sensing perspectives. In particular, I have elected to use surface meteorological observations and scenes from Landsat 5 and 7 covering the period from 1984-2013. Landsat has a long history of multispectral index development for different views on vegetation [Hardisky et al., 1983; Crist and Kauth, 1986; Gao, 1996; Sellers et al., 1996; Huete et al., 1997; Healey et al., 2005; Jin and Sader, 2005a] and their use for phenological analyses [Zhang et al., 2003; Ahl et al., 2006; Fisher and Mustard, 2007; White et al., 2014]. Remote sensing presents a wider view of the land surface, and in any given satellite-observed "forest" pixel there may be several species of trees, undergrowth, bare soil, roads and other development, and any number of additional land components, including open water. The satellite perspective thus provides a view not on the phenology of individual trees, even at Landsat resolution ( $30-\mathrm{m}$ pixels), but on "land surface phenology" [de Beurs and Henebry, 2010] as it is viewed in total over an area. This is especially the case for coarser multispectral remote sensing products: $\sim 8$-km pixels from AVHRR [Buitenwerf et al., 2015; Zhao et al., 2015]; 250-, 500-, and 1000-m pixels from MODIS [Huete et al., 2002; Friedl et al., 2010; Ganguly et al., 2010]; 1-km pixels from SPOT VEGETATION [Ivits et al., 2013]. These products are useful for the construction of high-temporal- but low-spatial-resolution land surface phenological analyses [Duchemin et al., 1999; Jenkins et al., 2002; Soudani et al., 2008; Liu et al., 2016]. Low-temporal-resolution platforms such as Landsat are useful for the development of multi- 
year, high-spatial-resolution phenological analyses [Fisher et al., 2006; Melaas et al., 2013], as I present here. These studies generally gather several years of growing-season images over an area of interest and fit mean phenological curves to those data, with often large residuals appearing in their results [Fisher and Mustard, 2007]. In this work, I exploit those residuals by combining them with climatological data to formalize the overall understanding of the links that may drive phenological variability. This climatologically-informed phenological analysis results in greatly improved predictions of observed, satellite-based land surface phenology with a greater sensitivity to the interannual variability of forest phenological timing and events.

\subsection{Orientation on Land-Atmosphere Interactions and Modeling}

In this work, I present a detailed modeling framework that is oriented on the accurate representation of forest phenological state and its sensitivity to climatological conditions, with the potential to make more realistic the representation of forest areas in such models and thus improve outcomes in forecasting and prediction activities. I am concerned in general with the representation of forest areas in land surface models (LSMs) that characterize land-atmosphere interactions in weather and climate modeling systems. Typically, LSMs in weather and climate modeling systems parameterize complex natural processes for mathematical simplicity, with vegetation biophysical traits simplified to a small number of parameters used in those calculations. However, fixed parameter values based on coarsely aggregated vegetation types yield an effectively stationary land surface boundary, ignoring the variability of these parameters during disturbance and recovery. Modeling outcomes may then deviate significantly from reality in areas where the land cover type does not change but its energetic and hydrological function is different from that bulk, categorical representation [Law et al., 2002]. Remote sensing-based phenology can be incorporated directly into the calculations of the LSMs that are coupled with regional weather and climate modeling systems [Sellers et al., 1996; Bonan et al., 2002; Sterling and Ducharne, 2008] and provide verisimilitude to the modeled vegetation biophysical traits including 
seasonal maxima of albedo, leaf area index (LAI), and stomatal conductance [Dorman and Sellers, 1989; Salvucci and Entekhabi, 2011].

Land surface models often tie several key mechanisms of vegetation function to LAI [Bonan, 1993], which follows well-known phenological patterns in the absence of disturbances [Jolly et al., 2005; Kucharik et al., 2006; Kathuroju et al., 2007; Stöckli et al., 2011]. Model vegetation parameterizations based on LAI may include canopy interception of precipitation [Gash, 1979; Gash et al., 1980], evaporation from the leaf surface [Stewart, 1977; Klaassen, 2001; Blyth and Harding, 2011], and transpiration rates [Federer, 1975; Ziemer, 1979; Herbst et al., 2007]. The atmospheric boundary layer $(\mathrm{ABL})$ is influenced by several key land surface characteristics: albedo (solar reflectance) as a primary control on surface energy balance, roughness as a primary control on land-atmosphere momentum fluxes, and moisture sources as an aspect of land-atmosphere water and energy exchange [Garratt, 1993; Collins and Avissar, 1994; Sun and Bosilovich, 1996; Liu et al., 2004; Santanello et al., 2005]. Heat and moisture exchange between the surface and free atmosphere shapes the stability of the ABL [Troen and Mahrt, 1986] and determines the relative ease by which fluxes through the ABL occur. As part of the landatmosphere system, vegetation exerts biological controls on surface fluxes of energy, moisture, and carbon [Schulze, 1986; Baldocchi et al., 2000; Juang et al., 2007; Katul et al., 2007, 2012; Siqueira et al., 2009]. Those biological controls are functions of plant physiology and morphology that change with vegetation growth phase over individual seasons and the plant's lifetime [Bond, 2000; McMillan and Goulden, 2008; Goulden et al., 2011]. Leaf morphology affects the interception of precipitation [Brubaker et al., 1993], the rates of transpiration and photosynthesis [Bloom et al., 1985; Chapin et al., 1987; Chaves, 1991], and the near-surface radiative balance through solar reflectance and absorption [Sellers et al., 1997; Barnes and Roy, 2008]. Seasonal forest phenology [Deguchi et al., 2006] is thus a primary control on physical interaction of the forest with weather and land surface energetic processes. It remains important to distinguish forest disturbances from seasonal phenology patterns [Reed et al., 1994; Dymond et al., 2002; Fisher et al., 2006] when we wish to assess acute disturbance impacts and their effects on ecosystem function. 
The spatial scale of weather and climate models is important to the proper representation of landatmosphere interactions. Many global models are applied at spatial resolutions that are too coarse to capture the physical mechanisms of these interactions [Dirmeyer et al., 2006; Salmun et al., 2009]. However, as many of these models progress to finer scales in their computing capabilities, and necessarily in their representation of the landscape, bulk parameterization methods are gradually being replaced. In some cases, LSM-based parameter estimation and optimization results [Salvucci and Entekhabi, 2011] are introduced to the model. Often this replacement includes externally specified temporally varying spatial datasets, such as aggregate approximations of the seasonal phenological cycle based on several years of remote sensing data [Tian et al., 2004; White and Nemani, 2006; Kathuroju et al., 2007; Xie et al., 2008]. More recently, dynamical vegetation models (DVMs) explicitly address phenology, seasonal cycles, and some disturbance types [Dickinson et al., 1998, 2008; Arora, 2002]. Even in the absence of disturbances, the annual phenological cycle is not fixed: times of key phenological phases, the duration of the growing season, and the speed and intensity of green-up and senescence can all vary on a year-to-year basis. My work here is intended to support the incorporation of high-resolution phenological variability and, eventually, various forest disturbance types into such model representations of the land surface.

\subsection{Forest Phenology}

At the scale of an individual tree, the phenology involves complex and dynamic interactions among processes that are both driven and constrained by environmental conditions: photosynthesis balances carbon assimilation with moisture loss and nutrient transport; carbon must be allocated among leaf and stem growth, wood production [Delpierre et al., 2016], chemical defenses, and reproductive processes; the tree must protect itself against temperature-related stresses such as freezing during spring and autumn transitions [Kim et al., 2014] and moisture-related stresses in hot and/or dry periods [Arend and Fromm, 2007; Anderegg et al., 2012]. The pace of photosynthesis is acutely sensitive to environmental conditions, especially temperature [Ali et al., 2015], and varies over time with phenophase. A tree must constantly 
adjust its allocation of resources (carbon, nutrients, moisture, sunlight) according to changing biological strategies, subject to biological and abiotic limitations, in a competitive environment.

Forest phenology, and especially the leaf life cycle on woody plants, is broadly representative of the role of forests in the global energy, water, carbon, and nutrient cycles. Green-up, senescence, and forest disturbance represent significant changes in land surface albedo and the surface energy balance, canopy carbon content, and the continuing ability of the forest to assimilate atmospheric carbon and provide atmospheric moisture through transpiration [Czikowsky and Fitzjarrald, 2004; Puma et al., 2013]. The availability of that moisture, in concert with numerous biophysical responses to temperature, determines the partitioning of absorbed energy between evaporation and transpiration [Lawrence et al., 2007] and sensible heating of the forest canopy and understory. Light intensity, temperature, and the availability of water and nutrients all determine the balance between gross primary productivity (via photosynthesis) and respiration [Migliavacca et al., 2015] on sub-daily time scales [Nemani et al., 2003; Richardson et al., 2007a, 2010; Puma et al., 2013; Sims et al., 2014], rendering over time the annual status of the forest as a source or sink of carbon in the global system [Richardson et al., 2012]. The duration of the growing season as an indicator of net primary productivity is a key factor in the magnitude of the forest carbon sink [Nemani et al., 2003; Twine and Kucharik, 2009; Desai, 2010], especially in temperate regions where extension of the growing season into a warming autumn season may be more important than spring green-up in the overall source/sink status of the forest region [Jeong et al., 2011, 2013; Hwang et al., 2014].

Phenology in midlatitude temperate forests is driven largely by the annual temperature cycle, and phenological events are typically ordered in "thermal time" through the growing season [Trudgill et al., 2005]. Meteorological and climatological factors thus significantly influence vegetation seasonal phenology at the land surface [Jolly et al., 2005; Ceccherini et al., 2014; Hwang et al., 2014; Koster et al., 2014; Xie et al., 2015] and explain a large fraction of observed year-to-year variability in temperate forest phenology [Fisher et al., 2007; Marchin et al., 2015], specifically as drivers of the annual growing season start, intensity, and duration. Additional influences on phenology range from phylogeny [Polgar 
and Primack, 2011; Panchen et al., 2014] to tree age [McMillan and Goulden, 2008]. Photosynthetic capacity in the forest canopy thus varies over the growing season and is acutely sensitive to environmental conditions including temperature [Ali et al., 2015], light intensity [Stuart-Haëntjens et al., 2015], and humidity [Laube et al., 2014b]. These environmental conditions induce risk-based trade-offs: the tree must protect itself against temperature-related stresses, including freezing during spring and autumn transitions [Kim et al., 2014] and potentially lethal summer heat [Teskey et al., 1987; Ruehr et al., 2009; Duarte et al., 2016], and moisture-related stresses during relatively dry periods [Arend and Fromm, 2007; Anderegg et al., 2012].

\subsection{Climate Change}

Changes in the climatology and related weather patterns of the Great Lakes region [Sousounis and Grover, 2002; Hayhoe et al., 2010; Huff and Thomas, 2014] are expected to affect both vegetation phenology [Saxe et al., 2001; Nemani et al., 2003; Jolly et al., 2005; Schwartz et al., 2006; Peñuelas et al., 2009; Groffman et al., 2012; Gunderson et al., 2012; Jeong et al., 2013; Richardson et al., 2013] and forest disturbance regimes [Hufkens et al., 2012; Filewood and Thomas, 2014]. Understanding climate change impacts on forest phenology requires the comprehensive characterization of recent climatological variability, as a basis to more accurately assess important trends, and developing an improved understanding of forest responses to that variability. Through their influence on the tree's capability for leaf growth and carbon assimilation, all of these can affect the role of the forest in the regional and global climate system through physical and chemical feedback mechanisms [Richardson et al., 2013]. Forests exhibit both direct and long-term indirect feedback responses to changes in climate [Heide, 1993; Pope et al., 2013; Marchin et al., 2015], complicating our ability to predict forest phenology, estimate carbon sequestration, and represent clearly the numerous land-atmosphere interactions within the climate system [Peñuelas et al., 2009; Richardson et al., 2013].

Proximity to large water bodies such as Lake Superior can affect local and regional temperature and precipitation patterns [Changnon and Jones, 1972; Scott and Huff, 1996; Hinkel and Nelson, 2012]. 
The interactions of land-lake processes with climate change will be complex; spatiotemporal warming patterns and consequential changes in forest phenology may vary considerably across the Great Lakes region [Reyer et al., 2013]. Climate change in the Great Lakes region is thus expected to proceed at different rates for different seasons, with greater average warming expected in winter than in summer [Hayhoe et al., 2007, 2010]. Precipitation regimes are expected to change, with a greater frequency of heavy precipitation events [Groisman et al., 2012] and a diminishing proportion of winter precipitation falling as snow [Feng and Hu, 2007; Mishra and Cherkauer, 2011]. Warming winters may interfere with dormancy periods for species with leaf bud differentiation and development requirements [Rohde and Bhalerao, 2007; Morin et al., 2009; Viherä-Aarnio et al., 2014; Williams et al., 2014], which can then affect the timing of spring budburst [Cannell and Smith, 1983; Murray et al., 1989]. Young trees and undergrowth can be affected by changes in winter precipitation regimes and seasonal snow cover, especially where snowpack insulates seedlings and soil from hard freezing [Drescher and Thomas, 2013].

A regional trend toward earlier spring green-up [Schwartz et al., 2006; Morin et al., 2009; Jeong et al., 2011] can have substantial consequences for the ecosystem: overall seasonal carbon uptake and sequestration may increase [Saxe et al., 2001; Millard et al., 2007], but new growth is also exposed to an increased likelihood of spring frost events [Hänninen, 1991]. The frequency and severity of spring frost events may change, though the nature of that change may depend on location and numerous influences (e.g. climatological teleconnections) that can vary on long timescales. Some species may readily adapt to changing early-season freezing regimes [Saxe et al., 2001] depending on the magnitude of interannual variability driving such changes. Winter warming along with greater variability in spring meteorological conditions may lead to more "false spring" and frost events that can damage leaves and severely hinder phenological processes through the remainder of the growing season [Rigby and Porporato, 2008; Augspurger, 2013; Peterson and Abatzoglou, 2014].

Uncertainty regarding climate change impacts on forests is even greater for autumn transitions, generally because the senescence process and its triggers remain poorly understood [Estiarte and Peñuelas, 2015; Gallinat et al., 2015]. Autumn senescence and deciduous leaf abscission occur with 
photosynthetic downregulation [Hörtensteiner, 2006; Guo, 2013] and nutrient conservation as defenses against freezing injury [Killingbeck, 1996; Niinemets and Tamm, 2005]. A regional trend toward later leaf senescence can lead to overall longer growing seasons [Jeong et al., 2011; Gunderson et al., 2012] and possibly to increased total primary productivity [Nemani et al., 2003; Twine and Kucharik, 2009]. However, individuals and species for which phenological triggers adjust more slowly to environmental changes may remain susceptible to both frost and drought stress later in the growing season [Saxe et al., 2001; Parida and Buermann, 2014]. Potentially longer heatwaves and drought periods can enhance moisture stress and increase the likelihood of tree mortality [Allen et al., 2015; Duarte et al., 2016; Adams et al., 2017]. Under drought conditions, a nutrient conservation process similar to winter preparation may drive leaf dormancy and senescence [Munné-Bosch and Alegre, 2004; Marchin et al., 2010], and chronic moisture stress can compromise the long-term capacity for carbon assimilation in these forests [Noormets et al., 2008; Anderegg et al., 2012, 2013, 2015; Brzostek et al., 2014].

\subsection{Analytical Approach and Plan of Dissertation}

Tree phenology can be strongly place-based, with often large spatial variability according to species, competition, growth and disturbance history, soils and nutrient availability, physiography, and microclimate. In this dissertation, I will build up to the analysis of meteorological and climatological influences on that phenological variability through various steps.

\subsubsection{Land-Atmosphere Modeling Example}

In Chapter 2 [Garcia et al., 2014], I present an example of the way the land surface is often represented in meteorological models, demonstrating differences in model results at high resolution (100m grid spacing) when the underlying land cover is changed. This experiment depicts a categorical change in land cover type, but is intended to suggest possibilities for better representation of land cover (especially forests) by moving away from the categorical, bulk parameter representation to observationbased and more location- and climate-sensitive specifications of important land surface parameters. With 
Landsat observations, and with detailed analysis of the phenological patterns and their variability as presented in this work, more accurate characterization of the land surface state could make LSMs more realistic in their representation of changing land-atmosphere interactions throughout the growing season, potentially leading to better weather forecasts and climate predictions.

\subsubsection{Study Area, Data Sources, and Model (Pre-) Processing}

In Chapter 3, I provide descriptions of my selected study area and the datasets that I employ in this work to inform the phenoclimatological model and analysis that is my overall goal. The forest areas around western Lake Superior are diverse in physiography, composition, condition, and use. Complex climate gradients on both large and small scales add a level of challenge to my study area selection from which several and varied effects on forest phenology will be analyzed. The challenge in detection and description of these complex interactions is one of the reasons for my study area selection. In this chapter I also describe several pre-processing and processing steps required for use of the available datasets toward the climatological and phenological analyses to be presented in Chapters 5 and 6 , respectively.

\subsubsection{Phenoclimatogical Modeling Framework}

In Chapter 4, I present the details of a novel, computationally-intensive phenological model that I have applied to Landsat-based time series of vegetation indexes, in combination with climatological observations and analyses, to describe the mean phenological season and its meteorologically-driven interannual variability across my study area. The theoretical basis of this model, including underlying assumptions, covers identification and separation of potential disturbance events from the Landsat time series, linear modeling of the mean phenological curve, and partial-least-squares regression (PLSR) modeling of phenological residuals against climatological observations. I also discuss the practicalities of model application on a pixel-by-pixel basis across five Landsat footprints, each with 200-250 scenes for the period of study, and the computational resources and methods used for that component of this work. 


\subsubsection{Climatological Analysis}

In Chapter 5 [Garcia and Townsend, 2016] I assess 1984-2013 climatological means, trends, and interannual variability in my study area forests around the western end of Lake Superior. I focus on describing changes in basic and derived climate indicators, from seasonal and annual temperature and precipitation to a novel definition of the climatological growing season. In accordance with the "thermal time" hypothesis, I employ several threshold-based metrics in common use to characterize seasonal climatology: chilling days $(C D)$ and freezing days $(F D)$ from autumn through spring, and growing degree-days $(G D D)$ from spring through autumn. I introduce an alternative definition of the climatological growing season based on the accumulation of $C D$ through the year, similar but not equivalent to the more traditional frost-free growing season. Using these and several derivative metrics, I assess recent changes in regional climatology for the 1984-2013 period and examine spatiotemporal variability in growing season influences, identifying specific areas where changes and trends have differed markedly from others during our study period. This study presents an examination of the climatological growing season and facilitates comparisons with the observed forest vegetation growing season using remote sensing methods.

\subsubsection{Remote Sensing and Phenological Modeling Analysis}

The Landsat record from successive TM/ETM+ platforms now exceeds 30 years with nearhomogeneous quality that can be employed for detailed exploration of hypotheses on forest ecology and health. Remote sensing observations can be linked to vegetation phenology [Dymond et al., 2002] through several spectral indexes with often targeted utility. The Normalized Difference Vegetation Index (NDVI) [Sellers et al., 1996] is still frequently employed because of its long history in remotely-sensed products, especially based on band-limited sensors. Despite this advantage of a long-term observational history over generally consistent spatial scales, $N D V I$ can be insensitive to moderate disturbances in dense vegetation [Huete et al., 2002]. Indices using Landsat TM/ETM+ SWIR bands are more affected than NDVI by leaf/canopy moisture content [Hunt et al., 1987, 2011; Yilmaz et al., 2008] and are thus more sensitive to 
vegetation stress and disturbance [Isaacson et al., 2012; Townsend et al., 2012]. In Chapter 6, I examine three vegetation indexes based on collected Landsat observations for the 1984-2013 period and will explore results from application of the phenological model outlined in Chapter 4. I will present numerous results relating the mean phenological season and its variability with regional climatology and its own variability, presented in Chapter 5. Chapter 6 of this dissertation is, to the best of my knowledge, the first application of Landsat observations with relatively homogeneous quality to the examination of forest and land surface phenology over a climatological period (30+ years) at high spatial resolution (30-m pixels). This is also likely the first study of forest phenology over a region of this size to combine remote sensing images and daily meteorological information, including climatological derivative fields, to inform the diagnosis of phenological spatial and temporal patterns. This work, based on freely-available datasets and published with open-source software, establishes possibilities for reproduction of this analysis in other regions as well as opportunities for extension, refinement, and parameterization of our methods for forest management and land surface modeling applications. 


\section{CHAPTER 2}

\section{LAND-ATMOSPHERE MODELING EXAMPLE}

Author's Note: much of this chapter has been published previously [Garcia et al., 2014].

Changes in vegetation state can cover a wide range, from incremental growth and senescence through the seasonal phenological cycle to full removal of the vegetation and a change in land cover entirely. It is therefore important to consider the extent to which vegetation state and overall land cover changes may modify weather-related and hydrologic processes at the land surface. With demonstrated differences in surface conditions and land-atmosphere fluxes between different forest states, it becomes important to consider the spatiotemporal variation of specific vegetation characteristics for the accurate representation of the land surface in coupled modeling systems for meteorology and climate. Most continental and global models are applied at spatial resolutions too coarse to capture the details of these land cover changes and are not formulated to account for the physical mechanisms of change processes [Dirmeyer et al., 2006]. Careful application of a scale-spanning modeling system to address both highresolution land characterization and large-scale atmospheric dynamics may provide a better understanding of the local (but widely accumulating) impacts of land cover change (including changing vegetation state) on weather and climate.

\subsection{Simulation Methods}

For this simulation experiment I employed the Weather Research and Forecasting (WRF) model with the Advanced Research WRF (ARW) dynamical core physics package [LeMone et al., 2010a, b; Trier et al., 2011]. At the land surface I employed the community Noah land surface model (LSM) [Pan and Mahrt, 1987; LeMone et al., 2008]. The Noah LSM is a second-generation land surface parameterization [Sellers et al., 1997] that simultaneously solves the energy and water balance at the atmospherevegetation-soil (or -snow) interface using corresponding flux-oriented parameters for each of these 


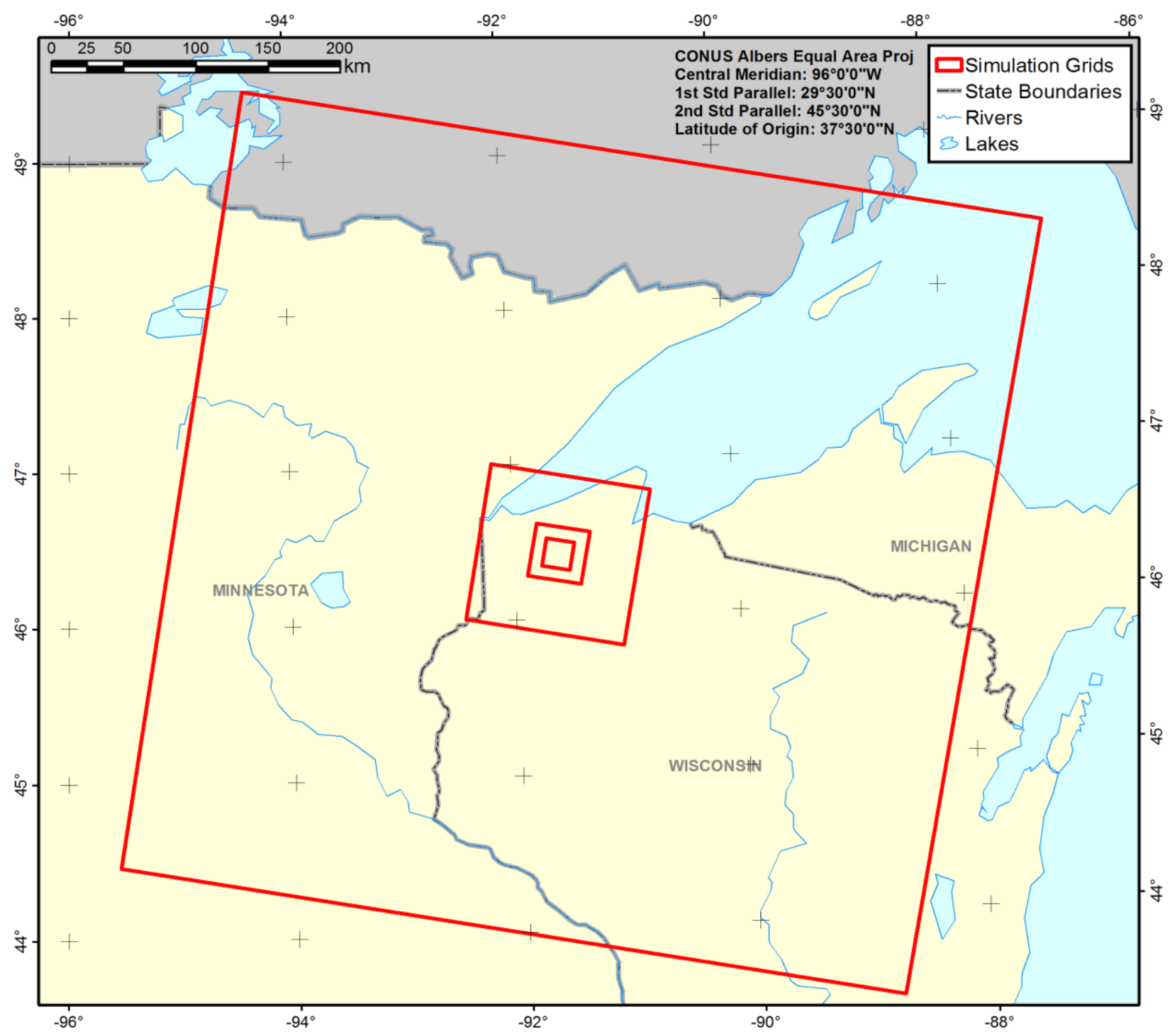

Figure 2.1: The four innermost grids in the WRF simulation domain; the outermost (coarsest) grid covers a large portion of North America and is not shown here. The center grid covers a $20 \times 20 \mathrm{~km}$ area and indicates the location and orientation of the finest simulation grid ("Grid 5") for which the scenario land cover maps are shown in Fig. 2.2.

layers. I employed LSM parameter values that were available in the standard Noah LSM look-up tables included in the WRF-Noah package. The WRF-Noah coupled modeling system is a tested, proven, and highly regarded tool that has been long in development and refinement.

My WRF simulation domain was defined by five nested grid layers in a telescoping configuration (Fig. 2.1). For these simulations, $\sim 27 \%$ of a $20 \times 20 \mathrm{~km}$ area was "converted" (in my land cover categorical map; see Fig. 2.2) from forest to grassland based on remote sensing analyses of clearcut harvest areas in the study area [Özdogan, 2014]. I specified the spatial resolutions of the finest 
(a)

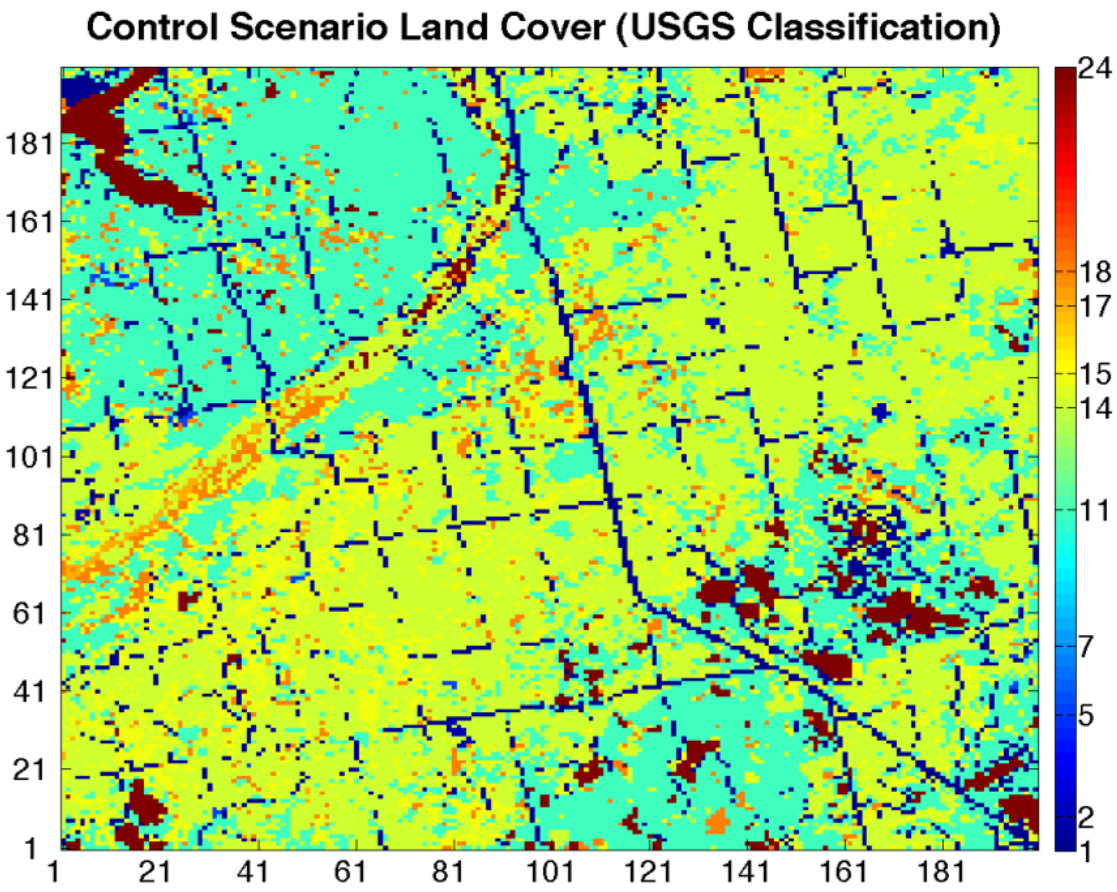

(b)

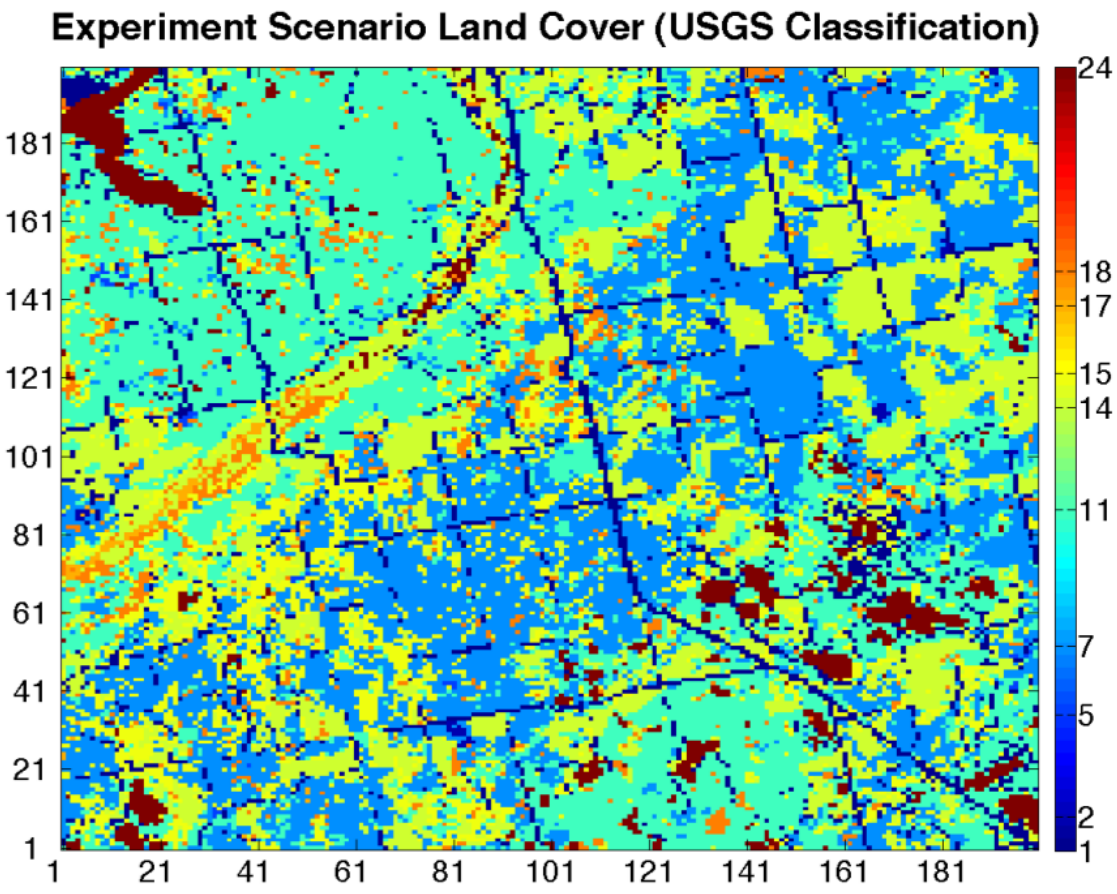

Figure 2.2: Land cover classifications for the finest WRF simulation grid ("Grid 5") covering $20 \times 20 \mathrm{~km}$ with 100-m pixels for the (a) control and (b) experiment scenarios. USGS classification index values are noted at right and referenced to the land cover categories listed in Table 2.1. 


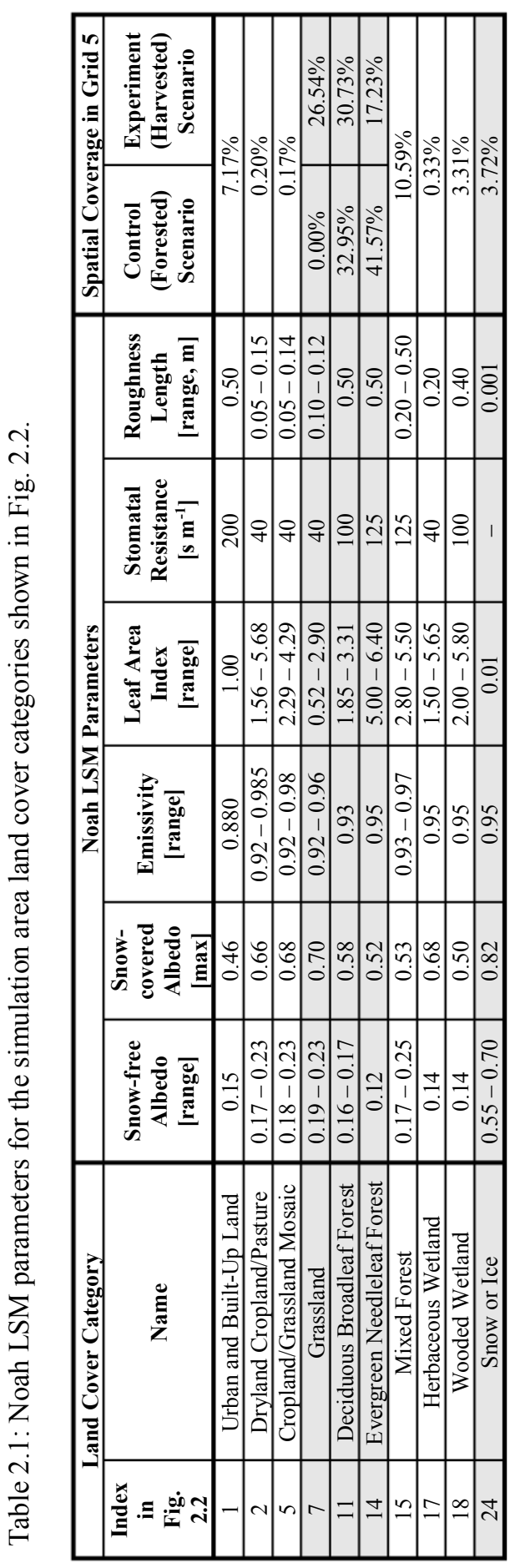


(innermost) grids to provide an adequate representation of forest harvest activities at the land surface, considering both the native resolution of Landsat images used for analysis of those activities (30-m pixels) and a characteristic size of forest ownership plots in many areas of the U.S. Specifically, a forest parcel that covers 16.2 hectares (40 acres) would occupy about 180 Landsat pixels in the land cover change analysis, providing a detailed view of the harvest activity. However, this translates to only $16100-$ m pixels in my finest WRF simulation grid and only a single 400-m pixel on the next-finest grid. I attempted to strike a balance between the apparent small-scale capabilities of the boundary layer and surface layer parameterizations [Horvath et al., 2012; Hu et al., 2013], the availability of high-resolution land cover information from Landsat, adequate representation of forest harvest areas on land parcel sizes characteristic to the region, and model developers' guidance regarding telescoped grid configurations in the simulation domain [Skamarock et al., 2008].

Synoptic atmospheric and surface forcing conditions for these simulations were derived from the North American Regional Reanalysis (NARR) dataset [Mesinger et al., 2006; Luo et al., 2007] at 3-hour intervals and a grid spacing of $0.33^{\circ}(\sim 32 \mathrm{~km})$. I selected a four-day period in February 2001 for demonstration of the differential impacts of snow cover on near-surface conditions in forested and harvested areas. Following the initial time, NARR forcing conditions are applied only to the outermost (coarsest) WRF simulation grid; synoptic-scale meteorological forcing is thus propagated to the internal grids only through the WRF model physics and the numerical formulation of grid nesting. Conditions on the internal grids at mesoscale and convective scales then evolve from established initial conditions in dynamical connection with large-scale atmospheric patterns and disturbances and are allowed to feed back to the continued evolution of the model atmosphere and surface conditions.

I executed these simulations on a supercomputing system in Boulder, Colorado. The simulations were undertaken as a pair to examine the differences between control ("forest") and experiment ("harvest") conditions on the innermost simulation grid ("Grid 5") using only standard Noah LSM parameter values for the specified vegetation types, an operation that I am looking to change with detailed phenological (and disturbance) analyses of Landsat time series. Inputs to the two scenarios in the 
simulation pair differed only in land cover on the two finest modeling grids with $100-\mathrm{m}$ and 400 -m grid spacing. My applied map of forest harvest areas (Fig. 2.2) was derived using Landsat images for the P26R28 footprint at five-year intervals for 1985-2010 following standard forest change detection methods

[Özdogan, 2014]. These methods employed pair-wise comparisons of KTTC transform components [Crist and Cicone, 1984; Crist and Kauth, 1986; Collins and Woodcock, 1996] within a support vector machine (SVM) framework [Huang et al., 2002; Mountrakis et al., 2011] to classify harvested areas. For the "experiment" scenario, pixels identified as harvested in the change map were classified with the NLCD Grassland/Herbaceous category; for the "control" scenario, all of these pixels were reclassified as Evergreen Forest. Standard Noah LSM parameter values, as well as spatial coverage within Grid 5, for each of the land cover types shown in Fig. 2.2 are listed in Table 2.1. Note that the land cover map used for the experiment scenario does not correspond to any specific land cover configuration in the study area during 1985-2010, but instead to the accumulated harvest activity in the study area over that period. Land cover on the coarser model grids did not differ across simulations.

\subsection{Simulation Results}

I spatially aggregated the time series of the several output variables of interest over (a) the area subjected to forest harvest within Grid 5, (b) the area of stable forest or other land cover within Grid 5, and (c) across all of Grid 5 (Table 2.2, main column divisions). These calculations produced time series of mean and variance of model output variables for each spatial area of aggregation. I then compared (a) all times, (b) daytime, and (c) nighttime periods (Table 2.2, sub-columns) to isolate any day/night differences and to detect indicators of nocturnal boundary layer development, especially in the harvested areas. I used a two-tailed Student's $t$-test and $F$-test to determine statistical significance of the differences between onedimensional time series of scenario results at these nine spatiotemporal resolutions. Color codes in Table 2.2 indicate the level of test significance. In addition, the time series for each spatial and temporal aggregation were differenced $($ difference $=$ experiment - control) and the mean and variance of that difference time series is listed in the cells of Table 2.2. Note that these aggregated and summarized values 
in Table 2.2 cover the three-day evaluation period of the simulation (following one "spin-up" day) and are not explicitly segregated to account for changing dynamical conditions within that time, such as the cold front passage.

\subsubsection{Surface Temperature and Wind Speed}

The nighttime reduction of surface temperature in the experiment scenario that is shown in Fig. 2.3a is statistically significant $(p<0.05)$ and the differences between experiment and control scenarios are shown in Fig. 2.3b. The greater near-surface mean wind speed in forest harvest areas for the experiment scenario is also statistically significant $(p<0.001)$ in all temporal aggregations and leads to some significant differences when averaged over the entire finest grid as well $(p<0.01)$. Time series of the surface wind speed in the area of forest harvest are shown in Fig. 2.3c, and differences between the two scenarios over the three areas of spatial aggregation are shown in Fig. $2.3 \mathrm{~d}$. At all levels of spatial and temporal aggregation, the near-surface wind speed mean and variance increased in the experiment (harvest) scenario.

The largest differences in surface temperature between control and experiment scenarios occur at

night, especially during relatively calm periods with near-surface wind speeds generally less than $4 \mathrm{~m} \mathrm{~s}^{-1}$. These can be related to the establishment of a cold nocturnal boundary layer that is apparent in harvest areas, which are $\sim 2 \mathrm{~K}$ colder than surrounding forest areas just prior to sunrise on 18 February and generally have near-surface wind speeds $\sim 1 \mathrm{~m} \mathrm{~s}^{-1}$ less than surrounding areas at the same time. Similar conditions are found just prior to sunrise on 20 February as well. Animated simulation results indicate on both of these mornings that greater daytime wind speeds propagate downward from the free atmosphere (above $\sim 900 \mathrm{hPa}$ ) and erode the stable nocturnal boundary layer within $\sim 30$ minutes after sunrise. Differences in near-surface wind speeds between the control and experiment scenarios are generally larger during daytime hours and through a period of frontal disturbance on 19 February. 


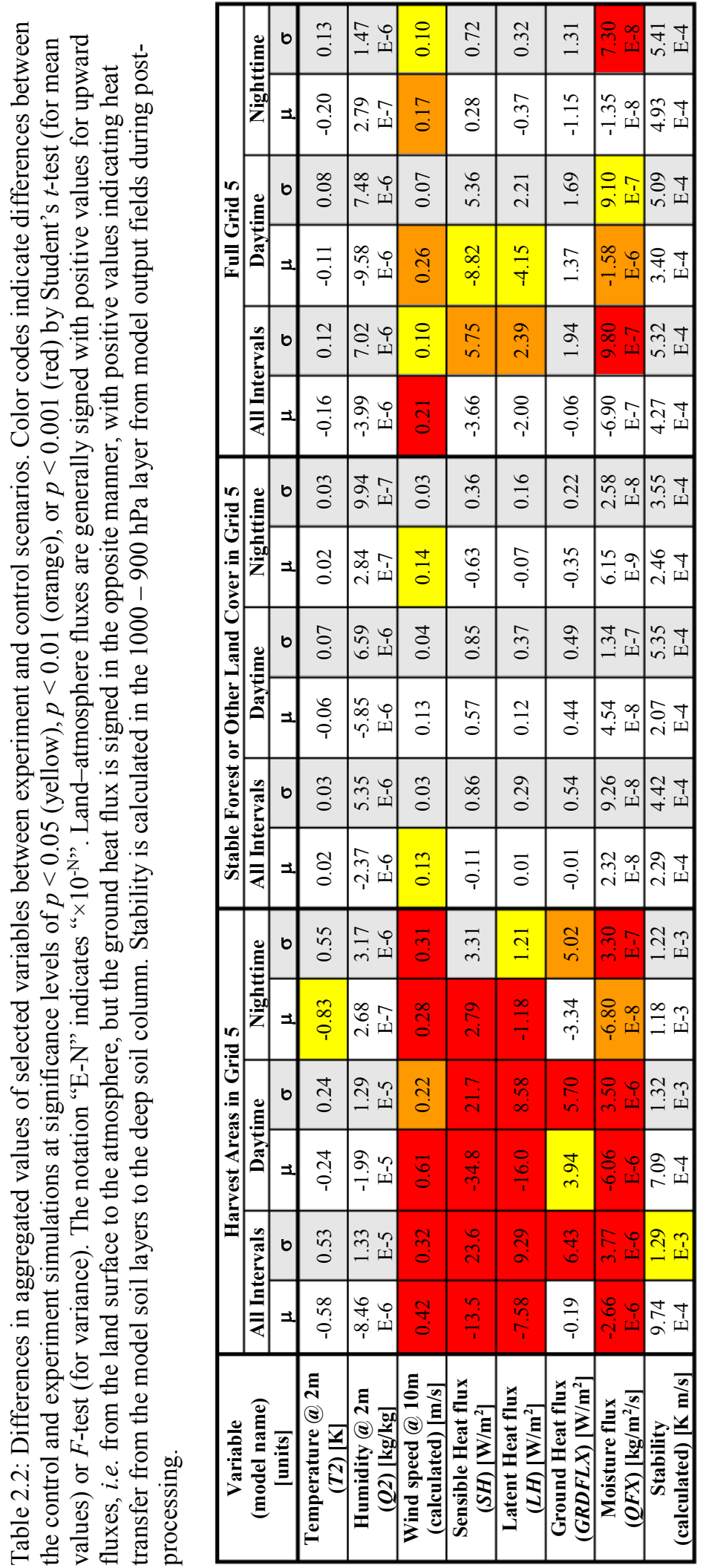


(a)

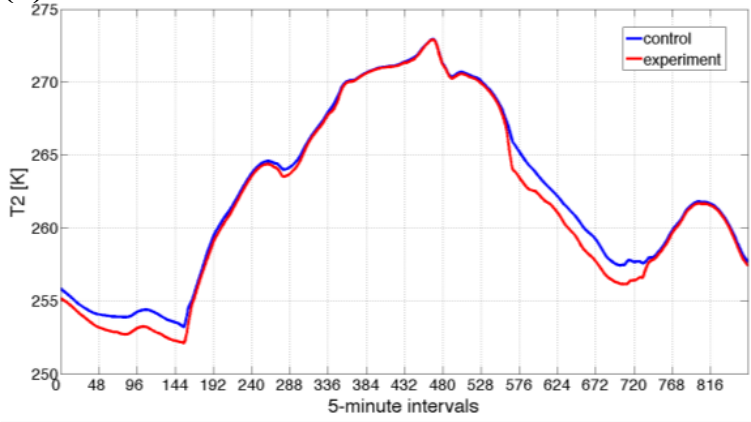

(c)

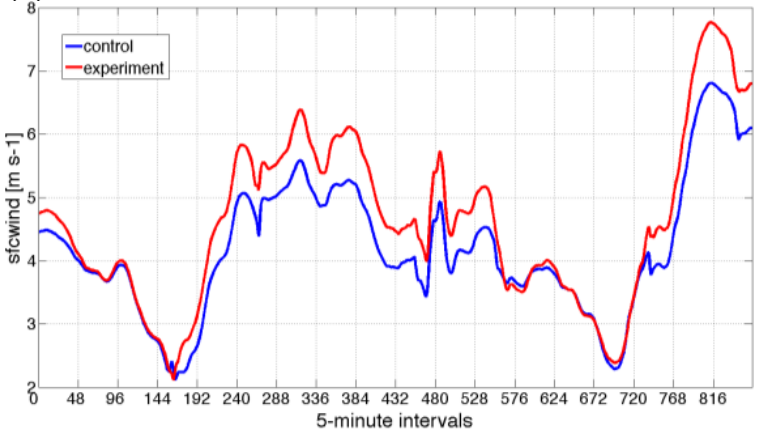

(b)

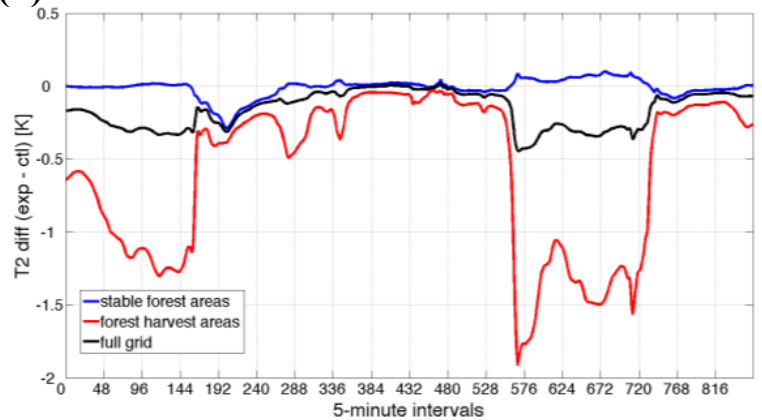

(d)

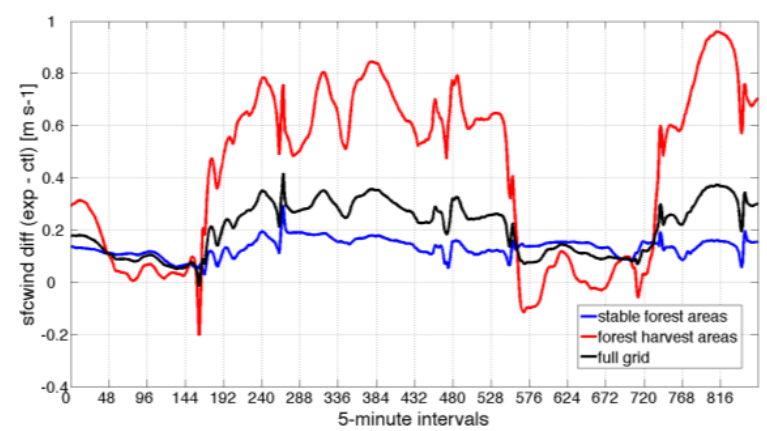

Figure 2.3: Time series of surface temperature (T2) and near-surface wind speed for (a) and (c) the control and experiment scenarios in the areas of forest harvest, and (b) and (d) the difference at each time between the two scenarios as aggregated over forest areas, harvest areas, and all of Grid 5 (Fig. 2.2).

\subsubsection{Surface Fluxes and Energy Balance}

Land-atmosphere fluxes also show the greatest differences between control and experiment scenarios during the daytime, with local energetic balance driving the surface fluxes through those hours. Insolation reaches peak daytime values of $\sim 650 \mathrm{~W} \mathrm{~m}^{-2}$, and the surface albedo of the forest and harvested areas (Table 2.1) accounts for much of the difference in energetic balance between scenarios. Within forest harvest areas, the sensible heat $(\mathrm{SH})$ flux in the experiment scenario is reduced by $\sim 65 \mathrm{~W} \mathrm{~m}^{-2}$ from the control scenario during the day, as shown in Fig. 2.4(a), although smaller differences are found during the period of frontal passage on 19 February. The experimental daytime latent heat $(L H)$ flux is likewise reduced from the control scenario by $25 \mathrm{~W} \mathrm{~m}^{-2}$ as shown in Fig 2.4(b), with larger differences during the date of frontal passage. Warmer temperatures on that day lead to a slight melting of the existing snow cover in the finest simulation grid. On the less disturbed days of the simulation period, the mid-day peak 

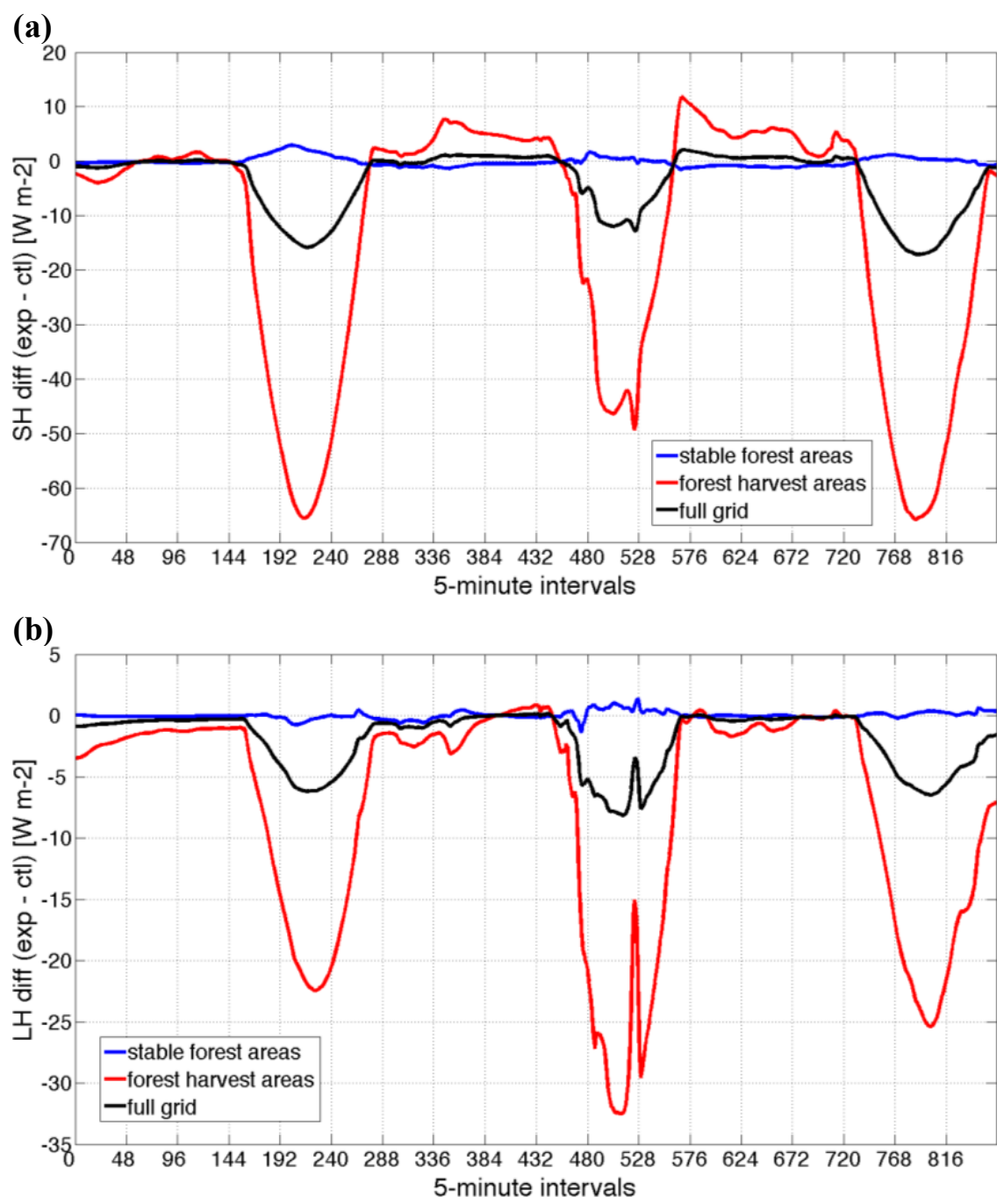

Figure 2.4: Time series differences between control and experiment scenarios of surface (a) sensible heat $(\mathrm{SH})$ flux and (b) latent heat $(\mathrm{LH})$ flux aggregated over forest areas, harvest areas, and all of Grid 5.

in $\mathrm{SH}$ flux is apparent in Fig. 2.5 where the pattern of forest harvest areas is clearly visible. The spatial pattern of the peak (mid-day) $L H$ flux is the same, although its magnitude in the cleared areas is smaller.

Figure 2.6 summarizes the modeled land surface energy balance for the areas in the finest grid of the simulation domain that were subjected to forest harvest. In these diagrams, fluxes (arrows) were calculated from model simulation results as the average of that flux component over the three-day period (including nights) and the residual of that calculation is assigned as the net land surface heat flux. The differences between control (forested) scenario in Fig. 2.6a and the experiment (harvested) scenario in 


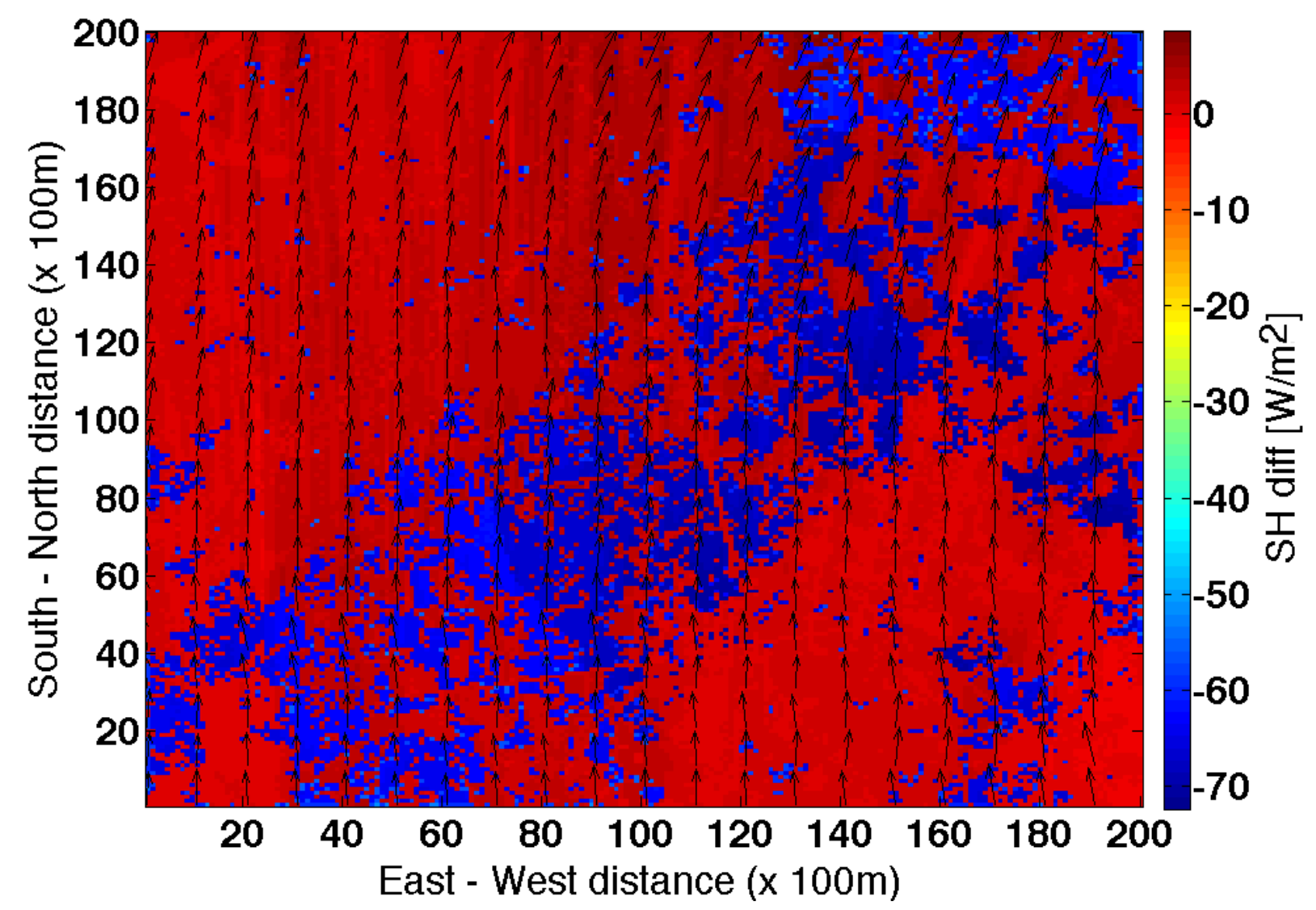

Figure 2.5: Mapped differences between experiment and control scenarios of surface sensible heat $(\mathrm{SH})$ flux at 1800 UTC (1200 LST) on 18 February 2001, showing the variation between forested and cleared areas in the finest simulation grid near the mid-day peak of surface heat fluxes. A map of latent heat $(\mathrm{LH})$ fluxes (not shown) is qualitatively similar in pattern, but of smaller magnitude in the cleared areas.

Fig. $2.6 \mathrm{~b}$ are driven primarily by the large difference in surface albedo and the partitioning of remaining energetic fluxes to sensible and latent heating.

The Bowen ratio, defined as the ratio of sensible to latent heat exchange or $B=S H / L H$, actually shifts between these two scenarios. For the forested conditions in Fig. 2.6a, $B=1.14$ suggests that the surface energy balance is affected almost equally by the temperature difference between land and atmosphere (driving $S H$ exchange) and by the use of energy in the snowpack for melting, evaporation and sublimation, and in the forest for transpiration processes (the paths of $L H$ exchange). For the harvest scenario in Fig. 2.6b, values of $S H$ and $L H$ are both smaller than in non-logged conditions (attributable in large part to the albedo difference between scenarios), but $B=0.5$ suggests a strong shift toward greater energy allocation to melting, evaporation, and sublimation processes in the exposed snowpack. The exposed snow surface in forest harvest areas effectively insulates the soil surface, which retains less of the 
(a)

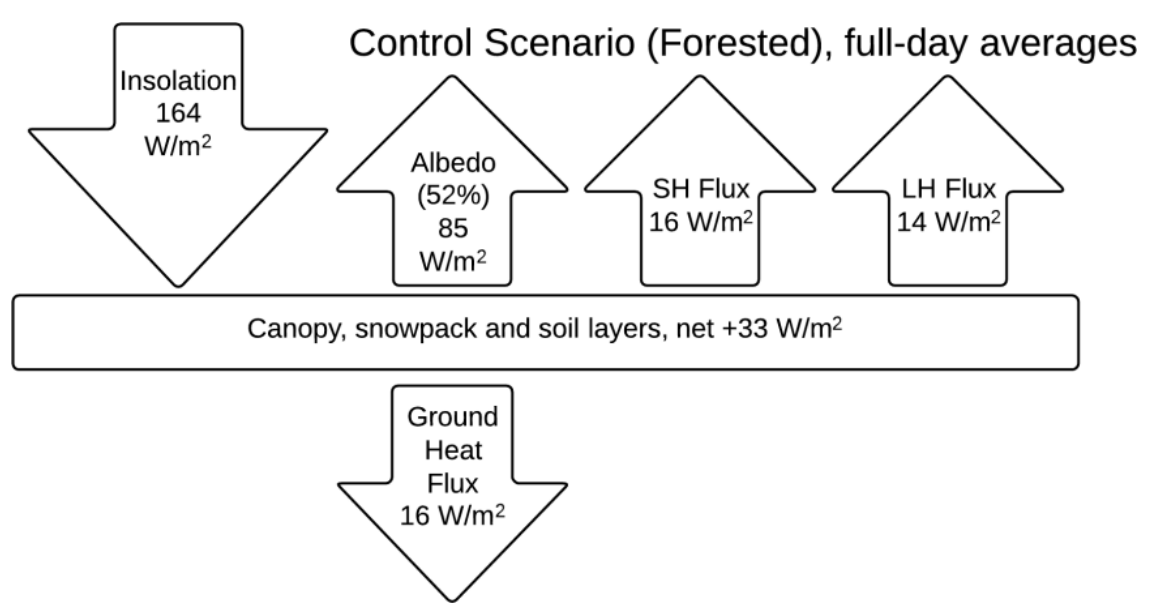

(b)

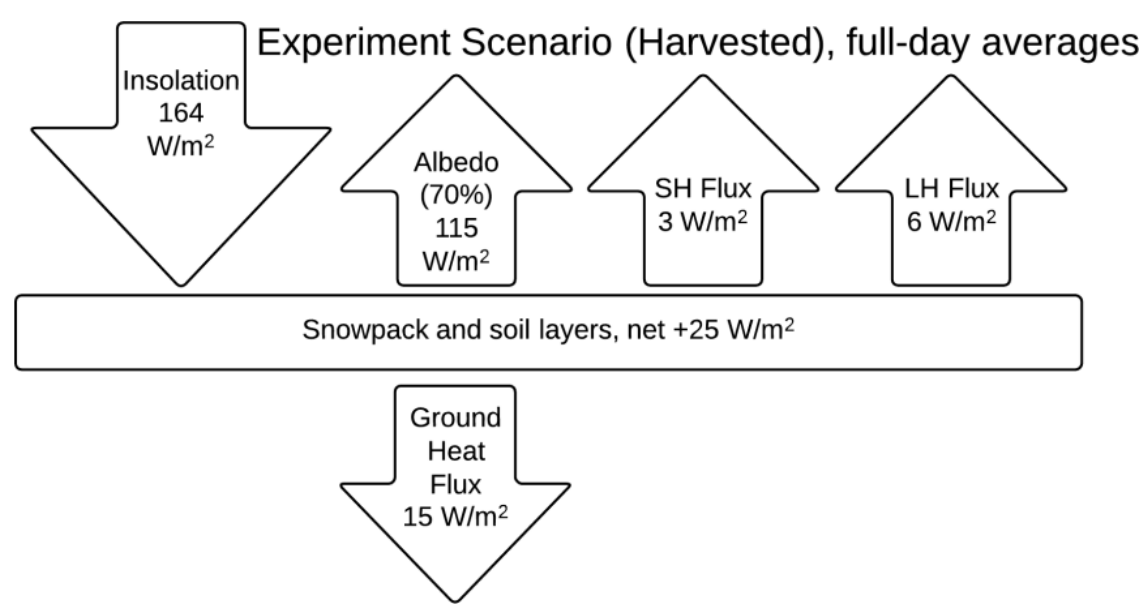

Figure 2.6: Energetic balance at the land surface for the three-day simulation period in areas subject to forest harvest (Fig. 2.2) for (a) control and (b) experiment conditions. $S H=$ sensible heat; $L H=$ latent heat.

insolation heat flux than the forested areas in the control scenario. Overall, these simulations suggest that forest clear-cutting is equivalent to a net local land surface heat flux of $-8 \mathrm{~W} \mathrm{~m}^{-2}$ under winter, snowcovered conditions.

\subsection{Discussion}

The most significant changes in surface states, flux variables, and boundary layer processes between intact and harvested forests resulted from the differences between input land cover maps and their representation by the LSM parameterization. For example, departures in temperature were tied most 
generally to the differences in albedo, emissivity, and snow depth between control (forested) and experiment (harvested) land covers, as well as the role of these conditions within the model in the energetic balance at the land surface. Differences in wind speed can be attributed to the diminished roughness of grass and snow surfaces compared to the forest canopy in the control scenario. Simulated latent heat fluxes were greater on 19 February than on previous or subsequent days, which can be attributed to higher surface temperatures around the time of frontal passage and a greater partition of available energy to melting and evaporation in the snow cover of exposed clearings. The strength of the front, characterized most simply by the cross-frontal temperature gradient, remained steady over time in the control scenario, but was weakened in the experiment upon passage over the harvested area. While frontal dynamics can be complex over heterogeneous surfaces, I attribute these differences primarily to the obvious variations in surface roughness between the forest and harvested (grassland/snow) cover types, as well as to the collection of colder, more stable air near the surface in the harvested areas prior to frontal passage (Table 2.2).

In forest harvest areas, the exposed snow layer effectively insulates the land surface, which retains less of the insolation heat flux than the full forest canopy in the control scenario. Overall, these simulations suggested that forest clear-cutting is equivalent to a climatologically significant net local land surface heat flux of $-8 \mathrm{~W} \mathrm{~m}^{-2}$ under winter, snow-covered conditions. These results were consistent with conceptual understanding of the impacts of forest harvest on the climate system in middle and high latitudes, particularly the cooling effect of exposed snow surfaces in winter [Snyder et al., 2004; Bonan, 2008]. The high-resolution experimental results at the local scale were also consistent with large-scale simulations of deforestation impacts obtained using coarser modeling grids [Snyder et al., 2004; Klingaman et al., 2008; Mishra et al., 2010b]. Extending this modeling capability to landscape- and stand-scale forest dynamics, these simulation results provide further support for the use of remote sensing-based land cover change products in analyses of land-atmosphere fluxes and energetic balance. In particular, the relevance of such efforts in forest regions subject to natural and anthropogenic disturbances is demonstrated, even where the changes are transient in time and discontinuous in space. 
Although the surface energy balance can be evaluated at an individual point within this model, in which a vegetation/soil column in the LSM does not communicate with its neighbors, wind-based atmospheric transfer of energy and momentum between locations can still alter the atmospheric kinematic and thermodynamic balance in the surrounding area. Harvest-induced changes in surface temperature, humidity, and wind speed in disturbed areas are thus tied dynamically to the surrounding intact forest. This points to the need to evaluate impacts of land cover disturbance at scales larger than the individual forest clearing, at which both the occurrence and pattern of disturbances become important. In this work, I have specifically addressed the local energy and moisture balance, but not the carbon cycle, at the land surface. This approach can be extended with alternative models that consider more completely the role of forest harvest in the intertwined energy, moisture, and carbon cycles of the global climate system. 


\section{CHAPTER 3}

\section{STUDY AREA AND DATA SOURCES}

Chapter 2 demonstrated the sensitivity of weather model results to the specification of the land surface using categorical land cover specifications and default values for important parameters. If we wish to move beyond representation of the land surface using "look-up-table" approaches, the important role of vegetation in the land-atmosphere system cannot be understated, and significant progress might be seen with improvements to the way vegetation parameters are specified or otherwise provided to the modeling system. This dissertation approaches the representation of forests in such models with the idea that vegetation should be represented not with simple land cover categories on the land surface, but with a continuum of land surface states that are conditioned on both biological and environmental conditions. Forest phenology is strongly related to weather and climate on local, regional, and global scales [Peñuelas et al., 2009; Richardson et al., 2013]. A detailed study of phenology must therefore bring together observations and measurements from several different fields. Weather and climate datasets, Landsatbased multispectral satellite observations, land cover analyses, and numerous additional datasets used in my analyses are described here, with pre-model processing details given for several of these information sources.

\subsection{Study Area and Regional Hydroclimate}

My study area covers $\sim 202,000 \mathrm{~km}^{2}$ of midlatitude evergreen and mixed forest around the western end of Lake Superior in the North American Upper Great Lakes (Fig. 3.1). This area covers five Landsat $5(\mathrm{TM})$ and $7(\mathrm{ETM}+)$ footprints in northeastern Minnesota, northern Wisconsin, and part of the Upper Peninsula of Michigan that are representative of the northern temperate/sub-boreal forest region in the U.S. and part of the Laurentian mixed forest province of North America. This region hosts diverse forest and wildlife species, numerous protected and managed areas including state and national forests, widespread forest-related industry, and extensive tourism and recreational opportunities. A "tension zone" 


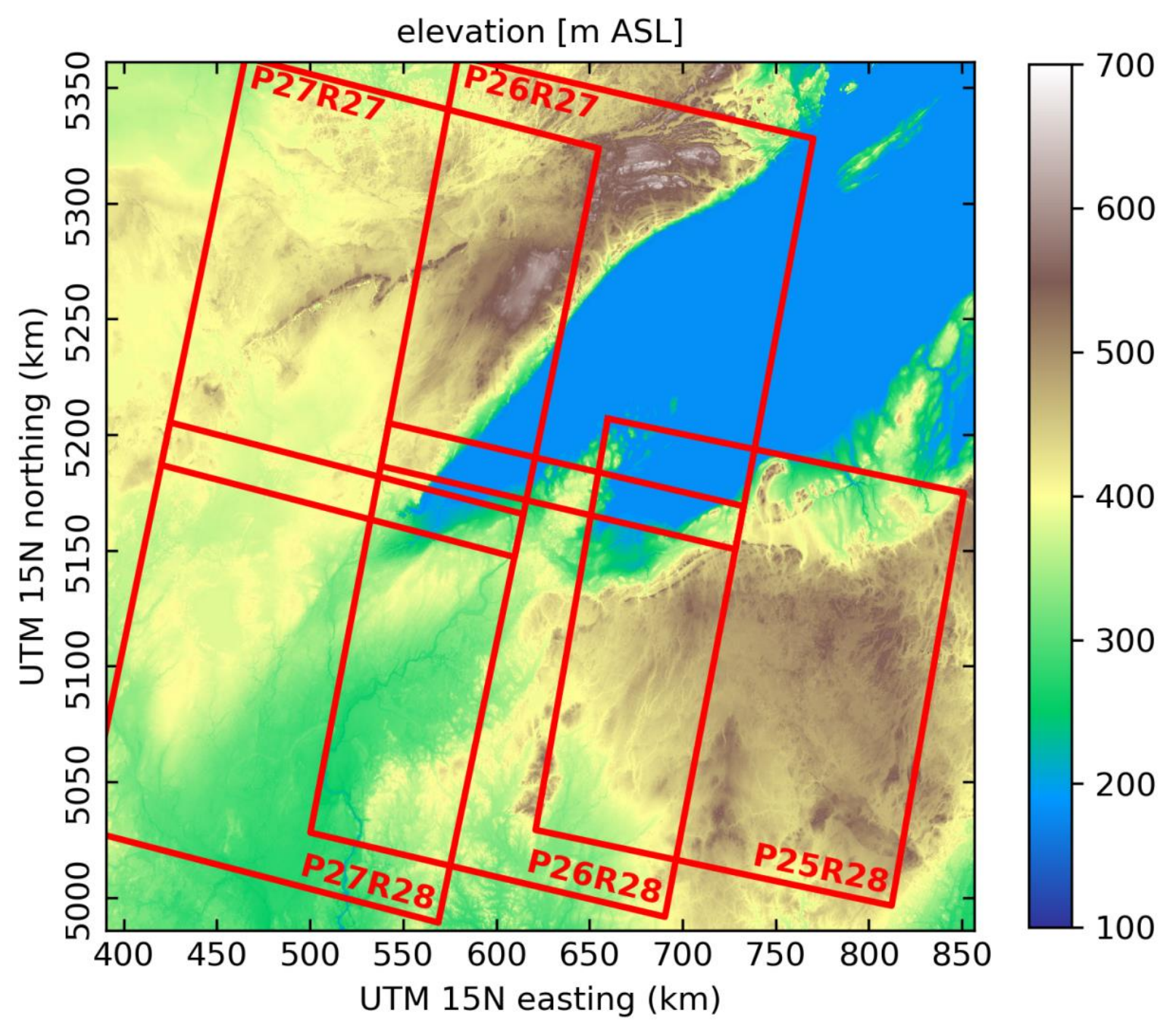

Figure 3.1: Study area topography with nominal Landsat footprint coverage. The surface elevation of Lake Superior is $\sim 183 \mathrm{~m}$ ASL.

[Curtis and McIntosh, 1951] that traverses the study area is defined by a combination of geographic transitions, from warm and dry continental interior to cool and wet lake-influenced landscapes. This tension zone marks the approximate southern extent of the Wisconsin Glacial Episode (c. 85 kya to 11 kya) with resulting gradients in soil types [Schaetzl et al., 2005; Danz et al., 2013] and now encompasses a gradient in natural vegetation types [Wheeler et al., 1992; Bockheim and Schlieman, 2014], from prairie and hardwood forests in the southwest (now mixed with agriculture) to sub-boreal evergreen and temperate mixed forests closer to Lake Superior. This transition is clearly visible in USGS National Land Cover Database (NLCD) products [Jin et al., 2013; Homer et al., 2015] and can be identified in USEPA 


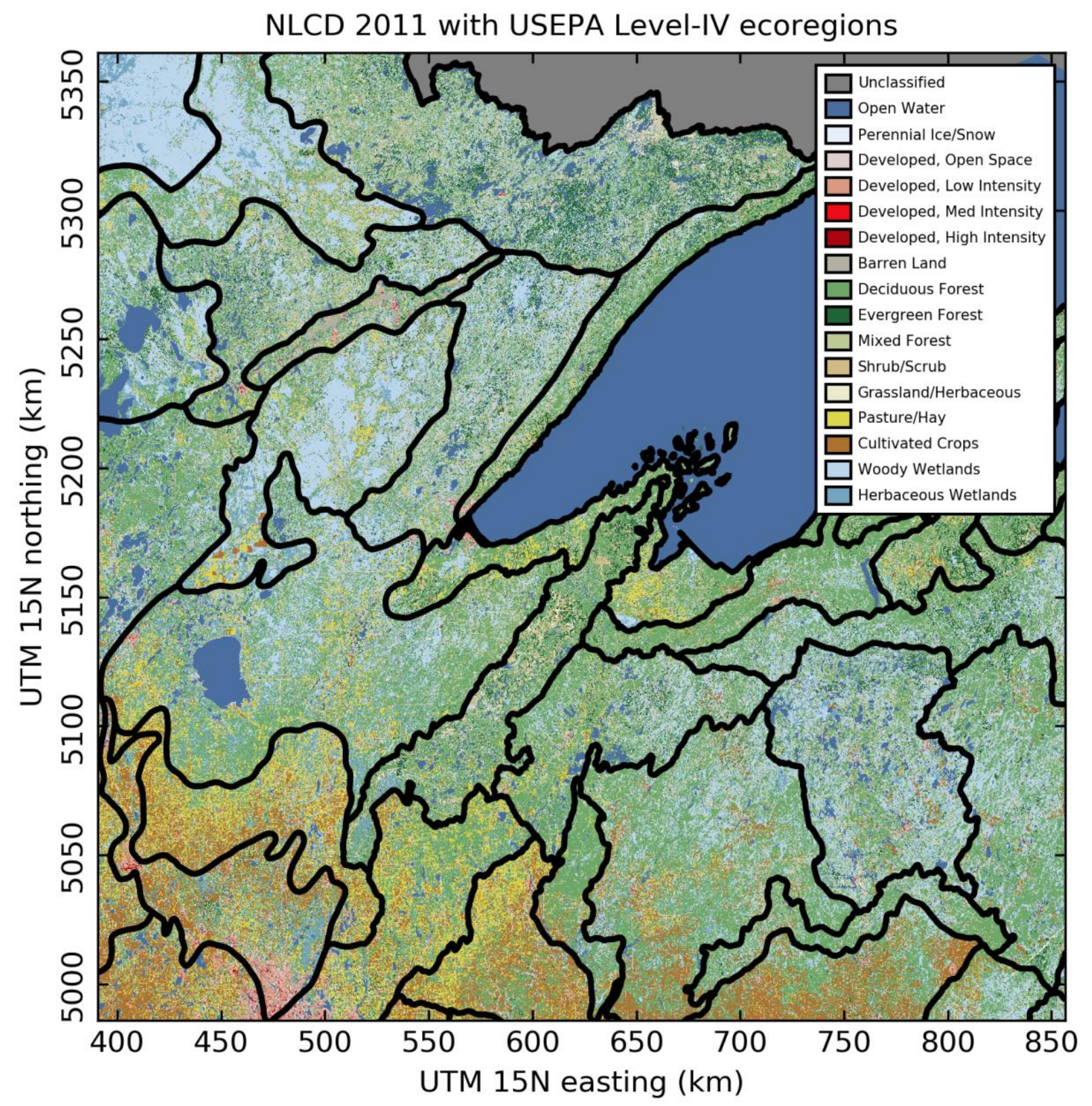

Figure 3.2: Study area USGS NLCD 2011 land cover categories with USEPA ecoregion boundaries.

Level-IV ecoregion maps of the study area [Omernik et al., 2000; Omernik, 2004; USEPA, 2011] (Fig.

$3.2)$.

My choice of study area reflects a particular challenge that arises from the complex geography of the region. Situated at the prairie-forest ecotone, this area borders the North American boreal forest but also has strong agricultural influences and is subject to land-lake interactions and climatological influences of the Great Lakes. Forests in this region display a wide variety in observed phenology and 
disturbance events, with further variety expected as a consequence of climate change impacts on all of these elements. The forests of the Upper Great Lakes are increasingly vulnerable to disturbance factors and tree mortality due to seasonal moisture stress and fire risk under changing climatic conditions [Irland et al., 2001]. This region is also sensitive to changing forest ecology and management practices, increasing recreational use and logging pressure, anthropogenic warming and climate change, and evolving conditions in the Great Lakes themselves including rapid recent warming of Lake Superior [Van Cleave et al., 2014]. All of these factors can affect the forest and its role in the carbon and water cycles in this region.

Upper Great Lakes climatology is influenced by the polar jet stream that frequently traverses the region and generally marks a continental-scale boundary between cold/dry polar air and warm/humid subtropical air along the primary midlatitude storm track. The jet stream over the region is directed by interactions between global circulations, synoptic dynamics, and climatological teleconnections [Rohli et al., 1999; Grise et al., 2013]. Both the upper-level jet stream and surface land-lake interactions drive surface temperature gradients, frontal positions, and storm meteorology [Payer et al., 2011]. Seasonality is a key factor in meteorological patterns at temperate latitudes, with synoptic variability dominating the Spring and Autumn transition seasons [Grover and Sousounis, 2002; Small and Islam, 2009; Small et al., 2010] and the Great Lakes providing a strong regional influence on temperature and precipitation patterns throughout the year.

With the position of the Great Lakes near the middle of the North American continent, jet stream and storm track patterns across this region are driven by numerous teleconnections including the PacificNorth America (PNA) pattern [Rodionov and Assel, 2001], the Arctic and North Atlantic Oscillations (AO and NAO, respectively) [Nie et al., 2008; Luo and Cha, 2012], and often the combination of these with the Pacific Ocean El Niño-Southern Oscillation (ENSO) and the Pacific Decadal Oscillation (PDO) [Bond and Harrison, 2000; Grise et al., 2013]. Climatological teleconnections have been correlated most strongly with Great Lakes Winter conditions [Rodionov and Assel, 2000, 2003; Wise et al., 2015] and seasonal lake ice cover [Assel and Rodionov, 1998; Assel et al., 2003; Bai et al., 2011, 2012; Benson et 
al., 2012; Bai and Wang, 2012; Wang et al., 2012]. The warm phase of the long-period Atlantic Multidecadal Oscillation (AMO) has been associated with cold Winters in eastern North America [Peings and Magnusdottir, 2014]. With a 4- to 7-year cycle, the ENSO cycle is often associated with warm/dry Winters in the Upper Midwest US during El Niño (warm) years and cold/wet Winters during La Niña (cold) episodes [Trenberth et al., 1998; McPhaden et al., 2006].

Several studies have associated a climatological regime shift in the Upper Great Lakes region around 1998 with influential long- and short-period oscillating teleconnections: the AMO entered a primarily warm phase around 1995, and the PDO entered a persistently cold phase in 1998 coincident with an anomalously strong El Niño episode during the 1997-1998 Winter [Bond et al., 2003; Peterson and Schwing, 2003; Jo et al., 2014]. Changes in Lake Superior ice cover, evaporation, water level, and water temperature regimes and trends have been identified around the same time [Van Cleave et al., 2014; Watras et al., 2014]. Great Lakes surface water temperatures have warmed faster than surrounding land surface temperatures [Austin and Colman, 2007, 2008; Van Cleave et al., 2014] and Lake Superior ranks highly among the fastest-warming large freshwater lakes in the world [O'Reilly et al., 2015]. This warming has driven changes in the lake ice regime: peak areal ice coverage has decreased [Howk, 2009; Wang et al., 2012], and observations indicate later ice onset in Winter and break-up in Spring [Assel, 2003; Howk, 2009; Assel et al., 2013]. Reduced lake ice cover allows an overall increase in lake surface evaporation through the early Winter [Brown and Duguay, 2010], resulting in greater Winter lake-effect precipitation in downwind areas [Scott and Huff, 1996; Wright et al., 2013].

\subsection{Weather and Climate Data}

Author's Note: much of this subsection has been published previously [Garcia and Townsend, 2016].

I developed climatological maps and statistics using daily minimum and maximum temperature ( $T_{\min }$ and $T_{\max }$ ) and precipitation $(P)$ observations from the merged Global Historical Climate NetworkDaily (GHCND) dataset [Durre et al., 2010; Menne et al., 2012] for 410 weather stations in the vicinity 


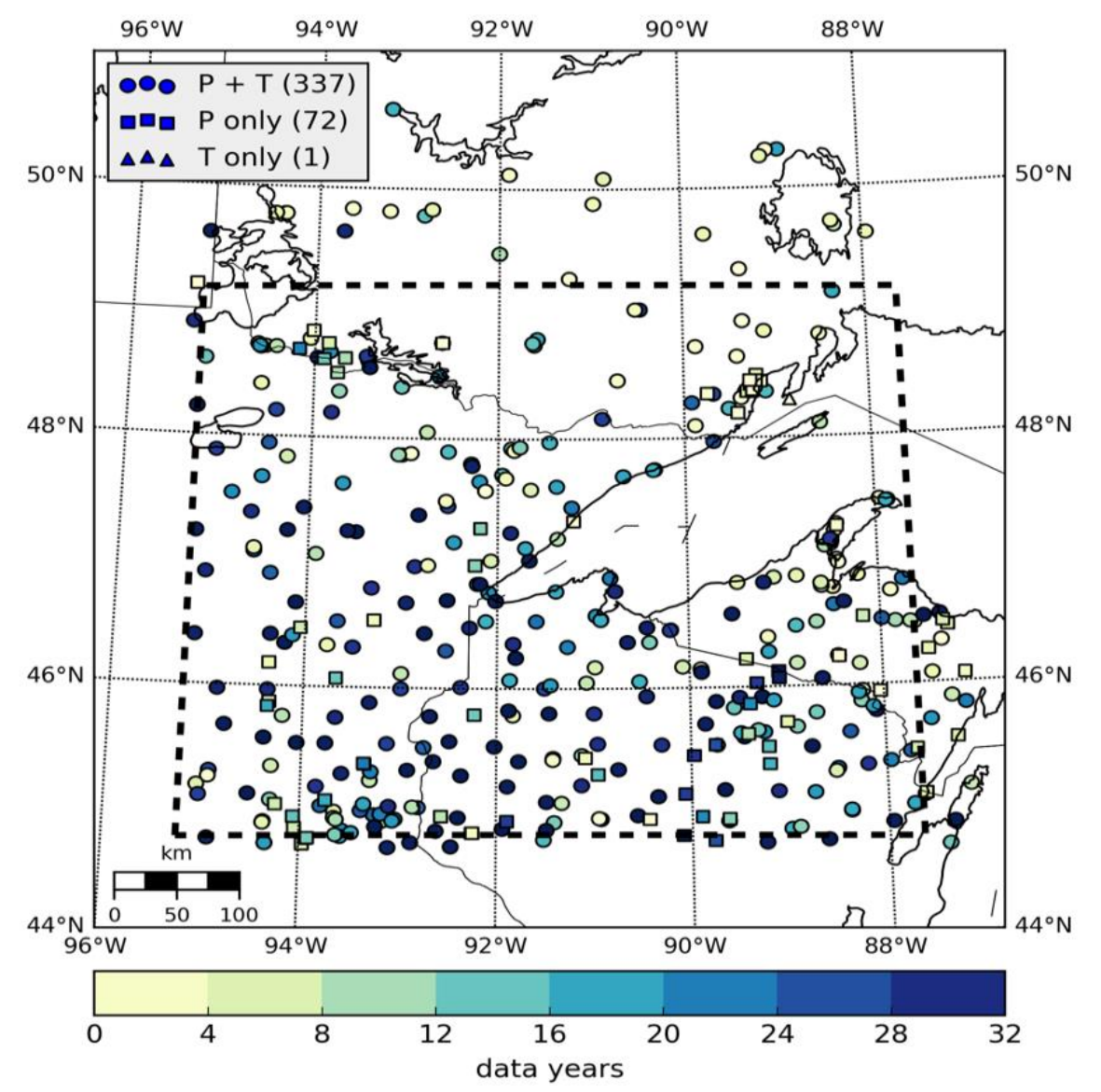

Figure 3.3: Locations of GHCND meteorological stations in and around the study area, indicated by the dashed line.

of western Lake Superior over the period from 1 January 1983 through 31 December 2013 (Fig. 3.3). My selected dataset included first-order (operated by the U.S. or Canadian National Weather Service) and officially-recognized cooperative weather stations but excluded volunteer reporting stations (primarily in the CoCoRaHS precipitation network) for lack of documentation on quality control procedures. A number of these stations, including 83 stations in Canada, are located outside of my Landsat study area but are included for more complete and accurate coverage of the study area in interpolated products.

Using GHCND quality assurance flags, I cleaned the dataset to exclude stations with no location information or with uncertain data quality. I then used all valid station data for each date to generate gridded daily $T_{\min }, T_{\max }$, and $P$ fields at 480 -m grid spacing using radial basis functions [Akkala et al., 2010]. This method is an exact multiquadric interpolator [Hardy, 1971, 1990; Franke et al., 1994] with results that compare favorably with other spatial interpolators [Garcia et al., 2008]. I evaluated spatial 
interpolation error characteristics by a jackknife procedure (Figs. 3.4 and 3.5) and found daily $T_{\min }$ bias (mean error) of $+0.022^{\circ} \mathrm{C}$ and mean absolute errors $(M A E)$ of $1.78^{\circ} \mathrm{C}$, daily $T_{\max }$ bias of $+0.038^{\circ} \mathrm{C}$ and $M A E$ of $1.60^{\circ} \mathrm{C}$, and daily $P$ bias of $+0.001 \mathrm{~cm}$ and $M A E$ of $0.152 \mathrm{~cm}$. These bias values are less than the reporting precision of the meteorological stations in the GHCND dataset $\left(0.1^{\circ} \mathrm{C}\right.$ and $0.025 \mathrm{~cm}$ for $T$ and $P$, respectively) and $M A E$ values fall generally within expected interpolation accuracy [Garcia et al., 2008]. Some weather stations cover almost the entire analysis period, while many were active for only portions of that period (Fig. 3.3). There is no explicit temporal interpolation of station values to cover periods of missing data at individual stations. I sought to generate the best possible temperature and precipitation maps on a daily basis using the available and valid station information for each day. All subsequent operations, including aggregation to seasonal averages or totals and statistical analyses for temporal trends, used this 31-year series of gridded daily observations.

I calculated the daily average temperature [Cannell and Smith, 1983] from the gridded fields as

$$
T_{\text {avg }}=\frac{T_{\min }+T_{\max }}{2}
$$

As a proxy for humidity, but in the absence of any humidity measurements, I also estimated the vapor pressure deficit (VPD) using the minimum and maximum daily temperatures [Thornton et al., 1997] as

$$
V P D=e_{s}\left(T_{a v g, d}\right)-e_{s}\left(T_{\min }\right)
$$

with the daytime-weighted average daily temperature [Running et al., 1987] calculated by

$$
T_{\text {avg,d }}=0.606 T_{\max }+0.394 T_{\min }
$$

and the standard formula for the saturation vapor pressure in units of Pascals (Pa)

$$
e_{S}(T)=610.78 \exp \left[\frac{17.269 T}{237.3+T}\right]
$$

Table 3.1 lists temperature-based meteorological indicators used in this study, primarily in Chapter 6, and Table 3.2 lists specific seasonal temperature-based indicators that are used specifically for the climatological analysis in Chapter 5. Likewise, Table 3.3 lists precipitation-based meteorological indicators used in Chapter 6, and Table 3.4 lists specific seasonal and annual precipitation-based 

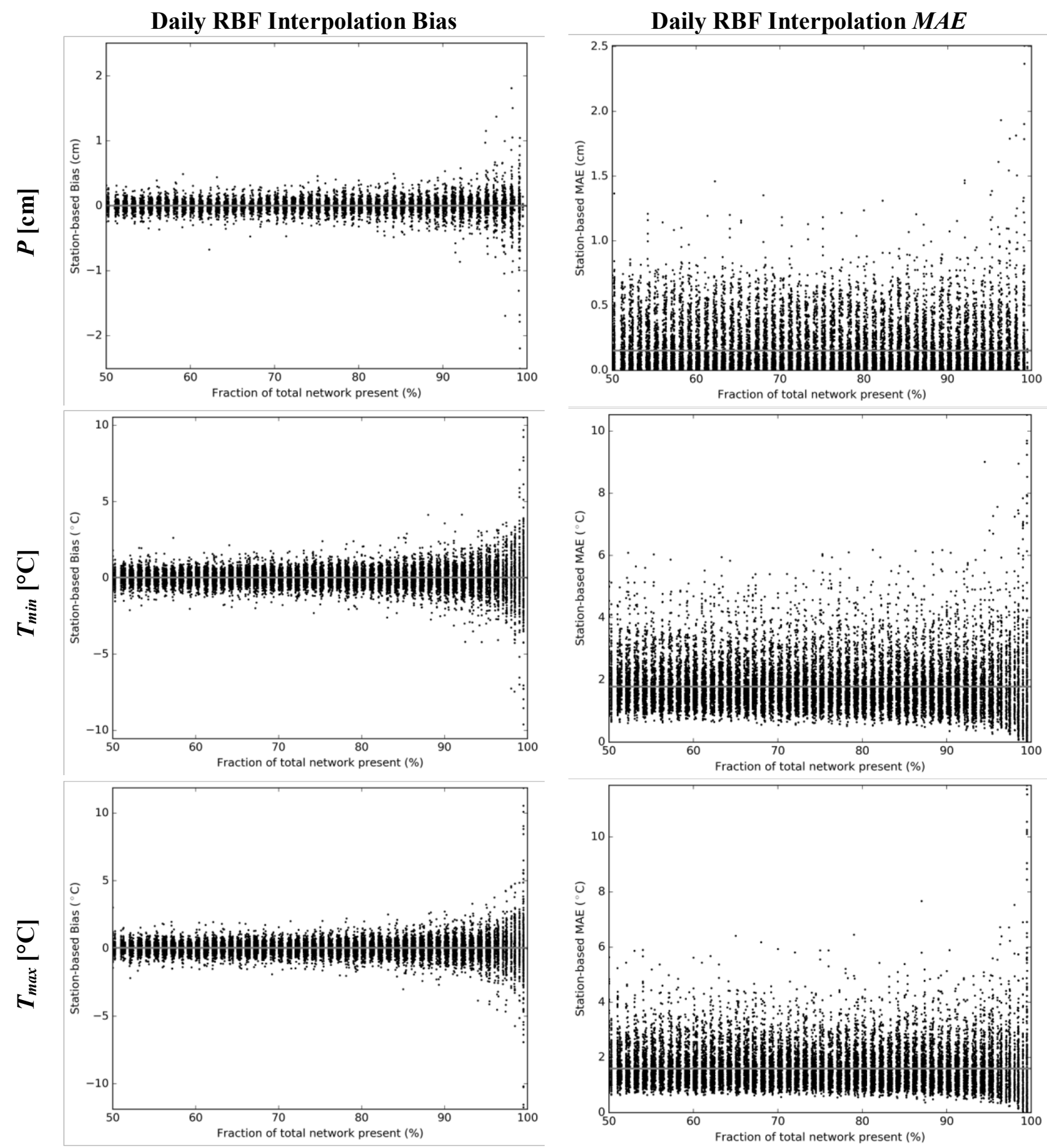

Figure 3.4: Meteorological station network interpolation accuracy based on a jackknife procedure with as much as $50 \%$ of the station network withheld. 

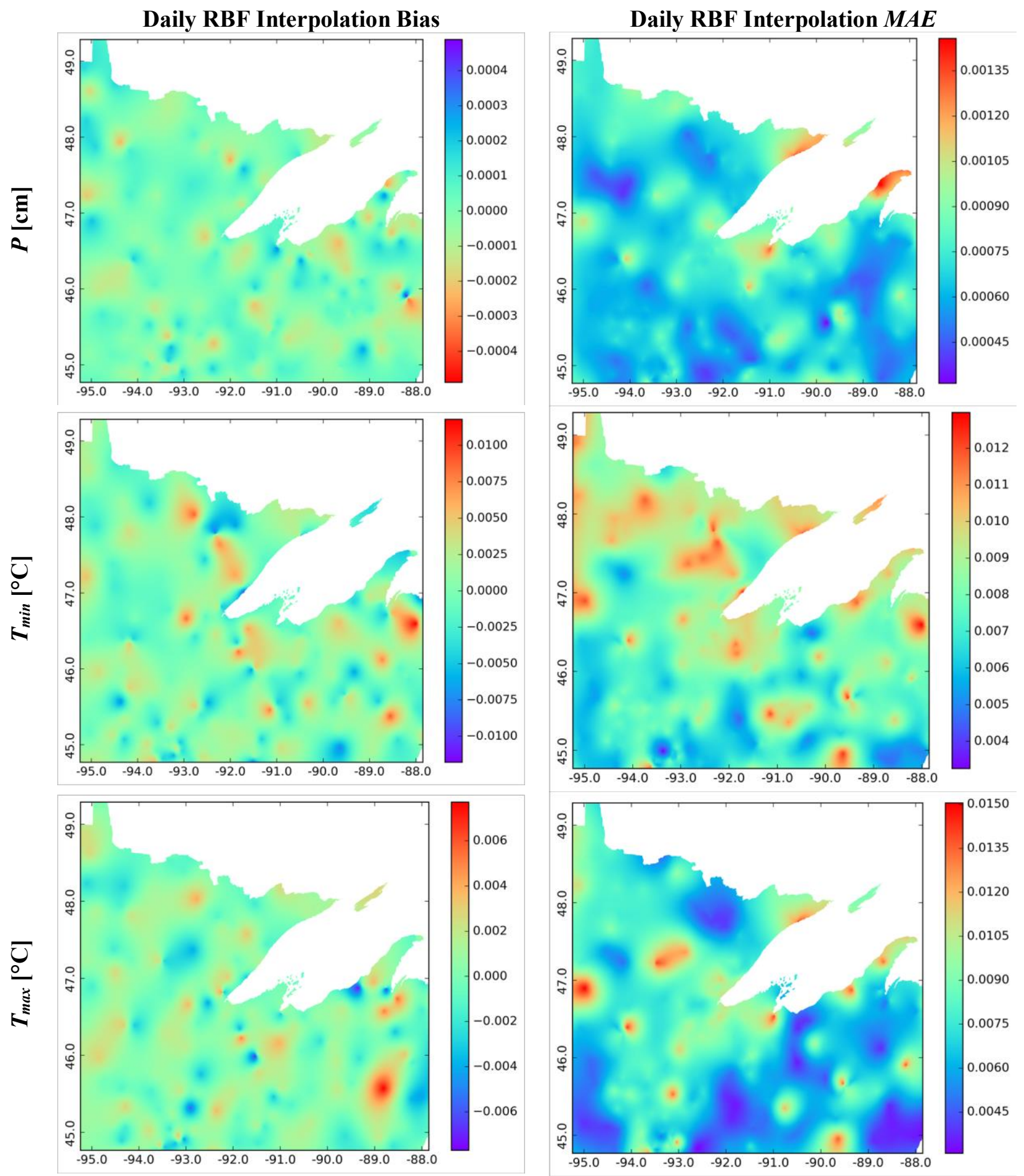

Figure 3.5: Meteorological station network interpolation error maps based on a jackknife ("leave-one-out") procedure. 
Table 3.1: Temperature-based meteorological indicators calculated on a daily basis for analyses in this work.

\begin{tabular}{|c|c|c|}
\hline Variable category and name & Units & Abbreviation \\
\hline \multicolumn{3}{|l|}{ Temperature indicators } \\
\hline Daily $T_{\min }$ & ${ }^{\circ} \mathrm{C}$ & grid_tmin \\
\hline 3-day mean $T_{\min }$ & ${ }^{\circ} \mathrm{C}$ & tmin $03 d \_a v g$ \\
\hline 3-day $T_{\min }$ variance & ${ }^{\circ} \mathrm{C}^{2}$ & tmin_03d_var \\
\hline 7-day mean $T_{\min }$ & ${ }^{\circ} \mathrm{C}$ & tmin_07d_avg \\
\hline 7-day $T_{\min }$ variance & ${ }^{\circ} \mathrm{C}^{2}$ & tmin_07d_var \\
\hline 15-day mean $T_{\min }$ & ${ }^{\circ} \mathrm{C}$ & tmin $15 \mathrm{~d}$ avg \\
\hline 15-day $T_{\min }$ variance & ${ }^{\circ} \mathrm{C}^{2}$ & $\operatorname{tmin} 15 \mathrm{~d}$ var \\
\hline 30-day mean $T_{\min }$ & ${ }^{\circ} \mathrm{C}$ & tmin 30d avg \\
\hline 30-day $T_{\min }$ variance & ${ }^{\circ} \mathrm{C}^{2}$ & tmin 30d var \\
\hline 60-day mean $T_{\min }$ & ${ }^{\circ} \mathrm{C}$ & tmin_60d_avg \\
\hline 60-day $T_{\min }$ variance & ${ }^{\circ} \mathrm{C}^{2}$ & tmin_60d_var \\
\hline 90-day mean $T_{\min }$ & ${ }^{\circ} \mathrm{C}$ & tmin_90d_avg \\
\hline 90-day $T_{\min }$ variance & ${ }^{\circ} \mathrm{C}^{2}$ & tmin_90d_var \\
\hline Daily $T_{\max }$ & ${ }^{\circ} \mathrm{C}$ & grid_tmax \\
\hline 3-day mean $T_{\max }$ & ${ }^{\circ} \mathrm{C}$ & tmax 03d avg \\
\hline 3-day $T_{\max }$ variance & ${ }^{\circ} \mathrm{C}^{2}$ & tmax_03d_var \\
\hline 7-day mean $T_{\max }$ & ${ }^{\circ} \mathrm{C}$ & tmax 07d avg \\
\hline 7-day $T_{\max }$ variance & ${ }^{\circ} \mathrm{C}^{2}$ & tmax 07d var \\
\hline 15-day mean $T_{\max }$ & ${ }^{\circ} \mathrm{C}$ & tmax_15d_avg \\
\hline 15-day $T_{\max }$ variance & ${ }^{\circ} \mathrm{C}^{2}$ & tmax_15d_var \\
\hline 30-day mean $T_{\max }$ & ${ }^{\circ} \mathrm{C}$ & tmax_30d_avg \\
\hline 30-day $T_{\max }$ variance & ${ }^{\circ} \mathrm{C}^{2}$ & tmax_30d_var \\
\hline 60-day mean $T_{\max }$ & ${ }^{\circ} \mathrm{C}$ & tmax_60d_avg \\
\hline 60-day $T_{\max }$ variance & ${ }^{\circ} \mathrm{C}^{2}$ & tmax $60 \mathrm{~d}$ var \\
\hline 90-day mean $T_{\max }$ & ${ }^{\circ} \mathrm{C}$ & tmax 90d avg \\
\hline 90-day $T_{\max }$ variance & ${ }^{\circ} \mathrm{C}^{2}$ & tmax 90d var \\
\hline Daily $T_{a v g}$ & ${ }^{\circ} \mathrm{C}$ & grid tavg \\
\hline 3-day mean $T_{a v g}$ & ${ }^{\circ} \mathrm{C}$ & tavg 03d avg \\
\hline 3-day $T_{a v g}$ variance & ${ }^{\circ} \mathrm{C}^{2}$ & tavg_03d_var \\
\hline 7-day mean $T_{a v g}$ & ${ }^{\circ} \mathrm{C}$ & tavg_07d_avg \\
\hline 7-day $T_{a v g}$ variance & ${ }^{\circ} \mathrm{C}^{2}$ & tavg_07d_var \\
\hline 15-day mean $T_{\text {avg }}$ & ${ }^{\circ} \mathrm{C}$ & tavg_15d_avg \\
\hline 15-day $T_{a v g}$ variance & ${ }^{\circ} \mathrm{C}^{2}$ & tavg_15d_var \\
\hline 30-day mean $T_{a v g}$ & ${ }^{\circ} \mathrm{C}$ & tavg 30d avg \\
\hline 30-day $T_{a v g}$ variance & ${ }^{\circ} \mathrm{C}^{2}$ & tavg 30d_var \\
\hline 60 -day mean $T_{a v g}$ & ${ }^{\circ} \mathrm{C}$ & tavg_60d_avg \\
\hline 60-day $T_{a v g}$ variance & ${ }^{\circ} \mathrm{C}^{2}$ & tavg_60d_var \\
\hline 90-day mean $T_{a v g}$ & ${ }^{\circ} \mathrm{C}$ & tavg_90d_avg \\
\hline 90-day $T_{a v g}$ variance & ${ }^{\circ} \mathrm{C}^{2}$ & tavg_90d_var \\
\hline \multicolumn{3}{|l|}{ Humidity indicators } \\
\hline Daily $V P D$ & $\mathrm{~Pa}$ & grid_vpd \\
\hline 3-day mean $V P D$ & $\mathrm{~Pa}$ & vpd 03d_avg \\
\hline 3-day $V P D$ variance & $\mathrm{Pa}^{2}$ & vpd 03d_var \\
\hline 7-day mean $V P D$ & $\mathrm{~Pa}$ & vpd_07d_avg \\
\hline 7-day $V P D$ variance & $\mathrm{Pa}^{2}$ & vpd_07d_var \\
\hline 15-day mean $V P D$ & $\mathrm{~Pa}$ & vpd_15d_avg \\
\hline 15 -day $V P D$ variance & $\mathrm{Pa}^{2}$ & vpd_15d_var \\
\hline 30-day mean $V P D$ & $\mathrm{~Pa}$ & vpd_30d_avg \\
\hline 30-day $V P D$ variance & $\mathrm{Pa}^{2}$ & vpd_30d_var \\
\hline 60-day mean $V P D$ & $\mathrm{~Pa}$ & vpd_60d_avg \\
\hline 60-day $V P D$ variance & $\mathrm{Pa}^{2}$ & vpd_60d_var \\
\hline 90-day mean $V P D$ & $\mathrm{~Pa}$ & vpd 90d avg \\
\hline 90-day $V P D$ variance & $\mathrm{Pa}^{2}$ & vpd 90d_var \\
\hline
\end{tabular}


Table 3.2: Temperature-based meteorological indicators calculated on a seasonal basis for analyses in this work. Seasons are evaluated according to the equinox ("veq" and "aeq") and solstice ("ssol" and "wsol") dates.

\begin{tabular}{|c|c|c|}
\hline Variable category and name & Units & Abbreviation \\
\hline \multicolumn{3}{|l|}{ Temperature indicators } \\
\hline Winter (90-day) mean $T_{\min }$ & ${ }^{\circ} \mathrm{C}$ & tmin 90d avg at veq \\
\hline Winter (90-day) $T_{\min }$ variance & ${ }^{\circ} \mathrm{C}^{2}$ & tmin 90d_var_at_veq \\
\hline Spring (90-day) mean $T_{\min }$ & ${ }^{\circ} \mathrm{C}$ & tmin_90d_avg_at_ssol \\
\hline Spring (90-day) $T_{\min }$ variance & ${ }^{\circ} \mathrm{C}^{2}$ & tmin_90d_var_at_ssol \\
\hline Summer (90-day) mean $T_{\min }$ & ${ }^{\circ} \mathrm{C}$ & tmin_90d_avg_at_aeq \\
\hline Summer (90-day) $T_{\min }$ variance & ${ }^{\circ} \mathrm{C}^{2}$ & tmin_90d_var_at_aeq \\
\hline Autumn (90-day) mean $T_{\min }$ & ${ }^{\circ} \mathrm{C}$ & tmin_90d_avg_at_wsol \\
\hline Autumn (90-day) $T_{\min }$ variance & ${ }^{\circ} \mathrm{C}^{2}$ & tmin 90d var at wsol \\
\hline Winter (90-day) mean $T_{\max }$ & ${ }^{\circ} \mathrm{C}$ & tmax 90d avg at veq \\
\hline Winter (90-day) $T_{\max }$ variance & ${ }^{\circ} \mathrm{C}^{2}$ & tmax 90d var at veq \\
\hline Spring (90-day) mean $T_{\max }$ & ${ }^{\circ} \mathrm{C}$ & tmax 90d avg at_ssol \\
\hline Spring (90-day) $T_{\max }$ variance & ${ }^{\circ} \mathrm{C}^{2}$ & tmax_90d_var_at_ssol \\
\hline Summer (90-day) mean $T_{\max }$ & ${ }^{\circ} \mathrm{C}$ & tmax_90d_avg_at_aeq \\
\hline Summer (90-day) $T_{\max }$ variance & ${ }^{\circ} \mathrm{C}^{2}$ & tmax_90d_var_at_aeq \\
\hline Autumn (90-day) mean $T_{\max }$ & ${ }^{\circ} \mathrm{C}$ & tmax_90d_avg_at_wsol \\
\hline Autumn (90-day) $T_{\max }$ variance & ${ }^{\circ} \mathrm{C}^{2}$ & tmax_90d_var_at_wsol \\
\hline Winter (90-day) mean $T_{a v g}$ & ${ }^{\circ} \mathrm{C}$ & tavg_90d_avg_at_veq \\
\hline Winter (90-day) $T_{a v g}$ variance & ${ }^{\circ} \mathrm{C}^{2}$ & tavg 90d var at veq \\
\hline Spring (90-day) mean $T_{a v g}$ & ${ }^{\circ} \mathrm{C}$ & tavg 90d avg at ssol \\
\hline Spring (90-day) $T_{a v g}$ variance & ${ }^{\circ} \mathrm{C}^{2}$ & tavg_90d_var_at_ssol \\
\hline Summer (90-day) mean $T_{a v g}$ & ${ }^{\circ} \mathrm{C}$ & tavg_90d_avg_at_aeq \\
\hline Summer (90-day) $T_{a v g}$ variance & ${ }^{\circ} \mathrm{C}^{2}$ & tavg_90d_var_at_aeq \\
\hline Autumn (90-day) mean $T_{a v g}$ & ${ }^{\circ} \mathrm{C}$ & tavg_90d_avg_at_wsol \\
\hline Autumn (90-day) $T_{a v g}$ variance & ${ }^{\circ} \mathrm{C}^{2}$ & tavg_90d_var_at_wsol \\
\hline
\end{tabular}

Table 3.3: Precipitation-based meteorological indicators calculated on a daily basis for the analyses in this work.

\begin{tabular}{|c|c|c|}
\hline Variable category and name & Units & Abbreviation \\
\hline \multicolumn{3}{|l|}{ Precipitation indicators } \\
\hline Daily precipitation & $\mathrm{cm}$ & grid prep \\
\hline 3-day total precipitation & $\mathrm{cm}$ & prep 03d sum \\
\hline 7-day total precipitation & $\mathrm{cm}$ & prcp_07d_sum \\
\hline 15-day total precipitation & $\mathrm{cm}$ & prcp_15d_sum \\
\hline 30-day total precipitation & $\mathrm{cm}$ & prcp_30d_sum \\
\hline 60-day total precipitation & $\mathrm{cm}$ & prep_60d_sum \\
\hline 90-day total precipitation & $\mathrm{cm}$ & prcp 90d sum \\
\hline 90-day precipitation days $(P>0)$ & $\mathrm{d}$ & prep 90d nd0_sum \\
\hline 90-day precipitation days $(P>10 \mathrm{~mm})$ & $\mathrm{d}$ & prep 90d nd10 sum \\
\hline 90-day precipitation days $(P>25 \mathrm{~mm})$ & $\mathrm{d}$ & prep_90d_nd25_sum \\
\hline 120-day total precipitation & $\mathrm{cm}$ & prep_120d_sum \\
\hline 180-day total precipitation & $\mathrm{cm}$ & prcp_180d_sum \\
\hline 365-day total precipitation & $\mathrm{cm}$ & prcp_365d_sum \\
\hline
\end{tabular}


Table 3.4: Precipitation-based meteorological indicators calculated on seasonal and annual bases for the analyses in this work. Seasons are evaluated according to the equinox ("veq" and "aeq") and solstice ("ssol" and "wsol") dates.

\begin{tabular}{|c|c|c|}
\hline Variable category and name & Units & Abbreviation \\
\hline \multicolumn{3}{|l|}{ Precipitation indicators } \\
\hline Winter (90-day) total precipitation & $\mathrm{cm}$ & prcp 90d sum at veq \\
\hline Winter (90-day) precipitation days $(P>0)$ & $\mathrm{d}$ & prep $90 \mathrm{~d}$ nd0 sum at veq \\
\hline Winter (90-day) moderate precipitation days $(P>10 \mathrm{~mm})$ & $\mathrm{d}$ & prep $90 \mathrm{~d}$ nd10 sum at veq \\
\hline Winter (90-day) heavy precipitation days $(P>25 \mathrm{~mm})$ & $\mathrm{d}$ & prep $90 \mathrm{~d}$ nd25 sum at veq \\
\hline Spring (90-day) total precipitation & $\mathrm{cm}$ & prep_90d_sum_at_ssol \\
\hline Spring (90-day) precipitation days $(P>0)$ & $\mathrm{d}$ & prcp_90d_nd0_sum_at_ssol \\
\hline Spring (90-day) moderate precipitation days $(P>10 \mathrm{~mm})$ & $\mathrm{d}$ & prep_90d_nd10_sum_at_ssol \\
\hline Spring (90-day) heavy precipitation days $(P>25 \mathrm{~mm})$ & $\mathrm{d}$ & prcp_90d_nd25_sum_at_ssol \\
\hline Summer (90-day) total precipitation & $\mathrm{cm}$ & prcp_90d_sum_at_aeq \\
\hline Summer (90-day) precipitation days $(P>0)$ & $\mathrm{d}$ & prcp_90d_nd0_sum_at_aeq \\
\hline Summer (90-day) moderate precipitation days $(P>10 \mathrm{~mm})$ & $\mathrm{d}$ & prep 90d nd10 sum at aeq \\
\hline Summer (90-day) heavy precipitation days $(P>25 \mathrm{~mm})$ & $\mathrm{d}$ & prep 90d nd25 sum at aeq \\
\hline Autumn (90-day) total precipitation & $\mathrm{cm}$ & prep 90d sum at wsol \\
\hline Autumn (90-day) precipitation days $(P>0)$ & $\mathrm{d}$ & prep 90d nd0 sum at wsol \\
\hline Autumn (90-day) moderate precipitation days $(P>10 \mathrm{~mm})$ & $\mathrm{d}$ & prep_90d_nd10_sum_at_wsol \\
\hline Autumn (90-day) heavy precipitation days $(P>25 \mathrm{~mm})$ & $\mathrm{d}$ & prep_90d_nd25_sum_at_wsol \\
\hline Annual (365-day) total precipitation (on $31 \mathrm{Dec}$ ) & $\mathrm{cm}$ & prcp_365d_at_eoy \\
\hline
\end{tabular}

indicators used for the climatological analysis in Chapter 5. All of the temperature-based cold-and warmseason indicators described below are listed in Table 3.5.

I calculated the accumulations of chilling days $(C D)$, chilling degree-days $(C D D)$, and growing degree-days $(G D D)$ [de Reaumur, 1735; Baskerville and Emin, 1969; Thompson and Moncrieff, 1982; Lechowicz, 1984] on a daily basis as:

$$
\begin{gathered}
C D=\sum_{i=0 \text { at } 1 \mathrm{Jul}}^{\text {current date }} d_{i} \text { with } d_{i}=\left\{\begin{array}{l}
1 \text { if } T_{\text {avg, } i}<T_{\text {base }} \\
0 \text { otherwise }
\end{array}\right. \\
C D D=\sum_{i=0 \text { at } 1 \text { Jul }}^{\text {current date }} d d_{i} \text { with } d d_{i}=\max \left[0,\left(T_{\text {base }}-T_{\text {avg }, i}\right)\right] \\
G D D=\sum_{i=0 \text { at 1 Jan }}^{\text {current date }} d d_{i} \text { with } d d_{i}=\max \left[0,\left(T_{\text {avg, } i}-T_{\text {base }}\right)\right]
\end{gathered}
$$

For these calculations, I used $T_{\text {base }}=5^{\circ} \mathrm{C}$ following numerous empirical studies of tree physiology and Spring phenology [Cannell and Smith, 1983; Murray et al., 1989; Hunter and Lechowicz, 1992; Fisher et al., 2007; Schenker et al., 2014; Viherä-Aarnio et al., 2014; Körner, 2015]. Accepted values of $T_{\text {base }}$ vary with species and setting, and those used for analysis of forest areas can differ greatly from values used in 
Table 3.5: Temperature-based cold-, warm-, and growing-season indicators calculated on daily and seasonal bases for the analyses in this work. All derived cold- and warm-season indicators use $T_{\text {avg }}$ unless specified otherwise. Seasons are evaluated according to the equinox ("veq" and "aeq") and solstice ("ssol" and "wsol") dates.

\begin{tabular}{|c|c|c|}
\hline Variable category and name & Units & Abbreviation \\
\hline \multicolumn{3}{|l|}{ Cold season indicators (accumulated from 1 July through 30 June) } \\
\hline$C D$ at end of Winter & $\mathrm{d}$ & chill d at veq \\
\hline$C D D$ at end of Winter & ${ }^{\circ} \mathrm{C} \cdot \mathrm{d}$ & chill dd at veq \\
\hline$F D$ (using $T_{\min }$ ) at end of Winter & $\mathrm{d}$ & tmin frz days at veq \\
\hline Spring $C D$ & $\mathrm{~d}$ & cd veq to ssol \\
\hline Spring $C D D$ & ${ }^{\circ} \mathrm{C} \cdot \mathrm{d}$ & cdd_veq to ssol \\
\hline Spring $F D$ (using $T_{\min }$ ) & $\mathrm{d}$ & tmin frz veq to ssol \\
\hline$C D$ at beginning of plateau & $\mathrm{d}$ & chill_d_at_ssol \\
\hline$C D D$ at beginning of plateau & ${ }^{\circ} \mathrm{C} \cdot \mathrm{d}$ & chill_dd_at_ssol \\
\hline$F D$ at beginning of plateau (using $T_{\min }$ ) & $\mathrm{d}$ & tmin_frz_days_at_ssol \\
\hline Summer $C D$ & $\mathrm{~d}$ & chill d at aeq \\
\hline Summer $C D D$ & ${ }^{\circ} \mathrm{C} \cdot \mathrm{d}$ & chill dd at aeq \\
\hline Summer $F D$ (using $T_{\min }$ ) & $\mathrm{d}$ & tmin frz days at aeq \\
\hline Autumn $C D$ & $\mathrm{~d}$ & cd aeq to wsol \\
\hline Autumn $C D D$ & ${ }^{\circ} \mathrm{C} \cdot \mathrm{d}$ & cdd aeq to wsol \\
\hline Autumn $F D$ (using $T_{\min }$ ) & $\mathrm{d}$ & tmin_frz_aeq_to_wsol \\
\hline$C D$ at end of Autumn & $\mathrm{d}$ & chill d at wsol \\
\hline$C D D$ at end of Autumn & ${ }^{\circ} \mathrm{C} \cdot \mathrm{d}$ & chill_dd_at_wsol \\
\hline$F D$ (using $T_{\min }$ ) at end of Autumn & $\mathrm{d}$ & tmin_frz_days_at_wsol \\
\hline Cold season intensity & ${ }^{\circ} \mathrm{C}$ & intensity Winter \\
\hline Date of last Spring freeze/frost (using $T_{\min }$ ) & DOY & doy last spring tmin frz \\
\hline$C D$ at last Spring freeze/frost (using $T_{\min }$ ) & $\mathrm{d}$ & cd last spring tmin frz \\
\hline$C D D$ at last Spring freeze/frost (using $T_{\min }$ ) & ${ }^{\circ} \mathrm{C} \cdot \mathrm{d}$ & cdd last_spring tmin frz \\
\hline Date of first Autumn freeze/frost (using $T_{\min }$ ) & DOY & doy first_autumn tmin frz \\
\hline$C D$ at first Autumn freeze/frost (using $T_{\min }$ ) & $\mathrm{d}$ & cd_first_autumn_tmin_frz \\
\hline$C D D$ at first Autumn freeze/frost (using $T_{\min }$ ) & ${ }^{\circ} \mathrm{C} \cdot \mathrm{d}$ & cdd_first_autumn_tmin_frz \\
\hline \multicolumn{3}{|c|}{ Warm season indicators (accumulated from 1 January through 31 December) } \\
\hline$G D D\left(T_{\text {base }}=5^{\circ} \mathrm{C}\right)$ & ${ }^{\circ} \mathrm{C} \cdot \mathrm{d}$ & grow_dd \\
\hline$G D D\left(T_{\text {base }}=0^{\circ} \mathrm{C}\right)$ & ${ }^{\circ} \mathrm{C} \cdot \mathrm{d}$ & grow dd base 0 \\
\hline$G D D$ at end of Winter & ${ }^{\circ} \mathrm{C} \cdot \mathrm{d}$ & grow dd at veq \\
\hline$G D D\left(T_{\text {base }}=0^{\circ} \mathrm{C}\right)$ at end of Winter & ${ }^{\circ} \mathrm{C} \cdot \mathrm{d}$ & grow dd base 0 at_veq \\
\hline$G D D$ at end of Spring & ${ }^{\circ} \mathrm{C} \cdot \mathrm{d}$ & grow_dd_at_ssol \\
\hline$G D D\left(T_{\text {base }}=0^{\circ} \mathrm{C}\right)$ at end of Spring & ${ }^{\circ} \mathrm{C} \cdot \mathrm{d}$ & grow_dd_base0_at_ssol \\
\hline Spring $G D D$ & ${ }^{\circ} \mathrm{C} \cdot \mathrm{d}$ & gdd_veq_to_ssol \\
\hline$G D D$ from beginning of Spring to beginning of $C D$ plateau & ${ }^{\circ} \mathrm{C} \cdot \mathrm{d}$ & gdd_veq to plateau \\
\hline Date at beginning of $C D$ plateau & DOY & doy_plateau_begin \\
\hline$G D D$ at beginning of $C D$ plateau & ${ }^{\circ} \mathrm{C} \cdot \mathrm{d}$ & gdd_plateau_begin \\
\hline$G D D$ at last Spring freeze/frost & ${ }^{\circ} \mathrm{C} \cdot \mathrm{d}$ & gdd last spring tmin frz \\
\hline$G D D$ at end of Summer & ${ }^{\circ} \mathrm{C} \cdot \mathrm{d}$ & grow dd at aeq \\
\hline$G D D\left(T_{\text {base }}=0^{\circ} \mathrm{C}\right)$ at end of Summer & ${ }^{\circ} \mathrm{C} \cdot \mathrm{d}$ & grow dd base 0 at aeq \\
\hline Summer $G D D$ & ${ }^{\circ} \mathrm{C} \cdot \mathrm{d}$ & gdd ssol to aeq \\
\hline$G D D$ accumulated during Spring and Summer & ${ }^{\circ} \mathrm{C} \cdot \mathrm{d}$ & gdd_veq_to_aeq \\
\hline$G D D$ from beginning of Autumn to end of $C D$ plateau & ${ }^{\circ} \mathrm{C} \cdot \mathrm{d}$ & gdd aeq to plateau end \\
\hline Date at end of $C D$ plateau & DOY & doy_plateau_end \\
\hline$G D D$ at end of $C D$ plateau & ${ }^{\circ} \mathrm{C} \cdot \mathrm{d}$ & gdd plateau end \\
\hline$G D D$ from end of $C D$ plateau to end of Autumn & ${ }^{\circ} \mathrm{C} \cdot \mathrm{d}$ & gdd_plateau_to_wsol \\
\hline$G D D$ at first Autumn freeze/frost & ${ }^{\circ} \mathrm{C} \cdot \mathrm{d}$ & gdd_first_autumn_tmin_frz \\
\hline$G D D$ at end of Autumn & ${ }^{\circ} \mathrm{C} \cdot \mathrm{d}$ & grow dd at wsol \\
\hline$G D D\left(T_{\text {base }}=0^{\circ} \mathrm{C}\right)$ at end of Autumn & ${ }^{\circ} \mathrm{C} \cdot \mathrm{d}$ & grow dd base 0 at_wsol \\
\hline Autumn $G D D$ & ${ }^{\circ} \mathrm{C} \cdot \mathrm{d}$ & gdd aeq to wsol \\
\hline \multicolumn{3}{|l|}{ Growing season indicators } \\
\hline Frost-free season duration & $\mathrm{d}$ & frost_free_season_days \\
\hline$G D D$ accumulated during frost-free season & ${ }^{\circ} \mathrm{C} \cdot \mathrm{d}$ & frost free season gdd \\
\hline$C D$ plateau duration & $\mathrm{d}$ & days plateau length \\
\hline$G D D$ accumulated during $C D$ plateau & ${ }^{\circ} \mathrm{C} \cdot \mathrm{d}$ & gdd plateau length \\
\hline Growing season intensity & ${ }^{\circ} \mathrm{C}$ & intensity plateau \\
\hline
\end{tabular}


agricultural applications [Skaggs and Irmak, 2012]. In general, there is an inverse relationship between the value of $T_{\text {base }}$ and the accumulation of $G D D$ required to attain certain phenophases such as budburst or flowering [Trudgill et al., 2005]. I recognize that $T_{\text {base }}$ and the biophysical efficiency of GDD and photoperiod use in early phenophases can vary widely among species and even within species across climatological settings, which might be evaluated using remote sensing indicators of phenophases. Such a sensitivity analysis remains beyond the intended scope of this work. For simplicity in my climatological analysis, I have selected a single value of $T_{\text {base }}$ in common use for temperate tree species to be applied across the study area. On this common basis, calculated $G D D$ accumulations can be combined with vegetation phenological observations to differentiate among fast- and slow-developing, or more and less cold-adapted, forest phenotypes.

I calculated numerous meteorological indicators on a daily basis for seasonal analyses in Chapter 5 and for use with Landsat scenes in Chapter 6 (Fig. 3.6). Among these indicators are the accumulation of freezing days $\left(F D\right.$, using $\left.T_{\text {base }}=0^{\circ} \mathrm{C}\right)$ through the cold season using both $T_{a v g}$ and $T_{\min }$ similar to eq. (3.5). Cold season variables $(C D, C D D$, and $F D)$ are accumulated from 1 July through the following Spring, and warm season variables $(G D D)$ are accumulated from 1 January through the calendar year. I calculated temperature statistics over various periods using the mean and variance of $T_{\min }, T_{\max }$, and $T_{\text {avg }}$, and total $P$ over numerous periods up to a full year. For seasonal analyses, I accumulated days within a 90-day period prior to equinox and solstice dates with any $(P>0)$, moderate $(1 \mathrm{~cm}<P \leq 2.5 \mathrm{~cm})$, and heavy $(P>2.5$ $\mathrm{cm})$ precipitation.

I defined the climatological growing season, which is to be compared with the vegetation growing season as determined by Landsat observations in Chapter 6, in two ways. The first is a traditional definition based on the time between last Spring frost and first Autumn frost dates using nighttime freezing temperatures $\left(T_{\min }<0^{\circ} \mathrm{C}\right)$ [Kunkel et al., 2004; Skaggs and Irmak, 2012; Yu et al., 2014; McCabe et al., 2015]. Frost dates and the duration of the frost-free season are useful for assessing long-term seasonal changes as well as acute indicators of possible vegetation freeze damage, especially in the transition from cold to warm seasons [Augspurger, 2013]. Alternatively, I also defined the growing 


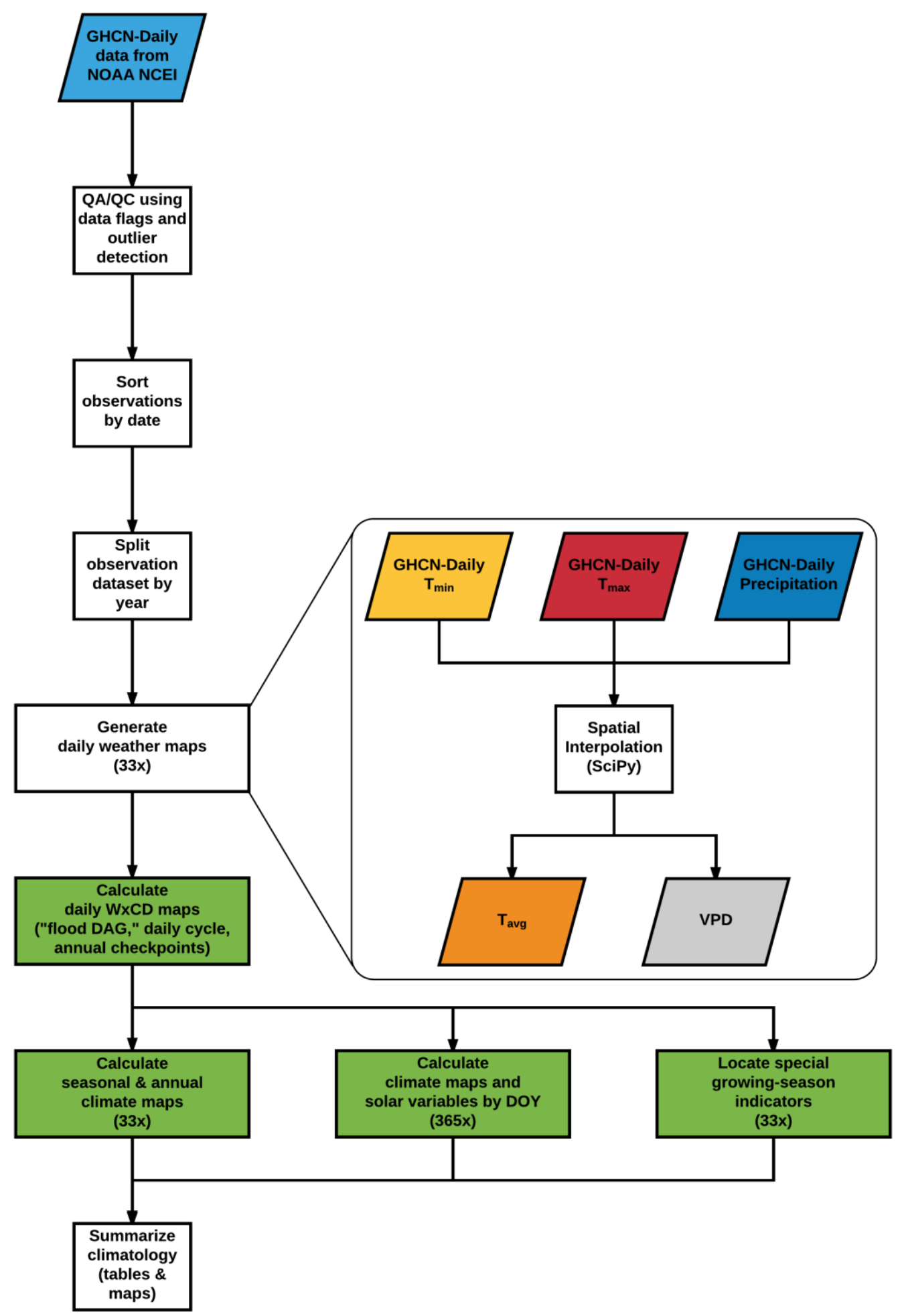

Figure 3.6: Climatological processing and analysis procedure. Wide process boxes are those procedures that were easily divided for distributed processing at $\mathrm{UW}$-Madison CHTC, as described in Chapter 4. 
season as a function of $C D$ accumulation. I observed that $C D$ accumulation reaches a warm-season plateau soon after $G D D$ accumulation begins in the Spring. The $C D$ accumulation then departs from that plateau in the Autumn just before $G D D$ accumulation ceases. Between these dates, $T_{\text {avg }} \geq T_{\text {base }}$ and vegetation defenses against freezing are limited while net primary productivity is generally dedicated to growth and reproduction [Schenker et al., 2014; Vitasse et al., 2014; Körner, 2015; Pagter et al., 2015]. The resulting plateau-based growing season is (by definition) several days longer than the frost-free season for the same year.

The differences between these season-starting and -ending dates are of great interest: within those periods, many forest species are vulnerable to environmental conditions that could affect vegetation carbon uptake over the entire growing season, as in the case of a late Spring frost, or bring the growing season to an early close with an Autumn frost event that triggers leaf senescence and the tree's Winter preparations. To evaluate this danger, I also calculated $G D D$ accumulation at the date of the last Spring frost. With more accumulated $G D D$ near the beginning of the growing season, there is a greater chance that the opening of flowers and leaves on many species makes them vulnerable to a freezing event that can adversely affect productivity through the remainder of the growing season. In some cases, severe late frost events have followed a "false Spring" period brought on by shifts in synoptic influences over several weeks, from cold to warm and then to cold again. Two such events within my study area, in 2007 and 2010, will be discussed in Chapter 5.

Using my alternative definition of the growing season, I also evaluated two aggregate seasonal measures. I defined the "cold season intensity" CSI as

$$
C S I=\frac{C D D_{\text {plateau beginning }}}{C D_{\text {plateau beginning }}}
$$

Cold season duration is represented well by $C D$, but that measure does not indicate the severity of the season: a particular Winter could be cold or relatively mild, but $C D$ will continue to accumulate as long as $T_{\text {avg }}<T_{\text {base. }}$ Conversely, accumulated $C D D$ alone has little phenological meaning unless it is related to a calendar duration of some importance. Together, $C D$ and $C S I$ provide a composite indication of both the 
duration and severity of the cold season. Two Winters with similar $C D$ accumulations may be differentiated by their $C D D$ or $C S I$ values, providing a distinction between cold and relatively mild seasons. Although trees may not recognize a difference between one sub-freezing temperature and another, with regional warming both $C D$ and $C S I$ (as calculated by these definitions) would diminish over time but at different rates. Warmer Winters can interfere with endodormancy ("Winter chill”) requirements for many forest species [Morin et al., 2009], leading to altered phenological cycles and reduced primary productivity in subsequent growing seasons.

Accordingly, my aggregate measure for the warm season is a "growing season intensity" GSI defined as

$$
G S I=\frac{G D D_{\text {plateau end }}-G D D_{\text {plateau beginning }}}{D O Y_{\text {plateau end }}-D O Y_{\text {plateau beginning }}}
$$

with DOY as the day-of-year and referring to the $C D$ plateau as discussed above. In this case, both $G D D$ and growing season duration remain relevant: phenophases (e.g. budburst, leaf size thresholds, maturity) are frequently associated with GDD accumulation [Cannell and Smith, 1983; Trudgill et al., 2005], and there is evidence that senescence timing depends on both inherent and external limits to leaf longevity, such as photoperiod [Kikuzawa et al., 2013]. This GSI metric is separately useful as an indicator of temperature-related influences throughout the growing season, such as transpiration moisture demand and, if precipitation is inadequate through the season, the likelihood of vegetation moisture stress [Koster et al., 2014]. Tracking GSI through the growing season, especially using its rate of change on a short-term basis, enables sub-seasonal monitoring of vegetation moisture status that, if stressed, could lead to reduced capacity for carbon uptake and growth [Teskey et al., 1987], leaf wilting [Munné-Bosch and Alegre, 2004; Marchin et al., 2010], and litter drying with evolving conditions conducive to forest fires [Yebra et al., 2013] and other disturbance agents.

There are a number of caveats to be noted regarding the time series analyses that I perform here. Although the 30-year period that I have examined generally meets the customary duration criterion for climatological analyses, it is a relatively short period for trend analysis. Within the study period, I have 
assumed and analyzed trends by linear regression instead of higher-order functions that may fit the examined time series better. Linear long-term trends ignore possible breaks and shifts within the 30 -year analysis. Although I do examine changes in (linear) trends across the 1997-1998 Winter season that can be extracted from these time series, there is a necessary trade-off where each of those trends covers a much shorter period and is therefore less likely to represent a trend of long-term climatological significance. Trends important to the physical system may not demonstrate statistical significance, and not all statistically significant trends indicate physical processes that can be adequately represented in conceptual or computational models of the land-lake-atmosphere system examined here. I am particularly sensitive to the likelihood that derived climate indicators, such as plateau-based growing season duration, incorporate more basic variables that may demonstrate their own significant trends, even though a trend in the derived indicator may not itself end up being significant. Such cases can sometimes be attributed to opposing trends in the constituent indicators.

\subsection{Landsat TM/ETM+ Scenes}

I identified and downloaded Landsat scenes for the five footprints in Fig. 3.1 over the 1984-2013 period using the USGS EROS Data Center's EarthExplorer web application (earthexplorer.usgs.gov). I selected each scene in the collection based on a combination of little (ideally, zero) snow cover and little cloud cover (generally $<50 \%$ ). In the P26R27 footprint, where approximately half of the footprint covers Lake Superior, I applied that cloud cover criterion over only the land area of the footprint. A complete list of scene identifiers collected for each of the five Landsat footprints examined here is given in the Appendix. Overall, I obtained 1146 Landsat scenes on 823 unique dates within the study period; Table 3.6 summarizes the Landsat scenes that I have employed for this work. Figure 3.7 shows the distribution of collected scenes in each footprint throughout the study period, and Fig. 3.8 summarizes the temporal distribution of those scenes through the year. The limited availability of suitable Landsat scenes in Spring and Autumn, primarily due to cloud cover in the transition seasons, can be expected to play a role in the analyses that follow, especially in the diagnosis of phenology near the start and end of the growing 
Table 3.6: Landsat scene counts by footprint (Fig. 3.1) and sensor.

\begin{tabular}{|c|c|c|c|c|c|c|c|}
\hline \multirow{3}{*}{$\begin{array}{c}\text { WRS-2 } \\
\text { Path/Row } \\
\text { Designation }\end{array}$} & \multirow{3}{*}{$\begin{array}{c}\begin{array}{c}\text { Landsat } 5 \\
\text { (TM) }\end{array} \\
1984-2011 \\
\end{array}$} & \multicolumn{2}{|c|}{ Landsat 7 (ETM+) } & \multirow{3}{*}{$\begin{array}{c}\text { Total } \\
\text { Images }\end{array}$} & \multirow{2}{*}{\multicolumn{3}{|c|}{ Images Per Year }} \\
\hline & & \multirow{2}{*}{$\begin{array}{c}\text { (SLC-on) } \\
1999-2003\end{array}$} & \multirow{2}{*}{$\begin{array}{r}\text { (SLC-off) } \\
2003-2013\end{array}$} & & & & \\
\hline & & & & & Avg & Min & Max \\
\hline P25R28 & 152 & 18 & 61 & 231 & 7.7 & 1 & 18 \\
\hline P26R27 & 133 & 17 & 52 & 202 & 6.7 & 2 & 13 \\
\hline P26R28 & 162 & 24 & 64 & 250 & 8.3 & 3 & 14 \\
\hline P27R27 & 145 & 21 & 39 & 205 & 6.8 & 1 & 11 \\
\hline P27R28 & 176 & 24 & 57 & 257 & 8.6 & 2 & 15 \\
\hline Total & 768 & 104 & 274 & 1146 & 7.6 & 1 & 18 \\
\hline
\end{tabular}

season. The model that I present in Chapter 4 is designed to overcome some limitations of data availability, but an even longer study with more scenes available in the transition seasons would likely help improve phenological model accuracy.

My Landsat pre-processing procedure is illustrated in Fig. 3.9. I processed each downloaded Landsat scene package (containing separate band images and metadata) using LEDAPS [Masek et al., 2008, 2012] to derive atmosphere-corrected surface reflectance values. I used the object-based Fmask algorithm [Zhu and Woodcock, 2012] to identify and mask clouds and surface cloud shadows. I then examined the images in order to determine the maximum geographic area covered by all of the images in a footprint and cropped each image in the collection to that extent. I then visually inspected and compared each image in the collection for geographic alignment and overall composition, which led to the exclusion of one downloaded scene in the P26R27 footprint because of geographic misregistration.

For each footprint in the study area, I derived a forest mask using the USGS NLCD 2001, 2006, and 2011 products that are discussed below. For each of these products, I selected the principal forest categories (deciduous forest, evergreen forest, mixed forest, and woody wetlands) for inclusion in my analyses and masked out all other land cover categories. The overall forest mask is based on the union of these three product-based masks, such that a pixel that was classified as forest (or woody wetland) in any of those NLCD products would be included in my analyses. This process masked out streams, rivers, and open water where those were indicated in the NLCD product, but I also used an image-based criterion for masking water locations: for each Landsat scene in each footprint, I calculated the Tasseled Cap wetness image (described below) and imposed a threshold at a high wetness value selected from a sample of 


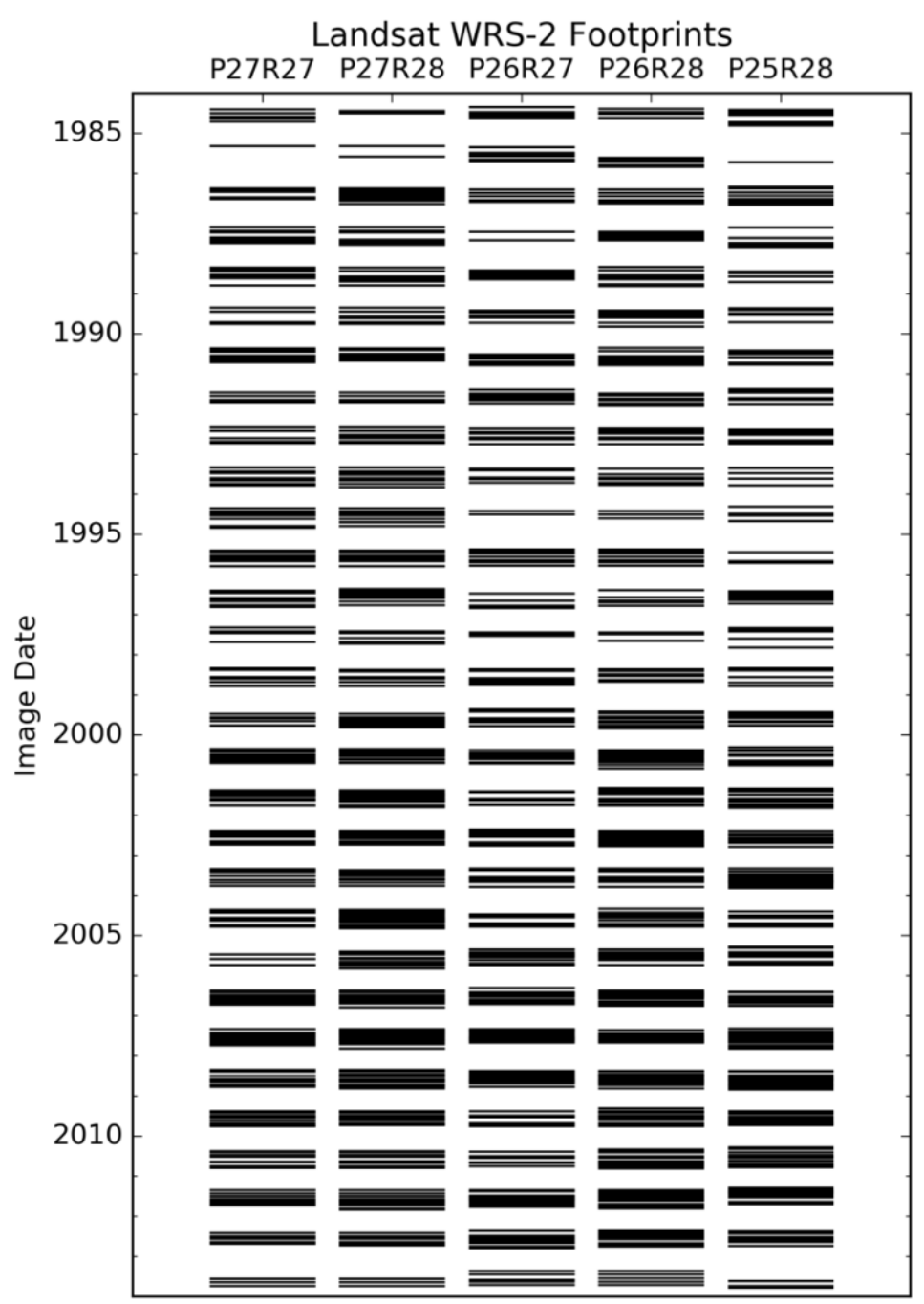

Figure 3.7: Landsat scene dates, by footprint. Full Landsat scene designators are given in the Appendix.

individual image histograms, masking out all areas above that threshold. Because of both seasonal and interannual variability in precipitation, and potentially due to changing surface hydrology and

groundwater conditions, many open water areas in the study area expanded and contracted over time, and these changes were evident in the Landsat scenes. I then used a union of water masks for all scenes in each footprint and combined that with the land cover mask, excluding riparian (and some lakeshore) areas that were noticeably inundated at various times during the study period.

Forest health and phenology have been observed by remote sensing for several decades using some standard spectral vegetation indexes (VIs). Previous remote sensing studies of regional and global phenology have employed observations from AVHRR ( 8-km pixels) over similar time spans [Buitenwerf 


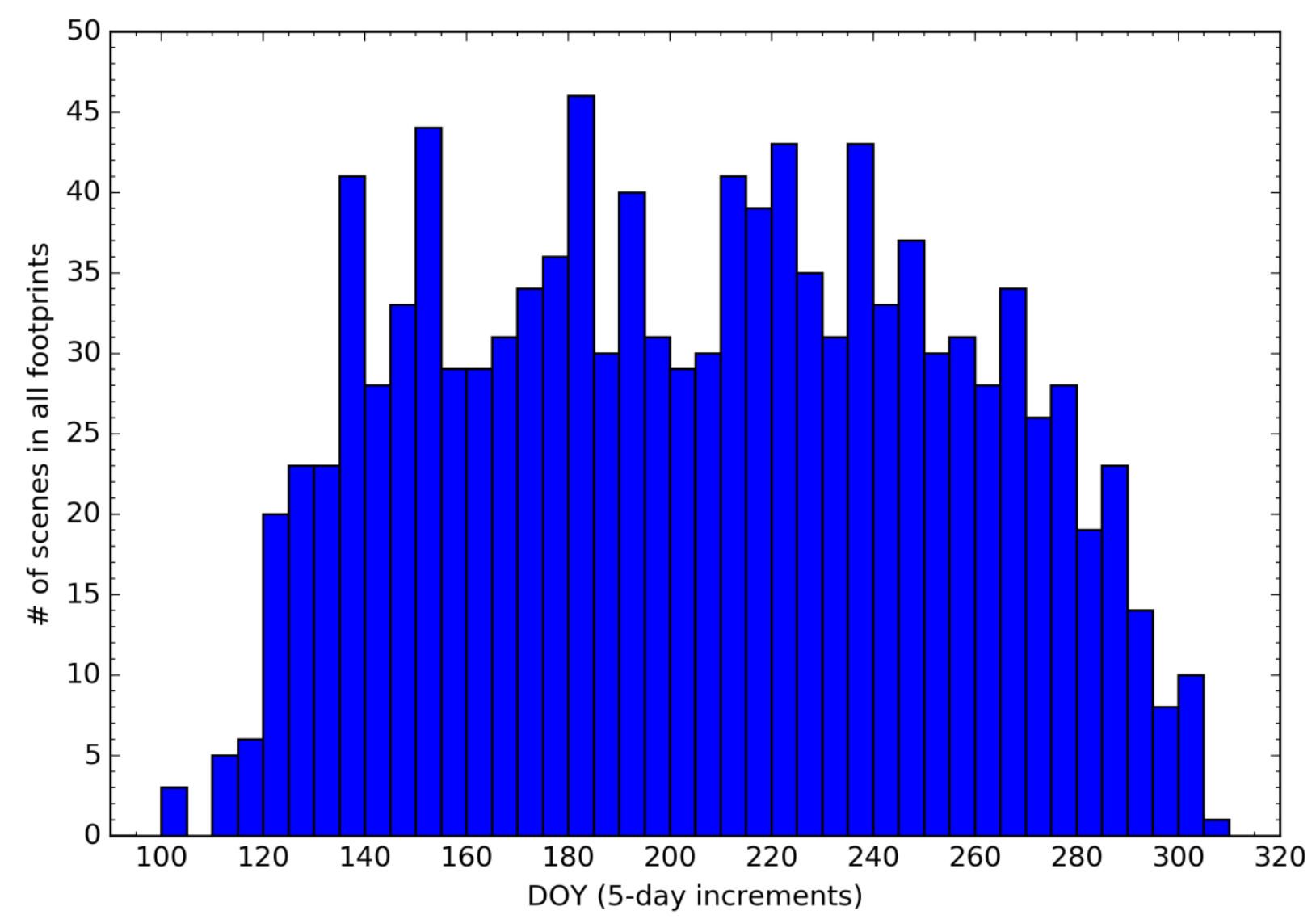

Figure 3.8: Distribution of collected Landsat scenes by day-of-year (DOY) for all footprints in the study area.

et al., 2015; Zhao et al., 2015], SPOT VEGETATION (1-km pixels) in the period since 1999 [Ivits et al., 2013], and MODIS (from 250-m to 1000-m pixels) since 2000 [Friedl et al., 2010; Ganguly et al., 2010]. Many analyses use the normalized difference vegetation index (NDVI) [Sellers et al., 1996], primarily because of its long-term availability from these observing platforms. The NDVI is calculated using the red (b3) and near-infrared (NIR, b4) Landsat surface reflectance bands:

$$
N D V I=\frac{b 4-b 3}{b 4+b 3}
$$

The enhanced vegetation index (EVI) [Huete et al., 1997], developed to reduce atmospheric aerosol effects on NDVI that can diminish the vegetation signal, is growing in use as a standard MODIS product [Huete et al., 2002]. The EVI is calculated using the blue (b1), red (b3), and NIR (b4) Landsat surface reflectance bands with several adjustment factors: 


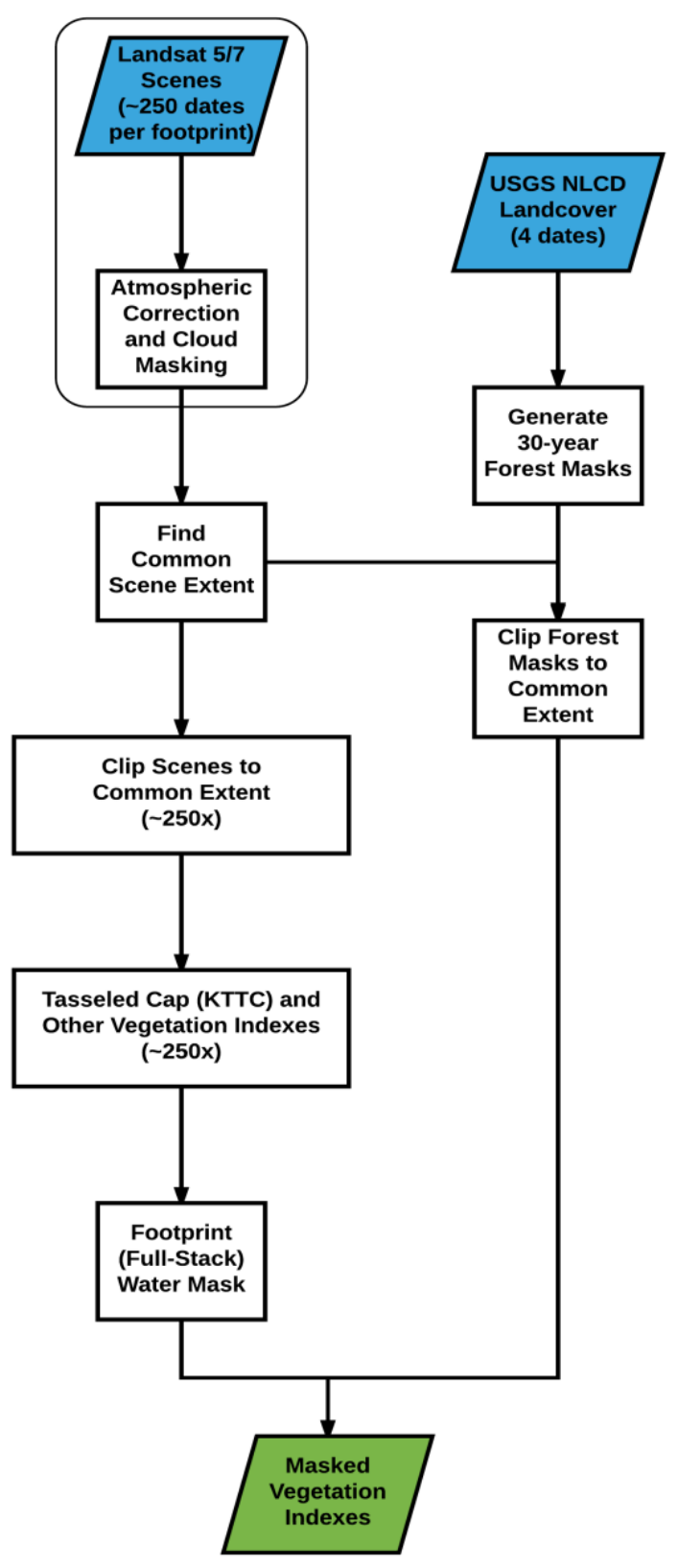

Figure 3.9: Landsat image processing procedure. Wide process boxes are those procedures that were easily divided for distributed processing at UWMadison CHTC, as described in Chapter 4. 


$$
E V I=G \frac{b 4-b 3}{b 4+C_{1} b 3-C_{2} b 1+L}
$$

using $G=2.5, C_{I}=6.0, C_{2}=7.5$, and $L=1.0$. Finally, the normalized difference infrared index (NDII) [Hardisky et al., 1983], variously referred to as the normalized difference moisture index (NDMI) [Jin and Sader, 2005] or the normalized difference wetness index (NDWI) [Gao, 1996], was developed to indicate possible vegetation moisture stress using a Landsat shortwave infrared (SWIR, b5) band:

$$
N D I I=\frac{b 4-b 5}{b 4+b 5}
$$

With SWIR sensitivity to foliar and canopy water content [Hunt et al., 1987, 2011; Yilmaz et al., 2008], NDII thus supports detection of conditions such as moisture stress and dry vegetation. These data can then be factored into phenology, forest health, and disturbance likelihood indicators that may not show such signals as early (or as clearly) as with NDVI [Isaacson et al., 2012].

It was for these reasons of sensitivity that I also used the Kauth-Thomas Tasseled Cap (KTTC) [Crist and Cicone, 1984; Crist, 1985, Crist and Kauth, 1986] transformation, with the idea that I would be able to identify possible disturbance events in three dimensions instead of just one dimension, as any of the individual spectral VIs allows. Working with this multidimensional response to vegetation change, Healey et al. [2005] used the KTTC as the basis for their forest-oriented Disturbance Index. The KTTC transformation coefficients using Landsat TM/ETM+ surface reflectance values [Crist, 1985] are:

$$
\begin{gathered}
B G T=0.2043 * b 1+0.4158 * b 2+0.5524 * b 3+0.5741 * b 4 \\
+0.3124 * b 5+0.2303 * b 7 \\
G R N=-0.1603 * b 1-0.2819 * b 2-0.4934 * b 3+0.7940 * b 4 \\
-0.0002 * b 5-0.1446 * b 7 \\
W E T=0.0315 * b 1+0.2021 * b 2+0.3102 * b 3+0.1594 * b 4 \\
-0.6806 * b 5-0.6109 * b 7
\end{gathered}
$$

for KTTC brightness, greenness, and wetness, respectively. As described above, I used the calculated WET component to mask surface water areas in each footprint. The WET component (often standardized 
over large areas) has been shown a useful proxy indicator for vegetation moisture content [Cohen, 1991; Toomey and Vierling, 2005] and like NDII is therefore ideal for disturbance detection [Collins and Woodcock, 1996; Jin and Sader, 2005; Hais et al., 2009]. The BGT and GRN components also have their specific applications that will be discussed in the model formulation below. Collins and Woodcock [1996] developed a multi-temporal Kauth-Thomas (MKT) transformation for change detection using "anniversary" image differencing, similar to the method in common use with individual VIs. In this work, I have sought to describe forest phenology and identify possible disturbances in greater dimensions of response than individual, standard VIs can provide and in a more robust, exacting, and informative framework than anniversary image differencing would allow. Combined use of standard VIs for phenological description and the KTTC components for disturbance identification support just such an approach.

\subsection{Land Cover and Additional Datasets}

To obtain some sense of the locations in my study area where phenological analysis would be useful and where land cover may have changed due to forest disturbances, I downloaded the USGS National Land Cover Database (NLCD) products [Jin et al., 2013; Homer et al., 2015] for 2001, 2006, and 2011. As described above, those pixels labeled forest (or woody wetlands) in any of these products were analyzed using the model described in Chapter 4; agricultural areas, shrub and grasslands, and urban areas were thus excluded from analysis. The NLCD 2011 product provides the base map in Fig. 3.2. I obtained topographic data for my study area from the USGS National Elevation Dataset (NED; ned.usgs.gov), which required stitching of individual tiles to obtain the base map shown in Fig. 3.1. To provide context for some results, Fig. 3.2 also includes the outlines of USEPA Level-IV ecoregions [Omernik et al., 2000; Omernik, 2004; USEPA, 2011] (www.epa.gov/eco-research/ecoregions) that are based on expert analyses of areas that generally share common physiography, climatology, and soil and vegetation types. 
To examine the possible effects of Lake Superior seasonal ice phenology on nearby land areas, I included observations of ice onset and break-up at Bayfield, Wisconsin, from two sources. I obtained records for 1984-2012 from the NOAA National Snow and Ice Data Center (NSIDC; www.nsidc.org) [Howk, 2009] and supplemented those with operations records from the nearby Madeline Island Ferry Line for 2011-2014 [Mary Ross, 2015, personal communication]. Both of these datasets indicate in their overlapping period that the area of Lake Superior near Bayfield did not completely freeze during the 2011-2012 Winter season, and ice-on/ice-off dates otherwise differ between these sources by only 1-2 days, thus I considered MIFL observations a reasonable proxy for Lake Superior ice cover records that were not yet available from the NSIDC for this work.

Finally, I evaluated the possible influences of global teleconnections on the regional climatology using monthly indices reported by the NOAA National Centers for Environmental Prediction (NCEP) Climate Prediction Center (CPC; www.cpc.ncep.noaa.gov) and the NOAA Earth Systems Research Laboratory (ESRL) Physical Sciences Division (PSD; www.esrl.noaa.gov/psd). From these records, I calculated three-month index averages for each season (DJF, MAM, JJA, and SON) for the Pacific Decadal Oscillation (PDO), the Pacific-North America (PNA) pattern, the Atlantic Multidecadal Oscillation (AMO), the Arctic Oscillation (AO), and the North Atlantic Oscillation (NAO). The El NiñoSouthern Oscillation (ENSO) index is traditionally summarized over an extended Winter (DJFM) period, for which I calculated the average index value in each of the reported equatorial Pacific Ocean regions (Niño-3 in the eastern Pacific, Niño-4 in the central Pacific, and Niño-3.4 overlapping parts of -3 and -4) in order to identify any differences in their influence. 


\section{CHAPTER 4}

\section{MODEL FORMULATION AND COMPUTATION}

This chapter presents a model of the possible relationship between the environment, represented with meteorological observations and calculated climatology, and vegetation phenology, represented using time series of Landsat-based spectral vegetation index images. We start by writing the relationship between climate and phenology as

$$
V(x, y, Y, D O Y)=f[W(x, y, Y, D O Y)]
$$

where $V$ is the calculated vegetation index value, $W$ is any of the climatological variables, $x$ and $y$ are coordinate (grid) locations, $Y$ is the year, and $D O Y$ is the ordinal day-of-year. From this relationship, all of the assumptions and implementation follow below. Both the theoretical background of this model and the practical challenges of applying the model over large areas ( $\sim 130$ million pixels in five $185 \mathrm{x} 185 \mathrm{~km}$ Landsat footprints) are addressed, with some notes on the scale and volume of computing resources employed for this work.

\subsection{Borrowing from Climatology}

Assumption 1: The long-term mean and short-term deviations of a variable are linearly separable.

Climatological analysis is the process of determining both the long-term means, in practice typically for a period of 30 years or more, and short-term deviations from the mean for various atmospheric states such as temperature or precipitation. In addition to the long-term mean, climatology may also account for a detectable trend and other long-term cycles over the analysis period. Just as important as the mean and trend is the measured variability about the mean that characterizes expected and extreme deviations. The separation of mean and variability is traditionally a linear operation, such that each is not expected to affect the other: even if the mean temperature increases over time, the statistics of observed variability about the mean do not change. This is known as the stationarity 
assumption and has been challenged by the speed of recent climate change but remains a useful first approximation for climatological analyses on minimal ( $\sim 30$-year) time scales as addressed here.

I have borrowed the linearity and stationarity assumptions from climatology for the description of forest phenology as well. Although this assumption may not always hold, in general it is expected that the mean varies slowly over time (possibly as a trend) and assumed that the mean and deviation components of a variable do not interact (which would indicate nonlinearity and, thus, nonstationarity). Numerous exceptions can occur with specific climatological conditions and in forest vegetation, especially considering tree growth and survival over long times and in the presence of disturbance agents. Regional climate change, as reported in Garcia and Townsend [2016], is one of the possible mechanisms of nonlinear interaction but generally occurs on slow time scales.

For the available weather and climate variables (Tables 3.1-3.5) and with an assumption of linear separability, a variable can be written as the sum of its mean and residual components:

$$
W(x, y, Y, D O Y)=\bar{W}(x, y, D O Y)+W^{\prime}(x, y, Y, D O Y)
$$

The mean value $\bar{W}$ is found by the average of $W$ at each $D O Y$ over the study period so that seasonal variability is accounted in the long-term mean for many of those variables. The residual $W^{\prime}$ can then be calculated and its use will be addressed below. The same assumption of separability is made for vegetation index time series calculated from the available Landsat image record:

$$
V(x, y, Y, D O Y)=\bar{V}(x, y, D O Y)+V^{\prime}(x, y, Y, D O Y)
$$

Again, note that $\bar{V}$ is the mean of $V$ for a specific $D O Y$ for all years in the observation record to account for seasonal variability in vegetation indices (that is, phenology), not the overall annual mean $V$ for a location. As above, deviations $V^{\prime}$ are specific to both year $Y$ (allowing for interannual variability in phenology) and DOY. From here I will drop the $(x, y)$ notation with the understanding that these operations are performed on a pixel-by-pixel basis, each with a unique geographic location and its own time series of $W$ and $V$. Two additional assumptions then follow for this work: that $\bar{V}$ is related to $\bar{W}$, and that $V^{\prime}$ is related to $W^{\prime}$. The model components exploiting these relationships are illustrated in Fig. 4.1. 

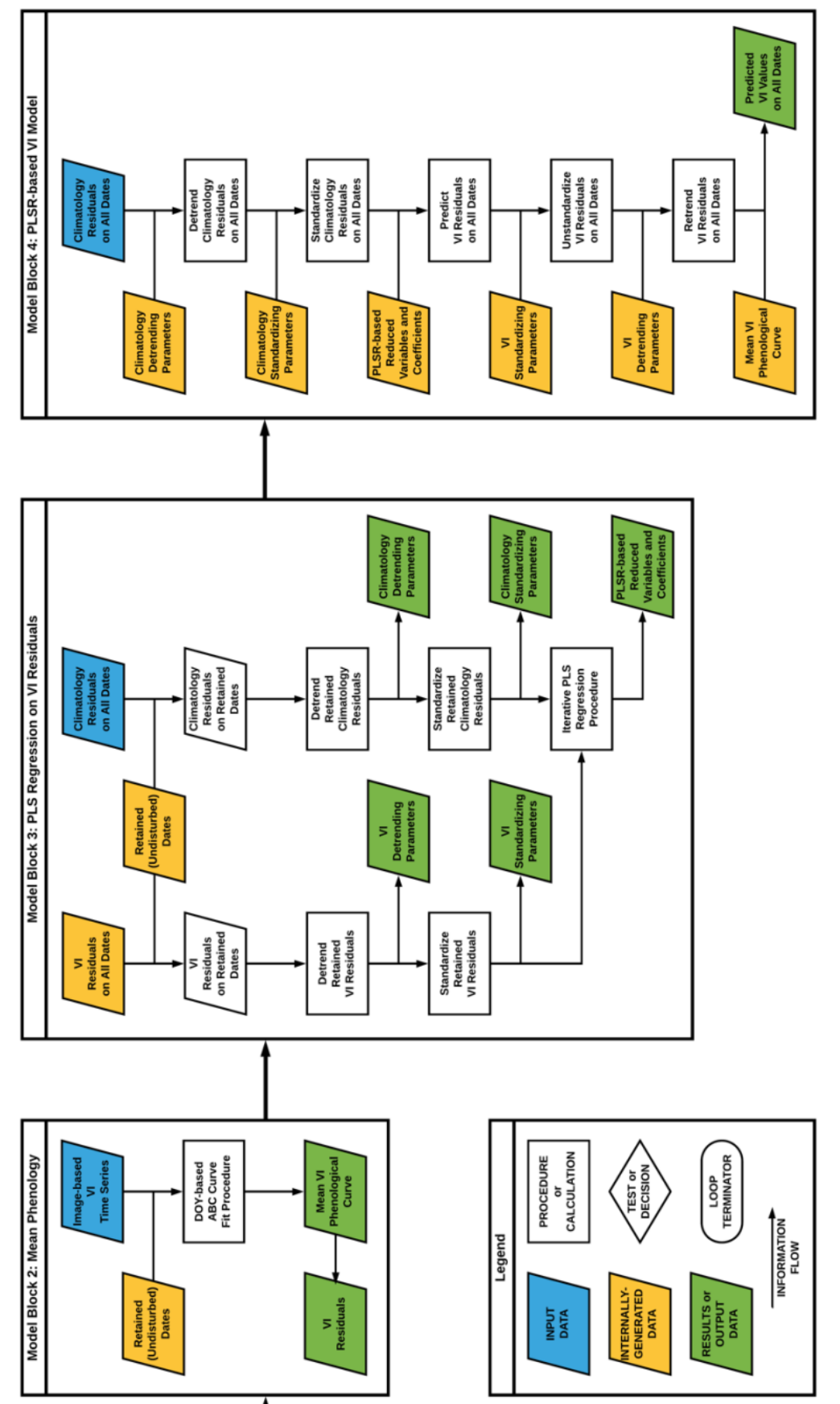

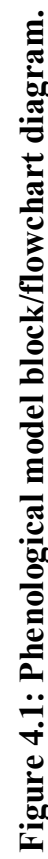




\subsection{Mean Phenology}

Assumption 2: Mean forest phenology is related to mean local climatology, or $\bar{V}=f(\bar{W})$.

Physically, this assumption suggests that the mean annual process of vegetation phenology is in approximate equilibrium with the mean annual progression of exogenous variables such as temperature, precipitation, light availability, and so forth. Changing climatological conditions can thus drive year-toyear changes in phenology. While these processes occur in tandem (but not necessarily in synchrony), it cannot yet be assured that the complex associations between specific aspects of plant physiology and environment at one time are the same as at another time in the plant life cycle. I therefore maintain this as a weak equilibrium assumption, at least within the 30 -year study period, that we explore further with some of our results below.

Given the unlikelihood of holding multiple observations of $V$ for every day over many years, the mean annual phenology $\bar{V}(D O Y)$ is found by fitting a curve to the available Landsat-based observations. In the first part of this analyses ("Model Block 1" in Fig. 4.1) I employed the Kauth-Thomas Tasseled Cap (KTTC) [Crist and Cicone, 1984; Crist, 1985; Crist and Kauth, 1986] components described in Chapter 3. The KTTC transform attempts to separate the principal features of the multi-spectral (6-band) Landsat scene into three approximately orthogonal components: brightness (as a measure of overall reflectance [McMillan and Goulden, 2008]), greenness (useful for distinction of deciduous phenophases and overall vegetation cover [Dymond et al., 2002]), and "darkness" (i.e. shadows) or wetness, useful for indications of open water areas, vegetation structure [Dymond et al., 2002], and moisture content [Cohen, 1991; Toomey and Vierling, 2005]. As demonstrated by Healy et al. [2005], these three components show strong but vastly different sensitivity to vegetation disturbances. Because the KTTC components are designed as orthogonal (and thus uncorrelated) representations of Landsat scene conditions, an anomaly may become evident to different effect in the different components across various disturbance types. The components, evaluated together, are thus useful for the identification of dates in the Landsat pixel time series that might represent anomalies from the seasonal phenological cycle, which is otherwise relatively consistent (barring complete land cover conversion) on a year-to-year basis. 
Plotting the values available for a given location against the $D O Y$ of the observation, each of these KTTC components lends itself easily to a fitted sinusoidal curve over the year. It is important to ensure that the fitted curve is periodic over the year; a linear or polynomial function is not necessarily continuous across the December-to-January (or other annual) transition. The applied curve for each KTTC component is a three-parameter function:

$$
\bar{V}(D O Y)=V_{m i d}+A \sin \left(2 \pi \frac{D O Y-S O S}{365}\right)
$$

with the midpoint value found as

$$
V_{\text {mid }}=\frac{1}{2}\left(V_{\max }+V_{\min }\right)
$$

and the amplitude as

$$
A=\frac{1}{2}\left(V_{\max }-V_{\min }\right)
$$

such that the functional parameters $V_{\min }, V_{\max }$, and the start-of-season $S O S$ are optimized together for each KTTC component. I then use the fitted KTTC phenological curves to detect outliers in the calculated residuals, which are sequestered as possible disturbances to be analyzed separately from the remaining observations.

I apply Chauvenet's criterion [Ross, 2003] to identify as outliers the far limbs of the residual distribution from the fitted KTTC curves. I elected to sequester those data that exceed the $99^{\text {th }}$ percentile (2.58 standard deviations) in departure from the mean for the given pixel, which are those observations that occur at $p<0.005$ and $p>0.995$ in the normal distribution of residuals. This procedure is applied iteratively: first, sinusoidal curves are fitted to each set of KTTC component observations; second, residuals for each component are calculated; third, outliers that occur in any KTTC component are sequestered from all KTTC components; finally (and returning to the first step), new sinusoidal curves are fitted to the remaining KTTC component observations. With each iteration, the model will also identify singular inclusions in the observation dataset that occur between two already sequestered dates, but which might not qualify as an outlier on its own. In algorithm tests, I found it was sometimes the case that an 
inclusion identified in one iteration would have become an outlier in a later iteration if not already sequestered. The process of identifying inclusions is particularly useful near the beginning and end of the growing season, when the effects of a possible forest disturbance might still be present even though the observation seems otherwise normal for that time of year. Iteration over these steps continues until no more outliers or inclusions are detected. This iterative procedure provides (1) a collection of retained observations that approximately conform to the mean annual phenological cycle, to which I will apply a regression modeling process to discern environmental influences on phenological variability, and (2) a collection of sequestered observations that deviate from the mean phenological cycle significantly enough that I can analyze them separately (in follow-on work) as possible disturbed forest conditions.

Using the retained observations, I then fit a mean phenological curve to the vegetation index values on a $D O Y$ basis. This step is designated "Model Block 2" in Fig. 4.1. For this work, I have selected NDII as my signal vegetation index, as discussed above, but the same process applies to the more common use of NDVI or EVI (as demonstrated below and in Chapter 6) or another greenness-oriented vegetation index. In early formulations of this Model Block I considered a traditional 4-parameter logistic curve (for fitting just one end of the growing season, green-up or senescence) [Zhang et al., 2003, 2004; Ahl et al., 2006; White et al., 2014], a 6-parameter double-logistic (DL) curve [Beck et al., 2006; Fisher et al., 2006] (although de Beurs and Henebry [2010] indicate that 8 parameters are required for this), and a 6-parameter modified double-logistic (MDL) curve [Fisher and Mustard, 2007]. All of these are frequently used to show seasonal phenological changes from vegetation index observations, but I observed that none allow a non-zero slope that is often observed between the growth (Spring) and senescence (Autumn) limbs of the annual phenological cycle. This "green-down" period during maturity was addressed with a 5-parameter logistic curve formulated by Elmore et al. [2012] to model a sloped Summer and more sharply senescent Autumn season together. That curve resembles, to a certain degree, the post-green-up portion of the 6-parameter DL curve used by Beck et al. [2006]. The 5-parameter Elmore et al. [2012] logistic curve was used in conjunction with a logistic Spring green-up curve by Melaas et al. [2013], though the two segments were not continuous across the mature season. I also 


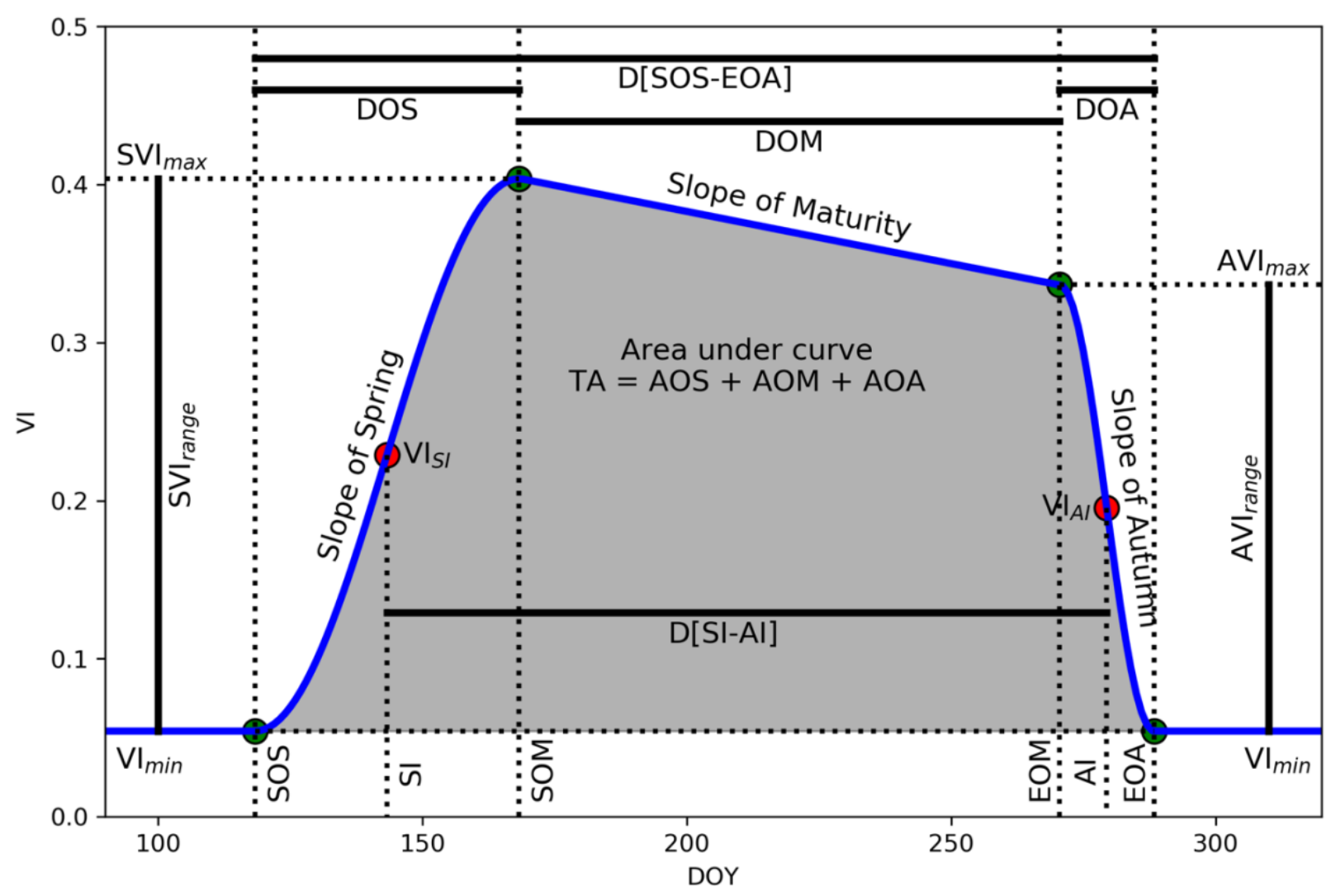

Figure 4.2: Schematic fitted 7-parameter asymmetric broken cosine (ABC) phenological curve with control points (green) and phenological indicators marked. Abbreviations are defined in Table 4.1.

attempted a MDL curve here, but proposed a piecewise definition to allow for different heights of the Spring and Autumn limbs, and drew a linear function between those. However, my own MDL curve sometimes produced an Autumn slope that was unrealistically shallow, especially where too few Autumn observations were available, and frequently resulted in non-periodic functions with a discontinuity in phenological minima across the December-to-January transition.

To address such challenges as asymmetry between green-up and senescence seasons, non-zero slope in the intervening mature period, continuity at the shoulders of the curve, and annual periodicity, I developed a piecewise-continuous function based on a sinusoidal curve in the Spring and Autumn seasons and a linear function through the Summer (mature) period. I have called this curve an "asymmetric broken cosine" (ABC) function. This $\mathrm{ABC}$ curve fitted to NDII observations on a DOY basis requires optimization on seven parameters, indicated with the four green control points in Fig. 4.2: $V_{\min }$ at the beginning and end of the growing season, the start-of-Spring $(S O S)$ date, the dates and vegetation index 
Table 4.1: Mean curve-based phenological indicators as illustrated in Fig. 4.2.

\begin{tabular}{|c|c|}
\hline Abbreviation & Description and [units] \\
\hline \multicolumn{2}{|r|}{ Direct Model Output } \\
\hline$V I_{\min }$ & annual minimum VI [-] \\
\hline SOS & DOY $[\mathrm{d}]$ at start of Spring \\
\hline$S V I_{\max }$ & VI [-] at Spring maximum (or curve break) \\
\hline$S O M$ & DOY [d] at $S V I_{\max }$ (start of Maturity) \\
\hline$A V I_{\max }$ & VI [-] at Autumn maximum (or curve break) \\
\hline$E O M$ & DOY [d] at $A V I_{\max }$ (end of Maturity) \\
\hline$E O A$ & DOY [d] at end of Autumn \\
\hline$M A E$ & fitted curve mean absolute error [-] (units of VI) \\
\hline RMSE & fitted curve root-mean-squared error [-] (units of VI) \\
\hline$r^{2}$ & fitted curve coefficient of determination [-] \\
\hline \multicolumn{2}{|r|}{ Derived Characteristics } \\
\hline$S V I_{\text {range }}$ & Spring VI range [-] $=S V I_{\max }-V I_{\min }$ \\
\hline$D O S$ & Duration $[\mathrm{d}]$ of Spring $=S O M-S O S$ \\
\hline$V I_{S I}$ & VI [-] at Spring Inflection $=\left(V I_{\min }+S V I_{\max }\right) / 2$ \\
\hline \multirow[t]{2}{*}{ SI } & DOY [d] at Spring Inflection $=(S O S+S O M) / 2$ \\
\hline & Slope $\left[\mathrm{d}^{-1}\right]$ of Spring curve $=\left(S V I_{\max }-V I_{\min }\right) / D O S$ \\
\hline$A O S$ & Area [d] under Spring curve $=\left(S V I_{\max }-V I_{\min }\right) \times D O S / 2$ \\
\hline \multirow[t]{2}{*}{$D O M$} & Duration [d] of Maturity $=E O M-S O M$ \\
\hline & Slope $\left[\mathrm{d}^{-1}\right]$ of Maturity curve $=\left(A V I_{\max }-S V I_{\max }\right) / D O M$ \\
\hline$A O M$ & Area [d] under Maturity curve $=\left(S V I_{\max }+A V I_{\max }\right) \times D O M / 2$ \\
\hline$A V I_{\text {range }}$ & Autumn VI range [-] $=A V I_{\max }-V I_{\min }$ \\
\hline$D O A$ & Duration $[\mathrm{d}]$ of Autumn $=E O A-E O M$ \\
\hline$V I_{A I}$ & VI [-] at Autumn Inflection $=\left(V I_{\min }+A V I_{\max }\right) / 2$ \\
\hline \multirow[t]{2}{*}{$A I$} & DOY [d] at Autumn Inflection $=(E O M+E O A) / 2$ \\
\hline & Slope $\left[\mathrm{d}^{-1}\right]$ of Autumn curve $=\left(A V I_{\max }-V I_{\min }\right) / D O A$ \\
\hline \multirow[t]{2}{*}{$A O A$} & Area [d] under Autumn curve $=\left(A V I_{\max }-V I_{\min }\right) \times D O A / 2$ \\
\hline & ummary Characteristics \\
\hline$V I_{\max }$ & Overall maximum [-] $=\max \left[S V I_{\max }, A V I_{\max }\right]$ \\
\hline$V I_{\text {range }}$ & Overall range [-] $=\max \left[S V I_{\text {range, }}, A V I_{\text {range }}\right]$ \\
\hline$T A$ & Total area under curve $[\mathrm{d}]=A O S+A O M+A O A$ \\
\hline$D[S O S-E O A]$ & Duration $[\mathrm{d}]$ of overall season $=E O A-S O S$ \\
\hline$D[S I-A I]$ & Duration [d] of inflection-based growing season $=A I-S I$ \\
\hline
\end{tabular}


values at the start and end of the mature phase ( $S O M$ and $S V I_{\max }, E O M$ and $A V I_{\max }$, respectively), and the end-of-Autumn $(E O A)$ date. Functional continuity near BOM and $E O M$ dates is obtained with an adaptive slope-matching method between the cosine and linear functions that meet there. The ABC curve has easily-calculated cosine and linear sections, allowing for the rapid computation of numerous additional growing-season indicators that are shown in Fig 4.2 and listed in Table 4.1. Observational values for these indicators will be presented in Chapter 6.

\subsection{Phenological Variability by Regression Analysis}

Assumption 3: Variability in phenology is related to deviations from local climatology, or $V^{\prime}=f\left(W^{\prime}\right)$.

This assumption is also related to the balance between plant physiology and environment, but on generally shorter (quasi-daily) time scales. It is expected that immediate weather and climatological conditions will interact with the immediate needs and capabilities of the forest canopy: sunlight, temperature, and moisture and nutrient demand. Some of these processes operate very rapidly, such as sunlight and temperature controls on photosynthetic rates, while others span seasonal scales, such as the accumulation of leaf area and canopy biomass over the first several months of the growing season. To address these at operational time scales, I have included temporal averages and other measures of the basic environmental variables over numerous periods (Table 4.1) with the goal that this analysis will identify the most relevant variables and time scales for overall forest phenological process interactions with environmental stressors.

Given that many of the weather and climate variables are derived from the same daily time series of temperature and precipitation, many of these variables are collinear and co-vary strongly. Instead of a multiple linear regression analysis, which may be poorly suited to such collinearity, my goal is to identify parsimonious linear relationships between predictors $(W)$ and response variables $(V)$ with a model that exploits this collinearity by reducing the number of relevant predictors. This suggests such methods as principal components regression (PCR) and partial-least-squares regression (PLSR) [Wold, 1975; Wold et $a l ., 1984,2001 a, b]$. Originally developed for problems in which the number of measured, possibly 
collinear predictors can far exceed the number of response variables [Kettaneh et al., 2005], PLSR has been applied extensively in chemometric analysis [Wold et al., 2001a, 2009] and has recently been employed in optical multispectral and hyperspectral remote sensing studies to estimate forest species composition [Wolter et al., 2008; Wolter and Townsend, 2011], canopy structure [Wolter et al., 2009] and foliar chemical constituents from hyperspectral observations [Serbin et al., 2014; Singh et al., 2015].

In both PCR and PLSR methods, independent latent predictors are derived by transformation of the available measured variables. However, PCR seeks to minimize correlation among latent predictors resulting in uncorrelated (orthogonal) components, while PLSR seeks to maximize covariance between the derived latent components [Wolter et al., 2008]. The PLSR process also incorporates the response variable(s) into this determination of covariance, whereas PCR derives latent components solely from the provided predictors. Unlike ordinary least-squares (OLS) regression methods, PLSR does not assume that the predictor variables have zero error [Wolter et al., 2009] and, in fact, the sources and error structures of those predictors may be unknown (although uncorrelated, normally distributed errors are still assumed). Wold et al. [1984] demonstrated that, in their experiments, PLSR provided better overall error characteristics in both validation and prediction results than PCR and several other regression methods.

This PLSR process is designated "Model Block 3" in Fig. 4.1. Using the functional $\bar{V}$ (derived above) and climatological $\bar{W}$ (calculated separately), both varying with $D O Y$, the residuals $V^{\prime}$ and $W^{\prime}$ are found using eqns. (4.2) and (4.3). For the PLSR process, the residual relation $V^{\prime}=f\left(W^{\prime}\right)$ can be written

$$
V^{\prime}(Y, D O Y)=\beta_{w^{\prime}, 0}+\sum_{i=1}^{m} \beta_{w^{\prime}, i} W_{i}^{\prime}(Y, D O Y)
$$

where the residuals can vary both with $D O Y$ and year over the entire study period. The unknown $\beta$ coefficients in the summation are unique to each of the $m$ weather and climate variables determined to be significant through PLS regression, and $\beta_{w^{\prime}, 0}$ is the value of the regression intercept resulting from that process. As a simplified representation of the iterative PLSR procedure, I will write

$$
\left[V^{\prime}(Y, D O Y), W_{1}^{\prime}(Y, D O Y), \ldots W_{m}^{\prime}(Y, D O Y)\right] \stackrel{P L S R}{\Longrightarrow}\left[\beta_{w^{\prime}, 0}, \beta_{w^{\prime}, 1}, \ldots \beta_{w^{\prime}, m}\right]
$$


where I have detrended (over the full study period) and standardized (within each variable's set of retained observations, with approximate normal distributions for all residuals) each input variable's residual time series $V^{\prime}$ and $W^{\prime}$ before regression [Wold et al., 1984]. The resulting $\beta$ coefficients are location- (pixel-) and variable-specific. The detrending and standardization parameters found at this step, based on the retained dataset, are saved for later modeling of $V^{\prime}$ on non-observation dates and, potentially, for application to the full $W^{\prime}$ dataset for the same location (pixel) and study period.

To obtain a parsimonious PLSR model for each pixel, instead of a combinatorics approach that would explore every possible subset of input variables and number of latent model components retained, I have employed an iterative procedure that tests each weather and climate variable for its contribution to the accuracy of the regression result. Starting with the full set of predictor variables, this algorithm discards the least-helpful variable (that is, the variable whose elimination provides the best incremental improvement in the chosen model evaluation statistic) at each iteration until the "best" regression model is found. This method is conceptually similar to the approach employed by Wolter et al., [2008] for estimation of forest basal area from Landsat scenes, iteratively eliminating image-based variables with low sensitivity to the response variable. While many applications have used the predicted residual sumof-squares (PRESS) statistic [Wolter et al., 2008] to guide PLSR model selection, I have chosen the corrected (second-order) Akaike Information Criterion (AIC) [Akaike, 1974; Burnham and Anderson, 2002]. Other metrics, such as the Bayesian Information Criterion (BIC) [Wit et al., 2012] and standard model error measures (MAE, RMSE, and PRESS), are available in the algorithm for model optimization, but none of those demonstrated a concave profile with a readily-identifiable optimum model at reasonable levels of model complexity that I found with the $A I C c$ (Fig. 4.3). The $A I C$ is calculated as

$$
A I C=2 m-2 \ln (\hat{L})
$$

where $m$ is the number of model parameters ( $W^{\prime}$ variables) and the maximum likelihood function for a normal distribution is similar to the probability density function: 


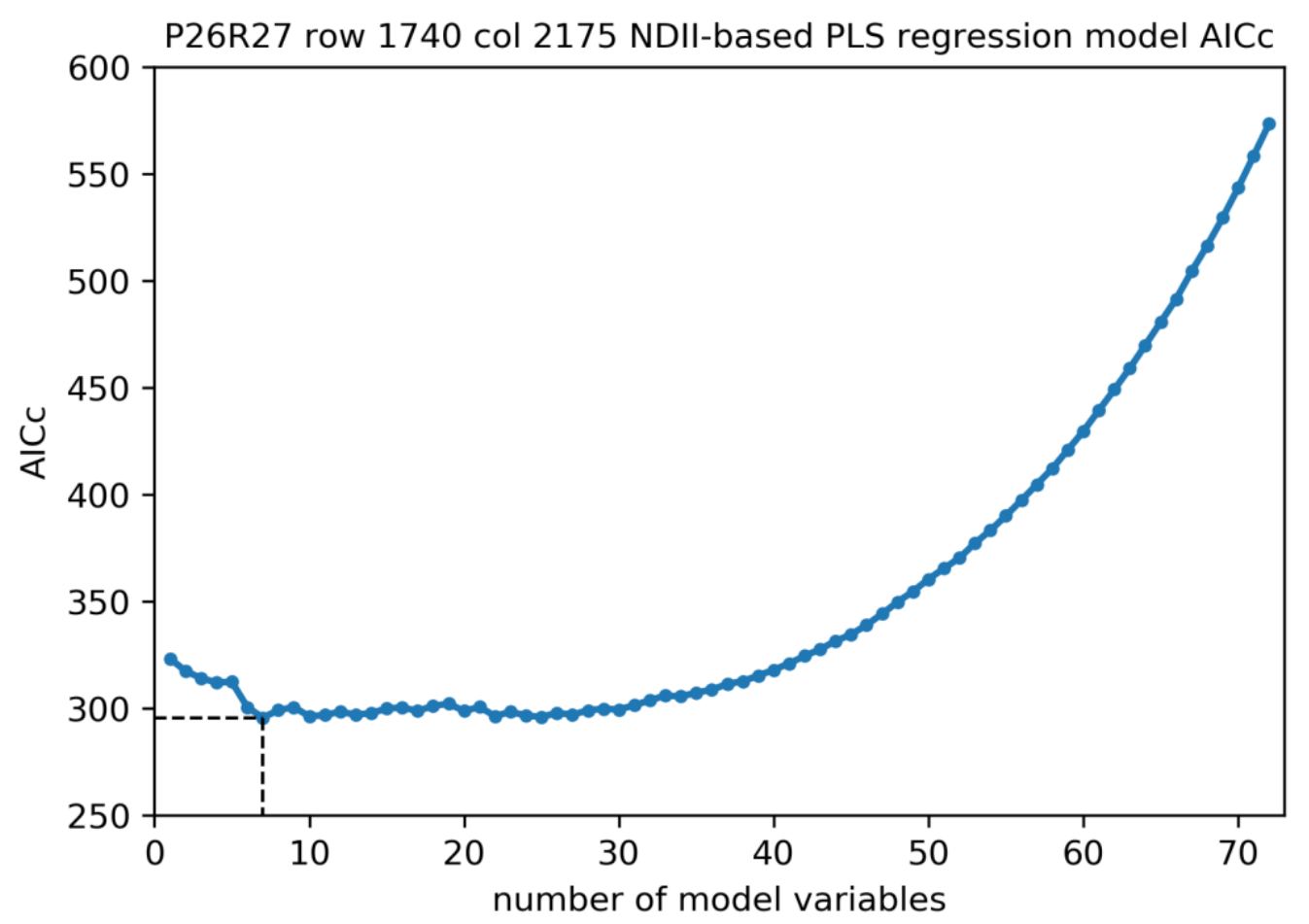

Figure 4.3: Illustration of PLSR model-based $A I C c$ vs. the number of model variables retained, showing the selection of minimum $A I C c$ and resulting model size (6 variables) for a single pixel.

$$
\widehat{L}=\left(\frac{1}{\sqrt{2 \pi s^{2}}}\right)^{n} \exp \left(-\frac{S S E}{2 s^{2}}\right)
$$

with mean squared error $s^{2}$ and the sum of squared (prediction) errors SSE (also known as PRESS) as

$$
s^{2}=\frac{S S E}{n}=\frac{1}{n} \sum_{i=1}^{n}\left(\widehat{V}_{l}^{\prime}-V_{i}^{\prime}\right)^{2}
$$

where $n$ is the number of observations in the response variable time series (i.e. the sample size) and $\widehat{V}^{\prime}$ is the predicted value of $V^{\prime}$ after the regression process. I then correct the value of $A I C$ for the resulting model complexity [Hurvich and Tsai, 1989; Cavanaugh, 1997] based on the numbers of observations $n$ and model parameters $m$ as

$$
A I C C=A I C+\frac{2(m+1)(m+2)}{n-m-2}
$$

In comparisons, the model with the lower value of $A I C c$ is considered to have greater information content. The best model at each iteration is thus the model with the lowest value of $A I C c$ in the collection. 
The parameter that was excluded from that selected model is then discarded from the set of model parameters, and the next iteration is begun. Once all parameters (variables) have been eliminated, the $A I C C$ values for the models that survived their respective iterations are compared (Fig. 4.3) and that with the lowest $A I C c$ is selected as the most parsimonious model for the examined pixel and response variable (Tasseled Cap component or other vegetation index). Algorithm tests using error metrics such as PRESS, $R M S E$, and $r^{2}$ categorically resulted in a final, "best" model retaining all 72 input variables, suggesting that either I didn't provide enough predictor variables to the PLSR process, the response variable didn't provide an adequate constraint on the PLSR process, or that the process allowed model overfitting. This procedure results in a set of $\beta$ coefficients and their corresponding weather and climate variables $W^{\prime}$ that is often considerably smaller than the input dataset while typically explaining a large proportion of variability in the response variable $V^{\prime}$. The number of regression model components (latent variables) may be specified explicitly for the PLS procedure. In my algorithm tests to optimize the number of model components $k$, using the PRESS statistic for model comparisons, the "best" PLSR model was consistently that with the most variables and the number of latent components specified by $k=m-1$, with $m$ as the number of weather and climate variables retained in the model. To reduce computational time, all model results shown below use this heuristic to specify the number of PLSR components retained. Given that I fixed the number of model components to the number of model variables in a simple but systematic manner, model overfitting is the most likely of the issues mentioned above. This is a detail of my PLSR application that I will improve in future versions of this model algorithm. On the other hand, my iterative elimination-based procedure using $A I C c$ for model comparisons, with a rulebased selection of model components, leads to a considerable savings in computation time: for each pixel in my study area ( $\sim 130$ million pixels in 5 Landsat footprints), the specified $m=72 W^{\prime}$ input variables (see Tables 3.1-3.5) would have required evaluating $2^{m} \sim 5 \times 10^{21}$ unique models by the combinatorics approach; using this iterative elimination procedure requires evaluating only $\sim 3 \times 10^{3}$ candidate models at each pixel. As will be mentioned below in a modeling example, retaining all of the input variables in 
the final PLSR model would demand considerable computational resources when applied over the entire study area.

Finally, in "Model Block 4" in Fig. 4.1 the phenological model then returns to the complete (uncensored) dataset, detrends and standardizes each of the weather and climate variables needed for that set of $\beta$ coefficients using the parameters found using the censored dataset, and applies the PLSR result as a model (PLSM) for the vegetation index at each image date in the dataset:

$$
\left[\beta_{w^{\prime}, 0}, \beta_{w^{\prime}, 1}, W_{1}^{\prime}(Y, D O Y), \ldots \beta_{w^{\prime}, m}, W_{m}^{\prime}(Y, D O Y)\right] \stackrel{P L S M}{\Longrightarrow}\left[\hat{V}^{\prime}(Y, D O Y)\right]
$$

where $\hat{V}^{\prime}$ is the predicted value of $V^{\prime}$ by this process. These predicted values are then de-standardized and re-trended, using the parameters found for the retained $V^{\prime}$ data, and added to the fitted (mean) curve to find the expected vegetation index value for a given date:

$$
\widehat{V}(Y, D O Y)=\bar{V}(D O Y)+\widehat{V}^{\prime}(Y, D O Y)
$$

Though I use the image-based dataset of $V^{\prime}$ and derived maps of $W^{\prime}$ for this application of the PLSM to evaluate the accuracy of our procedure, a set of climatological $W^{\prime}$ observations for the same location on any other dates within the study period could be prepared and applied to the same derived set of $\beta$ coefficients to estimate $\widehat{V}$ at those times. In other words, this procedure allows calculation of $\widehat{V}$ for any date in the study period, not just those dates for which I collected Landsat scenes. The procedure described here thus allows for various operations such as filling cloud-masked areas with estimated VI values, calculation of the full phenological curve on an annual basis for trend analysis, and comparison of estimated VI values with ground-based or other remote sensing data that were collected on non-Landsat dates.

\subsection{Example Model Results for a Single Pixel}

I applied this model to several individual pixels before deployment on the footprint scale. Summary results like those shown in Fig. 4.4 were invaluable in the ongoing development of the phenological model. Each part of this summary plot results from, or further informs, a component of the 

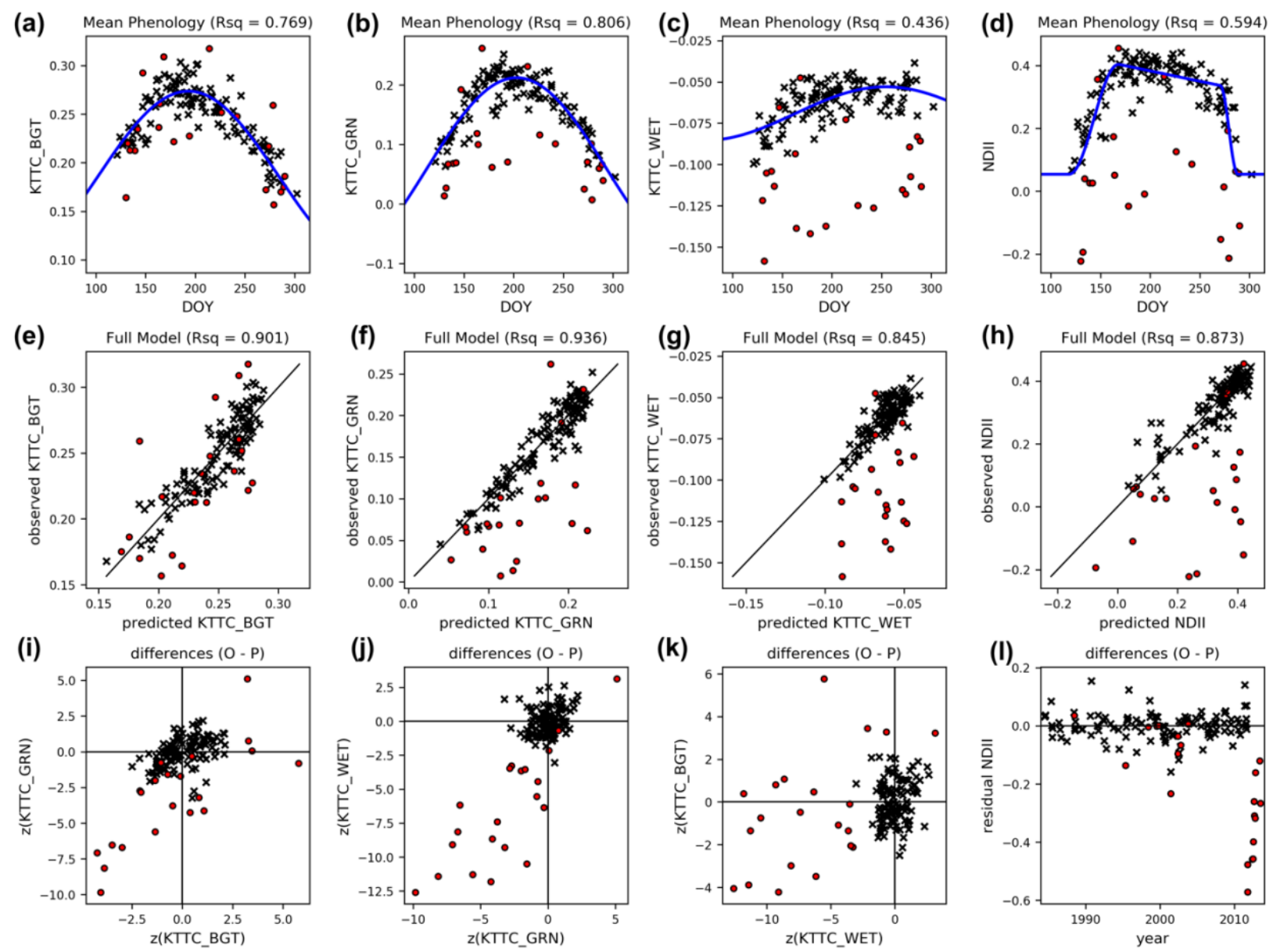

Figure 4.4: Example phenological model results for a single Landsat pixel in northeastern Minnesota. The model progression through these panels is described in the text, using Fig. 4.1 to reference the applied computational steps. Red circles represent sequestered (potential disturbance) data, and black $\times$ s represent the retained data. Blue lines in panels (a)-(d) are fitted curves (sinusoidal for KTTC components, ABC for NDII). The 1:1 line is shown in panels (e)-(g).

model shown in Fig. 4.1, which will be useful to review in the context of these results. A 12-panel summary plot (e.g. Fig. 4.4) can be produced from the collected model results for any location (pixel) in the study area, allowing highly specific investigations in areas of particular interest. In general, I have aggregated these results to the footprint scale for mapping and interpretation, as shown in Chapter 6. An individual, user-directed query into any of $\sim 130$ million single-pixel phenological model results is possible and would be useful but would require considerable computational development for the necessary data server and interface. Aside from aggregation of the salient model-based indicators to footprint and regional scale maps, it remains difficult to illustrate the scope and detail of model information produced in this work. 
Figure 4.4a-c illustrate the KTTC-based sinusoidal curve fitting (constrained to the growing season) and outlier elimination that comprises Block 1 in the model diagram (Fig. 4.1). The three KTTC indexes are fitted simultaneously, with an outlier in any KTTC index removed from all of the KTTC indexes. In this view, the sequestered outliers are marked with red circles, and the retained data are fitted with the blue sinusoidal curve. Because of this (self-imposed) rule of removing an outlier in any component from all components, some KTTC components may show data points that lie close to the fitted curve but were sequestered in another, associated component. This result conforms to the idea that disturbance events may produce different signatures in the three KTTC components depending on the disturbance impacts on canopy moisture content, greenness, and exposure of the underlying (often brighter) land surface [Healey et al., 2005].

Figure $4.4 \mathrm{~d}$ illustrates fitting the $\mathrm{ABC}$ curve described above to observed pixel $N D I I$ values as part of Block 2 in Fig. 4.1. Only the retained data for the pixel are used for the fitted curve by a leastsquares optimization procedure, and the sequestered data points are shown in red for comparison. As described in Chapter 3, the fitted curve then represents the 1984-2013 mean phenology for that location and VI through the growing season. The mean phenological curve derived for this pixel in Fig. $4.4 \mathrm{~d}$ is, in fact, the example schematic curve that is illustrated in Fig. 4.2. In this case, most of the sequestered disturbance events had lower values of $N D I I$ than expected for a given $D O Y$, though some outlier points are found above the curve in the Spring.

For all of the retained data, the difference between the actual VI value and the fitted curve (sinusoidal or $\mathrm{ABC}$ ) for the same $D O Y$ forms the residual values that are passed to the regression procedure in Block 3 of Fig. 4.1. This is where the described model splits in its orientation: as shown in Fig. 4.1, Block 3 develops a PLSR model for the desired VI and leads to Block 4, where the PLSR model (PLSM) is applied to determine expected VI values on the desired dates. The results of these PLSR and PLSM procedures are shown in panel (h) in Fig. 4.4, with the 1:1 line shown and relatively high model fit values. Alternatively, the KTTC components are subject to a procedure similar to that shown in Blocks 3 and 4 for the estimation of KTTC component values on sequestered (disturbed) dates in the time series, 
which are shown in Fig. 4.4e-g and are held for later analysis. The $r^{2}$ values on Fig. 4.4e-h were calculated using only the retained data points, with the predicted values at the sequestered data points shown for comparison. Note that these $r^{2}$ values in the middle row of Fig. 4.4 are the combined result of both the mean fitted curve and the PLSM applied to each vegetation index: it is not that the ABC phenological curve fitted to the retained $N D I I$ observations has an $r^{2}$ value of $\sim 0.87$ (it is actually much lower at $\sim 0.59$, as listed in the top row of Fig. 4.4 ), but that the complete phenological model uses the mean phenology and the observed climatological variability, through the PLSR and modeling process, to explain $\sim 87 \%$ of the observed variability in NDII (excluding suspected disturbances, i.e. sequestered points) in the collected scenes at that pixel.

Not shown in Fig. 4.1 is an additional computational block in which I then calculated the differences between expected (predicted) and observed values for each of the KTTC components and standardized those results, then extracted the values for the sequestered data points as measures of possible disturbance impact on those dates, as shown in Fig. 4.4i-k. The model procedures in Blocks 3, 4, and 5 are thus oriented on providing information on the VI-based phenology as well as possible disturbance events that have been extracted from the Landsat time series information for this pixel. Figure 4.5 illustrates the three-dimensional time series representation where the disturbance dates are again marked in red, and the blue line is a cubic spline fitted curve connecting sequential dates in the time series for this location. This visualization emphasizes the departure from the central region of comparative forest health when a disturbance occurs.

The procedures in model Blocks 3 and 4 are the same for VI or KTTC inputs, and for VIs are oriented on comparing the residuals in the retained NDII time series with departures from climatological conditions for the same dates, following Assumption 3 above. In this phenology-oriented PLS regression procedure, much more attention is given to the "best" model as determined by the method described above and illustrated previously in Fig. 4.3, which shows the NDII-based AICc results and PLS regression model selection for this same pixel. The NDII values calculated by PLSM for both retained and sequestered data points are shown in Fig. 4.4h, which indicates a relatively high correlation for this pixel. 


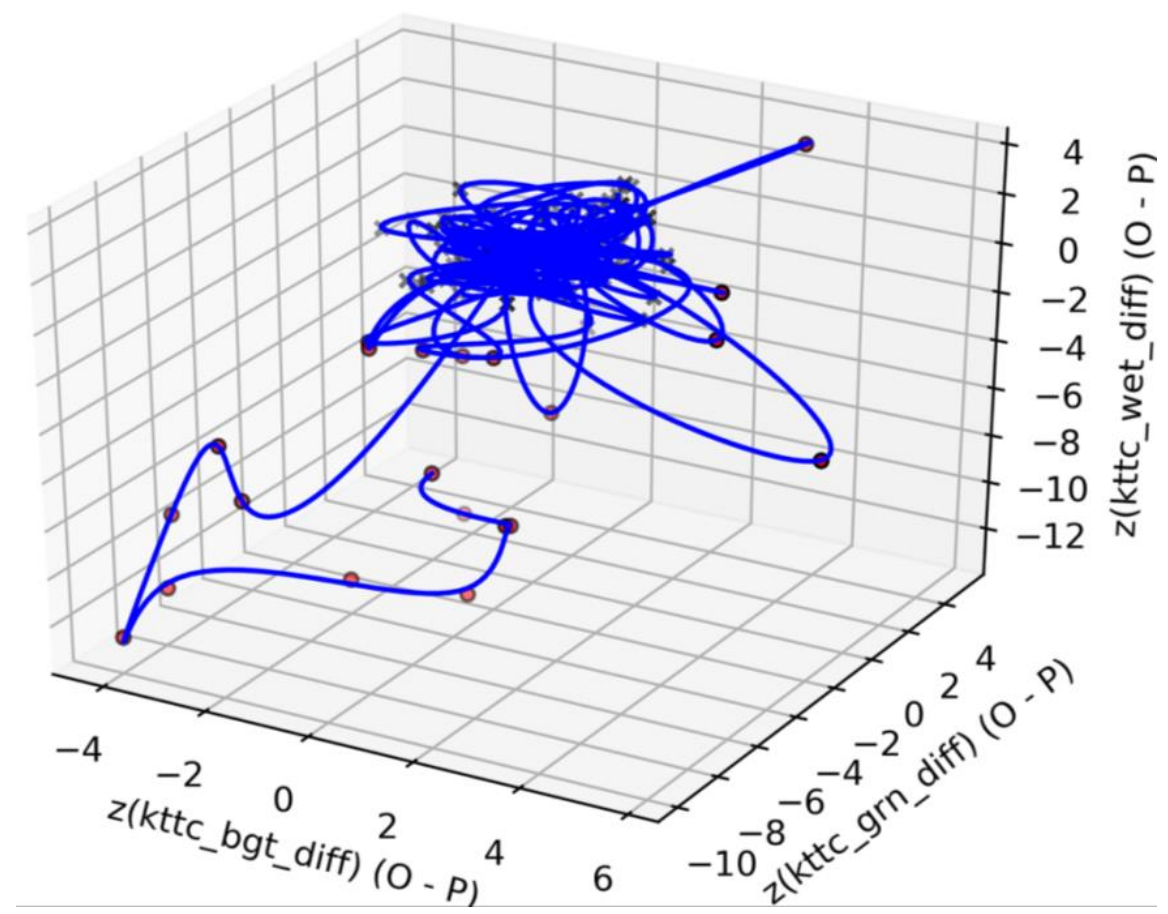

Figure 4.5: Time series of standardized Tasseled Cap residuals for the Landsat pixel shown in Fig. 4.4. As in panels (i)-(k) of that figure, sequestered disturbance dates are marked with red circles. The blue line is a fitted cubic spline curve connecting sequential dates in the time series.

Just as in Fig. 4.4d, NDII values during disturbance events generally fall below their corresponding expected values in Fig. 4.4h.

The complete time series of residuals (observed - PLSM values) for this pixel is shown in Fig. 4.41. Although some smaller disturbance events in this Landsat pixel appear at several times over the course of the study, Figs. $4.4 \mathrm{~h}$ and 4.5 show a large event at the end of the time series at this location. This is a pixel that I selected for examination (among several to help guide model development) inside the Pagami Creek fire area in northeastern Minnesota [Miesel et al., 2015; Kolka et al., 2017]. The Pagami Creek fire reached this location in the second week of September 2011 and was so severe by the time that no vegetation remained after the fire's passage. This sharp contrast between pre- and post-disturbance conditions affected all of the KTTC components and VIs examined in this work and provided a useful test case for both phenological model development and disturbance detection. The value of this time series approach is evident in Fig. 4.5: the apparent wander of the pixel trajectory in Tasseled Cap space after the occurrence of the fire, evident in the lower left portion of the figure, illustrates the punctuated nature of 
(a) Mean Phenology $(\mathrm{Rsq}=0.603)$

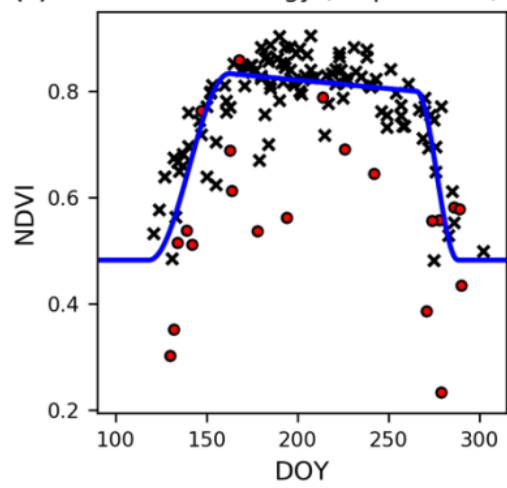

(d)
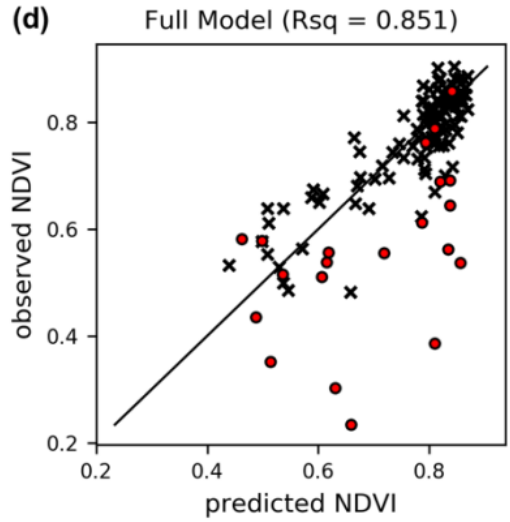

(b) Mean Phenology (Rsq $=0.742)$

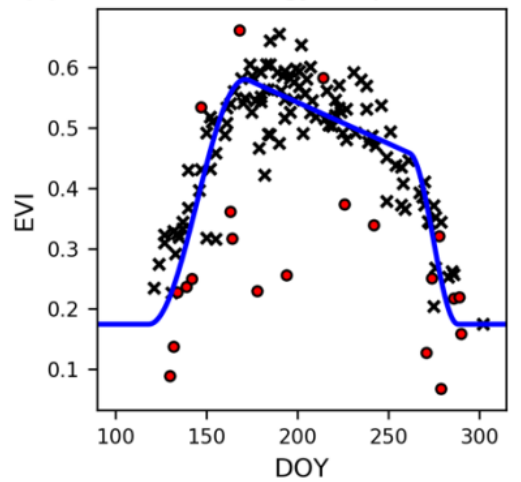

(e) Full Model $(\mathrm{Rsq}=0.950)$

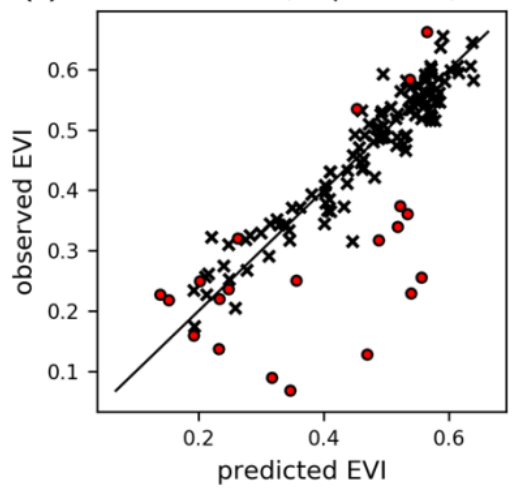

(c) Mean Phenology $(\mathrm{Rsq}=0.594)$

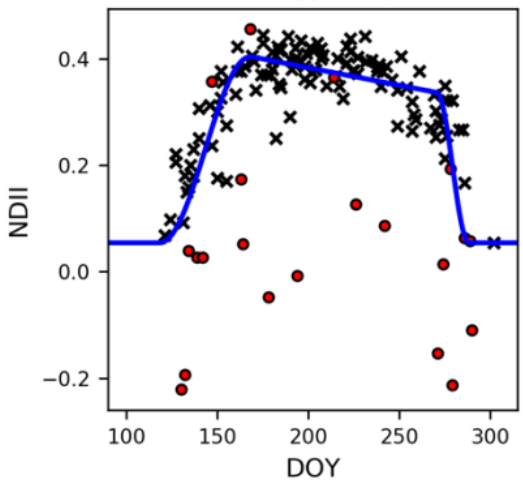

(f) $\quad$ Full Model $(\mathrm{Rsq}=0.873)$

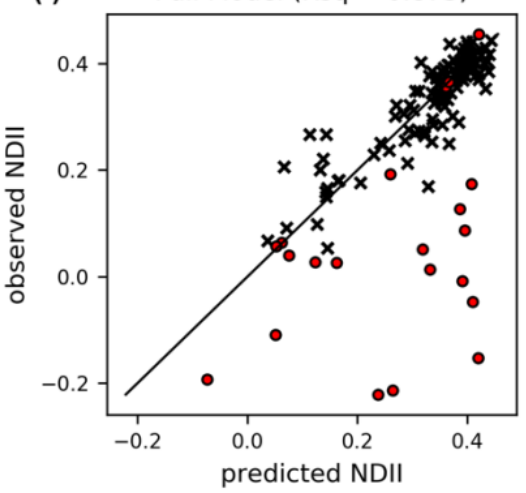

Figure 4.6: Goodness-of-fit results for the mean phenology fitted curve and the full phenological model (mean fitted curve + PLSR model) for NDVI-, EVI-, and NDII-based datasets at the same pixel shown in Figs. 4.4 and 4.5. Red circles represent sequestered (potential disturbance) data, and black $\times$ s represent the retained data. Blue lines in panels (a)-(c) are the fitted ABC curves. The 1:1 line is shown in panels (d)-(f).

forest recovery following a severe disturbance event. Tracking of the recovery process in disturbed forest locations is an important aspect in our understanding of forest disturbance dynamics, including their interactions with phenology and its role in forest development at multi-year to decadal time scales. This valuable information, though excluded from my phenological analysis in Chapter 6, is retained for analysis in ongoing and future work.

Finally, in preparation for the results that will be presented in Chapter 6, I also used NDVI and EVI to model this example pixel. The process of outlier identification and removal using only the KTTC components is the same among these results. The fitted mean phenological curves (Fig. 4.6) appear somewhat similar between $N D V I$ and $N D I I$, but the $E V I$-based curve appears quite different with a greater overall range and a steeper slope during the mature period. A comparison of the full model results (using both the mean phenological curve and the PLSR modeling component) with observed values of each VI 
Table 4.2: Model goodness-of-fit results for three VIs at the single pixel in P26R27 shown in Figs. 4.2-4.6.

\begin{tabular}{|c|c|c|c|c|c|}
\hline \multirow[b]{2}{*}{ VI } & \multirow[b]{2}{*}{$\begin{array}{c}\text { Retained } \\
\text { points }\end{array}$} & \multicolumn{3}{|c|}{$r^{2}$ (goodness-of-fit) values } & \multirow[b]{2}{*}{$\begin{array}{l}\text { \# of PLSR } \\
\text { variables in } \\
\text { final model }\end{array}$} \\
\hline & & $\begin{array}{c}\text { Mean } \\
\text { phenology } \\
\text { fitted curve }\end{array}$ & $\begin{array}{c}\text { Residuals of } \\
\text { phenology } \\
\text { (PLSR) }\end{array}$ & $\begin{array}{c}\text { Mean } \\
\text { phenology } \\
\text { + PLSR }\end{array}$ & \\
\hline$N D V I$ & 115 & 0.60 & 0.18 & 0.85 & 6 \\
\hline$E V I$ & 115 & 0.74 & 0.58 & 0.95 & 25 \\
\hline NDII & 115 & 0.59 & 0.33 & 0.87 & 7 \\
\hline
\end{tabular}

are also shown in Fig. 4.6, and the RMSE and $r^{2}$ values for each VI are listed in Table 4.2. While NDVI and NDII are similar in the accuracy of their mean phenological curves $\left(r^{2}=0.60\right.$ and 0.59 , respectively) and their full model results ( $r^{2}=0.85$ and 0.87 , respectively), PLSR modeling of the phenological residuals using NDII (7 variables, $\left.r^{2}=0.33\right)$ is clearly better than using $N D V I\left(6\right.$ variables, $\left.r^{2}=0.18\right)$ at the expense of only one additional model variable. Overall, EVI provides the best result for the mean phenological curve $\left(r^{2}=0.74\right)$, the climatology-based PLSR component of the model $\left(25\right.$ variables, $r^{2}=$ $0.58)$, and the overall full model results when compared against observations ( $\left.r^{2}=0.95\right)$. However, the added value of using EVI comes at the expense of a much larger PLSR model, which then requires both a larger volume of input data (with its necessary pre-processing for model use) and greater computational resources when applied over a large area. In Chapter 6, I will proceed with footprint- and regional-scale analyses using NDII, because this vegetation index has a demonstrated sensitivity [Hunt et al., 1987, 2011; Yilmaz et al., 2008] to aspects of the forest canopy that I seek to explain using climatological indicators: greenness, as an indicator of phenological phase, and moisture content, as an indicator of possible drought and vegetation stress.

\subsection{Computational Implementation}

In addition to the phenological model itself (Fig. 4.1), there are numerous steps to be completed for data preprocessing (as described in Chapter 3), domain discretization for efficient model application on distributed computing resources, and model post-processing to be completed for proper visualization of model and analytical results. 


\subsubsection{Programming}

All of the programming for this work used Python (www.python.org), Linux-based shell scripts, and the HTCondor (research.cs.wisc.edu/htcondor/) and DAGMan protocols for computational job submission and workflow management, respectively, on UW-Madison Center for High Throughput Computing (CHTC) and affiliated computing resources that are described below. All of Python's core functions and extended libraries for specialized operations are open-source. Curve fitting using a leastsquared-error routine, and the core regression component of the PLSR operations described above, are both available in the Python library SciPy (www.scipy.org). All plots in this work used the Python library Matplotlib (www.matplotlib.org). Portions of my code used for this work are already online, especially the code published with Garcia and Townsend [2016] that is available at megarcia.github.io/WxCD. The code that I used for preprocessing Landsat data is online at GitHub, and my phenological model code will be available at GitHub upon publication.

\subsubsection{Domain Discretization}

Pre-modeling steps, including the processing and merger of meteorological and Landsat datasets, required extensive programming and computing operations. The size and volume of arrays in these datasets required discretizing the geographic domain so that input processing, statistical modeling, and output processing and analyses could be completed in a timely manner using high-throughput scalable computing resources. Since the phenological model for a given pixel location does not consider the neighboring pixels, a natural discretization to individual Landsat pixels might be suggested. However, given the combination of meteorological datasets at 480-m resolution with Landsat images at 30-m resolution, discretization to individual Landsat pixels would require repetition of meteorological data where it spans adjacent pixels. Taking a single meteorological pixel (across all available variables) and its corresponding 16 x 16 grid of Landsat pixels (across the four calculated indices described above) as a "chunk" of the dataset (Fig. 4.7), I have decomposed each Landsat footprint in the study area to obtain several hundred thousand discrete data chunks, each of which can then be treated as an independent 

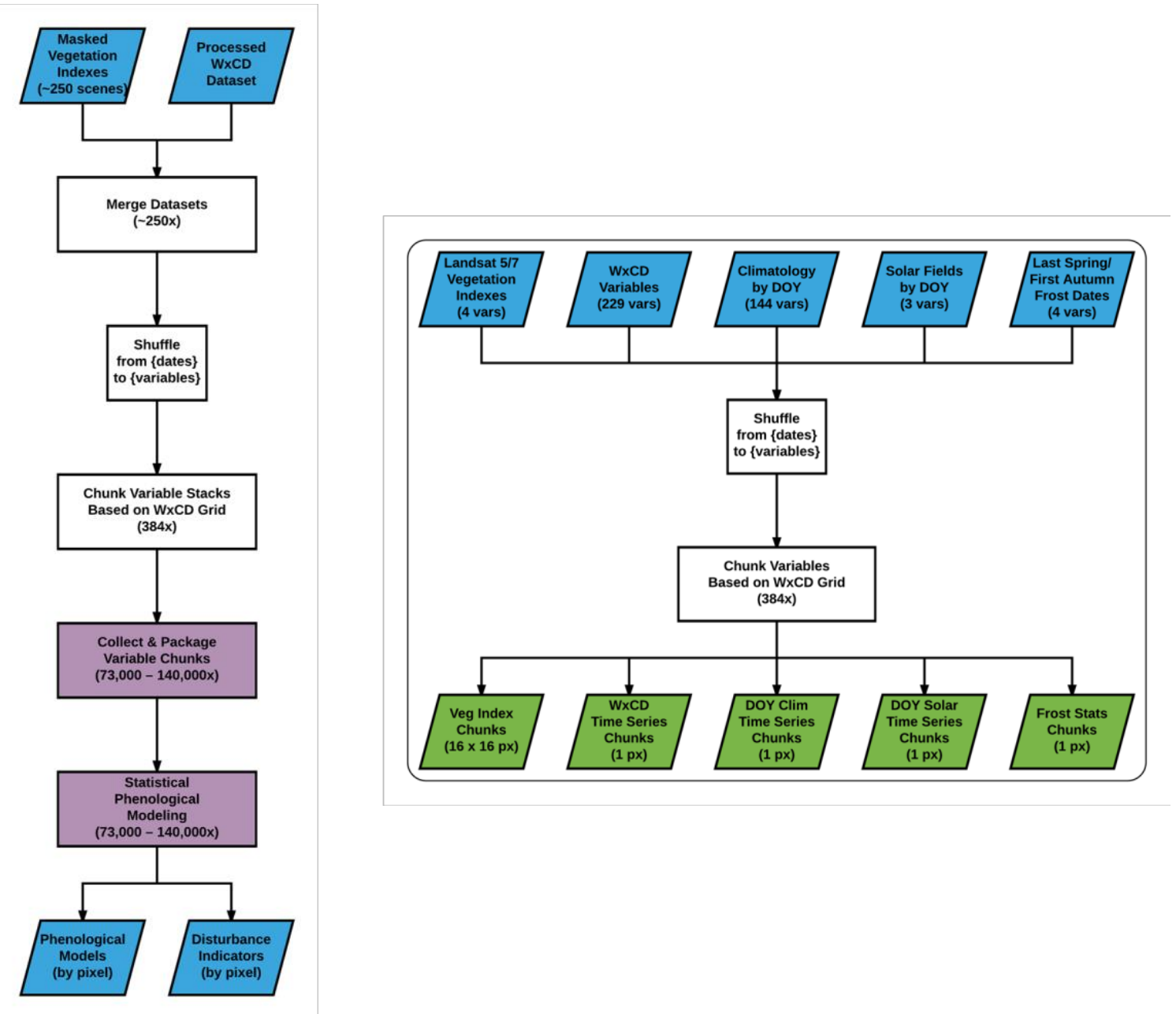

Figure 4.7: Data merger and domain discretization process in preparation for phenological modeling.

modeling task. Parts of overlapping Landsat footprints were modeled separately, primarily to allow a measure of model cross-validation based on the separate (though not necessarily independent) datasets present in each Landsat footprint image stack.

\subsubsection{Processing Implementation}

Each available pixel in a geographical chunk (Fig. 4.7) was modeled individually by the process described above (Fig. 4.1). Phenological modeling for a single data chunk (a bundle of up to 256 pixels) typically required up to 14 hours of processing time, depending on the number of Landsat pixels in the 
chunk, with water and non-forest pixels removed. As a task for serial computation or on a small network of distributed processors, this procedure applied over an entire Landsat footprint would have been timeprohibitive. The chunks and their constituent pixels do not need to be processed in any particular order, and the results of processing one pixel or chunk do not affect other (even neighboring) pixels or chunks in the study area. This aspect of task independence lends to this problem an ease of processing on the Open Science Grid (OSG; www.opensciencegrid.org) [Pordes et al., 2007; Altunay et al., 2011], a distributed computing environment designed for high-throughput tasks with low memory demands and relatively small processing times using "opportunistic" (spare, otherwise unused) scheduling at affiliated sites across the US [Jayatilaka et al., 2015]. For my computational needs I worked with the UW-Madison CHTC, with access to numerous on-campus computing clusters as well as the OSG. The CHTC DAGMan protocols facilitated both data and computational workflow management throughout this research.

Over the course of this work I consumed more than 13 million hours of processor time, with nearly half of that time ( $\sim 5.6$ million hours $)$ provided by OSG affiliates across the US and the remainder provided by CHTC and affiliated on-campus computing clusters. I designated all of the phenological model processing ( $\sim 8.8$ million hours) to OSG and on-campus clusters. My pre-model processing, to prepare datasets and decompose the geographic domain into individual chunks, and post-model processing, to recompose the results for footprint- and domain-level analyses, consumed significant processor time ( $\sim 5.1$ million hours) within CHTC's own distributed computing cluster and with access to high-capacity data storage units. I estimate that processing each Landsat footprint, including 30 years of pre-processed Landsat scenes ( $\sim 250$ image dates), the corresponding climatological input data, and the reconstituted model products, requires $\sim 500 \mathrm{~GB}$ of archival storage space (model input and output, based on compressed file sizes) and as much as 4 TB of volatile (actively-managed) storage space throughout the processing workflow that I have developed. If all intermediate data products are to be retained through the end of the workflow, processing of each footprint could require as much as $8 \mathrm{~TB}$ of total storage space before data reduction. 


\section{CHAPTER 5}

\section{REGIONAL CLIMATOLOGY}

Author's Note: much of this chapter has been published previously [Garcia and Townsend, 2016].

To support application of the phenoclimatological model described in Chapter 4, I undertook a detailed study of the climatology in my study area using the meteorological data sources described in Chapter 3. Instead of relying on existing climatological analyses that covered only part of my study area or time period of interest, this fresh climatological analysis allowed me to expand beyond standard temperature and precipitation measures to an exhaustive list of climatological derivative measures based on daily meteorological observations that could be tailored to my desired time period, locations, and subject of interest. Specifically, I was able to focus on an extensive accounting of cold-season and growing season measures to support the phenological analyses that will be presented in Chapter 6 .

\subsection{Temperature and Precipitation Indicators and Lake Superior Influences}

Area-averaged 1984-2013 mean daily temperature observations $\left(T_{\min }, T_{\max }\right.$, and $\left.T_{a v g}\right)$ through the year are shown in Fig. 5.1, which also provides a conceptual overview of key temperature-related climate variables that I analyze: $C D, F D$ and $G D D$ accumulations, mean last Spring and first Autumn frost dates, and mean $C D$ plateau beginning and ending dates. The area-averaged mean annual accumulations of $C D$ and $G D D$ are shown in Fig. 5.2 and are also marked with key dates related to the $C D$ plateau. Areaaveraged seasonal and annual precipitation $(P)$ totals for 1984-2013 are shown in Fig. 5.3. Mean daily precipitation throughout the year is shown in Fig. 5.4. The study-area-averaged climatological mean, variance, trend, and extreme values for a number of both seasonal and annual climatological indicators are summarized in Table 5.1.

Maps of annual average $T$ values and interannual trends are shown in Fig. 5.5. Cooler temperatures in Spring and Summer are concentrated on the Minnesota (northern) shore of Lake Superior, and the 


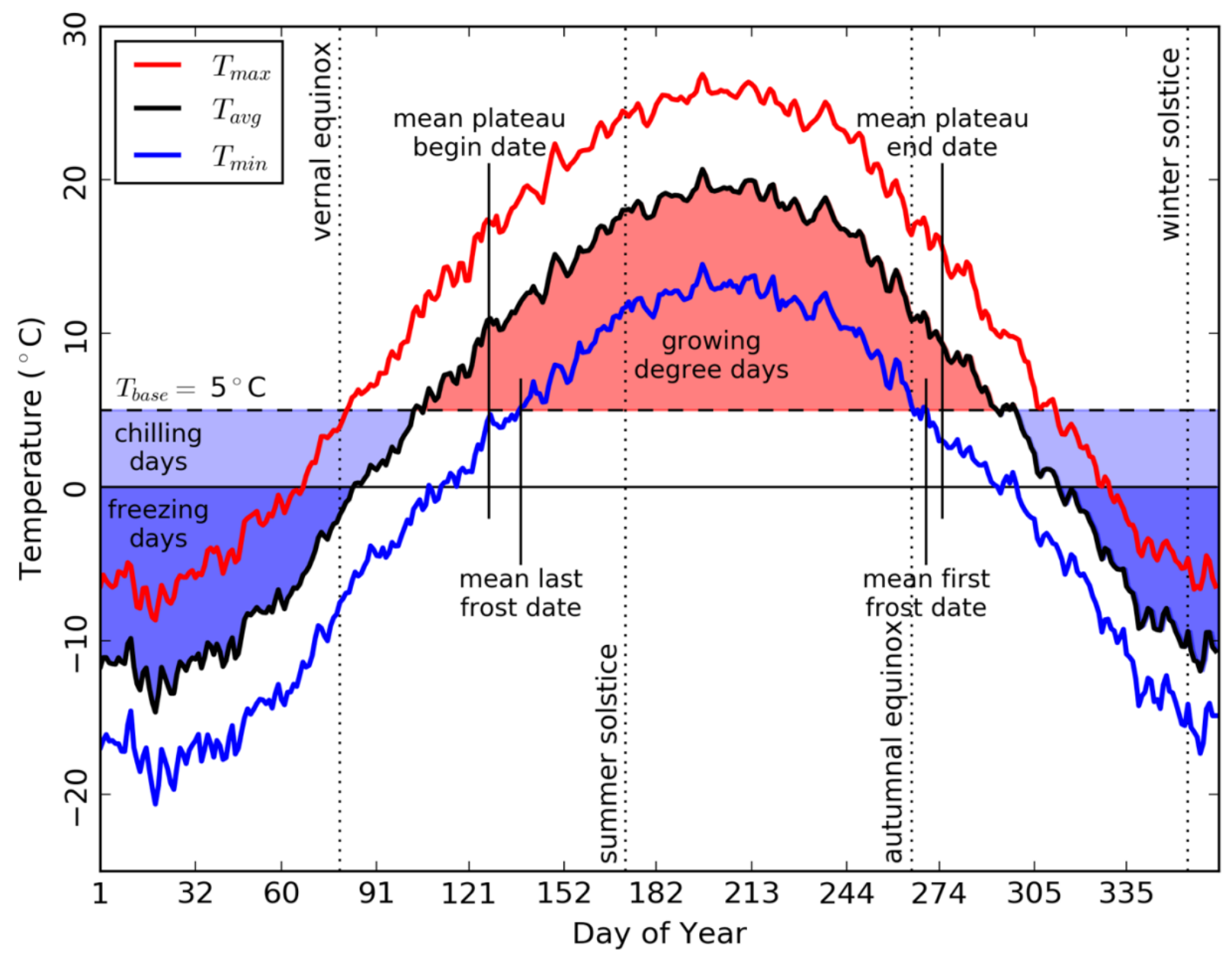

Figure 5.1: 1984-2013 mean daily temperatures averaged over the study area, with observed seasonal indicators marked. See Table 5.1 for indicator values and additional details.

coldest temperatures in all seasons are found in the land areas northwest of the lake (Fig. 5.5a, c, and e).

Warmer temperatures in Autumn and Winter are generally concentrated on the Wisconsin/ Michigan (southern) shore of Lake Superior, although some warm locations along the Minnesota shore are also apparent. The warmest temperatures in all seasons are found in the southwestern portion of the study area in primarily agricultural regions (see Fig. 3.2). The resulting spatial gradient in $T_{\text {avg }}$ is strongest in a general north-south orientation across the far western end of Lake Superior and more generally across the tension zone that traverses our study area. Long-term positive trends in $T_{\min }$ (Fig. 5.5b) are generally more widespread and stronger than those in $T_{\max }$ (Fig. 5.5d), which actually demonstrates some areas of longterm cooling both northwest and southeast of Lake Superior over the study period. 
Table 5.1: Climatological indicator statistics for the study period 1984-2013, derived from daily maps of interpolated $T$ and $P$ and averaged across the study area. Significant trends are indicated * at $p<0.05$ and ** at $p$ $<0.01$. El Niño years $(1987-1988,1992,1995,1998,2003,2010)$ are marked ${ }^{\#}$ and La Niña years $(1985,1989$, 1996, 1999-2001, 2006, 2008-2009, 2011-2012) are marked ${ }^{\dagger}$.

\begin{tabular}{|c|c|c|c|c|c|}
\cline { 2 - 6 } \multicolumn{1}{c|}{} & Mean & Std. Dev. & 30y Trend & Minimum & Maximum \\
\hline Temperature Indicators & $\mathbf{( 3 0 y ~ r e c )}$ & $\mathbf{( 3 0 y ~ r e c )}$ & $\mathbf{( u n i t s} / \mathbf{y r})$ & (Year) & (Year) \\
\hline Winter Average Temperature $\left[{ }^{\circ} \mathrm{C}\right]$ & -9.2 & 2.2 & +0.03 & $-13.2(1994)$ & $-4.3\left(2012^{\dagger}\right)$ \\
\hline - within-season variance $\left[{ }^{\circ} \mathrm{C}^{2}\right]$ & 49.5 & 13.8 & -0.41 & $25.1\left(2006^{\dagger}\right)$ & $81.3(1994)$ \\
\hline Spring Average Temperature $\left[{ }^{\circ} \mathrm{C}\right]$ & 10.0 & 1.4 & -0.04 & $7.5(2013)$ & $12.4\left(1987^{\#}\right)$ \\
\hline - within-season variance $\left[{ }^{\circ} \mathrm{C}^{2}\right]$ & 45.4 & 11.6 & -0.10 & $28.5\left(1998^{\#}\right)$ & $69.1\left(1996^{\dagger}\right)$ \\
\hline Summer Average Temperature $\left[{ }^{\circ} \mathrm{C}\right]$ & 18.3 & 0.9 & +0.03 & $15.6\left(1992^{\#}\right)$ & $19.9(2002)$ \\
\hline - within-season variance $\left[{ }^{\circ} \mathrm{C}^{2}\right]$ & 14.1 & 4.0 & +0.02 & $6.5\left(2009^{\dagger}\right)$ & $20.4\left(1988^{\#}\right)$ \\
\hline Autumn Average Temperature $\left[{ }^{\circ} \mathrm{C}\right]$ & 1.0 & 1.6 & +0.05 & $-2.7\left(1985^{\dagger}\right)$ & $4.2\left(2001^{\dagger}\right)$ \\
\hline - within-season variance $\left[{ }^{\circ} \mathrm{C}^{2}\right]$ & 69.8 & 26.8 & +0.18 & $27.9\left(2001^{\dagger}\right)$ & $121.7\left(19899^{\dagger}\right)$ \\
\hline Annual Average Temperature $\left[{ }^{\circ} \mathrm{C}\right]$ & 5.1 & 1.5 & +0.02 & $3.3\left(1996^{\dagger}\right)$ & $7.3\left(1998^{\#}\right)$ \\
\hline
\end{tabular}

\section{Precipitation Indicators}

\begin{tabular}{|l|c|c|c|c|c|}
\hline Winter Total Precipitation [cm] & 7.9 & 1.9 & +0.07 & $3.3\left(1987^{\#}\right)$ & $11.7(1997)$ \\
\hline - season precipitation days [d] & 86.2 & 2.8 & $+0.17^{* *}$ & $78.0\left(1987^{\#}\right)$ & $90.0(1997)$ \\
\hline Spring Total Precipitation [cm] & 23.3 & 5.5 & +0.17 & $12.8\left(1988^{\#}\right)$ & $35.7\left(2012^{\dagger}\right)$ \\
\hline - season precipitation days [d] & 85.9 & 3.4 & $+0.18^{* *}$ & $77.0(1984)$ & $90.0(1994)$ \\
\hline Summer Total Precipitation [cm] & 28.1 & 6.0 & $-0.30^{*}$ & $18.1\left(2012^{\dagger}\right)$ & $39.1\left(2010^{\#}\right)$ \\
\hline - season precipitation days [d] & 88.5 & 1.4 & -0.02 & $85.0\left(1989^{\dagger}\right)$ & $90.0(1984)$ \\
\hline Autumn Total Precipitation [cm] & 15.4 & 4.6 & +0.03 & $7.5\left(1989^{\dagger}\right)$ & $24.7\left(1996^{\dagger}\right)$ \\
\hline - season precipitation days [d] & 86.1 & 3.2 & +0.08 & $78.0\left(1999^{\dagger}\right)$ & $90.0\left(1995^{\#}\right)$ \\
\hline Annual Total Precipitation [cm] & 76.2 & 8.6 & -0.02 & $61.1\left(2006^{\dagger}\right)$ & $92.0(1991)$ \\
\hline
\end{tabular}

\section{Cold Season Indicators}

\begin{tabular}{|l|c|c|c|c|c|}
\hline Freezing Days (using $\left.T_{\min }\right)[\mathrm{d}]$ & 165.5 & 8.5 & -0.17 & $149.2\left(2010^{\#}\right)$ & $183.6\left(1996^{\dagger}\right)$ \\
\hline Chilling Days (using $T_{\text {avg }}$ ) [d] & 160.1 & 9.5 & -0.14 & $139.3\left(2010^{\#}\right)$ & $178.1\left(1996^{\dagger}\right)$ \\
\hline Cold Season Intensity [ $\left.{ }^{\circ} \mathrm{C}\right]$ & 11.5 & 1.3 & -0.03 & $9.2\left(2012^{\dagger}\right)$ & $13.9\left(1996^{\dagger}\right)$ \\
\hline Last Spring Freezing Night [DOY] & 129.2 & 5.6 & +0.00 & $119.2\left(1998^{\#}\right)$ & $139.2(2002)$ \\
\hline First Autumn Freezing Night [DOY] & 271.6 & 6.3 & +0.10 & $258.3(2007)$ & $285.6(2013)$ \\
\hline Lake Superior Ice-On Date [DOY] & 15.9 & 18.7 & $+1.15^{* *}$ & $-13(1986 / 90)$ & $69(2002)$ \\
\hline Lake Superior Ice-Off Date [DOY] & 88.3 & 12.2 & +0.69 & $63\left(2000^{\dagger}\right)$ & $115\left(1996^{\dagger}\right)$ \\
\hline Lake Superior Ice Duration [d] & 72.4 & 26.9 & -0.46 & $7(2002)$ & $109\left(1996^{\dagger}\right)$ \\
\hline
\end{tabular}

\begin{tabular}{|l|l|c|c|c|c|}
\hline Warm (Growing) Season Indicators \\
\hline Last Spring Freezing Night $\left[{ }^{\circ} \mathrm{C} \mathrm{d}\right]$ & 117.2 & 44.7 & -0.49 & $43.2\left(1996^{\dagger}\right)$ & $204.8\left(2000^{\dagger}\right)$ \\
\hline Frost-free Season [d] & 142.4 & 5.9 & +0.09 & $129.6\left(2011^{\dagger}\right)$ & $153.8\left(1998^{\#}\right)$ \\
\hline Beginning of CD Plateau [DOY] & 122.2 & 9.2 & +0.21 & $97.4\left(1987^{\#}\right)$ & $136.1(2002)$ \\
\hline End of CD Plateau [DOY] & 276.9 & 7.1 & $+0.36^{*}$ & $262.9(1991)$ & $289.4(2013)$ \\
\hline Plateau Length [d] & 154.7 & 9.6 & +0.15 & $135.2(1991)$ & $179.1\left(1987^{\#}\right)$ \\
\hline Plateau Growing Degree Days $\left[{ }^{\circ} \mathrm{C} \mathrm{d}\right]$ & 1796.7 & 134.2 & +1.58 & $1527.8(1993)$ & $2065.6\left(1987^{\#}\right)$ \\
\hline Plateau Intensity $\left[{ }^{\circ} \mathrm{C}\right]$ & 11.6 & 0.8 & -0.00 & $9.9\left(1992^{\#}\right)$ & $13.6(1991)$ \\
\hline
\end{tabular}



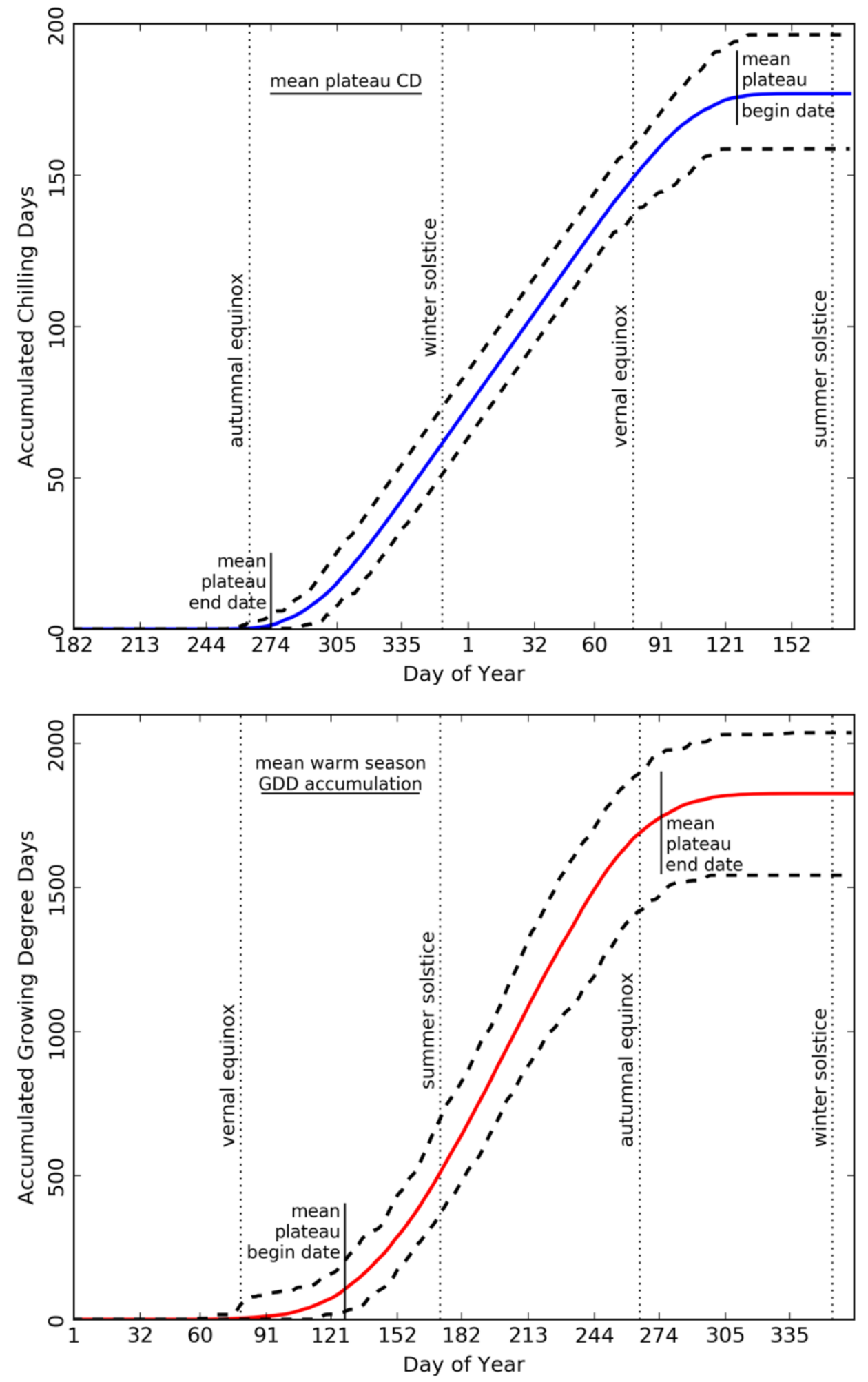

Figure 5.2: 1984-2013 mean $C D$ and $G D D$ accumulations averaged over the study area, with seasons marked. Dashed lines represent the maximum and minimum accumulations within our study period. See Table 5.1 for indicator values and additional details. 


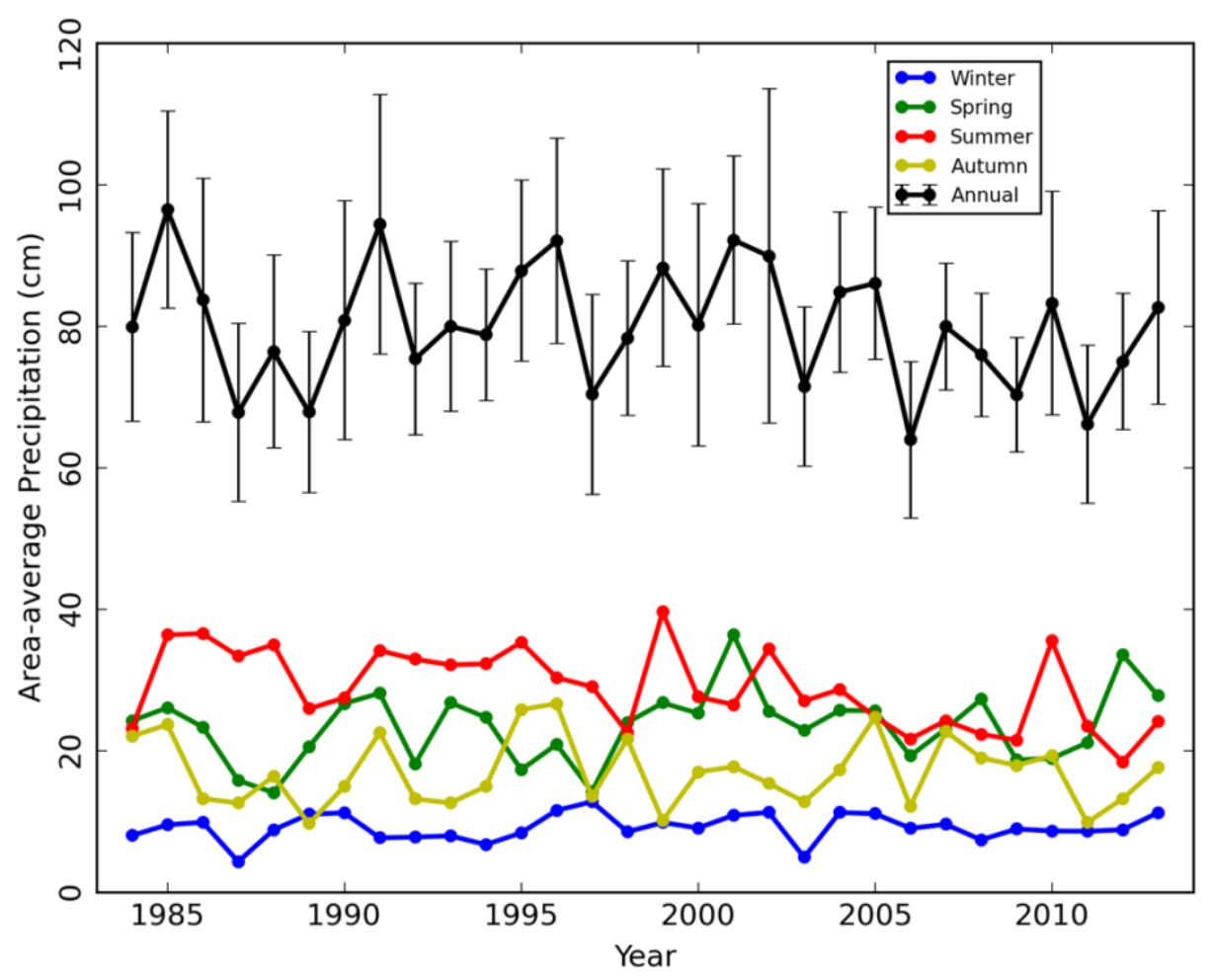

Figure 5.3: 1984-2013 seasonal and annual precipitation averaged over the study area. Error bars indicate spatial variability $( \pm 1 \sigma)$ in annual total precipitation.

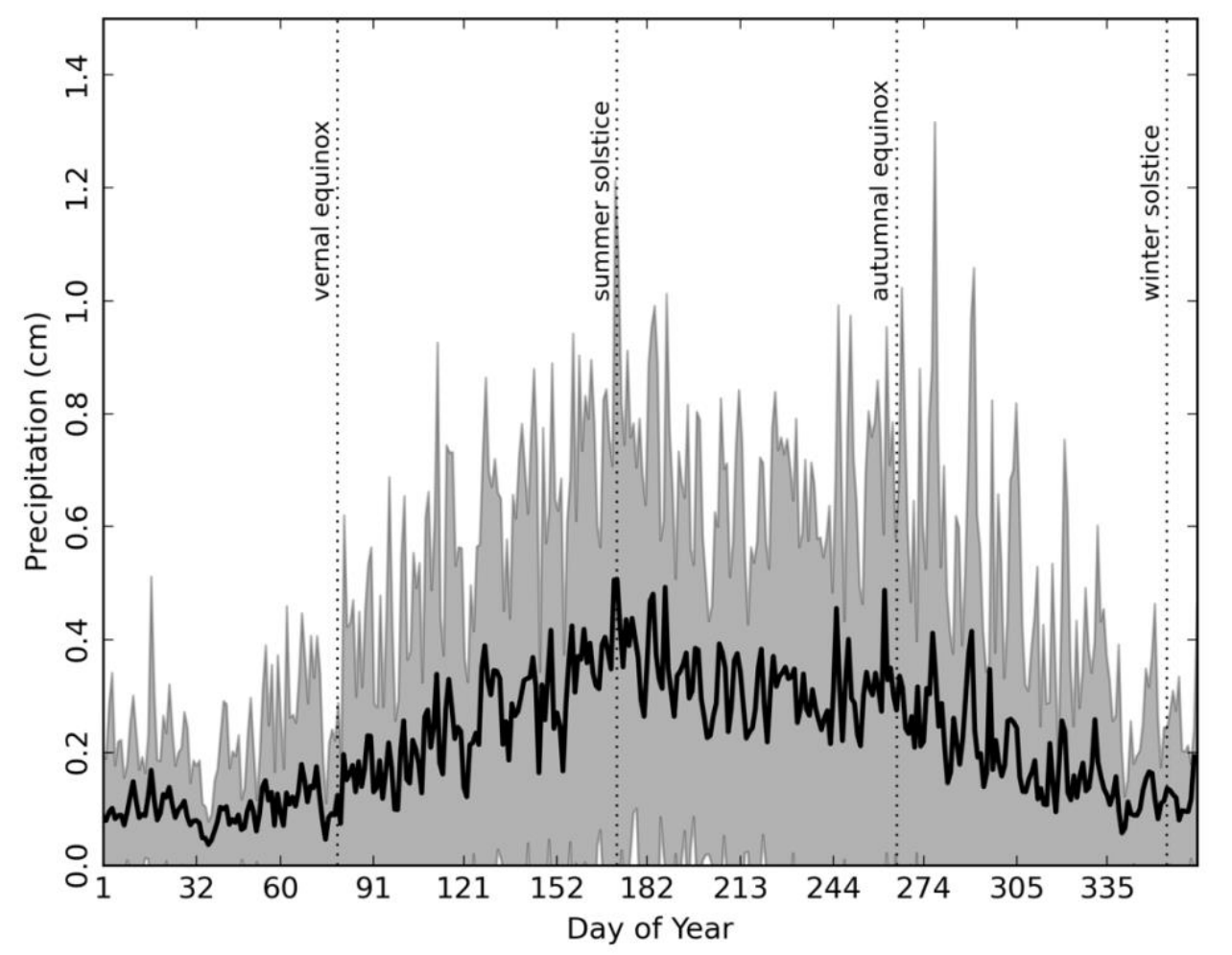

Figure 5.4: 1984-2013 mean annual cycle of daily precipitation. The gray region indicates year-to-year temporal variability $( \pm 1 \sigma)$ in daily precipitation averaged over the study area. 
(a) 30-y mean $T_{\min }\left[{ }^{\circ} \mathrm{C}\right]$

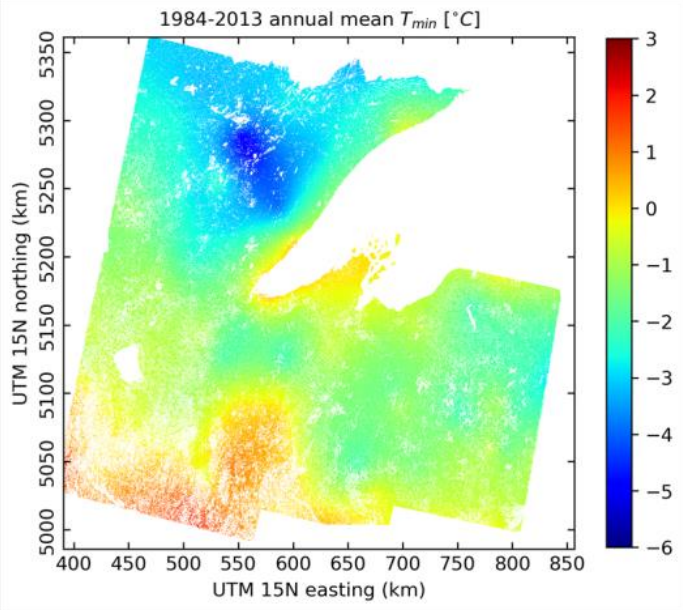

(c) 30-y mean $T_{\max }\left[{ }^{\circ} \mathrm{C}\right]$

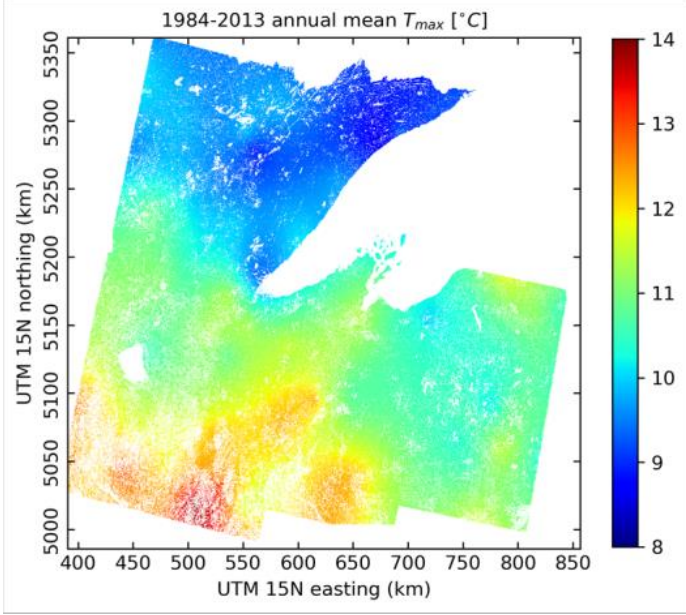

(e) 30-y mean $T_{\text {avg }}\left[{ }^{\circ} \mathrm{C}\right]$

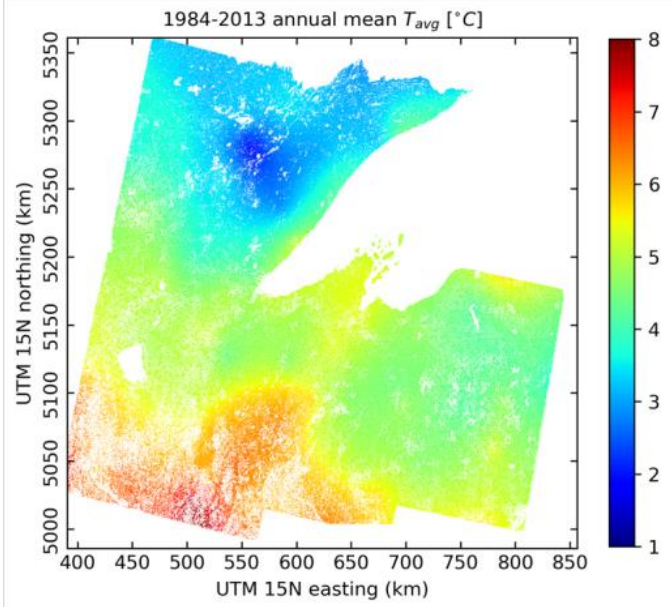

(b) 30-y $T_{\min }$ trend $\left[{ }^{\circ} \mathrm{C} / \mathrm{y}\right]$

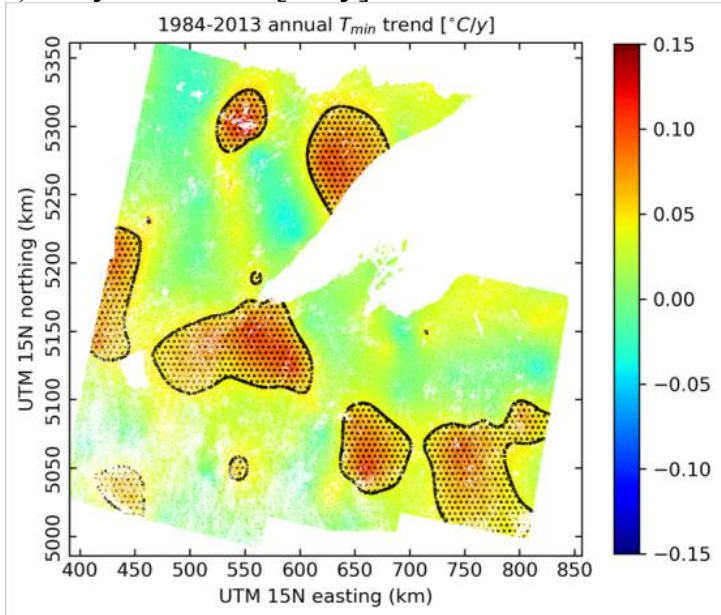

(d) 30-y $T_{\max }$ trend $\left[{ }^{\circ} \mathrm{C} / \mathrm{y}\right]$

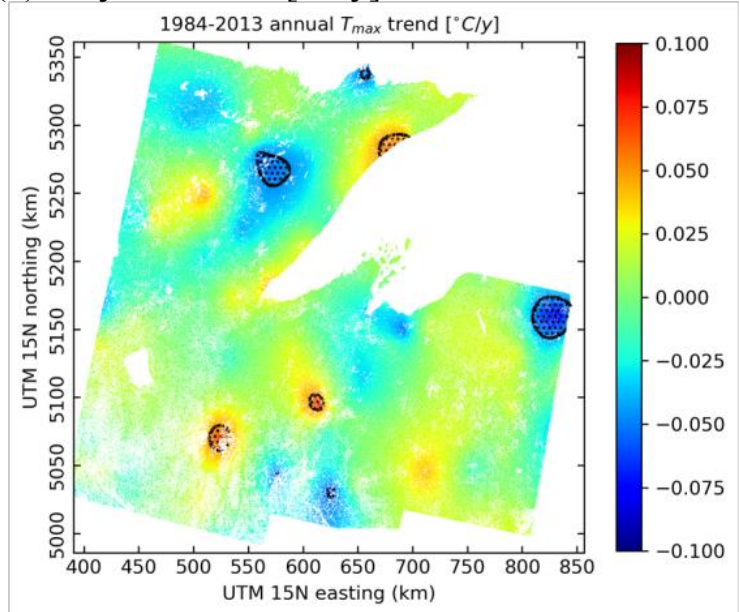

(f) 30 -y $T_{\text {avg }}$ trend $\left[{ }^{\circ} \mathrm{C} / \mathrm{y}\right]$

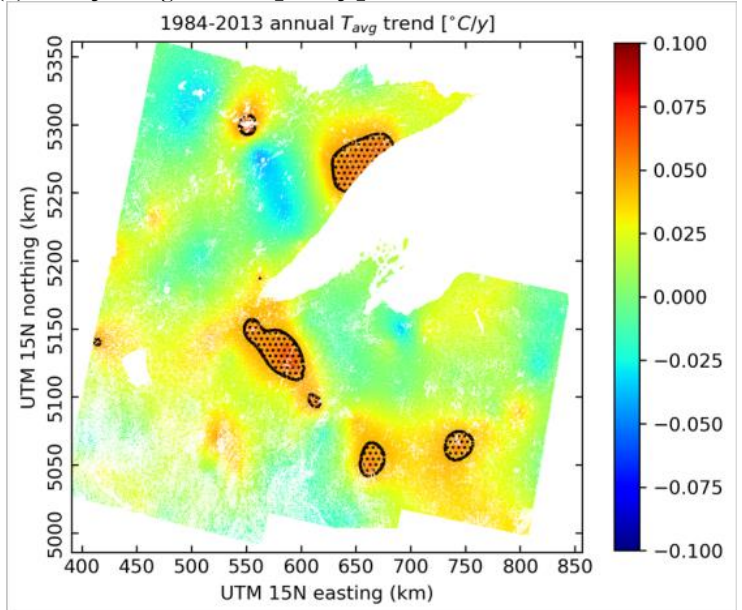

Figure 5.5: 1984-2013 mean annual $T_{\min }, T_{\max }$, and $T_{a v g}$ with trends. Areas of trend significance at $p<0.05$ in (b), (d), and (f) are stippled. 
From the area-average trends listed in Table 5.1 I calculated a 30-year net warming of $+0.56^{\circ} \mathrm{C}$ in annual mean $T_{\text {avg }}$ for my study area. Long-term cooling in Spring (net $-1.26^{\circ} \mathrm{C} / 30 \mathrm{y}$ ) has been offset by warming in all other seasons, especially Autumn (net $+1.54^{\circ} \mathrm{C} / 30 \mathrm{y}$ ), with the remainder of overall 30 year net warming divided almost evenly between the Winter and Summer seasons. Though none of these overall temperature trends demonstrate statistical significance at the $p<0.05$ level using area-averaged values, significant trends can be found in more localized portions of the study area. In particular, longterm warming is evident in $T_{\min }$ (Fig. 5.5b) and $T_{\text {avg }}$ (Fig. 5.5f) on the Minnesota shore of Lake Superior and following the approximate location of the tension zone across the study area. Seasonal averages and trends in $T_{\min }$ are shown in Fig. 5.6, where slightly warming Winter nights (the general time-of-day for $T_{\min }$ occurrence) are accompanied by clearly warming Summer and Autumn nights as well. Seasonal averages and trends for $T_{\max }$ in Fig. 5.7 indicate cooling Spring days (the usual time-of-day for $T_{\max }$ occurrence) and slight warming in Autumn days. Seasonal average $T_{\text {avg }}$ and their trends are shown in Fig. 5.8, where cooling generally covers the study area in Spring but warming dominates in Summer and Autumn. Effects in some areas due to lake proximity are evident in a number of these seasonal results, especially in the mean $T_{\min }$ in Winter and Autumn and in the mean $T_{\max }$ and $T_{\text {avg }}$ in most seasons, which will be discussed in greater detail below.

Maps of annual average $P$ values and interannual trends are shown in Fig. 5.9. Mean seasonal precipitation (Fig. 5.10) is lowest in Winter and highest in Summer, with generally ambiguous area-wide spatial gradients in those seasons, while spatial gradients in mean seasonal $P$ across the study area are generally north-to-south in Spring and west-to-east in Autumn, with comparable total $P$ in those seasons. These patterns result in a southeastward gradient in mean annual $P$ that is most prominent across Lake Superior (Fig. 5.9a). Note here the lake-effect influence on spatial distributions of $P$ in Winter, with the areas of heaviest Winter precipitation on the southern shore and immediately downwind (southeast) of Lake Superior. Winter precipitation is increasing in some areas, especially in the immediate vicinity of Lake Superior, but Summer precipitation exhibits an area-average trend of $-0.34 \mathrm{~cm} / \mathrm{y}(p<0.01)$, with areas of stronger trends along almost the entire lakeshore and extending in a number of directions from 
(a) Winter $T_{\min }$ mean $\left[{ }^{\circ} \mathrm{C}\right]$

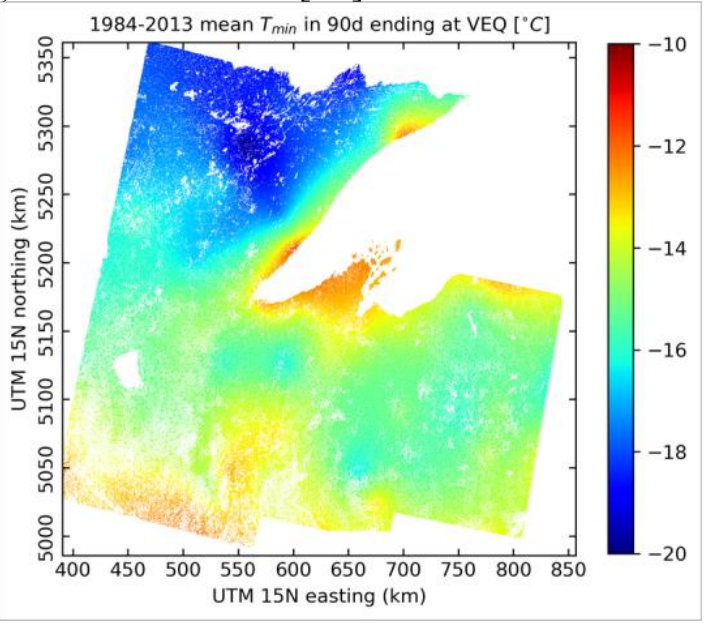

(c) Spring $T_{\min }$ mean $\left[{ }^{\circ} \mathrm{C}\right]$

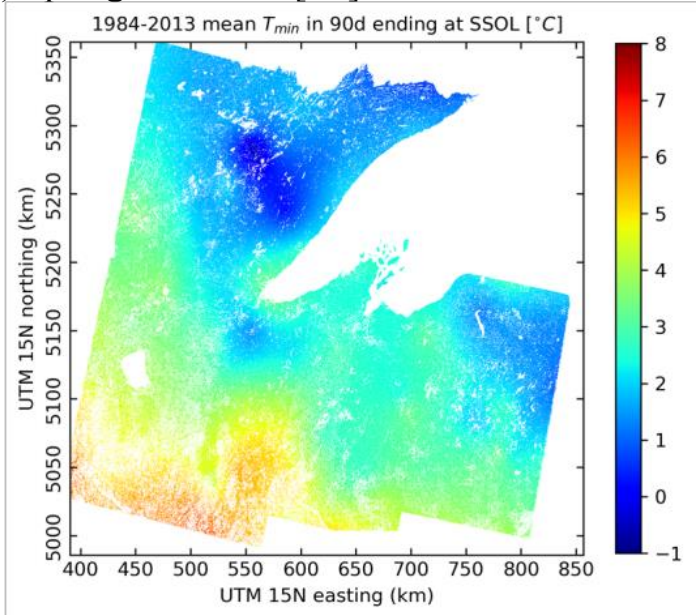

(b) Winter $T_{\min }$ trend $\left[{ }^{\circ} \mathrm{C} / \mathrm{y}\right]$

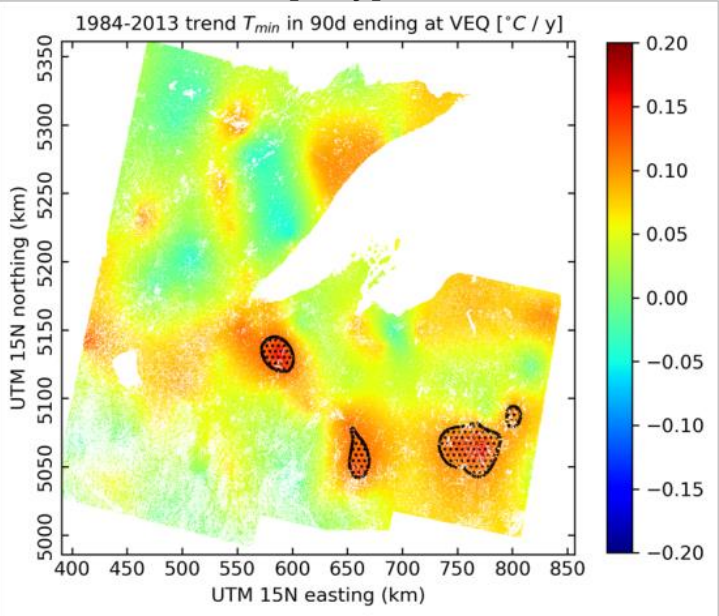

(d) Spring $T_{\min }$ trend $\left[{ }^{\circ} \mathrm{C} / \mathrm{y}\right]$

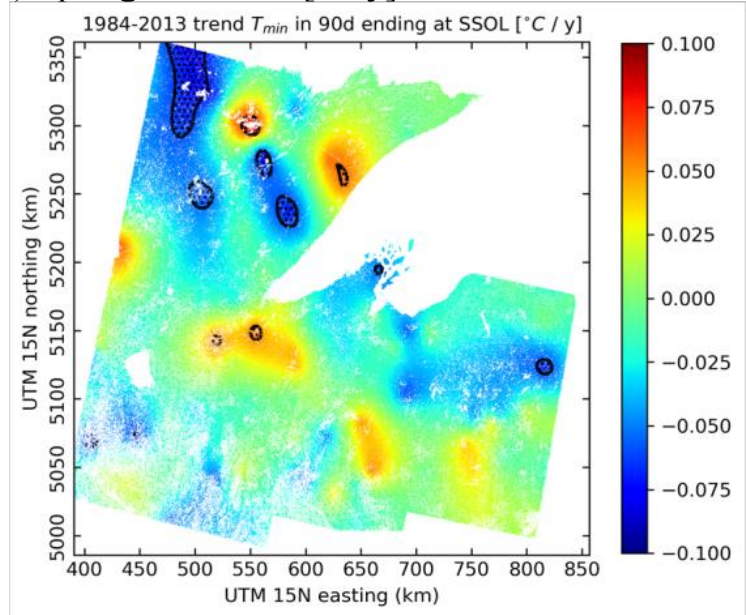

Figure 5.6: 1984-2013 mean seasonal $T_{\min }$ with trends. Areas of trend significance at $p<0.05$ in (b) and (d) are stippled. 
(e) Summer $T_{\min }$ mean $\left[{ }^{\circ} \mathrm{C}\right]$

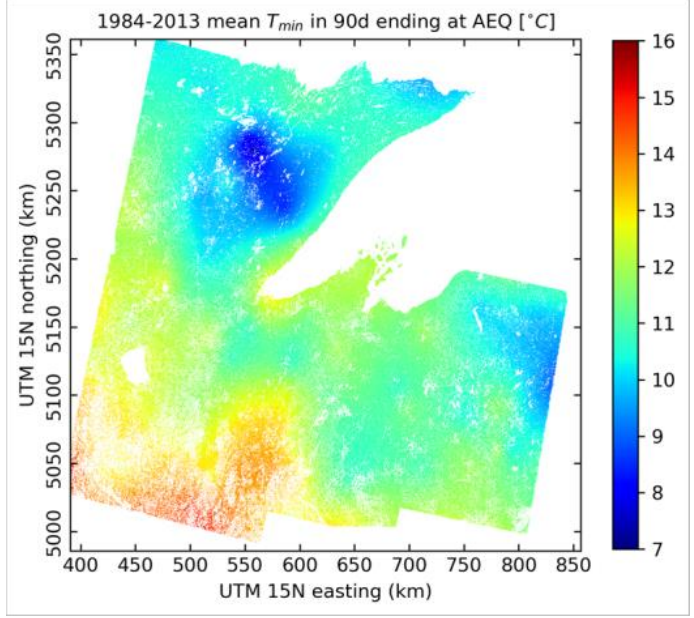

(g) Autumn $T_{\min }$ mean $\left[{ }^{\circ} \mathrm{C}\right]$

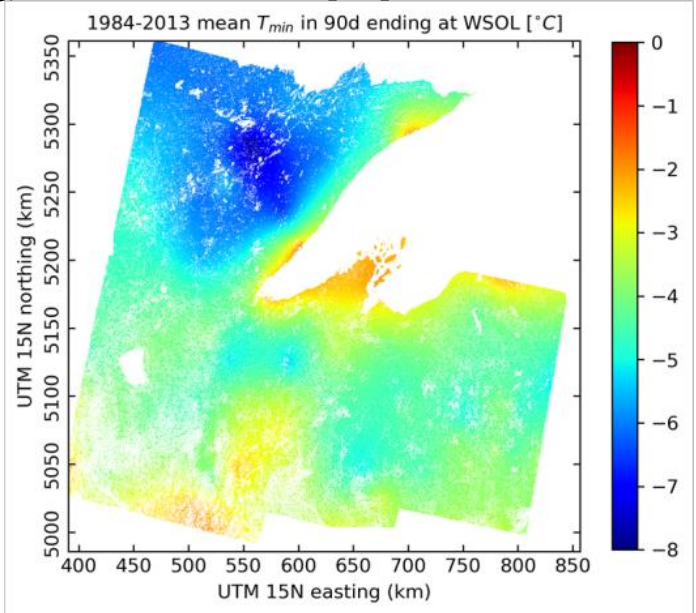

(f) Summer $T_{\min }$ trend $\left[{ }^{\circ} \mathrm{C} / \mathrm{y}\right.$ ]

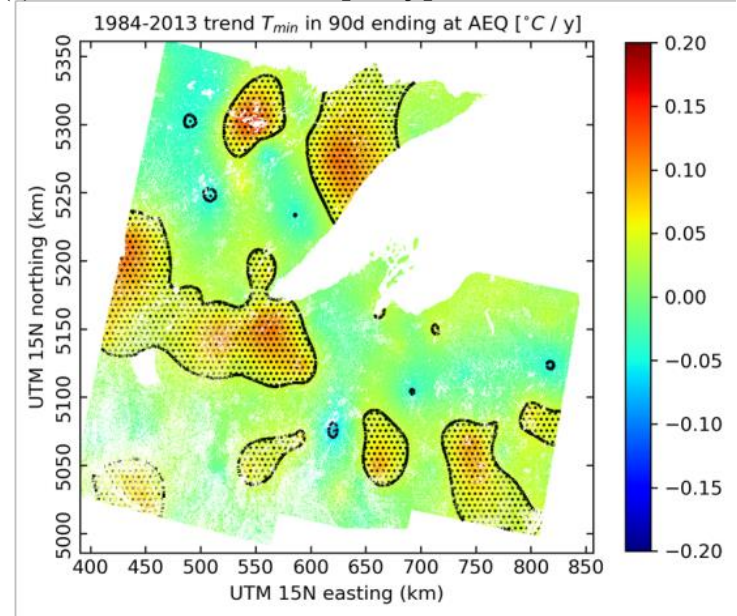

(h) Autumn $T_{\min }$ trend $\left[{ }^{\circ} \mathrm{C} / \mathrm{y}\right]$

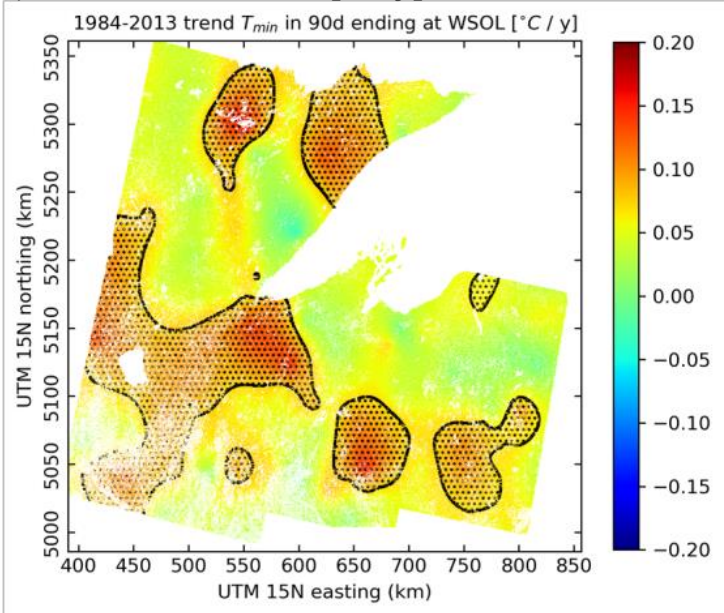

Figure 5.6 (continued): 1984-2013 mean seasonal $T_{\min }$ with trends. Areas of trend significance at $p<0.05$ in (f) and (h) are stippled. 
(a) Winter $T_{\max }$ mean $\left[{ }^{\circ} \mathrm{C}\right]$

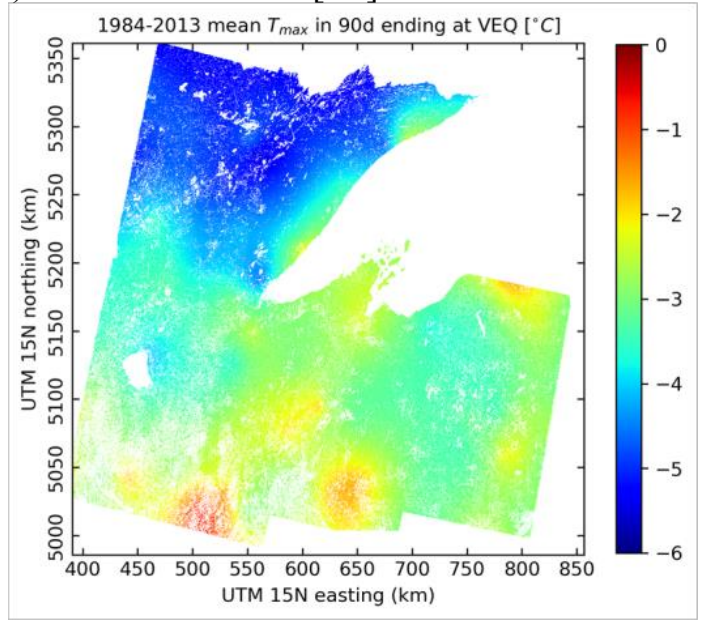

(c) Spring $T_{\max }$ mean $\left[{ }^{\circ} \mathrm{C}\right]$

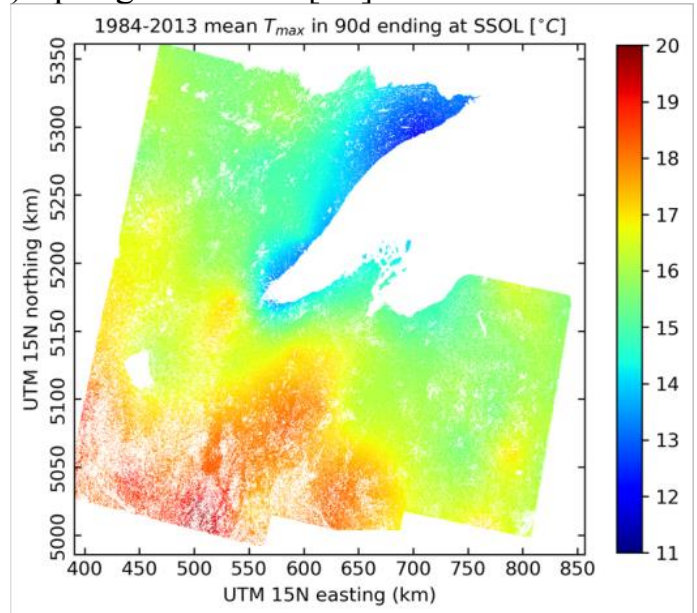

(b) Winter $T_{\max }$ trend $\left[{ }^{\circ} \mathrm{C} / \mathrm{y}\right]$

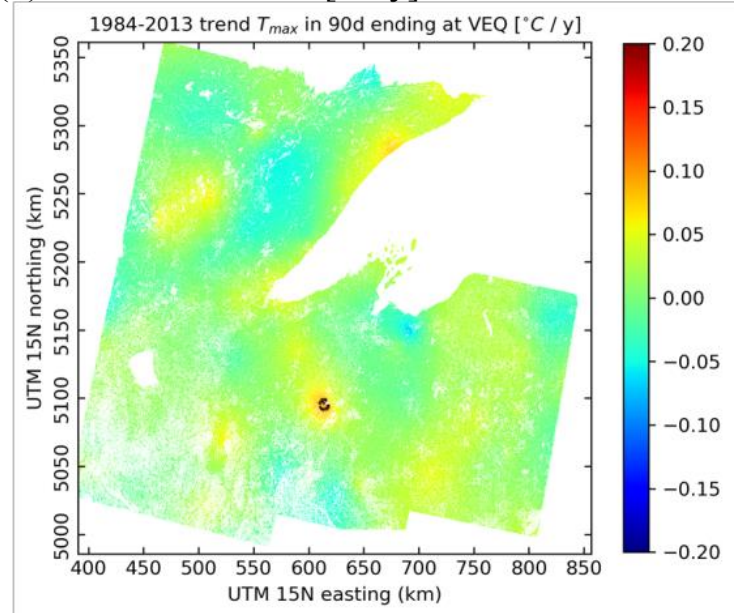

(d) Spring $T_{\max }$ trend $\left[{ }^{\circ} \mathrm{C} / \mathrm{y}\right]$

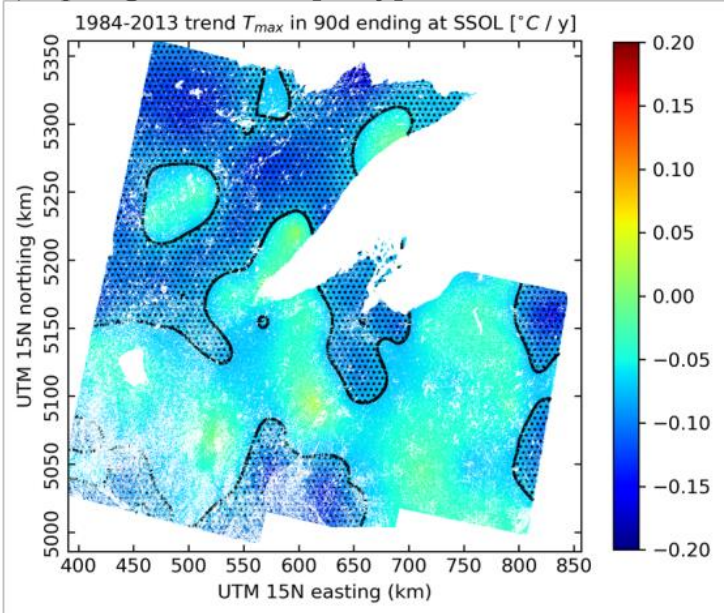

Figure 5.7: 1984-2013 mean seasonal $T_{\max }$ with trends. Areas of trend significance at $p<0.05$ in (b) and (d) are stippled. 
(e) Summer $T_{\max }$ mean $\left[{ }^{\circ} \mathrm{C}\right]$

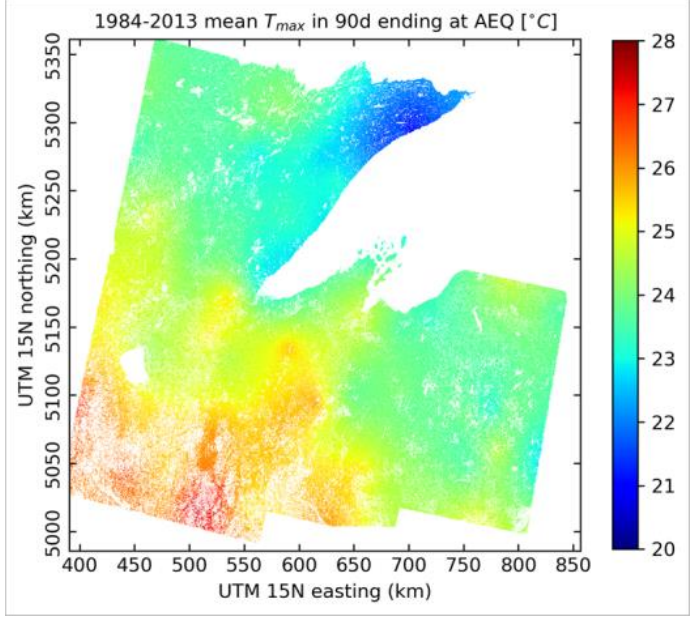

(g) Autumn $T_{\max }$ mean $\left[{ }^{\circ} \mathrm{C}\right]$

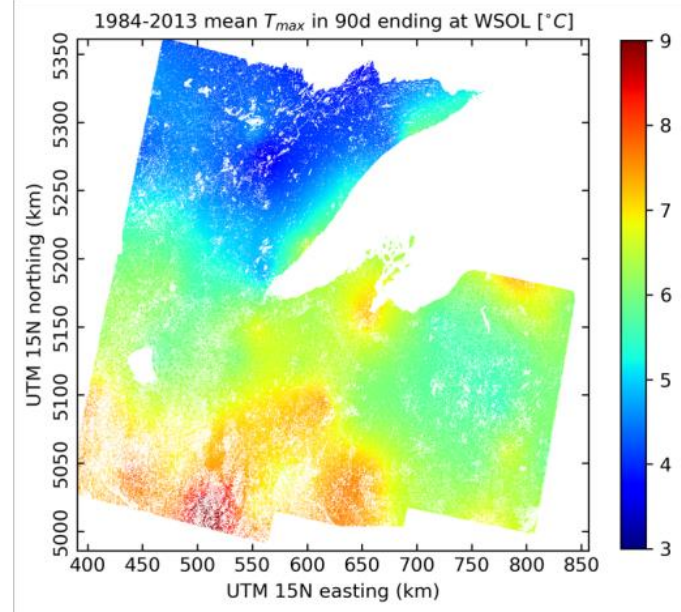

(f) Summer $T_{\max }$ trend $\left[{ }^{\circ} \mathrm{C} / \mathrm{y}\right]$

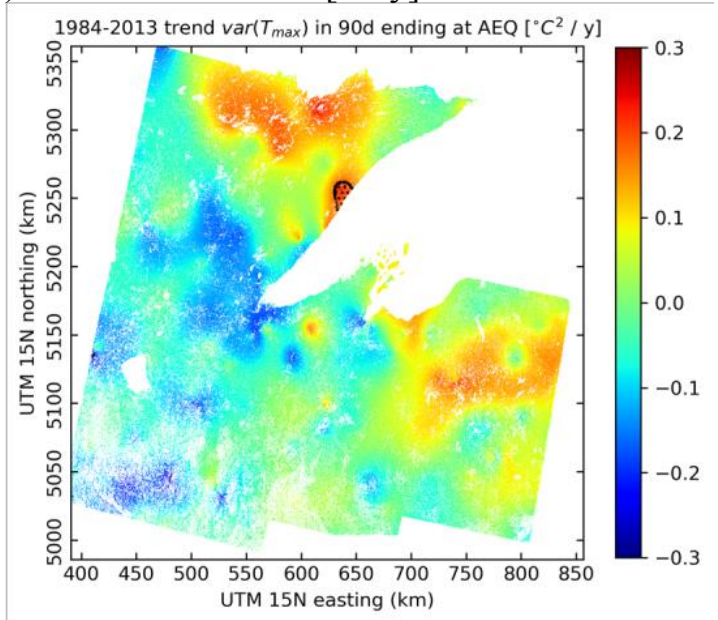

(h) Autumn $T_{\max }$ trend $\left[{ }^{\circ} \mathrm{C} / \mathrm{y}\right]$

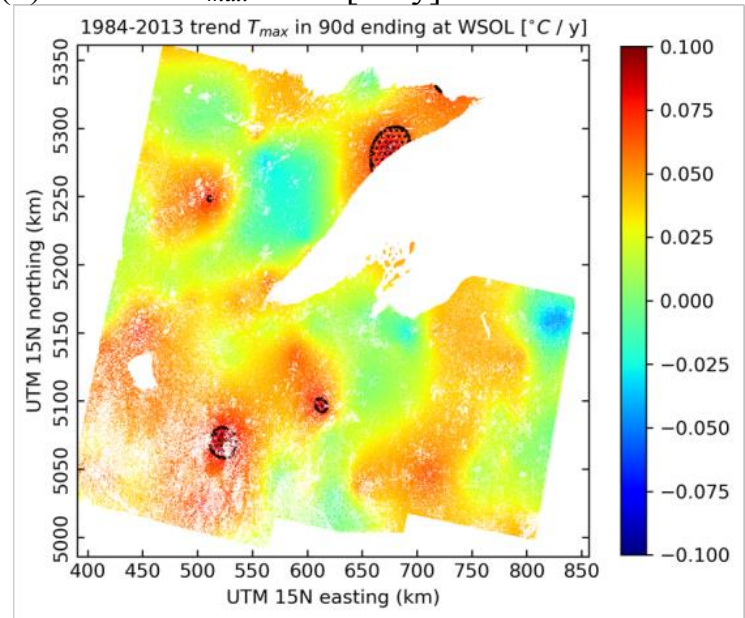

Figure 5.7 (continued): 1984-2013 mean seasonal $T_{\max }$ with trends. Areas of trend significance at $p<0.05$ in (f) and (h) are stippled. 
(a) Winter $T_{\text {avg }}$ mean $\left[{ }^{\circ} \mathrm{C}\right]$

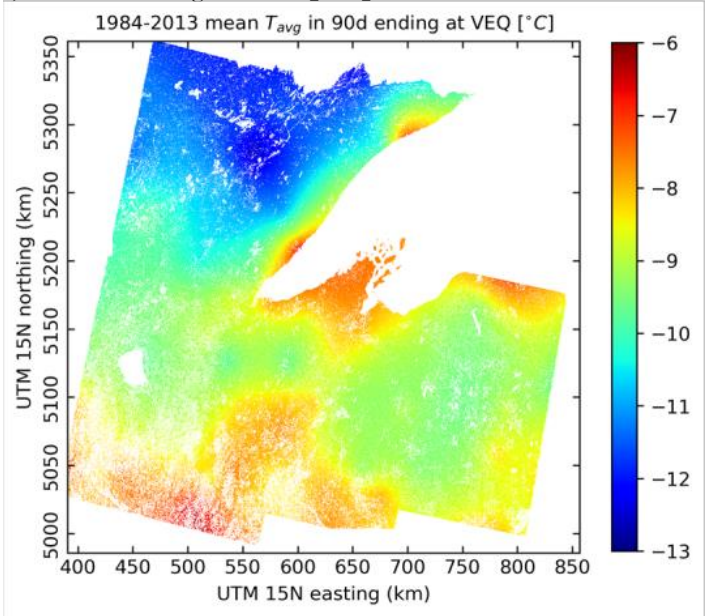

(c) Spring $T_{\text {avg }}$ mean $\left[{ }^{\circ} \mathrm{C}\right]$

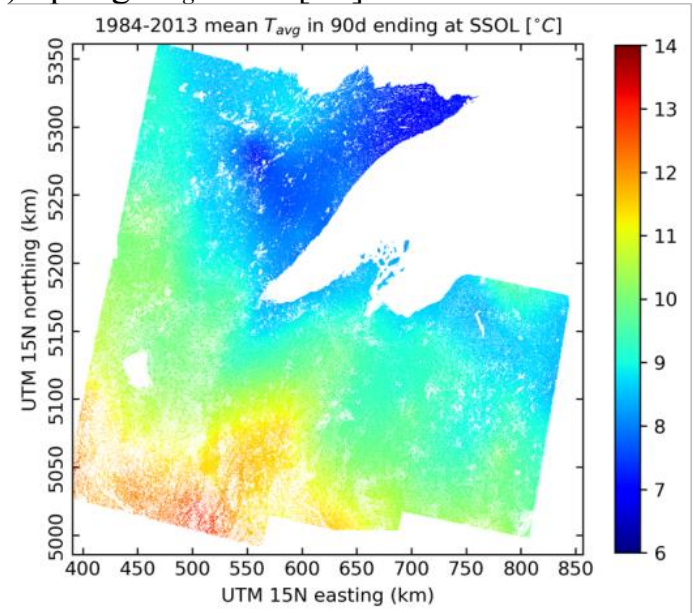

(b) Winter $T_{\text {avg }}$ trend $\left[{ }^{\circ} \mathrm{C} / \mathrm{y}\right]$

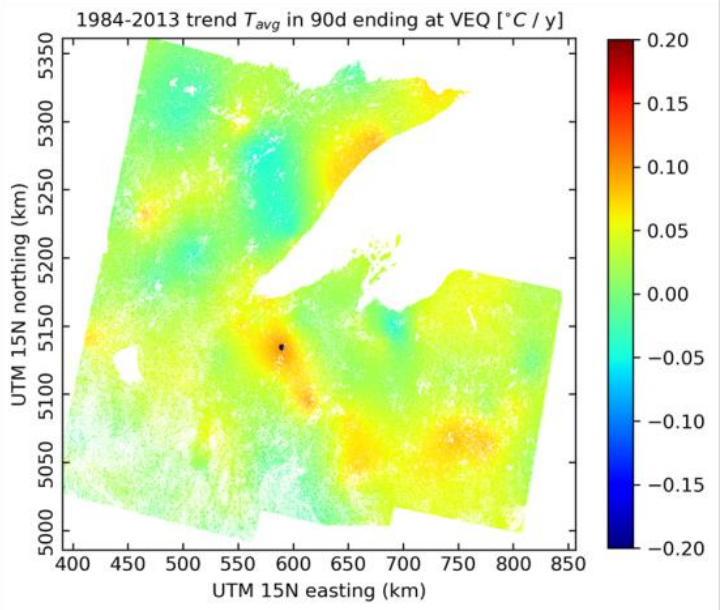

(d) Spring $T_{a v g}$ trend $\left[{ }^{\circ} \mathrm{C} / \mathrm{y}\right]$

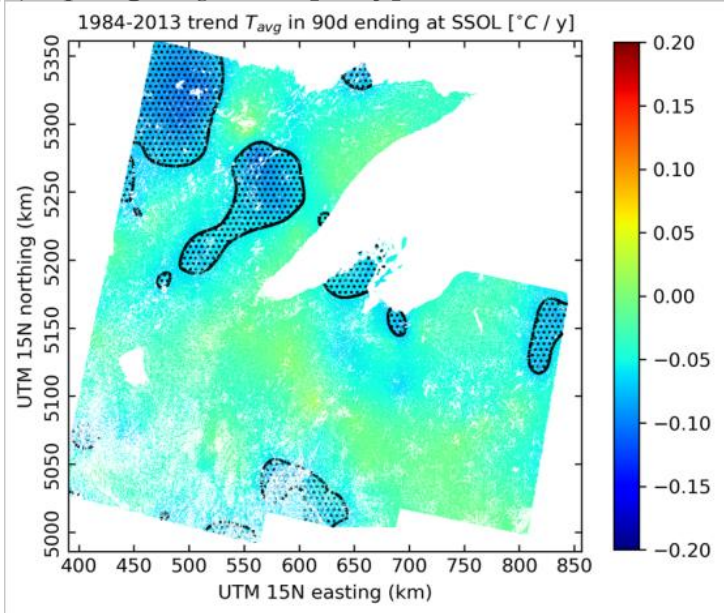

Figure 5.8: 1984-2013 mean seasonal $T_{\text {avg }}$ with trends. Areas of trend significance at $p<0.05$ in (b) and (d) are stippled. 
(e) Summer $T_{\text {avg }}$ mean $\left[{ }^{\circ} \mathrm{C}\right]$

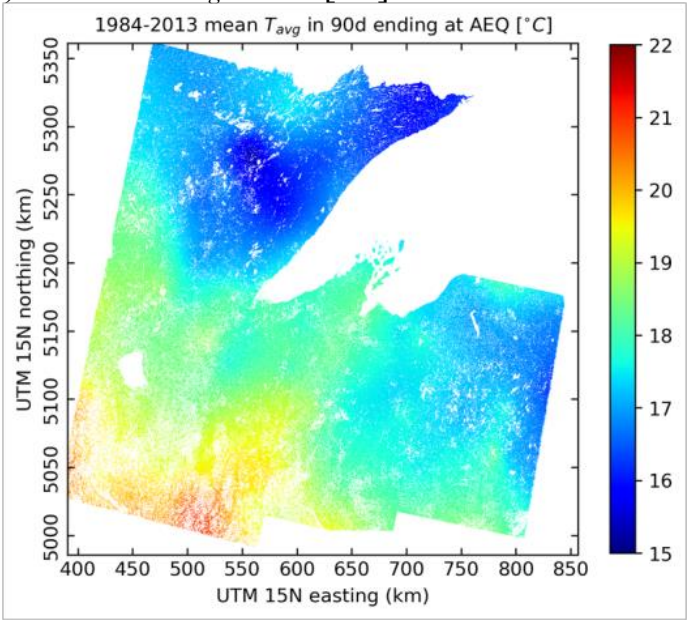

(g) Autumn $T_{a v g}$ mean $\left[{ }^{\circ} \mathrm{C}\right]$

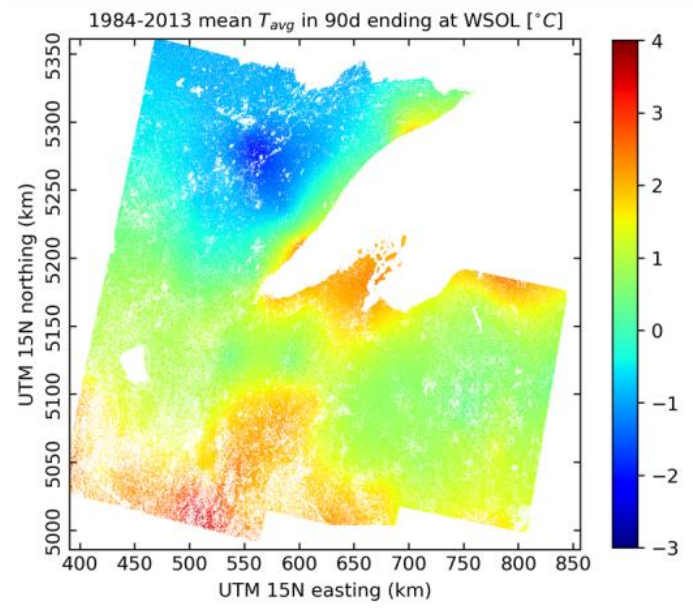

(f) Summer $T_{\text {avg }}$ trend $\left[{ }^{\circ} \mathrm{C} / \mathrm{y}\right]$

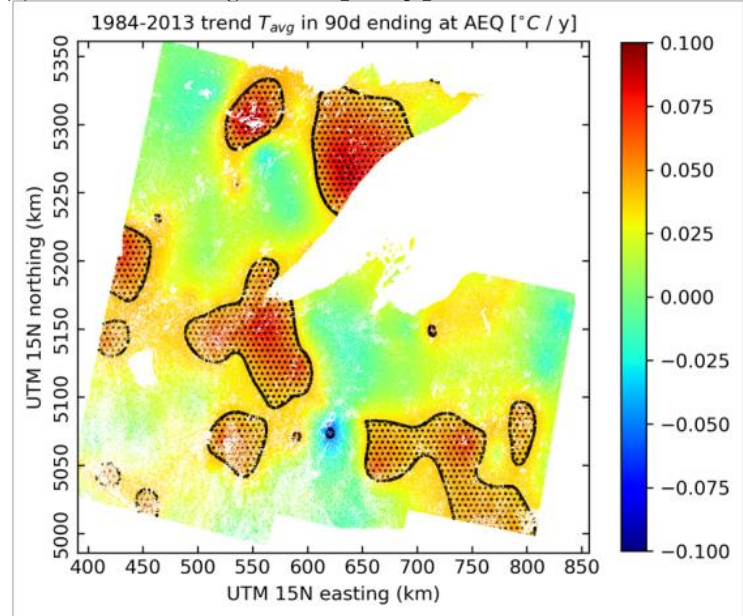

(h) Autumn $T_{a v g}$ trend $\left[{ }^{\circ} \mathrm{C} / \mathrm{y}\right]$

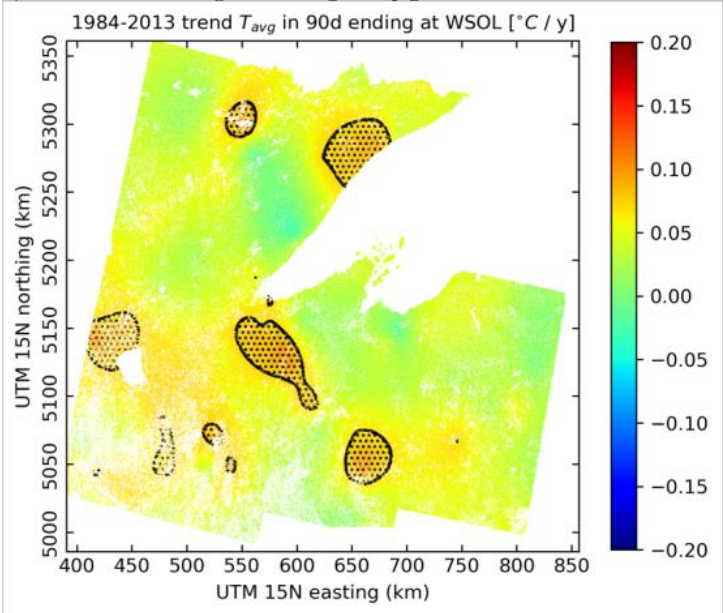

Figure 5.8 (continued): 1984-2013 mean seasonal $T_{a v g}$ with trends. Areas of trend significance at $p<0.05$ in (f) and $(\mathrm{h})$ are stippled. 
(a) 30-y mean $P[\mathrm{~cm}]$

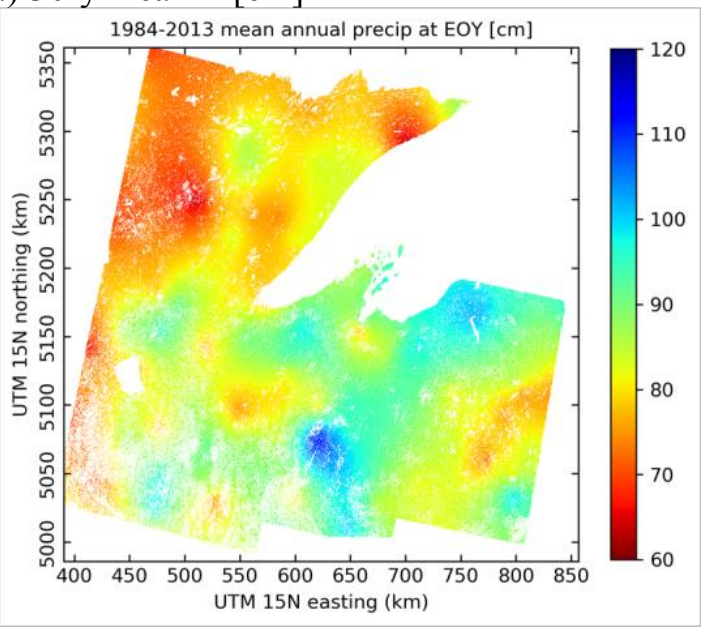

(b) $30-\mathrm{y} P$ trend $[\mathrm{cm} / \mathrm{y}]$

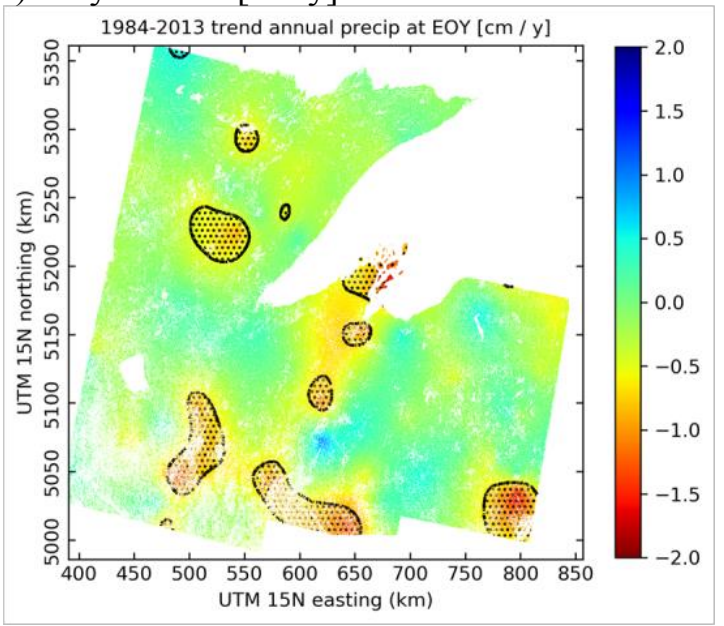

Figure 5.9: 1984-2013 mean annual $P$ with trends. Areas of trend significance at $p<0.05$ in (b) are stippled.

Lake Superior, especially along the tension zone in Wisconsin. Area-average reductions in Summer precipitation are consistent with an overall trend in Summer precipitation days $(P>0)$ of -0.25 days/y $(p$ $<0.001$ ). The resulting trends in total annual precipitation (Fig. 5.9b) are relatively neutral across much of the study area, with isolated locations of significant negative trends in areas where the Summer trends dominate, especially south and southeast of Lake Superior.

A number of results are consistent with the thermal influence of Lake Superior on regional climatological indicators. Higher Winter and lower Summer temperatures are found along the northern lakeshore than in the forest region farther to the northwest, with a longer frost-free season along that lakeshore by more than 4 days. Growing season transitions, as indicated by the beginning and end of the $C D$ plateau, are also delayed 2-3 days along the lakeshore in comparison with the forest areas to the northwest. The accumulation of area-wide Winter precipitation days is positively correlated with the date of Lake Superior ice-on conditions $(p<0.05)$ and negatively correlated with ice cover duration $(p<0.05)$. The number of Spring moderate precipitation days $(1 \mathrm{~cm}<P \leq 2.5 \mathrm{~cm})$ in areas downwind (south and southeast) of Lake Superior is negatively correlated with ice duration $(p<0.05)$, a result consistent with ice cover inhibiting lake evaporation and thus lake-effect precipitation in those areas. 
These results suggest that the seasonal ice cover on western Lake Superior plays an important role in temperature and precipitation patterns on adjacent land areas. Analyses indicate a trend in the date of Lake Superior ice-on conditions of +1.15 days $/ y(p<0.01)$, though it should be recognized that a linear trend cannot adequately account for the large interannual variability in lake ice phenology. Notably, during Autumn and Winter the area of greatest precipitation in the study area is concentrated southeast of Lake Superior where prevailing northwesterly winds carry lake-evaporated moisture onshore. Conversely, Spring and Summer $P$ appears relatively suppressed in the same area. Cold-season ice cover from midWinter through early Spring inhibits lake evaporation and lake effect precipitation; during the Summer, the lake is generally cooler than the surrounding land areas, inducing a stable atmospheric boundary layer and inhibiting the development of storms that could then move onshore [Changnon and Jones, 1972]. Even the small trends in lake ice phenology found here are enough to allow statistically significant increases in Winter precipitation (Fig. 5.10) southeast of Lake Superior. However, Spring precipitation seems also to increase in those same areas, which cannot be attributed to the slightly later lake ice breakup over time. In that case, these analyses may require a finer temporal division of seasonal precipitation in areas downwind of the lake so that portions of the Spring season before and after the observed ice-off date in each year are treated separately. Such a division could help demonstrate the transition from Winter (cold) to Spring (warm) weather patterns and phenological processes in the area of lake influence.

\subsection{Cold and Warm Season Indicators and Interseasonal Variability}

As expected, the greatest accumulation of $C D$ in the study area occurs in the forest areas northwest of Lake Superior (Fig. 5.11a). The 30-year trend in CD accumulation (Fig. 5.11b) is generally mixed across the study area, with only small areas of statistically significant reduction in $C D$ accumulation that 
(a) Winter $P$ mean $[\mathrm{cm}]$

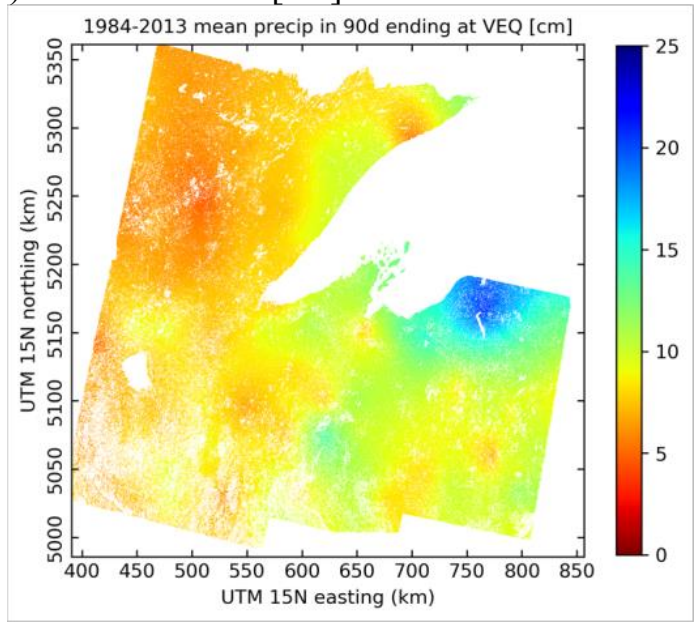

(c) Spring $P$ mean $[\mathrm{cm}]$

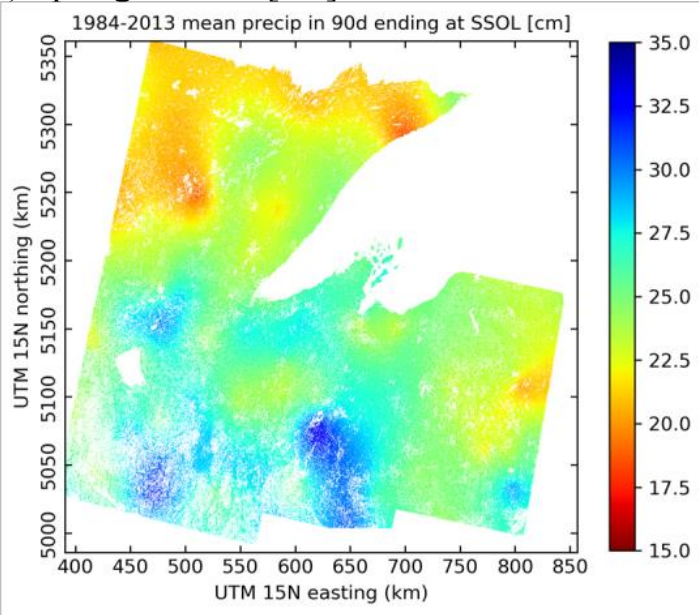

(b) Winter $P$ trend $[\mathrm{cm} / \mathrm{y}]$

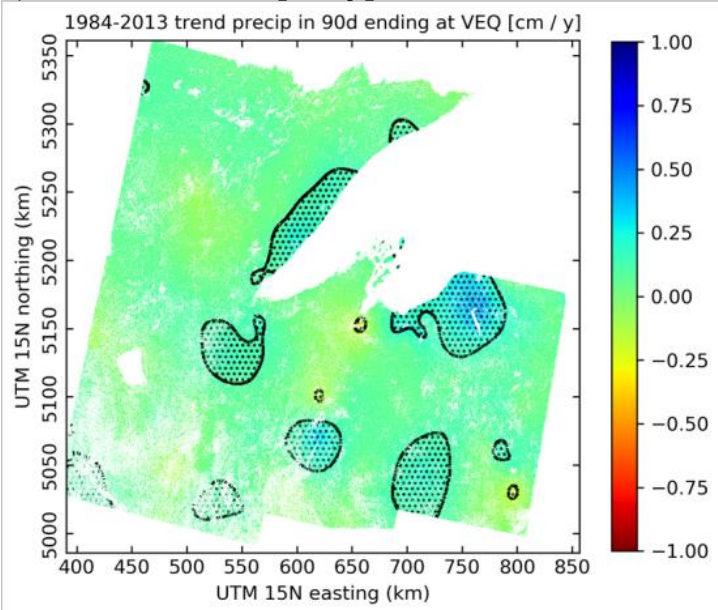

(d) Spring $P$ trend $[\mathrm{cm} / \mathrm{y}]$

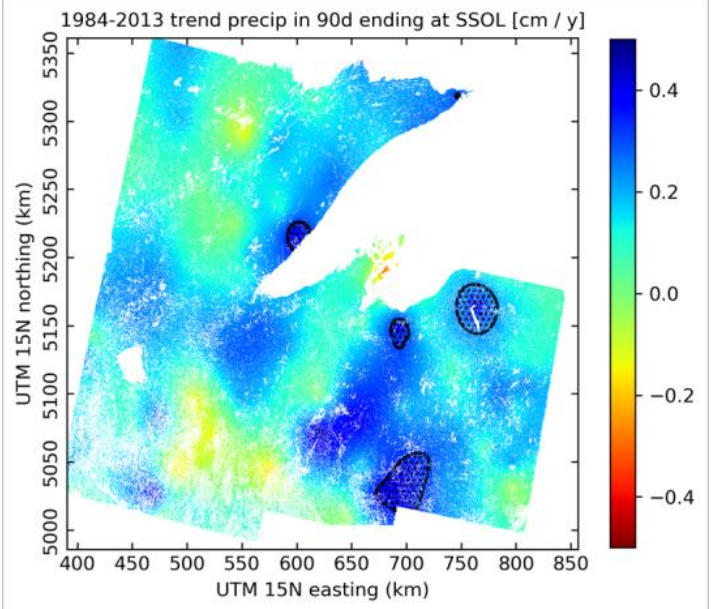

Figure 5.10: 1984-2013 mean seasonal $P$ with trends. Areas of trend significance at $p<0.05$ in (b) and (d) are stippled. 
(e) Summer $P$ mean $[\mathrm{cm}]$

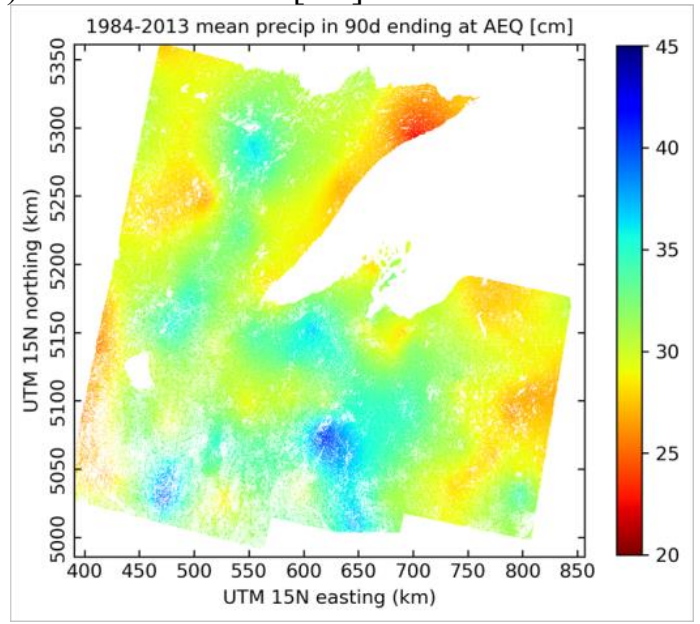

(g) Autumn $P$ mean $[\mathrm{cm}]$

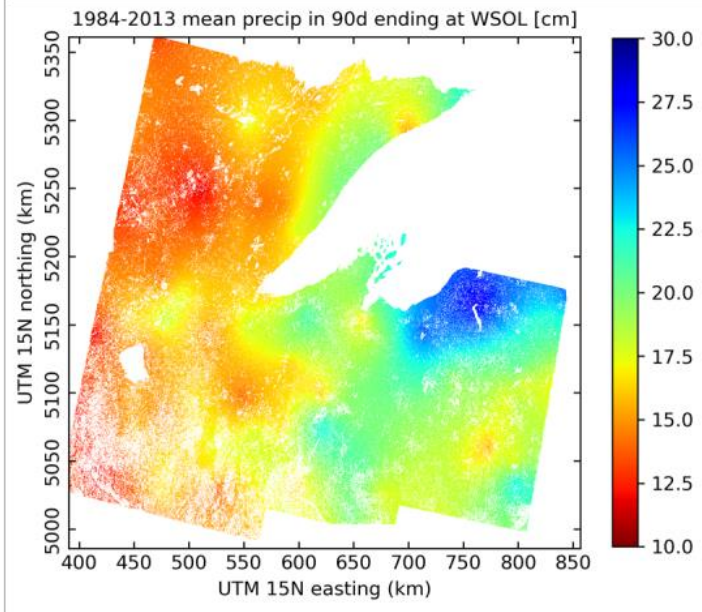

(f) Summer $P$ trend $[\mathrm{cm} / \mathrm{y}]$

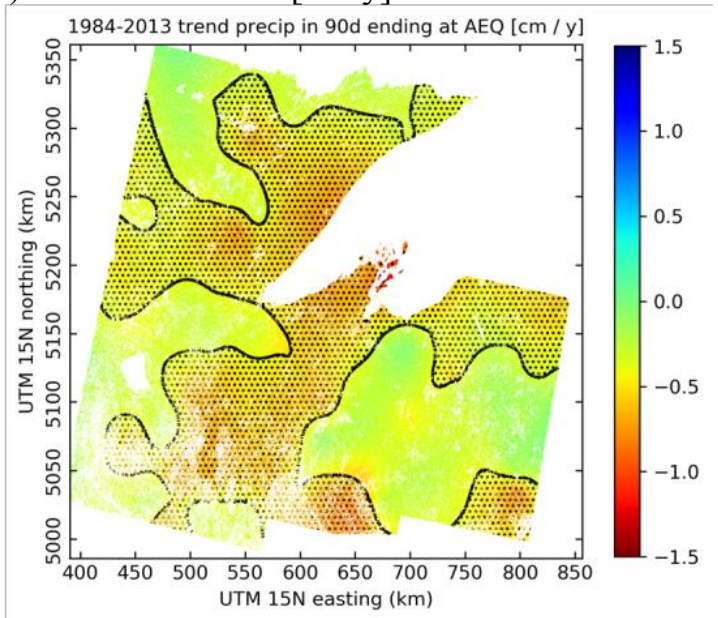

(h) Autumn $P$ trend $[\mathrm{cm} / \mathrm{y}]$

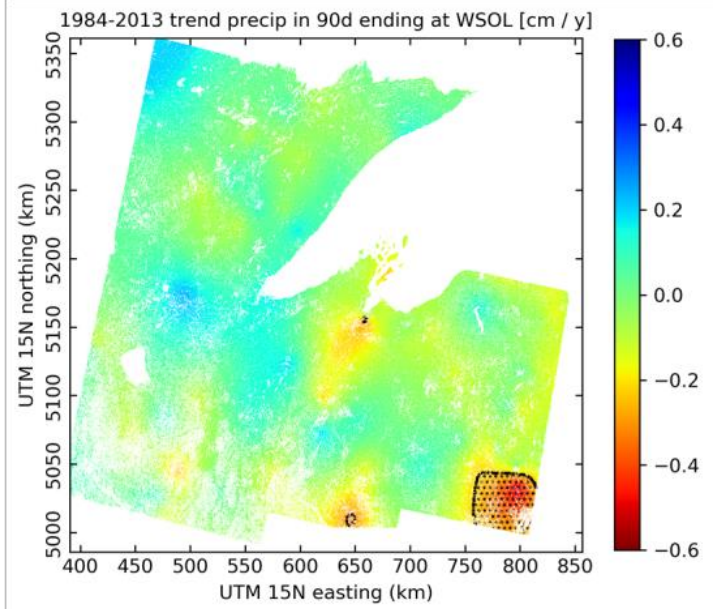

Figure 5.10 (continued): 1984-2013 mean seasonal $P$ with trends. Areas of trend significance at $p<0.05$ in (f) and (h) are stippled. 
(a) 30-y mean plateau $C D[\mathrm{~d}]$

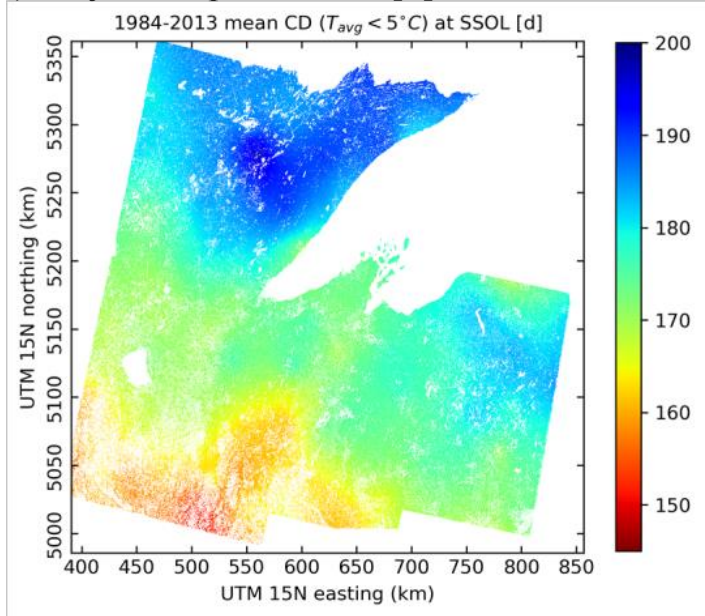

(c) 30-y mean $C S I\left[{ }^{\circ} \mathrm{C}\right]$

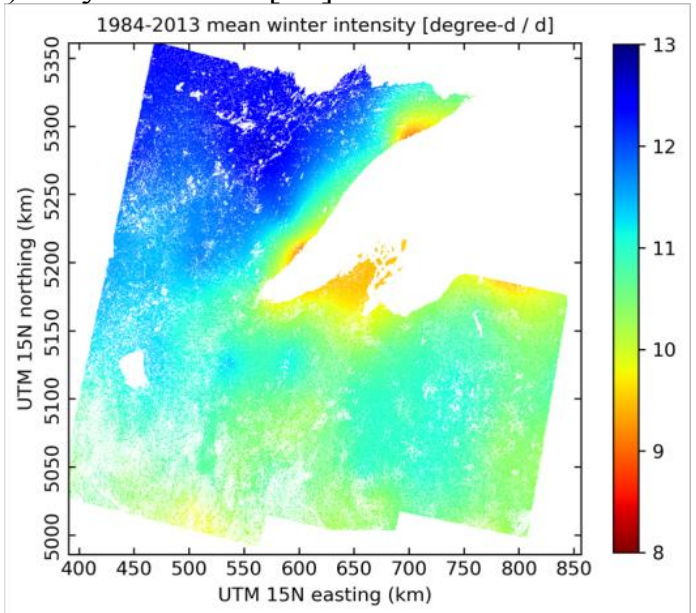

(b) 30 -y plateau $C D$ trend $[\mathrm{d} / \mathrm{y}]$

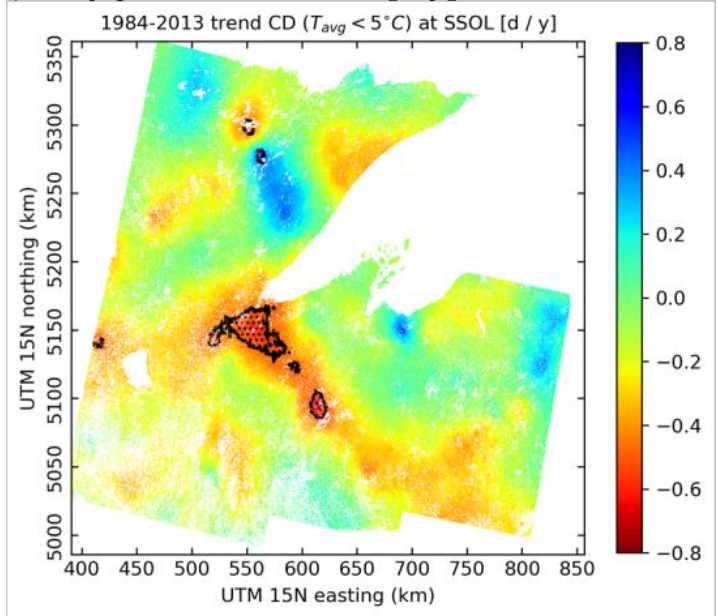

(d) 30-y CSI trend $\left[{ }^{\circ} \mathrm{C} / \mathrm{y}\right]$

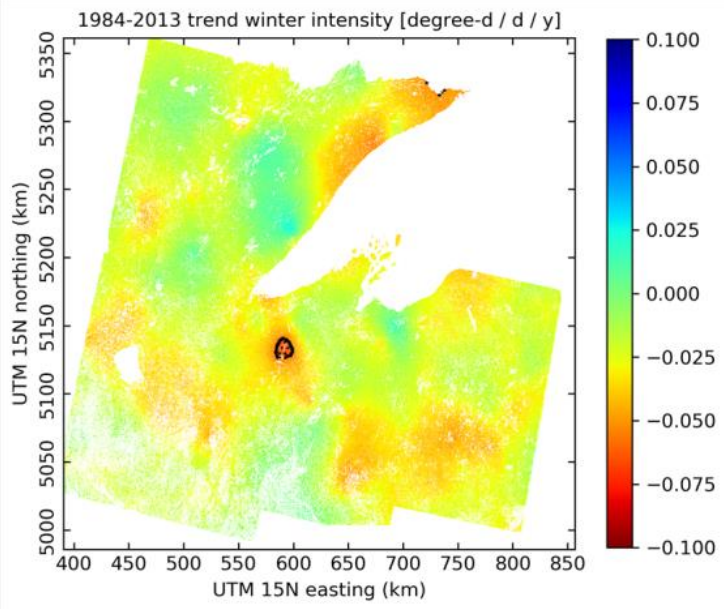

Figure 5.11: 1984-2013 mean derived cold season indicators with trends. Areas of trend significance at $p<$ 0.05 in (b) and (d) are stippled. 
are generally coincident with locations of Winter $T_{\min }$ and annual $T_{\text {avg }}$ warming. There is slightly greater spatial variability evident in the detail of 30-year mean CSI (Fig. 5.11c) which is lowest in the immediate vicinity of Lake Superior and in downwind regions. This result, along with decreasing CSI over time in many areas on the lakeshore (Fig. 5.11d), is consistent with the warming surface temperatures and changing ice phenology of Lake Superior.

The duration of the frost-free season (Fig. 5.12f), the traditional measure of the climatological growing season as described in Chapter 3 , shows an area-average trend of +0.32 days $/ y(p<0.05)$ over the 30-year period (Table 5.1) that is consistent with previous studies [Easterling, 2002; Kunkel et al., 2004; Yu et al., 2014; McCabe et al., 2015]. There is much spatial detail in maps of trends in the last Spring and first Autumn frost dates (Figs. 5.12b and 5.12d, respectively), with some areas experiencing statistically significant changes in both indicators consistent with lengthening of the frost-free season. Most of these areas occur in the forested areas northwest of Lake Superior and along the tension zone south and southeast of the lake.

Quite different patterns are found for the $C D$ plateau (Fig. 5.13), my alternative measure of the climatological growing season that was described in Chapter 3. The beginning of the $C D$ plateau changed little over time in the area average but shows a large area downwind of Lake Superior with significant trends toward a later start to the climatological growing season (Fig. 5.13b). The end of the $C D$ plateau showed an area-average trend of +0.27 days $/ y(p<0.05)$ over the 30 -year study period with large areas of significant trends (Fig. 5.13d) that are consistent with significant Autumn warming. These include areas on the Minnesota (northern) shore of Lake Superior and across agricultural areas in the southwest portion of our study area, but notably not in the region immediately southeast of the lake. Trends in the duration of the $C D$ plateau are strongly mixed across the study area (Fig. 5.13f), with an overall longer plateau evident in many areas where the end of the plateau now extends further into Autumn, but a shorter plateau in areas directly upwind and downwind of Lake Superior. Maps of plateau GDD accumulation, GSI, and their trends (Fig. 5.14) are consistent with slightly warmer climatological growing seasons over time, especially for isolated areas in immediate proximity to the lake. 
(a) 30-y mean last spring frost [DOY]

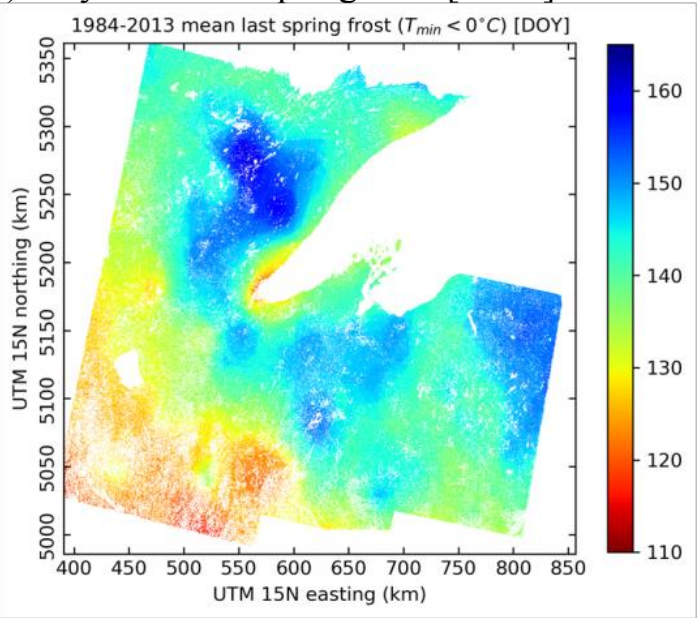

(c) 30-y mean first autumn frost [DOY]

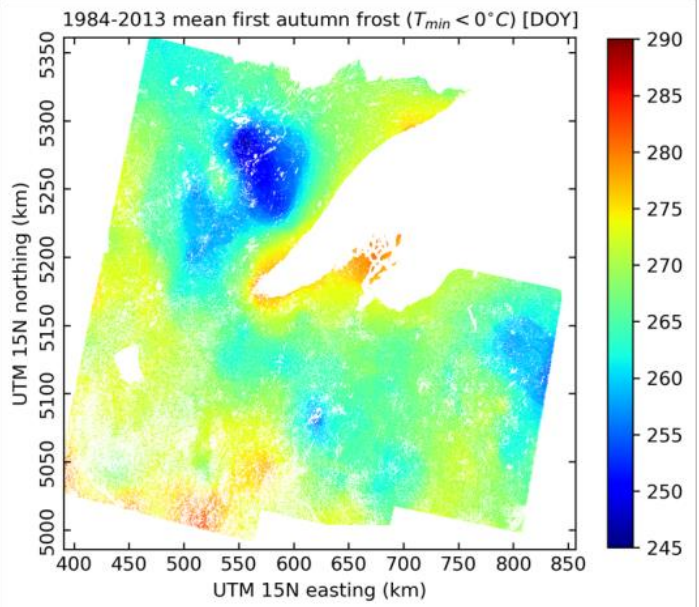

(e) 30-y mean frost-free season [d]

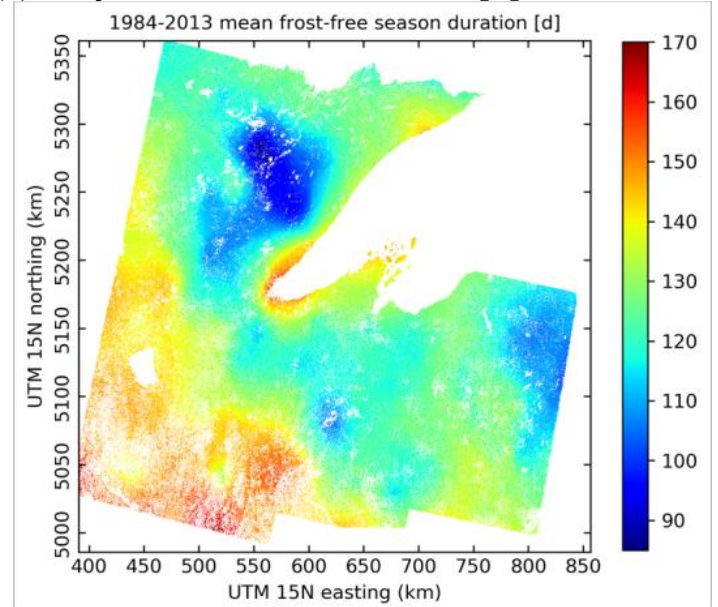

(b) 30-y last spring frost trend $[\mathrm{d} / \mathrm{y}]$

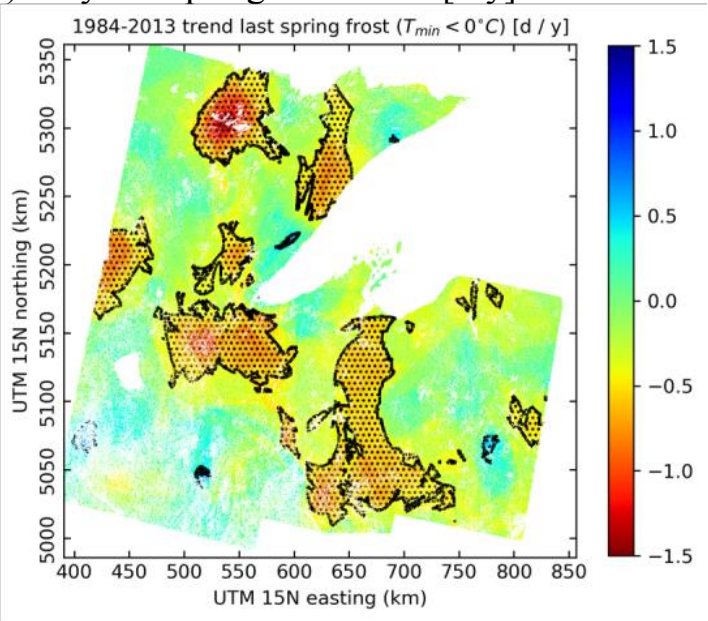

(d) 30-y first autumn frost trend [d/y]

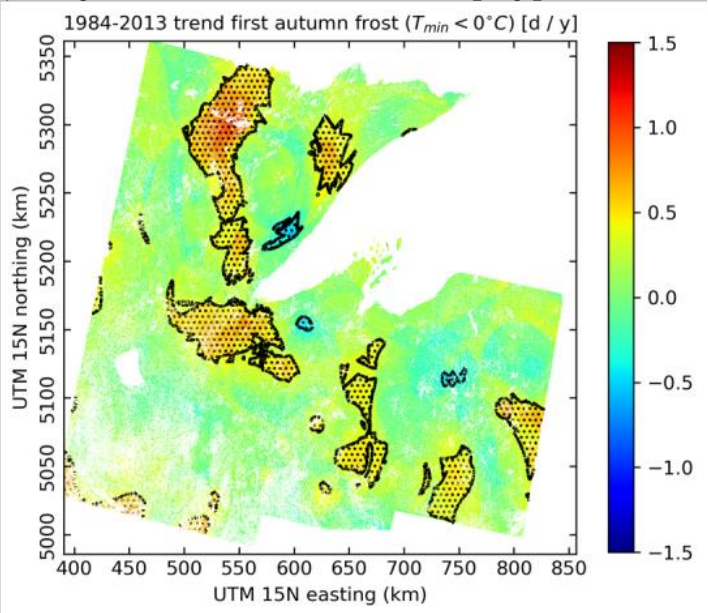

(f) 30-y frost-free season trend $[\mathrm{d} / \mathrm{y}]$

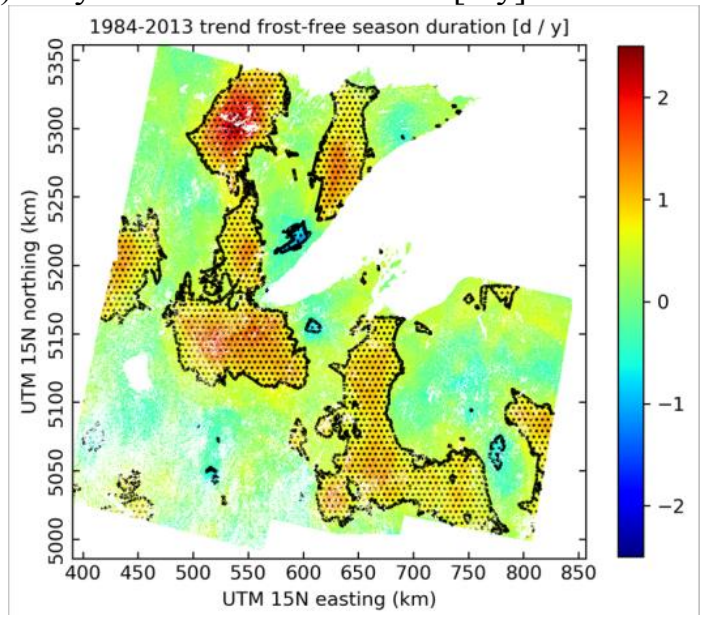

Figure 5.12: 1984-2013 mean frost-based growing season start, end, and duration with trends. Areas of trend significance at $p<0.05$ in (b), (d), and (f) are stippled. 
(a) 30-y mean $C D$ plateau start [DOY]

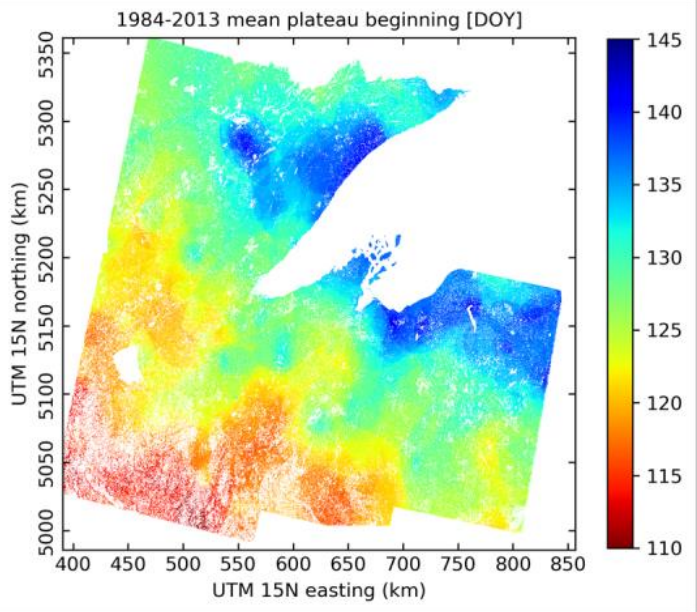

(c) 30-y mean $C D$ plateau end [DOY]

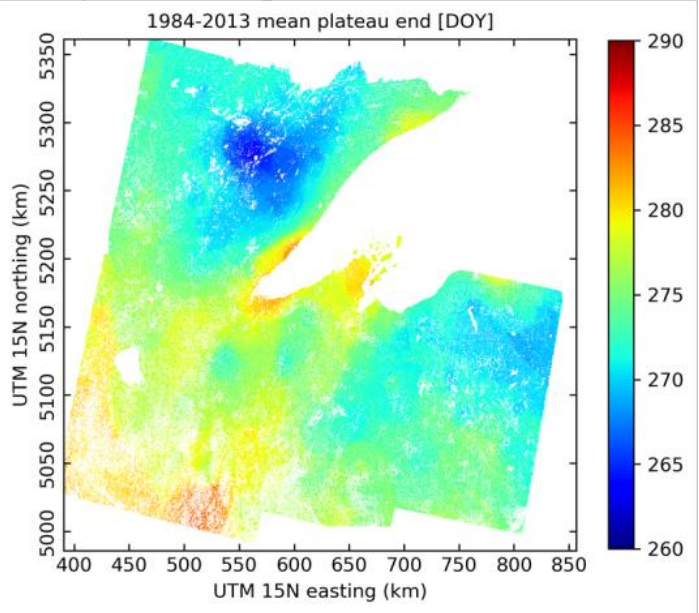

(e) 30-y mean $C D$ plateau duration [d]

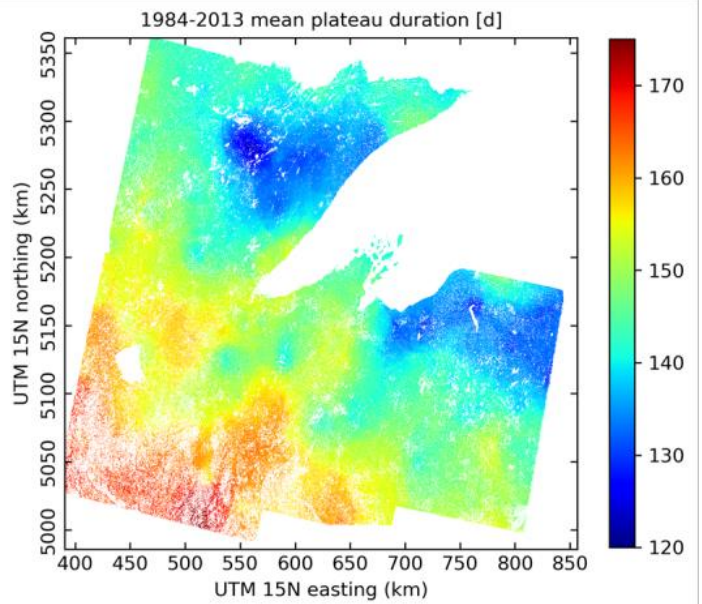

(b) 30-y $C D$ plateau start trend $[\mathrm{d} / \mathrm{y}]$

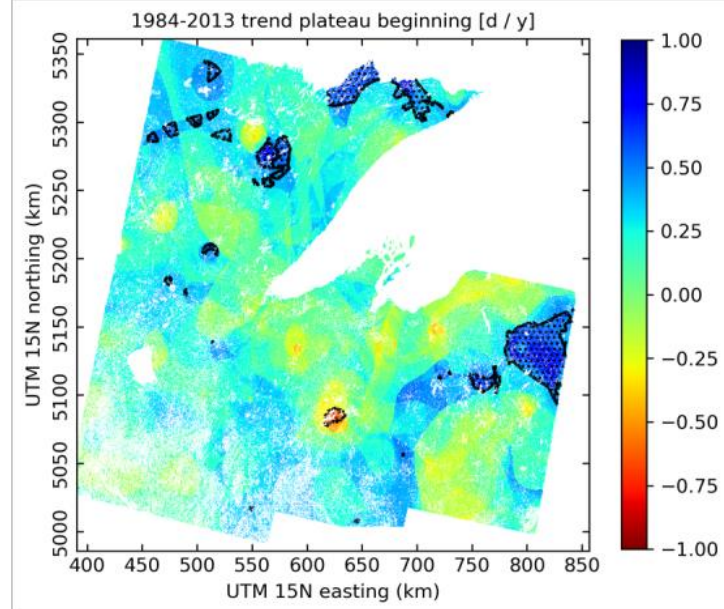

(d) 30-y $C D$ plateau end trend $[\mathrm{d} / \mathrm{y}]$

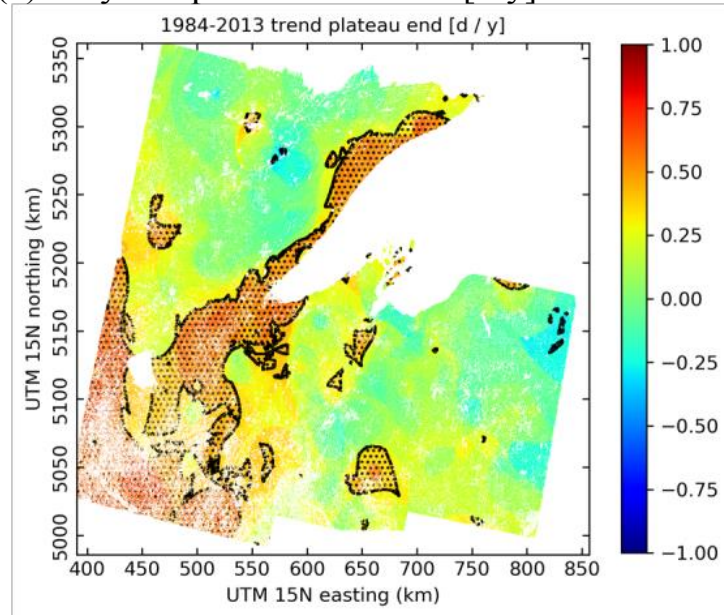

(f) 30-y $C D$ plateau duration trend $[\mathrm{d} / \mathrm{y}]$

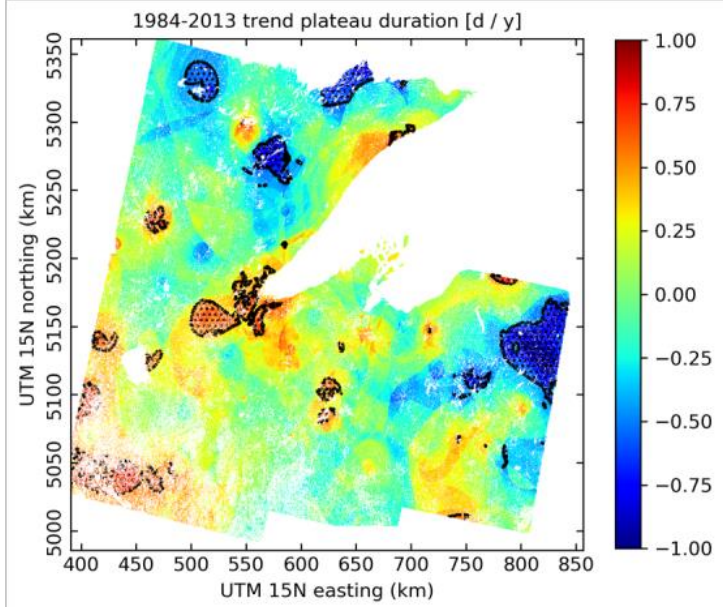

Figure 5.13: 1984-2013 mean $C D$ plateau-based growing season start, end, and duration with trends. Areas of trend significance at $p<0.05$ in (b), (d), and (f) are stippled. 
(a) 30-y mean plateau $G D D\left[{ }^{\circ} \mathrm{C}\right.$ d]

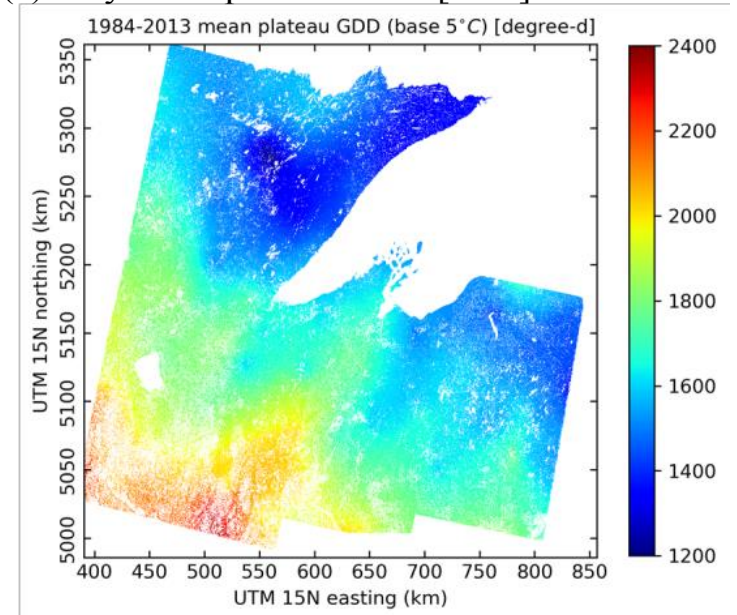

(c) 30-y mean $G S I\left[{ }^{\circ} \mathrm{C}\right]$

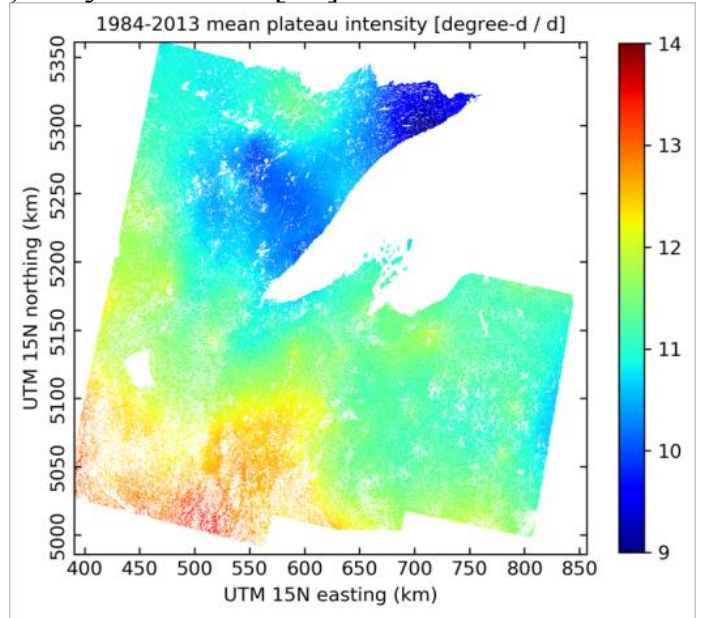

(b) 30-y plateau $G D D$ trend $\left[{ }^{\circ} \mathrm{C} \mathrm{d} / \mathrm{y}\right]$

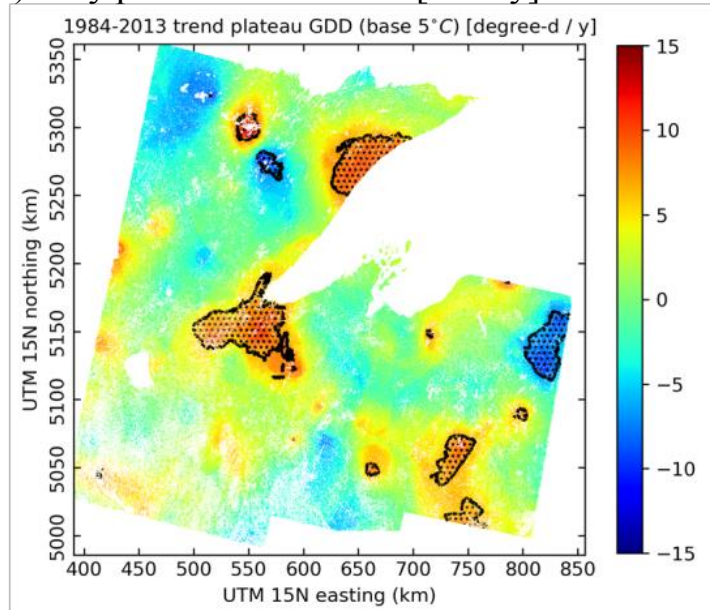

(d) 30-y GSI trend [ $\left.{ }^{\circ} \mathrm{C} / \mathrm{y}\right]$

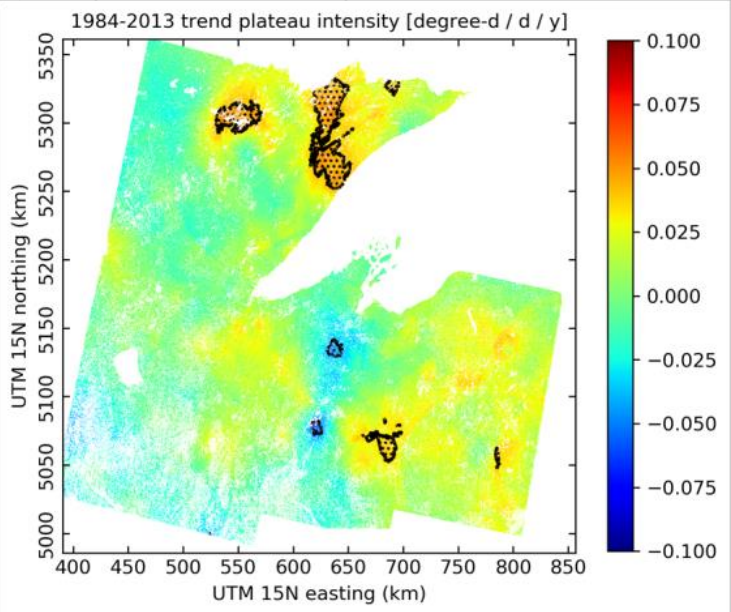

Figure 5.14: 1984-2013 mean $C D$ plateau-based growing season indicators with trends. Areas of trend significance at $p<0.05$ in (b) and (d) are stippled.

Throughout the year, seasonal mean average temperature is inversely correlated with the number of seasonal precipitation days $(P>0)$ but not with accumulated seasonal $P$, demonstrating that cloudy seasons are cooler seasons overall. Several significant $(p<0.05)$ interseasonal correlations among $T$ and $P$ indicators, especially between Winter and Spring conditions, are shown in Fig. 5.15. Winter $T$ and $P$ indicators are strongly tied to $T$ and the number of precipitation days in the following Spring. However, by this method there were no statistically significant interseasonal correlations that might be used to extend $T$ and $P$ predictability beyond Spring and through the remainder of the growing season. 


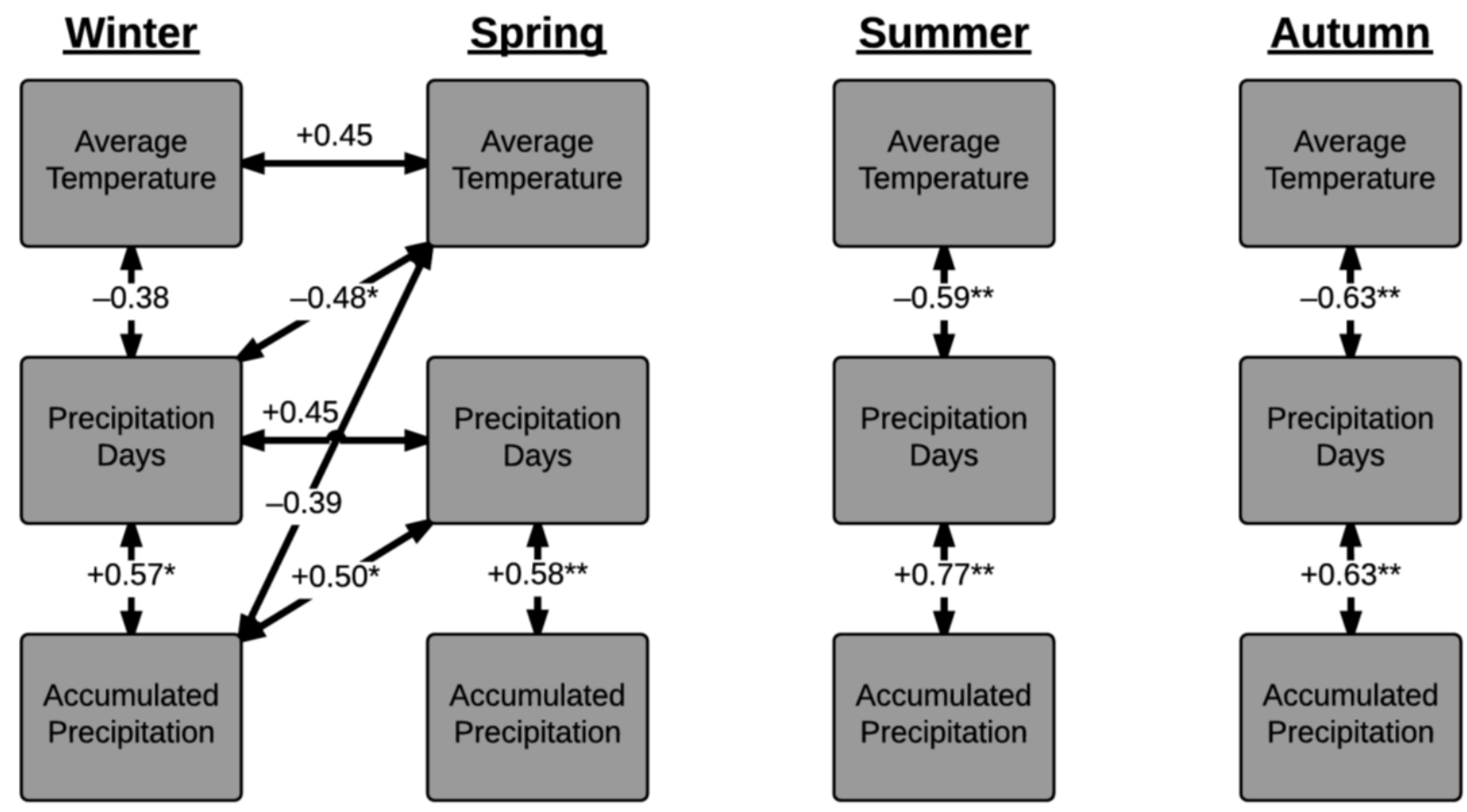

Figure 5.15: 1984-2013 intra- and interseasonal study area temperature and precipitation correlations. Only statistically significant correlations $(p<0.05, *$ at $p<0.01, * *$ at $p<0.001)$ are shown.

\subsection{Teleconnection Influences and a Climatological Regime Shift}

Two large-scale teleconnections examined here, the AMO and PDO, demonstrated regime transitions around the middle of our study period in the course of their long-period oscillations. NOAA observations indicated that the AMO index increased during 1984-2013 and around 1995 shifted to a warm $(\mathrm{AMO}+)$ phase that is generally associated with drier conditions in the Upper Midwest U.S.

[Enfield et al., 2001]. The PDO index generally decreased through the 30-year study period, with observations of distinct PDO+ periods (indicating warmer northeastern Pacific coastal waters) in the mid1980s and mid-1990s followed by PDO- anomalies (cool northeastern Pacific coastal waters) beginning around 1998. Significant correlations between various teleconnections and seasonal $T$ and $P$ in the study area are listed in Table 5.2. Results indicate that the AMO (PDO) index is positively (negatively) correlated with study area $T$, Winter $P$, and the date of Lake Superior ice onset. The AMO (PDO) index is negatively (positively) correlated with Summer $P$ and lake ice duration. Local correlations with Pacific teleconnections (PDO, ENSO, and PNA) appear strongest in Winter and Spring, with Pacific indices overall positively correlated with $T$ and negatively correlated with $P$ in the Upper Great Lakes region. A 
Table 5.2: Study area summarized significant $(p<0.05)$ teleconnection effects through the 1984-2013 study period. Traditional seasons are evaluated according to solstice/equinox dates; the growing season is taken as the CD plateau from approximately mid-spring to early autumn (Fig. 5.2); ENSO indices are evaluated only over winter seasons, and "Niño3" denotes the Region 3 (eastern equatorial Pacific Ocean) ENSO index for the winter beginning the calendar year. This chart should not be interpreted to suggest equal effects from the various teleconnection indices on a regional climatological indicator in a given time period.

\begin{tabular}{|c|c|c|c|c|c|c|c|c|c|c|}
\hline \multirow{2}{*}{$\begin{array}{c}\text { Teleconnection } \\
\text { Index }\end{array}$} & \multicolumn{2}{|c|}{ Winter } & \multicolumn{2}{c|}{ Spring } & \multicolumn{2}{c|}{ Summer } & \multicolumn{2}{c|}{ Autumn } & \multicolumn{2}{c|}{ Growing Season } \\
\cline { 2 - 11 } & $\boldsymbol{T}$ & $\boldsymbol{P}$ & $\boldsymbol{T}$ & $\boldsymbol{P}$ & $\boldsymbol{T}$ & $\boldsymbol{P}$ & $\boldsymbol{T}$ & $\boldsymbol{P}$ & Intensity & Duration \\
\hline AMO & + & + & + & & + & & + & & + & + \\
\hline PDO & & - & & - & - & & & & & \\
\hline Niño3 & + & - & + & - & & & & & & + \\
\hline PNA & + & & + & - & & & & & & \\
\hline AO & & & & & & & & - & & \\
\hline NAO & & & & & & & & & & - \\
\hline
\end{tabular}

long-term increase in Autumn PNA index values $(p<0.05)$, and positive values of the PNA index in general, has been associated with relatively dry conditions over continental North America [Leathers et al., 1991]. The often-related AO and NAO indices showed no significant correlation with study area surface climatology except in Autumn, when the AO index is negatively correlated with area-averaged $P$.

I am interested not only in the mean climatology but also its year-to-year variability and extremes, as these also influence forest phenology and growth [Bouriaud et al., 2005; Voelker et al., 2012]. It is often generalized for the Upper Midwest U.S. that El Niño (ENSO+) events foster warm/dry years and La Niña (ENSO-) events support cool/wet years: Table 5.1 lists extremes in many of the climatological indicators that can be associated with ENSO events within the study period. El Niño conditions that persisted through 1987 and 1988 produced the driest Winter and warmest growing season in the study period in conjunction with regional drought conditions [Trenberth et al., 1988; Weaver et al., 2009]. On the other hand, Lake Superior did not fully freeze during both the El Niño Winter of 1997-1998 [Changnon, 1999] and the La Niña Winter of 2011-2012 [Peterson et al., 2013; Dole et al., 2014], the latter event leading into one of the most spatially and temporally variable growing seasons during my study period [Hoerling et al., 2014] including significant drought conditions in the Upper Midwest U.S. [Wang et al., 2014]. Teleconnection variability may have influenced both the wettest year of the study period in 1985 (ENSO-, AMO-, and $\mathrm{PDO}+$ ) and the driest year in 2006 (ENSO-, AMO+ and PDO-), both La Niña years. 
A distinct shift in climatological regimes across several global and regional indicators was thus observed around 1998. Analyses indicated changes to, and even reversals of, statistically significant ( $p<$ 0.05) temporal trends for several climatological indicators between the 1984-1998 and 1998-2013 periods, as listed in Table 5.3. Specifically, paired maps of trends in Spring and Autumn $T_{a v g}$ and annual $P$ are shown in Fig. 5.16. Many locations with strong Spring cooling during 1984-1998 (Fig 5.16a) shifted to near-neutral trends in the latter half of the study period (Fig. 5.16b), which can be attributed primarily to a shift in the trend of Spring $T_{\max }$ with very little change in Spring $T_{\min }$ trends across much of the study area. Locations with weak Autumn warming during the earlier period (Fig. 5.16c) shifted to strong cooling trends in the 1998-2013 period (Fig. 5.16d), which may be attributed to sharp reversals in both Autumn $T_{\max }$ and Autumn $T_{\min }$ trends across the study area. Despite this reversal to statistically significant cooling in many locations, the strongest 30-year area-averaged seasonal warming still appears in Autumn (Fig. 5.17 and Table 5.1). Annual total $P$ shifted from increasing trends in isolated locations during the 1984-1998 period (Fig. 5.16e) to widespread decreasing trends across much of the study area in the 19982013 period (Fig. 5.16f). This shift can be attributed primarily to an area-average negative trend in Summer $P(p<0.01$, Table 5.1 and Fig. 5.10) that is also strongest in the latter half of the study period, and to a lesser extent diagnosed shifts to negative trends in Winter and Spring seasonal precipitation. Cold season shifts in trends are shown in Fig. 5.17, where the $C D$ totals shift to a generally diminishing trend northwest of Lake Superior in the 1998-2013 period, but the overall CSI clearly transitions from a warming trend in the 1984-1998 period to a cooling trend in the latter period. Figure 5.18 summarizes trend shifts in the two measures of growing season duration, with a transition from lengthening to diminishing frost-free seasons across much of the study area, but changes in the trends of $C D$ plateaubased growing season duration are more localized and mixed, with a shift toward longer $C D$ plateaus along the north shore of Lake Superior and in a band from the Bayfield peninsula toward the agricultural areas in the southwest corner of the study area, but a shift toward shorter $C D$ plateau-based growing seasons in the Wisconsin Northwoods area. Additional warm-season trends are summarized in Fig. 5.19, where the change in $C D$ plateau-oriented $G D D$ accumulations are generally mixed but overall are aligned 
Table 5.3: Climatological indicator statistics for the periods 1984-1998 and 1999-2013, derived from daily maps of interpolated $T$ and $P$ and averaged across the study area. Significant differences in mean values via Student's t-test are indicated $*$ at $p<0.05$, where the mean values of both periods are marked. Significant differences in variance values via Levene's test are indicated * at $p<0.05$, where the standard deviation values of both periods are marked. Significant trends are indicated * at $p<0.05$.

\begin{tabular}{|c|c|c|c|c|c|c|}
\hline & \multicolumn{3}{|c|}{ 1984-1998 } & \multicolumn{3}{|c|}{ 1999-2013 } \\
\hline & Mean & Std. Dev. & $15 y$ trend & Mean & Std. Dev. & $15 y$ trend \\
\hline Temperature Indicators & (15y rec) & (15y rec) & (units/yr) & (15y rec) & (15y rec) & (units/yr) \\
\hline Winter Average Temperature $\left[{ }^{\circ} \mathrm{C}\right]$ & -9.73 & 2.46 & -0.01 & -9.11 & 1.93 & +0.02 \\
\hline — within-season variance $\left[{ }^{\circ} \mathrm{C}^{2}\right]$ & 52.67 & 15.11 & -0.38 & 45.59 & 11.00 & -0.07 \\
\hline Spring Average Temperature $\left[{ }^{\circ} \mathrm{C}\right]$ & 9.78 & 1.35 & -0.14 & 9.41 & 1.39 & -0.04 \\
\hline - within-season variance $\left[{ }^{\circ} \mathrm{C}^{2}\right]$ & 47.05 & 12.04 & +0.82 & 41.44 & 9.87 & +0.45 \\
\hline Summer Average Temperature $\left[{ }^{\circ} \mathrm{C}\right]$ & 17.68 & 0.94 & +0.02 & 18.23 & 0.74 & -0.01 \\
\hline - within-season variance $\left[{ }^{\circ} \mathrm{C}^{2}\right]$ & 13.27 & 3.94 & -0.30 & 14.03 & 3.80 & +0.20 \\
\hline Autumn Average Temperature $\left[{ }^{\circ} \mathrm{C}\right]$ & 0.37 & 1.79 & +0.14 & 1.24 & 1.27 & -0.07 \\
\hline — within-season variance $\left[{ }^{\circ} \mathrm{C}^{2}\right]$ & 67.95 & 24.50 & -1.62 & 69.78 & 28.53 & +2.35 \\
\hline Annual Average Temperature $\left[{ }^{\circ} \mathrm{C}\right]$ & 4.53 & 1.09 & +0.00 & 4.94 & 0.89 & -0.03 \\
\hline
\end{tabular}

\begin{tabular}{|c|c|c|c|c|c|c|}
\hline Precipitation Indicators \\
\hline Winter Total Precipitation [cm] & 7.77 & 2.08 & +0.16 & 8.57 & 1.58 & +0.02 \\
\hline Spring Total Precipitation [cm] & $20.70^{*}$ & 4.70 & -0.17 & $24.56^{*}$ & 5.00 & -0.01 \\
\hline Summer Total Precipitation [cm] & $29.56^{*}$ & 4.65 & -0.26 & $25.63^{*}$ & 5.45 & -0.57 \\
\hline Autumn Total Precipitation [cm] & 16.01 & 5.18 & +0.21 & 15.39 & 4.08 & +0.17 \\
\hline Annual Total Precipitation [cm] & 75.36 & 8.62 & -0.07 & 75.76 & 7.57 & -0.39 \\
\hline
\end{tabular}

\section{Cold Season Indicators}

\begin{tabular}{|l|c|c|c|c|c|c|}
\hline Freezing Days (using $\left.T_{\min }\right)[\mathrm{d}]$ & 169.50 & 7.44 & +0.60 & 164.84 & 8.27 & +0.13 \\
\hline Chilling Days (using $T_{\text {avg }}$ ) [d] & 164.79 & 8.17 & +0.94 & 159.53 & 9.61 & +0.11 \\
\hline Cold Season Intensity [ $\left.{ }^{\circ} \mathrm{C}\right]$ & 11.93 & 1.33 & -0.07 & 11.37 & 1.31 & +0.05 \\
\hline Last Spring Freezing Night [DOY] & 129.97 & 5.06 & -0.05 & 130.02 & 5.43 & -0.08 \\
\hline First Autumn Freezing Night [DOY] & 270.43 & 5.25 & +0.39 & 270.46 & 6.84 & +0.30 \\
\hline
\end{tabular}

\begin{tabular}{|l|c|c|c|c|c|c|}
\hline Warm (Growing) Season Indicators \\
\hline Last Spring Freezing Night $\left[{ }^{\circ} \mathrm{C} \mathrm{d}\right]$ & 108.50 & 47.37 & $-6.45^{*}$ & 112.99 & 38.47 & -1.11 \\
\hline Frost-free Season [d] & 140.46 & 5.31 & +0.44 & 140.44 & 6.57 & +0.37 \\
\hline Beginning of CD Plateau [DOY] & 122.20 & 9.41 & +0.53 & 124.02 & 8.50 & +0.44 \\
\hline End of CD Plateau [DOY] & 274.85 & 7.46 & +0.60 & 278.46 & 5.85 & $+0.84^{*}$ \\
\hline Plateau Length [d] & 152.65 & $11.54^{*}$ & +0.08 & 154.44 & $6.54^{*}$ & -0.40 \\
\hline Plateau Growing Degree Days $\left[{ }^{\circ} \mathrm{C} \mathrm{d}\right]$ & 1721.32 & 149.39 & -0.45 & 1746.54 & 108.20 & +3.56 \\
\hline Plateau Intensity $\left[{ }^{\circ} \mathrm{C}\right]$ & 11.28 & 0.91 & -0.01 & 11.30 & 0.71 & -0.01 \\
\hline
\end{tabular}


(a) 1984-1998 spring $T_{\text {avg }}$ trend $\left[{ }^{\circ} \mathrm{C} / \mathrm{y}\right]$

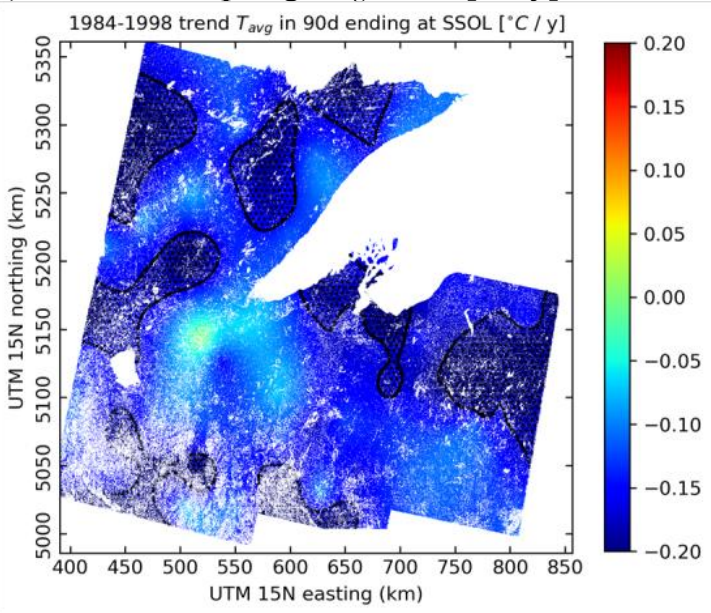

(c) 1984-1998 autumn $T_{\text {avg }}$ trend $\left[{ }^{\circ} \mathrm{C} / \mathrm{y}\right]$

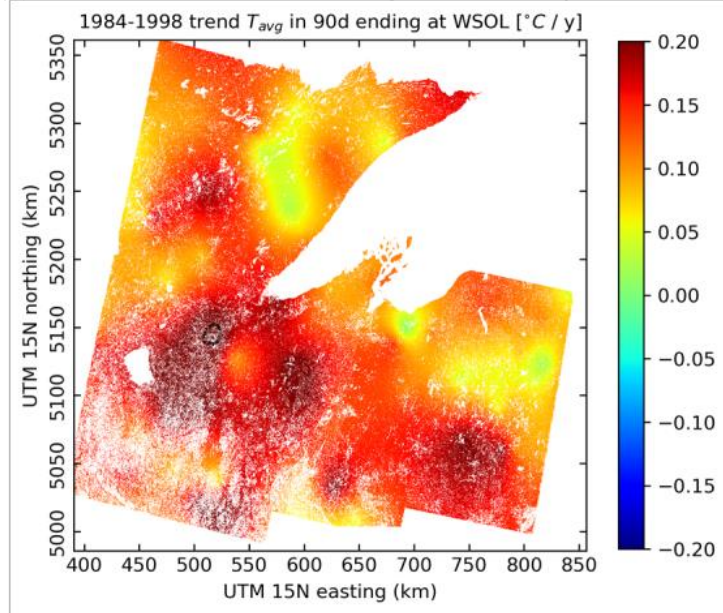

(e) 1984-1998 annual $P$ trend $[\mathrm{cm} / \mathrm{y}]$

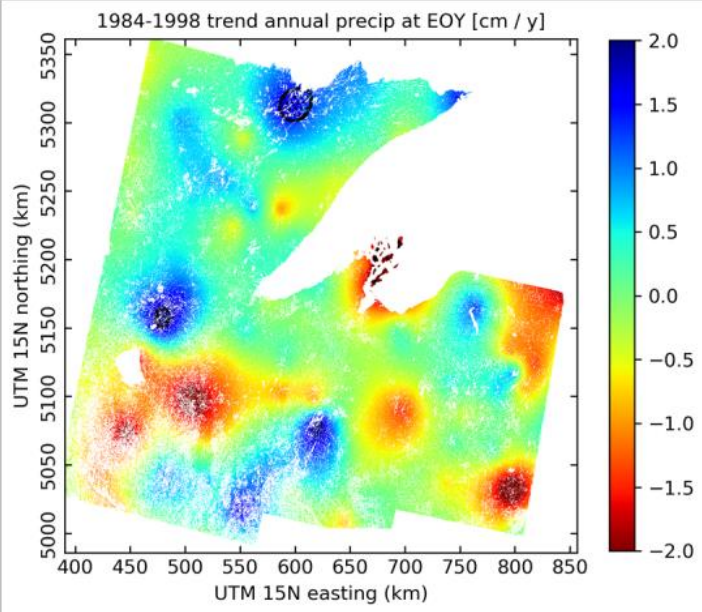

(b) $1998-2013$ spring $T_{\text {avg }}$ trend $\left[{ }^{\circ} \mathrm{C} / \mathrm{y}\right]$

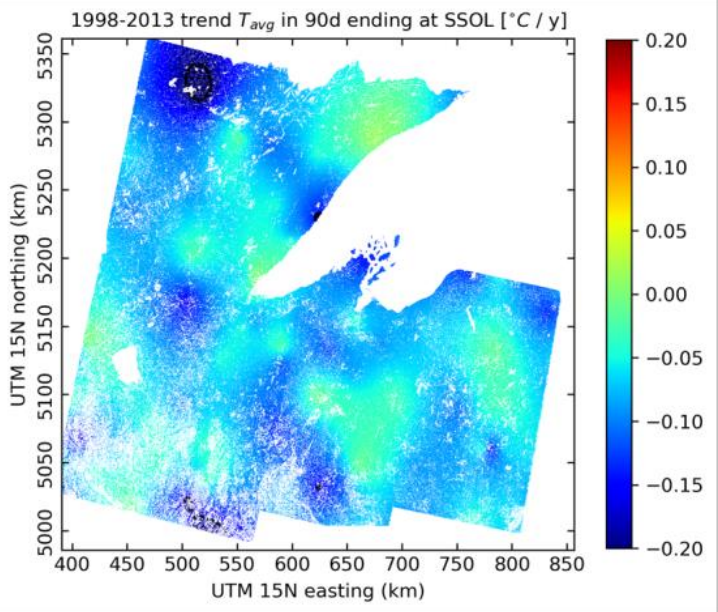

(d) 1998-2013 autumn $T_{\text {avg }}$ trend $\left[{ }^{\circ} \mathrm{C} / \mathrm{y}\right]$

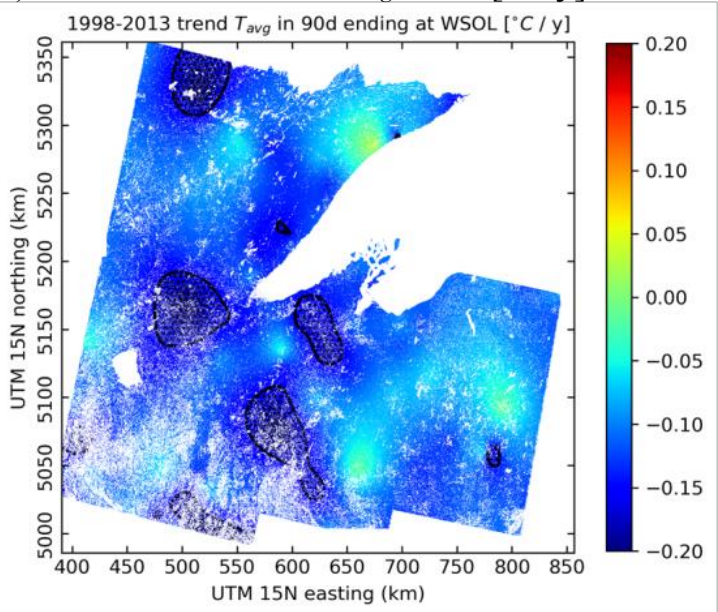

(f) 1998-2013 annual $P$ trend [cm/y]

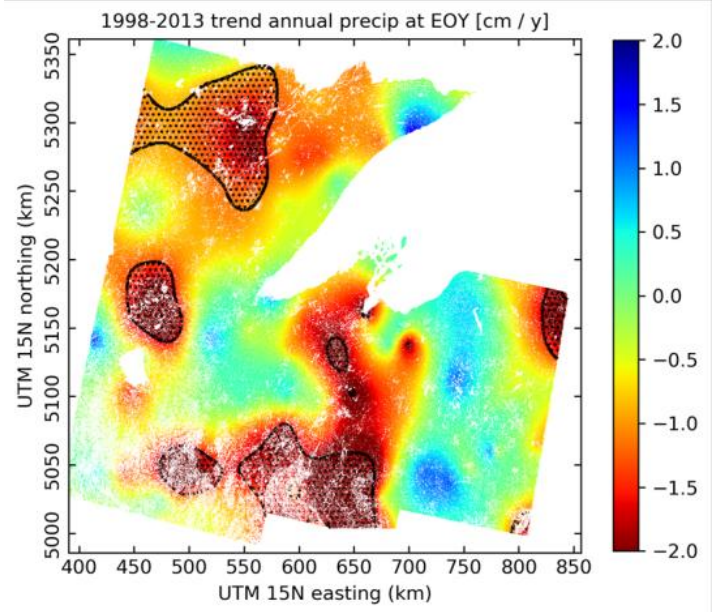

Figure 5.16: 1984-1998 and 1998-2013 trends in Spring and Autumn $T_{a v g}$ and annual $P$. Areas of trend significance at $p<0.05$ are stippled. 
(a) 1984-1998 $C D$ trend $[\mathrm{d} / \mathrm{y}]$

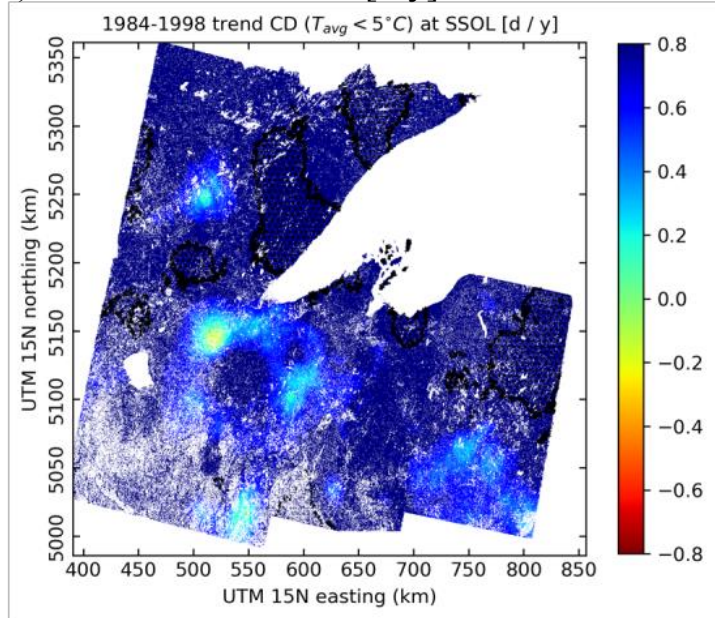

(c) 1984-1998 CSI trend $\left[{ }^{\circ} \mathrm{C} / \mathrm{y}\right]$

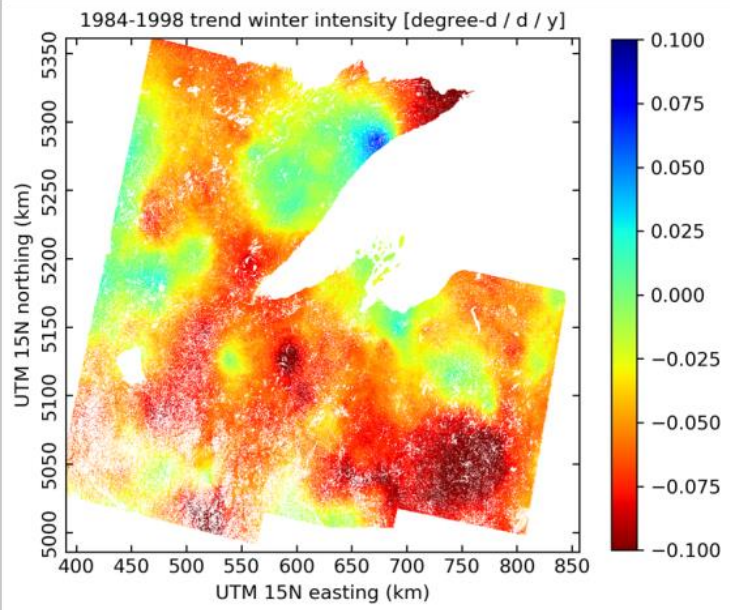

(b) $1998-2013 C D$ trend $[\mathrm{d} / \mathrm{y}]$

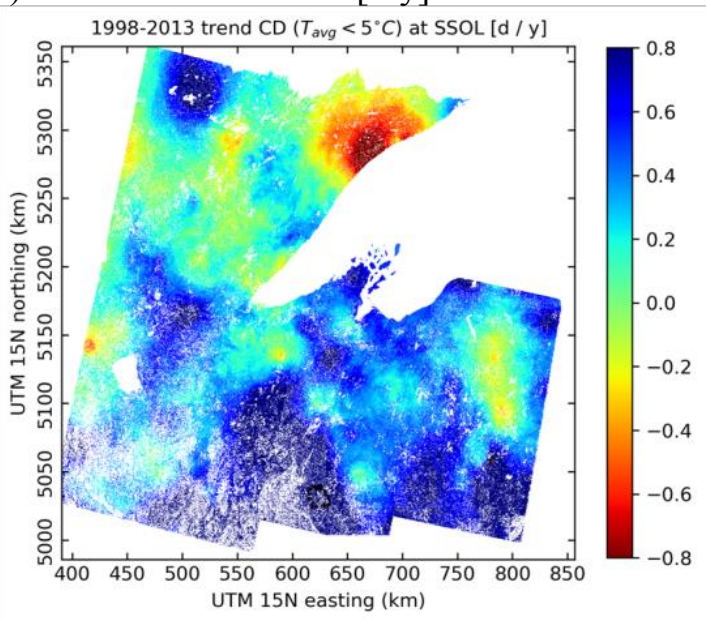

(d) $1998-2013 \mathrm{CSI}$ trend $\left[{ }^{\circ} \mathrm{C} / \mathrm{y}\right]$

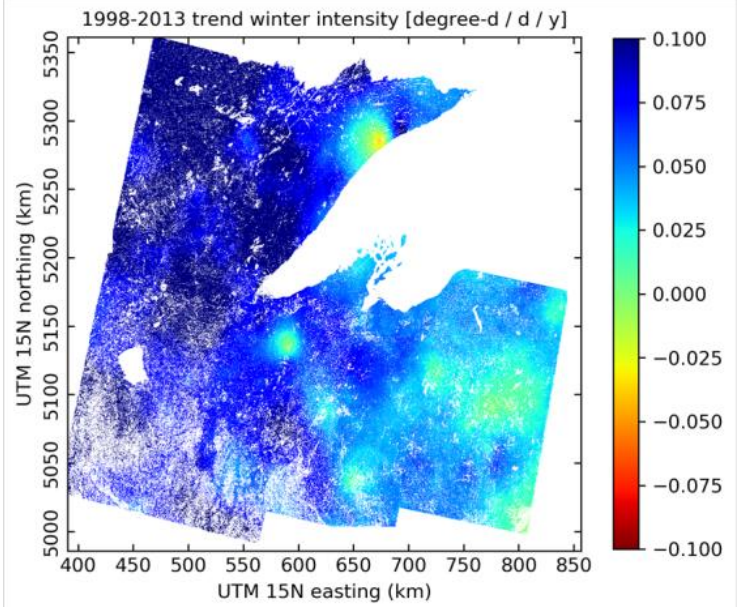

Figure 5.17: 1984-1998 and 1998-2013 trends in mean derived cold season indicators. Areas of trend significance at $p<0.05$ are stippled.

with those in Fig. 5.18c and 5.18d, but the shift in GSI trends are the opposite, suggesting trends toward slightly less intense growing seasons where their length is increasing and slightly more intense seasons where the $C D$ plateau trends toward shorter growing season length.

\subsection{Climatological and Growing Season Trends}

The overall area-average temperature increase that we estimated by linear regression is consistent with prior estimates of warming in this region over similar periods [Li et al., 2010; Groisman et al., 2012]. Results show a consistent continuation of trends reported for 1951-1980 climatic changes in the 
(a) 1984-1998 frost-free season trend $[\mathrm{d} / \mathrm{y}]$

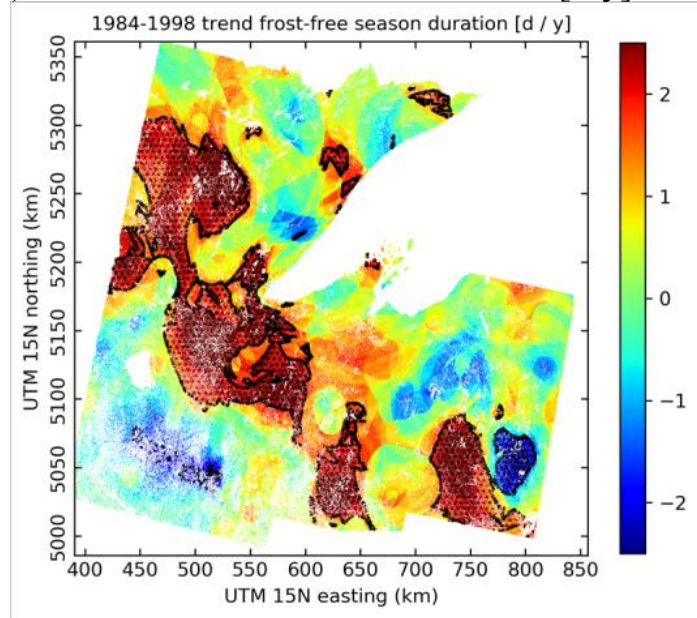

(c) 1984-1998 CD plateau duration trend [d/y]

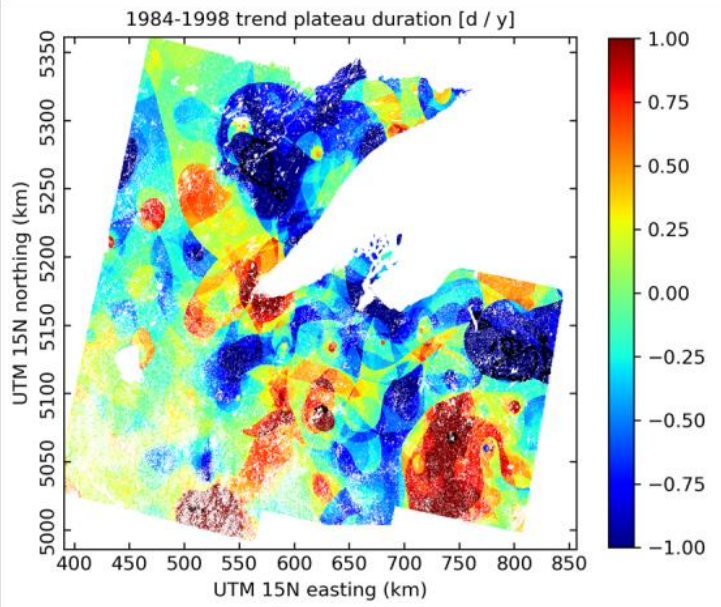

(b) 1998-2013 frost-free season trend $[\mathrm{d} / \mathrm{y}]$

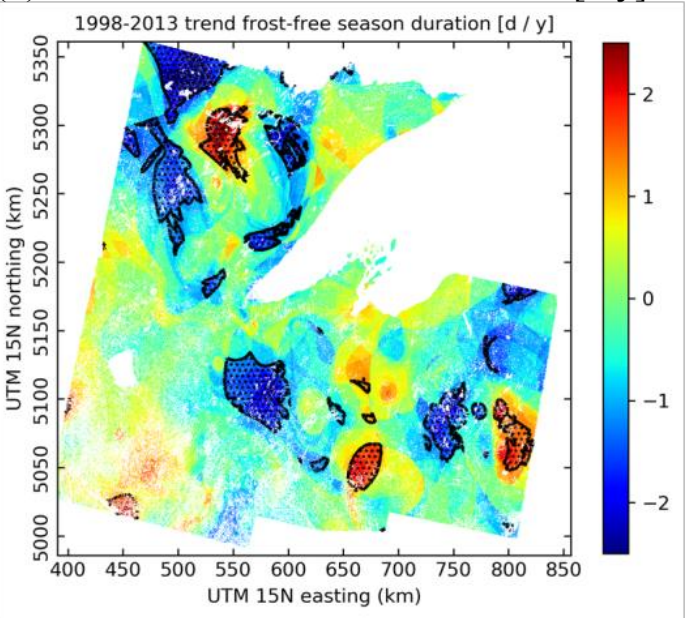

(d) 1998-2013 CD plateau duration trend [d/y]

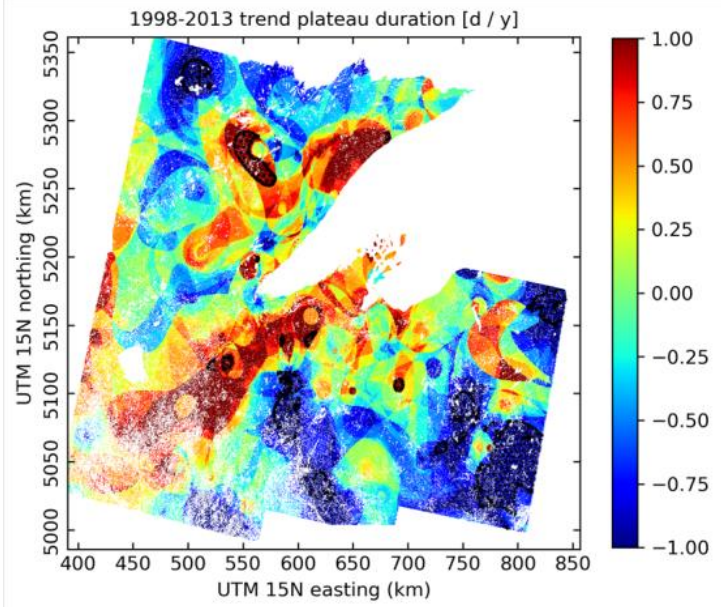

Figure 5.18: 1984-1998 and 1998-2013 trends in mean derived growing season indicators. Areas of trend significance at $p<0.05$ are stippled.

Great Lakes region reported by Scott and Huff [1996]: increased minimum temperatures in all seasons, decreased Spring and Summer maximum temperatures, slight decreases in Summer rainfall, and large Winter precipitation increases for lake-effect areas primarily southeast of Lake Superior and as Winter ice cover diminished. Choi et al. [2014] analyzed Serbin and Kucharik's [2009] climatological dataset for Wisconsin and showed statistically significant decreases in the frequency of heavy precipitation events during 1950-2006, especially southeast of Lake Superior in the same lake-effect areas where this analysis also found slight decreases for heavy precipitation days $(P>2.5 \mathrm{~cm})$ in Summer and Autumn. However, analyses here indicated slight increases in Winter and Spring heavy precipitation days in those areas. These differences with Choi et al. [2014] might be attributed to differing study periods but are likely 
(a) 1984-1998 plateau $G D D$ trend $\left[{ }^{\circ} \mathrm{C} \mathrm{d} / \mathrm{y}\right]$

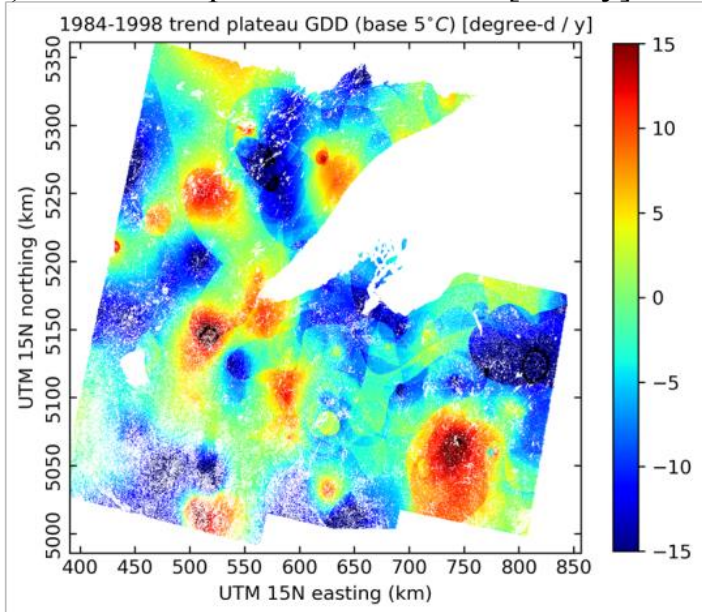

(c) $1984-1998 \mathrm{GSI}$ trend $\left[{ }^{\circ} \mathrm{C} / \mathrm{y}\right]$

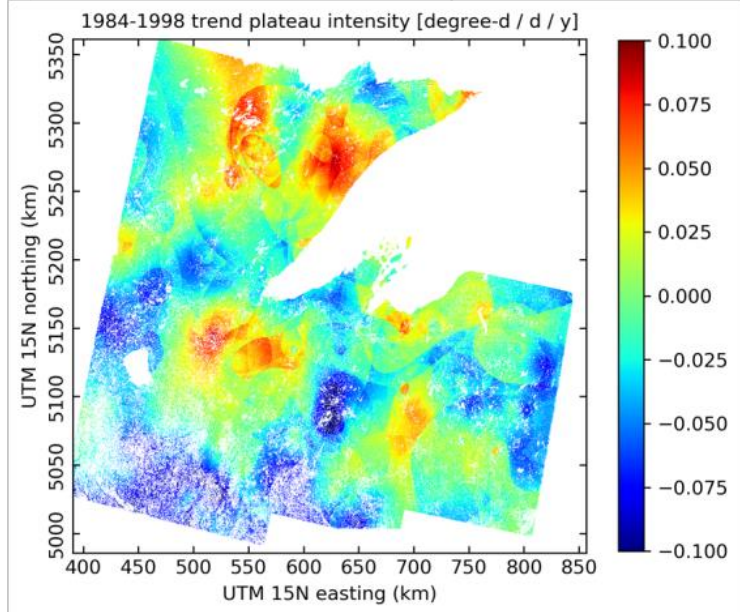

(b) 1998-2013 plateau $G D D$ trend $\left[{ }^{\circ} \mathrm{C} \mathrm{d} / \mathrm{y}\right]$

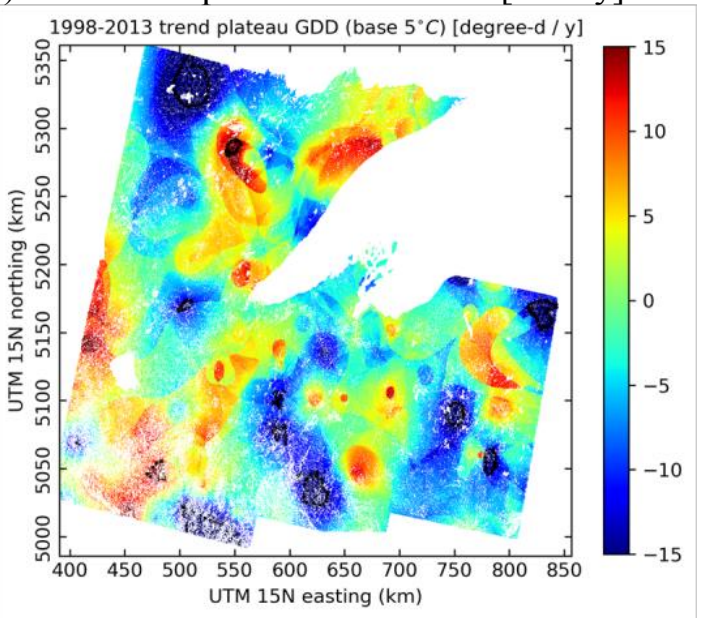

(d) $1998-2013 \mathrm{GSI}$ trend $\left[{ }^{\circ} \mathrm{C} / \mathrm{y}\right]$

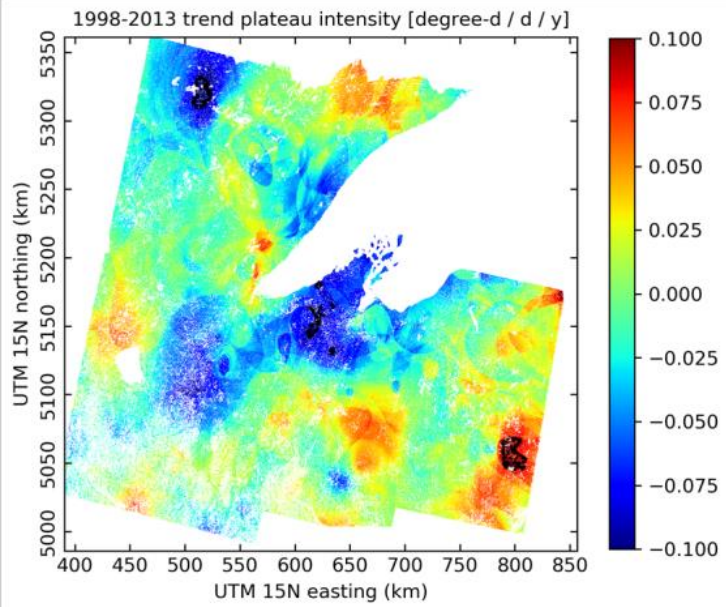

Figure 5.19: 1984-1998 and 1998-2013 trends in mean derived warm season indicators. Areas of trend significance at $p<0.05$ are stippled.

related to technical differences (e.g. study area boundaries, station data selection, interpolation methods, grid resolution) in the examined precipitation fields that can demonstrate large spatial variability.

The estimated $+0.56^{\circ} \mathrm{C}$ area-average temperature change, along with the accelerated warming of Lake Superior surface waters $\left(+2.5^{\circ} \mathrm{C}\right)$ [Austin and Colman, 2007, 2008; Van Cleave et al., 2014], clearly indicates regional warming over the 30 -year study period. Though the beginning of the CD plateau in Spring has changed little, the extension of the CD plateau later into Autumn is consistent with long-term temperature increases concentrated primarily in that season. Overall these results point to a longer climatological growing season due more to warm-season extension later into Autumn than earlier into Spring, consistent with findings by Jeong et al. [2011]. Annual total precipitation demonstrated a slight 
negative area-averaged trend over the study period, and a particularly strong negative trend since 1998 (Fig. 5.16) driven by a sharp decrease in Summer precipitation (Fig. 5.10). The above analyses of temperature trends support the small negative trends that we found for cold-season $C D$ and $F D$ and a consequent increase in the duration of the frost-free season. Along with observed influences of large-scale teleconnections (AMO+, PDO-, and increasing PNA), these changes suggest an overall drying trend [Parida and Buermann, 2014] within the study period for this region.

Area-wide climatological warming and drying during 1984-2013 means that forests in the study area may have experienced increasing moisture stress in this period, especially in Summer. It is important here to distinguish between changes affecting moisture availability, based primarily on $P$, and those that drive vegetation moisture demand, based primarily on $T$. Vegetation moisture stress occurs with the combination of these, when moisture availability is insufficient to meet moisture demand, and changes in either or both of those factors can lead to that stress. Conditions promoting moisture stress can inhibit transpiration and reduce growth [Teskey et al., 1987], promote leaf wilt and early senescence [MunnéBosch and Alegre, 2004; Marchin et al., 2010], enhance tree mortality [Anderegg et al., 2012, 2013], and reduce overall forest carbon uptake [Brzostek et al., 2014; Koster et al., 2014].

For tree species that adapt quickly to changing climate conditions and phenological cues, a longer climatological growing season may drive changes in growing season net primary productivity [Nemani et al., 2003; Twine and Kucharik, 2009]. A warmer Spring is typically associated with an earlier start to the vegetation growing season, provided adjustment to the timing and speed of leaf growth (subject to freezing risks) to these warmer conditions. Higher Autumn temperatures may support longer vegetation growing seasons for those forest species that can adjust their leaf longevity and senescence triggers to warmer Autumn conditions. However, we are still learning to identify and understand the many cues for leaf senescence that influence leaf phenology, including photoperiod and temperature [Kikuzawa et al., 2013; Ali et al., 2015] and biochemical limits on leaf longevity [Keenan and Richardson, 2015; Seki et al., 2015]. 


\subsection{Seasonal Transitions}

Climate during the shoulder seasons can be critical to vegetation phenology. A lack of statistically significant interseasonal correlations between Summer and the shoulder seasons (Table 5.2 and Fig. 5.15) leads to uncertainty regarding aspects of forest phenology such as the progression of green-up, seasonal peak greenness in early Summer, and deciduous Autumn senescence with follow-on effects for the related Winter feeding patterns of overwintering herbivores. Summer and Autumn conditions strongly influence seed production in many forest species, with consequences for wildlife feeding and reproduction patterns [Yang et al., 2010]. These growing season conditions affect biochemical processes that control leaf bud set and hardening prior to late Autumn freezing, with consequences for optimum productivity in the next growing season [Vitasse et al., 2014; Estiarte and Peñuelas, 2015]. Seasonal climatological conditions can thus affect forest phenology and overall primary productivity in the same season and well into the forest life cycle [Noormets et al., 2008; Anderegg et al., 2012, 2013, 2015; Brzostek et al., 2014].

Regarding late frost events and false Spring occurrences, I found an area-average trend of -2.1 degree-days/y $(p<0.05)$ in the accumulation of $G D D$ before the last Spring frost. This trend, along with a slight trend toward earlier last Spring freezing nights (Table 5.1), suggests a slowly diminishing risk of vegetation-damaging Spring frost events over our study period. This result is not necessarily consistent with a frequency-based examination of frost-based Spring vegetation damage [Augspurger, 2013] that suggested more frequent occurrences in the Midwest U.S. I also take particular note of false Spring and late frost events that were observed in the U.S. Upper Midwest in 2007 [Augspurger, 2009; Gu et al., 2008] and 2010 [Fereday et al., 2012; Hufkens et al., 2012; Filewood and Thomas, 2014; Ning and Bradley, 2014]. For these years the area-average accumulations of GDD before the last Spring frost were 147 and 156 degree-days, respectively, both very close to the 30 -year average for that metric, and with

earlier-than-normal last Spring frost dates in both years. By contrast, a Spring heatwave in 2012 [Ellwood et al., 2013; Peterson et al., 2013] led to an area-average accumulation of $200 G D D$ by the time of the last Spring frost, which also occurred earlier than the 30-year normal date, yet no particular frost-related damage was reported across the region. Hypothetically, higher-than-normal $G D D$ accumulations 
(suggesting greater likelihood of early leaf growth) would be expected before a possibly later-than-normal last Spring frost date for these events. However, false Springs in ENSO-neutral 2007 and El Niñoinfluenced 2010, but not in La Niña-influenced 2012, ran contrary to that expectation. Under that hypothesis, I further suspect that false Spring events occurred for portions of our study area in 1986 and 1992, as suggested in Table 5.1. I anticipate that continuing observational study of regional forest phenology will provide greater insight into these occurrences, given the potential importance of Spring late frost events to seasonal phenological patterns and their impact on tree growth.

The seasonal and inter-seasonal climatological relationships found above may promote some interesting phenological patterns. Within a season, the inverse relationship between average temperature and precipitation days (as a proxy for cloudy days) means that days with greater water availability (from precipitation) would also have lower evaporation and transpiration moisture demand. A cycle may be established in which forest soil and canopy moisture are allowed to build up during periods with cloudy/wet days and then deplete during periods with clear/dry days. Lower leaf primary productivity in cloudy periods may then be offset by enhanced productivity, supported by greater soil moisture availability, during clear periods. Increased soil moisture is also conducive to seed germination and seedling growth, especially for shade-tolerant species, and can thus have potential impacts on mixed forest understory structure and species distributions [Nowacki and Abrams, 2008].

Conversely, dry soils and forest litter can lead to inhibited soil respiration, slower litter decay, and potential interference with seed germination and seedling growth, also altering forest structure and composition over time [Gustafson and Sturtevant, 2013; Peters et al., 2015]. Reduced moisture availability can eventually lead to a shift of the surface energy balance away from latent heating (evaporation, transpiration) in the growing season to greater sensible heating at the surface and in the forest canopy, a positive feedback cycle that can enhance local warming and exacerbate forest canopy moisture stress [Anderegg et al., 2012]. A warmer Spring and earlier start to the growing season may therefore compensate for a cooler Summer in some years in terms of total growing season primary productivity, but the reverse is not necessarily true: a warm Summer may not compensate for a cool 
Spring and a late start to the growing season but can instead exacerbate temperature and moisture stresses that reduce forest productivity throughout the growing season.

\subsection{Lake Effects}

Climatological characteristics are distinguished across the western end of Lake Superior and reflect the influence of the lake on $T$ (northwestern shore) and $P$ (to the southeast), both of which can be linked to the thermal inertia of Lake Superior surface waters during seasonal transition periods. Numerous such influences generate complex interactions around the Upper Great Lakes, and the lakes themselves contribute to spatiotemporal variability in the system through their internal mixing regimes, providing some "memory" of conditions across seasons (via thermal inertia) and potentially over several years [Bennett, 1978; Gerten and Adrian, 2001; Piccolroaz et al., 2015]. Warm lake surface waters in Autumn and Winter enhance the land-lake temperature contrast, contributing also to strong $P$ gradients across the lake from lower accumulations in the northwest to higher totals to the southeast. Delayed ice formation in warm Winters allows a longer period of surface evaporation, feeding lake-effect $P$ maxima to the southeast through both Autumn and Winter. Conversely, Lake Superior ice cover that extends well into Spring in some years may contribute to diminished Spring $P$ as well as delayed last Spring frost dates for that area. Because of the thermal and moisture effects of the lake, I anticipate that vegetation phenological transitions in the Spring season for areas south and east of Lake Superior are typically delayed several days, possibly weeks, compared with those transitions west and north of the lake.

The influence of Lake Superior and its own changes over time on study area climatology is substantial, including both recent lake warming [Austin and Colman, 2007, 2008; Van Cleave et al., 2014; O'Reilly et al., 2015] and changing ice phenology [Assel, 2003; Howk, 2009; Wang et al., 2012; Assel et al., 2013]. Observational studies regarding the effects of the Great Lakes on their surrounding land areas [Li et al., 2010] remain essential to our growing understanding of land-atmosphere processes in the surrounding forest areas. Given their prevalence on the study area landscape (Fig. 3.2), the roles of smaller lakes in regional climatology and forest phenology are also of interest to this work [Johnson and 
Stefan, 2006; Plank and Shuman, 2009; Mishra and Cherkauer, 2011; Mishra et al., 2011a, 2011b].

Overall, while I remain interested in phenological events in the Autumn season and the potential effects of Lake Superior thermal inertia on delayed timing of those events in the vicinity of the lake, the Spring transition and its complexity because of that proximity is vital for understanding phenological transitions at the start of the vegetation growing season and will be an interesting point of focus in Chapter 6 .

\subsection{Summary and Discussion}

Using available weather station data, I have examined the mean seasonal and annual climatology, temporal trends, teleconnection correlations, and the potential influences of Lake Superior on surrounding land areas during 1984-2013. My study area is characterized by an extensive and ecologically important forest-and-lake landscape at the Upper Midwest U.S. prairie-forest ecotone, where I anticipate observable sensitivity to recent and ongoing climate change. Key conclusions from the above climatological analyses that may be applicable to my phenological analysis include:

- $\quad 30$-year net warming of $+0.56^{\circ} \mathrm{C}$ averaged over my study area;

- long-term warming in annual minimum and average temperatures;

- long-term Spring cooling, offset by long-term Autumn warming;

- long-term increases in Winter and Spring precipitation

- long-term Summer drying, especially in areas northwest of Lake Superior, and especially in the latter half of my study period;

- long-term changes in ice phenology on Lake Superior, including a diminishing duration of Winter ice cover, with seasonally-oriented effects on the surrounding land areas, especially Winter precipitation and Spring temperatures southeast of the lake;

- a shift in climatological regimes around 1998, midway through my study period, that led to sharp changes and even reversals of several long-term temperature and precipitation trends; 
- an overall extension of the climatological growing season, whether defined traditionally by the frost-free season or by my own new metric based on the warm-season plateau in accumulated chilling days, primarily into Autumn.

Several prominent global and hemispherical climatic teleconnections correlate in varying degrees with seasonal and interannual variability in regional hydroclimatology and with lake ice phenology, all of these factors affecting seasonal $T$ and $P$ patterns in the study area.

Understanding interannual climatological variability and the interactions of the land-atmosphere system in this study area is clearly more complex than reliance on any single element, such as teleconnections [Wise et al., 2015] or another external indicator, will allow. As an example, Weaver et al. [2009] analyzed the 1993 Upper Midwest Summer flood event, which was supported by both AO- and NAO - conditions and found that moisture from the distant Gulf of Mexico contributed significantly to large rainfall totals in the Great Lakes and Upper Mississippi River regions that year. An event or season can reach climatological extremes by the interaction and reinforcement of influences: the NAO phase, which can influence synoptic organization over the Great Lakes region but may not necessarily generate a significant event on its own, interacted with the alignment of AO-driven meridional flows to drive the northward transport of Gulf moisture along the entire length of the Mississippi River valley. Both distant and local influences can thus dominate regional weather and climatology at various times. Changes in Lake Superior ice cover regimes have contributed to greater Winter and Spring lake-effect $P$ in portions of the study area south and southeast of the lake. Lake Superior itself clearly affects $T$ and $P$ patterns in nearby land areas through both proximity and an interseasonal lag due to thermal inertia, with effects that are likely strong enough to alter forest phenology in those areas. 


\section{CHAPTER 6}

\section{MEAN PHENOLOGY AND INTERANNUAL VARIABILITY}

In Chapter 5, I examined 92 climatological indicators at seasonal and annual time scales (Tables 3.2, 3.4, and 3.5), including several indicators that have been used previously to assess changing climate extremes [Frich et al., 2002; Alexander et al., 2006]. I calculated daily accumulations of chilling days $(C D)$ and growing degree days $(G D D)$ [Baskerville and Emin, 1969; Thompson and Moncrieff, 1982] using a base temperature of $5^{\circ} \mathrm{C}$ following several empirical studies of tree physiology and spring phenology [Cannell and Smith, 1983; Murray et al., 1989; Hunter and Lechowicz, 1992; Fisher et al., 2007]. I also calculated accumulated freezing days $(F D)$ in a similar manner to $C D$ but with a base temperature of $0^{\circ} \mathrm{C}$. I then defined the vegetation growing season in two ways. The first was a traditional definition based on the last spring frost and first autumn frost dates $\left(\mathrm{T}_{\min }<0{ }^{\circ} \mathrm{C}\right)[$ McCabe et al., 2015], and the second as a function of chilling day $(C D)$ accumulation. By definition, the $C D$ plateau-based growing season is several days longer than the frost-free season for the same year. In cases where a late spring frost can damage new foliage, or an early autumn frost might hasten leaf senescence, the differences between season starting and ending dates obtained by these different methods are of great interest.

In this chapter, I apply the model described in Chapter 4 and the climatological findings of Chapter 5 to the analysis of Landsat-based phenological observations in my study area, first at the scale of a single footprint and then over the entire study area (Fig. 3.1). This examination at the footprint scale will include model results from all three of the principal vegetation indexes discussed in Chapter 3, so that the advantages and efficacy of each index are shown. I then selected one vegetation index for phenological model application across the study area, from which I present numerous results at that scale. I will compare phenological indicators with climatological measures, examine the influences of Lake Superior on surrounding forest areas, and discuss the challenges of phenological analysis in the spring and autumn transition seasons. 


\subsection{Results at the Footprint Scale using NDVI, EVI, and NDII}

For comparison, I repeated the phenological modeling process for all three of the individual vegetation indexes discussed in Chapter 3 (NDVI, EVI, and NDII) over one footprint (P26R27) in my study area. Following dataset collection, merging, and discretization of the geographical area, I applied the phenological model described in Chapter 4 on a pixel-by-pixel basis across the footprint. After model processing on distributed systems, I collected the results and reconstituted the footprint-scale maps for each model output parameter.

\subsubsection{Mean Phenology}

Footprint-scale maps showing the accuracy $(R M S E)$ and goodness-of-fit $\left(r^{2}\right)$ of the fitted mean phenological curve for each of the three vegetation indexes are shown in Fig. 6.1, with statistics for these and later figures summarized in Table 6.1. There are clear differences among the applied vegetation indexes, with the lowest overall (footprint-wide) RMSE and highest $r^{2}$ values provided by $E V I$, and the highest overall RMSE and lowest $r^{2}$ values provided by NDII. Generally, the highest RMSE and lowest $r^{2}$ values for each vegetation index are located in areas where major disturbances are known to have occurred during the study period. Many potential disturbances are sequestered from the observations in the course of the modeling process, leaving fewer scenes to support phenological curve fitting.

The Spring Inflection $(S I)$ and Autumn Inflection $(A I)$ dates (see Fig. 4.2 and Table 4.1) are important descriptors of vegetation processes and growing season phenology, describing the approximate beginning and end of the period during which the forest is actively assimilating carbon from the atmosphere. $S I$ and $A I$ dates derived from the mean phenological curve for each of the three vegetation indexes differ considerably across the study area (Fig. 6.2, with statistics listed in Table 6.2). The SI dates for $N D V I$ and $N D I I$ are broadly similar in geographic pattern $(r=0.685, p<0.001)$ and are overall earlier than those using the $E V I$-based phenological curve-fitting process. The latest $A I$ dates are produced by the $N D I I$-based curves, and the earliest $A I$ dates occur with $E V I$-based curves. It is in these results that 

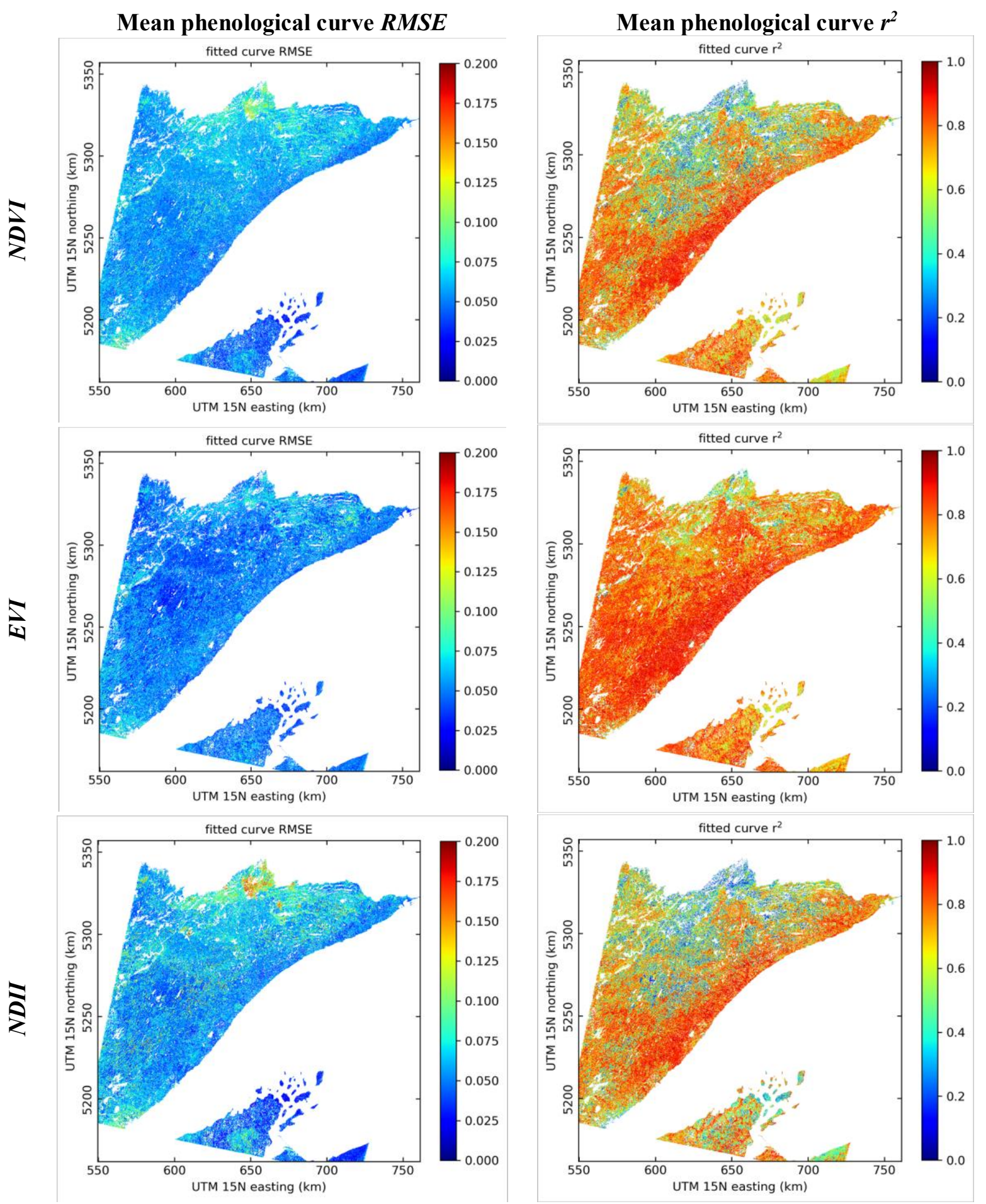

Figure 6.1: Comparison among fitted mean phenological curve accuracy measures for NDVI, EVI, and NDII over the P26R27 footprint. See also Table 6.1. 
Table 6.1: Accuracy measures for three VIs averaged over footprint P26R27, as shown in Figs. 6.1, 6.10, and 6.17 .

\begin{tabular}{|c|c|c|c|c|}
\hline VI & $\begin{array}{c}\text { Mean } \\
\text { phenology } \\
\text { fitted curve }\end{array}$ & $\begin{array}{c}\text { Residuals of } \\
\text { phenology } \\
\text { (PLSR) }\end{array}$ & $\begin{array}{c}\text { Mean } \\
\text { phenology } \\
\text { + PLSR }\end{array}$ & $\begin{array}{c}\text { Mean \# of } \\
\text { PLSR } \\
\text { variables }\end{array}$ \\
\hline \multirow{2}{*}{$N D V I$} & $\begin{array}{c}R M S E=0.656 \\
r^{2}=0.058\end{array}$ & $\begin{array}{c}R M S E=0.409 r^{2} \\
=0.762\end{array}$ & $\begin{array}{c}R M S E=0.987 r^{2} \\
=0.055\end{array}$ & $15.3 \pm 7.1$ \\
\hline$E V I$ & $\begin{array}{c}R M S E=0.772 r^{2} \\
=0.052\end{array}$ & $\begin{array}{c}R M S E=0.400 r^{2} \\
=0.769\end{array}$ & $\begin{array}{c}R M S E=0.980 r^{2} \\
=0.026\end{array}$ & $15.1 \pm 7.0$ \\
\hline \multirow{2}{*}{ NDII } & $\begin{array}{c}R M S E=0.618 r^{2} \\
=0.062\end{array}$ & $\begin{array}{c}R M S E=0.451 r^{2} \\
=0.735\end{array}$ & $\begin{array}{c}R M S E=0.944 r^{2} \\
=0.030\end{array}$ & $16.4 \pm 7.0$ \\
\hline
\end{tabular}

differentiation among forest types starts to become evident, with generally earlier $A I$ dates for all three vegetation indexes in a narrow band of largely deciduous forest along Lake Superior. Significant detail can be found in the full-scale versions of these figures, with near-lake areas, ridges and valleys, and known areas of aspen forest clearly delineated in the mean phenological $S I$ and $A I$ values.

The Durations of Spring and Maturity ( $D O S$ and $D O M$, respectively) derived from the mean phenological curve for each of the three vegetation indexes are shown in Fig. 6.3, with statistics listed in Table 6.2. For clarity, the DOS is not the duration of the astronomical or meteorological spring, but rather a phenological indicator based on the rising limb of the fitted mean Landsat-derived phenological curve (Fig. 4.2). Though the values for DOS follow similar geographic patterns across the three VIs ( $r=0.766$ for $N D V I$ vs. $E V I ; r=0.744$ for $E V I$ vs. NDII; $r=0.788$ for $N D V I$ vs. $N D I I$; all with $p<0.001$ ), this pattern is remarkably dissimilar from the geographic patterns of $S I$ for each index that are shown in Fig 6.2. The geographic patterns of $D O S$ and $D O M$ are similar but inversely correlated $(r=-0.720$ for $N D V I$; $r=-0.460$ for $E V I ; r=-0.723$ for NDII; all with $p<0.001)$. Positive correlations occur between $S I$ and $\operatorname{DOS}(r=0.391$ for NDVI; $r=0.211$ for EVI; $r=0.435$ for NDII; all with $p<0.001$ ). The values of DOM follow a somewhat similar geographic pattern to those of $A I(r=0.201$ for $N D V I ; r=0.680$ for $E V I ; r=$ 0.254 for $N D I I$; all with $p<0.001)$. However, $S I$ and $A I$ remain essentially uncorrelated $(r=0.182$ for NDVI; $r=-0.050$ for EVI; $r=0.010$ for NDII; all with $p<0.001)$. 

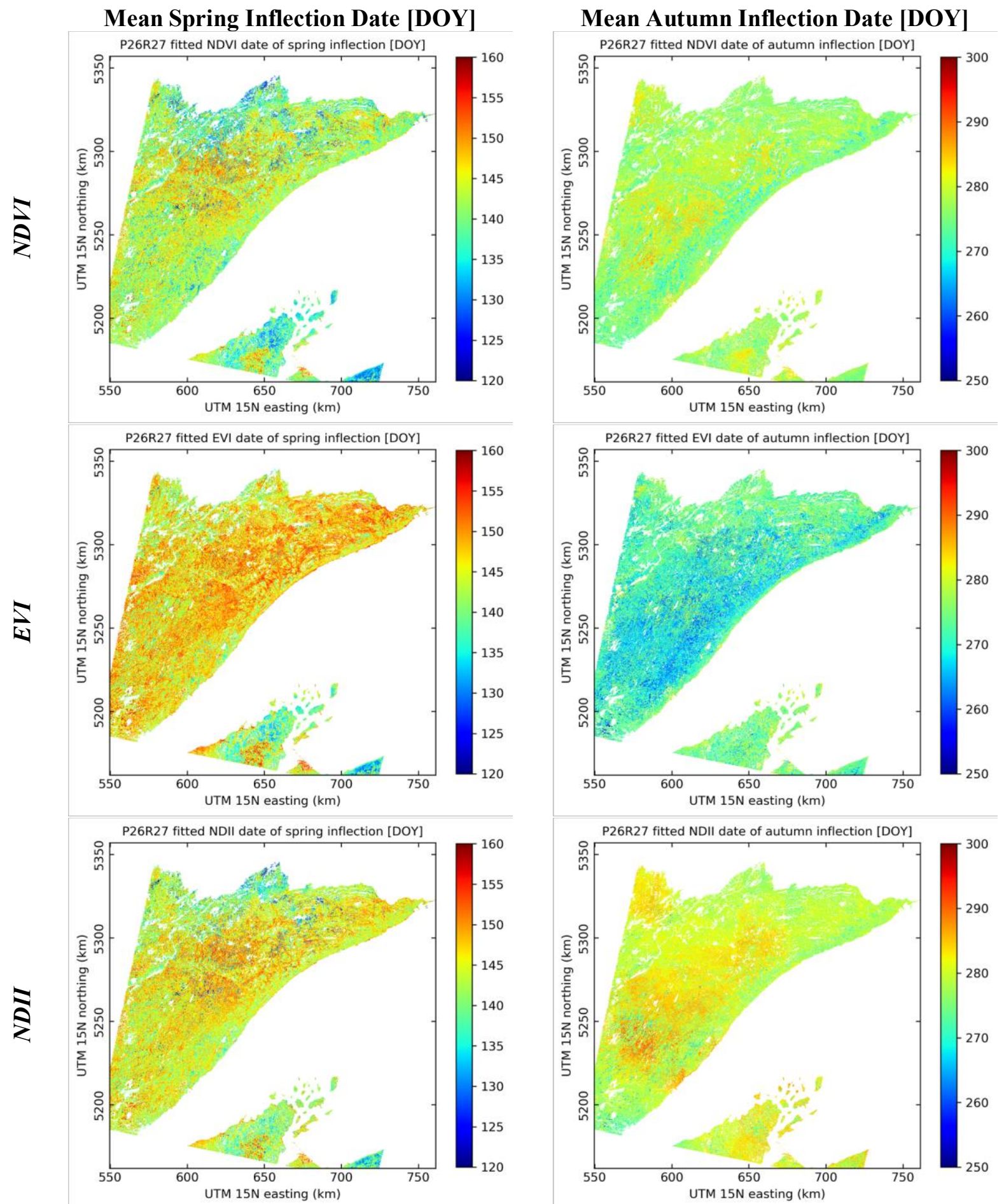

Figure 6.2: Comparison among fitted mean phenological curve key dates for NDVI, EVI, and NDII over the P26R27 footprint. See also Table 6.2. 

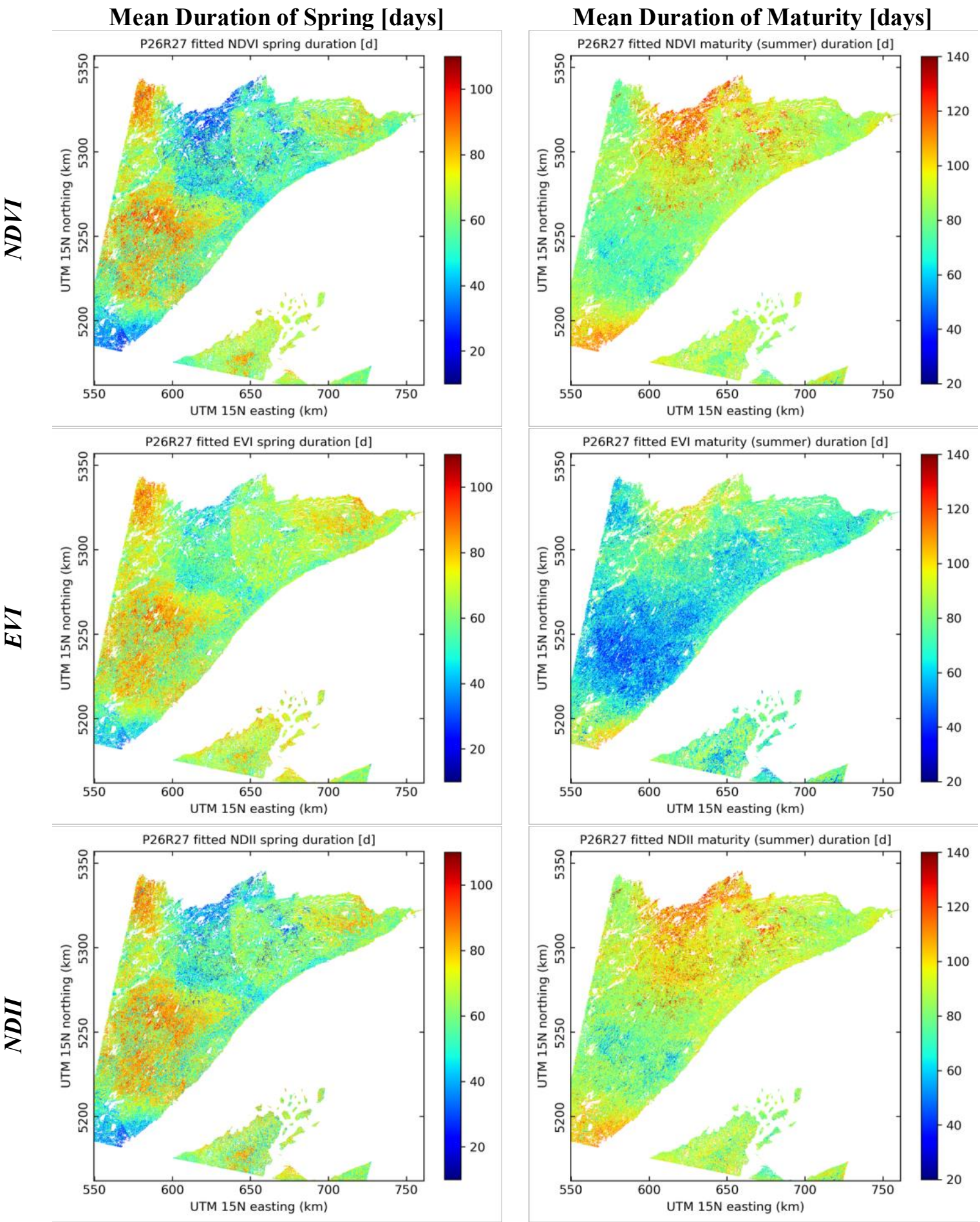

Figure 6.3: Comparison among fitted mean phenological curve key season durations for NDVI, EVI, and NDII over the P26R27 footprint. See also Table 6.2. 


\subsubsection{Comparisons with Climatology}

The phenological indicators described above, specifically the $S I$ dates and the DOS and DOM periods, are often associated closely with environmental cues such as Winter intensity, last Spring frost dates, Spring and Summer accumulation of $G D D$, high Summer temperatures, and low Summer precipitation. A number of other phenological indicators are also often associated with such environmental markers. It is therefore important to check model-diagnosed phenological indicators against potentially useful climatological indicators in order to take full advantage of those environment cues in understanding the seasonal phenological process.

My mean phenological curve (Fig. 4.2) produces 61 indicators (Table 4.1): mean and standard error (StdErr) values for each of 29 curve-based indicators, and three overall accuracy and goodness-of-fit indicators $\left(M A E, R M S E\right.$, and $\left.r^{2}\right)$. I compared mapped phenological indicators from NDVI-, EVI-, and NDII-based fitted curves to potential climatological influences using maps of 92 indicators of 1984-2013 mean climatology covering seasonal and annual time periods that were presented in Chapter 5 [ Garcia and Townsend, 2016] (Figs. 6.4-6.6). At the scale of footprint P26R27, none of the comparisons exceeds a Pearson correlation of \pm 0.5 , which holds for all of the other footprints examined here as well. Climatological indicators seem related to phenological model accuracy most strongly for NDVI and most weakly for $E V I$. The observed correlations between mean climatological and phenological indicators are generally strongest for $E V I$ and weakest for $N D V I$.

There are patterns in Figs. 6.4-6.6 of consistent positive and negative correlations between certain climatological indicators and vegetation phenology across all of the vegetation indexes, suggesting consistent relationships no matter which vegetation index is used to characterize that phenology. For example, accumulations of chilling days $(C D)$ appear almost always positively correlated with key phenological dates but negatively correlated with key phenological VI values; in other words, more coldseason chilling days generally delay (or prolong) Spring phenological processes and reduce the maximum intensity of "greenness" measured by a given vegetation index. The converse is apparent for accumulations of growing degree days: GDD correlates negatively with key phenological dates, 


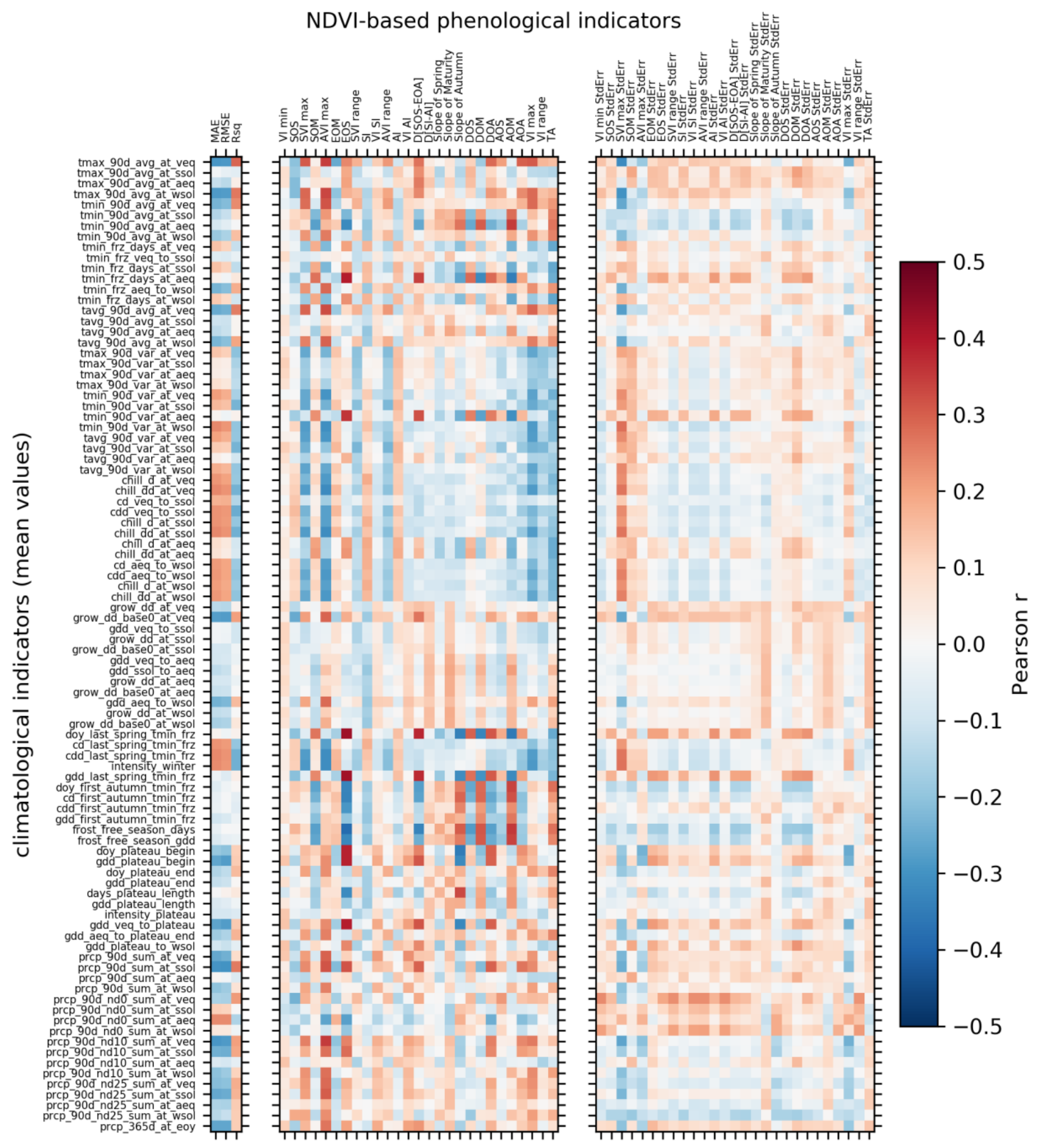

Figure 6.4: Summary of correlations for NDVI-based mean phenological curve indicators (left columns) and their standard errors (StdErr, right columns) with climatological indicator mean values for footprint P26R27. Climatological indicators are listed in Tables 3.1-3.5, and phenological indicators are listed in Table 4.1 and illustrated in Fig. 4.2. 


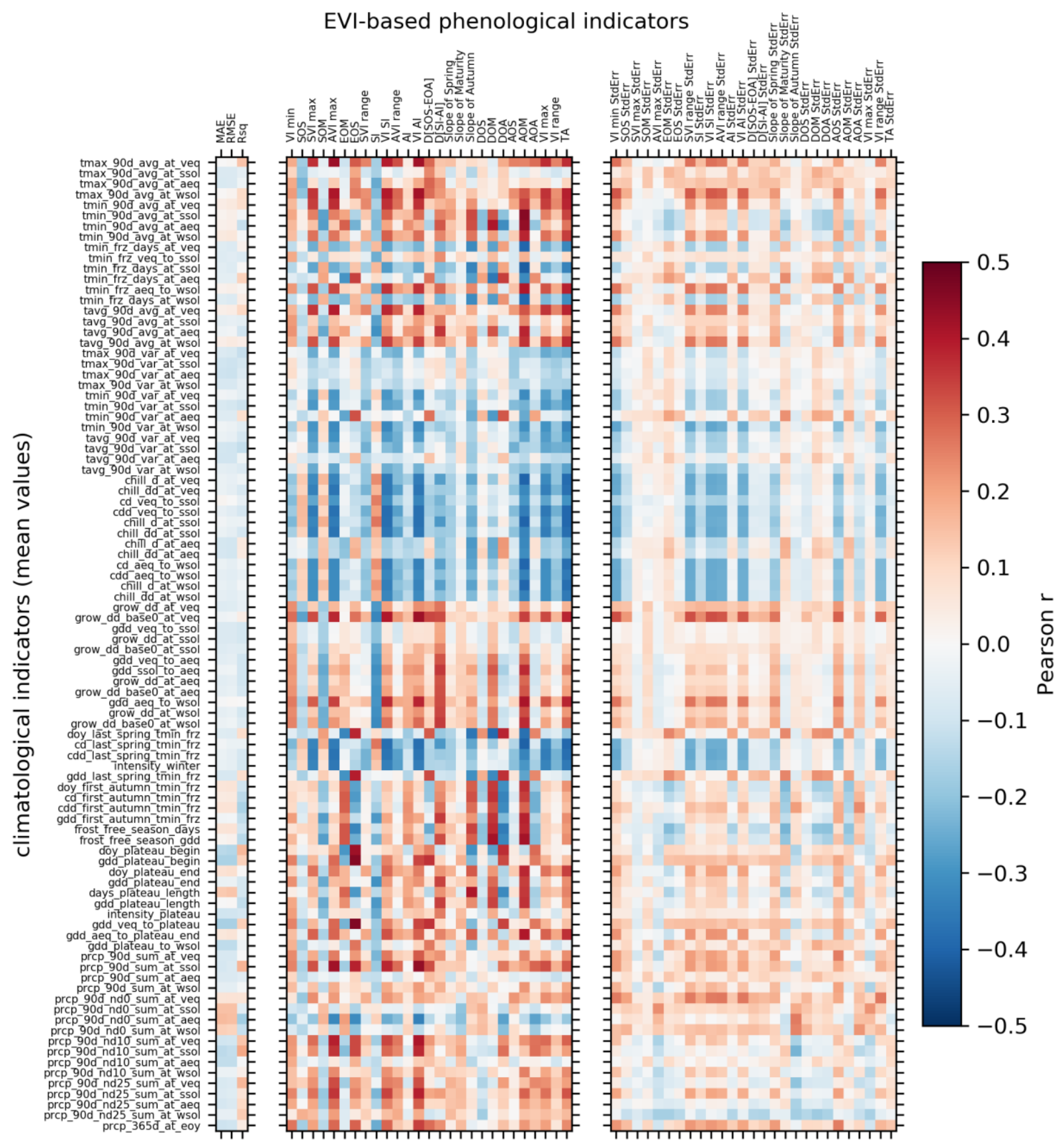

Figure 6.5: Summary of correlations for $E V I$-based mean phenological curve indicators (left columns) and their standard errors (StdErr, right columns) with climatological indicator mean values for footprint P26R27. Climatological indicators are listed in Tables 3.1-3.5, and phenological indicators are listed in Table 4.1 and illustrated in Fig. 4.2. 


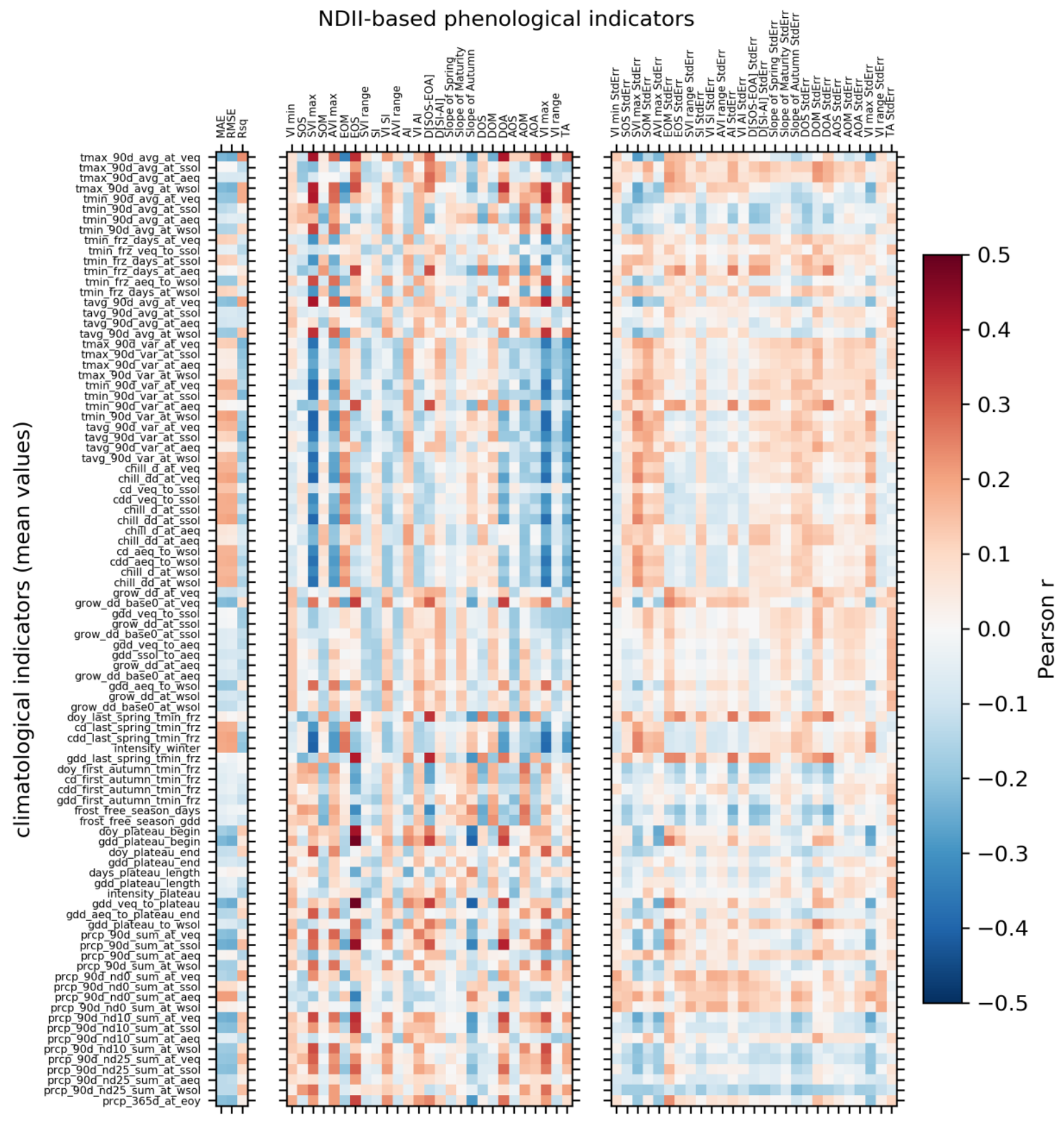

Figure 6.6: Summary of correlations for NDII-based mean phenological curve indicators (left columns) and their standard errors (StdErr, right columns) with climatological indicator mean values for footprint P26R27. Climatological indicators are listed in Tables 3.1-3.5, and phenological indicators are listed in Table 4.1 and illustrated in Fig. 4.2. 
Table 6.2: Statistics for comparison among VIs for the phenological indicators shown in Figs. 6.2 and 6.3.

\begin{tabular}{|c|c|c|c|c|}
\hline Phenological & \multirow{2}{*}{$\begin{array}{c}\text { Metric } \\
\text { Indicator }\end{array}$} & \multicolumn{3}{|c|}{ P26R27 mean phenological curve } \\
\cline { 2 - 5 } & & $\boldsymbol{N D V I}$ & $\boldsymbol{E} \boldsymbol{I}$ & $\boldsymbol{N D I I}$ \\
\hline \multirow{3}{*}{$S I[\mathrm{DOY}]$} & $\min$ & 111.7 & 113.5 & 111.7 \\
\cline { 2 - 5 } & $\max$ & 173.8 & 186.1 & 176.5 \\
\cline { 2 - 5 } & $\operatorname{mean}$ & 141.9 & 146.3 & 144.2 \\
\hline \multirow{3}{*}{$A I[\mathrm{DOY}]$} & $\min$ & 246.3 & 225.2 & 241.3 \\
\cline { 2 - 5 } & $\max$ & 296.4 & 295.2 & 297.1 \\
\cline { 2 - 5 } & $\operatorname{mean}$ & 277.1 & 271.3 & 280.3 \\
\hline \multirow{3}{*}{$D O S[\mathrm{~d}]$} & $\min$ & 1.7 & 1.9 & 3.6 \\
\cline { 2 - 5 } & $\max$ & 115.3 & 114.5 & 117.1 \\
\cline { 2 - 5 } & $\operatorname{mean}$ & 60.6 & 65.8 & 62.9 \\
\hline \multirow{3}{*}{$D O M[\mathrm{~d}]$} & $\min$ & 11.7 & 11.7 & 15.1 \\
\cline { 2 - 5 } & $\max$ & 147.4 & 147.1 & 147.7 \\
\cline { 2 - 5 } & mean & 87.8 & 69.1 & 90.0 \\
\hline
\end{tabular}

suggesting a hastening of phenological processes, and positively with key VI values, indicating support for greater VI-measured "greenness" through most of the growing season. In the maps of DOS (Fig. 6.3), a longer Spring occurs on elevated areas in the southern portion of the P26R27 footprint, where the topography leads to a slower accumulation of $G D D$ through the spring and summer seasons, while areas of lower topography farther north in the footprint likely accumulate $G D D$ more rapidly, with accordingly shorter DOS.

Temperature measures in these correlation grids show mixed correlations with phenological indicators, even though each cross-correlation combination remains consistent across the three vegetation indexes examined here. For instance, the Spring mean of $T_{\min }$ ("tmin_90d_avg_at_ssol" in Table 3.2) is positively correlated with the Spring peak VI value $\left(S V I_{\max }\right)$ but negatively correlated with the date of that spring peak $(S O M)$. This particular relationship holds for phenological curves based on NDVI, EVI, and NDII. Many of the prominent correlations in these footprint-scale analyses will examined in greater detail at the regional scale, below. 


\subsubsection{Phenological Variability}

Variability in some phenological indicators (indicated by the standard error estimate, either output from the mean phenological curve fitting procedure directly or calculated post hoc along with the numerous derivative indicators) is correlated with a number of climatological indicators (Figs. 6.4-6.6, right panels), but more weakly than mean phenology (left panels). The sign of each mean phenology correlation with mean climatology is overall consistent across all three of the vegetation indexes, but correlations using phenological variability have different signs across the vegetation indexes.

As part of the model I described in Chapter 4, I expect climatological and phenological variability to be related to some degree, with variations from the mean climatology potentially driving various deviations from the mean phenology. Attempting to relate the calculated variability in these phenological measures with the observed variability in potential climatological influences, I cross-correlated these phenological model indicators with 92 indicators of 1984-2013 climatological variability (using the standard deviation) covering seasonal and annual time periods, as with the mean climatology above (Figs. 6.7-6.9). In all of these combinations, the magnitude of correlations is as large as for the comparisons with climatological mean values, though not necessarily of the same sign. These results suggest that there is a modest relationship between climatological variability and modeled variability in mean phenology that could be used to help predict phenological variability.

The possible relationships between climatological and phenological variability are addressed more directly through the PLS regression modeling component of this work. It is, however, often difficult to summarize all of the results of a PLSR model with dozens of coefficients at each of millions of pixels across the footprint. There are some simple accuracy measures produced by the PLSR process that can be mapped to show the model fit, specifically RMSE and $r^{2}$ for each of the three vegetation indexes (Fig. 6.10). Contrary to the results for the fitted mean phenological curve, at the footprint scale the lowest $R M S E$ values and highest $r^{2}$ values are associated with modeling of NDII residuals. The largest $R M S E$ values across the footprint are generally associated with $E V I$, though modeling of NDVI produced slightly higher RMSE in the northern portion of the footprint and lower RMSE in the southern portion. A similar 


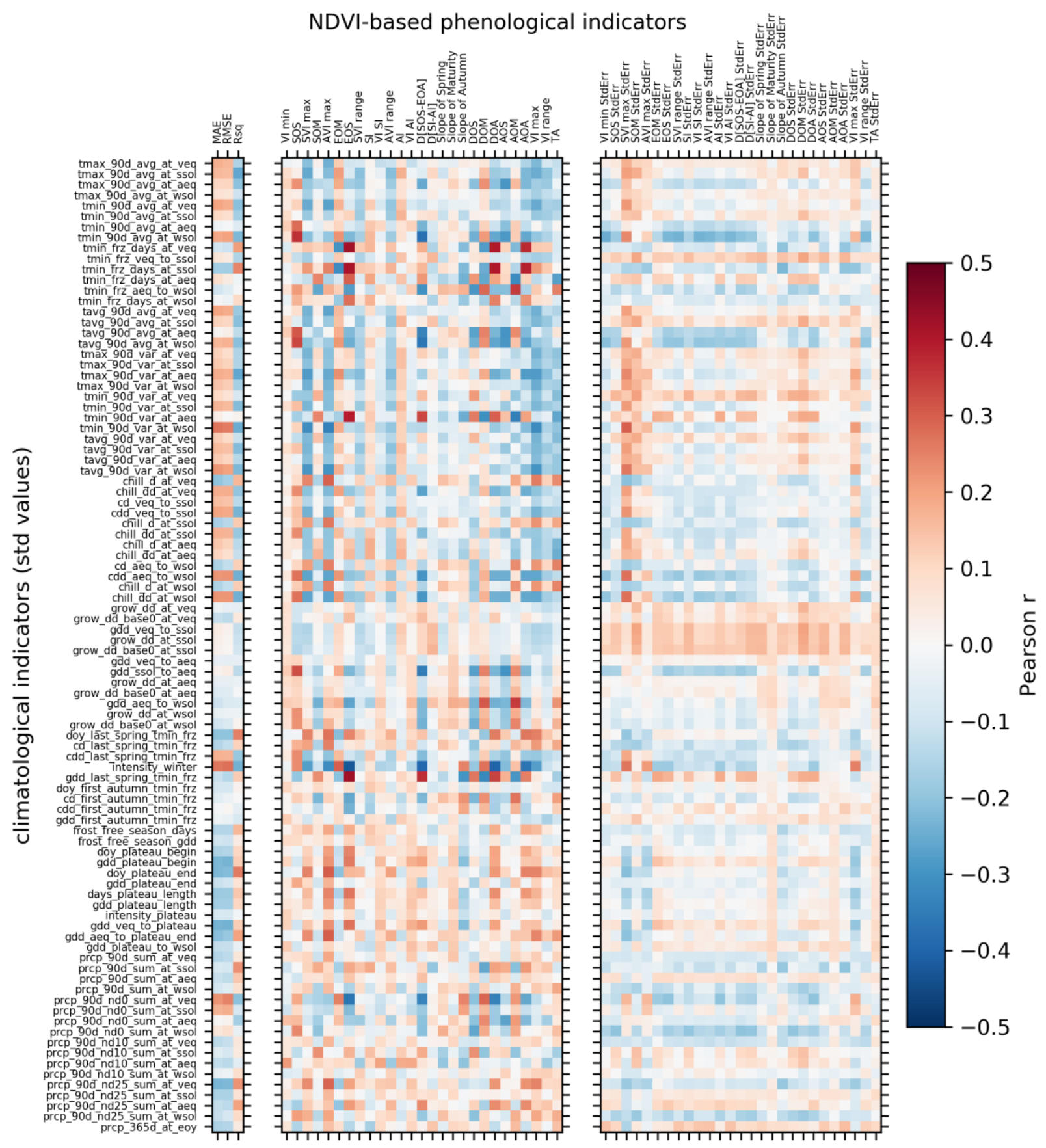

Figure 6.7: Summary of correlations for NDVI-based mean phenological curve indicators (left columns) and their standard errors (StdErr, right columns) with climatological indicator variance (StDev) values for footprint P26R27. Climatological indicators are listed in Tables 3.1-3.5, and phenological indicators are listed in Table 4.1 and illustrated in Fig. 4.2. 


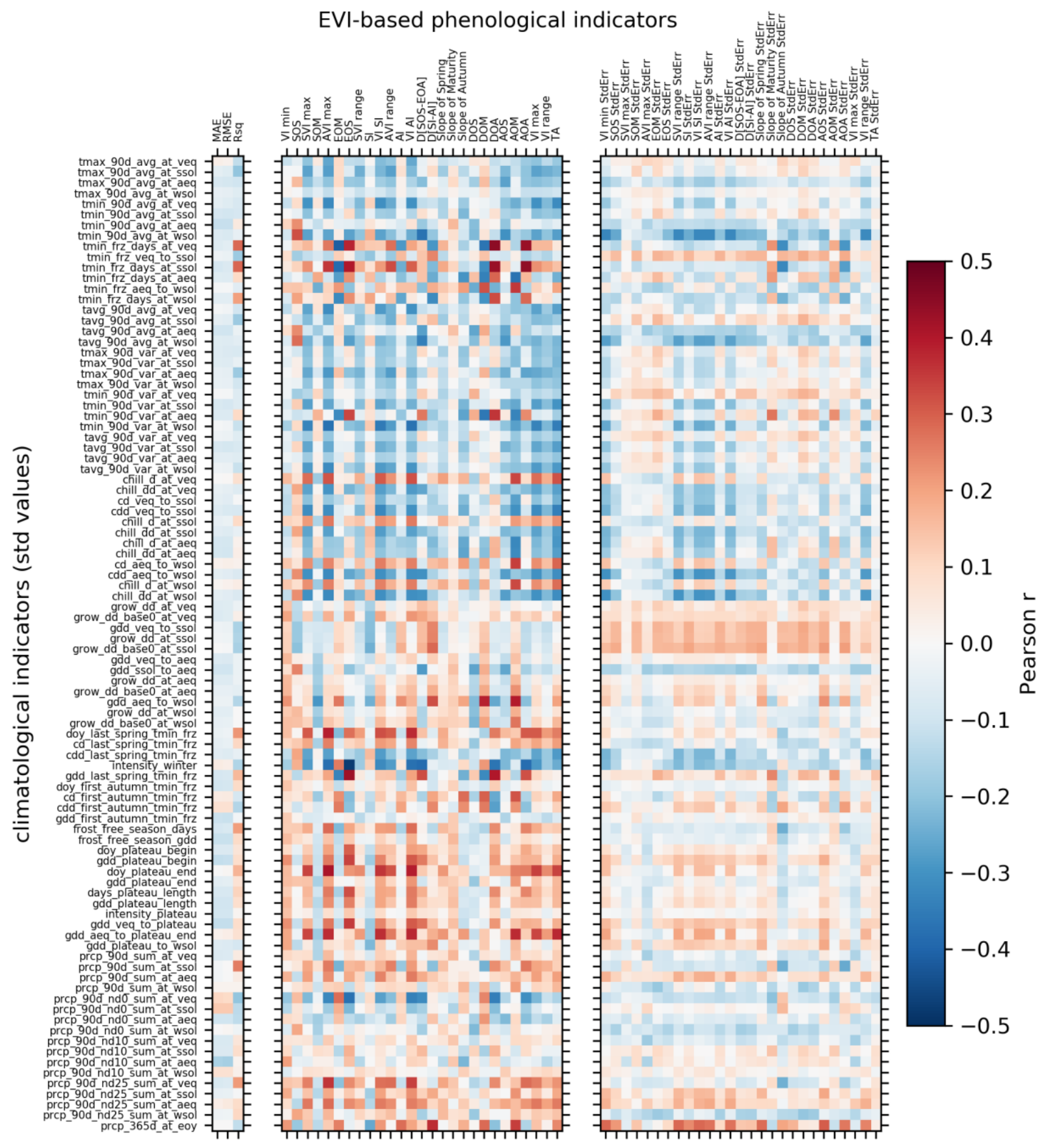

Figure 6.8: Summary of correlations for $E V I$-based mean phenological curve indicators (left columns) and their standard errors (StdErr, right columns) with climatological indicator variance (StDev) values for footprint P26R27. Climatological indicators are listed in Tables 3.1-3.5, and phenological indicators are listed in Table 4.1 and illustrated in Fig. 4.2. 


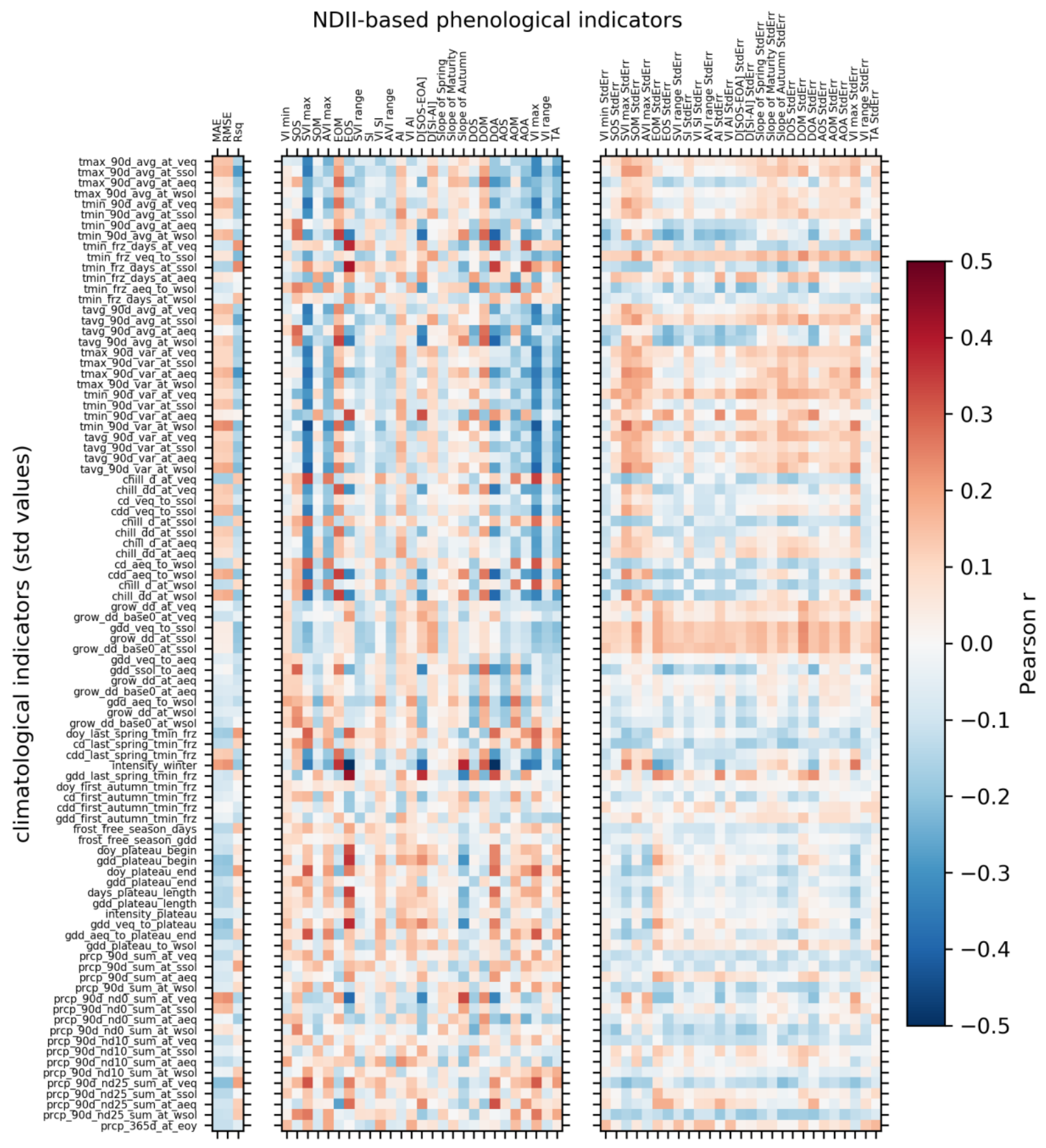

Figure 6.9: Summary of correlations for NDII-based mean phenological curve indicators (left columns) and their standard errors (StdErr, right columns) with climatological indicator variance (StDev) values for footprint P26R27. Climatological indicators are listed in Tables 3.1-3.5, and phenological indicators are listed in Table 4.1 and illustrated in Fig. 4.2. 

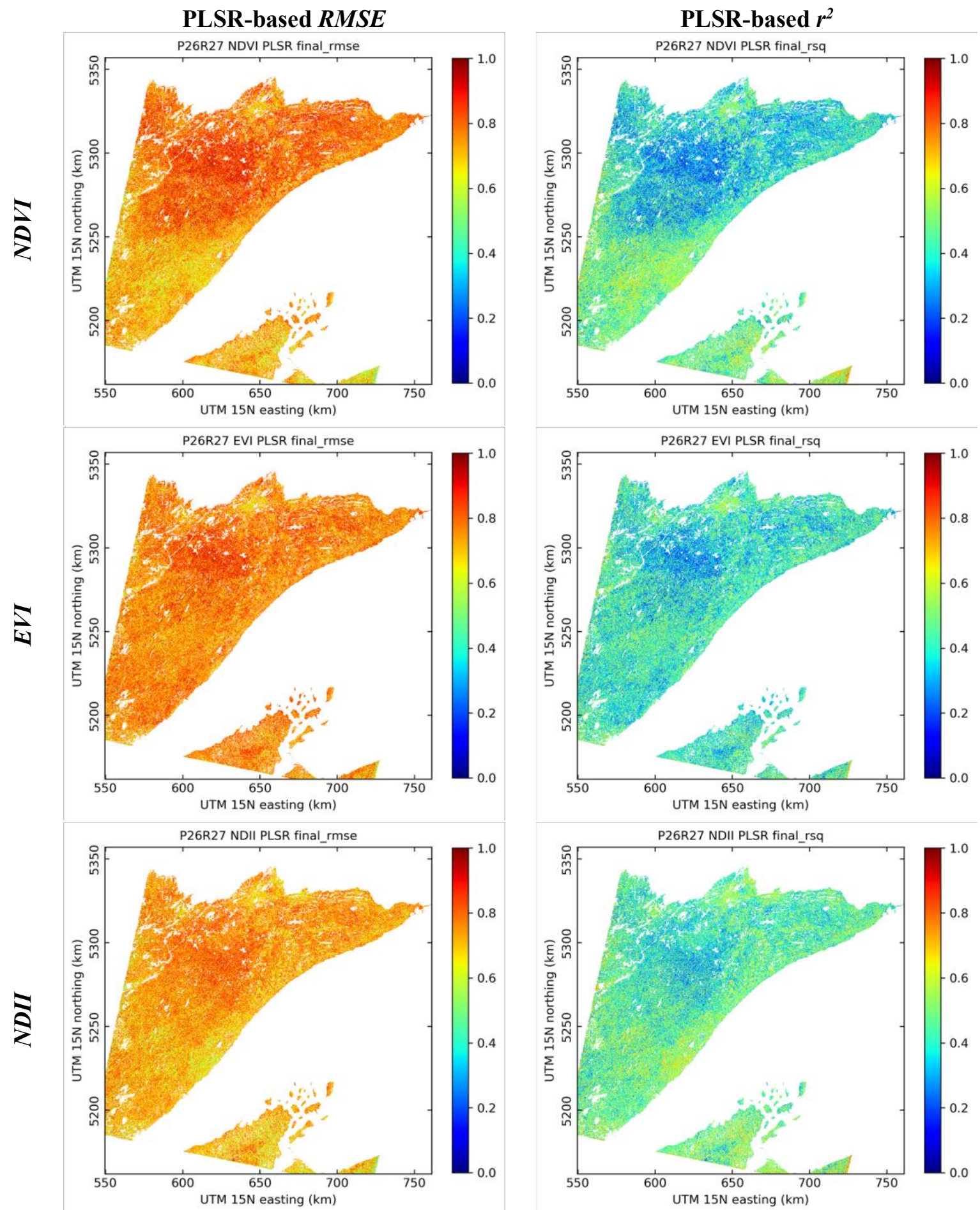

Figure 6.10: Comparison among PLS regression model accuracy and error measures for NDVI, EVI, and NDII over the P26R27 footprint. See also Table 6.1. 


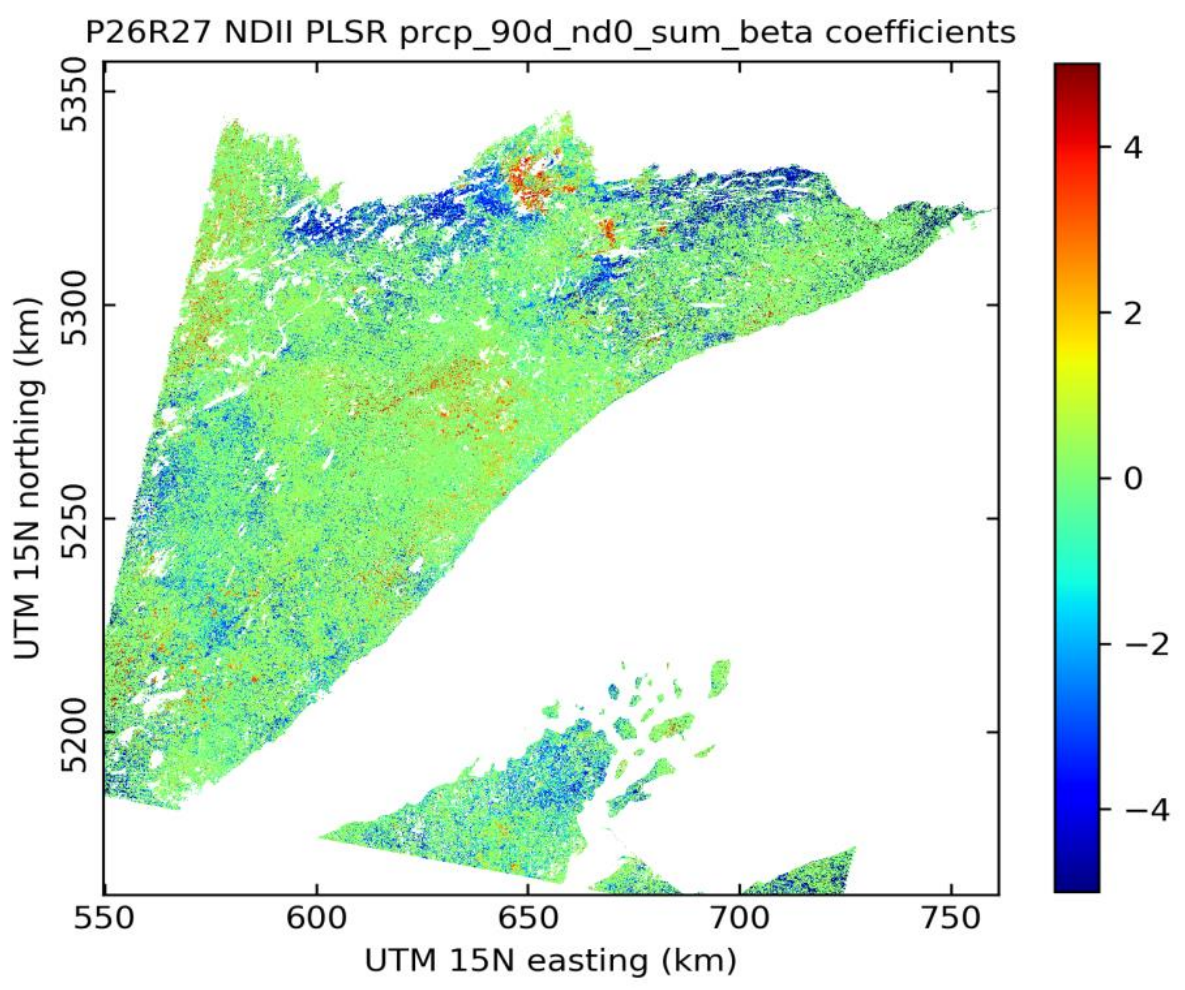

Figure 6.11: Example of standardized regression coefficients across the P26R27 footprint for the number of precipitation days within the 90 days prior to a Landsat scene ("prep_90d_nd0_sum" in Table 3.3) using the NDII-based PLSR model.

pattern is apparent in the $r^{2}$ results, with overall lower footprint-wide $r^{2}$ values for $E V I$, while $N D V I$ shows generally lower $r^{2}$ in the north and higher $r^{2}$ in the south.

To interpret the differences among models, I summarized the overall importance of each climatological variable to the final PLSR models across the footprint. For each pixel, the model provides a collection of climatological variables and their regression weights. From one pixel to another, the relative weights (standardized coefficients in the PLSM) for each variable can vary significantly, as shown for the number of precipitation days within the 90 days prior to a Landsat scene in Fig. 6.11. The order of variable rank is therefore not necessarily the best indicator of a variable's importance on the larger scale, but it is still useful here. Using these results, I tabulated the area (as a fraction of the overall footprint land area) covered by each climatological variable at each rank order of the absolute value of its standardized beta coefficient and plotted the relative coverage of the footprint at each rank, as shown for the NDII-based model in Fig. 6.12. For ease of interpretation, only those variables that cover more than 


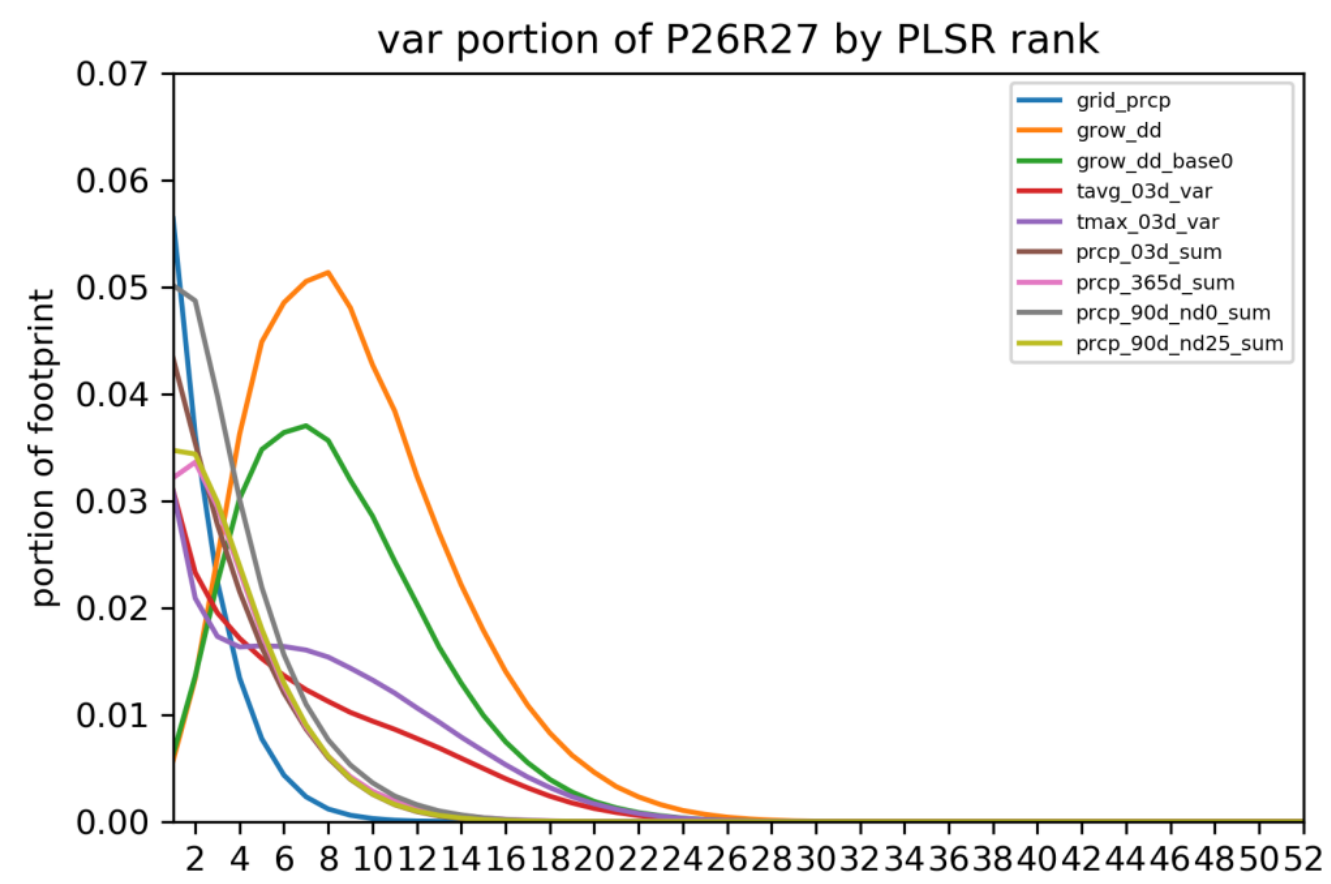

Figure 6.12: NDII-based PLSR results showing the area of footprint P26R27 covered by each climatological variable at each rank. For ease of interpretation, only those variables that cover more than $3 \%$ of the footprint at some rank are shown, with variable names as given in Tables 3.1, 3.3, and 3.5.

$3 \%$ of the footprint area at any rank are shown in that plot, but these areas are calculated for all of the climatological variables. I then calculated the area under this curve for each of the climatological variables and multiplied that result by the mean value of the standardized regression coefficient for that variable across the entire footprint at all ranks (e.g. Fig. 6.11). This resulted in an "effective" weight for each variable, which I then normalized across all variables to give a better sense of relative weights among all of the climatological variables (Figs. 6.13-6.15).

The general pattern of Fig. 6.12 is consistent across all three vegetation indexes, with 8-9 climatological variables covering $3 \%$ or more of the footprint area at some rank, though the actual variables themselves differ somewhat. In general, precipitation on the date of the Landsat scene ("grid_prcp" in Table 3.3) covers the largest portion of the top rank, but that coverage decays quickly with regression coefficient rank. The footprint portion covered by $G D D$, with either $T_{\text {base }}=5^{\circ} \mathrm{C}$ or $0^{\circ} \mathrm{C}$, generally grows until a peak at around 4-5\%, and then exhibits a longer decay with rank. By rank 30, 


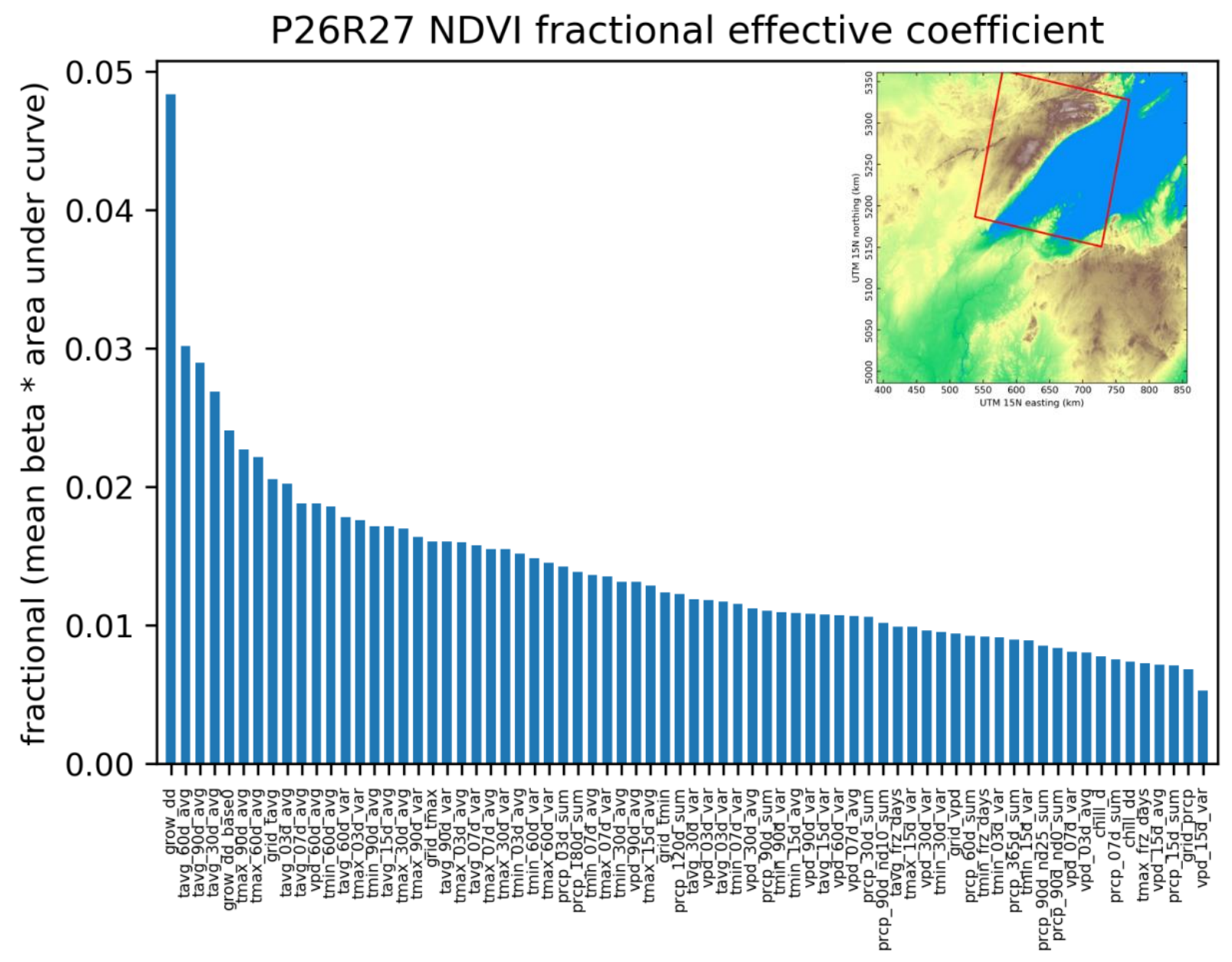

Figure 6.13: P26R27 PLSR overall effective coefficients using NDVI, with variable names as given in Tables 3.1, 3.3, and 3.5.

practically all variables have decayed to cover less than $1 \%$ of the footprint area, generally because the final PLSR model retains 30 or more variables at very few pixels in the footprint.

The average pixel, in footprint P26R27 and throughout the study area, retains only 15-20 of the initial 72 climatological variables provided to the PLS regression model process. Those variables that survive the iterative variable elimination process described in Chapter 3 are thus the indicators that may explain the larger portion of phenological variability over the footprint area. These most important indicators may be different among the three vegetation indexes used here at the footprint scale. For $N D V I$, the top climatological indicators are the accumulation of $G D D$ and the average values of $T_{a v g}$ over 60,90 , and 30 days prior to the Landsat scene date, in order of decreasing normalized effective regression 


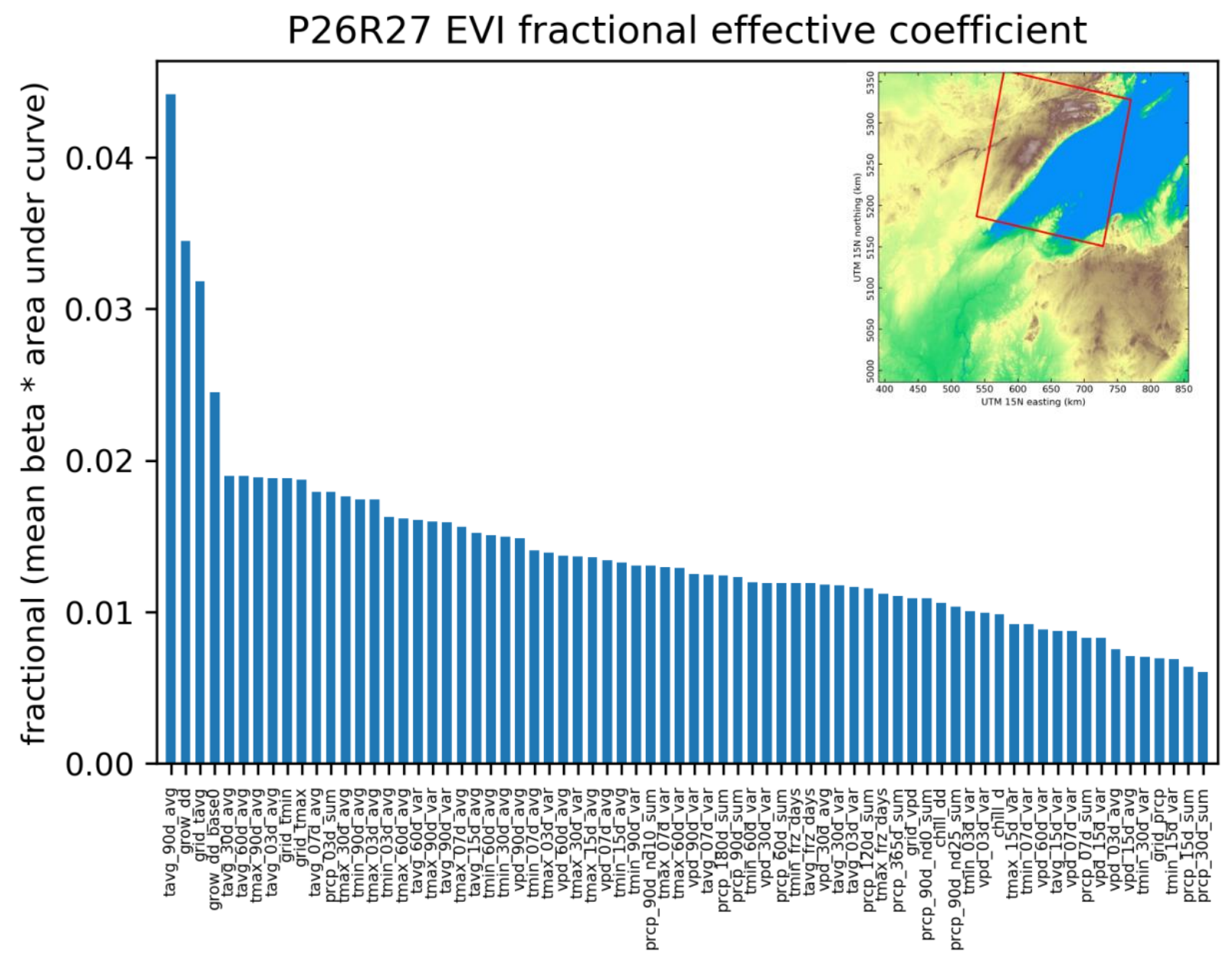

Figure 6.14: P26R27 PLSR overall effective coefficients using $E V I$, with variable names as given in Tables 3.1, 3.3, and 3.5.

coefficients (Fig. 6.13). For the EVI-based model (Fig. 6.14), the average values of $T_{\text {avg }}$ over 90 days prior to the Landsat scene date is the top indicator, followed in order by $G D D, T_{\text {avg }}$ on the day of the Landsat scene ("grid_tavg" in Table 3.1), and then $G D D$ with $T_{\text {base }}=0^{\circ} \mathrm{C}$. The $N D I I$-based model (Fig. 6.15) is dominated by $G D D$ as the top climatological indicator, followed by the average values of $T_{\text {avg }}$ over 90 and 60 days prior to the Landsat scene date and then $G D D$ with $T_{\text {base }}=0^{\circ} \mathrm{C}$.

Finally, I asked, how many variables would be needed to explain a given percentage of the observed phenological variability? The accumulation of these normalized ("effective") weights, in the same order of sorting (largest to smallest), for the NDII-based PLSR over the P26R27 footprint are shown in Fig. 6.16. To describe $100 \%$ of the phenological variability that can be explained using this method, which is approximately the overall model $r^{2}$ across the footprint (the remainder of the variability can 


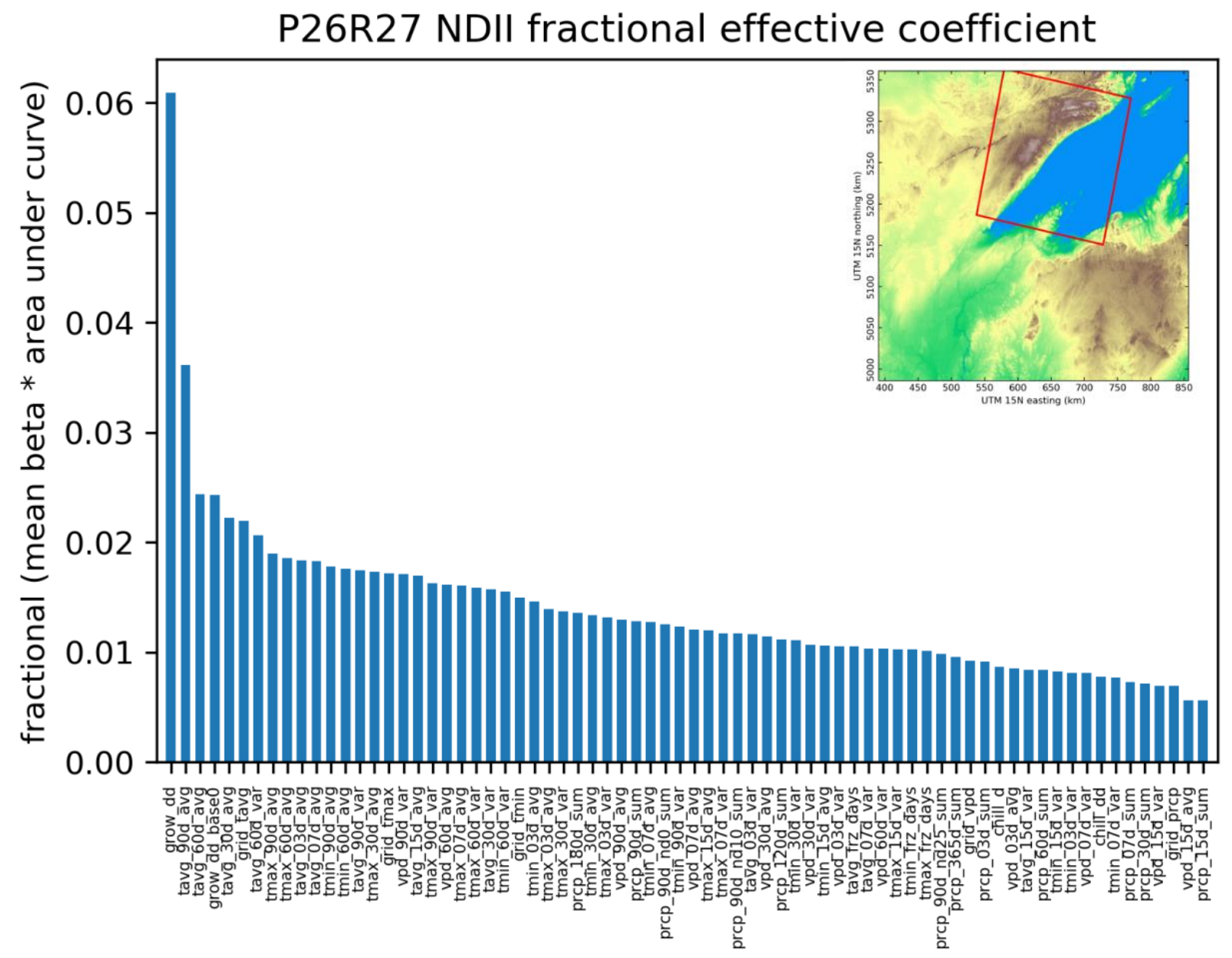

Figure 6.15: P26R27 PLSR overall effective coefficients using NDII, with variable names as given in Tables 3.1, 3.3, and 3.5.

likely be attributed to numerous factors, not least the biological processes inherent to the forest

phenology), would require all of the listed variables and potentially more that I have not considered here. However, fewer than half of the listed variables are needed to explain $50 \%$ of that variability.

Specifically, for the NDII-based model, only 24 of 72 variables $(33 \%)$ are needed to explain $50 \%$ of the phenological variability that is addressed with the PLSR process (Fig. 6.15).

\subsubsection{Phenological Prediction}

Error metrics for the full phenological model, which includes both the mean phenological curve and the climate-informed PLSR-based prediction of residuals, are shown for each of the VIs explored for footprint P26R27 in Fig. 6.17. These results can be compared with the error metrics for the mean 


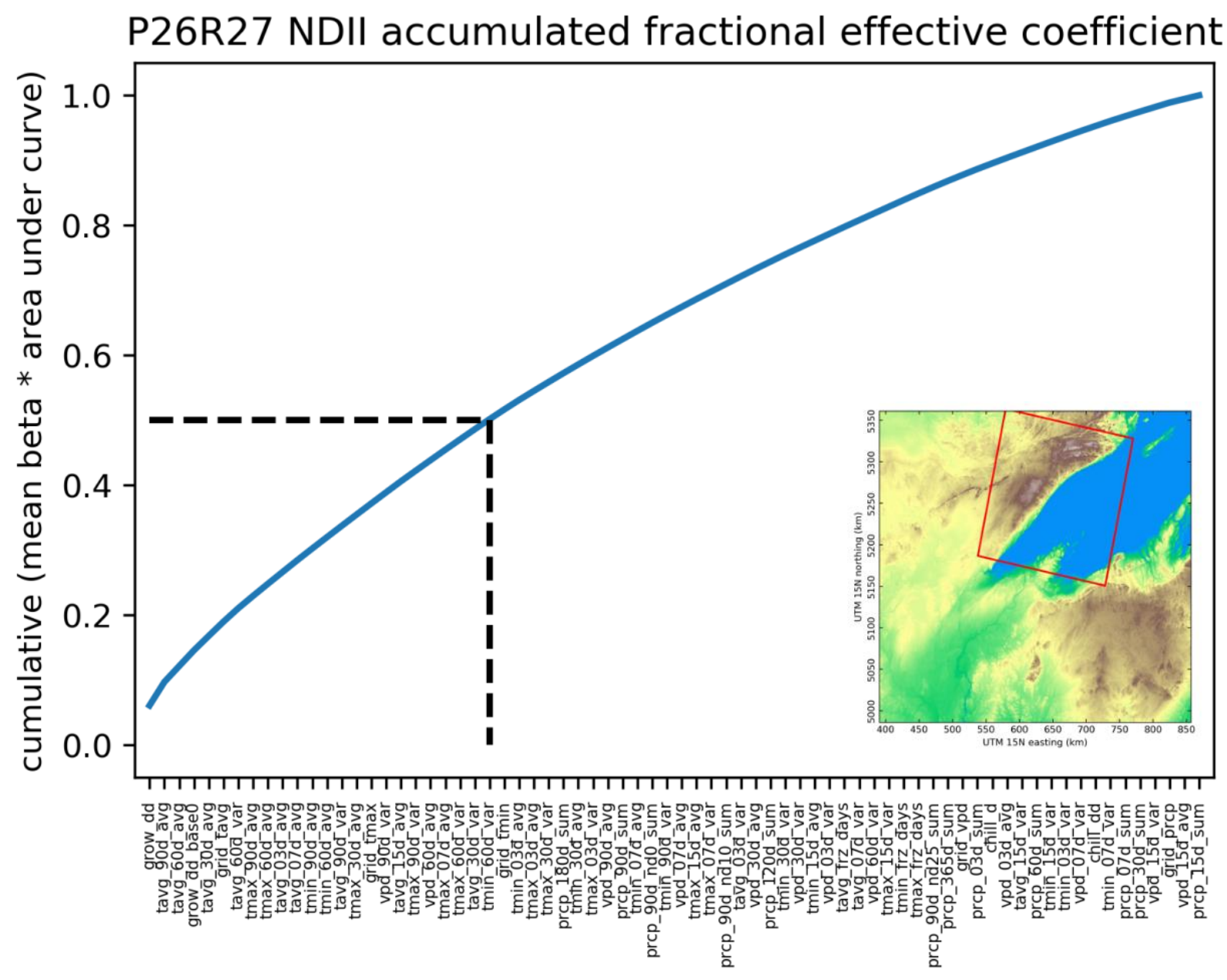

Figure 6.16: P26R27 PLSR accumulated normalized effective coefficients using NDII, with the variables required to achieve the $50 \%$ level indicated, and variable names as given in Tables 3.1, 3.3, and 3.5.

phenological curve alone that is shown in Fig. 6.1. The footprint-mean values for $R M S E$ and $r^{2}$ for both cases were summarized above in Table 6.1. If we considered only the mean phenological curve, EVI is clearly the best of the three vegetation indexes examined here. However, EVI is the vegetation index for which the goodness-of-fit $\left(r^{2}\right)$ improves the least $(+26.9 \%)$ when PLSR-based prediction of residuals is added to the overall modeling process, while the $r^{2}$ results for NDVI improve by $+50.5 \%$ and those for NDII improve by $52.8 \%$. In terms of RMSE, results for $N D V I$ show the least improvement, reducing the footprint-mean value by only $5.2 \%$, while the RMSE using EVI is reduced by $50.0 \%$ and using $N D I I$ is reduced by $51.6 \%$ with the addition of the PLSR-based component of the phenological model. Overall, these results suggest that NDII is more sensitive than NDVI and EVI to the climatological measures 
Full phenological model RMSE
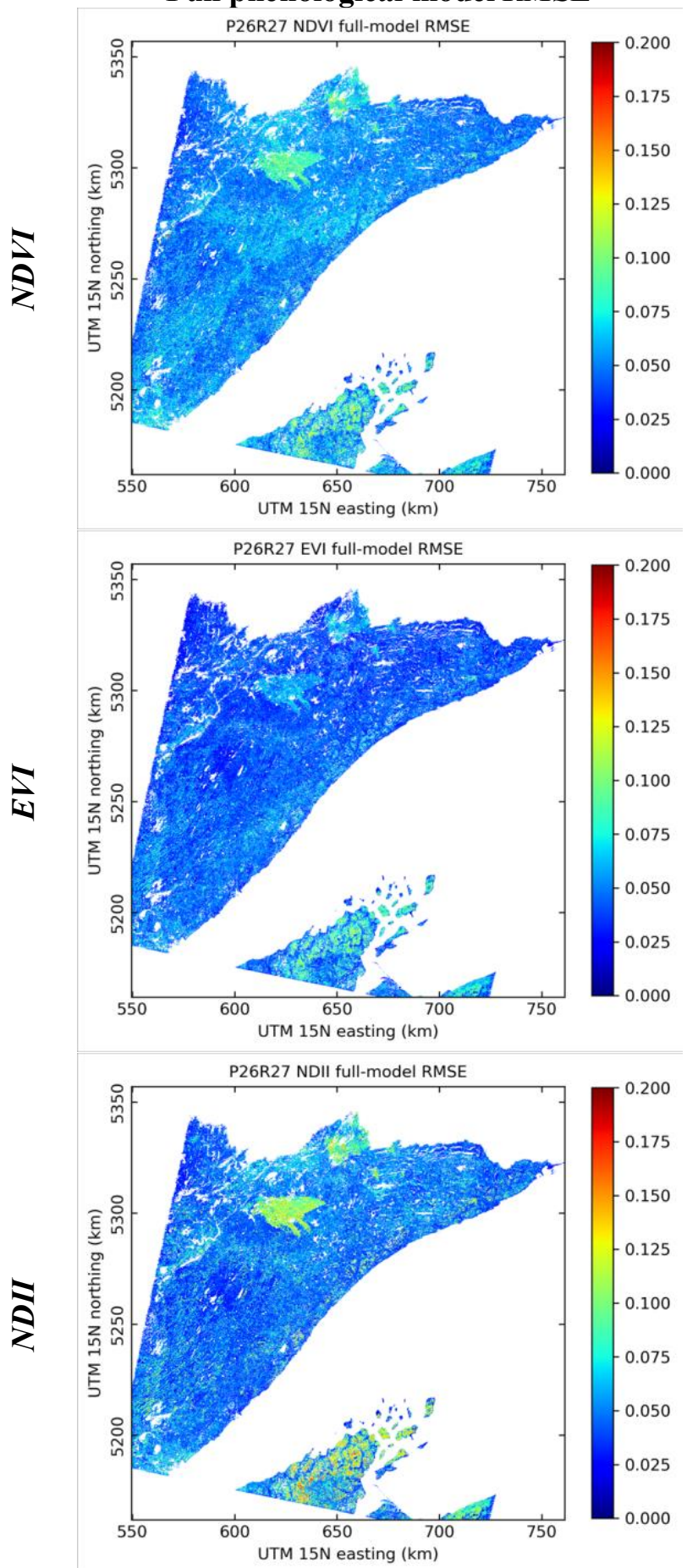

Full phenological model $r^{2}$
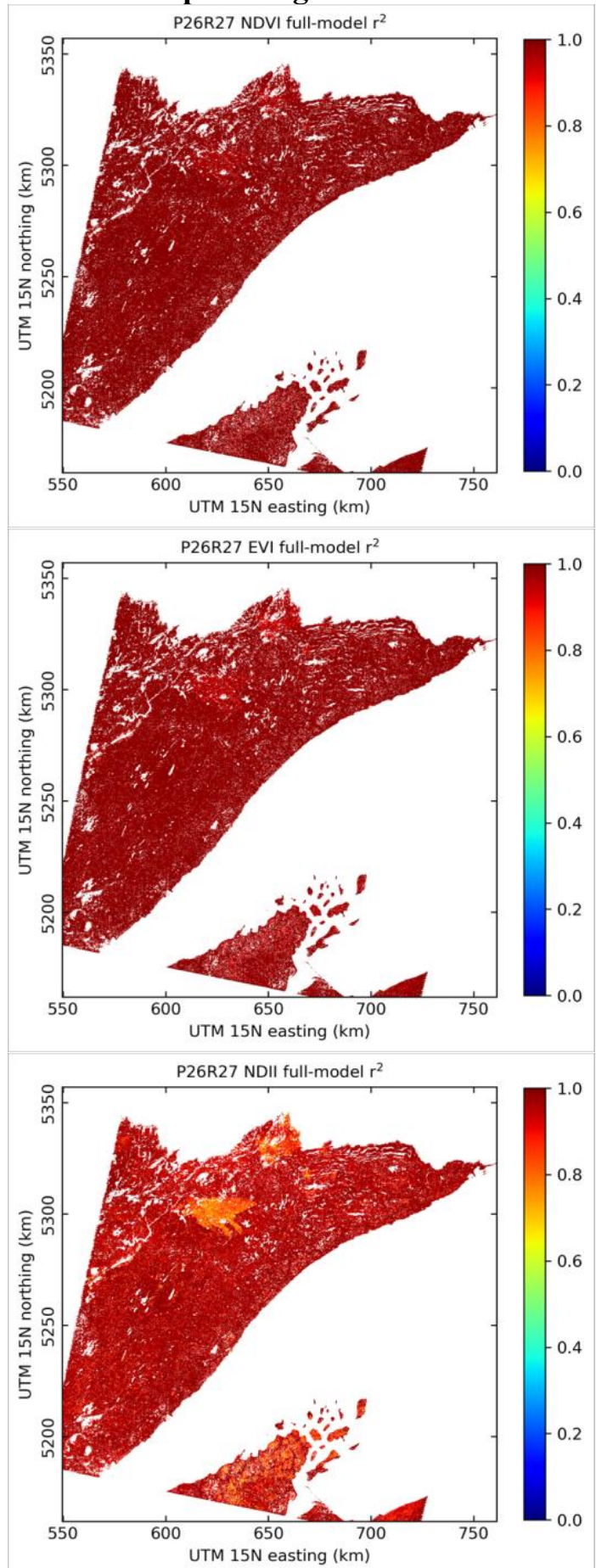

Figure 6.17: Comparison among full phenological model accuracy measures for NDVI, EVI, and NDII over the P26R27 footprint. The full model includes both the mean phenological curve and the PLSR-predicted residuals, with the combined results compared against observed VI values for the same location, as in Figs. 4.4 and 4.6. See also Figs. 6.1 and 6.10 and Table 6.1. 
introduced to the phenological prediction model by the PLSR-based process and may be the better choice of vegetation index for phenological analysis when environmental influences on year-to-year phenological variability are of great interest.

\subsection{Results at the Regional Scale using NDII}

\subsubsection{Mean Phenology}

Regional maps showing accuracy measures of the fitted mean phenological curve (RMSE in Fig. 6.18, $r^{2}$ in Fig. 6.19) for the NDII-based model show generally good results across the study area. There are some portions of the study area where the mean curve is less well-fit, especially in areas of known disturbance in far northeastern Minnesota, but also some areas in the Wisconsin Northwoods region and especially in the Wisconsin pine barrens (an area with extensive and regular harvest activities) and in lowland areas along the St. Louis River and where it meets Lake Superior. A number of wetlands areas in Minnesota (see Fig. 3.2) demonstrate low mean phenological curve goodness-of-fit values, a result that is different from other land cover types and that persists among the model results shown here.

Based on the fitted mean phenological curves, derived key phenological dates and NDII values are also mapped here. The date of Spring Inflection $(S I)$ is shown in Fig. 6.20, and the Duration of Spring (DOS) in Fig. 6.21. This is the duration of the rising limb of the phenological curve, which does not often conform to the period of astronomical spring between the Spring Equinox and Summer Solstice. The SI date occurs generally within a six-week window within that period, with earlier dates in the southern portions of the study area and later dates in northern and elevated areas. Again, the St. Louis River outlet plain is an anomaly, with generally later SI dates than its immediate surroundings. The duration of Spring is also shorter in that area and nearby areas of Minnesota to the northwest, but also in elevated areas of the Wisconsin Northwoods region. The differences between areas in Minnesota, generally northwest of Lake Superior, and areas in Wisconsin and Michigan that are southeast of Lake Superior become evident here in the duration of phenological Spring. However, the whole region evens out in Spring maximum 


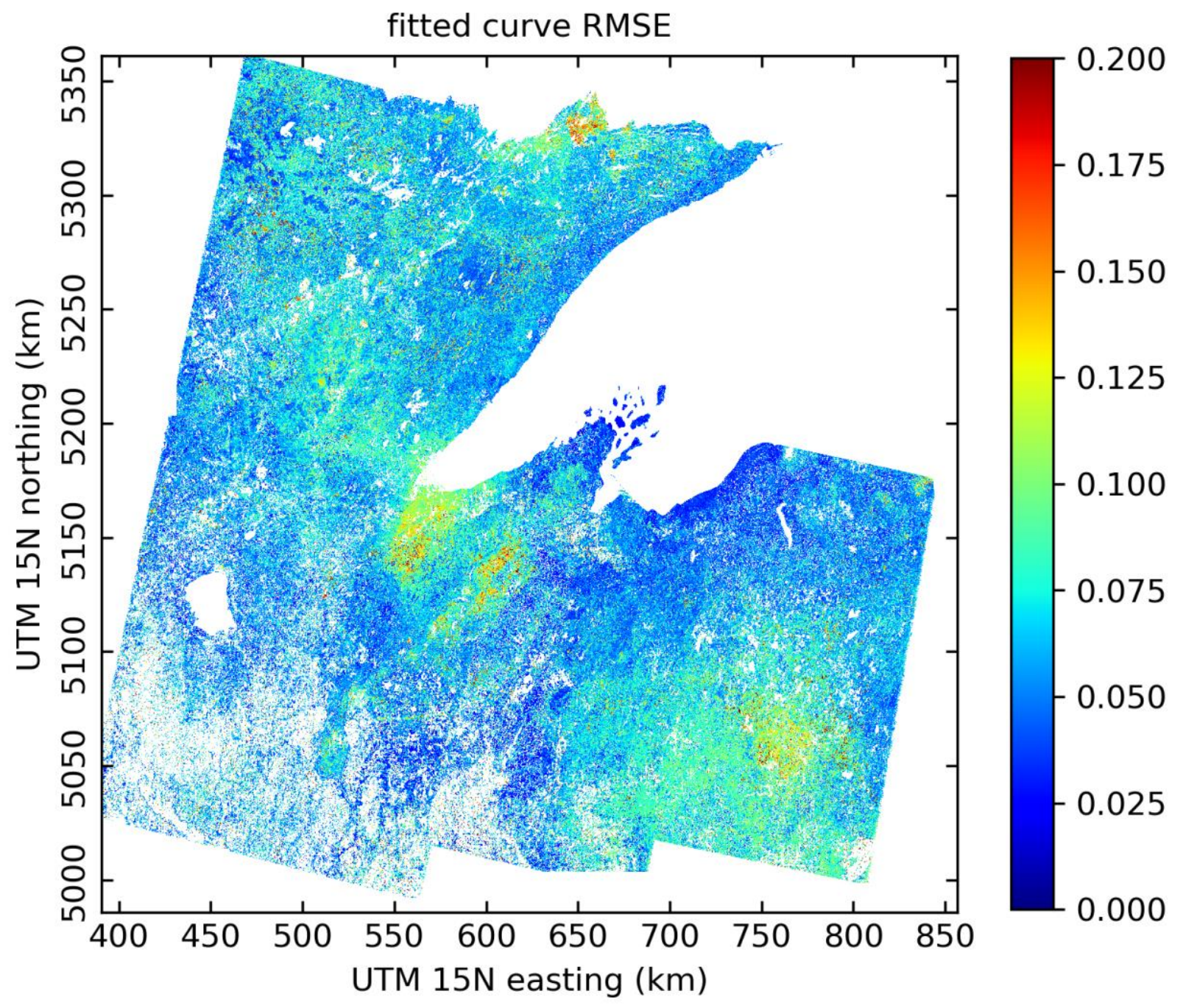

Figure 6.18: Regional mosaic of NDII-based mean phenological curve RMSE.

NDII values shown in Fig. 6.22, where the Minnesota wetlands and Wisconsin Northwoods regions are generally distinguished with well-mixed forests and thus peak NDII values, and the pine plantations in the Wisconsin pine barrens maintain generally low NDII values even at the height of Spring.

Where the phenological Spring is short, a compensating long summer mature period often occurs as shown in the map of the measured Duration of Maturity $(D O M)$ in Fig. 6.23. This leads to the downward break in the phenological curve at the time I have labeled the Autumn maximum, for which NDII values are shown in Fig. 6.24. The Autumn maximum NDII (just before senescence) is generally lower than the Spring maximum in NDII, leading to a slow green-down during the mature period, but this 


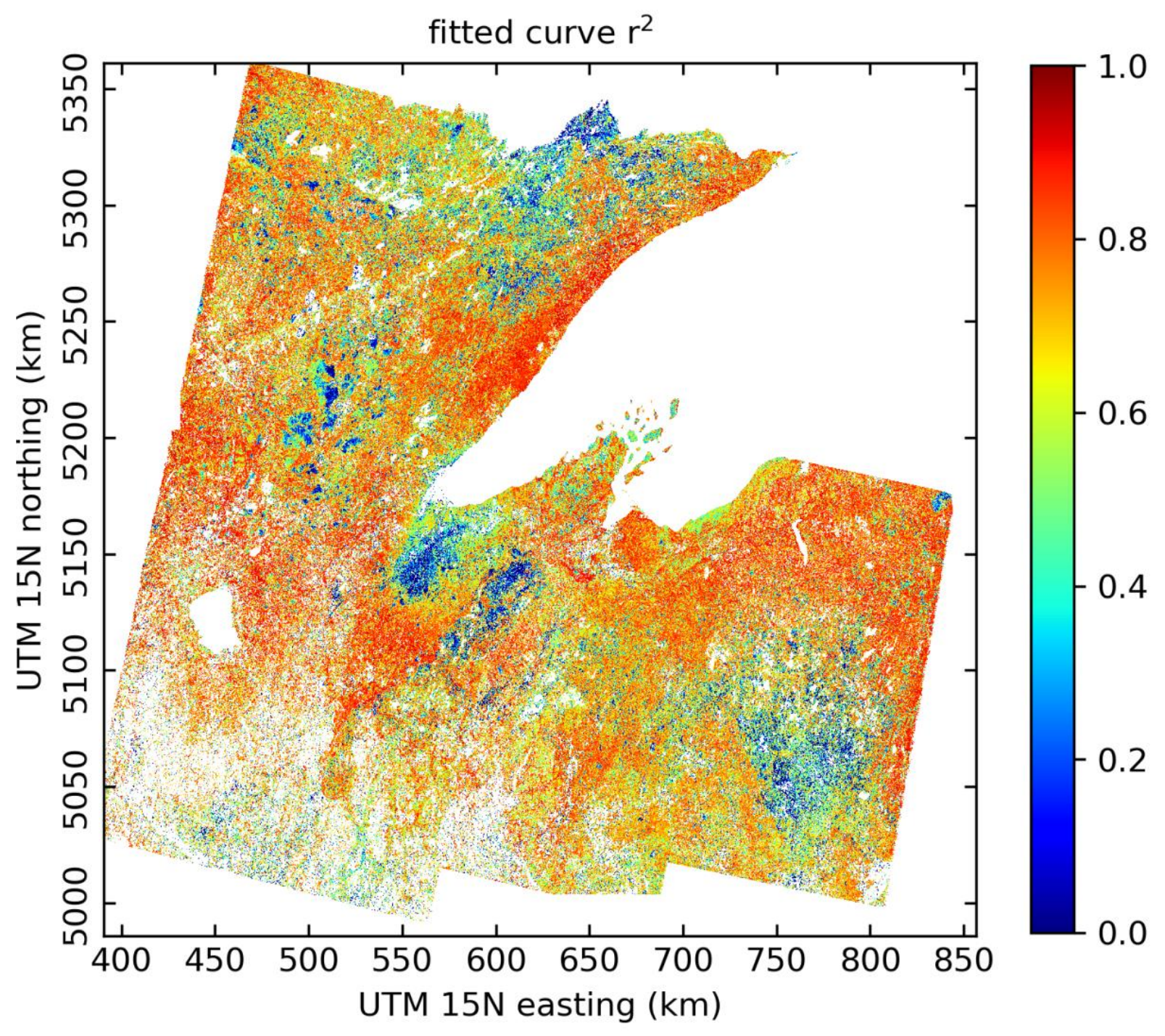

Figure 6.19: Regional mosaic of NDII-based mean phenological curve $r^{2}$.

is not as often the case in evergreen locations where $N D I I$ values may continue to rise until that Autumn break. The phenological fitted curve (Fig. 4.2) is designed to allow any such asymmetry in the seasons, with a forward (negative) or reverse (positive) slope to decreasing or increasing NDII values, respectively, in the mature period through the Summer.

The date of the Autumn Inflection (AI) is shown in Fig. 6.25. There is a better direct (positive) correlation between $A I$ and $D O S$, and a better inverse (negative) correlation between $A I$ and $D O M$, than between $A I$ and $S I$. Like $D O S$ and $D O M$, the $A I$ date shows a considerable correspondence with physiography (although there are no significant correlations with elevation, as discussed below). 


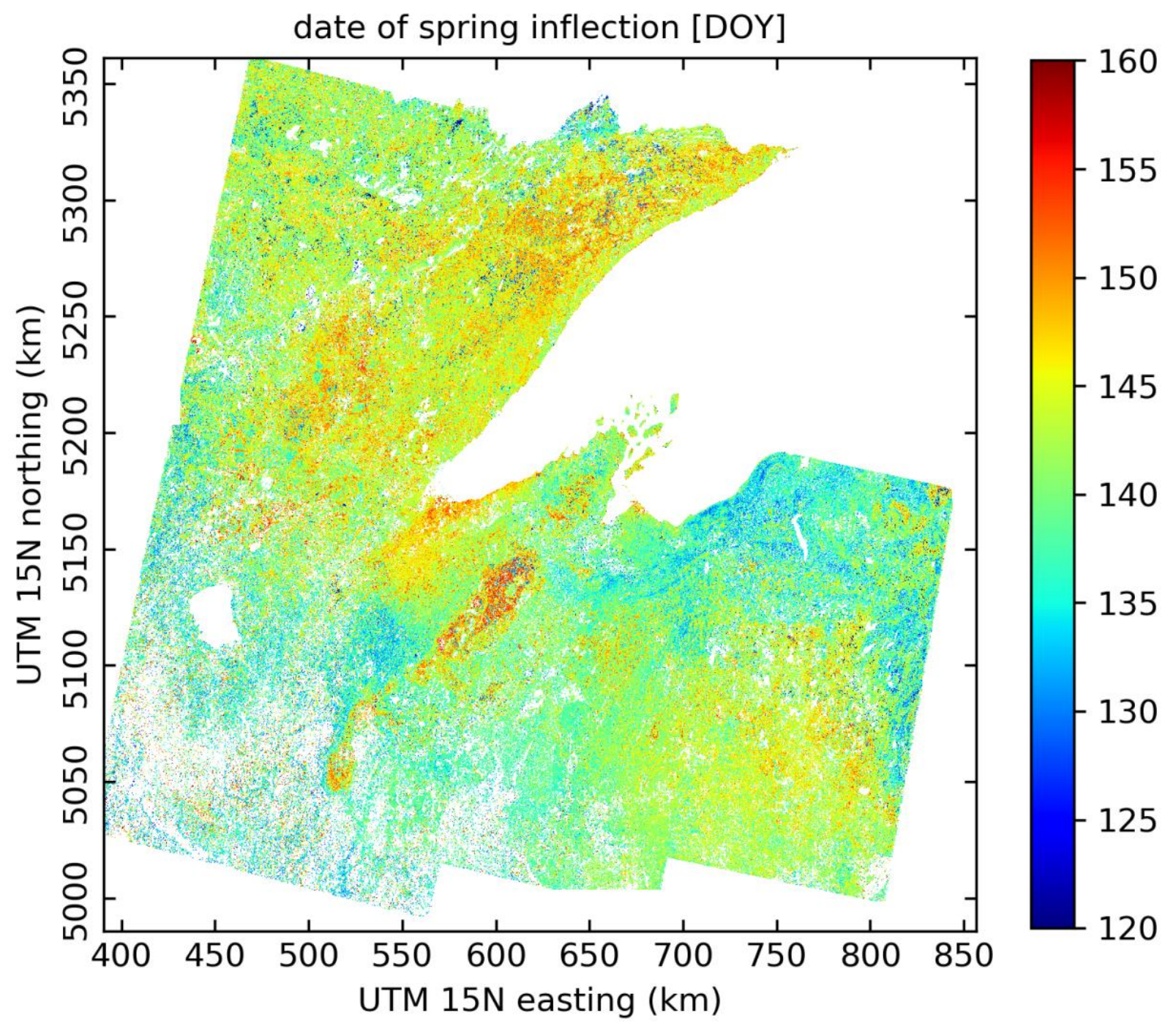

Figure 6.20: Regional mosaic of $N D I I$-based mean date of spring inflection (SI).

Comparing Fig. 6.25 with the topography in Fig. 3.1, the earliest $A I$ dates generally occur in broad valleys that have higher surrounding terrain on three sides, such as the St. Louis River lowlands at the edge of Lake Superior. Pooling cold air in these valleys, especially during more variable weather in the transition seasons, can hasten Autumn senescence in these locations. In at least the St. Louis River lowlands adjacent to Lake Superior, the same cold-air pooling (or even trapping in the Spring, as discussed below) may be responsible for overall later $S I$ dates there as well. This is reflected in the duration of the inflection-based growing season $(D[S I-A I])$ derived from the $N D I I$-based phenological curve that is shown in Fig. 6.26. 


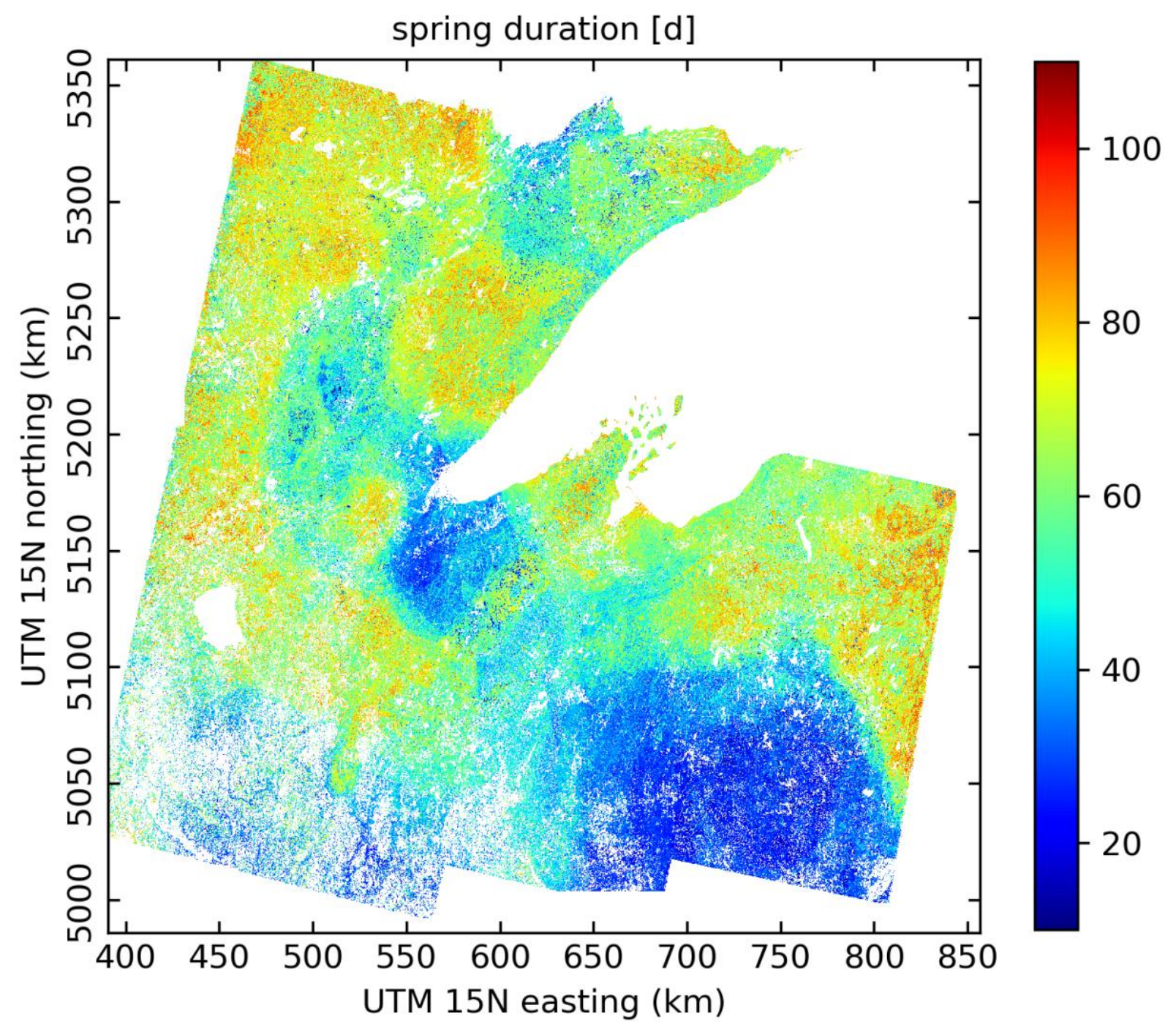

Figure 6.21: Regional mosaic of NDII-based mean duration of spring (DOS).

Of the many phenological parameters and indicators derived from the model that is described in Chapter 4, few depend more on the explicit assumptions of that model than the minimum NDII value. This model indicator is a direct result of the way that disturbed dates in a pixel time series are separated from the retained observations that are used to fit the mean phenological curve. Disturbances generally have the effect of lowering $N D I I$ values, and so the outlier sequestering process preferentially (though not explicitly) removes points below the NDII-based curve (Fig. 4.4). If fewer of those points were removed, the minimum $N D I I$ value would likely be lower than if more such points were sequestered from the time series. I have not tested the sensitivity of the sequestering criterion - that is an additional dimension of 


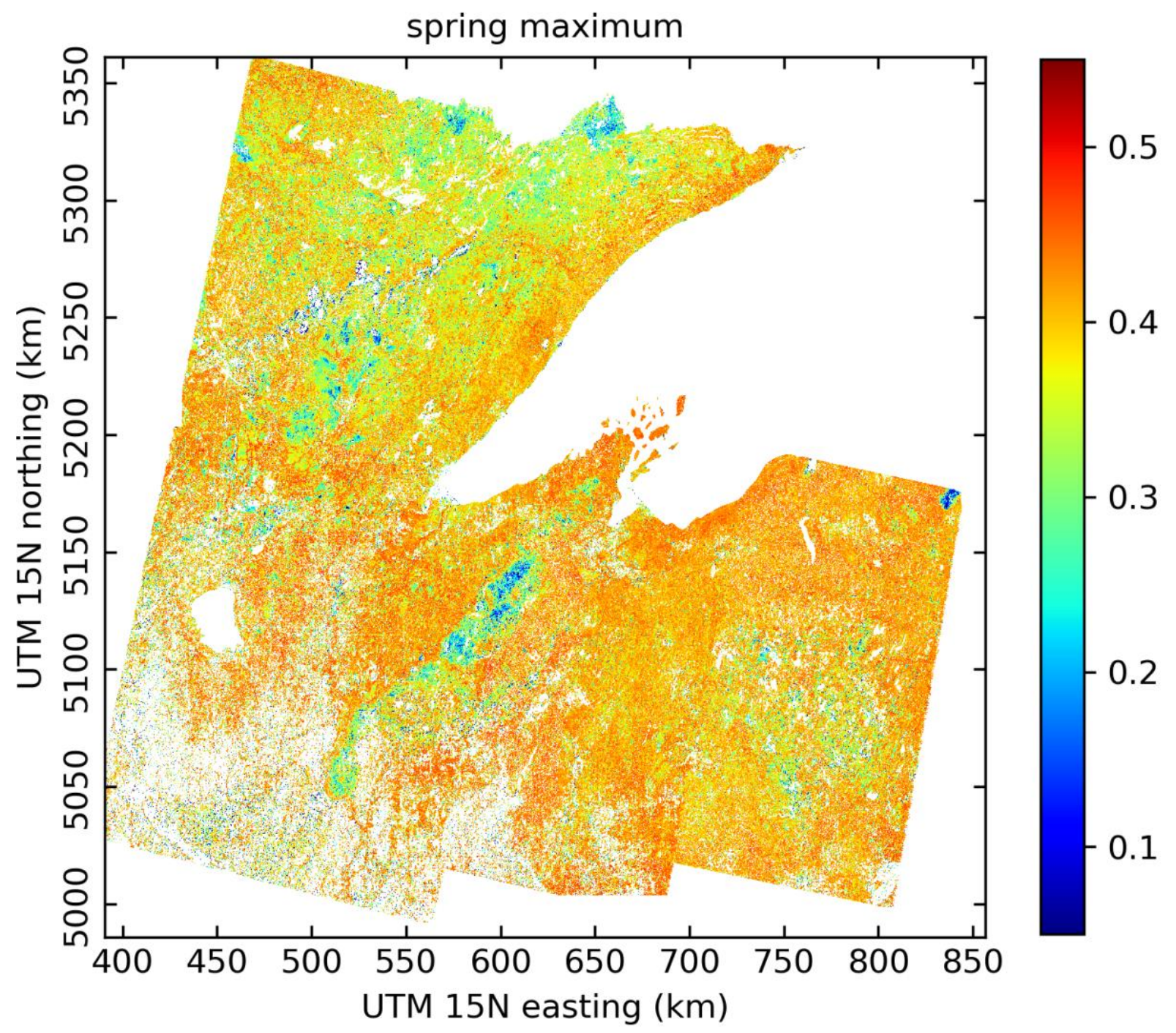

Figure 6.22: Regional mosaic of mean Spring NDII maxima.

variability beyond what could be accomplished in a reasonable time (see Section 4.5) — but I expect that a more liberal criterion would remove more points from the time series at each pixel and thus raise the minimum $N D I I$ value, yet the criterion is still applied without regard for location or other conditions, so the patterns in a regional map showing annual minimum NDII such as Fig. 6.27 should remain largely unchanged. The overall range of $N D I I$ values at a location, based on minimum $N D I I$ value and the larger of the $S V I_{\max }$ and $A V I_{\max }$ values, is shown in Fig. 6.28. These two maps correspond well with the maps of land cover shown in Fig. 3.2, indicating that minimum NDII values and the range of $N D I I$ values are 


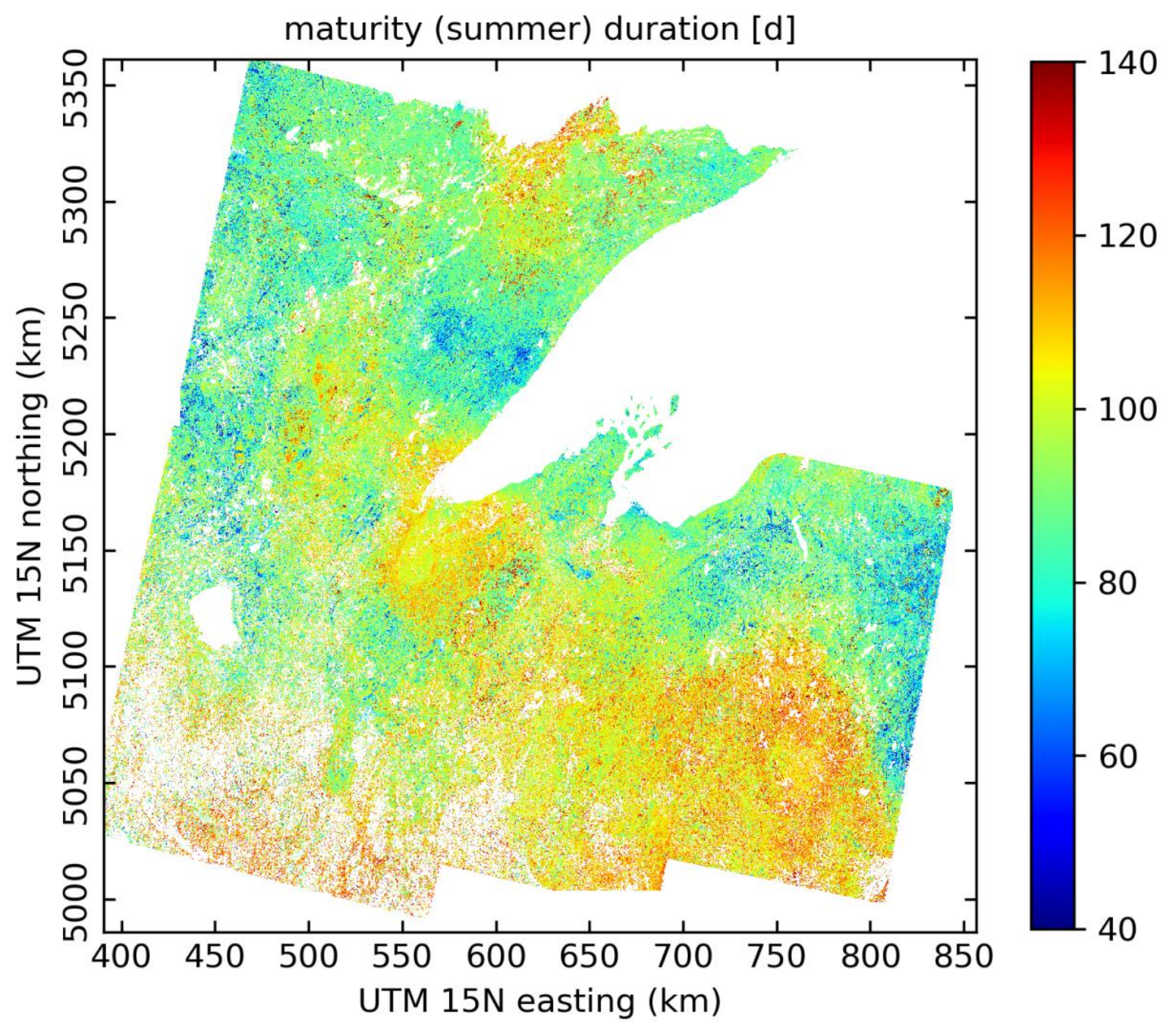

Figure 6.23: Regional mosaic of $N D I I$-based mean duration of maturity (DOM).

likely associated far more with vegetation type, and thus with various soil and nutrient conditions supporting that type of vegetation, than with other environmental (i.e. climatological) conditions.

Finally, as an exploratory indicator that could be useful in describing the entirely of the phenological growing season including both duration and dynamic range, the total area $(T A)$ under the NDII-based phenological curve is shown in Fig. 6.29. This integrative indicator combines the vegetation-type-oriented minimum $N D I I$ value and range of $N D I I$ values with the physiologically-based and climatologicallyoriented sharpness of the Spring green-up period ( $D O S$ and $\left.S V I_{\max }\right)$, the duration of the mature period $(D O M)$ and overall duration of the growing season $(D[S I-A I])$, and the sharpness of the Autumn break 


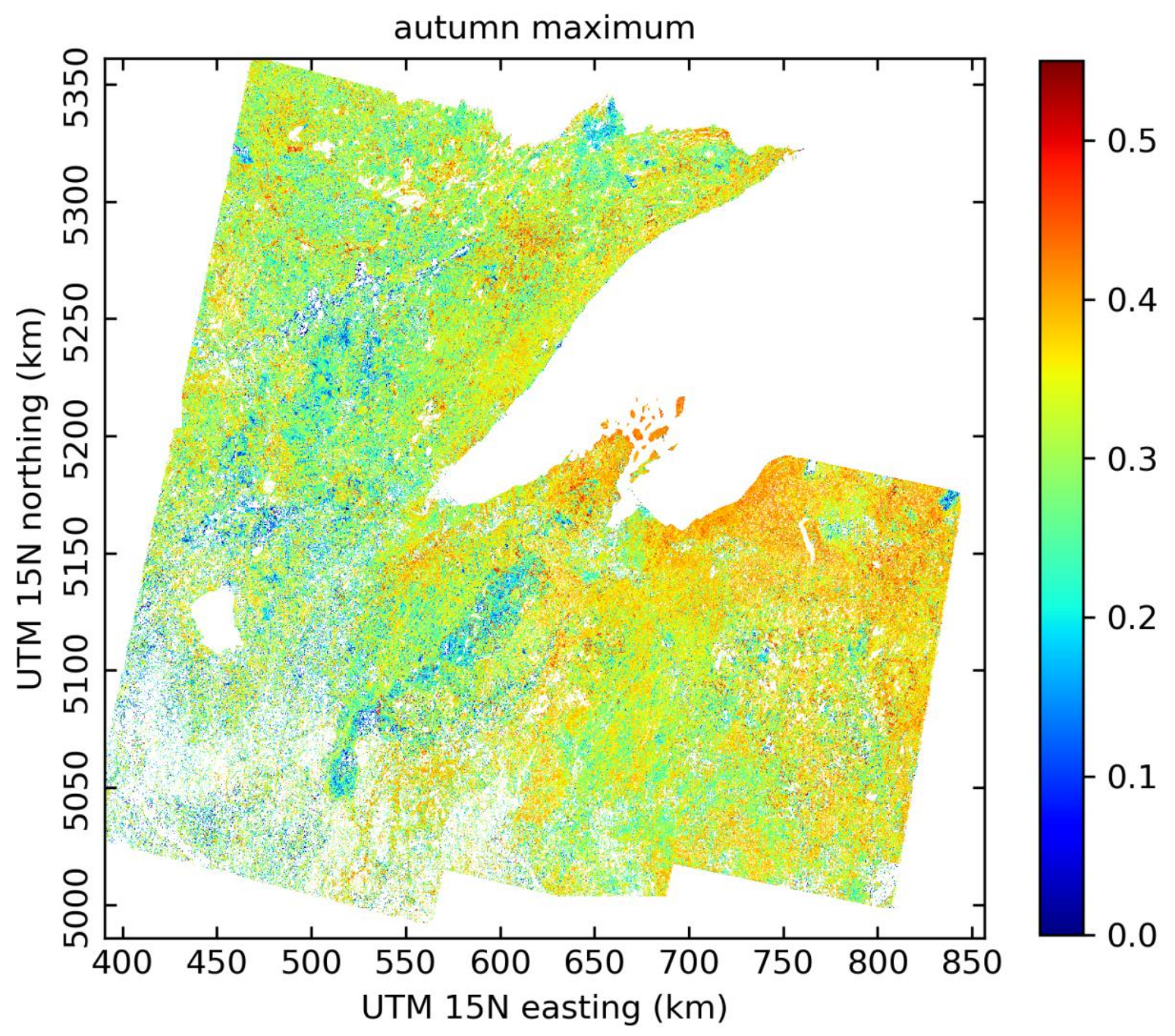

Figure 6.24: Regional mosaic of mean Autumn NDII maxima.

and senescence period $\left(A V I_{\max }\right.$ and $\left.D O A\right)$. As with any synthesized indicator that combines potentially compensating components, for example the trade-off between Spring and mature durations in many areas, there may be locations with similar $T A$ values but very different underlying conditions and other seasonal indicators. Researchers have attempted to link the growing season duration with ecosystem productivity (carbon assimilation) [White et al., 1999; White and Nemani, 2003; Desai, 2010; Fu et al., 2017], with mixed results. I suggest that the $T A$, with its incorporation of both growing season duration and VI range, may be a more accurate indicator of the overall vegetation productivity through the growing season. If the 


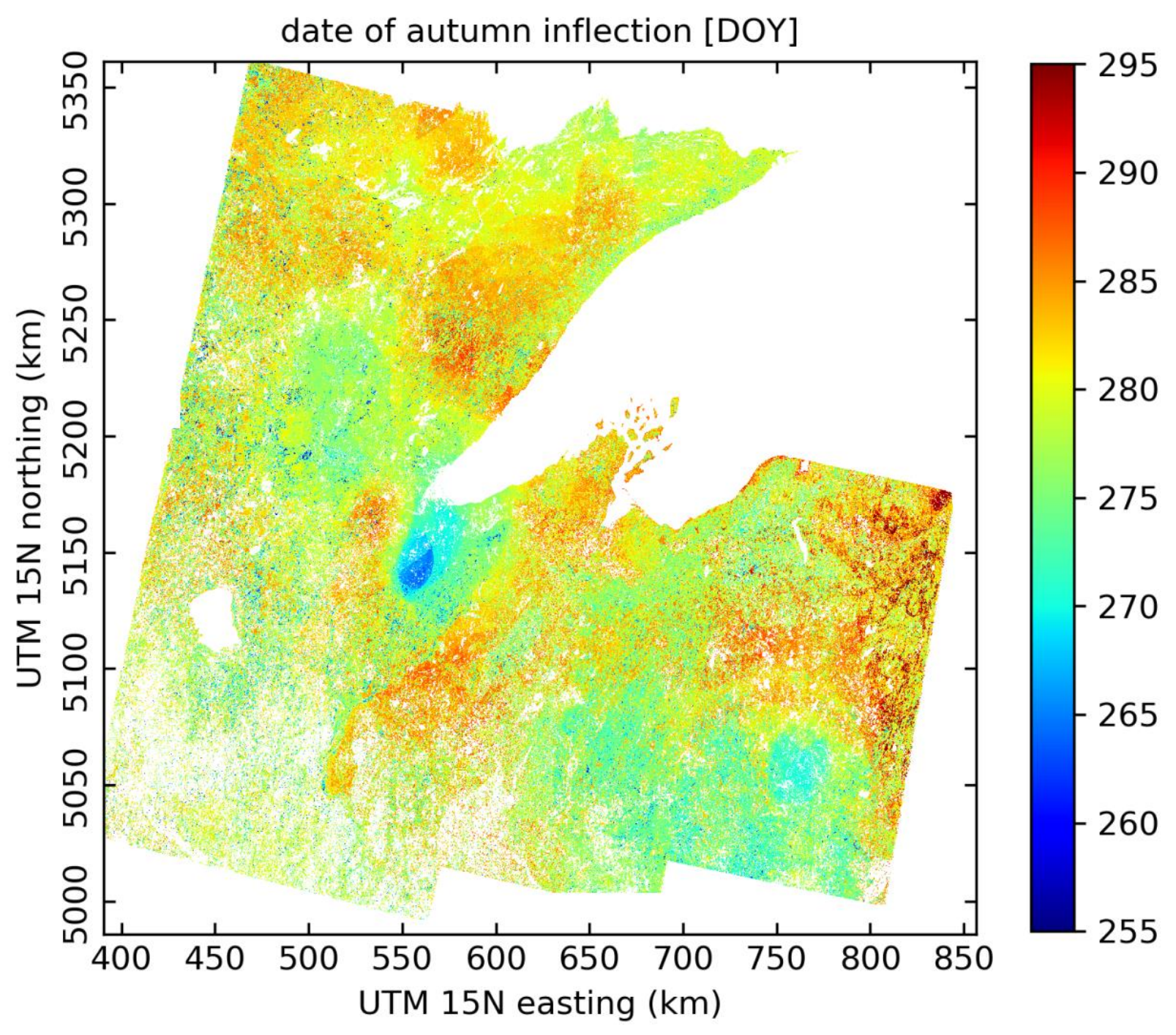

Figure 6.25: Regional mosaic of $N D I I$-based mean date of autumn inflection $(A I)$.

$T A$ can be related to seasonal carbon uptake, that indicator could be a useful way to describe the geographic variability of mixed forest regions in carbon cycle models.

\subsubsection{Mosaic Accuracy}

The maps shown in Figs. 6.18-6.29 were constructed from results on the scale of individual footprints, as shown for P26R27 in Figs. 6.1-6.3. The "best" mosaicking method, i.e. the method that seemed to produce the least-apparent image artifacts at individual footprint edges, was simply to average the available values at an overlap location (pixel). For most variables, this produced nearly seamless 


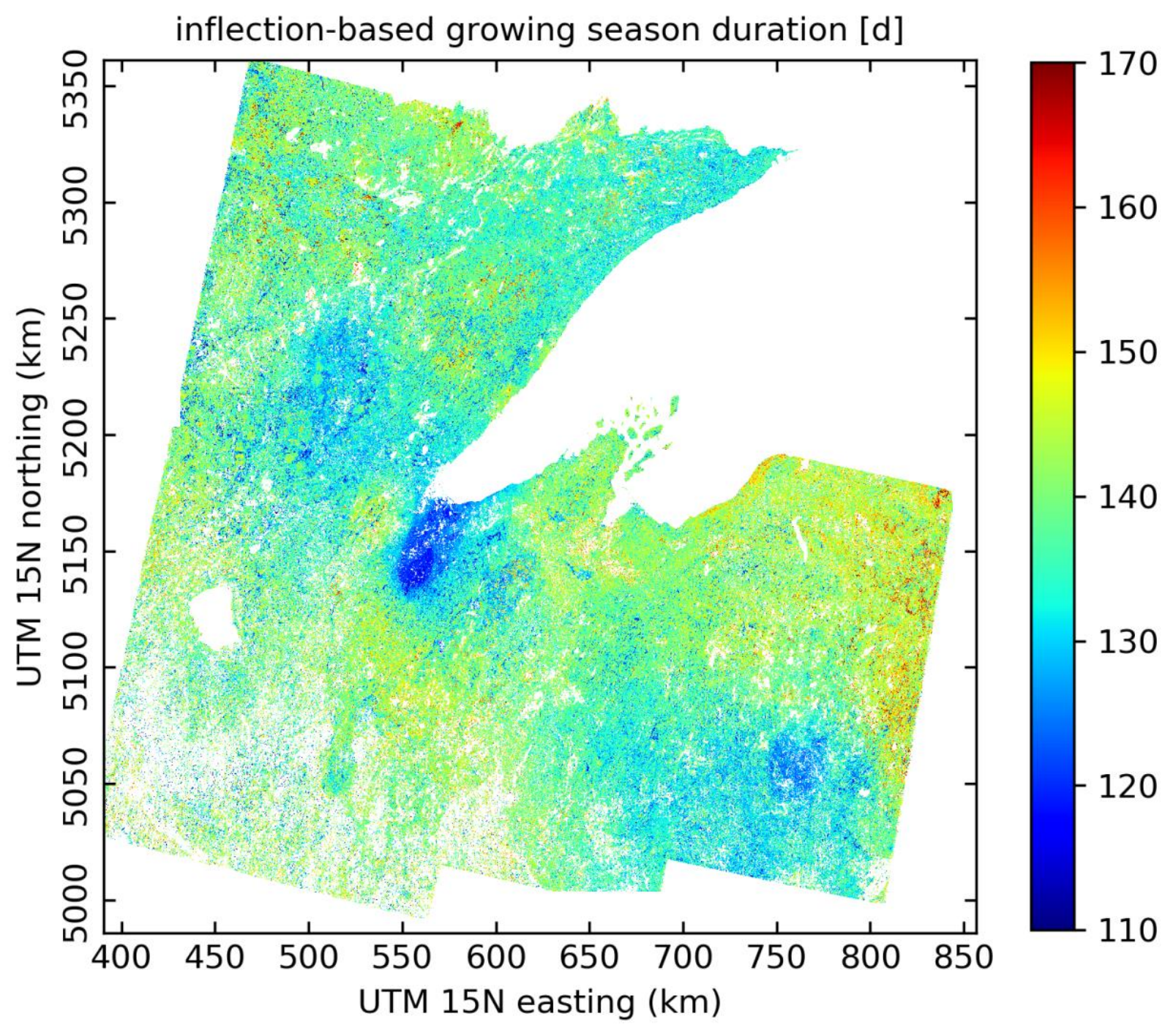

Figure 6.26: Regional mosaic of NDII-based mean growing season (D/SI-AII).

regional maps of phenological indicators, and it is these mosaic maps that are compared with regional climatological indicators below. For a sense of the suitability and accuracy of this averaging approach, root-mean-squared-difference $(R M S D)$ and correlation $\left(r^{2}\right)$ statistics for the basic parameters of the fitted phenological curve in each area of footprint overlap (see Fig. 3.1) are listed in Table 6.3. Heat maps and regressions showing regional overlap agreement at key phenological dates ( $S I$ and $A I$ ) and their VI values $\left(V I_{S I}\right.$ and $\left.V I_{A I}\right)$ are shown in Fig. 6.30. Not all parameters compare well in the footprint overlap areas, but some of the best overall comparisons are those in locations where two different Landsat paths overlap. In those locations, because of Landsat's 16-day repeat time and with two sensors in orbit through much of 


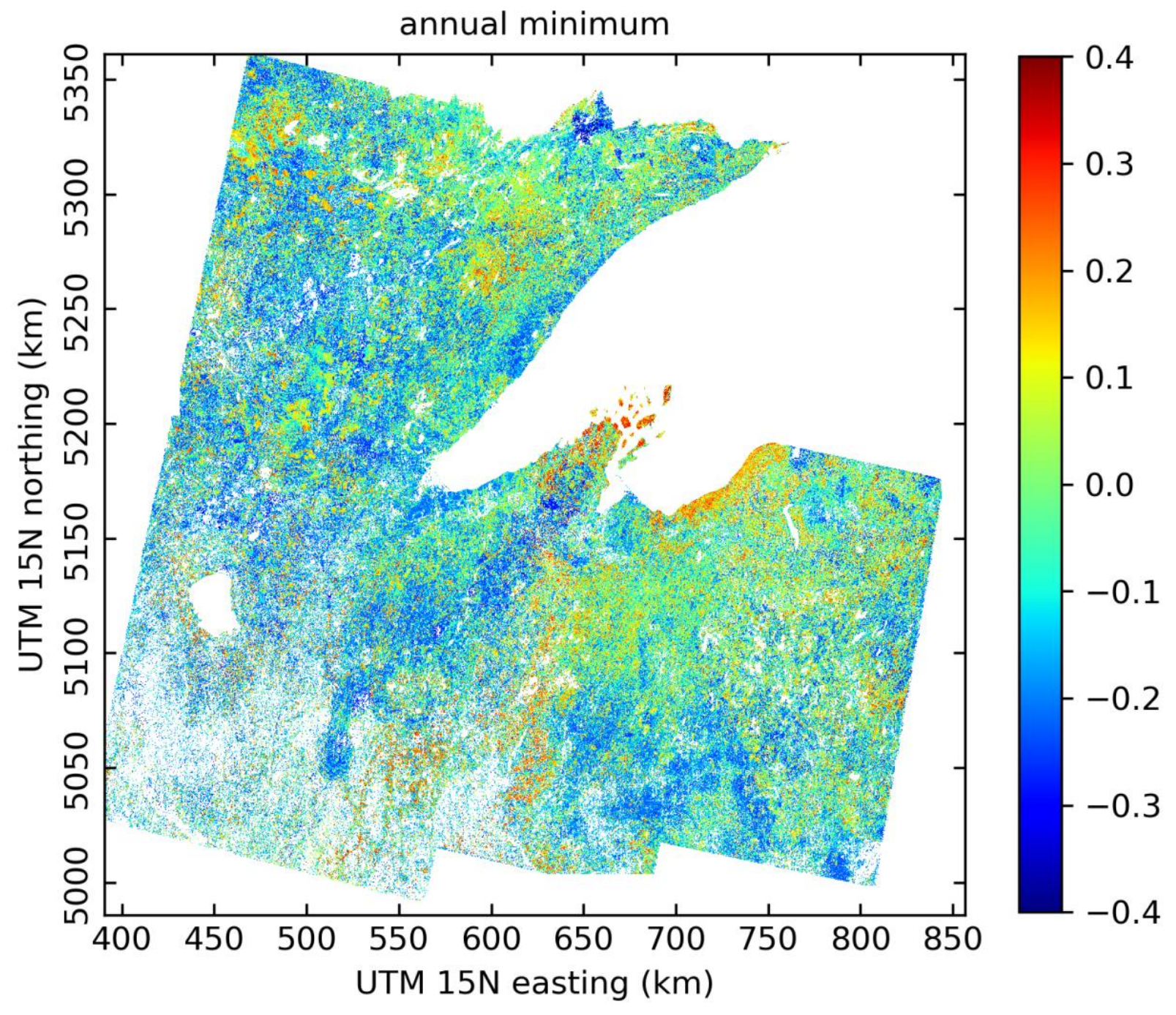

Figure 6.27: Regional mosaic of mean phenological NDII minima.

my study period, the closest in time that two adjacent scenes can be is 2-9 days. The parameters of the phenological fitted curves in adjacent footprints (Landsat paths) are thus derived from mutually exclusive time series, and yet their results generally agree at a high level of correlation. As will be discussed in Chapter 7, in future work I should combine overlapping footprints to produce harmonized time series on a pixel-by-pixel basis before the phenological model is applied, potentially leading to greater levels of accuracy in derived phenological indicators. 


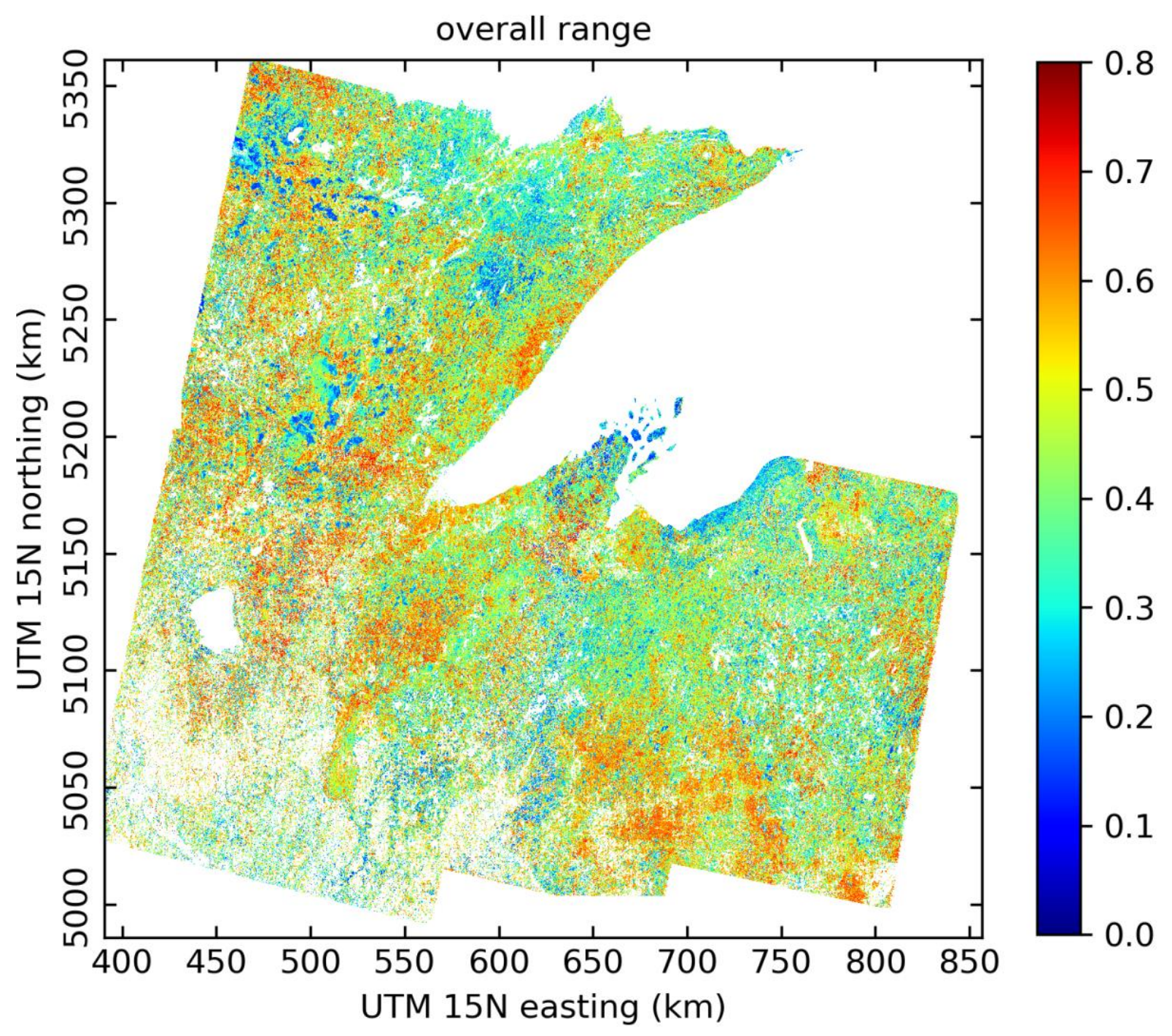

Figure 6.28: Regional mosaic of mean phenological NDII range.

\subsubsection{Spatial Patterns and Lake Effects}

A number of spatial patterns in the mean phenology result for my study area have been mentioned above. The phenological curve goodness-of-fit is generally high except in areas of known disturbances (e.g. harvest, fire, insect outbreaks) and very short growing seasons such as the outlet of the St. Louis River and in numerous wetland locations in eastern Minnesota. Topographic relief is strongly evident in the durations of Spring and Maturity, but in opposite patterns between northeastern Minnesota and northeastern Wisconsin. Spring and Autumn maximum NDII values are highest in areas of primarily deciduous forest and lowest in evergreen forest, while the opposite is generally the case for annual 


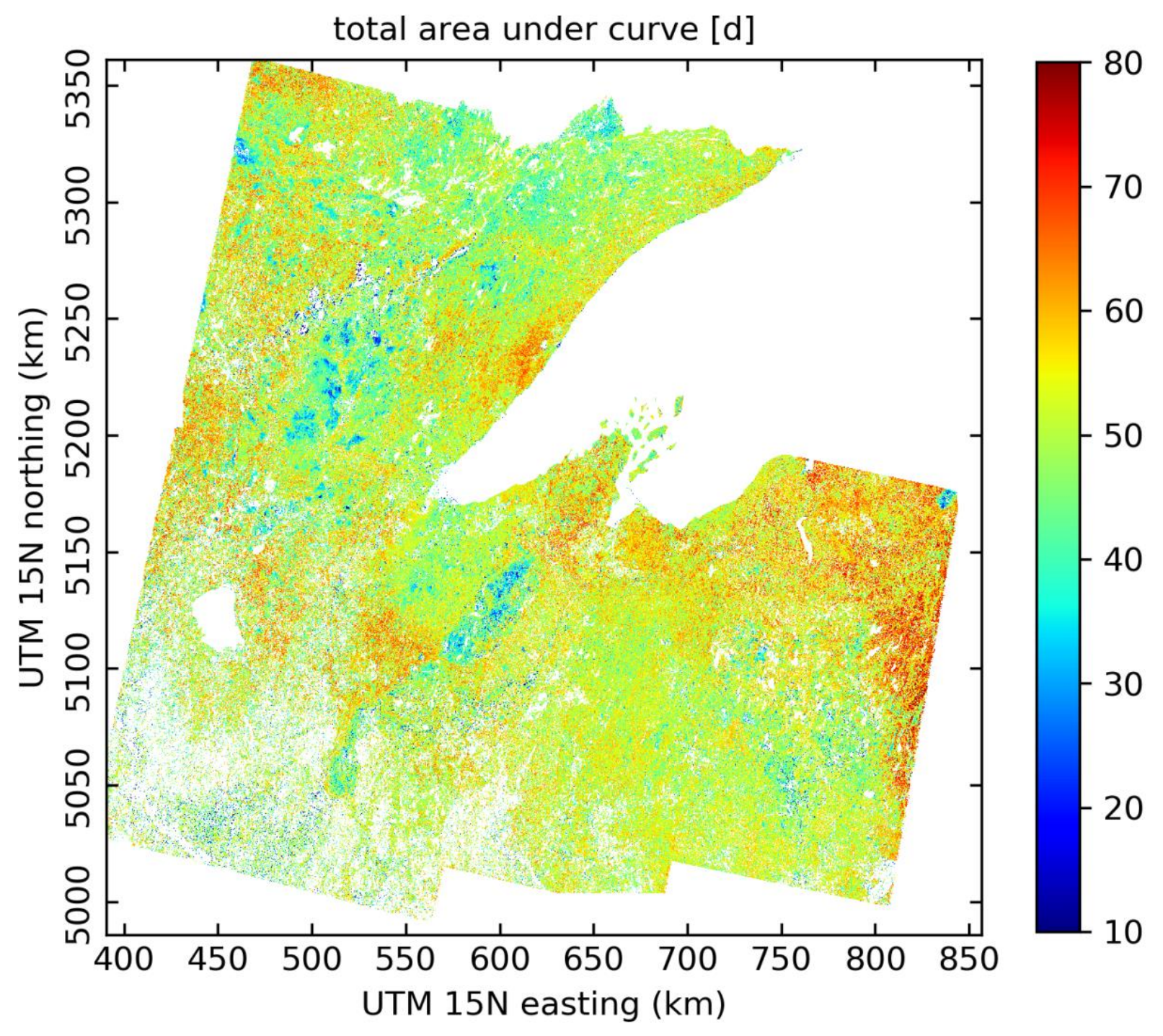

Figure 6.29: Regional mosaic of $N D I I$-based mean total area under the fitted curve (TA).

minimum NDII values, such that the overall annual range of $N D I I$ is greatest in deciduous forest and least in evergreen forest. Several effects of Lake Superior on the surrounding land areas were discussed at length in Chapter 5, and several of these interactions are reflected in phenological patterns around the Lake as well. The short growing season in the St. Louis River lowlands has been mentioned above. At least in the Spring, the delayed date of green-up (as compared with immediately surrounding areas) may be due to the pooling of cold air flowing inland from an often-frozen (or otherwise winter-chilled) Lake 
(a)

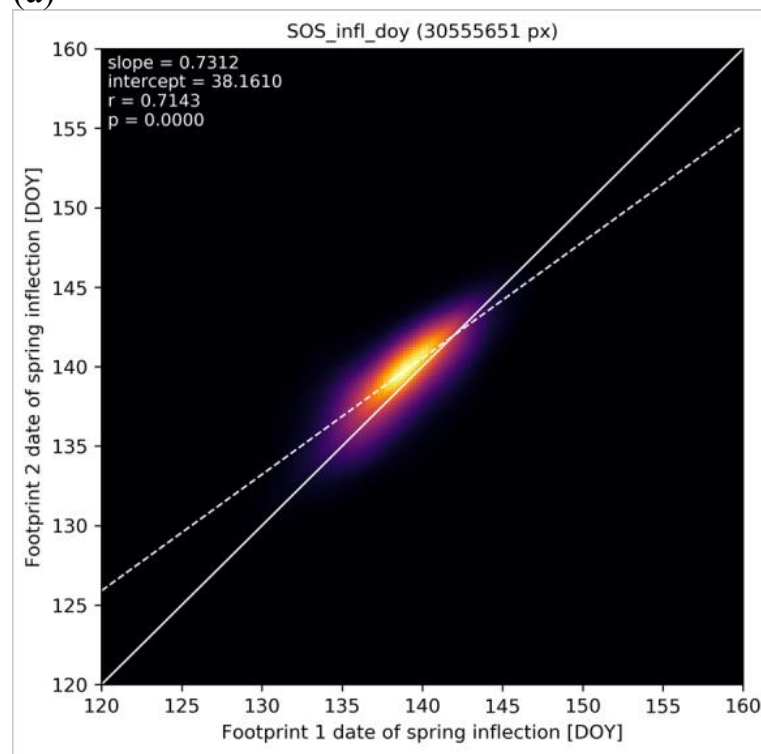

(c)

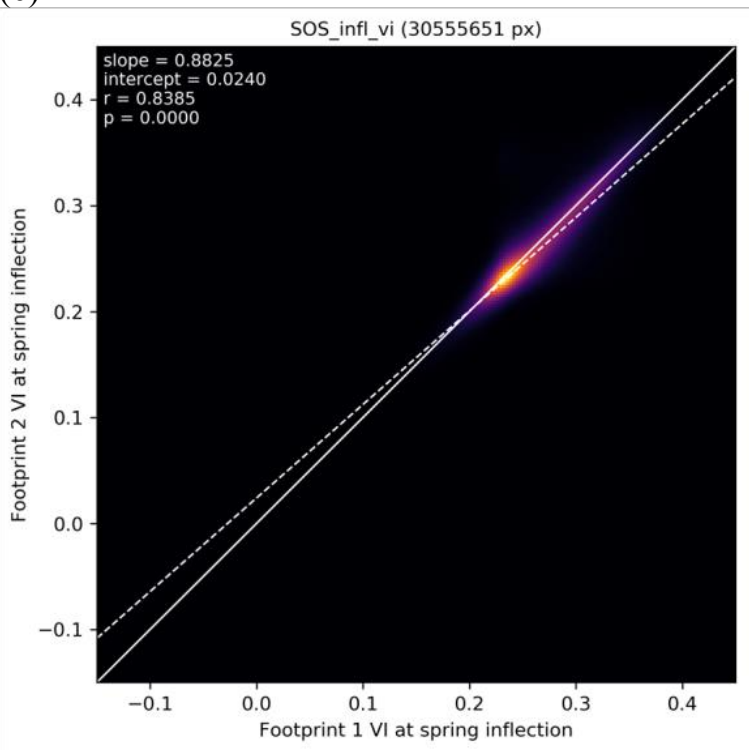

(b)

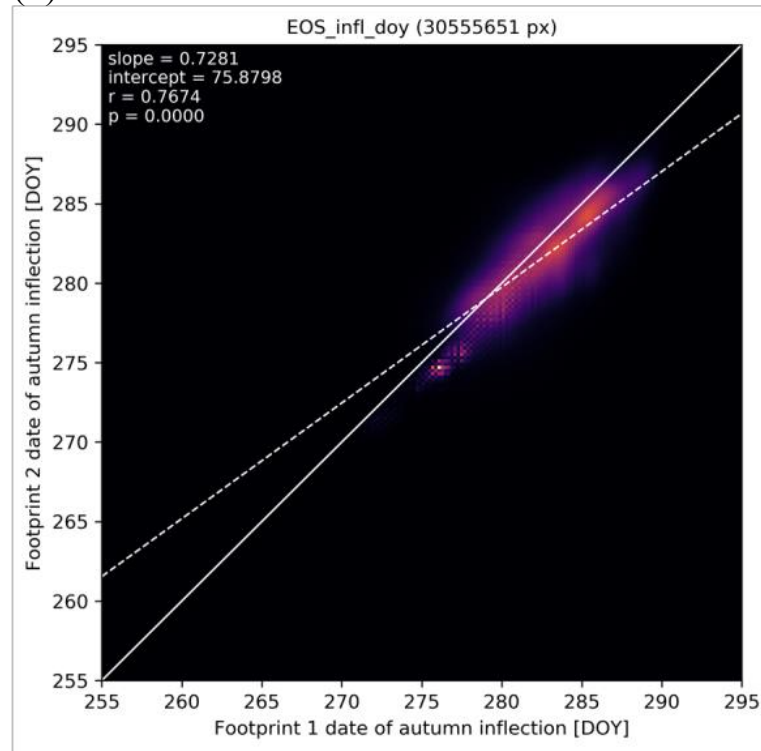

(d)

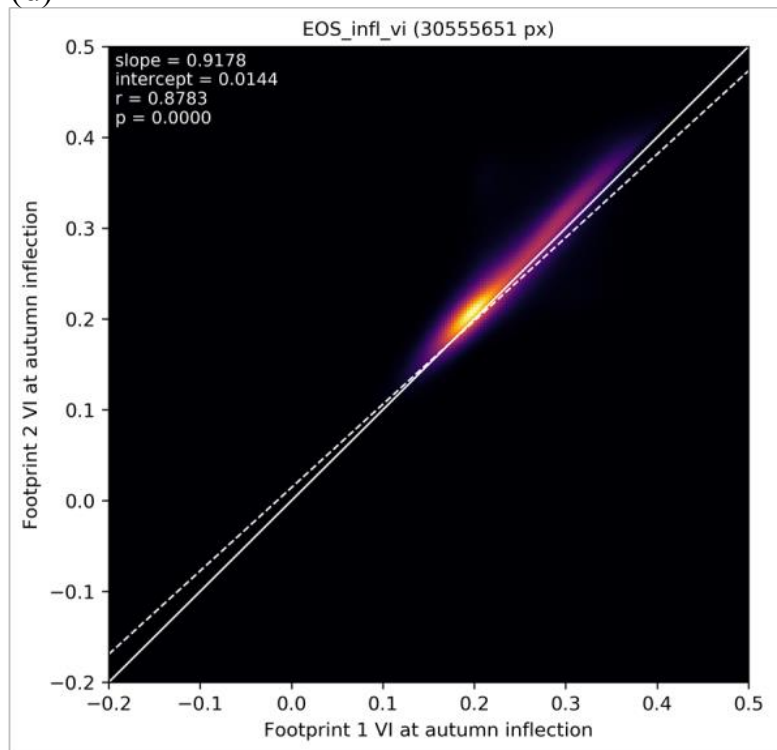

Figure 6.30: Comparison of fitted mean phenology curve key dates and NDII values in areas of footprint overlaps: (a) Spring Inflection DOY (SI, see Fig. 6.20); (b) Autumn Inflection DOY (AI, see Fig. 6.25); (c) $N D I I$ at SI; (d) NDII at $A I$. Linear regressions are shown as dashed lines, to be compared with the 1:1 (solid) lines. Mosaic overlap accuracy details for other phenological indicators are given in Table 6.3. 


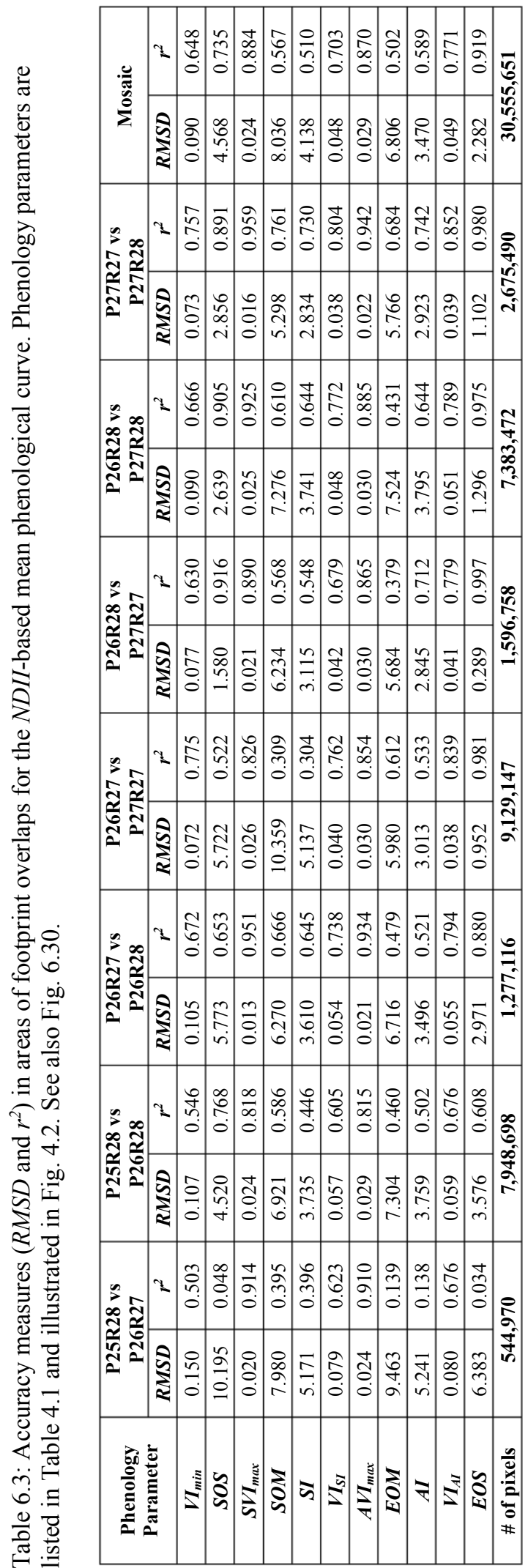


Superior. In the Autumn, however, the summer-warmed Lake cannot account for early senescence in that area, and cold-air pooling there would be more of a meteorological than lake-related process. Nighttime cold-air drainage over a large area into that location near Lake Superior during the Autumn transition could help explain the earlier $A I$ dates and shorter growing season duration in that location.

Several other indications of Lake Superior influence on the surrounding phenology are also evident. In northeastern Minnesota, generally upwind of the Lake (given a climatological wind direction from northwest to southeast over the region, as indicated in Chapter 5), a relatively narrow band (only 10$20 \mathrm{~km}$ across) along the North Shore experiences earlier SI, shorter DOS, higher Spring NDII maxima and greater NDII ranges (suggesting largely deciduous forest types), and larger $T A$ than further inland. However, that strip along the North Shore also demonstrates earlier $A I$ than its surroundings and thus a growing season duration little different from the forests farther inland. Comparing these results with the map in Fig. 3.2, it is easy to see why USEPA analysts designated that narrow strip along Lake Superior's North Shore as its own ecoregion, distinct in character from the surrounding areas.

Much attention was also given in Chapter 5 to the areas along the southern shore of Lake Superior, generally downwind of the lake in an area of greater total annual precipitation and mixed seasonal effects. This lake-effect area is much larger than that to the northwest of Lake Superior, with somewhat more diffuse indications in the observed phenology. Derived DOS are generally longer than areas farther inland (to the south) as winds off of the chilled and/or frozen Lake prevent the rapid accumulation of $G D D$. Derived $D O M$ are overall shorter than areas to the south, but the onshore flow of warm winds in Autumn seems to sustain distinctly higher Autumn NDII values and later $A I$ dates than are found in surrounding locations, extending the growing season farther into Autumn and producing a slightly longer average growing season duration than found on the North Shore. Higher NDII minima and greater overall NDII ranges in locations immediately adjacent to Lake Superior suggest that those forest areas are predominately evergreen, which can take greater advantage of a lake-influenced phenological 
shift from the meteorological seasons than deciduous species, with greater survivability in cold Spring conditions and by exploiting lower light conditions in both early Spring and late Autumn.

\subsubsection{Comparisons with Climatology}

Thousands of correlations between NDII-based phenological indicators and climatological mean indicators across the study area are summarized in Fig. 6.31, similar to the cross-correlation tables shown above for P26R27. The mosaic-wide cross-correlations differ substantively from those limited to the P26R27 footprint, with less influence of climatological indicators on overall fitted phenological curve (as measured using MAE, RMSE, and $r^{2}$ ). On the one hand, correlations of 90-day temperature averages, seasonal precipitation totals, chilling/freezing days $(C D, C D D$, and $F D)$, and measures of growing degree days $(G D D)$ with date- and duration-oriented NDII-derived Spring phenological indicators (e.g. SOS, SI, $D O S$, and $S O M$ ) are generally stronger on the regional scale than in P26R27 alone. However, these climatological indicators are less correlated with key NDII magnitudes through the growing season (e.g. Spring and Autumn maximum NDII, overall NDII range) than in P26R27. Correlations between phenological indicators and the values of 90-day temperature variance are also much less strong at the regional scale. For example, $N D I I$-based mean growing season $(D[S I-A I])$ on the regional scale is hardly correlated at all with either the mean frost-based growing season duration or the alternative mean $C D$ plateau-based growing season duration (Fig. 6.32). The mean accumulated $G D D$ for each of these climatological growing seasons are also barely correlated with the NDII-based mean growing season (Fig. 6.33). These are surprising results, given that several indicators of phenological timing at the seasonal scale (e.g. SI, DOS, SOM, and DOM) are correlated better with a number of more basic climatological measures than the with frost-based and plateau-based beginning and end dates.

$D O S$ is negatively correlated with the mean accumulation of Spring $G D D$ (Fig. 6.34a), as would be expected: a warm Spring suggests a more rapid, and thus shorter, phenological green-up period, while 


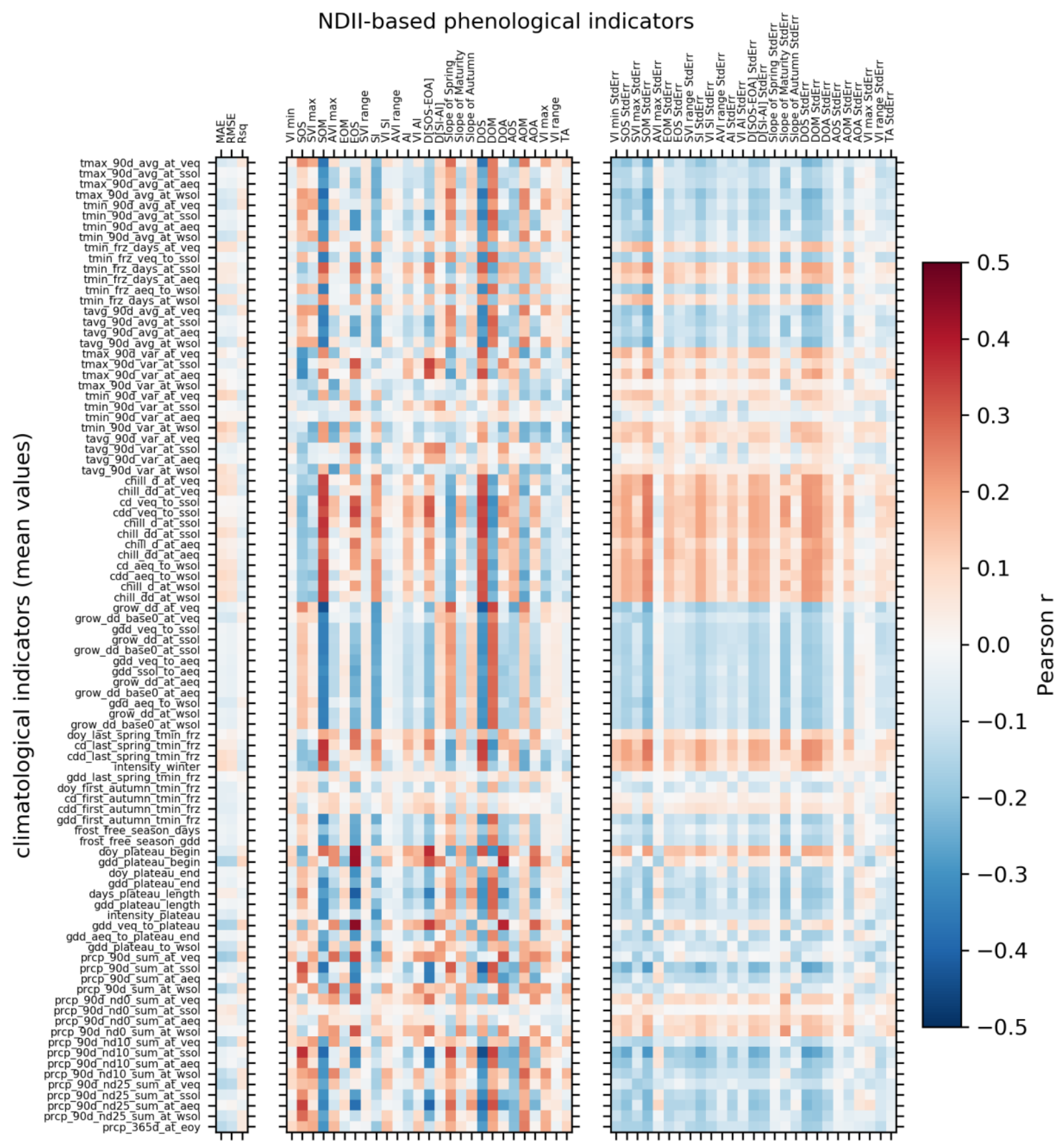

Figure 6.31: Summary of correlations for NDII-based mean phenological curve indicators (left columns) and their standard errors (StdErr, right columns) with climatological indicator mean values for regional mosaics. Climatological indicators are listed in Tables 3.1-3.5, and phenological indicators are listed in Table 4.1 and illustrated in Fig. 4.2. 
$\begin{array}{ll}\text { (a) } D[S I-A I] \text { vs. Frost-free season duration } & \text { (b) } D[S I-A I] \text { vs. Plateau duration }\end{array}$
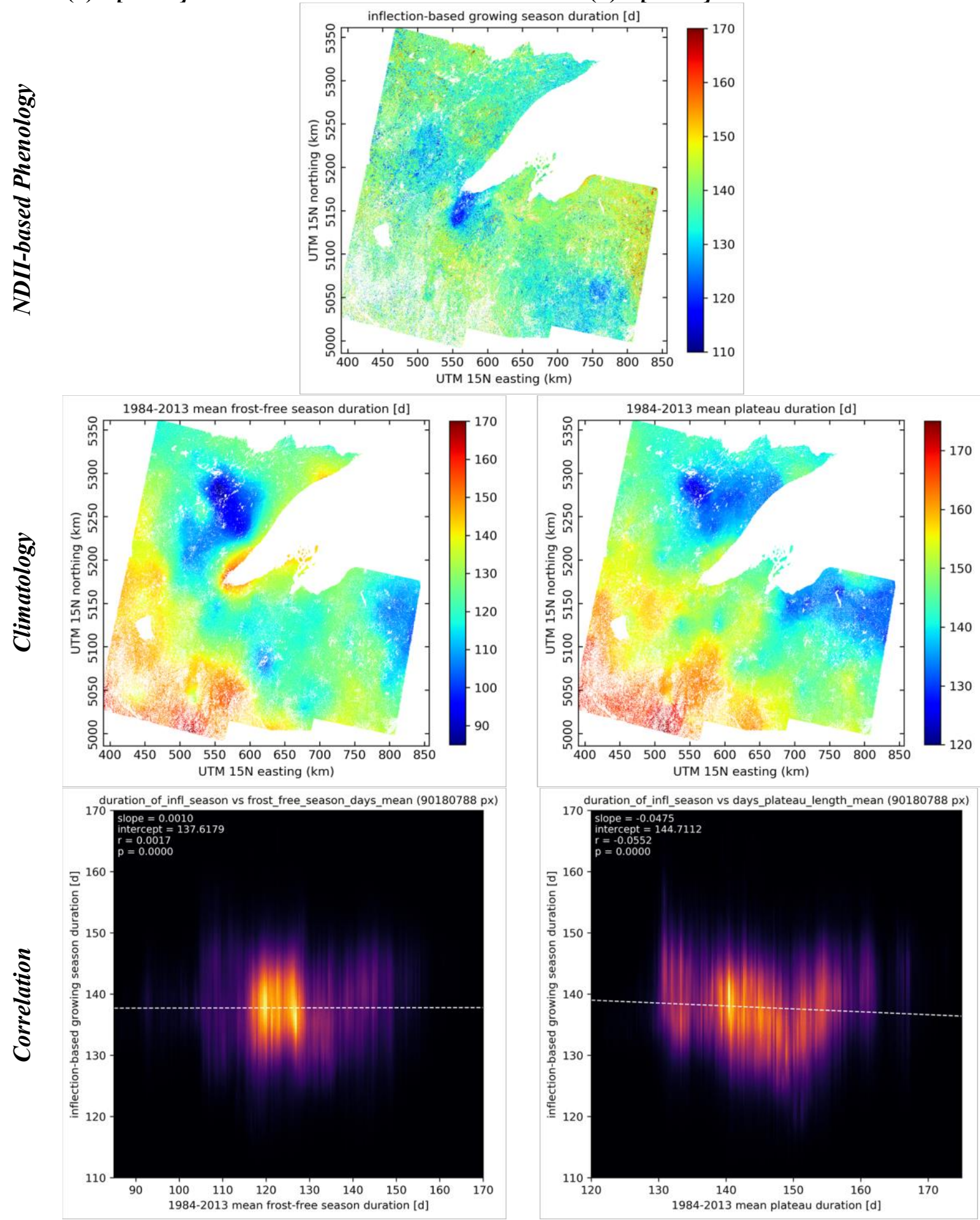

Figure 6.32: Correlations between (a) $D[S I-A I]$ and frost-free season duration, and (b) $D[S I-A I]$ and plateau duration. 
(a) $D[S I-A I]$ vs. Frost-free season $G D D$

(b) $D[S I-A I]$ vs. Plateau GDD
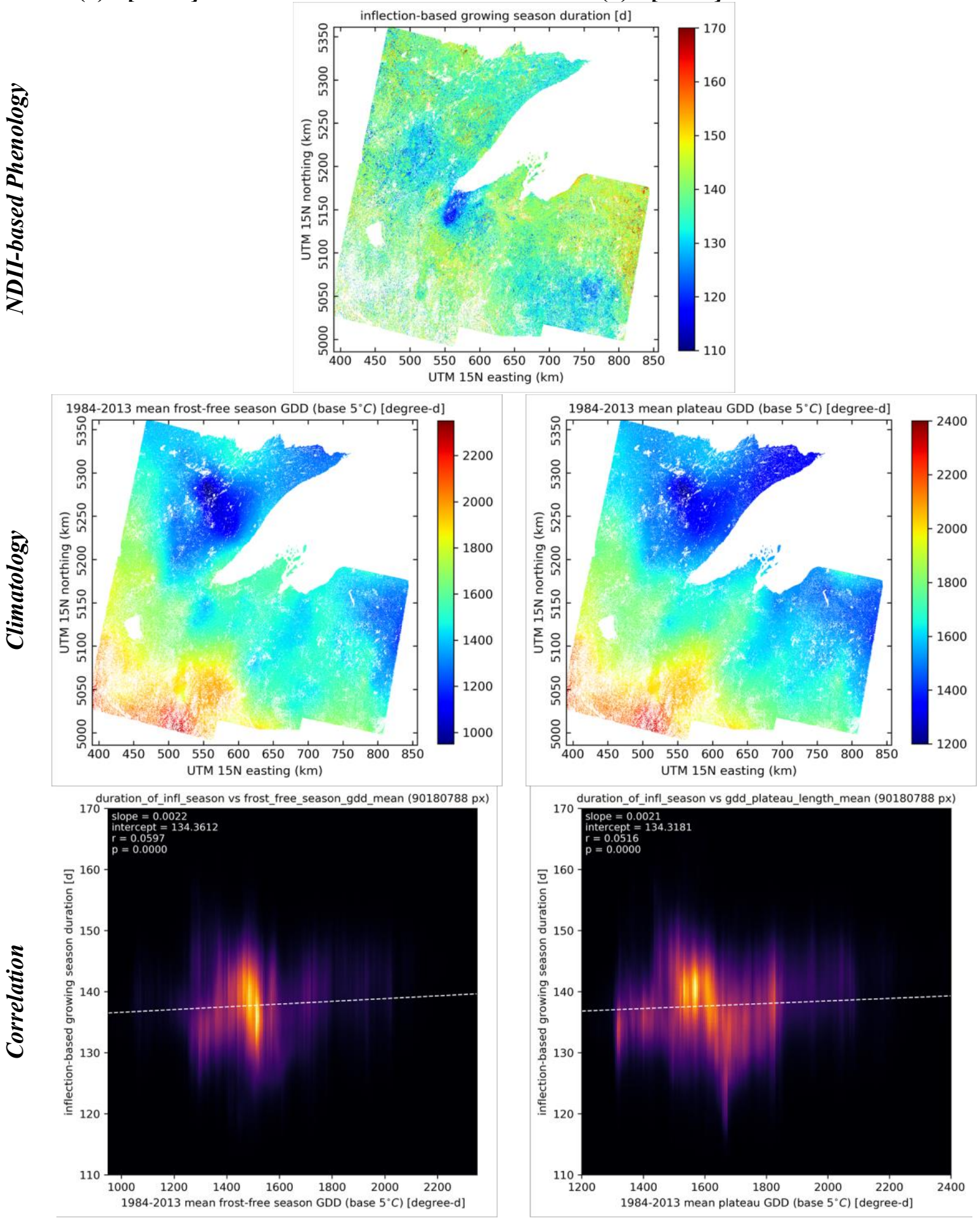

Figure 6.33: Correlations between (a) $D[S I-A I]$ and frost-free season $G D D$, and (b) $D[S I-A I]$ and plateau GDD. 
(a) $D O S$ vs. Spring $G D D$

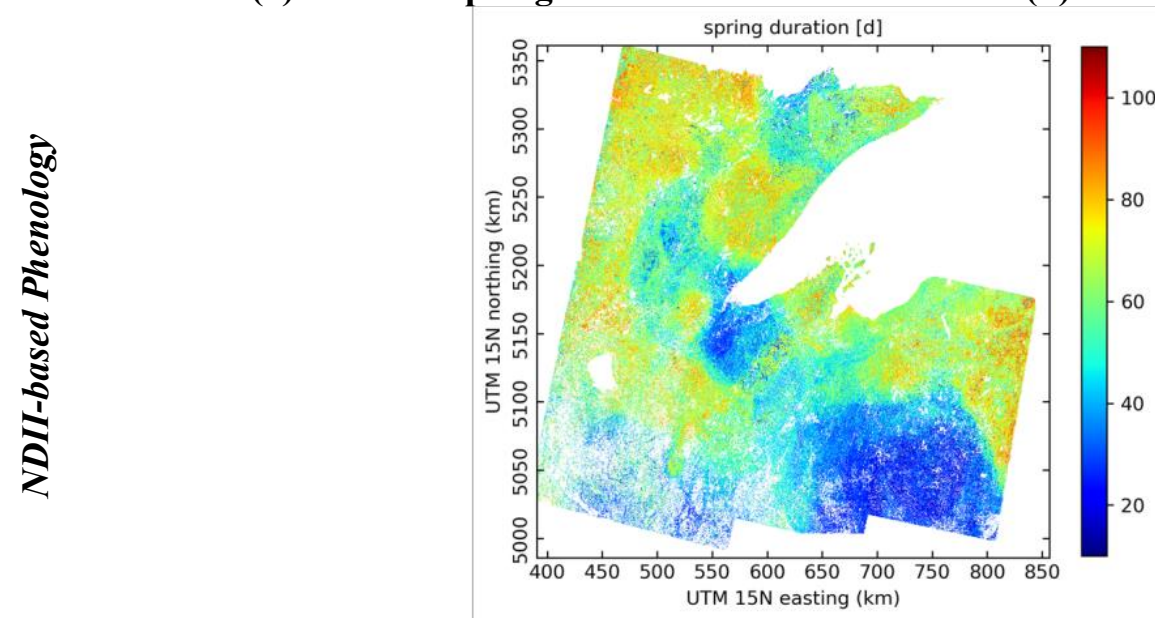

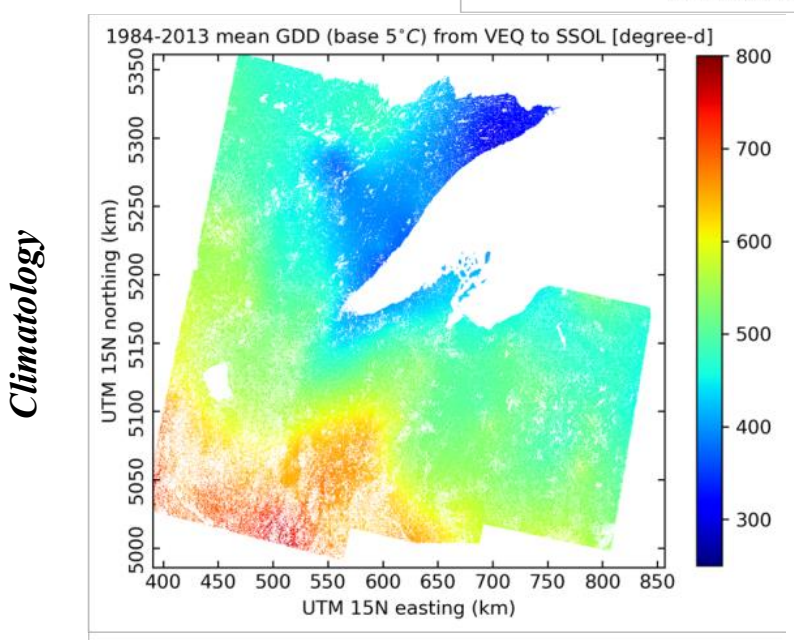

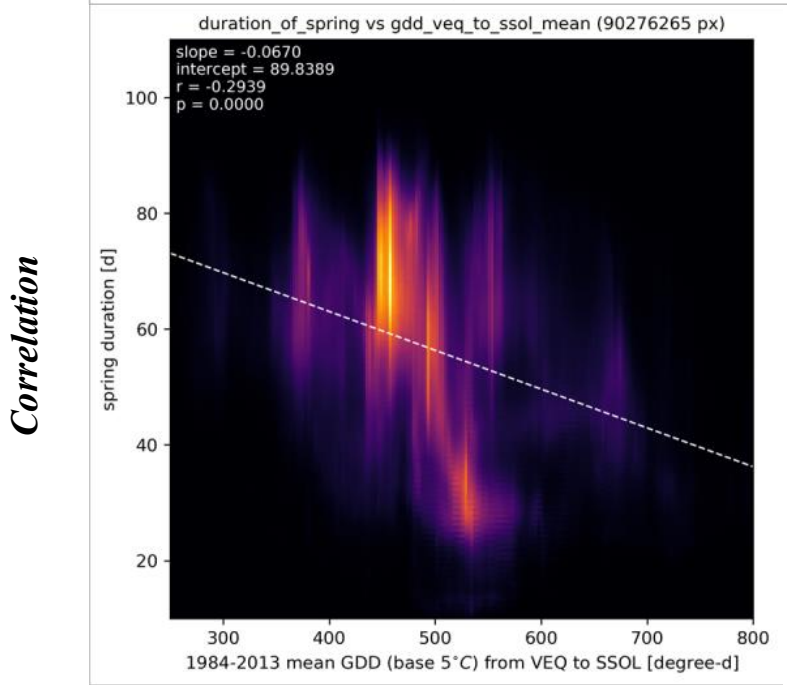

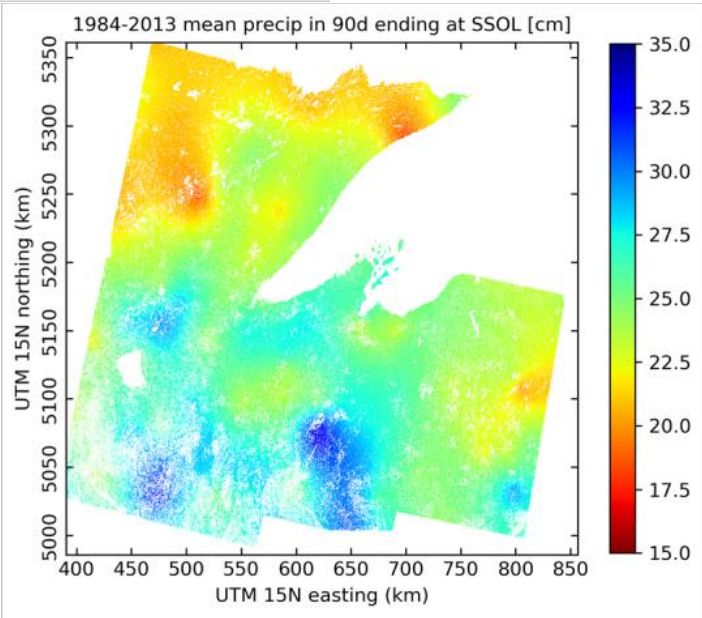

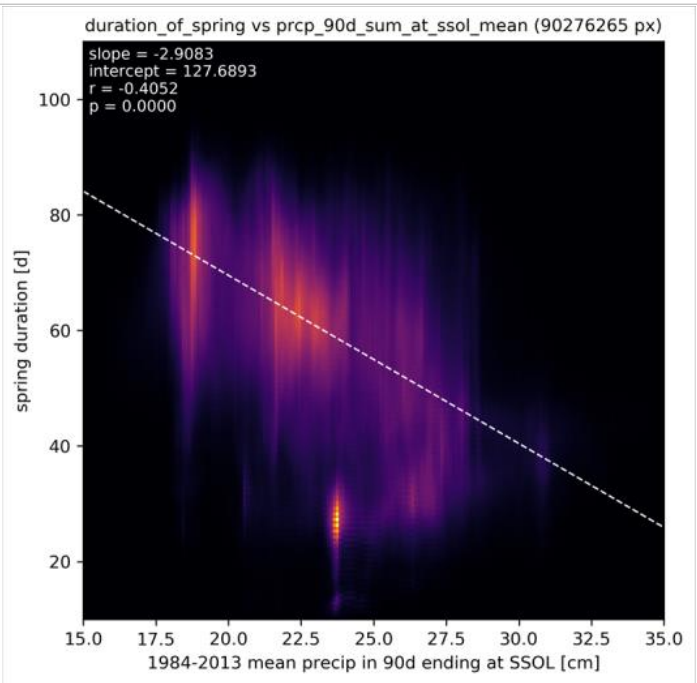

Figure 6.34: Correlations between (a) DOS and Spring GDD, and (b) DOS and Spring $P$. 
a cool Spring can draw out the duration of the green-up phase. Figure $6.34 \mathrm{~b}$ shows that $D O S$ is also negatively correlated with the mean Spring precipitation. These associations suggest that greater water availability may facilitate the green-up phase in the forest phenological cycle, and potentially that a dry Spring could hinder that phase. A seasonal or long-term drought, or even just a low-precipitation year, can affect tree health and growth [Noormets et al., 2008; Anderegg et al., 2012, 2013, 2015; Brzostek et al., 2014], especially in the early and middle growing season when the timing of water availability is most critical for wood and leaf biomass development [Delpierre et al., 2016].

The $S O M$ mean date is well correlated with numerous climatological indicators. Over the longer term, the intensity of the Winter (cold) season as it stretches into climatological Spring can indicate suppressed Spring temperatures and an overall delay in SOM (Fig. 6.35a). However, it also appears that an earlier mean $S O M$ is correlated with greater mean annual precipitation (Fig. 6.35b), which follows from the principle that greater total precipitation is generally associated with higher average temperatures (see Chapter 5). On the shorter term, positive correlations between SOM and the last date in Spring at which $T_{\min }<0^{\circ} \mathrm{C}$ and the mean date at which the $C D$-based plateau begins are shown in Fig. 6.36. A warmer meteorological Spring season is associated with earlier dates for both of those climatological indicators, and thus an earlier phenological transition from forest green-up into the mature period. The summary in Fig. 6.31 suggests that mean DOM is correlated better than the mean inflection-based growing season duration $(D[S I-A I])$ with climatological growing season indicators. Associations between mean $D O M$ and the same climatological growing season $G D D$ indicators that were examined in Fig. 6.33 are shown in Fig. 6.37, with much stronger positive correlations indicated. For a number of these associations, the relationship may be better fit with a sigmoidal curve than a linear regression, suggesting the operation of threshold dynamics in phenological responses to accumulation-based climatological indicators such as $G D D$. The mean $D O M$ is also positively correlated with the duration of the $C D$-based plateau (Fig. 6.38a; compare with Fig. 6.33) and, interestingly, with the mean total Summer precipitation 
(a) $S O M$ vs. Winter Intensity

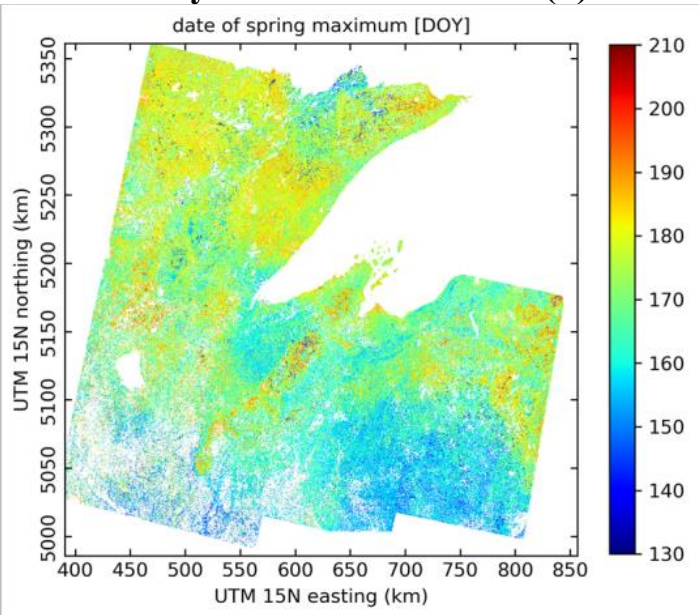

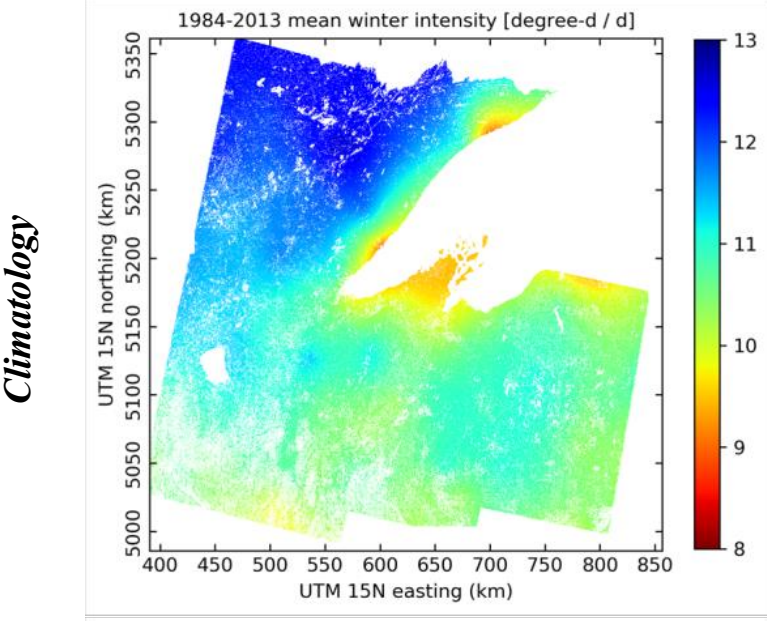

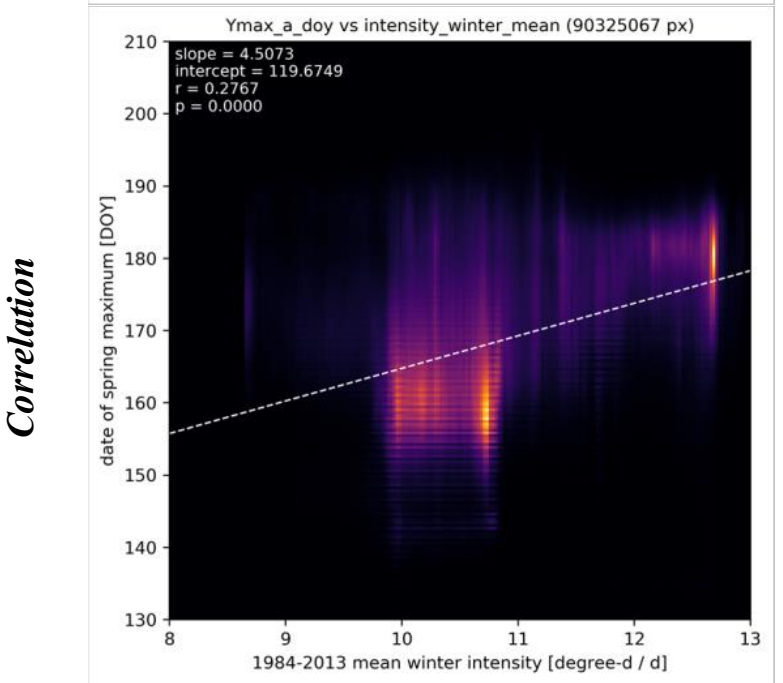

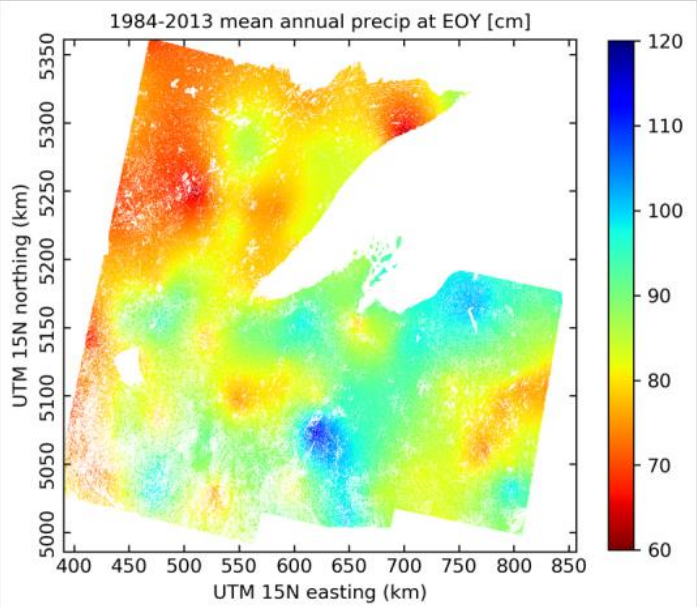

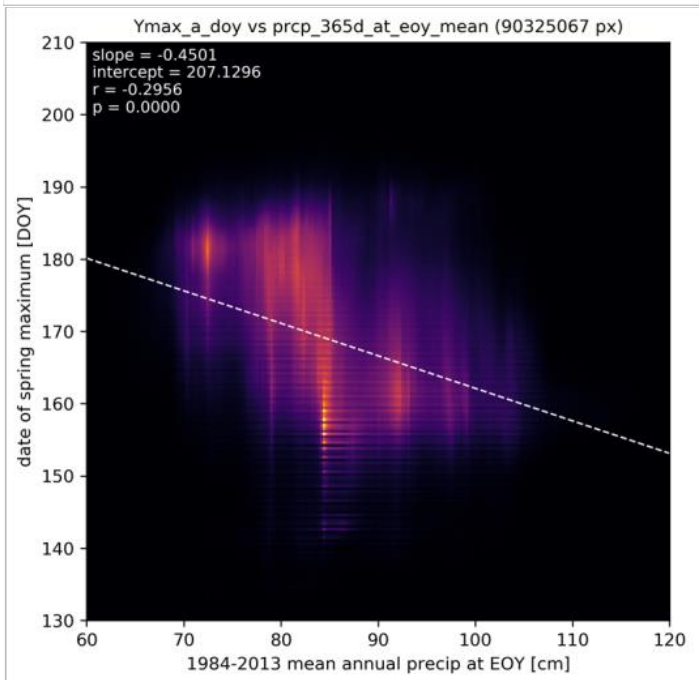

Figure 6.35: Correlations between (a) SOM and Winter Intensity, and (b) $S O M$ and annual $P$. 
$\begin{array}{ll}\text { (a) } S O M \text { vs. Last Spring } T_{\min } \text { freeze } & \text { (b) } S O M \text { vs. Plateau start date }\end{array}$
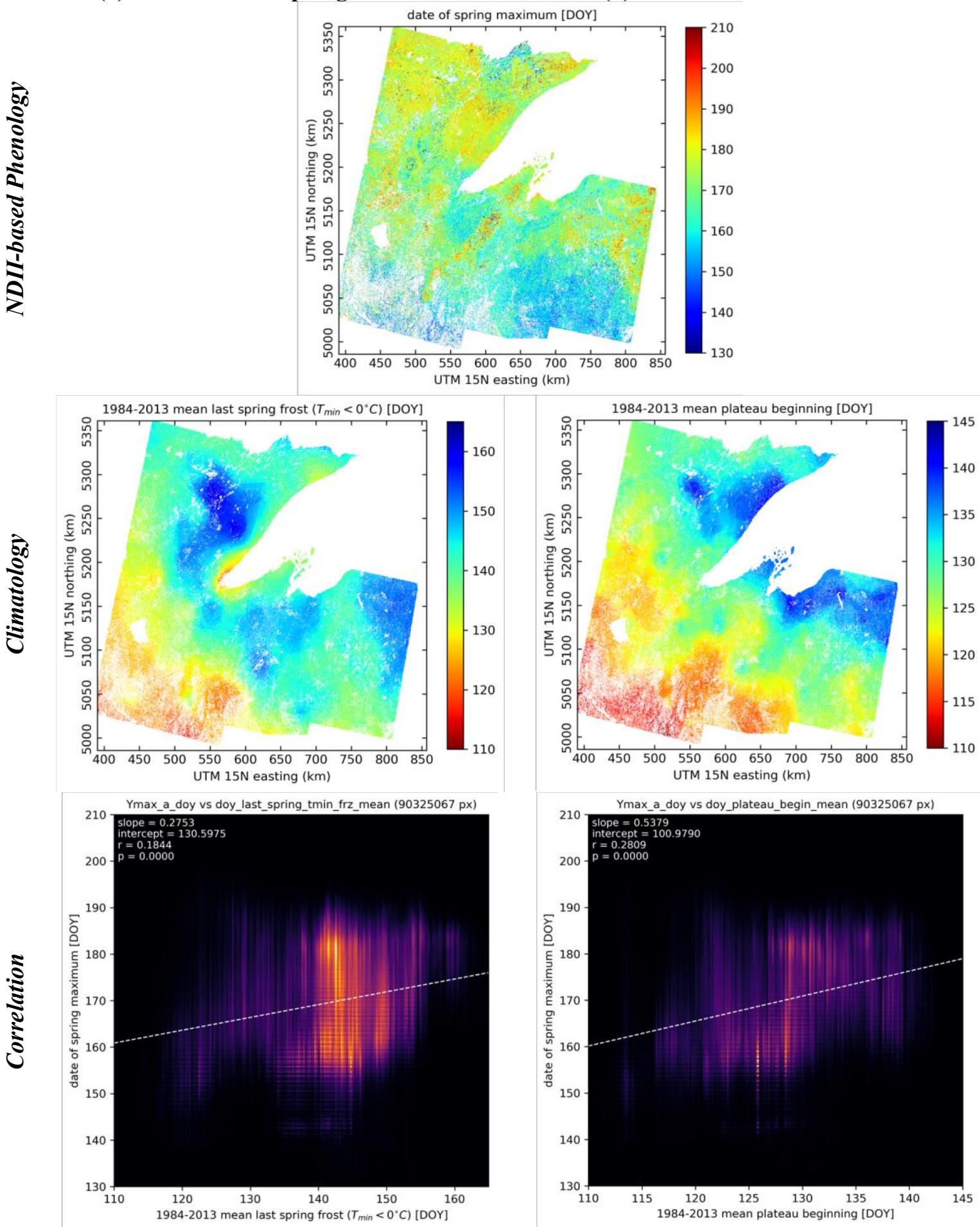

Figure 6.36: Correlations between (a) $S O M$ and last Spring $T_{\min }$ freeze date, and (b) $S O M$ and plateau start date. 
(a) $D O M$ vs. Frost-free season $G D D$

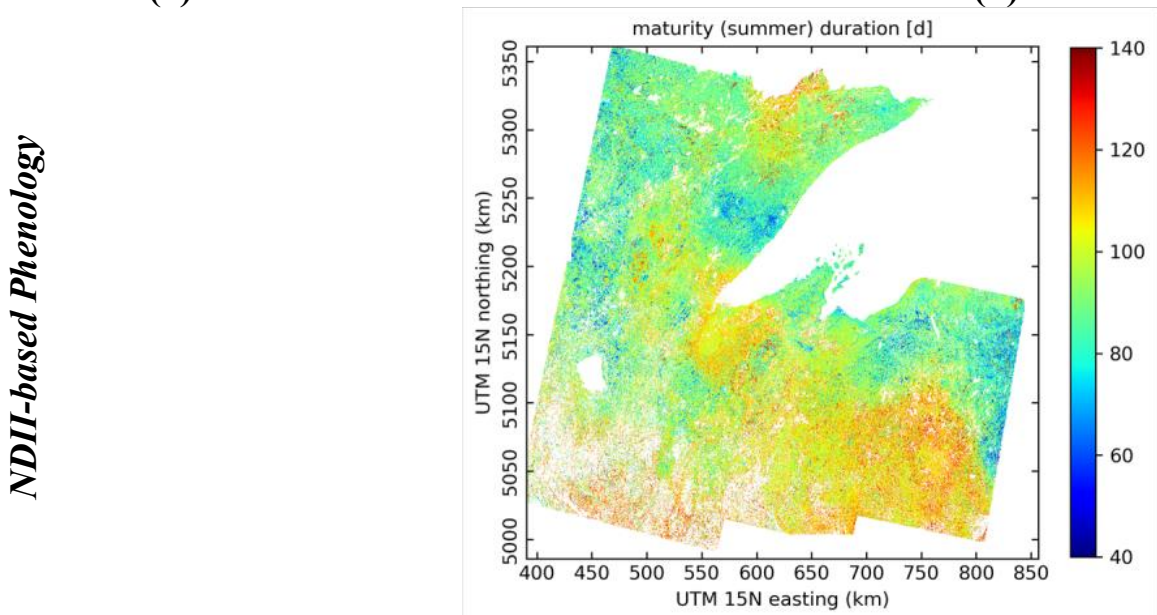

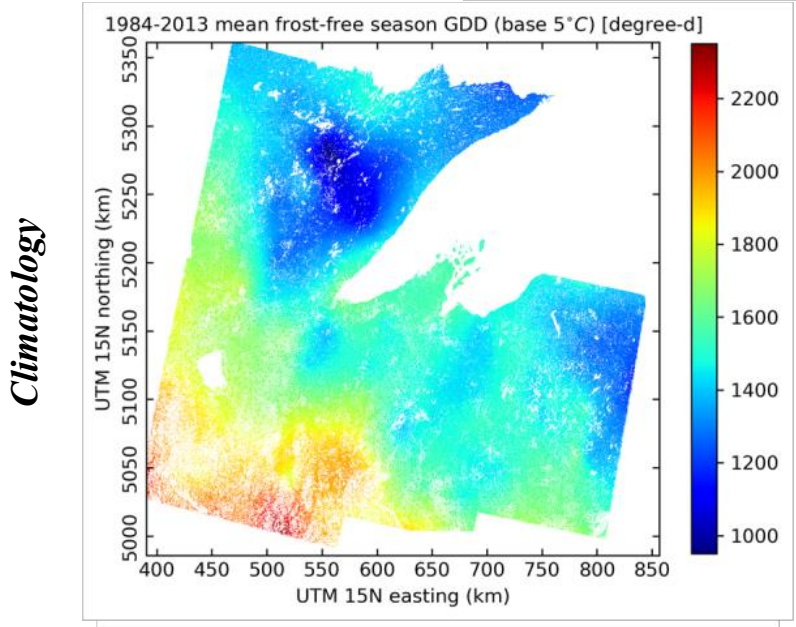

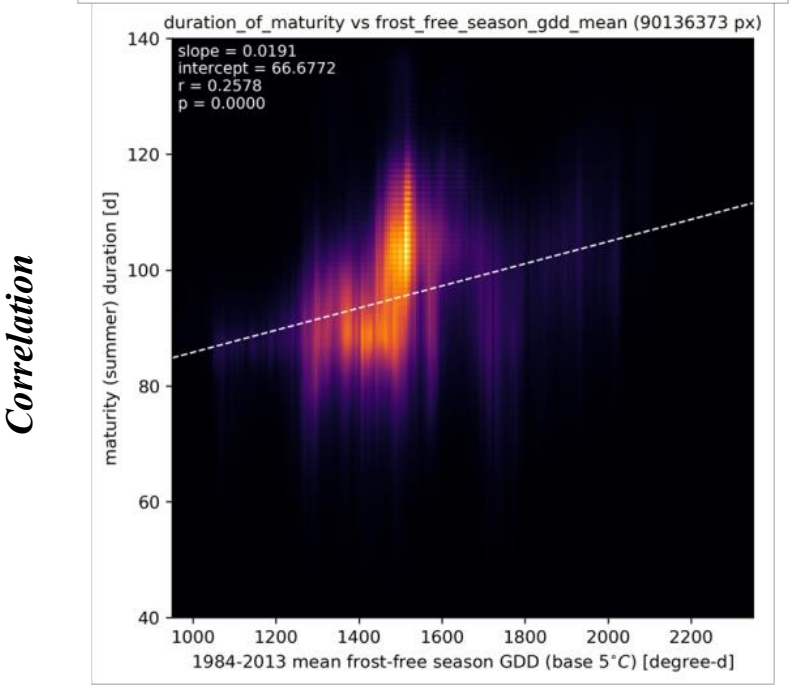

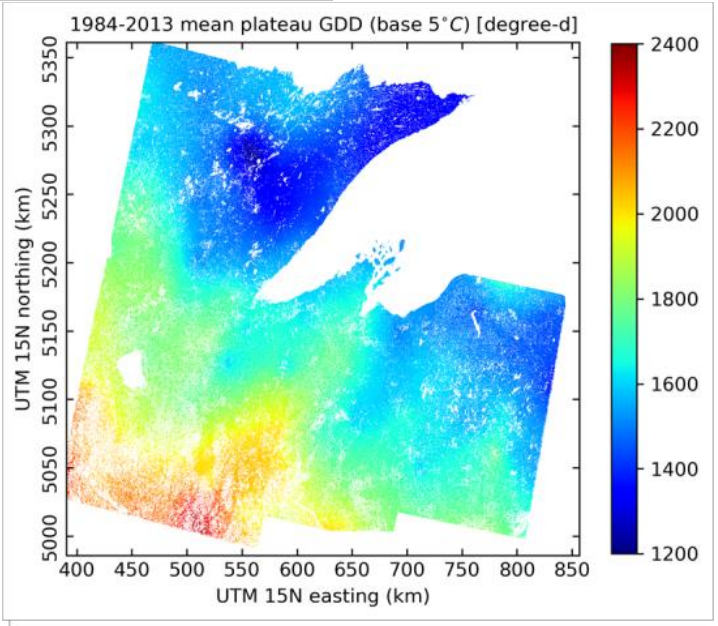

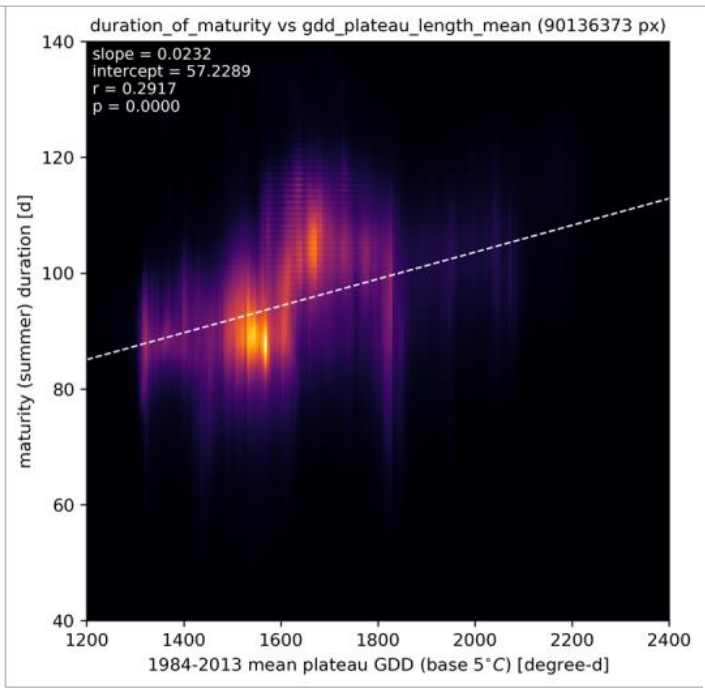

Figure 6.37: Correlations between (a) DOM and frost-free season GDD, and (b) $D O M$ and plateau GDD. 
(a) DOM vs. Plateau duration

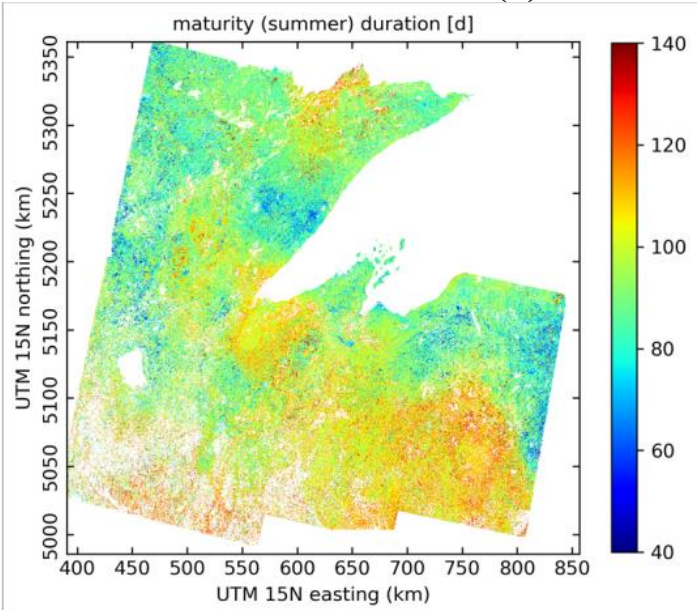

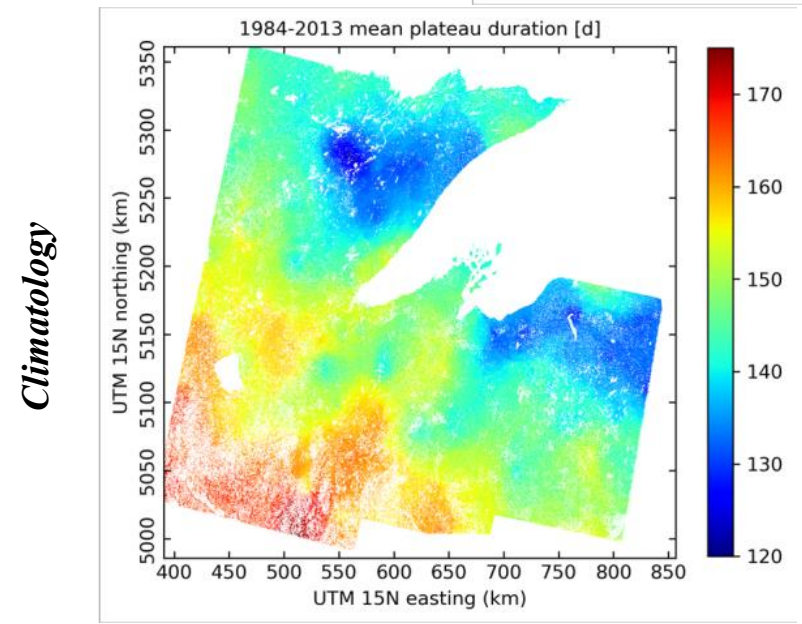

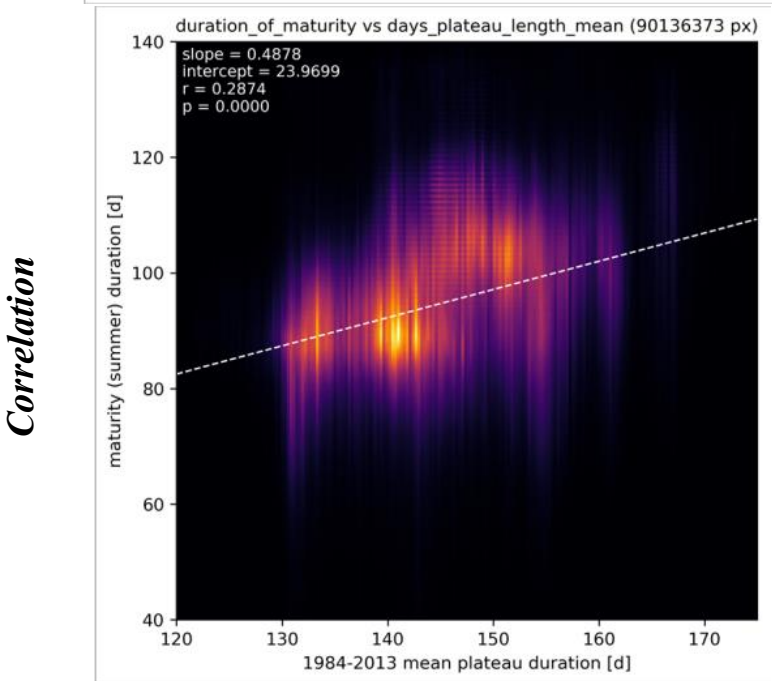

(b) $D O M$ vs. Summer $P$
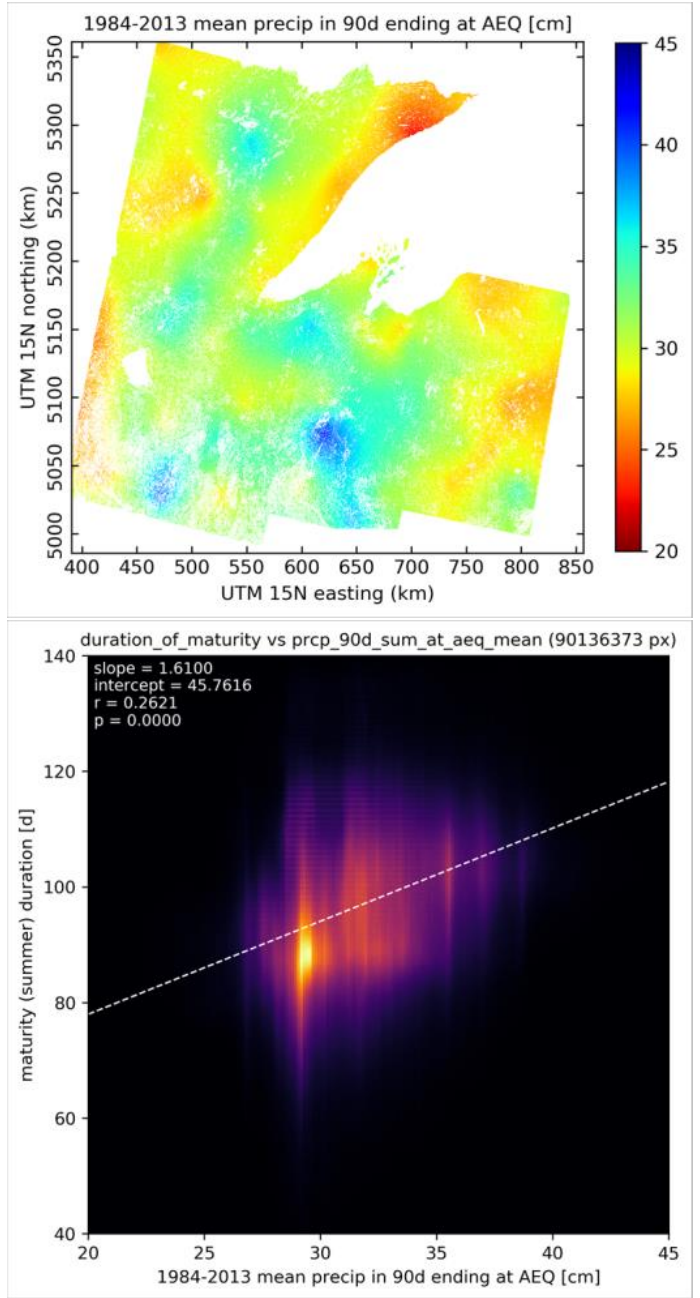

Figure 6.38: Correlations between (a) DOM and plateau duration, and (b) $D O M$ and Summer $P$. 
(Fig. 6.38b). Just as a dry Summer has been associated with early phenological senescence in many areas [Munné-Bosch and Allegre, 2004; Marchin et al., 2010], a wet Summer may allow a longer mature period before senescence begins. This is especially crucial in a warmer Summer, also positively correlated with greater $D O M$ (Fig. 6.39a), given a greater demand on transpiration and a greater risk of vegetation water stress in the warmest period of the growing season. A longer plateau-based growing season is associated with a shorter $D O A$, as shown in Fig. $6.39 \mathrm{~b}$. The mean $D O A$ appears well-correlated with Summer precipitation and with Autumn precipitation days, as shown in Fig. 6.40. In these results, a dry Summer is associated with a prolonged period of phenological senescence, whereas a wet Summer is generally associated with a shorter $D O A$ period, perhaps because of the sharpness of that meteorological transition into Autumn. Conversely, Fig. 6.40b indicates that more precipitation days in meteorological Autumn can draw out that senescence process to some degree.

\subsubsection{Overall Variability}

Along with the mean phenological indicators discussed above and shown in Figs. 6.20-6.29, I examined the observed variability in various measures of the growing season using the standard error either reported by the phenological model for each of the principal curve-fit parameters or calculated for derived indicators. The regional mosaic of the NDII-based date of spring inflection $(S I)$ standard error (StdErr) is shown in Fig. 6.41 and indicates the greatest variability in two main regions: northeastern Minnesota, over in slightly elevated terrain just west of Lake Superior and in known areas of extensive disturbances, and along the south shore of Lake Superior and farther inland to the south and in higher terrain to the east, where the Lake strongly influences Spring phenology as discussed above. These two general areas of Spring phenological variability are also indicated in the map of the duration of spring $(D O S)$ standard error in Fig. 6.42. Greater variability in the duration of maturity $(D O M)$ occurs in those areas again as well as wetland areas of northern Minnesota, in the St. Louis River lowlands area, and in parts of the northwestern Wisconsin pine barrens, as shown in Fig. 6.43. The same areas also exhibit greater variability in the autumn inflection $(A I)$ date, shown in Fig. 6.44. Figure 6.45 shows that the 
(a) DOM vs. Plateau Intensity
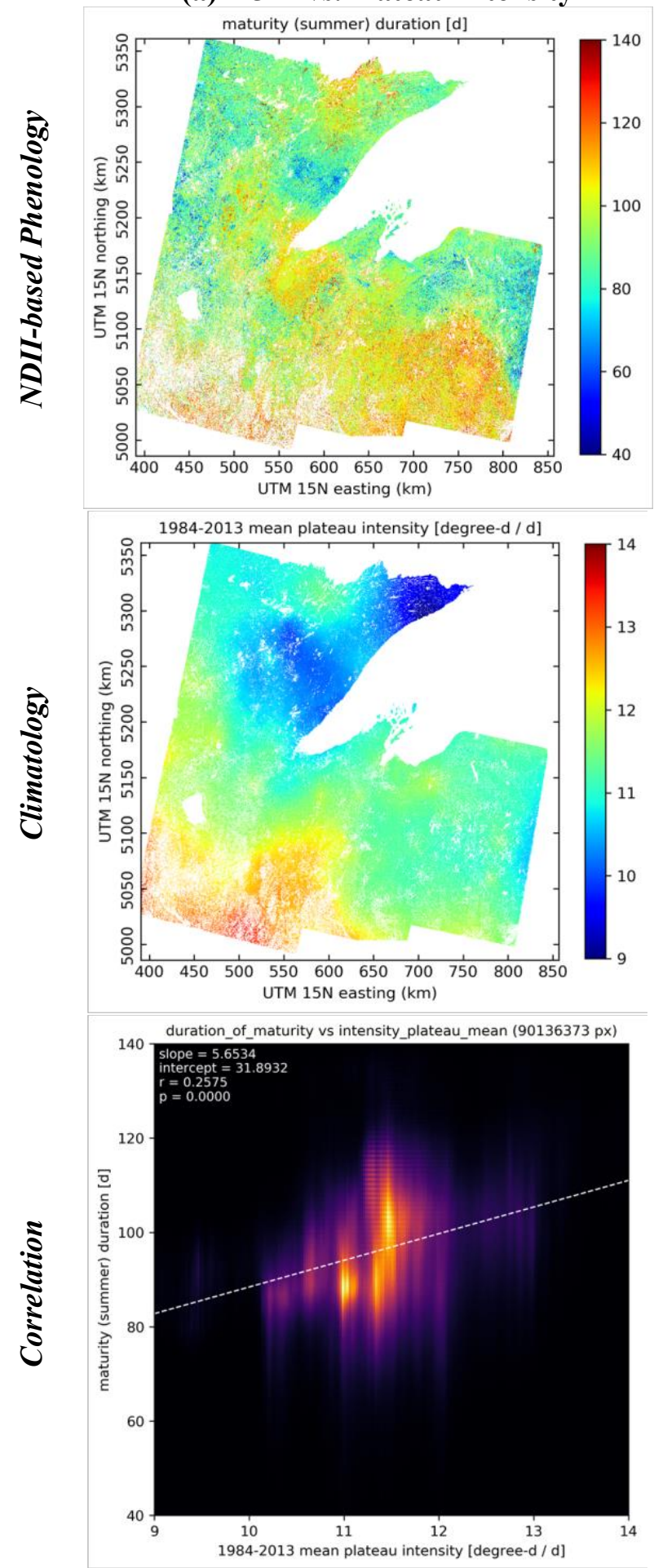

(b) $\mathrm{DOA}$ vs. Plateau end date
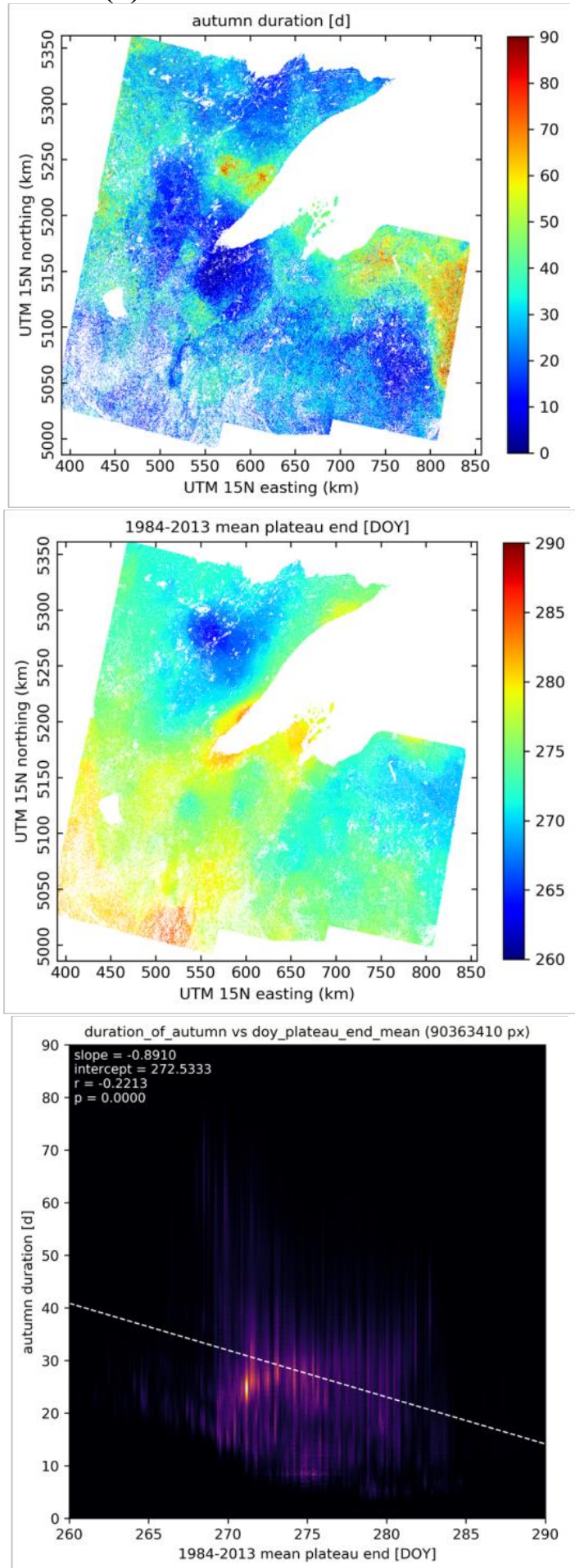

Figure 6.39: Correlations between (a) DOM and GSI, and (b) DOA and plateau end date. 
(a) $D O A$ vs. Summer $P$

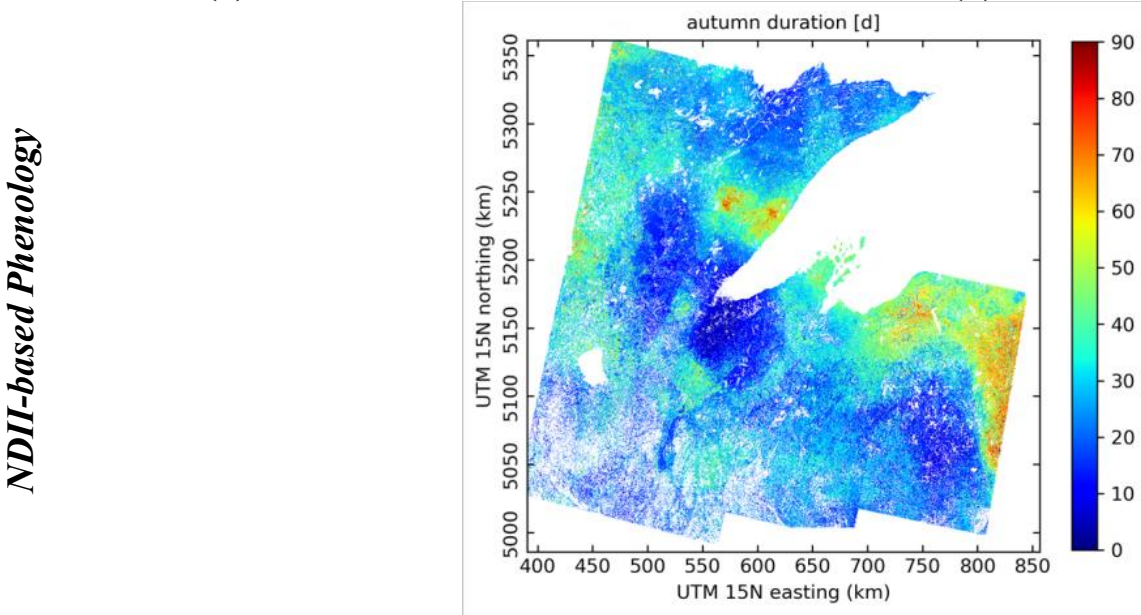

(b) $D O A$ vs. Autumn $P$ days
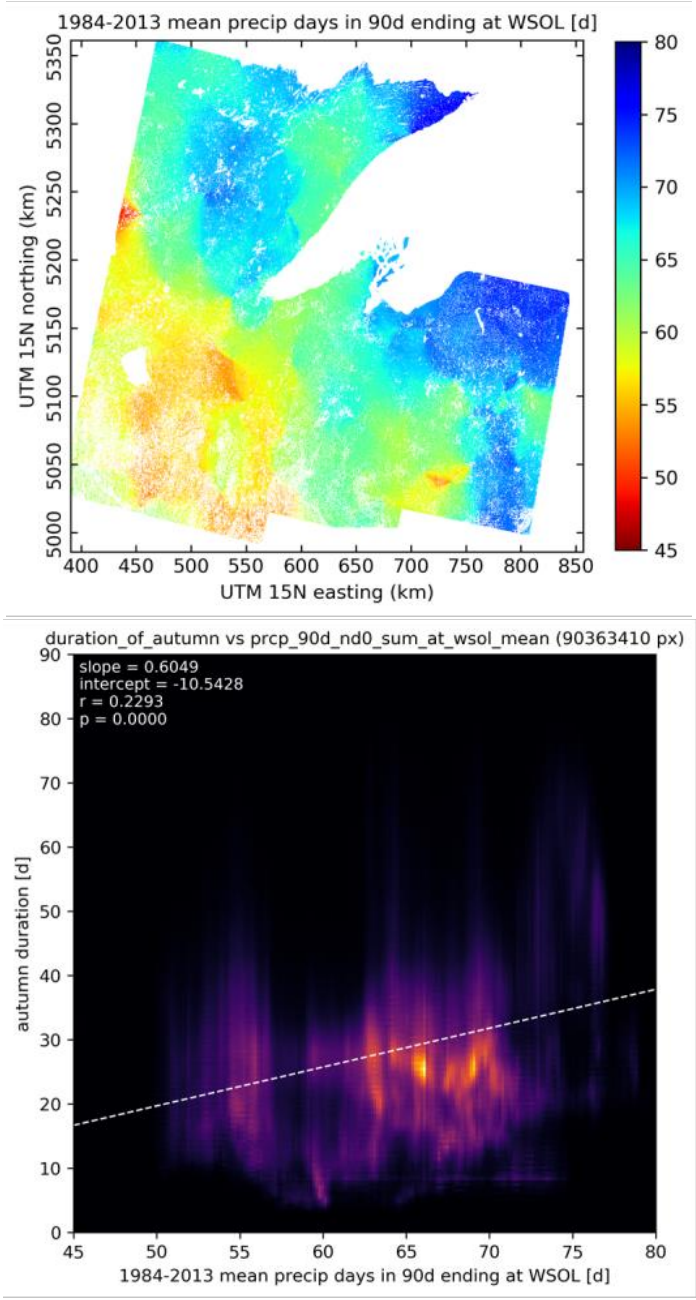

Figure 6.40: Correlations between (a) $D O A$ and Summer $P$, and (b) $D O A$ and Autumn $P$ days. 


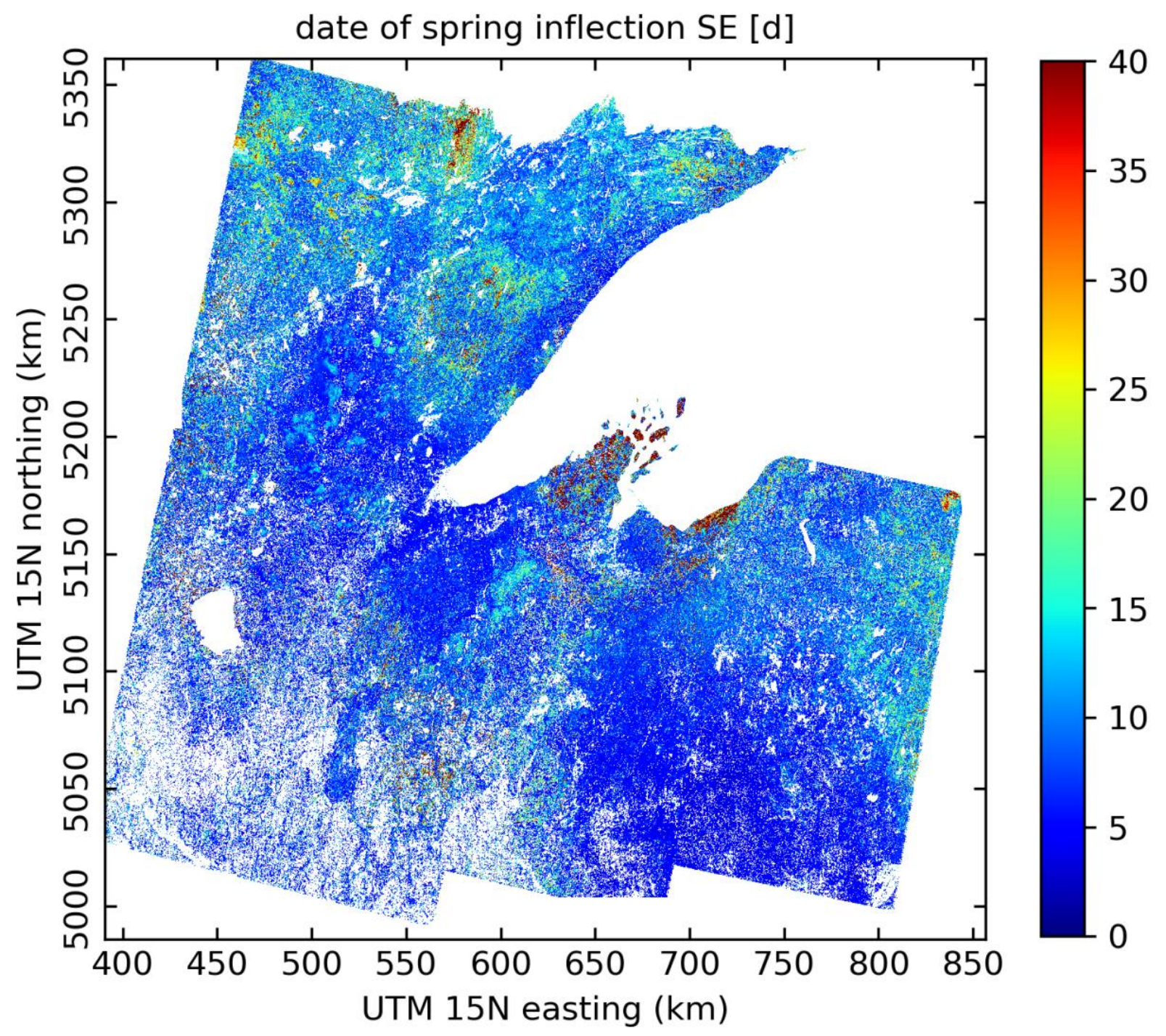

Figure 6.41: Mosaic NDII-based date of spring inflection (SI) standard error (StdErr). Compare with Fig. 6.20.

variability in these seasonal indicators all contribute to a standard error in the duration of inflection-based growing season $(D[S I-A I])$ of 2-4 weeks in those generally widespread areas, and more than seven weeks in isolated locations such as the St. Louis River lowlands and the nearby, lake-affected south shore of Lake Superior. However, large standard errors in the NDII-based total area under the fitted curve $(T A)$ is much more limited in geographic scope as shown in Fig. 6.46, where isolated areas of high variability are limited primarily to the northernmost areas of northeastern Minnesota, the St. Louis River lowlands that have seen extensive discussion thus far, and the varied terrain of the Wisconsin Northwoods region.

In an effort to explain some of this observed phenological variability, I correlated the standard 


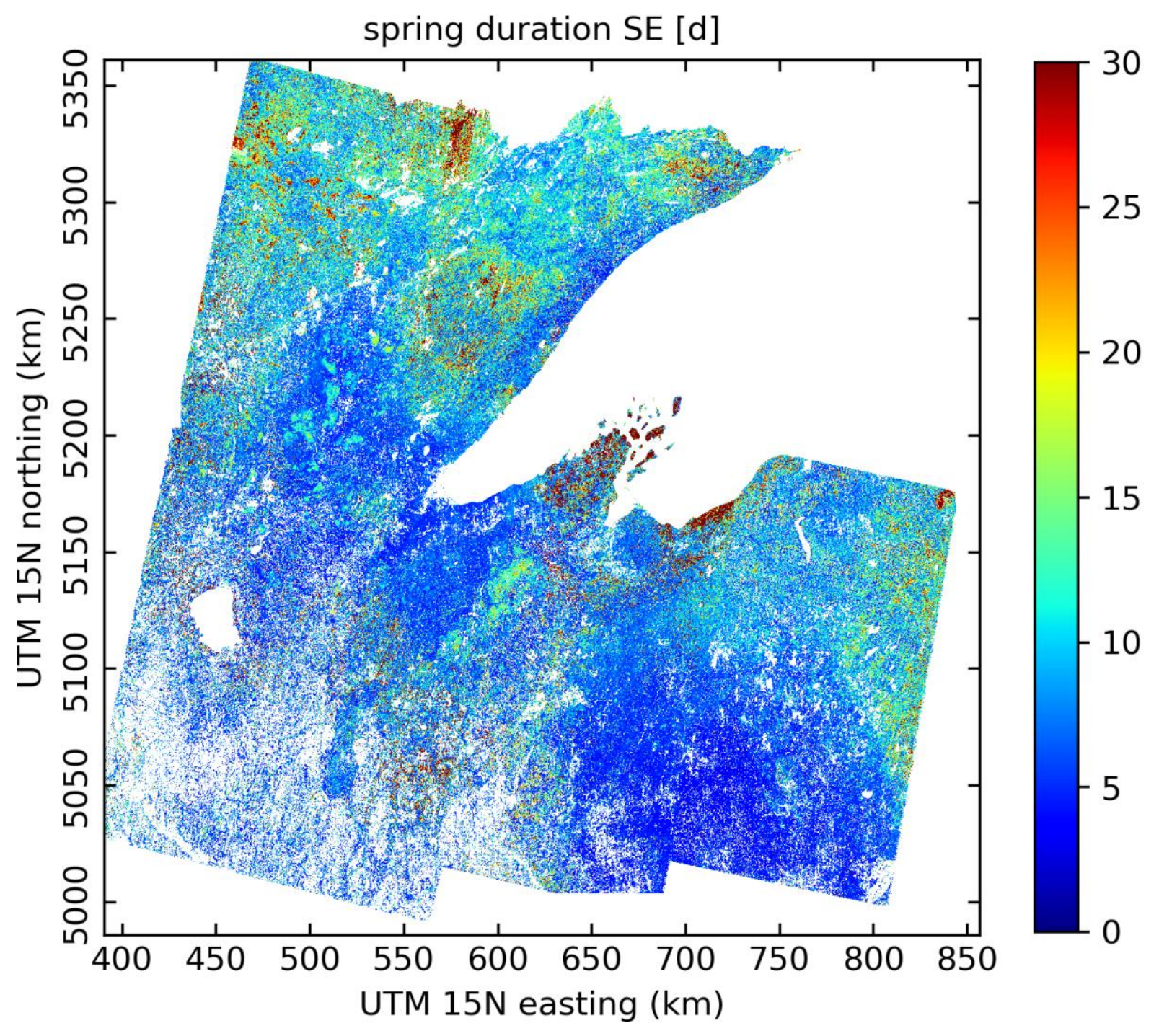

Figure 6.42: Mosaic NDII-based duration of spring (DOS) standard error (StdErr). Compare with Fig. 6.21.

errors of NDII-based phenological indicators with seasonal and annual climatological indicators as in Fig. 6.31 , but this time with the standard deviations from that climatological analysis. These correlations are summarized in Fig. 6.47 where the SOM, DOS and DOM again stand out, here for high correlations with climatological variability (StDev) instead of with the climatological mean indicators. None of the correlations related to variability on the right side of the figure are particularly strong, but their overall systematic correspondence warrants mention. Variability in seasonal temperature averages, $G D D$ accumulations, the durations of the climatological growing seasons (both the frost-free season and the $C D$-based plateau), seasonal and annual precipitation accumulations, and the seasonal numbers of days 


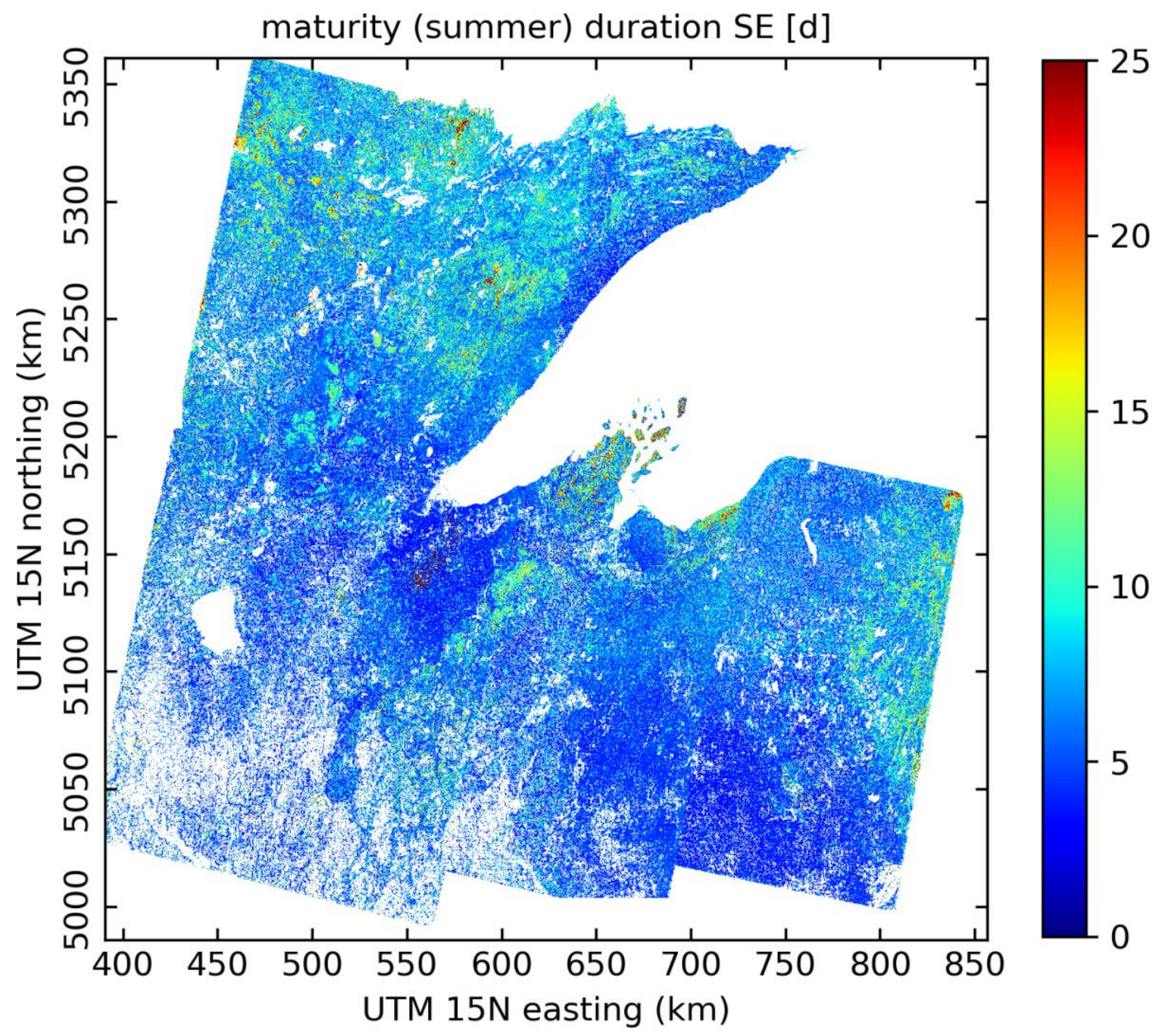

Figure 6.43: Mosaic NDII-based duration of maturity (DOM) standard error (StdErr). Compare with Fig. 6.23.

with moderate and heavy precipitation are all generally negatively correlated with variability in the model-based phenological indicators. Measures of the cold season such as various $F D$ accumulations, $C D$ and $C D D$ accumulations, and winter intensity, as well as the timing of the last spring frost and the beginning of the $C D$-based plateau and the seasonal numbers of precipitation days (when used as a proxy for cloudy days, which are generally associated with cooler weather) are all positively correlated with phenological variability. Warmth seems to stabilize the phenological growing season, reducing variance from the long-term mean cycle, while cold leads to greater variability and thus greater uncertainty in the progression of any single growing season. 


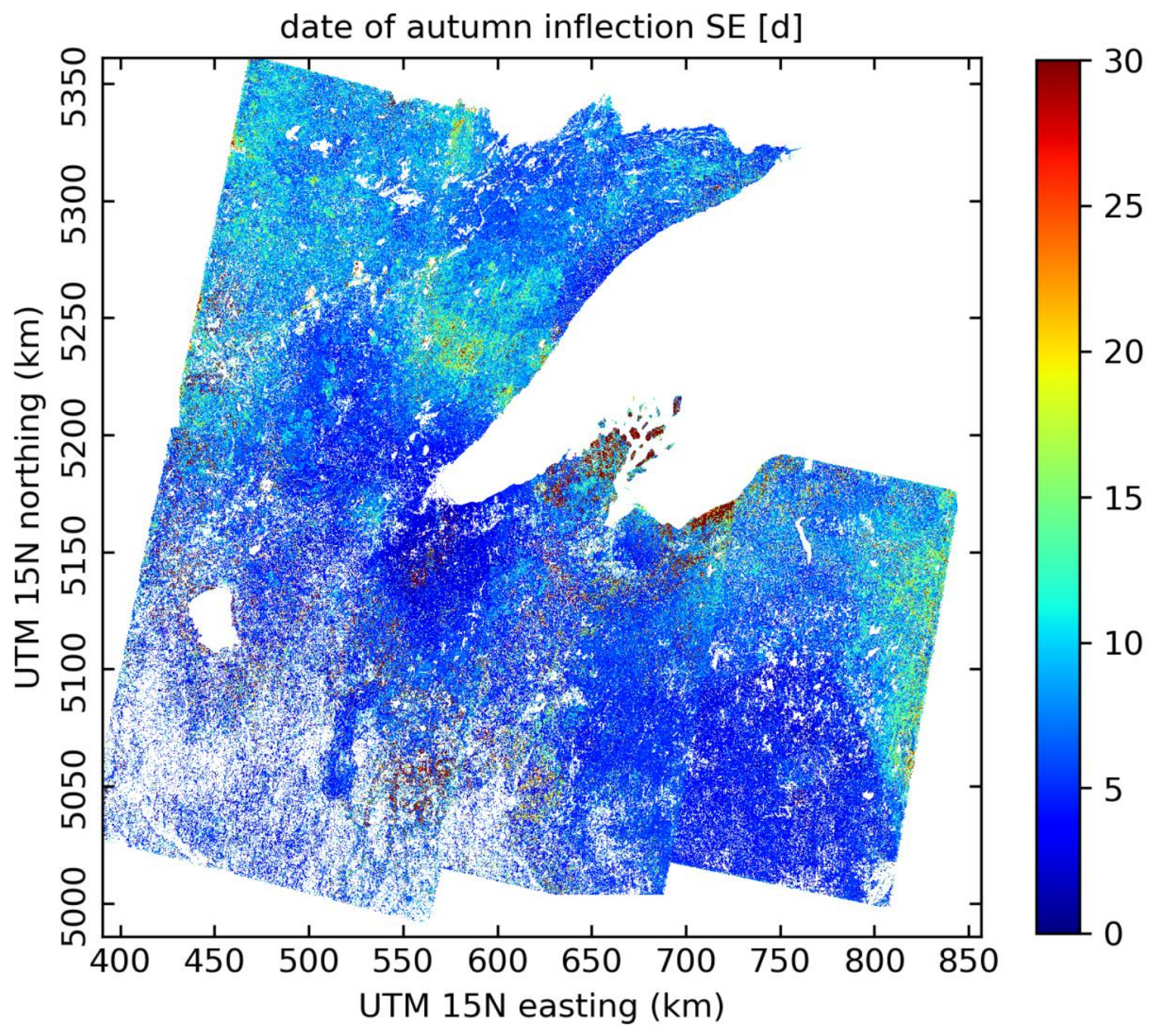

Figure 6.44: Mosaic NDII-based date of autumn inflection $(A I)$ standard error (StdErr). Compare with Fig. 6.25.

Error metrics for the PLSR modeling of phenological residuals are shown in Figs. 6.48 (RMSE) and $6.49\left(r^{2}\right)$. As with footprint P26R27 above (see Fig. 6.15), I calculated the normalized effective PLSR weights for the other footprints in my study area. These are not mosaicked results, but their evaluation on a footprint basis provides an indication of how the relative importance of the most influential climatological indicators on phenological variability changes across the study area. Normalized effective regression weights, are shown for footprint P25R28 in Fig. 6.50 and, proceeding from east to west, footprint P26R28 in Fig. 6.51, footprint P27R27 in Fig. 6.52, and footprint P27R28 in Fig. 6.53. Among these figures of normalized effective PLS regression-based coefficients, several footprints are much 


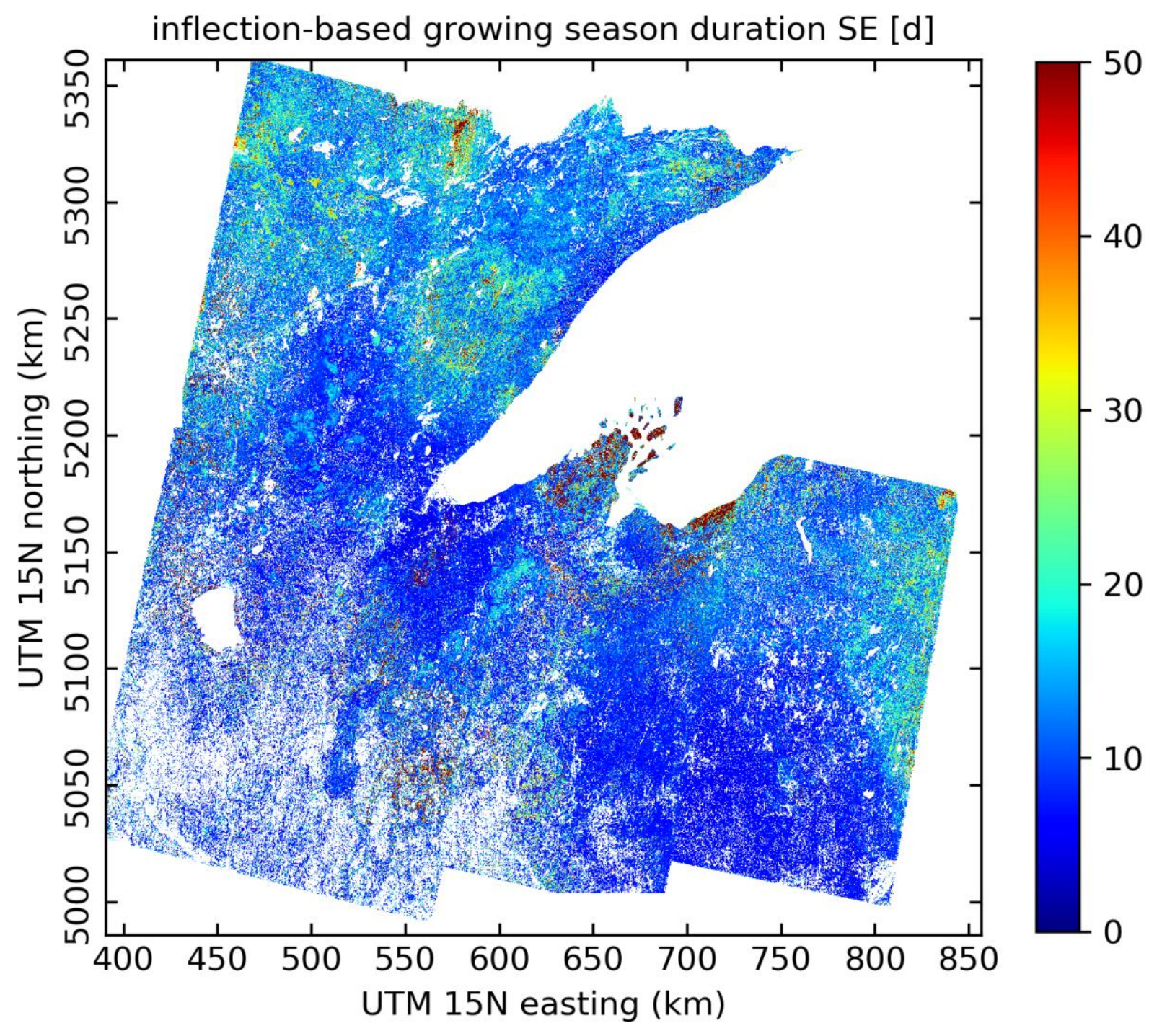

Figure 6.45: Mosaic NDII-based duration of inflection-based growing season (D/SI-AII) standard error (StdErr). Compare with Fig. 6.26.

smoother in the distribution of relative climatological influence (e.g. footprint P25R28) while others are much sharper in their distributions, with just one (in P26R27 and P27R28), two (in P26R28), or three (in P27R27) prominent indicators followed by a relatively smooth distribution of influence among the remainder of the indicators. The northern and western footprints seem to demonstrate this sharp division between top and lower indicators, while the southern and eastern footprints show the relatively smoother distributions of influence across the climatological indicators. 


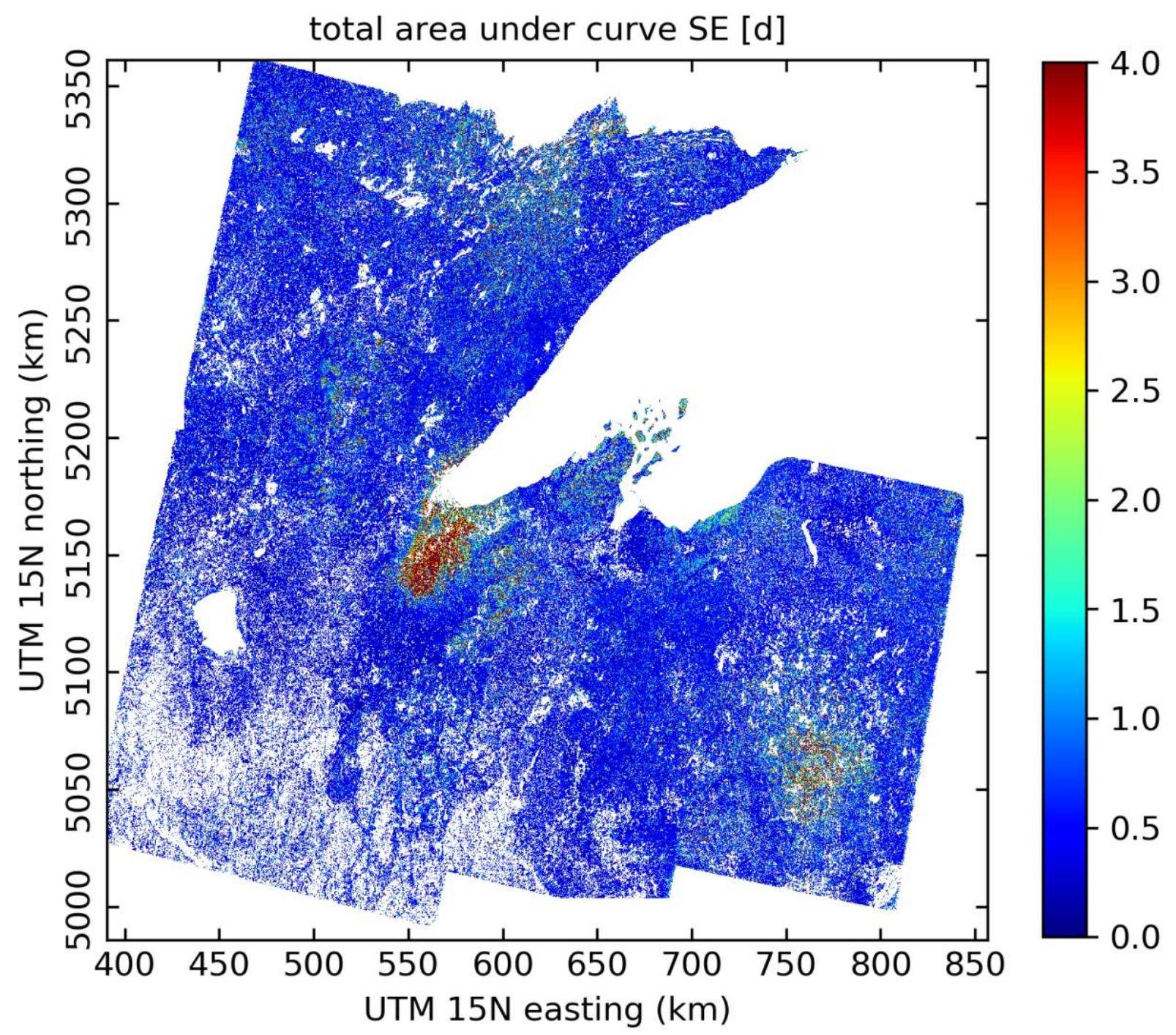

Figure 6.46: Mosaic NDII-based total area under fitted curve (TA) standard error (StdErr). Compare with Fig. 6.29.

Based on a $50 \%$ threshold on the cumulative phenological variability explained by these factors, as demonstrated for P26R27 (see Fig. 6.16), the top-weighted climatological indicators for each footprint are listed in Table 6.4. In the northwestern footprints (P26R27, P27R27, and P27R28) GDD is ranked as the most influential climatological indicators, while $G D D$ with $T_{\text {base }}=0^{\circ} \mathrm{C}$ and the 60 - and 90-day averages for $T_{\text {avg }}$ rank higher than $G D D$ in the southeastern footprints (P25R28 and P26R28). Numerous additional indicators are found to rank highly, though in different orders, across all footprints. Some climatological indicators stand out because of their relative importance in just one or two footprints. For example, $C D D$, the 60 -day average of $V P D$ (as a proxy for humidity), and the 365-day total precipitation 


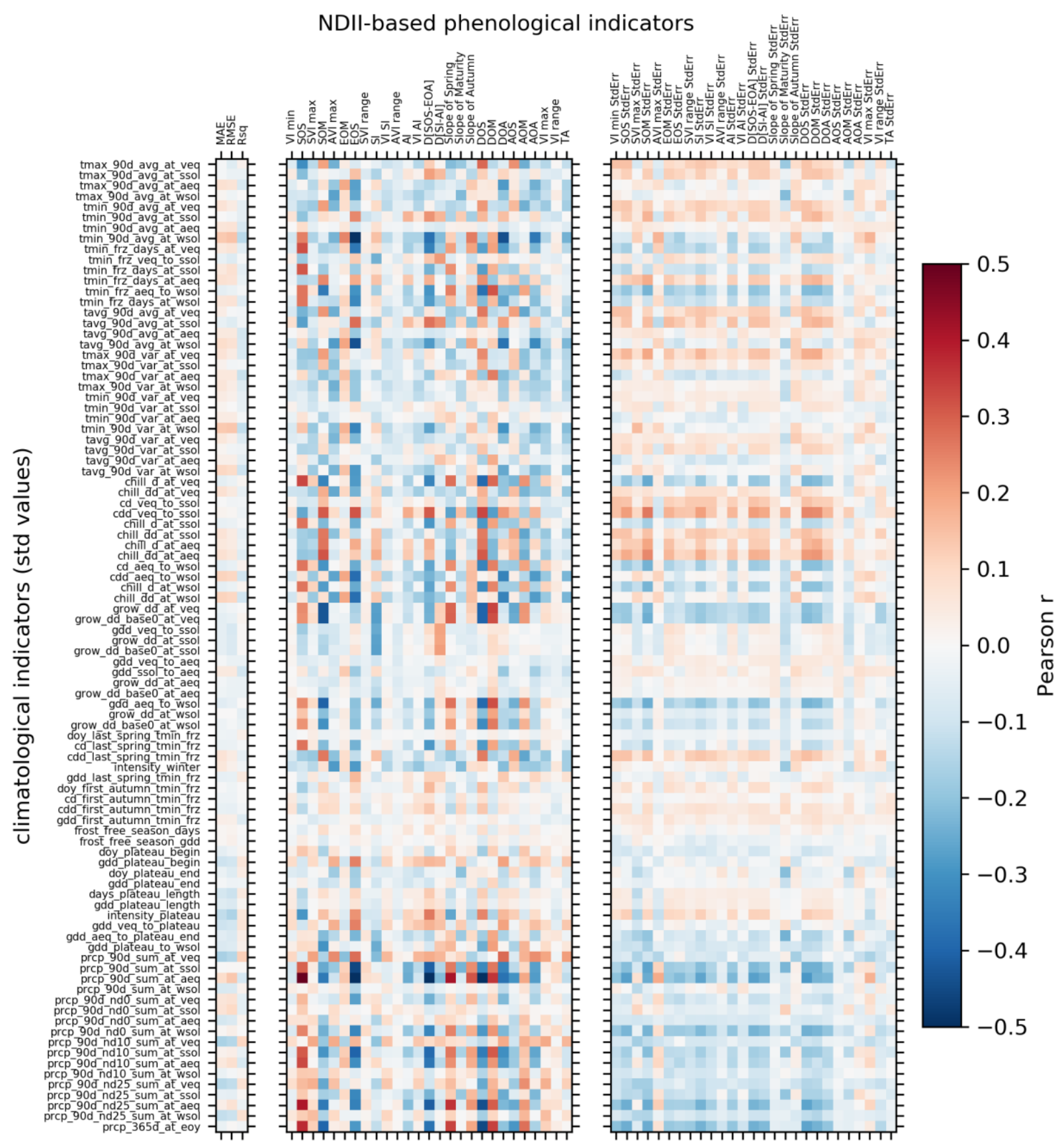

Figure 6.47: Summary of correlations for $N D I I$-based mean phenological curve indicators (left columns) and their standard errors (StdErr, right columns) with climatological indicator variance (StDev) values for regional mosaics. Climatological indicators are listed in Tables 3.1-3.5, and phenological indicators are listed in Table 4.1 and illustrated in Fig. 4.2. 


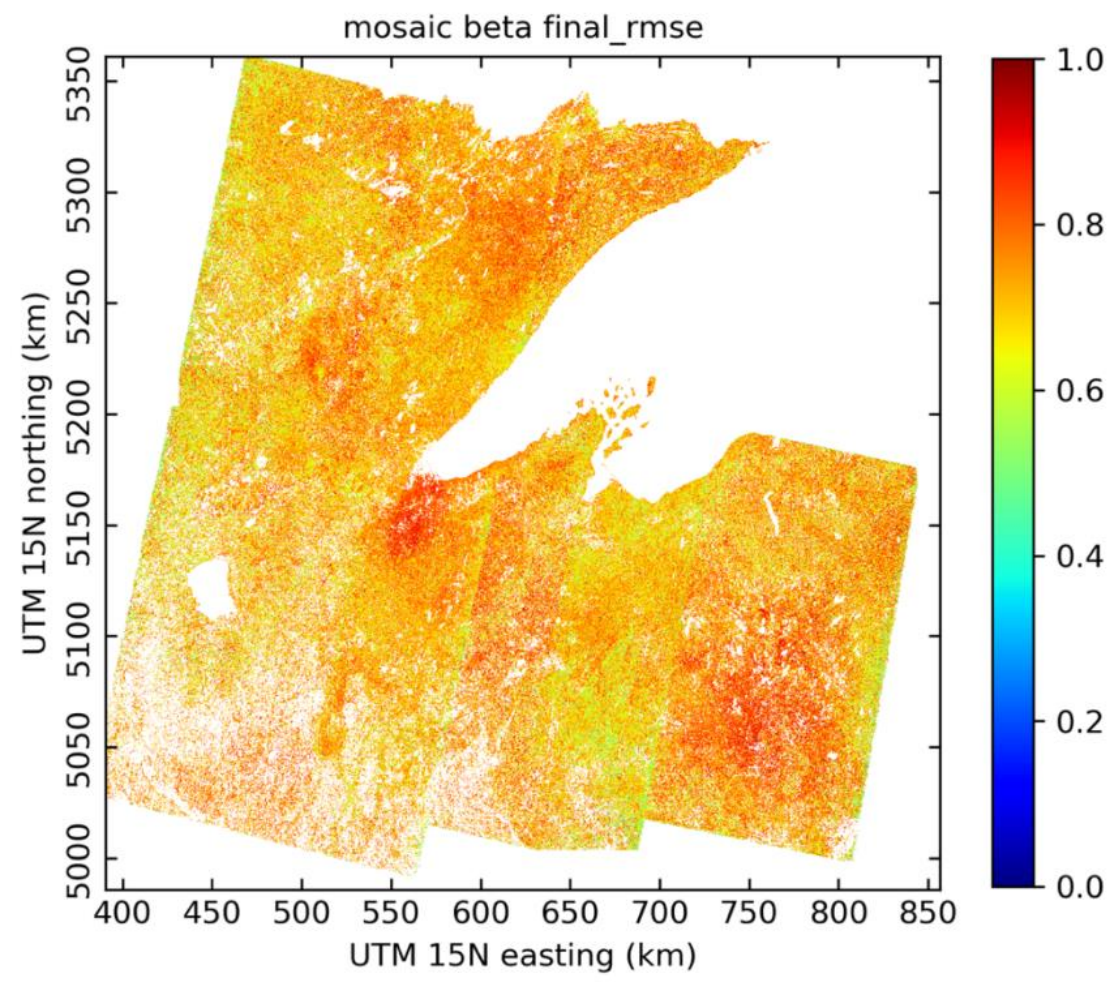

Figure 6.48: Mosaic PLSR-based RMSE based on NDII residuals.

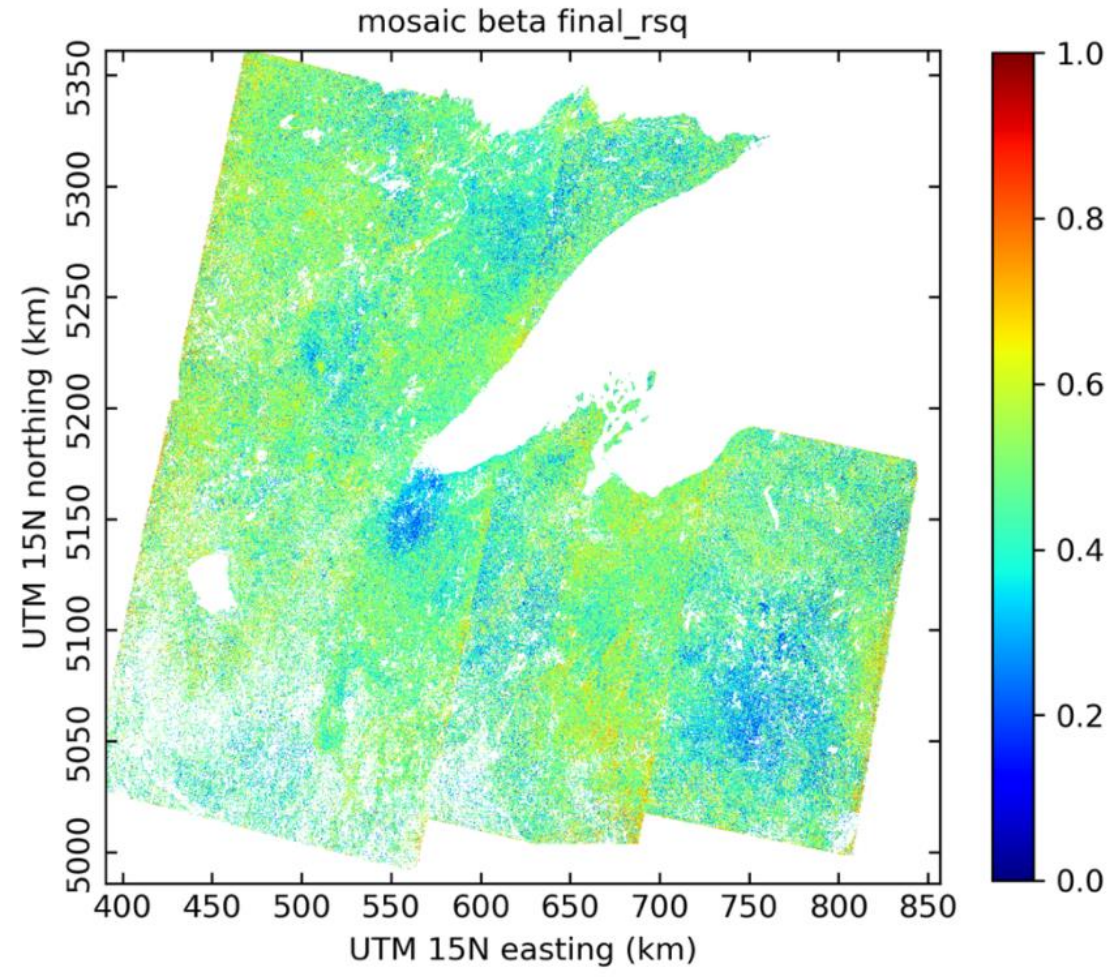

Figure 6.49: Mosaic PLSR-based $r^{2}$ based on NDII residuals. 
P25R28 NDII fractional effective coefficient

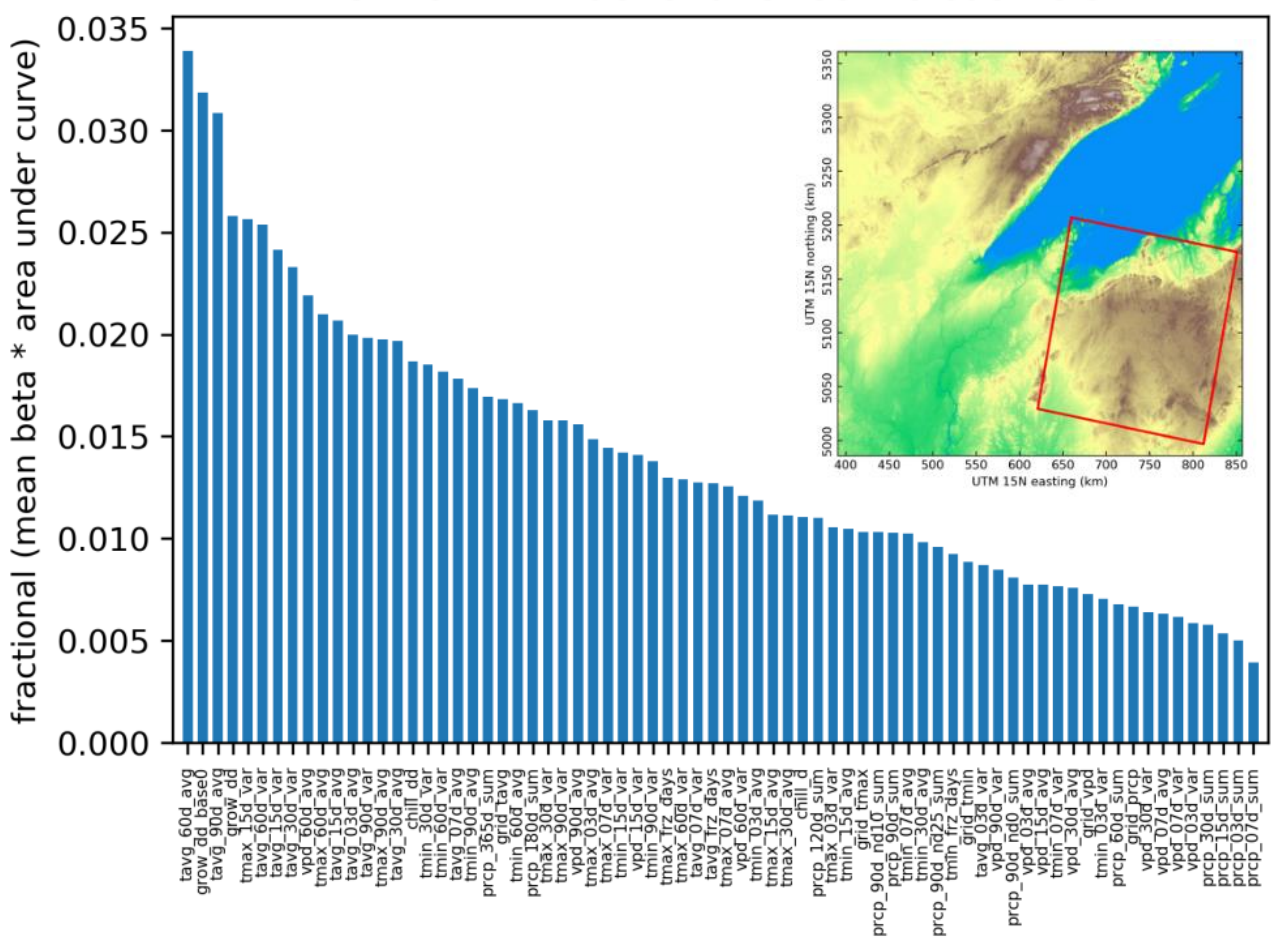

Figure 6.50: P25R28 PLSR overall effective coefficients using NDII, with variable names as given in Tables 3.1, 3.3, and 3.5.

P26R28 NDII fractional effective coefficient

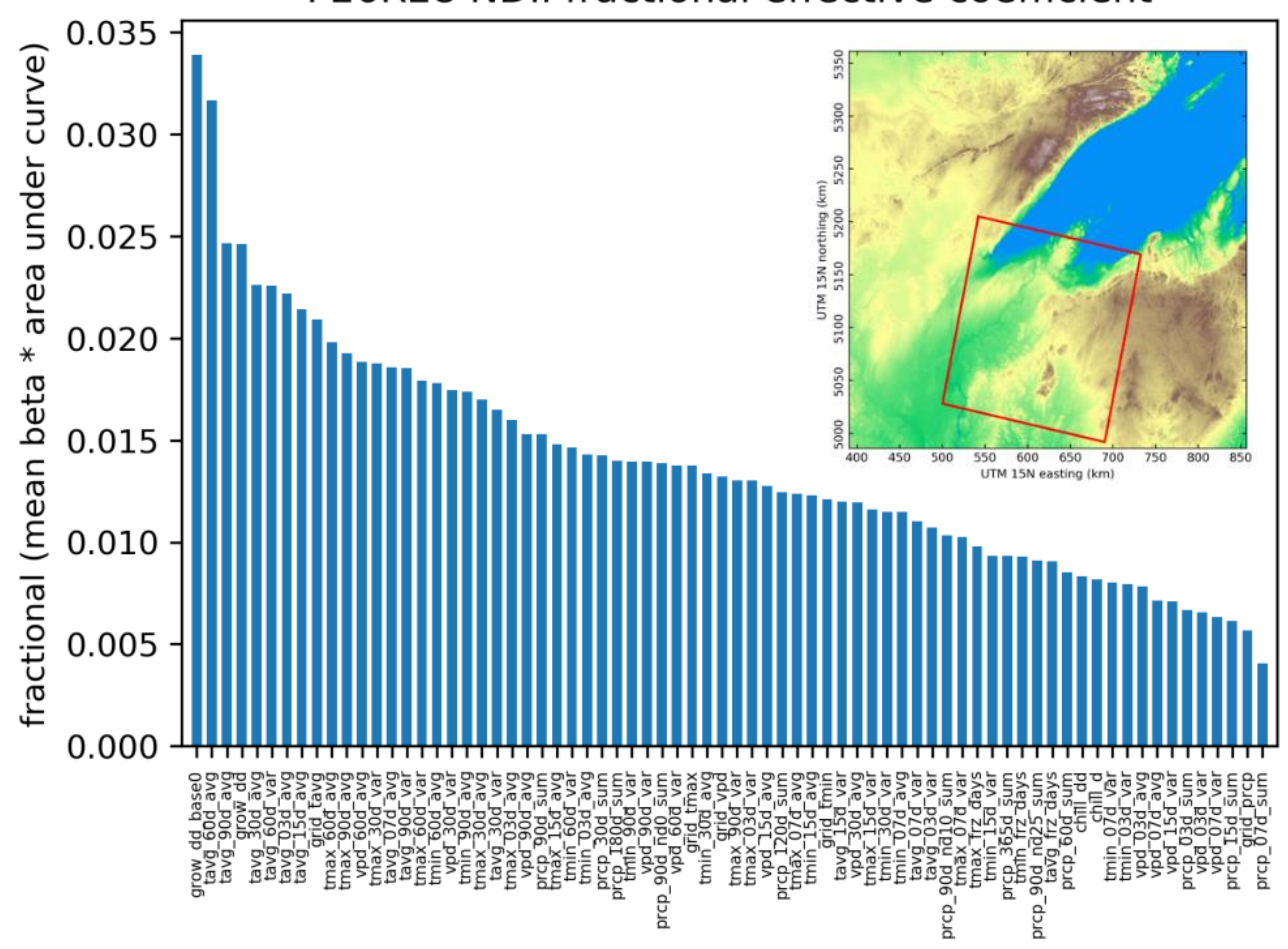

Figure 6.51: P26R28 PLSR overall effective coefficients using NDII, with variable names as given in Tables 3.1, 3.3, and 3.5. 
P27R27 NDII fractional effective coefficient

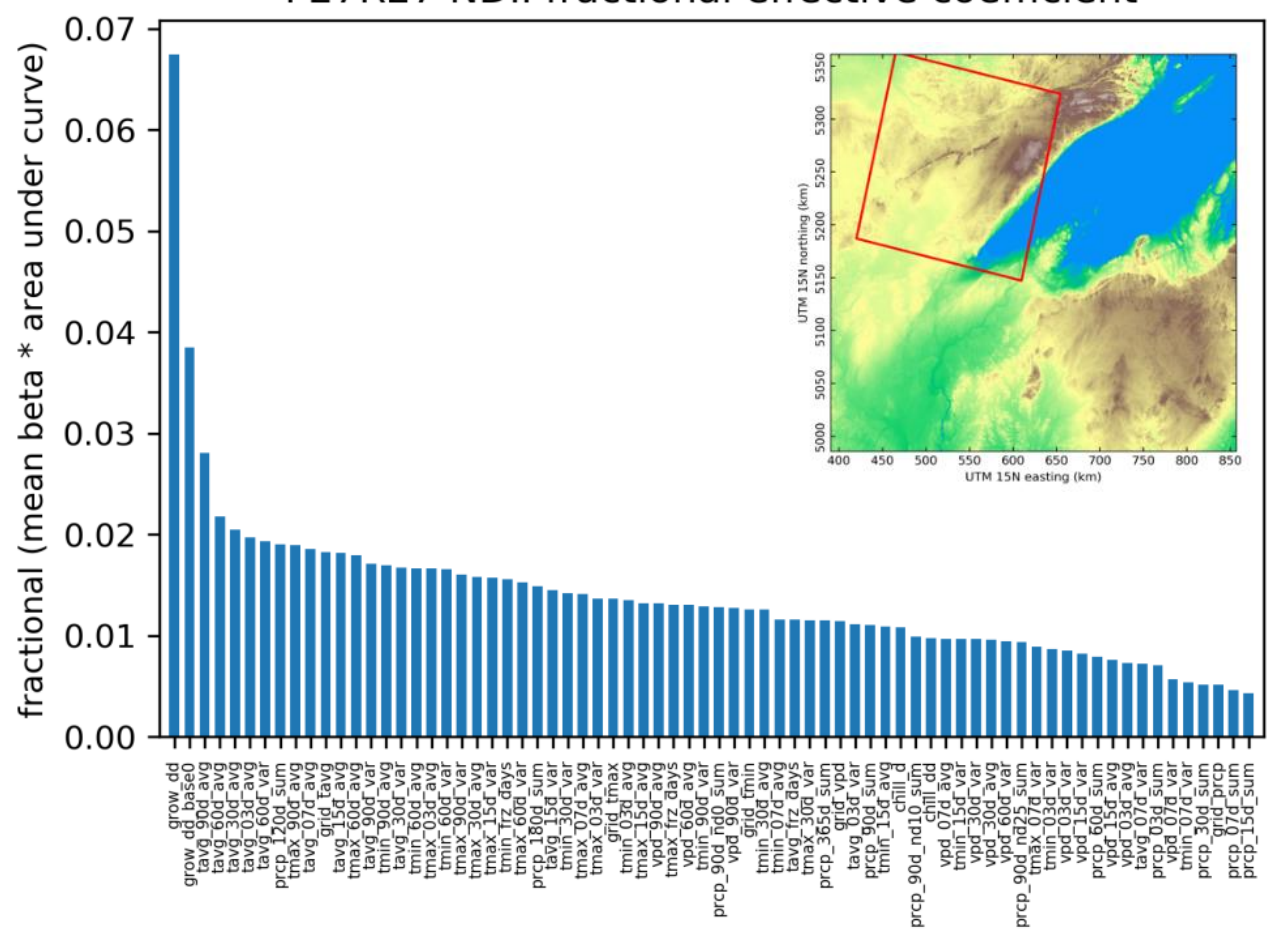

Figure 6.52: P27R27 PLSR overall effective coefficients using NDII, with variable names as given in Tables 3.1, 3.3, and 3.5.

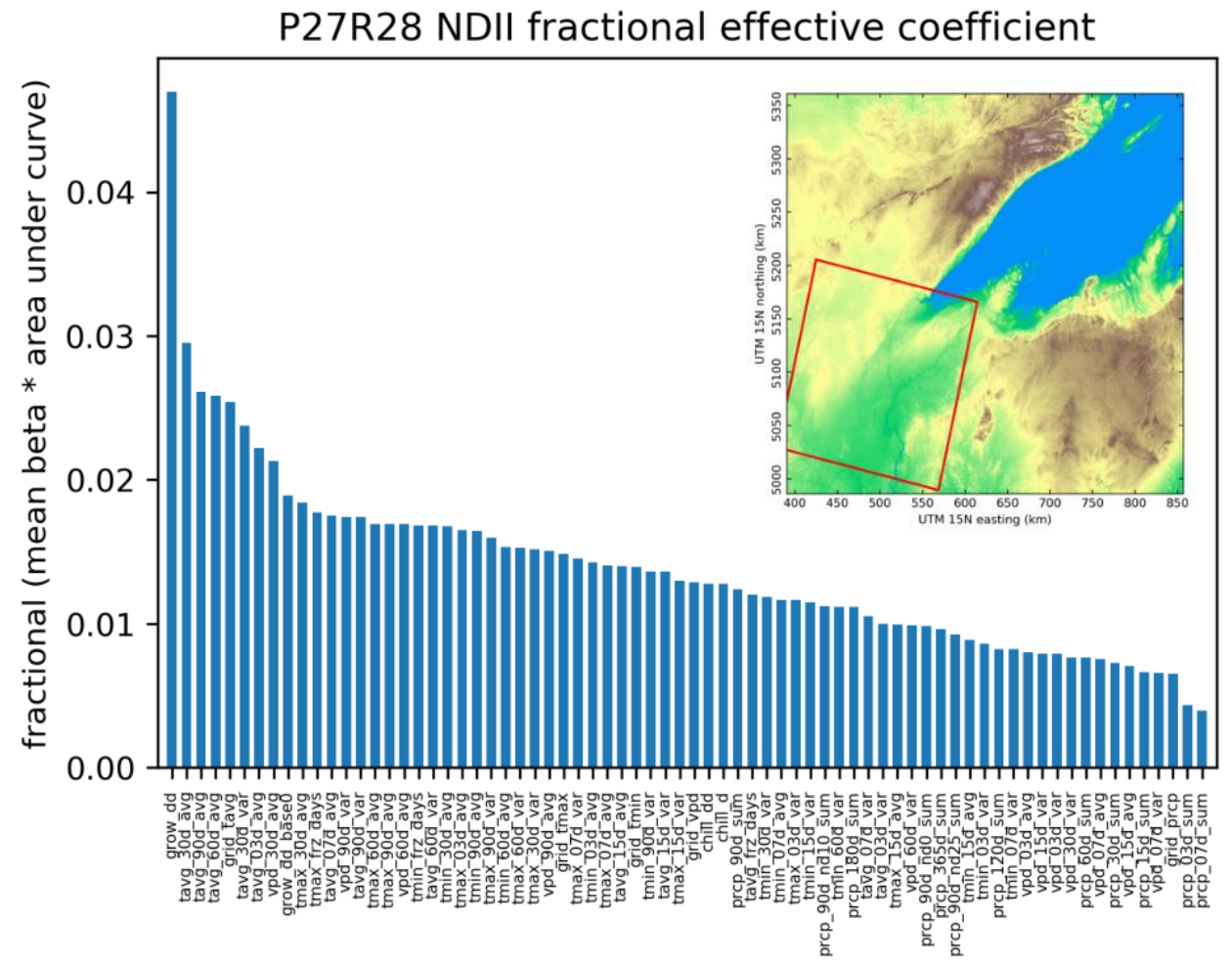

Figure 6.53: P27R28 PLSR overall effective coefficients using NDII, with variable names as given in Tables 3.1, 3.3, and 3.5. 
Table 6.4: Minimum number of climatological indicators (with their normalized effective PLSR coefficients) required to reach a threshold of $50 \%$ of the phenological variability explained by the PLSR model in each footprint. Indicator abbreviations are listed and defined in Tables 3.1-3.5. See also Fig. 6.50 for footprint P25R28, Figs. 6.15 and 6.16 for footprint P26R27, Fig. 6.51 for footprint P26R28, Fig. 5.52 for footprint P27R27, and Fig. 6.53 for footprint P27R28.

\begin{tabular}{|c|c|c|c|c|}
\hline P25R28 & P26R27 & P26R28 & P27R27 & P27R28 \\
\hline tavg 60d avg (0.034) & grow dd $(0.061)$ & grow dd base $0(0.034)$ & grow dd $(0.067)$ & grow dd (0.047) \\
\hline grow_dd_base $0(0.032)$ & tavg_90d_avg $(0.036)$ & tavg_60d_avg $(0.032)$ & grow_dd_base $0(0.039)$ & tavg $30 \mathrm{~d}$ avg $(0.030)$ \\
\hline tavg_90d_avg (0.031) & tavg_60d_avg $(0.024)$ & tavg_90d_avg $(0.025)$ & tavg $90 \mathrm{~d}$ avg $(0.028)$ & tavg $90 \mathrm{~d}$ avg $(0.026)$ \\
\hline grow_dd $(0.026)$ & grow_dd_base0 $0(0.024)$ & grow_dd $(0.025)$ & tavg_60d_avg $(0.022)$ & tavg_60d_avg $(0.026)$ \\
\hline tmax_15d_var $(0.026)$ & tavg_30d_avg (0.022) & tavg_30d_avg $(0.023)$ & tavg_30d_avg $(0.021)$ & grid_tavg $(0.025)$ \\
\hline tavg_60d_var $(0.025)$ & grid_tavg $(0.022)$ & tavg_60d_var $(0.023)$ & tavg_03d_avg $(0.020)$ & tavg_30d_var (0.024) \\
\hline tavg_15d_var (0.024) & tavg_60d_var (0.021) & tavg_03d_avg $(0.022)$ & tavg_60d_var (0.019) & tavg_03d_avg $(0.022)$ \\
\hline tavg $30 \mathrm{~d}$ var $(0.023)$ & $\operatorname{tmax} 90 \bar{d}$ avg $(0.019)$ & $\operatorname{tavg} 15 \mathrm{~d}$ avg $(0.021)$ & prcp $120 \overline{\mathrm{d}} \operatorname{sum}(0.019)$ & vpd $30 \mathrm{~d}$ avg $(0.021)$ \\
\hline vpd_60d_avg (0.022) & tmax_60d_avg $(0.019)$ & grid_tavg $(0.021)$ & tmax_90d_avg (0.019) & grow dd base $0(0.019)$ \\
\hline $\operatorname{tmax} 60 \overline{\mathrm{d}} \operatorname{avg}(0.021)$ & tavg $03 \mathrm{~d}$ avg $(0.018)$ & $\operatorname{tmax} 60 \mathrm{~d}$ avg $(0.020)$ & tavg $07 \mathrm{~d}$ avg $(0.019)$ & $\operatorname{tmax} 30 \overline{\mathrm{d}} \operatorname{avg}(0.018)$ \\
\hline tavg_15d_avg $(0.021)$ & tavg_07d_avg (0.018) & tmax_90d_avg $(0.019)$ & grid_tavg $(0.018)$ & tmax_frz_days $(0.018)$ \\
\hline tavg_03d_avg $(0.020)$ & tmin_90d_avg $(0.018)$ & vpd_-60d_avg (0.019) & tavg_15d_avg (0.018) & tavg_07d_avg (0.018) \\
\hline tavg_90d_var $(0.020)$ & $\operatorname{tmin}-60 \mathrm{~d}$-avg $(0.018)$ & tmax $30 \overline{\mathrm{d}}$-var $(0.019)$ & tmax_60 d avg $(0.018)$ & vpd_90d_var $(0.017)$ \\
\hline tmax_90d_avg $(0.020)$ & tavg_90d_var (0.017) & tavg_07d_avg (0.019) & tavg_90d_var (0.017) & tavg_90d_var $(0.017)$ \\
\hline $\operatorname{tavg} 30 \mathrm{~d}$ avg $(0.020)$ & $\operatorname{tmax} 30 \bar{d}$ avg $(0.017)$ & tavg 90d var (0.019) & $\operatorname{tmin} 90 \mathrm{~d}$ avg $(0.017)$ & $\operatorname{tmax} 60 \overline{\mathrm{d}} \operatorname{avg}(0.017)$ \\
\hline chill dd (0.019) & grid tmax $(0.017)$ & $\operatorname{tmax} 60 \overline{\mathrm{d}} \operatorname{var}(0.018)$ & $\operatorname{tavg} 30 \mathrm{~d}^{-} \operatorname{var}(0.017)$ & $\operatorname{tmax} 90 \mathrm{~d}^{-}$avg $(0.017)$ \\
\hline tmin_30d_var $(0.019)$ & vpd_90d_var $(0.017)$ & tmin_60d_avg $(0.018)$ & $\operatorname{tmin} 600 \bar{d} \_$avg $(0.017)$ & vpd_tod_āvg (0.017) \\
\hline tmin_60d_var $(0.018)$ & tavg_15d_avg (0.017) & vpd_30d_var (0.018) & tmax_03d_avg $(0.017)$ & tmin_frz_days $(0.017)$ \\
\hline tavg_07d_avg (0.018) & tmax_90d_var $(0.016)$ & tmin_90d_avg $(0.017)$ & $\operatorname{tmin} 60 \mathrm{~d} \_\operatorname{var}(0.017)$ & tavg_60d_var (0.017) \\
\hline tmin_90d_avg $(0.017)$ & vpd_60d_avg (0.016) & tmax_30d_avg (0.017) & tmax_90d_var $(0.016)$ & tmin_30d_avg $(0.017)$ \\
\hline prcp_365d_sum $(0.017)$ & tmax_ $07 \bar{d} \_a v g(0.016)$ & tavg_30d_var (0.017) & tmax_30d_avg $(0.016)$ & tmax_03d_avg $(0.017)$ \\
\hline grid_tavg $(0.017)$ & tmax_60d_var $(0.016)$ & tmax_03d_avg $(0.016)$ & $\operatorname{tmax} 15 \mathrm{~d}$ var $(0.016)$ & $\operatorname{tmin} 90 \mathrm{~d}$ avg $(0.016)$ \\
\hline \multirow{3}{*}{ tmin_60d_avg (0.017) } & $\operatorname{tavg} 30 \mathrm{~d} \operatorname{var}(0.016)$ & vpd $\overline{9} 0 \mathrm{~d}$ avg (0.015) & $\operatorname{tmin}$ frz days $(0.016)$ & $\operatorname{tmax} 90 \bar{d} \operatorname{var}(0.016)$ \\
\hline & tmin_60d_var $(0.016)$ & prcp_90d_sum $(0.015)$ & tmax_60)_var (0.015) & tmin_60d_avg $(0.015)$ \\
\hline & & tmax_15d_avg (0.015) & & tmax_60d_var $(0.015)$ \\
\hline Count: $23(50.47 \%)$ & Count: $24(50.16 \%)$ & Count: $25(50.37 \%)$ & Count: $24(50.50 \%)$ & Count: $25(50.91 \%)$ \\
\hline
\end{tabular}

appear important only in the P25R28 footprint, possibly due to both cold- and warm-season effects of

Lake Superior on downwind areas. As discussed above, the extension of cold weather into the Spring can

play a significant role in the timing of early growing-season indicators in that area where lake-effects are

prominent. Precipitation totals over shorter durations appear important in other footprints: the 120-day

precipitation appears relatively high in the list for footprint P27R27, and the 90-day precipitation appears

much lower on the list in footprint P26R28. The count of $F D$ using $T_{\min }$ (i.e. freezing nights, generally)

appears in the list of influential indicators for footprint P27R27, but the count of $F D$ using $T_{\max }$ (i.e.

freezing days, generally) appears influential (and higher on the list) for footprint P27R28.

As for the mean phenological and climatological indicators shown above in Figs. 6.32-6.40, I

examined some of the more salient individual correlations between phenological and climatological

variability that appear in Fig. 6.47. The variability (StdErr) in DOS, shown above in Fig. 6.42, is

compared with the variability (StDev) in Spring $C D D$ in Fig. 6.54a and with that in Spring total 
precipitation in Fig. 6.54b. The positive correlation between Spring $C D D$ and $D O S$ variabilities is to be expected; as suggested above, seasonal cold leads to greater variability and thus greater uncertainty in the progression of any single growing season. Figure 6.55 a shows that variability in the date of $S O M$ is correlated with variability in the Spring accumulation of $C D D$. Conversely, as for heat (also as suggested above), greater variability in Spring precipitation seems to have a stabilizing effect on $D O S$ variability (Fig. 6.54b), and greater variability in total annual precipitation is related to a more stable inflection-based growing season (Fig. 6.55b). Figure 6.56 indicates that greater variability in Autumn $G D D$ accumulations and in Autumn precipitation days are both associated with reduced variability in $D O M$, likely through meteorological influences at the onset of the senescence phase in the phenological cycle.

\subsubsection{Phenological Prediction}

Region-wide error metrics for the overall prediction capability of the NDII-based full phenological model, which includes the fitted mean phenological curve and climate-informed PLSRbased prediction of residuals, are shown in Figs. 6.57 and 6.58 for $R M S E$ and $r^{2}$, respectively. With the addition of the climatologically-influenced model component, study area mean RMSE is reduced from 0.067 (Fig. 18) to 0.060 (Fig. 6.57), mean $r^{2}$ improves from 0.635 (Fig. 6.19) to 0.936 (Fig. 6.58), an increase of $47.4 \%$, and mean $M A E$ (not shown) is reduced from 0.049 to 0.034 . There are no locations in my study area where the goodness-of-fit of the predicted phenology is not improved by this climatesensitive modeling process, even taking into account the inclusion of sequestered (disturbance) observations in the observation time series. These disturbance areas are more apparent in the study area map of RMSE, especially in northeastern Minnesota and in lake effect areas on the southern shore of Lake Superior, where increases in $R M S E$ are shown. Continued exclusion of those disturbed observations would leave an even clearer indication of overall improvement to regional error metrics with the inclusion of the climate-sensitive component of this phenological model. Overall, I can confidently conclude that 

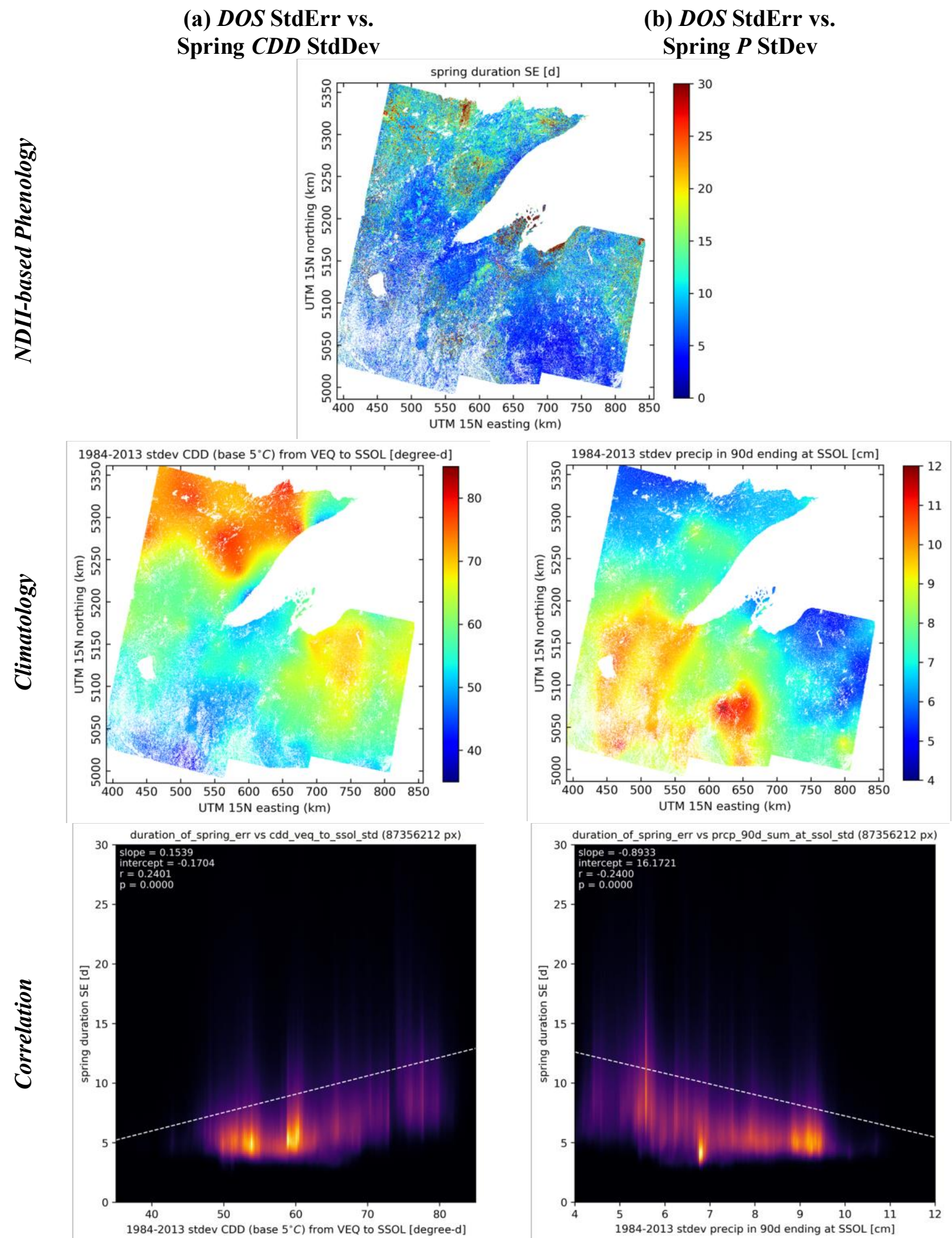

Figure 6.54: Correlations between (a) DOS StdErr and Spring CDD StDev, and (b) DOS StdErr and Spring $P$ StDev. 
(a) SOM StdErr vs. Spring CDD StdDev
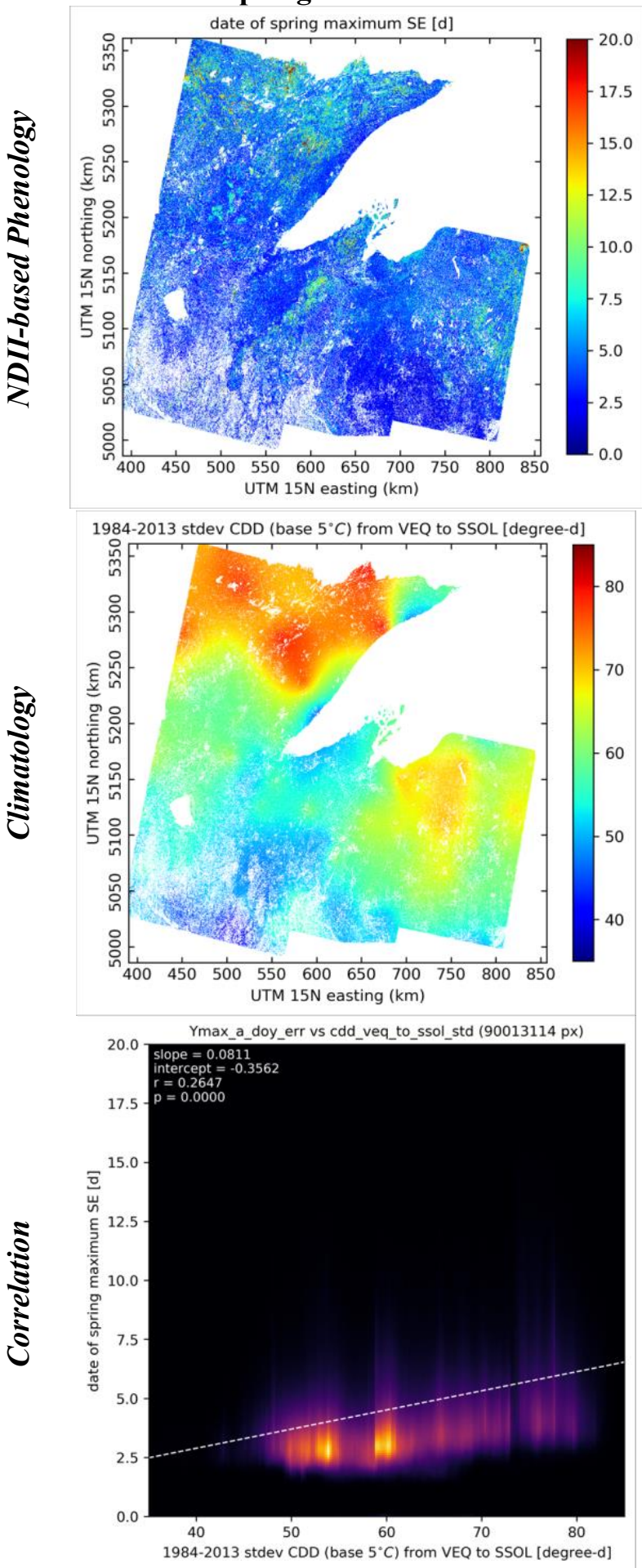

(b) D[SI-AI] StdErr vs. Annual P StDev
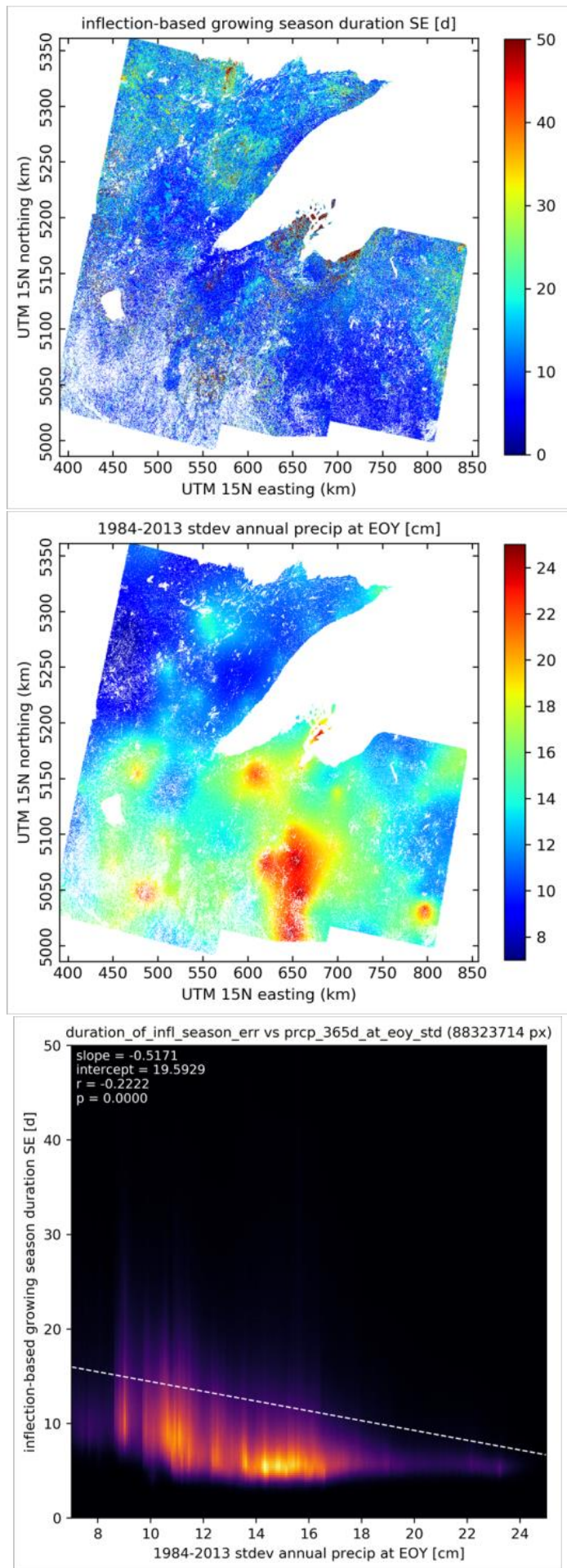

Figure 6.55: Correlations between (a) SOM StdErr and Spring CDD StDev, and (b) D[SI-AI] StdErr and annual $P$ StDev. 
(a) DOM StdErr vs. Autumn GDD StdDev (b) DOM StdErr vs.

Autumn $P$ days StDev
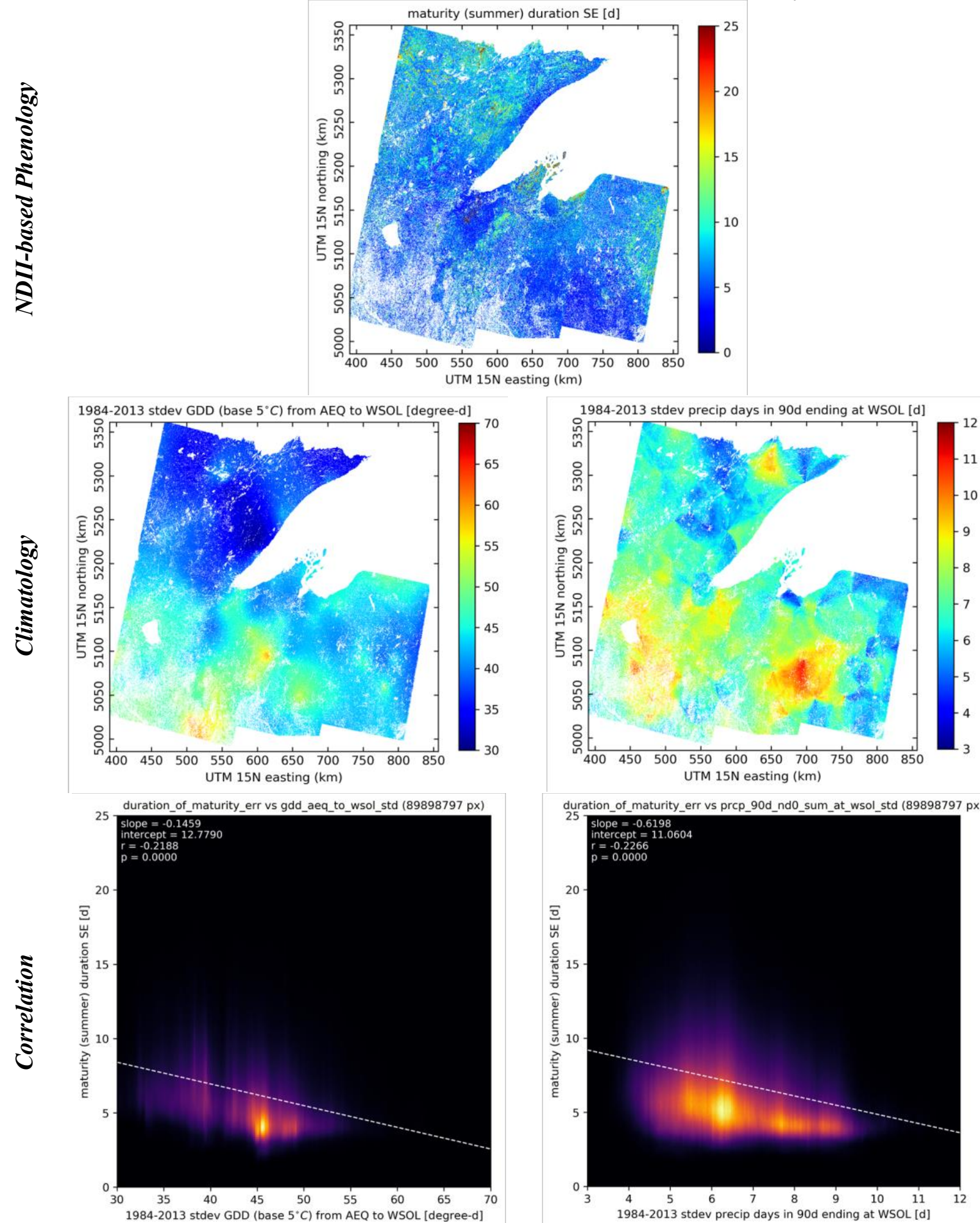

Figure 6.56: Correlations between (a) DOM StdErr and Autumn GDD StDev, and (b) DOM StdErr and Autumn $P$ StDev. 


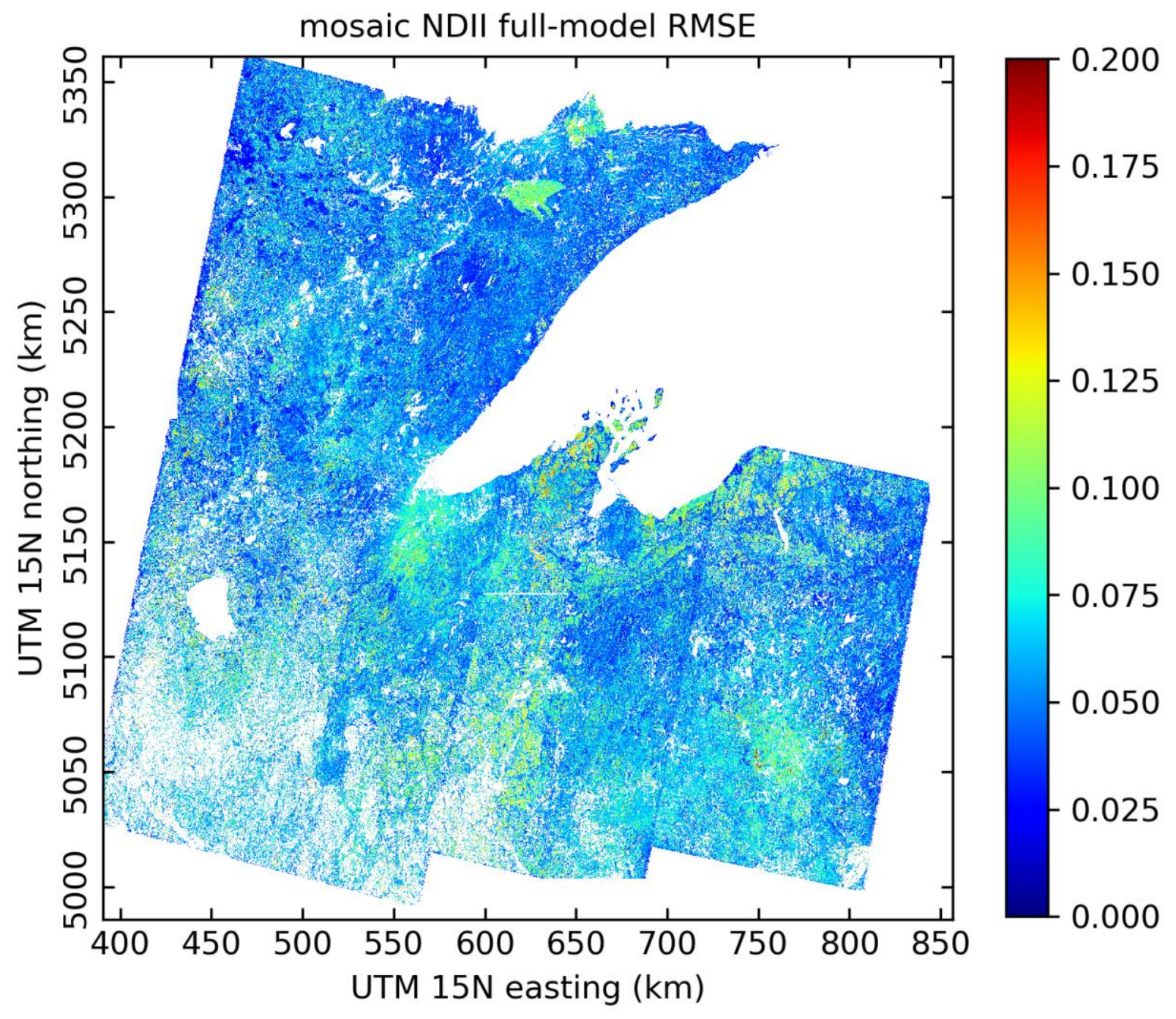

Figure 6.57: Mosaic NDII-based full phenological model RMSE. Compare with the mean phenological curve RMSE in Fig. 6.18 and the PLSR-based RMSE in Fig. 6.48.

analysis process can improve predictive capability for any vegetation index used, and that significant improvements in climate-sensitive forest phenological prediction are possible when using NDII as the signal vegetation index.

\subsection{Summary and Discussion}

While I have attempted to avoid causal language in my analysis of Landsat-derived, NDII-based phenological model results and their correlations with key climatological indicators, there is little doubt 


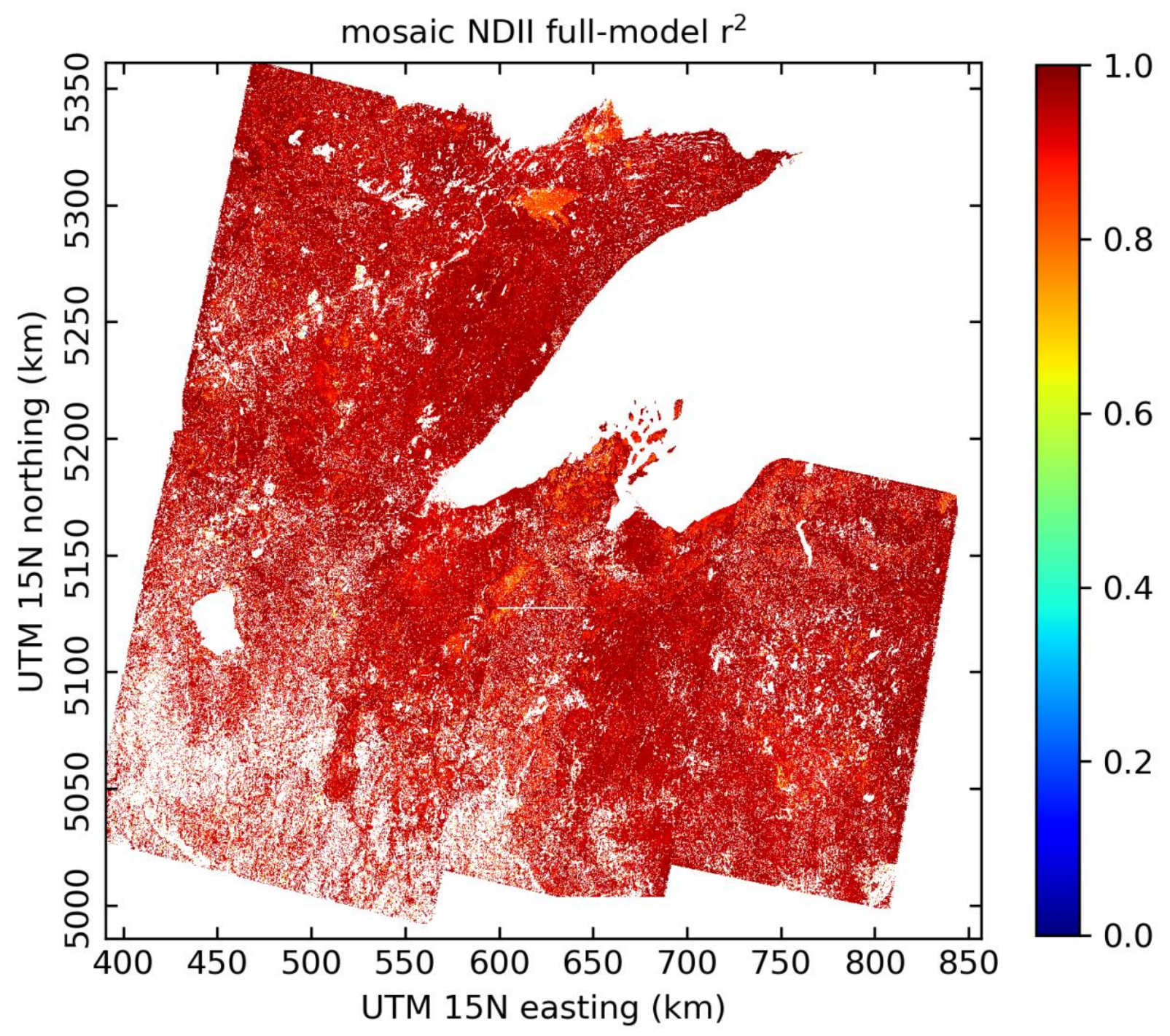

Figure 6.58: Mosaic NDII-based full phenological model $r^{2}$. Compare with the mean phenological curve $r^{2}$ in Fig. 6.19 and the PLSR-based $r^{2}$ in Fig. 6.49.

that the progression of the annual phenological cycle is closely related to seasonal temperatures and the availability of sunlight and moisture to facilitate leaf expansion, photosynthesis, and growth processes. Key indicators in seasonal meteorology and climatology thus exert an influence on forest phenology, while biology plays the role of interpreting and responding to those cues. I cannot account in this model for forest (and individual tree) biology and phenotypic traits, especially with their wide variation on the basis of genetics (including species), growth and disturbance (including stress) history, resource competition, nutrient availability, and lifelong environmental influences. The model of seasonal forest 
phenology that I have presented and examined here thus explains a portion, but not all, of the observed forest phenological variability by focusing on the long-term mean and short-term variability of those climatological influences within the limits of my study period.

With that caveat, there are a number of key findings and conclusions to be drawn from the analyses presented above, including:

- large differences among vegetation indexes (NDVI, EVI, and NDII) in resulting long-term mean phenological curves and in the degrees to which climatologically-based PLSR models using each index could explain residual, interannual variability;

- geographical patterns in NDII minima and seasonal NDII range that are consistent with land cover types, especially areas of relatively stable deciduous and evergreen forest;

- $\quad$ strong correlations between measures of mean Spring (green-up) and Summer (mature) phenological periods with climatological indicators;

- a longer Winter season is generally associated with a slower accumulation of $G D D$ in Spring, resulting in a longer period of green-up, and typically with lower maximum values of $N D I I$ at the phenological transition to the mature period;

- rapid accumulation of $G D D$ during a warm Spring season is generally associated with a shorter period of green-up and higher maximum NDII values at the transition to maturity;

- topographic features, where elevation generally results in cooler seasonal temperatures and thus a shorter growing season, are clearly evident in the mean phenology;

- the phenological growing season, based on the time and/or $G D D$ accumulation between the inflection points in the green-up and senescence limbs of the phenological curve, is not statistically correlated with either the frost-based or $C D$ plateau-based climatological growing seasons; 
- the duration of the mature phenological period, between green-up and senescence, is positively correlated with the frost-based climatological growing season and even more so with the plateau-based climatological growing season;

- the date of the phenological transition to the mature phenological phase is correlated with the date of the last Spring frost (freezing night) and even more so with the start date of the $C D$ plateau;

- a shorter phenological green-up period is associated with (and may be facilitated by) greater Spring precipitation, and the date of transition to the mature period generally comes earlier in overall wet years;

- a longer mature period (before the transition to senescence) is associated with greater Summer precipitation, and a dry Summer is (although less strongly) associated with an earlier transition to phenological senescence;

- few strong correlations are available to help explain the timing or duration of phenological senescence, although a wet Summer and fewer precipitation days in Autumn are both associated with a shorter phenological Autumn;

- PLSR-based modeling of phenological residuals using climatological indicators can significantly improve the simulation of observed NDII values throughout the growing season and may provide a greater level of accuracy to LSMs than the mean phenology alone.

A number of these findings can explain crucial variability in the transition seasons, specifically the greenup and senescence phenophases.

\subsubsection{Phenological Variability in Spring}

The general course of the phenological Spring can be outlined from the correlations and associations described above, especially the opposite correlations of $S O S$ and $S O M$ with climatological indicators. A climatological factor that is associated with an earlier SOM (at the end of phenological 
Spring), such as a greater accumulation of Spring $G D D$, is generally associated (in my study area) with a later $S O S$ and a shorter DOS. It is also generally associated with a greater Spring NDII maximum, and all of these lead to a steeper Slope of Spring on the phenological curve. The SI occurs earlier, though this does not necessarily mean a longer growing season $(D[S I-A I])$. Provided the Slope of Spring does not exceed the forest's capacity for Spring growth, given adequate moisture and nutrients, an earlier transition into maturity is the expected result of a warm Spring season. Although this might seem an intuitive conclusion, many of these relationships among Spring phenological indicators and with Spring seasonal climatology have not been previously elucidated.

A climatological factor associated with a later SOM, such as greater CSI (i.e. a colder Winter), is apparently associated with an earlier $S O S$ in my study area. This leads to a longer $D O S$, but is also associated with a lower Spring NDII maximum, adding up to a shallower Slope of Spring. However, the resulting later $S I$ does not necessarily mean that a shorter inflection-based growing season $(D[S I-A I])$ will ensue. This collection of correlations presents some seemingly counterintuitive associations, for which the mean phenology curve is likely at fault: the earlier SOS may be a result of limitations imposed on the curve-fitting procedure, and the colder Winter may have led to snow lasting longer into the Spring, which would have led to masking or overall exclusion from the Landsat scene collection that I developed, potentially depriving the model of crucial early-season data. It is possible that this model artifact also leads to the lower perceived Spring NDII maximum but it is also possible that canopy development through a slow Spring simply reaches a lower maximum before the SOM. While an adequate period of cold weather over the Winter is required for many species to meet endodormancy requirements before Spring budbreak [Rohde and Bhalerao, 2007; Viherä-Aarnio et al., 2014], the patterns and mechanisms by which overall Winter intensity (instead of duration) might influence subsequent Spring growth and maximum canopy greenness would be an interesting subject for future work. 


\subsubsection{Phenological Variability in Autumn}

Significantly fewer strong correlations between Autumn phenological indicators and climatological indicators are evident in Figs. 6.31 and 6.45. A few of those most prominent associations, all oriented on the mean DOA, have been discussed above and shown in Figs. 6.39 and 6.40. Phenological senescence in Autumn may well be the end result of a full growing season [Keenan and Richardson, 2015] with numerous and complex associations between the Spring and mature periods and climatological influences, but it remains unlikely that the onset of senescence is not associated with environmental cues. One of the most commonly cited senescence cues is photoperiod [Keskitalo et al., 2005; Fracheboud et al., 2009; Bauerle et al., 2012], which diminishes rapidly through the Autumn season (though no faster than it increases rapidly in the Spring, of course) and might cross a threshold below which the tree's investment in photosynthesis is no longer adequately returned. Another frequentlycited cue is the onset of the cold season, when lower temperatures mean slower photosynthetic activity and, again, the tree's return on investment becomes too low. A combination of these mechanisms has also been proposed [Delpierre et al., 2009; Archetti et al., 2013]. Part of the senescence process in many species involves the withdrawal of chlorophyll and other nutrients from leaves into the tree [Nordell and Karlsson, 1995; Killingbeck, 1996; Niinemets and Tamm, 2005] in preparation for use next spring, exposing other leaf colors in part as a byproduct of preparations for winter and the subsequent growing season. The environmental cues prompting these phenological transitions remain poorly understood [Estiarte and Peñuelas, 2015; Gallinat et al., 2015].

I propose here that, given the possibility of threshold-oriented processes around the end of the mature period $(E O M)$, the onset of phenological senescence may be a nonlinear process that can arise from a number of environmental cues, likely in combination, and only a fraction of which can be explained by the associations (via linear regression methods, instead of nonlinear analyses) with climatological indicators that I have explored here. Given the active participation of the tree in senescence as it recovers nutrients from the leaves for later re-use, in bud formation for release next Spring, and in the abscission process, there are a number of biological process that may far outweigh climatological 
influences on Autumn phenology as I have measured it here. Far from being a neglected season in phenological research [Gallinat et al., 2015], forest senescence in Autumn remains a rich area for exploration, experimentation, and future work. 


\section{CHAPTER 7}

\section{DISCUSSION AND CONCLUSIONS}

The research presented in this dissertation outlined methods to characterize climatological seasonality and analyze climatological influences on forest phenology using the 1984-2013 Landsat record. From these methods and analyses, I outline potential approaches to using land surface and remote sensing observations to better characterize the effects of varying phenology and its deviations on landatmosphere balance and exchange processes.

\subsection{Summary}

In Chapter 1 of this dissertation, I provided necessary background to orient this work on the improvement of forest representation in land surface models (LSMs) that support weather and climate modeling efforts. These models often use categorical forest types with static parameters linked to leaf area, which plays a crucial role in the calculations of surface energy balance and moisture exchange. Instead, the forest may be represented better by first accounting for the long-term mean phenological cycle, as many models already do, and then by using climatological information to more accurately characterize interannual phenological variability.

In Chapter 2 [Garcia et al. 2014], I presented an idealized simulation using a coupled landatmosphere modeling system to investigate the differences in land surface conditions and heat fluxes between forested and harvested (clear-cut) areas under Winter conditions. Though this represents an extreme case of changed vegetation state - a stand-replacing forest disturbance, rather than the incremental changes over an undisturbed forest phenological cycle - it provides some guidance regarding

model capabilities as well as the magnitudes of possible changes to surface fluxes that might be expected in further simulations under growing-season conditions. As described in Chapter 1, a number of vital LSM parameters and processes are tied to vegetation growth and phenological state, including land surface albedo, which exerts a dominating control on the surface energy balance, and vegetation 
transpiration, which links the water and carbon cycles at the land surface. More accurate representation of that phenological state, not just the mean phenological cycle but also the specific climatologically-driven variability about that mean in a given year, may lead to more accurate results from such experimental simulations as well as in weather forecasting and climate modeling systems.

In Chapter 3, I described the data sources for my study area, approximately $202,000 \mathrm{~km}^{2}$ covering five Landsat footprints around the extensively-forested western end of Lake Superior. These data contribute to the quantification of added value that climatology-based analyses can provide to the representation of forest phenology using remotely sensed imagery. The mixed parametric/statistical model that I used for this work was described in Chapter 4, with details regarding model assumptions and theoretical formulations leading to practical details of the model implementation. I also described there the computational considerations and resources used for application of this phenological model over $\sim 130$ million Landsat pixels in five footprints across my study area using 30 years of satellite images throughout the growing season.

To support that modeling framework application to my study area, Chapter 5 [Garcia and Townsend, 2016] described a detailed regional climatological analysis over the period 1984-2013 and congruous with the area and period of record of Landsat data used. I highlighted several notable trends consistent with regional warming and Summer drying in much of my study area. I examined spatiotemporal variability in the climatological growing season around the western end of Lake Superior, demonstrating the role of lake thermal inertia on seasonal conditions and transitions in surrounding land areas. A number of climatological indicators demonstrated clear impacts from proximity to Lake Superior, especially Spring temperatures and year-round precipitation patterns. The strongest of these influences affect the timing and pace of Spring and Autumn climatological transitions, the same times that the strongest phenological transitions are observed in green-up and senescence phases. Key conclusions regarding the potential effects of spatial and temporal climatological patterns in temperature and precipitation on forest phenology across my study area were discussed. 
Chapter 6 presented the results of my phenological model developed for this work. Phenological model calculations for the $\sim 130$ million Landsat pixels across my study area, including pre- and postprocessing tasks using a distributed computing environment, consumed more than 13 million computational hours and provided detailed analyses of mean land surface phenology and its variability at levels of detail that likely have not been examined previously for this region. I discussed numerous mean phenological indicators, such as the durations of Spring green-up and Summer maturity, as well as the date of transition between those, at the footprint scale based on model calculations using three different vegetation indexes (NDVI, EVI, and NDII). I also presented results of PLSR-based analyses of phenological variability using each of these vegetation indexes, with indications of the most influential climatological indicators across the footprint. Using NDII, I extended these analyses to the other Landsat footprints in my study area and presented these results at the regional scale. At both scales, I examined the correlations between key phenological metrics and climatological indicators. Two definitions of the climatological growing season, both the frost-based and $C D$ plateau-based seasons, are statistically uncorrelated with expected phenological indicators of the growing season, especially the start and duration of the growing season based on phenological inflection points. However, analyses identified a number of interesting correlations, especially in Spring and Summer, and even more interestingly with several precipitation measures, an advancement on phenological studies that are often focused solely on seasonal temperature measures.

\subsection{Conclusions}

A wide variety of environmental factors contribute to seasonal and year-to-year variability in forest phenology around the Upper Great Lakes. In this work, I have identified several trends showing recent and likely ongoing changes to the climatological growing season and the availability of moisture to regional forests. My results also show large spatial variability in trends such as Autumn and Winter warming, diminishing Summer precipitation, and the extension of the climatological growing season into Autumn. Drying Summers may lead to moisture stress that can impair forest carbon uptake and may 
render the forest more vulnerable to disturbance factors. Three overarching conclusions regarding my phenological analyses can be drawn from this work:

\section{Long-term climate change, and changes in seasonal temperature and precipitation regimes, have} affected (and continue to influence) forest growing season phenology around the Upper Great Lakes, potentially altering the role of those forests in the regional water and carbon cycles.

My new definition of the climatological growing season, based on a warm-season plateau in accumulated chilling days, provides information that can be combined with observations of the traditional frost-free growing season to show the speed, and potential dangers to vegetation, of the transition seasons. The timing and rates of Spring green-up and Autumn senescence can vary widely and have significant impacts on the seasonal carbon assimilation capacity of affected forest areas. The extension of warm periods into Autumn can alter the environmental cues for leaf senescence and the preparation of trees for Winter conditions, potentially affecting phenology in the following growing season as well. While meteorology changes with the season, and climate factors change from year to year, trees in the forest maintain a "memory" of these conditions through the impacts on phenology and growth in subsequent seasons and thus over their lifetime.

\section{The typical representation of land surface (especially forest) phenology in weather and climate} models, using a mean phenological curve, can be improved significantly with the consideration of interannual variability in those meteorological aspects of the environment that are known to influence seasonal forest phenology.

Knowledge of the mean land surface seasonal phenological cycle, based on a curve fitted to remote sensing observations and as commonly employed in LSMs, provides a certain measure of accuracy to land-atmosphere modeling efforts. My full model results, using both the mean phenological curve and climatology-based PLSR modeling of phenological residuals, showed considerable improvements in representation of the forest phenological state (as measured using remotely-sensed 
vegetation indexes) over the mean phenological curve alone. My analyses explored correlations between mean phenological indicators, derived from Landsat-based image time series, and climatological influences that can help explain the timing of forest canopy Spring green-up and the transition into the Summer (mature) period. Correlation-based analyses are less able to explain Autumn phenology, when senescence could be more biologically- than environmentally-driven after the physiological effects and stresses of a full growing season.

\section{Physiographic gradients, as in topography or along lakeshores, can exert significant influence on} nearby climatological conditions and thus phenological cycles, and such heterogeneity must be represented at appropriate characteristic scales in land surface models in order to capture those effects.

Contributing to the complexity of my study area, Lake Superior exerts an identifiable influence on temperature, precipitation, and thus forest phenology that varies with the seasons in surrounding (and especially downwind) land areas. Smaller lakes throughout my study area exhibit similar influences on their surrounding areas as well, but at magnitudes that vary with the lakes and their physiographic context. The warming Lake Superior, with its changing Winter ice phenology, contributes to Winter warming in the immediate vicinity of the lakeshore. One of the largest influences of Lake Superior on regional phenoclimatology is the contribution of longer ice-free periods in Autumn and Winter to lakeeffect precipitation in large forest areas south and southeast of the lake. The extension of cold periods later into Spring seems to prolong the green-up process in those areas at the time of year when seasonal leaf expansion and wood production are vital to the relative competitive advantages of different species in mixed forests, affecting both carbon sequestration and resource optimization to sustain optimum growth rates through the remainder of the warm season. Forest management in the context of climate change must consider this wide range of factors affecting the critical green-up and senescence periods bounding the growing season.

Finally, my work provides valuable support for an observational effort to understand long-term changes in vegetation phenology, including seasonal environmental cues and the influences of 
climatological variability on forest phenology. Observations of phenological processes inform our developing understanding of forest responses to recent and ongoing climate change. Feedbacks from surface conditions (including lakes, land cover, land use, snow cover, vegetation state, and forest disturbances) to the climate system [Sobolowski et al., 2010; Richardson et al., 2013; Rydzik and Desai, 2014] are integral to the co-evolution of the land-atmosphere system and thus important to any examination of regional phenoclimatology.

\subsection{Model Improvements}

This work represents an advancement in modeling Landsat phenology from climate indicators. Based on this work, I recommend testing a number of improvements to my phenological model and suggest potential applications of the analytical method I have presented here. The model itself is based on three explicit assumptions, most fundamentally the assumption of linear separability between mean and residual components, that should be tested for validity. It is well known that this assumption is not necessarily true in climatological analyses [Milly et al., 2008; Katz, 2010; Lins and Cohn, 2011], given the acceleration of climate change and its related effects in regional land-lake-atmosphere systems. Threshold responses among indicators, suggested in several of the correlations presented in Chapter 5, may indicate nonlinear behavior in the associations between climatology and phenology that I seek to represent with accuracy and fidelity. In such cases, if nonlinearity is an essential aspect of the physical system that is regularly observed, the mean and variability of an indicator may no longer be separable. Consequently, the system may become more complex to represent in, and analyze with, a statistical model. Simplifying assumptions make such analyses possible, but only to the extent of their validity.

Further, there are several aspects of my model application that call for focused improvement. First, the identification of outliers (here considered as possible disturbances) in the vegetation index time series for an individual pixel should be reconsidered with a more robust foundation, both statistically and physically. The simple criterion for sequestering outliers that I used is based entirely on the time series sample available at a given location, and not on some larger estimate of the population from which that 
series would theoretically be sampled. In this case, I would start by substituting for the existing method a more formal statistical test for outliers such as the generalized extreme Studentized deviate (ESD) method [Rosner, 1983] that can identify any number of possible outliers in a sample, an important distinction over some tests that require specification of the known number of outliers. Given the persistence of disturbance effects over time, on which disturbance identification and attribution could depend, whole segments of a single-pixel time series may be removed in order to analyze the "undisturbed" phenological cycle, and it is not known a priori (or necessarily constant from one pixel to the next) how many observations in the time series are thus affected.

Related to the identification of outliers for individual pixel locations, a check for consistency of possible disturbance events with those identified at neighboring pixels would be a valuable, but computationally costly, improvement to the model application. I am currently pursuing this spatial analysis only in post-processing, as I explore the possibilities for disturbance identification in the sequestered data points from all of the pixels processed. Though it may lengthen the time of model computation, distinction of signal and noise in the vegetation index time series at each pixel is of paramount importance so that all of the "legitimate" times are properly considered in the phenological analysis, and yet all of the legitimate disturbance events are also removed for separate analysis. Both phenology and disturbances constitute the signal at a given location; removal of noise is commonly a statistical problem, while distinction within the remaining time series for different land surface states, in this case the healthy and disturbed forest periods, requires a more physically-based approach to which my model aspires.

Next, my implementation of the PLSR process is different from relevant examples such as Wolter et al. [2008, 2009], who used a jackknife ("leave-one-out") method in combination with a more complete examination of the PLSR component space and the development of progressively more complex models to find an optimal model. As a consequence, my PLSR implementation very likely results in overfitting of the retained data and could benefit from testing against (and closer conformance to) established practices. As explained in Chapter 3, I encountered a number of instances and results in the course of model 
development that suggested some non-standard solutions in order to arrive at an acceptable result. In my own model development, using PRESS as a model metric led to poor results and necessitated my exploration of alternative metrics to aid model selection. My use of $A I C c$ was a novel approach and likely a suitable choice, especially given its use in comparing regression-based models in other applications, but that does not explain why PRESS led to poor results. As well, some applications of PLSR [Singh et al., 2015] have employed a method-specific technique that evaluates the calculated "variable importance of projection" $(V I P)$ to identify the most influential indicators (hyperspectral wavelengths in Singh et al.) in the regression process. The VIP is a model statistic that is not currently available in the SciPy implementation of the PLSR procedure and would require additional development (inside or outside of that SciPy module) for use here. Its utility in the interpretation of PLSR results, however, may far outweigh the effort invested in adding this model diagnostic.

In addition to my use of a non-traditional metric for PLSR-based model selection, I also made a choice explained in Chapter 3 regarding the number of model components (latent variables) retained. This was an imposition on the model that was intended to reduce the overall computation time but likely also led to an overestimation of model quality in the results: fixing the number of PLSR components to the number of weather and climate variables in a given regression iteration, instead of a more proper analysis of the variation in model errors with the number of regression components retained, almost certainly resulted in model overfitting. A lesser number of retained PLSR components may have provided a more parsimonious model within acceptable measures of accuracy. The methods employed by Wolter et al. [2008, 2009] provide useful guides toward improvement of the PLSR application in my model.

Finally, in the course of model application, it became apparent in my footprint-scale calculations that, where multiple and overlapping remote sensing footprints are used, individual footprint time series should be combined in those overlapping areas before a pixel's information is passed to the modeling algorithm. This is expected to produce a more accurate phenological curve for those pixels and would obviate the need for footprint mosaicking after the modeling is completed. In some locations in my study area, phenological modeling of pixels with $\sim 300-500$ available Landsat scenes may have been possible 
instead of just $\sim 150$ scenes per pixel (after cloud and cloud shadow masking), as when I applied the model on a footprint basis. Overall, and as in many applications of remote sensing time series, more collected scenes would help improve the model results to some degree. In particular, more scenes and even partial scenes (when cloud cover is extensive but not $100 \%$ ) would be useful in the transitions seasons to help better define the mean phenological curve and its variability at critical times in the growing season.

Going beyond phenological analyses, a key limitation to the detection of forest disturbance using Landsat is the issue of image timing [Masek et al., 2008; Thomas et al., 2011; Zhu et al., 2012; Zhu and Woodcock, 2014; Frantz et al., 2016]. The use of relatively clear, cloud-free images is important to change detection analysis, but the interval between images also determines the types of disturbances that can be detected and diagnosed. For quantification of permanent (or long-term) land cover change, a 5year interval as employed for NLCD updates may be sufficient. For the detection of forest fire severity, or the timing of forest harvest, an annual time series would be more useful and provide greater accuracy in post-disturbance analysis. For the observation of forest tent caterpillar outbreak events, known to have occurred at least twice in my study area during the period of analysis, a series of images at intervals of 1-2 months with at least one image around the time of greatest defoliation is necessary for the detection of that disturbance, otherwise it might be missed entirely using only satellite imagery. Extension of this work to the detection and attribution of forest disturbances, starting with those sequestered observations from the model analysis as explained in Chapter 3, will depend critically on both the frequency and the timing of collected Landsat observations.

\subsection{Applications}

There are numerous tasks to which the methods developed in this work may be applied beyond the analyses presented here and similar phenoclimatological analyses in other locations. At the least, my method of phenological modeling can be used to estimate VI values (assuming the absence of disturbance factors) in locations of cloud cover or otherwise missing data (e.g. Landsat 7 SLC-off gaps) in collected 
scenes. Because the PLSR-based method presented in Chapter 4 is sensitive to interannual variability in meteorological and climatological influences and their effects on VI residuals from the long-term mean phenological curve for a specific location, these estimated VI values would be tailored to the date and climatological context of that missing pixel in the Landsat time series, and thus also consistent with the variability among surrounding pixels for which data is available in that scene.

The estimation of VI residuals is not limited only to Landsat scene dates by this method: this approach could be used to derive the entire growing season VI profile for any year within (or potentially outside of) my study period. Though this might become computationally challenging over large areas, individual pixels of interest (or as representatives of larger areas) could be identified and, given the longterm meteorological records and climatological analyses that I have presented in Chapters 3 and 5, the daily VI values for those locations could be estimated using the mean phenological curves and climatesensitive PLSR coefficients presented in Chapters 4 and 6 . These estimates could then be compared with data collected on non-scene dates, such as daily phenocam observations where those are collected [Richardson et al., 2007b, 2009a; Keenan et al., 2014; Toomey et al., 2015; Robinson et al., 2017]. My next planned publication to be developed from this dissertation work will pursue exactly that objective, describing the phenological model developed in Chapter 4 and then demonstrating its capability to represent interannual phenological variability at selected locations in northeastern Minnesota, including comparisons of estimated VI values with data from a phenocam site in the Boundary Waters Canoe Area Wilderness near the northern edge of my analysis region.

Numerous field observations and measurement programs are undertaken every year by local, state, and national foresters to monitor forest health, suitability for harvesting, wildlife habitat, invasive species status, and other needs. Some of those observations related to the forest canopy, such as leaf area in the growing season, might be extended through the entire growing season and to unmeasured locations using the phenological modeling method demonstrated here. In association with species-specific models for tree growth and forest succession over the life cycle of a forest stand, longer-term estimates of forest canopy characteristics such as $L A I$ might be developed for use in management and planning applications. In 
addition to comparisons with other remote sensing (e.g. MODIS and Sentinel) observations and groundbased (e.g. phenocam and canopy) measurements, there is an opportunity for the association of estimated VI values and their indicated phenophases with surface ecosystem flux measurements at various forest locations, including an AmeriFlux site in northern Wisconsin [Yuan et al., 2007, 2010; Randerson et al., 2009; Richardson et al., 2009b, 2012] that is within my study area. Observed and estimated phenophases might then be used to derive the times and magnitudes of carbon sources and sinks in those and, by extension, similar locations. Better accounting for changing carbon fluxes over the growing season and from year to year could further help refine estimates of forest stand growth and succession, the availability of critical wildlife habitat, forest stand harvest planning, and our overall understanding of forest ecosystem processes.

One of my long-term objectives for this work is to develop a better understanding of recent climate and forest phenological variability in support of the development and validation of land surface models that incorporate both permanent and transient land cover change as well as seasonal vegetation processes [White et al., 1997]. There is an increasing need for improved accuracy in representation of land surface states and processes as regional and global climate models progress from relatively coarse $(\Delta \mathrm{x}=10-100$ $\mathrm{km}$ [Prein et al., 2015]) to finer representative scales. A better understanding of the coupled landatmosphere system at a range of spatial and temporal scales will enable the capability to model feedbacks between forest phenology and climate change at local scales [Peñuelas et al., 2009; Richardson et al., 2013; Brzostek et al., 2014], essential for accurate assessments of forest carbon states and their spatiotemporal variability [Desai et al., 2008; Jeong et al., 2013; Schwartz et al., 2013; Lu et al., 2015]. Ultimately, the long-term status of a forest area as a carbon source or sink, and the magnitude of that status, is determined by the daily progression of phenophases over a growing season and the interannual variability of that seasonal phenology over the period of interest. Better knowledge of how vegetation phenology varies with meteorological conditions and climatological regimes, coupled with existing (and still improving) knowledge on the relationships between phenology and vegetation biochemical 
processes, will lead to significant advances in the representation of forest carbon balance in LSMs and the improved representation of land-atmosphere flux processes in weather and climate models.

In regions such as the Great Lakes, we must exercise caution: most land-atmosphere models do not yet incorporate even large lakes in the physical system, producing significant errors in the simulation of regional temperatures and moisture fluxes [Bryan et al., 2015]. A number of researchers have pursued better parameterizations of lake processes for use in energy- and water-balance models [Plank and Shuman, 2009; Mishra et al., 2010] and for proper representation of lakes in regional climate models [Gula and Peltier, 2012; Bennington et al., 2014; Mallard et al., 2014]. It is important that large and small lakes, their surface temperatures and winter ice phenology, and their influences on vegetation in the surrounding areas are properly included in analyses of climate change effects on temperature and precipitation patterns and trends, so that regional modeling efforts [Hayhoe et al., 2010; Gula and Peltier, 2012; Mallard et al., 2014; Harding and Snyder, 2015; Notaro et al., 2015] can provide more accurate insight and guidance to scientists and decision-makers.

My analyses indicate that climatological influences are strongly heterogeneous across my study region but may be relatively homogeneous over short distances of $\sim 100 \mathrm{~km}$ or less. Analysis of climate trends in this area requires detailed examination of patterns at the sub-regional scale. We cannot assume that climatology and its influences on forest areas southeast of Lake Superior are similar to those in areas northwest of the lake, or even that climatology is consistent throughout the ecoclimatic tension zone that traverses my study area. Along the shores of Lake Superior, an analysis of climatological influences on forest phenology and health must include localized details that vary with the seasons. Different regimes of land-atmosphere interaction among these regions may require differing approaches to the identification of dominant influences on forest phenology, the causes of forest disturbances, and the processes involved in post-disturbance forest recovery. Recent and continued climatic changes may promote altered trajectories of forest health (e.g. due to moisture stress and other disturbance factors) and forest species composition (favoring more temperate and drought-resistant species) [Duveneck et al., 2014a, 2014b], complicating options for regional forest management [Rittenhouse and Rissman, 2015]. Trophic interactions between 
forest vegetation, insects, and wildlife become more uncertain with altered timing of phenological events [Foster et al., 2013; Roberts et al., 2015]. Forest managers with responsibility for planning Winter and dry-season harvest territories, or for undertaking intervention activities to mitigate forest disturbances due to insect pests and fire, can benefit from any potential improvements in seasonal predictability. 


\section{REFERENCES}

Adams, H.D., and 61 coauthors, 2017: A multi-species synthesis of physiological mechanisms in droughtinduced tree mortality. Nature Ecol. Evol., 1, 1285-1291, doi: 10.1038/s41559-017-0248-x.

Ahl, D.E., S.T. Gower, S.N. Burrows, N.V. Shabanov, R.B. Myneni, and Y. Knyazikhin, 2006: Monitoring spring canopy phenology of a deciduous broadleaf forest using MODIS. Rem. Sens. Env., 104, 88-95, doi: 10.1016/j.rse.2006.05.003.

Akaike, H., 1974: A new look at the statistical model identification. IEEE Trans. Auto. Control, 19 (6), 716-723, doi: 10.1109/TAC.1974.1100705.

Akkala, A., V. Devabhaktuni, and A. Kumar, 2010: Interpolation techniques and associated software for environmental data. Env. Prog. Sust. Energy, 29, 134-141, doi: 10.1002/ep.10455.

Alexander, L.V., and 23 coauthors, 2006: Global observed changes in daily climate extremes of temperature and precipitation. J. Geophys. Res. Atmos., 111, paper D05109, doi: 10.1029/2005JD006290.

Ali, A.A., C. Xu, A. Rogers, N.G. McDowell, B.E. Medlyn, R.A. Fisher, S.D. Wullschleger, P.B. Reich, J.A. Vrugt, W.L. Bauerle, L.S. Santiago, and C.J. Wilson, 2015: Global-scale environmental control of plant photosynthetic capacity. Ecol. Appl., 25, 2349-2365, doi: 10.1890/14-2111.1.

Allen, C.D., D.D. Breshears, and N.G. McDowell, 2015: On underestimation of global vulnerability to tree mortality and forest die-off from hotter drought in the Anthropocene. Ecosphere, 6, paper 129, doi: 10.1890/ES15-00203.1.

Altunay, M., and co-authors, 2011: A Science Driven Production Cyberinfrastructure- the Open Science Grid. J. Grid Comp., 9, 201-218, doi: 10.1007/s10723-010-9176-6.

Anderegg, W.R.L., J.M. Kane, and L.D.L. Anderegg, 2012: Consequences of widespread tree mortality triggered by drought and temperature stress. Nature Clim. Ch., 3, 30-36, doi: 10.1038/nclimate1635. 
Anderegg, W.R.L., L. Plavcová, L.D.L. Anderegg, U.G Hacke, J.A. Berry, and C.B. Field, 2013:

Drought's legacy: Multiyear hydraulic deterioration underlies widespread aspen forest die-off and portends increased future risk. Glob. Ch. Biol., 19, 1188-1196. doi: 10.1111/gcb.12100.

Anderegg, W.R.L., C. Schwalm, F. Biondi, J.J. Camarero, G. Koch, M. Litvak, K. Ogle, J.D. Shaw, E. Shevliakova, A.P. Williams, A. Wolf, E. Ziaco, and S. Pacala, 2015: Pervasive drought legacies in forest ecosystems and their implications for carbon cycle models. Science, 349, 528-532, doi: 10.1126/science.aab1833.

André, J.-C., P. Bougeault, J.-F. Mahfouf, P. Mascart, J. Noilhan, J.-P. Pinty, P.G. Jarvis, W. Kohsiek, J.R. Milford, J.I.L. Morison, P. Rowntree, D.A. Warrilow, W.J. Shuttleworth, and A. HendersonSellers, 1989: Impact of forests on mesoscale meteorology [and discussion]. Phil. Trans. Royal Soc. London B, 324, 407-422, doi: 10.1098/rstb.1989.0055.

Archetti, M., A.D. Richardson, J. O'Keefe, and N. Delpierre, 2013: Predicting climate change impacts on the amount and duration of autumn colors in a New England forest. PLoS ONE, 8, paper e57373, doi: 10.1371 journal.pone.0057373.

Arend, M., and J. Fromm, 2007: Seasonal change in the drought response of wood cell development in poplar. Tree Physiol., 27, 985-992, doi: 10.1093/treephys/27.7.985.

Arora, V., 2002: Modeling vegetation as a dynamic component in soil-vegetation-atmosphere transfer schemes and hydrological models. Rev. Geophys., 40, paper 1006, doi: 10.1029/2001RG000103.

Assel, R.A., 2003: Great Lakes Ice Cover, First Ice, Last Ice, and Ice Duration: Winters 1973-2002. NOAA Technical Memorandum GLERL-125, Great Lakes Environmental Research Laboratory, Ann Arbor, Michigan.

Assel, R. and S. Rodionov, 1998: Atmospheric teleconnections for annual maximum ice cover on the Laurentian Great Lakes. Int. J. Climatol., 18, 425-442, doi: 10.1002/(SICI)10970088(19980330)18:4<425::AID-JOC258>3.0.CO;2-Q.

Assel, R., K. Cronk, and D. Norton, 2003: Recent trends in Laurentian Great Lakes ice cover. Clim. Ch., 57, 185-204, doi: 10.1023/A:1022140604052. 
Assel, R.A., J. Wang, A.H. Clites, and X. Bai, 2013: Analysis of Great Lakes Ice Cover Climatology: Winters 2006-2011. NOAA Technical Memorandum GLERL-157, Great Lakes Environmental Research Laboratory, Ann Arbor, Michigan.

Augspurger, C.K., 2009: Spring 2007 warmth and frost: Phenology, damage and refoliation in a temperate deciduous forest. Functional Ecol., 23, 1031-1039, doi: 10.1111/j.13652435.2009.01587.x.

Augspurger, C.K., 2013: Reconstructing patterns of temperature, phenology, and frost damage over 124 years: Spring damage risk is increasing. Ecology, 94, 41-50, doi: 10.1890/12-0200.1.

Austin, J.A., and S.M. Colman, 2007: Lake Superior summer water temperatures are increasing more rapidly than regional air temperatures: A positive ice-albedo feedback. Geophys. Res. Lett., 34, paper L06604, doi: 10.1029/2006GL029021.

Austin, J., and S. Colman, 2008: A century of temperature variability in Lake Superior. Limnol. Oceanogr., 53, 2724-2730, doi: 10.4319/10.2008.53.6.2724.

Bai, X., and J. Wang, 2012: Atmospheric teleconnection patterns associated with severe and mild ice cover on the Great Lakes, 1963-2011. Wat. Qual. Res. J. Can., 47, 421-435, doi: 10.2166/wqrjc.2012.009.

Bai, X., J. Wang, Q. Liu, D. Wang, and Y. Liu, 2011: Severe ice conditions in the Bohai Sea, China, and mild ice conditions in the Great Lakes during the 2009/10 winter: Links to El Niño and a strong negative Arctic oscillation. J. Appl. Meteor. Climatol., 50, 1922-1935, doi: 10.1175/2011JAMC2675.1.

Bai, X., J. Wang, C. Sellinger, A. Clites, and R. Assel, 2012: Interannual variability of Great Lakes ice cover and its relationship to NAO and ENSO. J. Geophys. Res. Oceans, 117, paper C03002, doi: 10.1029/2010JC006932.

Baldocchi, D., F.M. Kelliher, T.A. Black, and P. Jarvis, 2000: Climate and vegetation controls on boreal zone energy exchange. Glob. Ch. Biol., 6, 69-83, doi: 10.1046/j.1365-2486.2000.06014.x. 
Barnes, C.A., and D.P. Roy, 2008: Radiative forcing over the conterminous United States due to contemporary land cover land use albedo change. Geophys. Res. Lett., 35, paper L09706, doi: 10.1029/2008GL033567.

Baskerville, G.L., and P. Emin, 1969: Rapid estimation of heat accumulation from maximum and minimum temperatures. Ecology, 50, 514-517, doi: 10.2307/1933912.

Bauerle, W.L., R. Oren, D.A. Way, S.S. Qian, P.C. Stoy, P.E. Thornton, J.D. Bowden, F.M. Hoffman, and R.F. Reynolds, 2012: Photoperiodic regulation of the seasonal pattern of photosynthetic capacity and the implications for carbon cycling. Proc. Nat. Acad. Sci., 109, 8612-8617, doi:10.1073/pnas.1119131109.

Beck, P.S.A., C. Atzberger, K.A. Høgda, B. Johansen, and A.K. Skidmore, 2006: Improved monitoring of vegetation dynamics at very high latitudes: A new method using MODIS NDVI. Rem. Sens. Env., 100, 321-334, doi: 10.1016/j.rse.2005.10.021.

Bennett, E.B., 1978: Characteristics of the thermal regime of Lake Superior. J. Great Lakes Res., 4, 310319, doi: 10.1016/S0380-1330(78)72200-8.

Bennington, V., M. Notaro, and K.D. Holman, 2014: Improving climate sensitivity of deep lakes within a regional climate model and its impact on simulated climate. J. Clim., 27, 2886-2911, doi: 10.1175/JCLI-D-13-00110.1.

Benson, B.J., J.J. Magnuson, O.P. Jensen, V.M. Card, G. Hodgkins, J. Korhonen. D.M. Livingstone, K.M. Stewart, G.A. Weyhenmeyer, and N.G. Granin, 2012: Extreme events, trends, and variability in Northern Hemisphere lake-ice phenology (1855-2005). Clim. Ch., 112, 299-323, doi: 10.1007/s10584-011-0212-8.

Bloom, A.J., F.S. Chapin III, and H.A. Mooney, 1985: Resource limitation in plants - An economic analogy. Ann. Rev. Ecol. System., 16, 363-392, doi: 10.1146/annurev.ecolsys.16.1.363.

Blyth, E., and R.J. Harding, 2011: Methods to separate observed global evapotranspiration into the interception, transpiration and soil surface evaporation components. Hydrol. Proc., 25, $4063-$ 4068, doi: 10.1002/hyp.8409. 
Bockheim, J.G., and S.A. Schliemann, 2014: Soil richness and endemism across an environmental transition zone in Wisconsin, USA. Catena, 113, 86-94, doi: 10.1016/j.catena.2013.09.011.

Bonan, G.B., 1993: Importance of leaf area index and forest type when estimating photosynthesis in boreal forests. Rem. Sens. Env., 43, 303-314, doi: 10.1016/0034-4257(93)90072-6.

Bonan, G.B., 2008: Forests and climate change: Forcings, feedbacks, and the climate benefits of forests. Science, 320, 1444-1449, doi: 10.1126/science.1155121.

Bonan, G.B., K.W. Oleson, M. Vertenstein, S. Levis, X. Zeng, Y. Dai, R.E. Dickinson, and Z.-L. Yang, 2002: The land surface climatology of the Community Land Model coupled to the NCAR Community Climate Model. J. Clim., 15, 3123-3149, doi: 10.1175/15200442(2002)015<3123:TLSCOT>2.0.CO;2.

Bond, B.J., 2000: Age-related changes in photosynthesis of woody plants. Trends Plant Sci., 5, 349-353, doi: 10.1016/S1360-1385(00)01691-5.

Bond, N.A., and D.E. Harrison, 2000: The Pacific decadal oscillation, air-sea interaction and central north Pacific winter atmospheric regimes. Geophys. Res. Lett., 27, 731-734, doi: 10.1029/1999GL010847.

Bond, N.A., J.E. Overland, M. Spillane, and P. Stabeno, 2003: Recent shifts in the state of the North Pacific. Geophys. Res. Lett., 30, 2183, doi: 10.1029/2003GL018597.

Bouriaud, O., J.-M. Leban, D. Bert, and C. Deleuze, 2005: Intra-annual variations in climate influence growth and wood density of Norway spruce. Tree Physiol., 25, 651-660, doi: 10.1093/treephys/25.6.651.

Brown, L.C., and C.R. Duguay, 2010: The response and role of ice cover in lake-climate interactions. Prog. Phys. Geog., 34, 671-704, doi: 10.1177/0309133310375653.

Brubaker, K.L., D. Entekhabi, and P.S. Eagleson, 1993: Estimation of continental precipitation recycling. J. Clim., 6, 1077-1089, doi: 10.1175/1520-0442(1993)006<1077:EOCPR>2.0.CO;2. 
Bryan, A.M., A.L. Steiner, and D.J. Posselt, 2015: Regional modeling of surface-atmosphere interactions and their impact on Great Lakes hydroclimate. J. Geophys. Res. Atmos., 120, 1044-1064, doi: 10.1002/2014JD022316.

Brzostek, E.R., D. Dragoni, H.P. Schmid, A.F. Rahman, D. Sims, C.A. Wayson, D. Johnson, and R.P. Phillips, 2014: Chronic water stress reduces tree growth and the carbon sink of deciduous hardwood forests. Glob. Ch. Biol., 20, 2531-2539, doi: 10.1111/gcb.12528.

Buitenwerf, R., L. Rose, and S.I. Higgins, 2015: Three decades of multi-dimensional change in global leaf phenology. Nature Clim. Ch., 5, 364-368, doi: 10.1038/nclimate2533.

Burnham, K.P., and D.R. Anderson, 2002: Model Selection and Multimodel Inference: A Practical Information-Theoretic Approach (2nd ed.), Springer-Verlag, ISBN 0-387-95364-7.

Cannell, M.G.R., and R.I. Smith, 1983: Thermal time, chill days and prediction of budburst in Picea sitchensis. J. Appl. Ecol., 20, 951-963, doi: 10.2307/2403139.

Cavanaugh, J.E., 1997: Unifying the derivations of the Akaike and corrected Akaike information criteria. Stat. Prob. Lett., 31, 201-208, doi: 10.1016/s0167-7152(96)00128-9.

Ceccherini, G., N. Gobron, and M. Migliavacca, 2014: On the response of European vegetation phenology to hydroclimatic anomalies. Rem. Sens., 6, 3143-3169, doi: 10.3390/rs6043143.

Changnon, S.A., 1999: Impacts of 1997-98 E1 Niño generated weather in the United States. Bull. Amer. Meteor. Soc., 80, 1819-1827, doi: 10.1175/1520-0477(1999)080<1819:IOENOG>2.0.CO;2.

Changnon Jr., S.A., and D.M.A. Jones, 1972: Review of the influences of the Great Lakes on weather. Wat. Resour. Res., 8, 360-371, doi: 10.1029/WR008i002p00360.

Chapin, F.S., III, A.J. Bloom, C.B. Field, and R.H. Waring, 1987: Plant responses to multiple environmental factors. BioScience, 37, 49-57, doi: 10.2307/1310177.

Chaves, M.M., 1991: Effects of water deficits on carbon assimilation. J. Exp. Botany, 42, 1-16, doi: 10.1093/jxb/42.1.1. 
Choi, W., R. Tareghian, J. Choi, and C.-S. Hwang, 2014: Geographically heterogeneous temporal trends of extreme precipitation in Wisconsin, USA during 1950-2006. Int. J. Climatol., 34, 2841-2852, doi: $10.1002 /$ joc. 3878 .

Cohen, W.B., 1991: Response of vegetation indices to changes in three measures of leaf water stress. Photogram. Eng. Rem. Sens., 57, 195-202, ISSN 0099-1112.

Collins, D.C., and R. Avissar, 1994: An evaluation with the Fourier amplitude sensitivity test (FAST) of which land-surface parameters are of greatest importance in atmospheric modeling. J. Clim., 7, 681-703, doi: 10.1175/1520-0442(1994)007<0681:AEWTFA>2.0.CO;2.

Collins, J.B., and C.E. Woodcock, 1996: An assessment of several linear change detection techniques for mapping forest mortality using multitemporal Landsat TM data. Rem. Sens. Env., 56, 66-77, doi: $10.1016 / 0034-4257(95) 00233-2$.

Crist, E.P., 1985: A TM Tasseled Cap equivalent transformation for reflectance factor data. Rem. Sens. Env., 17, 301-306, doi: 10.1016/0034-4257(85)90102-6.

Crist, E.P., and R.C. Cicone, 1984: A physically-based transformation of Thematic Mapper data - the TM Tasseled Cap. IEEE Trans. Geosci. Rem. Sens., 22, 256-263, doi: 10.1109/tgrs.1984.350619.

Crist, E.P., and R.J. Kauth, 1986: The Tasseled Cap de-mystified. Photogram. Eng. Rem. Sens., 52, 8186, ISSN 0099-1112.

Curtis, J.T., and R.P. McIntosh, 1951: An upland forest continuum in the prairie-forest border region of Wisconsin. Ecology, 32, 476-496, doi: 10.2307/1931725.

Czikowsky, M.J., and D.R. Fitzjarrald, 2004: Evidence of seasonal changes in evapotranspiration in eastern U.S. hydrological records. J. Hydrometeorol., 5, 974-988, doi: 10.1175/15257541(2004)005<0974:EOSCIE $>2.0$. CO;2.

Danz, N.P., L.E. Frelich, P.B. Reich, and G.J. Niemi, 2013: Do vegetation boundaries display smooth or abrupt spatial transitions along environmental gradients? Evidence from the prairie-forest biome boundary of historic Minnesota, USA. J. Veg. Sci., 24, 1129-1140, doi: 10.1111/jvs.12028. 
de Beurs K.M., and G.M., Henebry, 2010: Spatio-temporal statistical methods for modelling land surface phenology. In: Hudson I., Keatley M. (eds), Phenological Research. Springer, Dordrecht. ISBN 978-90-481-3335-2, doi: 10.1007/978-90-481-3335-2_9.

Deguchi, A., S. Hattori, and H.-T. Park, 2006: The influence of seasonal changes in canopy structure on interception loss: Application of the revised Gash model. J. Hydrol., 318, 80-102, doi: 10.1016/j.jhydrol.2005.06.005.

Delpierre, N., D. Berveiller, E. Granda, and E. Dufrêne, 2016: Wood phenology, not carbon input, controls the interannual variability of wood growth in a temperate oak forest. New Phytol., 210, 459-470, doi: 10.1111/nph.13771.

Delpierre, N., E. Dufrêne, K. Soudani, E. Ulrich, S. Cecchini, J. Boé, and C. François, 2009: Modelling interannual and spatial variability of leaf senescence for three deciduous tree species in France. Ag. For. Meteorol., 149, 938-948, doi: 10.1016/j.agrformet.2008.11.014.

de Reaumur, R.A.F., 1735: Observations du thermometre, faites à Paris pendant l'année 1735, comparées avec celles qui ont été faites sous la Ligne, à l'lsle de France, à Alger, et en qualques-unes de nos Isles de l'Amerique. Mem. Acad. Royale des Sci., Paris, 545-576.

Desai, A.R., 2010: Climatic and phenological controls on coherent regional interannual variability of carbon dioxide flux in a heterogeneous landscape. J. Geophys. Res., 115, paper G00J02, doi: 10.1029/2010JG001423.

Desai, A.R., A. Noormets, P.V. Bolstad, J. Chen, B.D. Cook, K.J. Davis, E.S. Euskirchen, C. Gough, J.G. Martin, D.M. Ricciuto, H.P. Schmid, J. Tang, and W. Wang, 2008: Influence of vegetation and seasonal forcing on carbon dioxide fluxes across the Upper Midwest, USA: Implications for regional scaling. Ag. For. Meteorol., 148, 288-308, doi: 10.1016/j.agrformet.2007.08.001.

Dickinson, R.E., Y. Tian, Q. Liu, and L. Zhou, 2008: Dynamics of leaf area for climate and weather models. J. Geophys. Res., 113, paper D16115, doi: 10.1029/2007JD008934.

Dickinson, R.E., M. Shaikh, R. Bryant, and L. Graumlich, 1998: Interactive canopies for a climate model. J. Clim., 11, 2823-2836, doi: 10.1175/1520-0442(1998)011<2823:ICFACM>2.0.CO;2. 
Dirmeyer, P.A., R.D. Koster, and Z. Guo, 2006: Do global models properly represent the feedback between land and atmosphere? J. Hydrometeorol., 7, 1177-1198, doi: 10.1175/JHM532.1.

Dixon, R.K., A.M. Solomon, S. Brown, R.A. Houghton, M.C. Trexier, and J. Wisniewski, 1994: Carbon pools and flux of global forest ecosystems. Science, 263, 185-190, doi: 10.1126/science.263.5144.185.

Dole, R., M. Hoerling, A. Kumar, J. Eischeid, J. Perlwitz, X.-W. Quan, G. Kiladis, R. Webb, D. Murray, M. Chen, K. Wolter, and T. Zhang, 2014: The making of an extreme event: Putting the pieces together. Bull. Amer. Meteor. Soc., 95, 427-440, doi: 10.1175/BAMS-D-12-00069.1.

Dorman, J.L., and P.J. Sellers, 1989: A global climatology of albedo, roughness length and stomatal resistance for atmospheric general-circulation models as represented by the Simple Biosphere model (SiB). J. Appl. Meteorol., 28, 833-855, doi: 10.1175/15200450(1989)028<0833:agcoar $>2.0 . \operatorname{co} ; 2$.

Drescher, M., and S.C. Thomas, 2013: Snow cover manipulations alter survival of early life stages of cold-temperate tree species. Oikos, 122, 541-554, doi: 10.1111/j.1600-0706.2012.20642.x.

Duarte, A.G., G. Katata, Y. Hoshika, M. Hossain, J. Kreuzwieser, A. Arneth, and N.K. Ruehr, 2016: Immediate and potential long-term effects of consecutive heat waves on the photosynthetic performance and water balance in Douglas-fir. J. Plant Physiol., 205, 57-66, doi: 10.1016/j.jplph.2016.08.012.

Duchemin, B., J. Goubier, and G. Courrier, 1999: Monitoring phenological key stages and cycle duration of temperate deciduous forest ecosystems with NOAA/AVHRR data. Rem. Sens. Env., 67, 68-82, doi: 10.1016/S0034-4257(98)00067-4.

Durre, I., M.J. Menne, B.E. Gleason, T.G. Houston, and R.S. Vose, 2010: Comprehensive automated quality assurance of daily surface observations. J. Appl. Meteorol. Climatol., 49, 1615-1633, doi: 10.1175/2010JAMC2375.1. 
Duveneck, M.J., R.M. Scheller, M.A. White, S.D. Handler, and C. Ravenscroft, 2014a: Climate change effects on northern Great Lake (USA) forests: A case for preserving diversity. Ecosphere, 5, article no. 23, doi: 10.1890/ES13-00370.1.

Duveneck, M.J., R.M. Scheller, and M.A. White, 2014b: Effects of alternative forest management on biomass and species diversity in the face of climate change in the northern Great Lakes region (USA). Can. J. For. Res., 44, 700-710, doi: 10.1139/cjfr-2013-0391.

Dymond, C.C., D.J. Mladenoff, and V.C. Radeloff, 2002: Phenological differences in Tasseled Cap indices improve deciduous forest classification. Rem. Sens. Env., 80, 460-472, doi: 10.1016/s0034-4257(01)00324-8.

Easterling, D.R., 2002: Recent changes in frost days and the frost-free season in the United States. Bull. Amer. Meteor. Soc., 83, 1327-1332, doi: 10.1175/15200477(2002)083<1327:RCIFDA>2.3.CO;2.

Elmore, A.J., S.M. Guinn, B.J. Minsley, and A.D. Richardson, 2012: Landscape controls on the timing of spring, autumn, and growing season length in mid-Atlantic forests. Glob. Ch. Biol., 18, 656-674, doi: 10.1111/j.1365-2486.2011.02521.x.

Ellwood, E.R., S.A. Temple, R.B. Primack, N.L. Bradley, and C.C. Davis, 2013: Record-breaking early flowering in the eastern United States. PLoS ONE, 8, paper e53788, doi: 10.1371/journal.pone.0053788.

Enfield, D.B., A.M. Mestas-Nuñez, and P.J. Trimble, 2001: The Atlantic multidecadal oscillation and its relation to rainfall and river flows in the continental U.S. Geophys. Res. Lett., 28, 2077-2080, doi: 10.1029/2000GL012745.

Estiarte, M., and J. Peñuelas, 2015: Alteration of the phenology of leaf senescence and fall in winter deciduous species by climate change: Effects on nutrient proficiency. Glob. Ch. Biol., 21, 10051017, doi: $10.1111 / \mathrm{gcb} .12804$.

Federer, C.A., 1975: Evapotranspiration. Rev. Geophys., 13, 442-445, doi: 10.1029/RG013i003p00442. 
Feng, S., and Q. Hu, 2007: Changes in winter snowfall/precipitation ratio in the contiguous United States. J. Geophys. Res. Atmos.,112, paper D15109, doi: 10.1029/2007JD008397.

Fereday, D.R., A. Maidens, A. Arribas, A.A. Scaife, and J.R. Knight, 2012: Seasonal forecasts of northern hemisphere winter 2009/10. Env. Res. Lett., 7, paper 034031, doi: 10.1088/17489326/7/3/034031.

Filewood, B. and S.C. Thomas, 2014: Impacts of a spring heat wave on canopy processes in a northern hardwood forest. Glob. Ch. Biol., 20, 360-371, doi: 10.1111/gcb.12354.

Fisher, J.I., and J.F. Mustard, 2007: Cross-scalar satellite phenology from ground, Landsat, and MODIS data. Rem. Sens. Env., 109, 261-273, doi: 10.1016/j.rse.2007.01.004.

Fisher, J.I., J.F. Mustard, and M.A. Vadeboncoeur, 2006: Green leaf phenology at Landsat resolution: Scaling from the field to the satellite. Rem. Sens. Env., 100, 265-279, doi: 10.1016/j.rse.2005.10.022.

Fisher, J.I., A.D. Richardson, and J.F. Mustard, 2007: Phenology model from surface meteorology does not capture satellite-based greenup estimations. Glob. Ch. Biol., 13, 707-721, doi: 10.1111/j.1365-2486.2006.01311.x.

Foster, J.R., P.A. Townsend, and D.J. Mladenoff, 2013: Mapping asynchrony between gypsy moth egghatch and forest leaf-out: Putting the phenological window hypothesis in a spatial context. Forest Ecol. Manage., 287, 67-76, doi: 10.1016/j.foreco.2012.09.006.

Franke, R., H. Hagen, and G.M. Nielson, 1994: Least squares surface approximation to scattered data using multiquadratic functions. Adv. Comput. Math., 2, 81-99, doi: 10.1007/BF02519037.

Frantz, D., A. Röder, T. Udelhoven, and M. Schmidt, 2016: Forest disturbance mapping using dense synthetic Landsat/MODIS time-series and permutation-based Disturbance Index detection. Rem. Sens., 8, 277, doi: 10.3390/rs8040277.

Fracheboud, Y., V. Luquez, L. Björkén, A. Sjödin, H. Tuominen, and S. Jansson, 2009: The control of autumn senescence in European aspen. Plant Physiol., 149, 1982-1991, doi: $10.1104 /$ pp.108.133249. 
Frich, P., L.V. Alexander, P. Della-Marta, B. Gleason, M. Haylock, A.M.G. Klein Tank, and T. Peterson, 2002: Observed coherent changes in climatic extremes during the second half of the twentieth century. Clim. Res., 19, 193-212, doi: 10.3354/cr019193.

Friedl, M.A., D. Sulla-Menashe, B. Tan, A. Schneider, N. Ramankutty, A. Sibley, and X.M. Huang, 2010: MODIS collection 5 global land cover: Algorithm refinements and characterization of new datasets. Rem. Sens. Env., 114, 168-82, doi: 10.1016/j.rse.2009.08.016.

Fu, Z., P.C. Stoy, Y. Luo, J. Chen, J. Sun, L. Montagnani, G. Wohlfahrt, A.F. Rahman, S. Rambal, C. Bernhofer, J. Wang, G. Shirkey, and S. Niu, 2017: Climate controls over the net carbon uptake period and amplitude of net ecosystem production in temperate and boreal ecosystems. Ag. For. Meteorol., 243, 9-18, doi: 10.1016/j.agrformet.2017.05.009.

Gallinat, A.S., R.B. Primack, and D.L. Wagner, 2015: Autumn, the neglected season in climate change research. Trends Ecol. Evol., 30, 169-176, doi: 10.1016/j.tree.2015.01.004.

Ganguly, S., M.A. Friedl, B. Tan, X. Zhang, and M. Verma, 2010: Land surface phenology from MODIS: Characterization of the Collection 5 global land cover dynamics product. Rem. Sens. Env., 114, 1805-1816, doi: 10.1016/j.rse.2010.04.005.

Gao, B., 1996: NDWI-A normalized difference water index for remote sensing of vegetation liquid water from space. Rem. Sens. Env., 58, 257-266, doi: 10.1016/S0034-4257(96)00067-3.

Garcia, M., M. Özdogan, and P.A. Townsend, 2014: Impacts of forest harvest on cold season land surface conditions and land-atmosphere interactions in northern Great Lakes states. J. Adv. Model. Earth Sys., 6, 923-937, doi: 10.1002/2014MS000317.

Garcia, M., C.D. Peters-Lidard, and D.C. Goodrich, 2008: Spatial interpolation of precipitation in a dense gauge network for monsoon storm events in the southwestern United States. Water Resour. Res., 44, W05S13, doi:10.1029/2006WR005788.

Garcia, M., and P.A. Townsend, 2016: Recent climatological trends and potential influences on forest phenology around western Lake Superior, USA. J. Geophys. Res. Atmos., 121, doi: 10.1002/2016JD025190. 
Garratt, J.R., 1993: Sensitivity of climate simulations to land-surface and atmospheric boundary-layer treatments-A review. J. Clim., 6, 419-448, doi: 10.1175/15200442(1993)006<0419:SOCSTL >2.0.CO;2.

Gash, J.H.C., 1979: An analytical model of rainfall interception by forests. Q. J. Roy. Meteorol. Soc., 105, 43-55, doi: 10.1002/qj.49710544304.

Gash, J.H.C., I.R. Wright, and C.R. Lloyd, 1980: Comparative estimates of interception loss from three coniferous forests in Great Britain. J. Hydrol., 48, 89-105, doi: 10.1016/0022-1694(80)90068-2.

Gerten, D., and R. Adrian, 2001: Differences in the persistency of the North Atlantic Oscillation signal among lakes. Limnol. Oceanogr., 46, 448-455, doi: 10.4319/lo.2001.46.2.0448.

Goulden, M.L., A.M.S. McMillan, G.C. Winston, A.V. Rocha, K.L. Manies, J.W. Harden, and B.P. Bond-Lamberty, 2011: Patterns of NPP, GPP, respiration, and NEP during boreal forest succession. Glob. Ch. Biol., 17, 855-871, doi: 10.1111/j.1365-2486.2010.02274.x.

Grise, K.M., S.-W. Son, and J.R. Gyakum, 2013: Intraseasonal and interannual variability in North American storm tracks and its relationship to equatorial Pacific variability. Mon. Wea. Rev., 141, 3610-3625, doi: 10.1175/MWR-D-12-00322.1.

Groffman, P.M., and 20 coauthors, 2012: Long-term integrated studies show complex and surprising effects of climate change in the northern hardwood forest. BioScience, 62, 1056-1066, doi: 10.1525/bio.2012.62.12.7.

Groisman, P.Y., R.W. Knight, and T.R. Karl, 2012: Changes in intense precipitation over the central United States. J. Hydrometeorol., 13, 47-66, doi: 10.1175/JHM-D-11-039.1.

Grover, E.K., and P.J. Sousounis, 2002: The influence of large-scale flow on fall precipitation systems in the Great Lakes basin. J. Clim., 15, 1943-1956, doi: 10.1175/15200442(2002)015<1943:TIOLSF>2.0.CO;2.

Gu, L., P.J. Hanson, W. Mac Post, D.P. Kaiser, B. Yang, R. Nemani, S.G. Pallardy, and T. Meyers, 2008: The 2007 eastern US spring freeze: Increased cold damage in a warming world? BioScience, 58, 253-262, doi: 10.1641/B580311. 
Gula, J., and W.R. Peltier, 2012: Dynamical downscaling over the Great Lakes basin of North America using the WRF regional climate model: The impact of the Great Lakes system on regional greenhouse warming. J. Clim., 25, 7723-7742, doi: 10.1175/JCLI-D-11-00388.1.

Gunderson, C.A., N.T. Edwards, A.V. Walker, K.H. O'Hara, C.M. Campion, and P.J. Hanson, 2012: Forest phenology and a warmer climate-growing season extension in relation to climatic provenance. Glob. Change Biol., 18, 2008-2025, doi: 10.1111/j.1365-2486.2011.02632.x.

Guo, Y., 2013: Towards systems biological understanding of leaf senescence. Plant Mol. Biol., 82, 519528, doi: 10.1007/s11103-012-9974-2.

Gustafson, E.J., and B.R. Sturtevant, 2013: Modeling forest mortality caused by drought stress: Implications for climate change. Ecosystems, 16, 60-74, doi: 10.1007/s10021-012-9596-1.

Hais, M., M. Jonasova, J. Langhammer, and T. Kucera, 2009: Comparison of two types of forest disturbance using multitemporal Landsat TM/ETM plus imagery and field vegetation data. Rem. Sens. Env., 113, 835-845, doi: 10.1016/j.rse.2008.12.012.

Hänninen, H., 1991: Does climatic warming increase the risk of frost damage in northern trees? Plant, Cell \& Env., 14, 449-454, doi: 10.1111/j.1365-3040.1991.tb01514.x.

Harding, K.J., and P.K. Snyder, 2015: The relationship between the Pacific-North American teleconnection pattern, the Great Plains low-level jet, and North Central U.S. heavy rainfall events. J. Clim., 28, 6729-6742, doi: 10.1175/JCLI-D-14-00657.1.

Hardisky, M.A., Klemas, and R.M. Smart, 1983: The influence of soil salinity, growth form, and leaf moisture on the spectral radiance of Spartina alterniflora canopies. Photogram. Eng. Rem. Sens., 49, 77-83, ISSN 0099-1112.

Hardy, R.L., 1971: Multiquadric equations of topography and other irregular surfaces. J. Geophys. Res., 76, 1905-1915, doi: 10.1029/JB076i008p01905.

Hardy, R.L., 1990: Theory and applications of the multiquadric-biharmonic method. Comput. Math. Appl., 19, 163-208, doi: 10.1016/0898-1221(90)90272-L. 
Hayhoe, K., C. Wake, T. Huntington, L. Luo, M. Schwartz, J. Sheffield, E. Wood, B. Anderson, J. Bradbury, A. DeGaetano, T. Troy, and D. Wolfe, 2007: Past and future changes in climate and hydrological indicators in the U.S. Northeast. Clim. Dyn., 28, 381-407, doi: 10.1007/s00382-0060187-8.

Hayhoe, K., J. VanDorn, T. Croley II, N. Schlegal, and D. Wuebbles, 2010: Regional climate change projections for Chicago and the US Great Lakes. J. Great Lakes Res., 36 (Suppl. 2), 7-21, doi: 10.1016/j.jglr.2010.03.012.

Healey, S.P., W.B. Cohen, Y. Zhiqiang, and O.N. Krankina, 2005: Comparison of Tasseled Cap-based Landsat data structures for use in forest disturbance detection. Rem. Sens. Env., 97, 301-310, doi: 10.1016/j.rse.2005.05.009.

Heide, O.M., 1993: Dormancy release in beech buds (Fagus sylvatica) requires both chilling and long days. Physiologia Plantarum, 89, 187-191, doi: 10.1111/j.1399-3054.1993.tb01804.x.

Herbst, M., J.M. Roberts, P.T.W. Rosier, M.E. Taylor, and D.J. Gowing, 2007: Edge effects and forest water use: A field study in a mixed deciduous woodland. For. Ecol. Manage., 250, 176-186, doi: 10.1016/j.foreco.2007.05.013.

Hinkel, K.M., and F.E. Nelson, 2012: Spatial and temporal aspects of the lake effect on the southern shore of Lake Superior. Theor. Appl. Climatol., 109, 415-428, doi: 10.1007/s00704-012-0585-2.

Hoerling, M., J. Eischeid, A. Kumar, R. Leung, A. Mariotti, K. Mo, S. Schubert, and R. Seager, 2014: Causes and predictability of the 2012 Great Plains drought. Bull. Amer. Meteor. Soc., 95, 269282, doi: 10.1175/BAMS-D-13-00055.1.

Homer, C.G., J.A. Dewitz, L. Yang, S. Jin, P. Danielson, G. Xian, J. Coulston, N.D. Herold, J.D. Wickham, and K. Megown, 2015: Completion of the 2011 National Land Cover Database for the conterminous United States-Representing a decade of land cover change information. Photogram. Eng. Rem. Sens., 81, 345-354, ISSN 0099-1112.

Hörtensteiner, S., 2006: Chlorophyll degradation during senescence. Ann. Rev. Plant Biol., 57, 55-77, doi: 10.1146/annurev.arplant.57.032905.105212. 
Horvath, K., D. Koracin, R. Vellore, J.H. Jiang, and R. Belu, 2012: Sub-kilometer dynamical downscaling of near-surface winds in complex terrain using WRF and MM5 mesoscale models. J. Geophys. Res. Atmos., 117, doi: 10.1029/2012jd017432.

Howk, F., 2009: Changes in Lake Superior ice cover at Bayfield, Wisconsin. J. Great Lakes Res., 35, 159-162, doi: 10.1016/j.jglr.2008.11.002.

Hu, X.-M., P.M. Klein, and M. Xue, 2013: Evaluation of the updated YSU planetary boundary layer scheme within WRF for wind resource and air quality assessments. J. Geophys. Res. Atmos., 118, doi:10.1002/jgrd.50823.

Huang, C., L.S. Davis, and J.R.G. Townshend, 2002: An assessment of support vector machines for land cover classification. Int. J. Rem. Sens., 23, 725-749, doi: 10.1080/01431160110040323.

Huete, A.R., K. Didan, T. Miura, E.P. Rodriguez, X. Gao, and G. Ferreira, 2002: Overview of the radiometric and biophysical performance of the MODIS vegetation indices. Rem. Sens. Env., 83, 195-213, doi: 10.1016/S0034-4257(02)00096-2.

Huete, A.R., H.Q. Liu, K. Batchily, and W.J. van Leeuwen, 1997: A comparison of vegetation indices over a global set of TM images for EOS-MODIS. Rem. Sens. Env., 59, 440-451, doi: 10.1016/S0034-4257(96)00112-5.

Huff, A., and A. Thomas, 2014: Lake Superior Climate Change Impacts and Adaptation. Prepared for the Lake Superior Lakewide Action and Management Plan - Superior Work Group. Available at http://www.epa.gov/glnpo/lakesuperior/index.html.

Hufkens, K., M.A. Friedl, T.F. Keenan, O. Sonnentag, A. Bailey, J. O'Keefe, and A.D. Richardson, 2012: Ecological impacts of a widespread frost event following early spring leaf-out. Glob. Ch. Biol., 18, 2365-2377, doi: 10.1111/j.1365-2486.2012.02712.x.

Hunt, E.R., L. Li, M.T. Yilmaz, and T.J. Jackson, 2011: Comparison of vegetation water contents derived from shortwave-infrared and passive-microwave sensors over central Iowa. Rem. Sens. Env., 115, 2376-2383, doi: 10.1016/j.rse.2011.04.037. 
Hunt, E.R., B.N. Rock, and P.S. Nobel, 1987: Measurement of leaf relative water content by infrared reflectance. Rem. Sens. Env., 22, 429-435, doi: 10.1016/0034-4257(87)90094-0.

Hunter, A.F., and M.J. Lechowicz, 1992: Predicting the timing of budburst in temperate trees. J. Appl. Ecol., 29, 597-604, doi: 10.2307/2404467.

Hurvich, C.M., and C.-L. Tsai, 1989: Regression and time series model selection in small samples. Biometrika, 76, 297-307, doi: 10.1093/biomet/76.2.297.

Hwang, T., L.E. Band, C.F. Miniat, C. Song, P.V. Bolstad, J.M. Vose, and J.P. Love, 2014: Divergent phenological response to hydroclimate variability in forested mountain watersheds. Glob. Ch. Biol., 20, 2580-2595, doi: 10.1111/gcb.12556.

Irland, L.C., D. Adams, R. Alig, C.J. Betz, C.-C. Chen, M. Hutchins, B.A. McCarl, K. Skog, and B.L. Sohngen, 2001: Assessing socioeconomic impacts of climate change on US forests, woodproduct markets, and forest recreation. BioScience, 51, 753-764, doi: 10.1641/00063568(2001)051[0753:asiocc]2.0.co;2.

Isaacson, B.N., S.P. Serbin, and P.A. Townsend, 2012: Detection of relative differences in phenology of forest species using Landsat and MODIS. Landscape Ecol., 27, 529-543, doi:10.1007/s10980012-9703-x.

Ivits, E., M. Cherlet, W. Mehl, and S. Sommer, 2013: Ecosystem functional units characterized by satellite observed phenology and productivity gradients: A case study for Europe. Ecol. Ind., 27, 17-28, doi: 10.1016/j.ecolind.2012.11.010.

Jayatilaka, B., T. Levshina, M. Rynge, C. Sehgal, and M. Slyz, 2015: The OSG open facility: A sharing ecosystem. J. Phys. Conf. Series, 664, 032016, doi: 10.1088/1742-6596/664/3/032016.

Jenkins, J.P., B.H. Braswell, S.E. Frolking, and J.D. Aber, 2002: Detecting and predicting spatial and interannual patterns of temperate forest springtime penology in the eastern U.S. Geophys. Res. Lett., 29, paper 2201, doi: 10.1029/2001GL014008. 
Jeong, S.-J., C.-H. Ho, H.-J. Gim, and M.E. Brown, 2011: Phenology shifts at start vs. end of growing season in temperate vegetation over the Northern Hemisphere for the period 1982-2008. Glob. Ch. Biol., 17, 2385-2399, doi: 10.1111/j.1365-2486.2011.02397.x.

Jeong, S.-J., D. Medvigy, E. Shevliakova, and S. Malyshev, 2013: Predicting changes in temperate forest budburst using continental-scale observations and models. Geophys. Res. Lett., 40, 359-364, doi: 10.1029/2012GL054431.

Jin, S., and S.A. Sader, 2005: Comparison of time series tasseled cap wetness and the normalized difference moisture index in detecting forest disturbances. Rem. Sens. Env., 94, 364-372, doi: 10.1016/j.rse.2004.10.012.

Jin, S., L. Yang, P. Danielson, C. Homer, J. Fry, and G. Xian, 2013: A comprehensive change detection method for updating the National Land Cover Database to circa 2011. Rem. Sens. Env., 132, 159175, doi: 10.1016/j.rse.2013.01.012.

Jo, H.-S., S.-W. Yeh, and B.P. Kirtman, 2014: Role of the western tropical Pacific in the North Pacific regime shift in the winter of 1998/1999. J. Geophys. Res. Oceans, 119, 6161-6170, doi: 10.1002/2013JC009527.

Johnson, S.L., and H.G. Stefan, 2006: Indicators of climate warming in Minnesota: Lake ice covers and snowmelt runoff. Clim. Ch., 75, 421-453, doi: 10.1007/s10584-006-0356-0.

Jolly, W.M., R. Nemani, and S.W. Running, 2005: A generalized, bioclimatic index to predict foliar phenology in response to climate. Glob. Ch. Biol., 11, 619-632, doi: 10.1111/j.13652486.2005.00930.x.

Juang, J.-Y., G.G. Katul, A. Porporato, P.C. Stoy, M.S. Siqueira, M. Detto, H.-S. Kim, and R. Oren, 2007: Eco-hydrological controls on summertime convective rainfall triggers. Glob. Ch. Biol., 13, 887-896, doi: 10.1111/j.1365-2486.2007.01315.x.

Kathuroju, N., M.A. White, J. Symanzik, M.D. Schwartz, J.A. Powell, and R.R. Nemani, 2007: On the use of the Advanced Very High Resolution Radiometer for development of prognostic land surface phenology models. Ecol. Model., 201, 144-156, doi: 10.1016/j.ecolmodel.2006.09.011. 
Katul, G.G., R. Oren, S. Manzoni, C. Higgins, and M.B. Parlange, 2012: Evapotranspiration: A process driving mass transport and energy exchange in the soil-plant-atmosphere-climate system. Rev. Geophys., 50, paper RG3002, doi: 10.1029/2011RG000366.

Katul, G., A. Porporato, and R. Oren, 2007: Stochastic dynamics of plant-water interactions. Ann. Rev. Ecol. Evol. System., 38, 767-791, doi: 10.1146/annurev.ecolsys.38.091206.095748.

Katz, R.W., 2010: Statistics of extremes in climate change. Clim. Ch., 100, 71, doi: 10.1007/s10584-0109834-5.

Keenan, T.F., B. Darby, E. Felts, O. Sonnentag, M.A. Friedl, K. Hufkens, J. O'Keefe, S. Klosterman, J.W. Munger, M. Toomey, and A.D. Richardson, 2014: Tracking forest phenology and seasonal physiology using digital repeat photography: A critical assessment. Ecol. Appl., 24, 1478-1489, doi: 10.1890/13-0652.1.

Keenan, T.F., and A.D. Richardson, 2015: The timing of autumn senescence is affected by the timing of spring phenology: Implications for predictive models. Glob. Ch. Biol., 21, 2634-2641, doi: 10.1111/gcb.12890.

Keskitalo, J., G. Bergquist, P. Gardeström, and S. Jansson, 2005: A cellular timetable of autumn senescence. Plant Physiol., 139, 1635-1648, doi: 10.1104/pp.105.066845.

Kettaneh, N. A. Berglund, and S. Wold, 2005: PCA and PLS with very large data sets. Comp. Stat. Data Analysis, 48, 69-85, doi: 10.1016/j.csda.2003.11.027.

Kikuzawa, K., Y. Onoda, I.J. Wright, and P.B. Reich, 2013: Mechanisms underlying global temperaturerelated patterns in leaf longevity. Glob. Ecol. Biogeogr., 22, 982-993, doi: 10.1111/geb.12042.

Killingbeck, K.T., 1996: Nutrients in senesced leaves: Keys to the search for potential resorption and resorption proficiency. Ecology, 77, 1716-1727, doi: 10.2307/2265777.

Kim, Y., J.S. Kimball, K. Didan, and G.M. Henebry, 2014: Response of vegetation growth and productivity to spring climate indicators in the conterminous United States derived from satellite remote sensing data fusion. Ag. For. Meteorol., 194, 132-143, doi:

10.1016/j.agrformet.2014.04.001. 
Klaassen, W., 2001: Evaporation from rain-wetted forest in relation to canopy wetness, canopy cover, and net radiation. Wat. Resour. Res., 37, 3227-3236, doi: 10.1029/2001WR000480.

Klingaman, N.P., J. Butke, D.J. Leathers, K.R. Brinson, and E. Nickl, 2008: Mesoscale simulations of the land surface effects of historical logging in a moist continental climate regime. J. Appl. Meteorol. Climatol., 47, 2166-2182, doi: 10.1175/2008JAMC1765.1.

Kolka, R.K., B.R. Sturtevant, J.R. Miesel, A. Singh, P.T. Wolter, S. Fraver, T.M. DeSutter. and P.A. Townsend, 2017: Emissions of forest floor and mineral soil carbon, nitrogen and mercury pools and relationships with fire severity for the Pagami Creek Fire in the Boreal Forest of northern Minnesota. Int. J. Wildland Fire, 26, 296-305, doi: 10.1071/WF16128.

Körner, C., 2015: Paradigm shift in plant growth control. Curr. Opinion Plant Biol., 25, 107-114, doi: 10.1016/j.pbi.2015.05.003.

Koster, R.D., G.K. Walker, G.J. Collatz, and P.E. Thornton, 2014: Hydroclimatic controls on the means and variability of vegetation phenology and carbon uptake. J. Clim., 27, 5632-5652, doi: 10.1175/JCLI-D-13-00477.1.

Kucharik, C.J., C.C. Barford, M. El Maayar, S.C. Wofsy, R.K. Monson, and D.D. Baldocchi, 2006: A multiyear evaluation of a dynamic global vegetation model at three AmeriFlux forest sites: Vegetation structure, phenology, soil temperature, and $\mathrm{CO}_{2}$ and $\mathrm{H}_{2} \mathrm{O}$ vapor exchange. Ecol. Model., 196, 1-31, doi: 10.1016/j.ecolmodel.2005.11.031.

Kunkel, K.E., D.R. Easterling, K. Hubbard, and K. Redmond, 2004: Temporal variations in frost-free season in the United States: 1895-2000. Geophys. Res. Lett., 31, paper L03201, doi: 10.1029/2003GL018624.

Laube, J., T.H. Sparks, N. Estrella, J. Höfler, D.P. Ankerst, and A. Menzel, 2014a: Chilling outweighs photoperiod in preventing precocious spring development. Glob. Ch. Biol., 20, 170-182, doi: $10.1111 /$ gcb.12360. 
Laube, J., T.H. Sparks, N. Estrella, and A. Menzel, 2014b: Does humidity trigger tree phenology?

Proposal for an air humidity based framework for bud development in spring. New Phytol., 202, 350-355, doi: 10.1111/nph.12680.

Law, B.E., and 32 coauthors, 2002: Environmental controls over carbon dioxide and water vapor exchange of terrestrial vegetation. Ag. For. Meteorol., 113, 97-120, doi: 10.1016/S01681923(02)00104-1.

Lawrence, D.M., P.E. Thornton, K.W. Oleson, and G.B. Bonan, 2007: The partitioning of evapotranspiration into transpiration, soil evaporation, and canopy evaporation in a GCM: Impacts on land-atmosphere interaction. J. Hydrometeorol., 8, 862-880, doi: 10.1175/JHM596.1.

Leathers, D.J., B. Yarnal, and M.A. Palecki, 1991: The Pacific/North American teleconnection pattern and United States climate. Part I: Regional temperature and precipitation associations. J. Clim., 4, 517-528, doi: 10.1175/1520-0442(1991)004<0517:TPATPA>2.0.CO;2.

Lechowicz, M.J., 1984: Why do temperate deciduous trees leaf out at different times? Adaptation and ecology of forest communities. Am. Naturalist, 124, 821-842, doi: 10.1086/284319.

LeMone, M.A., F. Chen, M. Tewari, J. Dudhia, B. Geerts, Q. Miao, R.L. Coulter, and R.L. Grossman, 2010a: Simulating the IHOP_2002 fair-weather CBL with the WRF-ARW-Noah modeling system. Part I: Surface fluxes and CBL structure and evolution along the eastern track. Mon. Wea. Rev., 138, 722-744, doi: 10.1175/2009MWR3003.1.

LeMone, M.A., F. Chen, M. Tewari, J. Dudhia, B. Geerts, Q. Miao, R.L. Coulter, and R.L. Grossman, 2010b: Simulating the IHOP_2002 fair-weather CBL with the WRF-ARW-Noah modeling system. Part II: Structures from a few kilometers to $100 \mathrm{~km}$ across. Mon. Wea. Rev., 138, 745764, doi: 10.1175/2009MWR3004.1.

LeMone, M.A., M. Tewari, F. Chen, J.G. Alfieri, and D. Niyogi, 2008: Evaluation of the Noah land surface model using data from a fair-weather IHOP_2002 day with heterogeneous surface fluxes. Mon. Wea. Rev., 136, 4915-4941, doi: 10.1175/2008MWR2354.1. 
Li, X., S. Zhong, X. Bian, W.E. Heilman, Y. Luo, and W. Dong, 2010: Hydroclimate and variability in the Great Lakes region as derived from the North American Regional Reanalysis, J. Geophys. Res. Atmos., 115, paper D12104, doi: 10.1029/2009JD012756.

Lins, H.F., and T.A. Cohn, 2011: Stationarity: Wanted dead or alive? J. Am. Wat. Resour. Assoc., 47, 475-480, doi: 10.1111/j.1752-1688.2011.00542.x

Liu, Y., H.V. Gupta, S. Sorooshian, L.A. Bastidas, and W.J. Shuttleworth, 2004: Exploring parameter sensitivities of the land surface using a locally coupled land-atmosphere model. J. Geophys. Res., 109, paper D21101, doi: 10.1029/2004JD004730.

Liu, Y., C. Wu, D. Peng, S. Xu, A. Gonsamo, R.S. Jassal, M. Altaf Arain, L. Lu, B. Fang, and J.M. Chen, 2016: Improved modeling of land surface phenology using MODIS land surface reflectance and temperature at evergreen needleleaf forests of central North America. Rem. Sens. Env., 176, 152162, doi: 10.1016/j.rse.2016.01.021.

Lu, X., D.W. Kicklighter, J.M. Melillo, J.M. Reilly, and L. Xu, 2015: Land carbon sequestration within the conterminous United States: Regional- and state-level analyses. J. Geophys. Res. Biogeosci., 120, doi: $10.1002 / 2014 J G 002818$.

Luo, D., and J. Cha, 2012: The North Atlantic oscillation and the North Atlantic jet variability: Precursors to NAO regimes and transitions. J. Atmos. Sci., 69, 3763-3787, doi: 10.1175/JAS-D-12-098.1.

Luo, Y., E.H. Berbery, K.E. Mitchell, and A.K. Betts, 2007: Relationships between land surface and nearsurface atmospheric variables in the NCEP North American Regional Reanalysis. $J$. Hydrometeorol., 8, 1184-1203, doi: 10.1175/2007JHM844.1.

Mallard, M.S., C.G. Nolte, O.R. Bullock, T.L. Spero, and J. Gula, 2014: Using a coupled lake model with WRF for dynamical downscaling. J. Geophys. Res. Atmos., 119, 7193-7208, doi: 10.1002/2014JD021785.

Marchin, R., H. Zeng, and W. Hoffmann, 2010: Drought-deciduous behavior reduces nutrient losses from temperate deciduous trees under severe drought. Oecologia, 163, 845-854, doi: 10.1007/s00442010-1614-4. 
Marchin, R. M., C.F. Salk, W.A. Hoffmann, and R.R. Dunn, 2015: Temperature alone does not explain phenological variation of diverse temperate plants under experimental warming. Glob. Ch. Biol., 21, 3138-3151, doi: 10.1111/gcb.12919.

Masek, J.G., C. Huang, R. Wolfe, W. Cohen, F. Hall, J. Kutler, and P. Nelson, 2008: North American forest disturbance mapped from a decadal Landsat record. Rem. Sens. Env., 112, 2914-2926, doi: 10.1016/j.rse.2008.02.010.

Masek, J.G., E.F. Vermote, N. Saleous, R. Wolfe, F.G. Hall, F. Huemmrich, F. Gao, J. Kutler, and T.K. Lim, 2012: LEDAPS Landsat Calibration, Reflectance, Atmospheric Correction Preprocessing Code. Model product, available on-line from Oak Ridge National Laboratory Distributed Active Archive Center, Oak Ridge, Tennessee, USA, doi: 10.3334/ORNLDAAC/1080.

McCabe, G.J., J.L. Betancourt, and S. Feng, 2015: Variability in the start, end, and length of frost-free periods across the conterminous United States during the past century. Int. J. Climatol., 35, 46734680, doi: $10.1002 /$ joc. 4315 .

McMillan, A.M.S., and M.L. Goulden, 2008: Age-dependent variation in the biophysical properties of boreal forests. Glob. Biogeochem. Cyc., 22, paper GB2023, doi: 10.1029/2007GB003038.

McPhaden, M.J., S.E. Zebiak, and M.H. Glantz, 2006: ENSO as an integrating concept in Earth science. Science, 314, 1740-1745, doi: 10.1126/science.1132588.

Melaas, E.K., M.A. Friedl, and Z. Zhu, 2013: Detecting interannual variation in deciduous broadleaf forest phenology using Landsat TM/ETM+ data. Rem. Sens. Env., 132, 176-185, doi: 10.1016/j.rse.2013.01.011.

Menne, M.J., I. Durre, R.S. Vose, B.E. Gleason, and T.G. Houston, 2012: An overview of the Global Historical Climatology Network-Daily database. J. Atmos. Oceanic Technol., 29, 897-910, doi: 10.1175/JTECH-D-11-00103.1

Mesinger, F., G. DiMego, E. Kalnay, K. Mitchell, P.C. Shafran, W. Ebisuzaki, D. Jović, J. Woollen, E. Rogers, E.H. Berbery, M.B. Ek, Y. Fan, R. Grumbine, W. Higgins, H. Li, Y. Lin, G. Manikin, D. 
Parrish, and W. Shi, 2006: North American Regional Reanalysis. Bull. Am. Meteor. Soc., 87, 343360, doi: 10.1175/BAMS-87-3-343.

Miesel, J.R., W.C. Hockaday, R.K. Kolka, and P.A. Townsend, 2015: Soil organic matter composition and quality across fire severity gradients in coniferous and deciduous forests of the southern boreal region. J. Geophys. Res. Biogeosci., 120, 1124-1141, doi: 10.1002/2015JG002959.

Migliavacca, M., and 19 coauthors, 2015: Influence of physiological phenology on the seasonal pattern of ecosystem respiration in deciduous forests. Glob. Ch. Biol., 21, 363-376, doi:10.1111/gcb.12671.

Millard, P., M. Sommerkorn, and G.-A. Grelet, 2007: Environmental change and carbon limitation in trees: A biochemical, ecophysiological and ecosystem appraisal. New Phytol., 175, 11-28, doi: 10.1111/j.1469-8137.2007.02079.x.

Milly, P.C.D., J. Betancourt, M. Falkenmark, R.M. Hirsch, Z.W. Kundzewicz, D.P. Lettenmaier, and R.J. Stouffer, 2008: Stationarity is dead: Whither water management? Science, 319, 573-574, doi: 10.1126/science. 1151915 .

Mishra, V., and K.A. Cherkauer, 2011: Influence of cold season climate variability on lakes and wetlands in the Great Lakes region. J. Geophys. Res. Atmos., 116, paper D12111, doi: 10.1029/2010JD015063.

Mishra, V., K.A. Cherkauer, and L.C. Bowling, 2010a: Parameterization of lakes and wetlands for energy and water balance studies in the Great Lakes region. J. Hydrometeorol., 11, 1057-1082, doi: 10.1175/2010JHM1207.1.

Mishra, V., K.A. Cherkauer, and L.C. Bowling, 2011a: Changing thermal dynamics of lakes in the Great Lakes region: Role of ice cover feedbacks. Glob. Planet. Ch., 75, 155-172, doi: 10.1016/j.gloplacha.2010.11.003.

Mishra, V., K.A. Cherkauer, L.C. Bowling, and M. Huber, 2011b: Lake ice phenology of small lakes: Impacts of climate variability in the Great Lakes region. Glob. Planet. Ch., 76, 166-185, doi: 10.1016/j.gloplacha.2011.01.004. 
Mishra, V., K.A. Cherkauer, D. Niyogi, M. Lei, B.C. Pijanowski, D.K. Ray, L.C. Bowling, and G. Yang, 2010b: A regional scale assessment of land use/land cover and climatic changes on water and energy cycle in the upper Midwest United States. Int. J. Climatol., 30, 2025-2044, doi: 10.1002/joc.2095.

Morin, X., M.J. Lechowicz, C. Augspurger, J. O'Keefe, D. Viner, and I. Chuine, 2009: Leaf phenology in 22 North American tree species during the 21st century. Glob. Ch. Biol., 15, 961-975, doi: 10.1111/j.1365-2486.2008.01735.x.

Morisette, J.T., A.D. Richardson, A.K. Knapp, J.I. Fisher, E.A. Graham, J. Abatzoglou, B.E. Wilson, D.D. Breshears, G.M. Henebry, J.M. Hanes, and L. Liang, 2009: Tracking the rhythm of the seasons in the face of global change: Phenological research in the 21st century. Front. Ecol. Env., 7, 253-260, doi: 10.1890/070217.

Mountrakis, G., J. Im, and C. Ogole, 2011: Support vector machines in remote sensing: A review. ISPRS J. Photogram. Rem. Sens., 66, 247-259, doi: 10.1016/j.isprsjprs.2010.11.001.

Munné-Bosch, S., and L. Alegre, 2004: Die and let live: Leaf senescence contributes to plant survival under drought stress. Functional Plant Biol., 31, 203-216, doi: 10.1071/FP03236.

Murray, M.B., M.G.R. Cannell, and R.I. Smith, 1989: Date of budburst of fifteen tree species in Britain following climatic warming. J. Appl. Ecol., 26, 693-700, doi: 10.2307/2404093.

Nemani, R.R., C.D. Keeling, H. Hashimoto, W.M. Jolly, S.C. Piper, C.J. Tucker, R.B. Myneni and S.W. Running, 2003: Climate-driven increases in global terrestrial net primary production from 1982 to 1999. Science, 300, 1560-1563, doi: 10.1126/science.1082750.

Nie, J., P. Wang, W. Yang, and B. Tan, 2008: Northern hemisphere storm tracks in strong AO anomaly winters. Atmos. Sci. Lett., 9, 153-159, doi: 10.1002/asl.186.

Niinemets, Ü., and Ü. Tamm, 2005: Species differences in timing of leaf fall and foliage chemistry modify nutrient resorption efficiency in deciduous temperate forest stands. Tree Physiol., 25, 1001-1014, doi: 10.1093/treephys/25.8.1001 
Ning, L., and R.S. Bradley, 2014: Winter precipitation variability and corresponding teleconnections over the northeastern United States. J. Geophys. Res. Atmos., 119, 7931-7945, doi: 10.1002/2014JD021591.

Noormets, A., S.G. McNulty, J.L. DeForest, G. Sun, Q. Li, and J. Chen, 2008: Drought during canopy development has lasting effect on annual carbon balance in a deciduous temperate forest. New Phytol., 179, 818-828, doi:10.1111/j.1469-8137.2008.02501.x.

Nordell, K.O., and P.S. Karlsson, 1995: Resorption of nitrogen and dry matter prior to leaf abscission: Variation among individuals, sites and years in the mountain birch. Functional Ecol., 9, 326-333, doi: $10.2307 / 2390581$.

Notaro, M., V. Bennington, and S. Vavrus, 2015: Dynamically downscaled projections of lake-effect snow in the Great Lakes basin. J. Clim., 28, 1661-1684, doi: 10.1175/JCLI-D-14-00467.1.

Nowacki, G.J., and M.D. Abrams, 2008: The demise of fire and "mesophication" of forests in the eastern United States. BioSci., 58, 123-138, doi: 10.1641/b580207.

Omernik, J.M., 2004: Perspectives on the nature and definitions of ecological regions. Env. Manage., 34 (Supp. 1), s27-s38, doi: 10.1007/s00267-003-5197-2.

Omernik, J.M., S.S. Chapman, R.A. Lillie, and R.T. Dumke, 2000: Ecoregions of Wisconsin. Trans. Wisc. Acad. Sci. Arts Lett., 88, 77-103.

O'Reilly, C. M., and 63 coauthors, 2015: Rapid and highly variable warming of lake surface waters around the globe. Geophys. Res. Lett., 42, doi: 10.1002/2015GL066235.

Özdogan, M., 2014: A practical and automated approach to large area forest disturbance mapping with remote sensing. PLoS ONE, 9, doi: 10.1371/journal.pone.0078438.

Pagter, M., U.B. Andersen, and L. Andersen, 2015: Winter warming delays dormancy release, advances budburst, alters carbohydrate metabolism and reduces yield in a temperate shrub. AoB Plants, 7, paper plv024, doi: 10.1093/aobpla/plv024.

Pan, H.-L., and L. Mahrt, 1987: Interaction between soil hydrology and boundary-layer development. Bound. Lay. Meteorol., 38, 185-202, doi: 10.1007/BF00121563. 
Pan, Y., R.A. Birdsey, J. Fang, R. Houghton, P.E. Kauppi, W.A. Kurz, O.L. Phillips, A. Shvidenko, S.L. Lewis, J.G. Canadell, P. Ciais, R.B. Jackson, S.W. Pacala, A.D. McGuire, S. Piao, A. Rautiainen, S. Sitch, and D. Hayes, 2011: A large and persistent carbon sink in the world's forests. Science, 333, 988-993, doi: 10.1126/science.1201609.

Panchen, Z.A., R.B. Primack, B. Nordt, E.R. Ellwood, A.-D. Stevens, S.S. Renner, C.G. Willis, R. Fahey, A. Whittemore, Y. Du, and C.C. Davis, 2014: Leaf out times of temperate woody plants are related to phylogeny, deciduousness, growth habit and wood anatomy. New Phytol., 203, 12081219, doi: 10.1111/nph.12892.

Parida, B.R., and W. Buermann, 2014: Increasing summer drying in North American ecosystems in response to longer nonfrozen periods. Geophys. Res. Lett., 41, 5476-5483, doi: 10.1002/2014GL060495.

Payer, M., N.F. Laird, R.J. Maliawco Jr., and E.G. Hoffman, 2011: Surface fronts, troughs, and baroclinic zones in the Great Lakes region. Wea. Forecast., 26, 555-563, doi: 10.1175/WAF-D-10-05018.1.

Peings, Y., and G. Magnusdottir, 2014: Forcing of the wintertime atmospheric circulation by the multidecadal fluctuations of the North Atlantic Ocean. Env. Res. Lett., 9, paper 034018, doi: 10.1088/1748-9326/9/3/034018.

Peñuelas, J., T. Rutishauser, and I. Filella, 2009: Phenology feedbacks on climate change. Science, 324, 887-888, doi: 10.1126/science. 1173004 .

Peters, M.P., L.R. Iverson, and S.N. Matthews, 2015: Long-term droughtiness and drought tolerance of eastern US forests over five decades. For. Ecol. Manage., 345, 56-64, doi: 10.1016/j.foreco.2015.02.022.

Peterson, T.C., M.P. Hoerling, P.A. Stott, and S.C. Herring, 2013: Explaining extreme events of 2012 from a climate perspective. Bull. Amer. Meteor. Soc., 94, S1-S74, doi: 10.1175/BAMS-D-1300085.1.

Peterson, A.G., and J.T. Abatzoglou, 2014: Observed changes in false springs over the contiguous United States. Geophys. Res. Lett., 41, 2156-2162, doi: 10.1002/2014GL059266. 
Peterson, W.T., and F.B. Schwing, 2003: A new climate regime in Northeast Pacific ecosystems.

Geophys. Res. Lett., 30, 1896, doi: 10.1029/2003GL017528.

Piccolroaz, S., M. Toffolon, and B. Majone, 2015: The role of stratification on lakes' thermal response: The case of Lake Superior. Wat. Resour. Res., 51, 7878-7894, doi: 10.1002/2014WR016555.

Plank, C., and B. Shuman, 2009: Drought-driven changes in lake areas and their effects on the surface energy balance of Minnesota's lake-dotted landscape. J. Clim., 22, 4055-4065, doi: 10.1175/2009JCLI1978.1.

Polgar, C.A., and R.B. Primack, 2011: Leaf-out phenology of temperate woody plants: From trees to ecosystems. New Phytol., 191, 926-941, doi: 10.1111/j.1469-8137.2011.03803.x.

Pope, K.S., V. Dose, D. Da Silva, P.H. Brown, C.A. Leslie, and T.M. DeJong, 2013: Detecting nonlinear response of spring phenology to climate change by Bayesian analysis. Glob. Ch. Biol., 19, 15181525, doi: 10.1111/gcb.12130.

Pordes, R., and co-authors, 2007: The Open Science Grid. J. Phys. Conf. Ser., 78, 012057, doi: 10.1088/1742-6596/78/1/012057.

Prein, A.F., et al., 2015: A review on regional convection-permitting climate modeling: Demonstrations, prospects, and challenges. Rev. Geophys., 53, 323-361, doi: 10.1002/2014RG000475.

Puma, M.J., R.D. Koster, and B.I. Cook, 2013: Phenological versus meteorological controls on landatmosphere water and carbon fluxes. J. Geophys. Res. Biogeosci., 118, 14-29, doi: 10.1029/2012JG002088.

Randerson, J.T., F.M. Hoffman, P.E. Thornton, N.M. Mahowald, K. Lindsay, Y.-H. Lee, C.D. Nevison, S.C. Doney, G. Bonan, R. Stöckli, S. Covey, S.W. Running, and I.Y. Fung, 2009: Systematic assessment of terrestrial biogeochemistry in coupled climate-carbon models. Glob. Ch. Biol., 15, 2462-2484, doi: 10.1111/j.1365-2486.2009.01912.x.

Reed, B.C., J.F. Brown, D. Vanderzee, T.R. Loveland, J.W. Merchant, and D.O. Ohlen, 1994: Measuring phenological variability from satellite imagery. J. Veg. Sci., 5, 703-714, doi: 10.2307/3235884. 
Reyer, C.P.O., and 20 coauthors, 2013: A plant's perspective of extremes: Terrestrial plant responses to changing climatic variability. Glob. Ch. Biol., 19, 75-89, doi: 10.1111/gcb.12023.

Richardson, A.D., and 30 coauthors, 2012: Terrestrial biosphere models need better representation of vegetation phenology: results from the North American Carbon Program Site Synthesis. Glob. Ch. Biol., 18, 566-584. doi: 10.1111/j.1365-2486.2011.02562.x.

Richardson, A.D., and 20 coauthors, 2010: Influence of spring and autumn phenological transitions on forest ecosystem productivity. Phil. Trans. Roy. Soc. B, 365, 3227-3246, doi: 10.1098/rstb.2010.0102.

Richardson, A.D., B.H. Braswell, D.Y. Hollinger, J.P. Jenkins, and S.V. Ollinger, 2009a: Near-surface remote sensing of spatial and temporal variation in canopy phenology. Ecol. Appl., 19, 14171428, doi: 10.1890/08-2022.1.

Richardson, A.D., D.Y. Hollinger, J.D. Aber, S.V. Ollinger, and B.H. Braswell, 2007a: Environmental variation is directly responsible for short- but not long-term variation in forest-atmosphere carbon exchange. Glob. Ch. Biol., 13, 788-803, doi: 10.1111/j.1365-2486.2007.01330.x.

Richardson, A.D., D.Y. Hollinger, D.B. Dail, J.T. Lee, J.W. Munger, and J. O'keefe, 2009b: Influence of spring phenology on seasonal and annual carbon balance in two contrasting New England forests. Tree Phys., 29, 321-331, doi: 10.1093/treephys/tpn040.

Richardson, A.D., J.P. Jenkins, B.H. Braswell, D.Y. Hollinger, S.V. Ollinger, and M.-L. Smith, 2007b: Use of digital webcam images to track spring green-up in a deciduous broadleaf forest. Oecologia, 152, 323-334, doi: 10.1007/s00442-006-0657-z.

Richardson, A.D., T.F. Keenan, M. Migliavacca, Y. Ryu, O. Sonnentag, and M. Toomey, 2013: Climate change, phenology, and phenological control of vegetation feedbacks to the climate system. $\mathrm{Ag}$. For. Meteorol., 169, 156-173, doi: 10.1016/j.agrformet.2012.09.012.

Rigby, J.R., and A. Porporato, 2008: Spring frost risk in a changing climate. Geophys. Res. Lett., 35, paper L12703, doi: 10.1029/2008GL033955. 
Rittenhouse, C.D., and A.R. Rissman, 2015: Changes in winter conditions impact forest management in north temperate forests. J. Env. Manage., 149, 157-167, doi: 10.1016/j.jenvman.2014.10.010.

Roberts, A.M.I., C. Tansey, R.J. Smithers, and A.B. Phillimore, 2015: Predicting a change in the order of spring phenology in temperate forests. Glob. Ch. Biol., 21, 2603-2611, doi: 10.1111/gcb.12896.

Robinson, P.N., B.W. Allred, M.O. Jones, A. Moreno, J.S. Kimball, D.E. Naugle, T.A. Erickson, and A.D. Richardson, 2017: A dynamic Landsat derived Normalized Difference Vegetation Index (NDVI) product for the conterminous United States. Rem. Sens., 9, paper 863, doi:

$10.3390 /$ rs 9080863.

Rodionov, S., and R. Assel, 2000: Atmospheric teleconnection patterns and severity of winters in the Laurentian Great Lakes basin. Atmos.-Ocean, 38, 601-635, doi: 10.1080/07055900.2000.9649661.

Rodionov, S., and R. Assel, 2001: A new look at the Pacific/North American Index. Geophys. Res. Lett., 28, 1519-1522, doi: 10.1029/2000GL012185.

Rodionov, S., and R.A. Assel, 2003: Winter severity in the Great Lakes region: a tale of two oscillations. Clim. Res., 24, 19-31, doi: 10.3354/cr024019.

Rohde, A., and R.P. Bhalerao, 2007: Plant dormancy in the perennial context. Trends Plant Sci., 12, 217223, doi: 10.1016/j.tplants.2007.03.012.

Rohli, R.V., A.J. Vega, M.R. Binkley, S.D. Britton, H.E. Heckman, J.M. Jenkins, Y. Ono, and D.E. Sheeler, 1999: Surface and $700 \mathrm{hPa}$ atmospheric circulation patterns for the Great Lakes basin and eastern North America and relationship to atmospheric teleconnections. J. Great Lakes Res., 25, 45-60, doi: 10.1016/S0380-1330(99)70716-1.

Rosner, B., 1983: Percentage points for a generalized ESD many-outlier procedure. Technometrics, 25, 165-172, doi: $10.2307 / 1268549$.

Ross, S.M., 2003: Peirce's criterion for the elimination of suspect experimental data. J. Eng. Tech., 20, 38-41, ISSN 07479964. 
Ruehr, N.K., C.A. Offermann, A. Gessler, J.B. Winkler, J.P. Ferrio, N. Buchmann, and R.L. Barnard, 2009: Drought effects on allocation of recent carbon: From beech leaves to soil $\mathrm{CO}_{2}$ efflux. New Phytol., 184, 950-961. doi: 10.1111/j.1469-8137.2009.03044.x.

Running, S.W., R.R. Nemani, and R.D. Hungerford, 1987: Extrapolation of synoptic meteorological data in mountainous terrain and its use for simulating forest evapotranspiration and photosynthesis. Can. J. For. Res., 17, 472-483, doi: 10.1139/x87-081.

Rydzik, M., and A.R. Desai, 2014: Relationship between snow extent and midlatitude disturbance centers. J. Clim., 27, 2971-2982, doi: 10.1175/JCLI-D-12-00841.1.

Salmun, H., A. Molod, J. Albrecht, and F. Santos, 2009: Scales of variability of surface vegetation: Calculation and implications for climate models. J. Geophys. Res., 114, paper G02007, doi: 10.1029/2008JG000762.

Salvucci, G.D., and D. Entekhabi, 2011: An alternate and robust approach to calibration for the estimation of land surface model parameters based on remotely sensed observations. Geophys. Res. Lett., $\mathbf{3 8 ,}$ doi: $10.1029 / 2011 \mathrm{~g} 1048366$.

Santanello, J.A., M.A. Friedl, and W.P. Kustas, 2005: An empirical investigation of convective planetary boundary layer evolution and its relationship with the land surface. J. Appl. Meteorol., 44, 917932, doi: 10.1175/JAM2240.1.

Saxe, H., M.G.R. Cannell, Ø. Johnsen, M.G. Ryan, and G. Vourlitis, 2001: Tree and forest functioning in response to global warming. New Phytol., 149, 369-399, doi: 10.1046/j.1469-8137.2001.00057.x.

Schaetzl, R.J., B.D. Knapp, and S.A. Isard, 2005: Modeling soil temperatures and the mesic-frigid boundary in the central Great Lakes region, 1951-2000. Soil Sci. Soc. Am. J., 69, 2033-2040, doi: $10.2136 /$ sssaj2004.0349.

Schenker, G., A. Lenz, C. Körner, and G. Hoch, 2014: Physiological minimum temperatures for root growth in seven common European broad-leaved tree species. Tree Physiol., 34, 302-313, doi: 10.1093/treephys/tpu003. 
Schulze, E.-D., 1986: Carbon dioxide and water vapor exchange in response to drought in the atmosphere and in the soil. Ann. Rev. Plant Physiol., 37, 247-274, doi: 10.1146/annurev.pp.37.060186.001335.

Schwartz, M.D., R. Ahas, and A. Aasa, 2006: Onset of spring starting earlier across the Northern Hemisphere. Glob. Ch. Biol., 12, 343-351, doi: 10.1111/j.1365-2486.2005.01097.x.

Schwartz, M.D., J.M. Hanes, and L. Liang, 2013: Comparing carbon flux and high-resolution spring phenological measurements in a northern mixed forest. Ag. For. Meteorol., 169, 136-147, doi: 10.1016/j.agrformet.2012.10.014.

Scott, R.W., and F.A. Huff, 1996: Impacts of the Great Lakes on regional climate conditions. J. Great Lakes Res., 22, 845-863, doi: 10.1016/S0380-1330(96)71006-7.

Seki, M., T. Yoshida, and T. Takada, 2015: A general method for calculating the optimal leaf longevity from the viewpoint of carbon economy. J. Math. Biol., 71, 669-690, doi: 10.1007/s00285-0140830-7.

Sellers, P.J., R.E. Dickinson, D.A. Randall, A.K. Betts, F.G. Hall, J.A. Berry, G.J. Collatz, A.S. Denning, H.A. Mooney, C.A. Nobre, N. Sato, C.B. Field, and A. Henderson-Sellers, 1997: Modeling the exchanges of energy, water, and carbon between continents and the atmosphere. Science, $\mathbf{2 7 5}$, 502-509, doi: 10.1126/science.275.5299.502.

Sellers, P.J., C.J. Tucker, G.J. Collatz, S.O. Los, C.O. Justice, D.A. Dazlich, and D.A. Randall, 1996: A revised land surface parameterization (SiB2) for atmospheric GCMs. Part II: The generation of global fields of terrestrial biophysical parameters from satellite data. J. Clim., 9, 706-737, doi: 10.1175/1520-0442(1996)009<0706:ARLSPF>2.0.CO;2.

Serbin, S.P., and C.J. Kucharik, 2009: Spatiotemporal mapping of temperature and precipitation for the development of a multidecadal climatic dataset for Wisconsin. J. Appl. Meteorol. Climatol., 48, 742-757, doi: 10.1175/2008JAMC1986.1. 
Serbin, S.P., A. Singh, B.E. McNeil, C.C. Kingdon, and P.A. Townsend, 2014: Spectroscopic determination of leaf morphological and biochemical traits for northern temperate and boreal tree species. Ecol. Appl., 24, 1651-1669, doi: 10.1890/13-2110.1.

Sims, D.A., E.R. Brzostek, A.F. Rahman, D. Dragoni, and R.P. Phillips, 2014: An improved approach for remotely sensing water stress impacts on forest C uptake. Glob. Ch. Biol., 20, 2856-2866, doi: 10.1111/gcb.12537.

Singh, A., S.P. Serbin, B.E. McNeil, C.C. Kingdon, and P.A. Townsend, 2015: Imaging spectroscopy algorithms for mapping canopy foliar chemical and morphological traits and their uncertainties. Ecol. Appl., 25, 2180-2197, doi: 10.1890/14-2098.1.

Siqueira, M., G. Katul, A. Porporato, 2009: Soil moisture feedbacks on convection triggers: The role of soil-plant hydrodynamics. J. Hydrometeorol., 10, 96-112, doi: 10.1175/2008JHM1027.1.

Skaggs, K.E., and S. Irmak, 2012: Long-term trends in air temperature distribution and extremes, growing degree-days, and spring and fall frosts for climate impact assessments on agricultural practices in Nebraska. J. Appl. Meteorol. Climatol., 51, 2060-2073, doi: 10.1175/JAMC-D-11-0146.1.

Skamarock, W.C., J.B. Klemp, J. Dudhia, D.O. Gill, D.M. Barker, M.G. Duda, X.-Y. Huang, W. Wang, and J.G. Powers, 2008: A Description of the Advanced Research WRF Version 3. NCAR Technical Note TN-475+STR, 125 pp.

Small, D., and S. Islam, 2009: A synoptic view of trends and decadal variations in autumn precipitation across the United States from 1948 to 2004. J. Geophys. Res. Atmos., 114, paper D10102, doi: 10.1029/2008JD011579.

Small, D., S. Islam, and M. Barlow, 2010: The impact of a hemispheric circulation regime on fall precipitation over North America. J. Hydrometeorol., 11, 1182-1189, doi: 10.1175/2010JHM1273.1.

Snyder, P.K., C. Delire, and J.A. Foley, 2004: Evaluating the influence of different vegetation biomes on the global climate. Clim. Dyn., 23, 279-302, doi: 10.1007/s00382-004-0430-0. 
Sobolowski, S., G. Gong, and M. Ting, 2010: Modeled climate state and dynamic responses to anomalous North American snow cover. J. Clim., 23, 785-799, doi: 10.1175/2009JCLI3219.1.

Soudani, K., G. le Maire, E. Dufrêne, C. François, N. Delpierre, E. Ulrich, and S. Cecchini, 2008: Evaluation of the onset of green-up in temperate deciduous broadleaf forests derived from Moderate Resolution Imaging Spectroradiometer (MODIS) data. Rem. Sens. Env., 112, 26432655, doi: 10.1016/j.rse.2007.12.004.

Sousounis, P.J., and E.K. Grover, 2002: Potential future weather patterns over the Great Lakes region. $J$. Great Lakes Res., 28, 496-520, doi: 10.1016/S0380-1330(02)70602-3.

Sterling, S., and A. Ducharne, 2008: Comprehensive data set of global land cover change for land surface model applications. Glob. Biogeochem. Cyc., 22, paper GB3017, doi: 10.1029/2007GB002959.

Stewart, J.B., 1977: Evaporation from the wet canopy of a pine forest. Wat. Resour. Res., 13, 915-921, doi: 10.1029/WR013i006p00915.

Stöckli, R., T. Rutishauser, I. Baker, M.A. Liniger, and A.S. Denning, 2011: A global reanalysis of vegetation phenology. J. Geophys. Res., 116, paper G03020, doi: 10.1029/2010JG001545.

Strassmann, K., F. Joos, and G. Fischer, 2008: Simulating effects of land use changes on carbon fluxes: Past contributions to atmospheric $\mathrm{CO}_{2}$ increases and future commitments due to losses of terrestrial sink capacity. Tellus B, 60, 583-603, doi: 10.1111/j.1600-0889.2008.00340.x.

Stuart-Haëntjens, E.J., P.S. Curtis, R.T. Fahey, C.S. Vogel, and C.M. Gough, 2015: Net primary production of a temperate deciduous forest exhibits a threshold response to increasing disturbance severity. Ecology, 96, 2478-2487, doi: 10.1890/14-1810.1.

Sun, W.-Y., and M.G. Bosilovich, 1996: Planetary boundary layer and surface layer sensitivity to land surface parameters. Bound. Lay. Meteorol., 77, 353-378, doi: 10.1007/BF00123532.

Teskey, R.O., B.C. Bongarten, B.M. Cregg, P.M. Dougherty, and T.C. Hennessey, 1987: Physiology and genetics of tree growth response to moisture and temperature stress: An examination of the characteristics of loblolly pine (Pinus taeda L.). Tree Physiol., 3, 41-61, doi: 10.1093/treephys/3.1.41. 
Thomas, N.E., C. Huang, S.N. Goward, S. Powell, K. Rishmawi, K. Schleeweis, A. Hinds, 2011: Validation of North American forest disturbance dynamics derived from Landsat time series stacks. Rem. Sens. Env., 115, 19-32, doi: 10.1016/j.rse.2010.07.009.

Thompson, A.J., and S.M. Moncrieff, 1982: Prediction of bud burst in Douglas fir by degree-day accumulation. Can. J. For. Res., 12, 448-452, doi: 10.1139/x82-069.

Thornton, P.E., S.W. Running, and M.A. White, 1997: Generating surfaces of daily meteorological variables over large regions of complex terrain. J. Hydrol., 190, 214-251, doi: 10.1016/S00221694(96)03128-9.

Tian, Y., R.E. Dickinson, L. Zhou, and M. Shaikh, 2004: Impact of new land boundary conditions from Moderate Resolution Imaging Spectroradiometer (MODIS) data on the climatology of land surface variables. J. Geophys. Res., 109, paper D20115, doi: 10.1029/2003JD004499.

Toomey, M., and 23 coauthors, 2015: Greenness indices from digital cameras predict the timing and seasonal dynamics of canopy-scale photosynthesis. Ecol. Appl., 25, 99-115, doi: 10.1890/140005.1.

Toomey, M., and L.A. Vierling, 2005: Multispectral remote sensing of landscape level foliar moisture: Techniques and applications for forest ecosystem monitoring. Can. J. For. Res., 35, 1087-1097, doi: $10.1139 / \mathrm{x} 05-043$.

Townsend, P.A., A. Singh, J.R. Foster, N.J. Rehberg, C.C. Kingdon, K.N. Eshleman, and S.W. Seagle, 2012: A general Landsat model to predict canopy defoliation in broadleaf deciduous forests. Rem. Sens. Env., 119, 255-265, doi: 10.1016/j.rse.2011.12.023.

Trenberth, K.E., G.W. Branstator, and P.A. Arkin, 1988: Origins of the 1988 North American drought. Science, 242, 1640-1645, doi: 10.1126/science.242.4886.1640.

Trenberth, K.E., G.W. Branstator, D. Karoly, A. Kumar, N.-C. Lau, and C. Ropelewski, 1998: Progress during TOGA in understanding and modeling global teleconnections associated with tropical sea surface temperatures. J. Geophys. Res. Oceans, 103, 14,291-14,324, doi: 10.1029/97JC01444. 
Trier, S.B., M.A. LeMone, F. Chen, and K.W. Manning, 2011: Effects of surface heat and moisture exchange on ARW-WRF warm-season precipitation forecasts over the central United States. Wea. Forecast., 26, 3-25, doi: 10.1175/2010WAF2222426.1.

Troen, I.B., and L. Mahrt, 1986: A simple model of the atmospheric boundary layer; sensitivity to surface evaporation. Bound. Lay. Meteorol., v. 37, pp. 129-148, doi: 10.1007/BF00122760.

Trudgill D.L., A. Honek, D. Li, and N.M. van Straalen, 2005: Thermal time — concepts and utility. Annals Appl. Biol., 146, 1-14, doi: 10.1111/j.1744-7348.2005.04088.x.

Twine, T.E., and C.J. Kucharik, 2009: Climate impacts on net primary productivity trends in natural and managed ecosystems of the central and eastern United States. Ag. For. Meteorol., 149, 21432161, doi: 10.1016/j.agrformet.2009.05.012.

[USEPA] U.S. Environmental Protection Agency, 2011: Level III and IV ecoregions of the continental United States. U.S. EPA, National Health and Environmental Effects Research Laboratory, Corvallis, Oregon. Available online at https://www.epa.gov/eco-research/level-iii-and-ivecoregions-continental-united-states.

Van Cleave, K., J.D. Lenters, J. Wang, and E.M. Verhamme, 2014: A regime shift in Lake Superior ice cover, evaporation, and water temperature following the warm El Niño winter of 19971998. Limnol. Oceanogr., 59, 1889-1898, doi: 10.4319/1o.2014.59.6.1889.

Viherä-Aarnio, A., S. Soutinen, J. Partanen, and R. Häkkinen, 2014: Internal development of vegetative buds of Norway spruce trees in relation to accumulated chilling and forcing temperatures. Tree Physiol., 24, 547-556, doi: 10.1093/treephys/tpu038.

Vitasse, Y., A. Lenz, and C. Körner, 2014: The interaction between freezing tolerance and phenology in temperate deciduous trees. Front. Plant Sci., 5, article no. 541, doi: 10.3389/fpls.2014.00541.

Voelker, S.L., P.-E. Noirot-Cosson, M.C. Stambaugh, E.R. McMurry, F.C. Meinzer, B. Lachenbruch, and R.P. Guyette, 2012: Spring temperature responses of oaks are synchronous with North Atlantic conditions during the last deglaciation. Ecol. Monogr., 82, 169-187, doi: 10.1890/11-0848.1. 
Wang, H., S. Schubert, R. Koster, Y. Ham, and M. Suarez, 2014: On the role of SST forcing in the 2011 and 2012 extreme U.S. heat and drought: A study in contrasts. J. Hydrometeor., 15, 1255-1273, doi: 10.1175/JHM-D-13-069.1.

Wang, J., X. Bai, H. Hu, A. Clites, M. Colton, and B. Lofgren, 2012: Temporal and spatial variability of Great Lakes ice cover, 1973-2010. J. Clim., 25, 1318-1329, doi: 10.1175/2011JCLI4066.1.

Watras, C.J., J.S. Read, K.D. Holman, Z. Liu, Y.-Y. Song, A.J. Watras, S. Morgan, and E.H. Stanley, 2014: Decadal oscillation of lakes and aquifers in the upper Great Lakes region of North America: Hydroclimatic implications. Geophys. Res. Lett., 41, 456-462, doi: 10.1002/2013GL058679.

Weaver, S.J., A. Ruiz-Barradas, and S. Nigam, 2009: Pentad evolution of the 1988 drought and 1993 flood over the Great Plains: An NARR perspective on the atmospheric and terrestrial water balance. J. Clim., 22, 5366-5384, doi: 10.1175/2009JCLI2684.1.

Wheeler, G.A., E.J. Cushing, E. Gorham, T. Morley, and G.B. Ownbey, 1992: A major floristic boundary in Minnesota: An analysis of 280 taxa occurring in the western and southern portions of the state. Can. J. Botany, 70, 319-333, doi: 10.1139/b92-043.

White, M.A. and R.R. Nemani, 2003: Canopy duration has little influence on annual carbon storage in the deciduous broad leaf forest. Glob. Ch. Biol., 9, 967-972. doi: 10.1046/j.1365-2486.2003.00585.x.

White, M.A., and R.R. Nemani, 2006: Real-time monitoring and short-term forecasting of land surface phenology. Rem. Sens. Env., 104, 43-49, doi: 10.1016/j.rse.2006.04.014.

White, K., J. Pontius, and P. Schaberg, 2014: Remote sensing of spring phenology in northeastern forests: A comparison of methods, field metrics and sources of uncertainty. Rem. Sens. Env., 148, 97-107, doi: 10.1016/j.rse.2014.03.017.

White, M.A., S.W. Running, and P.E. Thornton, 1999: The impact of growing-season length variability on carbon assimilation and evapotranspiration over 88 years in the eastern US deciduous forest. Int. J. Biometeorol., 42, 139-145, doi: 10.1007/s004840050097. 
White, M.A., P.E. Thornton, and S.W. Running, 1997: A continental phenology model for monitoring vegetation responses to interannual climatic variability. Glob. Biogeochem. Cyc., 11, 217-234, doi: 10.1029/97GB00330.

Williams, C.M., H.A.L. Henry, and B.J. Sinclair, 2014: Cold truths: How winter drives responses of terrestrial organisms to climate change. Biol. Rev., 90, 214-235, doi: 10.1111/brv.12105.

Wise, E.K., M.L. Wrzesien, M.P. Dannenberg, and D.L. McGinnis, 2015: Cool-season precipitation patterns associated with teleconnection interactions in the United States. J. Appl. Meteorol. Climatol., 54, 494-505, doi: 10.1175/JAMC-D-14-0040.1.

Wit, E., E. van den Heuvel, and J.-W. Romeyn, 2012: 'All models are wrong...': An introduction to model uncertainty. Statistica Neerlandica, 66, 217-236, doi: 10.1111/j.1467-9574.2012.00530.x.

Wold, H., 1975: Soft modeling by latent variables: The nonlinear iterative partial least squares approach. In J. Gani (Ed.), Perspectives in Probability and Statistics: Papers in Honour of M.S. Bartlett on the Occasion of His Sixty-Fifth Birthday, 117-142, London: Academic Press, ISBN 9780122744501 , OCLC 499454648.

Wold, S., M. Høy, H. Martens, J. Trygg, F. Westad, J. MacGregor, and B.M. Wise, 2009: The PLS model space revisited. J. Chemometrics, 23, 67-68, doi: 10.1002/cem.1171.

Wold, S., A. Ruhe, H. Wold, and W.J. Dunn, 1984: The collinearity problem in linear regression. The Partial Least Squares (PLS) approach to generalized inverses. SIAM J. Sci. Stat. Comp., 5, 735743.

Wold, S., M. Sjöström, and L. Eriksson, 2001a: PLS-regression: A basic tool of chemometrics. Chem. Int. Lab. Sys., 58, 109-130, doi: 10.1016/S0169-7439(01)00155-1.

Wold, S., J. Trygg, A. Berglund, and H. Antti, 2001b: Some recent developments in PLS modeling. Chem. Int. Lab. Sys., 58, 131-150, doi: 10.1016/S0169-7439(01)00156-3.

Wolkovich, E.M., and A.K. Ettinger, 2014: Back to the future for plant phenology research. New Phytol., 203, 1021-1024, doi: 10.1111/nph.12957. 
Wolter, P.T., P.A. Townsend, C.C. Kingdon, and B.R. Sturtevant, 2008: Remote sensing of the distribution and abundance of host species for spruce budworm in northern Minnesota and Ontario. Rem. Sens. Env., 112, 3971-3982, doi: 10.1016/j.rse.2008.07.005.

Wolter, P.T., P.A. Townsend, and B.R. Sturtevant, 2009: Estimation of forest structural parameters using 5 and 10-meter SPOT-5 satellite data. Rem. Sens. Env., 113, 2019-2036, doi:

10.1016/j.rse.2009.05.009.

Wolter, P.T., and P.A. Townsend, 2011: Multi-sensor data fusion for estimating forest species composition and abundance in northern Minnesota. Rem. Sens. Env., 115, 671-691, doi: 10.1016/j.rse.2010.10.010.

Wright, D.M., D.J. Posselt, and A.L. Steiner, 2013: Sensitivity of lake-effect snowfall to lake ice cover and temperature in the Great Lakes region. Mon. Wea. Rev., 141, 670-689, doi: 10.1175/MWRD-12-00038.1.

Xie, Y., K.F. Ahmed, J.M. Allen, A.M. Wilson, and J.A. Silander Jr., 2015: Green-up of deciduous forest communities of northeastern North America in response to climate variation and climate change. Landscape Ecol., 30, 109-123, doi: 10.1007/s10980-014-0099-7.

Xie, Y., Z. Sha, and M. Yu, 2008: Remote sensing imagery in vegetation mapping: A review. J. Plant Ecol., 1, 9-23, doi: 10.1093/jpe/rtm005.

Yang, L.H., K.F. Edwards, J.E. Byrnes, J.L. Bastow, A.N. Wright, and K.O. Spence, 2010: A metaanalysis of resource pulse-consumer interactions. Ecol. Monogr., 80, 125-151, doi: 10.1890/081996.1.

Yebra, M., P.E. Dennison, E. Chuvieco, D. Riaño, P. Zylstra, E.R. Hunt Jr., F.M. Danson, Y. Qi, and S. Jurdao, 2013: A global review of remote sensing of live fuel moisture content for fire danger assessment: Moving towards operational products. Rem. Sens. Env., 136, 455-468, doi: 10.1016/j.rse.2013.05.029. 
Yilmaz, M.T., E.R. Hunt, and T.J. Jackson, 2008: Remote sensing of vegetation water content from equivalent water thickness using satellite imagery. Rem. Sens. Env., 112, 2514-2522, doi: 10.1016/j.rse.2007.11.014.

Yu, L., S. Zhong, X. Bian, W.E. Heilman, and J.A. Andresen, 2014: Temporal and spatial variability of frost-free seasons in the Great Lakes region of the United States. Int. J. Climatol., 34, 3499-3514, doi: $10.1002 /$ joc.3923.

Yuan, W., S. Liu, G. Yu, J.-M. Bonnefond, J. Chen, K. Davis, A.R. Desai, A.H. Goldstein, D. Gianelle, F. Rossi, A.E. Suyker, and S.B. Verma, 2010: Global estimates of evapotranspiration and gross primary production based on MODIS and global meteorology data. Rem. Sens. Env., 114, 14161431, doi: 10.1016/j.rse.2010.01.022.

Yuan, W., and 15 coauthors, 2007: Deriving a light use efficiency model from eddy covariance flux data for predicting daily gross primary production across biomes. Ag. For. Meteorol., 143, 189-207, doi: 10.1016/j.agrformet.2006.12.001.

Zhang, X., M.A. Friedl, C.B. Schaaf, and A.H. Strahler, 2004: Climate controls on vegetation phenological patterns in northern mid- and high latitudes inferred from MODIS data. Glob. Ch. Biol., 10, 1133-1145, doi: 10.1111/j.1365-2486.2004.00784.x

Zhang, X., M.A. Friedl, C.B. Schaaf, A.H. Strahler, J.C.F. Hodges, F. Gao, B.C. Reed, and A. Huete, 2003: Monitoring vegetation phenology using MODIS. Rem. Sens. Env., 84, 471-475, doi: 10.1016/S0034-4257(02)00135-9.

Zhao, J., H. Zhang, Z. Zhang, X. Guo, X. Li, and C. Chen, 2015: Spatial and temporal changes in vegetation phenology at middle and high latitudes of the Northern Hemisphere over the past three decades. Rem. Sens., 7, 10,973-10,995, doi: 10.3390/rs70810973.

Zhu, Z., and C.E. Woodcock, 2012: Object-based cloud and cloud shadow detection in Landsat imagery. Rem. Sens. Env., 118, 83-94, doi: 10.1016/j.rse.2011.10.028.

Zhu, Z., and C.E. Woodcock, 2014: Continuous change detection and classification of land cover using all available Landsat data. Rem. Sens. Env., 144, 152-171, doi: 10.1016/j.rse.2014.01.011. 
Zhu, Z., C.E. Woodcock, and P. Olofsson, 2012: Continuous monitoring of forest disturbance using all available Landsat imagery. Rem. Sens. Env., 122, 75-91, doi: 10.1016/j.rse.2011.10.030.

Ziemer, R.R., 1979: Evaporation and transpiration. Rev. Geophys., 17, 1175-1186, doi: 10.1029/RG017i006p01175. 


\section{APPENDIX}

Table A1: Collected P25R28 USGS Landsat scene descriptors (231 scenes, see also Table 3.6).

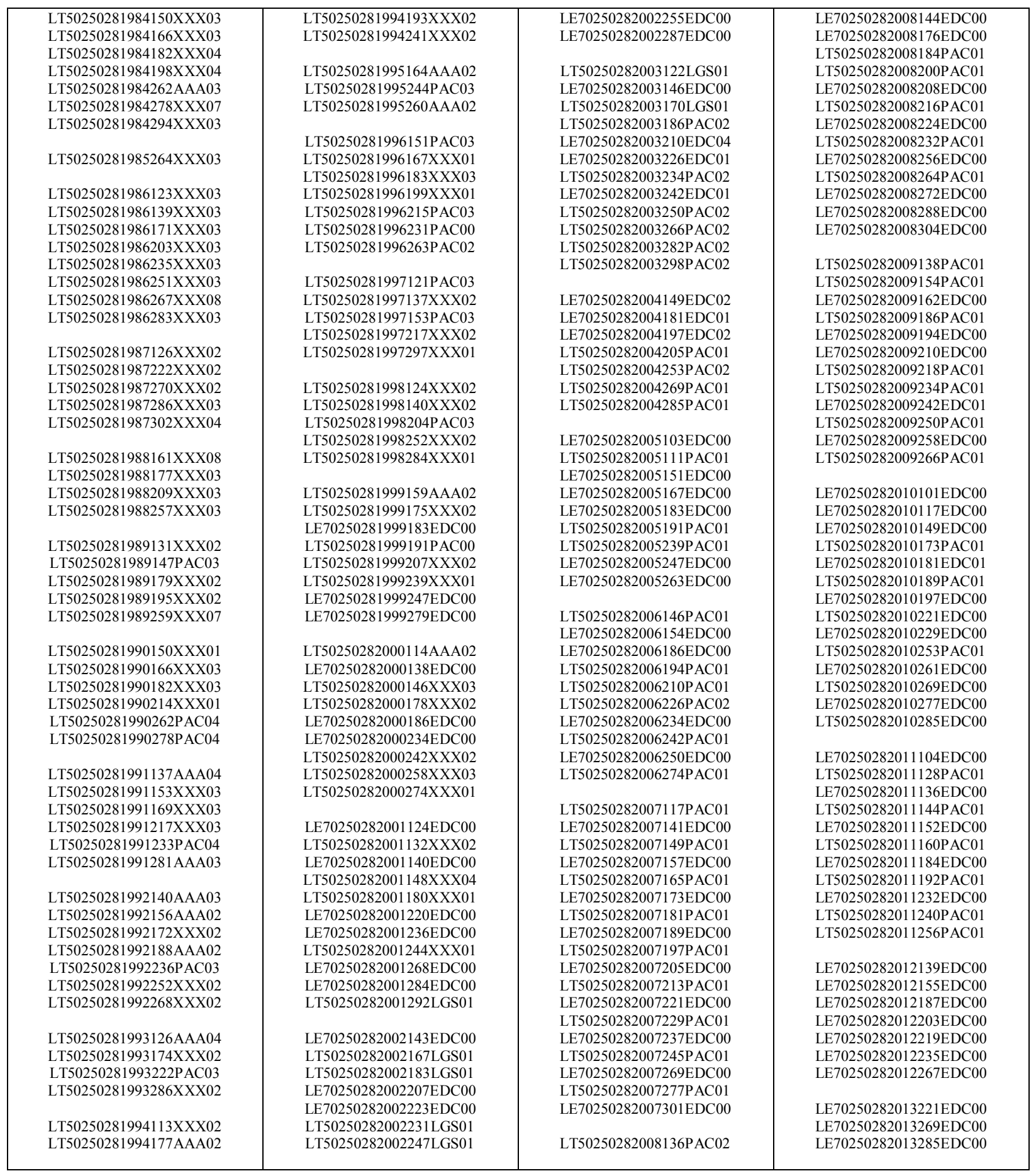


Table A2: Collected P26R27 USGS Landsat scene descriptors (202 scenes, see also Table 3.6).

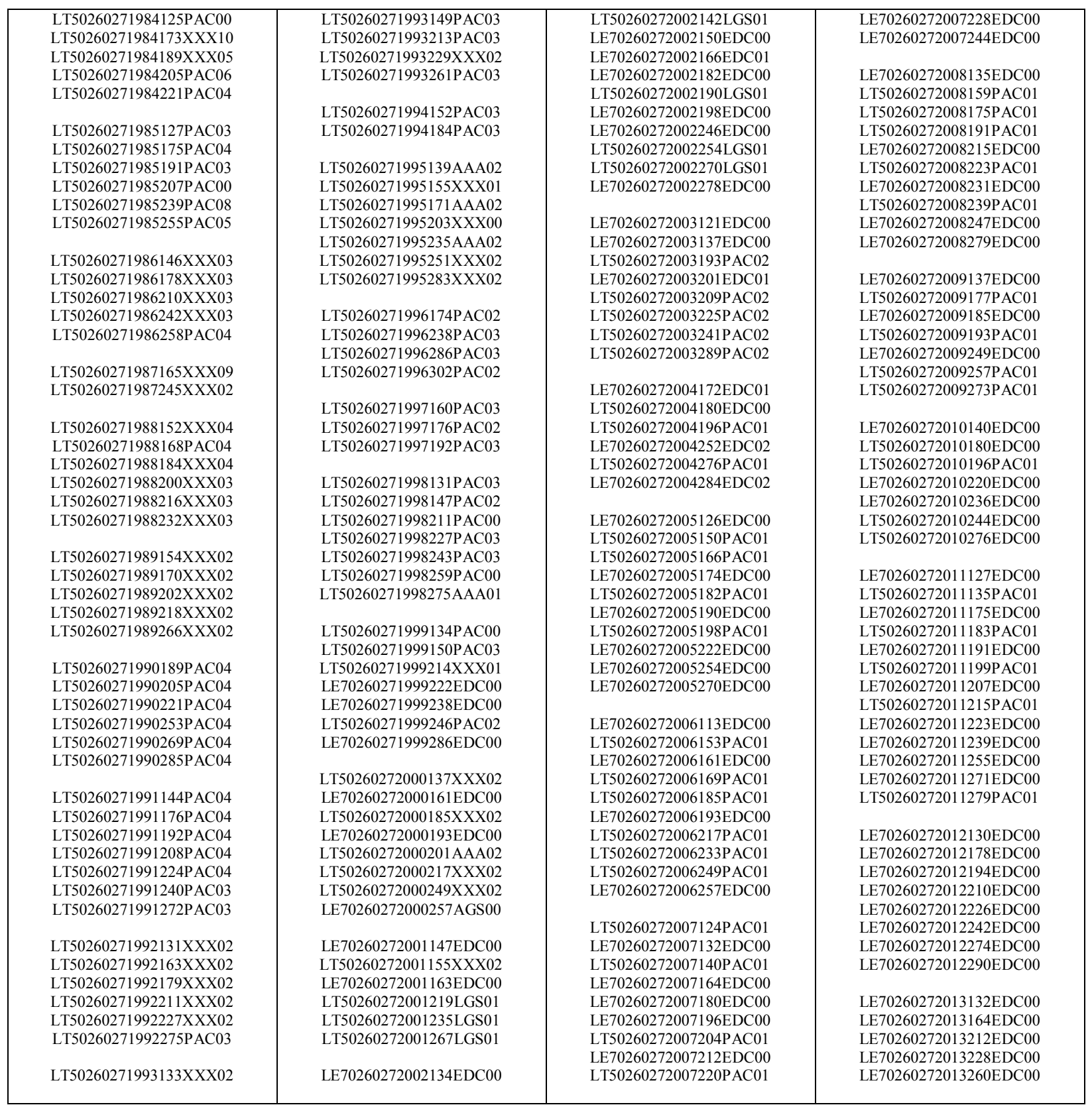


Table A3: Collected P26R28 USGS Landsat scene descriptors (250 scenes, see also Table 3.6).

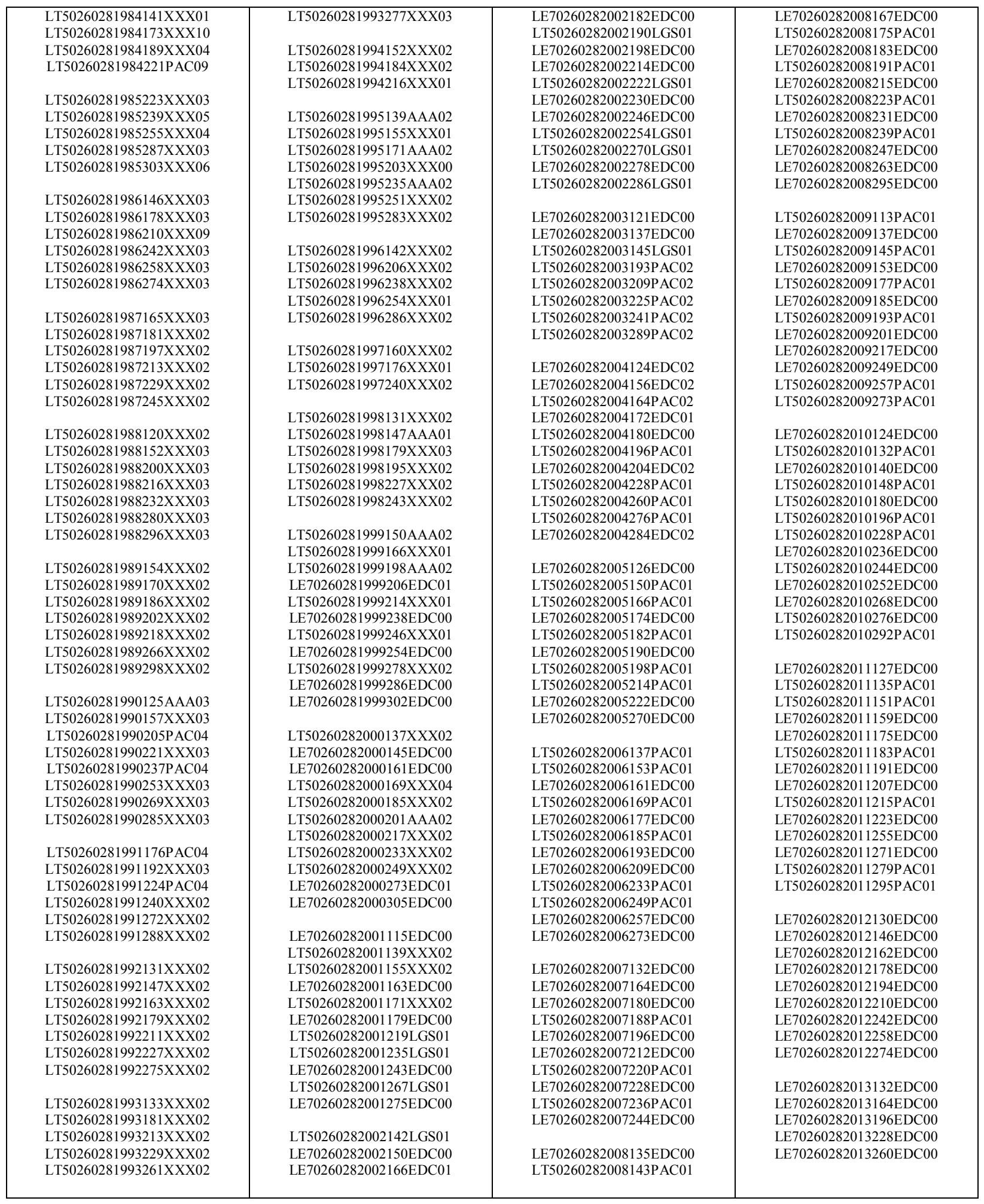


Table A4: Collected P27R27 USGS Landsat scene descriptors (205 scenes, see also Table 3.6).

\begin{tabular}{|c|c|c|c|}
\hline LT50270271984148PAC09 & LT50270271993220PAC03 & LE70270272001154EDC00 & LE70270272007187EDC00 \\
\hline LT50270271984180XXX13 & LT50270271993236PAC03 & LE70270272001170EDC00 & LT50270272007195PAC01 \\
\hline LT50270271984212PAC05 & LT50270271993268XXX02 & LT50270272001178XXX01 & LT50270272007211PAC01 \\
\hline LT50270271984228PAC03 & LT50270271993284PAC03 & LE70270272001186EDC01 & LT50270272007227PAC01 \\
\hline \multirow[t]{2}{*}{ LT50270271984260PAC00 } & & LT50270272001194LGS01 & LT50270272007243PAC01 \\
\hline & LT50270271994127PAC03 & LE70270272001218EDC00 & LT50270272007259PAC 01 \\
\hline \multirow[t]{2}{*}{ LT50270271985118XXX05 } & LT50270271994159P AC03 & LT50270272001226LGS01 & LE70270272007267EDC00 \\
\hline & LT50270271994175PAC03 & LE70270272001234EDC00 & \\
\hline LT50270271986137XXX02 & LT50270271994191PAC03 & LT50270272001274LGS01 & LE70270272008126EDC00 \\
\hline LT50270271986153XXX03 & LT50270271994223XXX01 & & LE70270272008142EDC00 \\
\hline LT50270271986169XXX03 & LT50270271994287XXX02 & LE70270272002141EDC00 & LT50270272008182PAC01 \\
\hline LT50270271986217XXX03 & LT50270271994303PAC03 & LE70270272002157EDC00 & LT50270272008214PAC01 \\
\hline \multirow[t]{2}{*}{ LT50270271986233XXX03 } & & LT50270272002165LGS01 & LE70270272008222EDC00 \\
\hline & LT50270271995146XXX01 & LT50270272002181LGS01 & LT50270272008230PAC01 \\
\hline LT50270271987124XXX02 & LT50270271995162XXX03 & LT50270272002197LGS01 & LE70270272008238EDC00 \\
\hline LT50270271987156PAC03 & LT50270271995194XXX03 & LE70270272002237EDC00 & LT50270272008262PAC01 \\
\hline LT50270271987172XXX02 & LT50270271995210XXX02 & LE70270272002253EDC00 & LE70270272008270EDC00 \\
\hline LT50270271987220XXX02 & LT50270271995226XXX02 & LE70270272002269EDC00 & LT50270272008278PAC01 \\
\hline LT50270271987236XXX04 & LT50270271995242XXX01 & & \\
\hline LT50270271987252XXX02 & LT50270271995290XXX02 & LE70270272003128EDC00 & LT50270272009136PAC01 \\
\hline \multirow[t]{2}{*}{ LT50270271987268XXX07 } & & LT50270272003136LGS01 & LE70270272009144EDC00 \\
\hline & LT50270271996149XXX02 & LE70270272003144EDC00 & LT50270272009152PAC01 \\
\hline LT50270271988127PAC04 & LT50270271996165PAC01 & LT50270272003152EDC02 & LE70270272009176EDC00 \\
\hline LT50270271988143PAC04 & LT50270271996213PAC03 & LT50270272003184PAC02 & LT50270272009184PAC01 \\
\hline LT50270271988159AAA05 & LT50270271996229PAC00 & LT50270272003216PAC02 & LE70270272009192EDC00 \\
\hline LT50270271988191XXX03 & LT50270271996245PAC02 & LE70270272003224EDC01 & LT50270272009200PAC 01 \\
\hline LT50270271988207XXX03 & LT50270271996277PAC02 & LT50270272003248PAC02 & LE70270272009224EDC00 \\
\hline LT50270271988223PAC04 & LT50270271996293PAC02 & LT50270272003280PAC02 & LT50270272009248PAC01 \\
\hline \multirow[t]{2}{*}{ LT50270271988287PAC04 } & & & LT50270272009264PAC01 \\
\hline & LT50270271997119PAC03 & LE70270272004131EDC01 & LE70270272009272EDC00 \\
\hline LT50270271989129XXX02 & LT50270271997151PAC03 & LT50270272004139PAC03 & \\
\hline LT50270271989161PAC03 & LT50270271997167PAC02 & LE70270272004147EDC01 & LT50270272010139PAC01 \\
\hline LT50270271989257XXX03 & LT50270271997247PAC03 & LT50270272004155PAC02 & LE70270272010147EDC00 \\
\hline \multirow[t]{2}{*}{ LT50270271989273PAC03 } & & LT50270272004203PAC01 & LT50270272010171EDC00 \\
\hline & LT50270271998122PAC03 & LT50270272004219PAC01 & LT50270272010187EDC00 \\
\hline LT50270271990132PAC04 & LT50270271998138PAC05 & LE70270272004227EDC02 & LT50270272010235PAC 01 \\
\hline LT50270271990148PAC04 & LT50270271998202PAC02 & LT50270272004267PAC01 & LE70270272010275EDC00 \\
\hline LT50270271990164PAC04 & LT50270271998218PAC02 & LT50270272004283PAC01 & LE70270272010291EDC00 \\
\hline LT50270271990196PAC04 & LT50270271998250PAC02 & & \\
\hline LT50270271990212PAC04 & LT50270271998282PAC05 & LT50270272005173PAC01 & LT50270272011126PAC01 \\
\hline LT50270271990228PAC04 & & LE70270272005213EDC00 & LT50270272011158PAC01 \\
\hline LT50270271990244PAC04 & LT50270271999173AAA02 & LT50270272005269PAC01 & LE70270272011182EDC00 \\
\hline \multirow[t]{2}{*}{ LT50270271990260XXX03 } & LT50270271999205XXX04 & & LT50270272011206PAC01 \\
\hline & LE70270271999213EDC00 & LE70270272006136EDC00 & LT50270272011222PAC01 \\
\hline LT50270271991167PAC04 & LT50270271999237PAC02 & LE70270272006152EDC00 & LT50270272011238PAC01 \\
\hline LT50270271991199PAC04 & LE70270271999277EDC00 & LT50270272006176PAC03 & LT50270272011254PAC01 \\
\hline LT50270271991231PAC04 & & LE70270272006184EDC00 & LE70270272011262EDC00 \\
\hline LT50270271991247XXX04 & LT50270272000128XXX01 & LT50270272006192PAC01 & \\
\hline \multirow[t]{2}{*}{ LT50270271991263PAC03 } & LE70270272000136EDC00 & LT50270272006208PAC01 & LE70270272012153EDC00 \\
\hline & LT50270272000144XXX02 & LT50270272006224PAC01 & LE70270272012185EDC00 \\
\hline LT50270271992122PAC03 & LE70270272000152EDC00 & LE70270272006232EDC00 & LE70270272012201EDC00 \\
\hline LT50270271992154XXX02 & LT50270272000176XXX02 & LE70270272006248EDC00 & LE70270272012233EDC00 \\
\hline LT50270271992218XXX02 & LE70270272000200EDC00 & LT50270272006256PAC04 & LE70270272012249EDC00 \\
\hline LT50270271992250AAA02 & LE70270272000216EDC00 & LE70270272006264EDC00 & \\
\hline LT50270271992266AAA02 & LE70270272000232EDC00 & & LE70270272013203EDC00 \\
\hline & LE70270272000248EDC00 & LE70270272007123EDC00 & LE70270272013235EDC00 \\
\hline LT50270271993124PAC03 & LT50270272000256XXX02 & LE70270272007155EDC00 & LE70270272013267EDC00 \\
\hline LT50270271993156XXX02 & & LT50270272007163PAC01 & \\
\hline LT50270271993172PAC04 & LE70270272001138EDC00 & LE70270272007171EDC00 & \\
\hline
\end{tabular}


Table A5: Collected P27R28 USGS Landsat scene descriptors (257 scenes, see also Table 3.6).

\begin{tabular}{|c|c|c|c|}
\hline LT50270281984164AAA03 & LT50270281994175XXX02 & & LE70270282007171EDC00 \\
\hline \multirow[t]{2}{*}{ LT50270281984180XXX04 } & LT50270281994191AAA02 & LE70270282002141EDC00 & LE70270282007187EDC00 \\
\hline & LT50270281994223XXX01 & LE70270282002157EDC00 & LT50270282007195PAC01 \\
\hline LT50270281985118XXX05 & LT50270281994255XXX02 & LT50270282002165LGS01 & LT50270282007211PAC01 \\
\hline LT50270281985214XXX03 & LT50270281994287XXX02 & $\begin{array}{l}\text { LE70270282002173EDC00 } \\
\text { LT50270282002181LGS01 }\end{array}$ & $\begin{array}{l}\text { LE70270282007219EDC00 } \\
\text { LT50270282007227PAC01 }\end{array}$ \\
\hline LT50270281986137XXX02 & LT50270281995146XXX01 & LT50270282002197LGS01 & LT50270282007243PAC01 \\
\hline LT50270281986153XXX03 & LT50270281995162XXX03 & LT50270282002213LGS01 & LT50270282007259PAC01 \\
\hline LT50270281986169XXX03 & LT50270281995194XXX03 & LE70270282002237EDC00 & LE70270282007299EDC00 \\
\hline LT50270281986185XXX02 & LT50270281995210XXX02 & LE70270282002253EDC00 & \\
\hline LT50270281986201XXX03 & LT50270281995226XXX01 & LE70270282002269EDC00 & LE70270282008126EDC00 \\
\hline LT50270281986217XXX03 & LT50270281995242XXX01 & & LE70270282008142EDC00 \\
\hline LT50270281986233XXX03 & LT50270281995290XXX02 & LT50270282003136LGS01 & LT50270282008166PAC01 \\
\hline LT50270281986249XXX03 & & LE70270282003144EDC00 & LT50270282008182PAC01 \\
\hline \multirow[t]{2}{*}{ LT50270281986281XXX03 } & LT50270281996133AAA01 & LT50270282003152EDC02 & LE70270282008190EDC00 \\
\hline & LT50270281996149XXX02 & LT50270282003168LGS01 & LT50270282008214PAC01 \\
\hline LT50270281987124XXX02 & LT50270281996165XXX00 & LT50270282003184PAC02 & LE70270282008222EDC00 \\
\hline LT50270281987156XXX02 & LT50270281996181XXX02 & LE70270282003208EDC01 & LT50270282008230PAC01 \\
\hline LT50270281987172XXX02 & LT50270281996197XXX02 & LT50270282003216PAC02 & LE70270282008238EDC00 \\
\hline LT50270281987236XXX02 & LT50270281996213AAA01 & LE70270282003224EDC01 & LT50270282008262PAC01 \\
\hline LT50270281987252XXX02 & LT50270281996245AAA01 & LT50270282003248PAC02 & LE70270282008270EDC00 \\
\hline LT50270281987268XXX07 & LT50270281996277XXX01 & LT50270282003280PAC02 & LT50270282008278PAC01 \\
\hline \multirow[t]{2}{*}{ LT50270281987284XXX03 } & & & LT50270282008294PAC01 \\
\hline & LT50270281997151XXX02 & LE70270282004131EDC01 & \\
\hline LT50270281988127XXX03 & LT50270281997167XXX01 & LT50270282004139PAC03 & LT50270282009136PAC01 \\
\hline LT50270281988159AAA04 & LT50270281997215XXX02 & LE70270282004147EDC01 & LE70270282009144EDC00 \\
\hline LT50270281988207XXX03 & LT50270281997247XXX02 & LT50270282004155PAC02 & LT50270282009152PAC01 \\
\hline LT50270281988223XXX03 & LT50270281997263AAA01 & LT50270282004171LGS01 & LE70270282009176EDC00 \\
\hline LT50270281988239AAA04 & & LE70270282004195EDC02 & LE70270282009192EDC00 \\
\hline LT50270281988255XXX03 & LT50270281998138AAA03 & LT50270282004203PAC01 & LT50270282009200PAC01 \\
\hline \multirow{2}{*}{ LT50270281988287XXX03 } & LT50270281998154XXX01 & LE70270282004211EDC02 & LE70270282009208EDC00 \\
\hline & LT50270281998202XXX01 & LT50270282004219PAC01 & LT50270282009216PAC01 \\
\hline LT50270281989129XXX03 & LT50270281998218XXX01 & LE70270282004227EDC02 & LE70270282009224EDC00 \\
\hline LT50270281989161XXX02 & LT50270281998250AAA01 & LT50270282004235PAC01 & LT50270282009248PAC01 \\
\hline LT50270281989209XXX02 & LT50270281998282AAA03 & LE70270282004243EDC02 & LE70270282009256EDC00 \\
\hline LT50270281989225XXX02 & & LT50270282004267PAC01 & LT50270282009264PAC01 \\
\hline LT50270281989257XXX02 & LT50270281999173AAA02 & LT50270282004283PAC01 & \\
\hline \multirow[t]{2}{*}{ LT50270281989273XXX02 } & LT50270281999205XXX04 & LT50270282004299PAC01 & LT50270282010139PAC01 \\
\hline & LE70270281999213EDC00 & & LE70270282010147EDC00 \\
\hline LT50270281990132XXX03 & LE70270281999229EDC01 & LE70270282005149EDC00 & LT50270282010171EDC00 \\
\hline LT50270281990148XXX03 & LT50270281999237AAA01 & LT50270282005157PAC01 & LE70270282010179EDC00 \\
\hline LT50270281990180XXX01 & LE70270281999245EDC00 & LT50270282005173PAC01 & LT50270282010187EDC00 \\
\hline LT50270281990196XXX03 & LE70270281999261EDC00 & LT50270282005205PAC01 & LE70270282010227EDC00 \\
\hline LT50270281990212PAC04 & LT50270281999269XXX01 & LE70270282005213EDC00 & LT50270282010235PAC01 \\
\hline LT50270281990228XXX03 & LE70270281999277EDC00 & LT50270282005237PAC01 & LE70270282010243EDC00 \\
\hline \multirow[t]{2}{*}{ LT50270281990244PAC04 } & LE70270281999293EDC00 & LE70270282005245EDC00 & LE70270282010275EDC00 \\
\hline & & LT50270282005253PAC01 & LE70270282010291EDC00 \\
\hline LT50270281991167AAA04 & LT50270282000128XXX01 & LE70270282005261EDC00 & \\
\hline LT50270281991199XXX03 & LE70270282000136EDC00 & LT50270282005269PAC01 & LT50270282011126PAC01 \\
\hline LT50270281991231XXX03 & LT50270282000144XXX02 & LE70270282005293EDC00 & LT50270282011158PAC01 \\
\hline LT50270281991247XXX04 & LE70270282000152EDC00 & LT50270282005301PAC01 & LE70270282011182EDC00 \\
\hline \multirow[t]{2}{*}{ LT50270281991263XXX02 } & LT50270282000160AAA02 & & LT50270282011206PAC01 \\
\hline & LE70270282000184EDC00 & LE70270282006136EDC00 & LT50270282011222PAC01 \\
\hline LT50270281992122PAC03 & LT50270282000192AAA02 & LT50270282006144PAC01 & LE70270282011230EDC00 \\
\hline LT50270281992154XXX02 & LE70270282000216EDC00 & LE70270282006152EDC00 & LT50270282011238PAC01 \\
\hline LT50270281992186XXX02 & LT50270282000224XXX02 & LT50270282006176PAC03 & LT50270282011254PAC01 \\
\hline LT50270281992202AAA02 & LE70270282000248EDC00 & LE70270282006184EDC00 & LE70270282011262EDC00 \\
\hline LT50270281992218XXX02 & LT50270282000256XXX02 & LT50270282006192PAC01 & LE70270282011294EDC00 \\
\hline LT50270281992250AAA02 & & LT50270282006208PAC01 & LT50270282011302PAC01 \\
\hline \multirow[t]{2}{*}{ LT50270281992266AAA02 } & LE70270282001138EDC00 & LE70270282006216EDC00 & \\
\hline & LE70270282001154EDC00 & LT50270282006224PAC01 & LE70270282012153EDC00 \\
\hline LT50270281993124XXX02 & LE70270282001170EDC00 & LE70270282006232EDC00 & LE70270282012185EDC00 \\
\hline LT50270281993156XXX02 & LT50270282001178XXX01 & LE70270282006248EDC00 & LE70270282012201EDC00 \\
\hline LT50270281993172AAA03 & LE70270282001186EDC01 & LT50270282006256PAC04 & LE70270282012233EDC00 \\
\hline LT50270281993188XXX02 & LT50270282001194LGS01 & LT50270282006288PAC01 & LE70270282012249EDC00 \\
\hline LT50270281993220XXX02 & LT50270282001210LGS01 & & LE70270282012265EDC00 \\
\hline LT50270281993236XXX02 & LE70270282001218EDC00 & LE70270282007123EDC00 & \\
\hline LT50270281993268XXX02 & LT50270282001226LGS01 & LT50270282007131PAC01 & LE70270282013203EDC00 \\
\hline LT50270281993300XXX02 & LE70270282001234EDC00 & LE70270282007139EDC00 & LE70270282013235EDC00 \\
\hline & LT50270282001242LGS01 & LT50270282007147PAC01 & LE70270282013267EDC00 \\
\hline LT50270281994127XXX02 & LT50270282001274LGS01 & LE70270282007155EDC00 & \\
\hline LT50270281994159XXX03 & LT50270282001290LGS01 & LT50270282007163PAC01 & \\
\hline
\end{tabular}

